\title{
Interaktion und Interpretation in russischsprachigen Threads
}

\author{
Dissertation \\ zur Erlangung des philosophischen Doktorgrades an der \\ Philosophischen Fakultät der Georg-August-Universität \\ Göttingen
}

\author{
vorgelegt von \\ Genia Böhnisch (geb. Kapustina) aus Kiev
}

Göttingen 2018 
1. Gutachter: Prof. Dr. Uwe Junghanns

2. Gutachterin: Prof. em. Dr. Gerhild Zybatow

Tag der mündlichen Prüfung: 17.08.2018 


\section{$\underline{\text { Inhaltsverzeichnis }}$}

$\begin{array}{ll}\text { Vorwort } & 5\end{array}$

$\begin{array}{ll}\text { Danksagung } & 5\end{array}$

Glossar 6

$\begin{array}{ll}\text { Notationskonventionen } & 9\end{array}$

$\begin{array}{ll}\text { 1. Einleitung } & 10\end{array}$

2. Theoretische Ansätze 14

2.1 Ansatz der interaktionalen Soziolinguistik 14

2.2 Eigener theoretischer Ansatz 29

3. Kommunikation $\quad 40$

3.1 Kommunikation und ihre Merkmale 40

3.2 Konversationsverlauf 53

3.3 Turn Taking $\quad 82$

3.4 Schlussfolgerungen $\quad 86$

4. Der Weg zur korrekten Interpretation 89

4.1 Hintergrundwissen in Internetthreads $\quad 89$

4.2 Kontextualisierungshinweise in Internetthreads 97

4.2.1 Hinweise auf Prosodie 99

4.2.2 Farbliche Markierung 113

4.2.3 Hinweise auf Handlungen, Gestik und Mimik 114

4.2.3.1 Akronyme 114

4.2.3.2 Emoticons 115

4.2.3.3 Regieanweisungen $\quad 141$

$\begin{array}{ll}\text { 4.2.4 Schlussfolgerungen } & 148\end{array}$ 
5. Schritt-für-Schritt Diskussion eines authentischen Beispiels 154

6. Fazit

223

7. Bibliographie

233

8. Anhang

238 


\section{Vorwort}

Diese Dissertation ist in Folge meiner Beschäftigung mit der russischen Internetsprache im Rahmen meiner Masterarbeit entstanden. Während viele Phänomene der Internetsprache eine Untersuchung wert sind, weckten vor allem soziolinguistische Fragen mein Interesse. Über einen Verweis im HSK Band „Soziolinguistik“ stieß ich auf die Monographie von J. Gumperz, die schließlich die theoretische Basis dieser Dissertation wurde. Während Gumperz sich mit mündlicher Kommunikation beschäftigte, interessierten mich vor allem die Fragen, wie Kommunikation im Internet funktioniert und wie es trotz fehlender Signale, wie Intonation, Mimik oder Gestik, zur korrekten Interpretation schriftlicher Äußerungen kommt. Im Rahmen der vorliegenden Dissertation wurden diesen Fragen verschiedene Interaktionen aus zwei russischsprachigen Threads unterzogen.

\section{Danksagung}

Mein besonderer Dank gilt meinem Doktorvater Uwe Junghanns, der mir jederzeit mit gutem Rat zur Seite gestanden hat. Ohne seine Betreuung, Unterstützung und stete Hilfe wäre diese Dissertation nicht entstanden.

Ich danke ebenso allen Jungslavistinnen und Jungslavisten sowie den Teilnehmern des Kolloqiuums zur slavistischen Linguistik in Göttingen, die mich durch ihre Nachfragen, Anregungen und Kommentare immer weitergebracht haben.

Für die Teilnahme an meinen Experimenten möchte ich mich bei Elena Grimmig, Genriyeta Kapustina, Marianna Leonova, Olga Liebich und Margarita Tkatsch bedanken. Ohne ihre Bereitschaft, zu jeder Zeit Konversationsbeispiele vorzulesen und sich dabei aufzeichnen zu lassen, wären wichtige Teile dieser Arbeit nicht zu Stande gekommen.

Des Weiteren gebührt mein Dank meiner Familie, die mich immer unterstützt hat, hier insbesondere meiner Großmutter, die in mir das Interesse an Sprachen überhaupt erst geweckt hatte und meinem Großvater, der mich Durchhaltevermögen gelehrt hat. Ich danke auch meinem Ehemann für anregende Diskussionen bezüglich unterschiedlicher Aspekte dieser Dissertation. 


\section{Glossar}

Äußerung = Bestandteil einer Mitteilung; entspricht einer Äußerung in der mündlichen Kommunikation.

Beitrag $=$ Post bzw. Posting eines Schreibers. Entspricht dem Turn/Gesprächsschritt in der mündlichen Kommunikation, da er ebenfalls durch einen Partizipantenwechsel begrenzt ist. In der Threadkommunikation kann ein Beitrag aus mehreren thematisch unterschiedlichen Mitteilungen bestehen.

Chatgruppe $=$ Eine Gruppe, die sich in einem Chatraum unterhält. Der Chatraum kann sowohl privat, d.h. nur für ausgewählte Gesprächsteilnehmer, oder öffentlich, d.h. jeder registrierte Nutzer kann sich am Gespräch beteiligen, sein.

Emoticon $=$ Auch Smiley genannt, wobei das abgebildete Emoticon nicht immer ein Lachen darstellt. Die Foren haben dabei häufig eine Seite mit einer Auswahl an eigenen Emoticons, z.B. auf folgender Seite für das zweite Forum: http://forum.xakepok.net/misc.php?do=showsmilies.

Funktionen in Foren $=$ Die User eines Forums können Funktionen inne haben. Die typischerweise auftretenden sind:

- Moderator $=$ unterste hierarchische Funktion. Ein Moderator achtet auf die Einhaltung der Regeln in einem Subforum, Subsubforum und den dazugehörigen Threads; er kann Beiträge redigieren, löschen oder in andere thematisch passende Threads verschieben. Er kann Usern für Fehlverhalten Verwarnungen ausstellen oder bei sehr schweren Verstößen den (die) Administrator(en) darum bitten, diese des Forums zu verweisen.

- Supermoderator $=$ hierarchische Funktion über der des Moderators. Bei einem Supermoderator handelt es sich um einen Moderator, der jedoch das gesamte Forum mit all seinen Subforen überwacht. Gleichzeitig beaufsichtigt er die Tätigkeit der Moderatoren.

- Administrator $=$ höchste hierarchische Funktion. Der Administrator ist dafür zuständig, dass das Forum von der technischen Seite her funktioniert. $\mathrm{Zu}$ seinen Aufgaben zählen auch die Anlegung von Subforen sowie die Ernennung der Supermoderatoren und Moderatoren, deren Arbeit er beaufsichtigt. Bei dem 
Administrator können sich sowohl User über Moderatoren bzw. Supermoderatoren beschweren als auch umgekehrt. Ein Administrator kann die Moderatoren bzw. Supermoderatoren ihres Postens entheben, aber auch User des Forums verweisen.

Gesprächsteilnehmer/-partizipant $=$ In dieser Dissertation sind damit Personen gemeint, die sich aktiv am Gespräch beteiligen, indem sie Beiträge zur Konversation verfassen.

Regieanweisung $=$ Mit ihnen gibt der Schreiber meist parallel zur Äußerung ausgeführte Handlungen bzw. die Art und Weise, wie eine Äußerung realisiert werden soll, an. In der Regel werden Regieanweisungen von Schreibern mit Asterisken * eingerahmt und so von der vorhergehenden und/oder folgenden Äußerung abgesetzt.

Internetforum $=$ Ein Ort zum Austausch von Gedanken oder Informationen zu einem Thema; besteht aus verschiedenen Subforen, die thematisch von einander getrennt sind und wiederum Subsubforen, die aus Threads bestehen, bilden. Exemplarisch am zweiten Forum (http://forum.xakepok.net/main.php) dargestellt: XaKeRoK = Internetforum; z.B. News = Subforum; IT-Новости, O Xakepok.su = Subsubforen; Прекращается основная поддержка Windows 7 = Thread aus dem Subsubforum „IT-Новости“.

Konventionen $=$ Verhaltensweisen bzw. Merkmale der Kommunikation, die implizit bleiben, aber allen Usern des jeweiligen Forums bewusst sind und von diesen als gültig angenommen werden.

Konversation $=$ Interaktion von Teilnehmern auf sprachlicher Ebene. In der vorliegenden Dissertation auch nur mit Interaktion bezeichnet.

Leser $=$ Derjenige, der einen Beitrag liest; entspricht in schriftlich realisierten Gesprächen dem Hörer in mündlichen Gesprächen. Nicht jeder Leser ist derjenige, an den die Äußerung gerichtet ist oder derjenige, der auf den Beitrag des Schreibers reagiert.

Mitteilung $=$ Thematisch zusammenhängender Bestandteil eines Beitrags, der sich aus mindestens einer Äußerung zusammensetzt.

Reaktion $=$ Reaktion des Hörers/Lesers auf die Mitteilung oder Äußerung des Sprechers/Schreibers in sprachlicher oder außersprachlicher Gestalt (z.B. durch Gestik oder Mimik). 
Regel $=$ Verhaltensvorschriften, die in den Foren meistens auf einer eigenen Seite aufgeführt werden. Durch Regeln wird festgelegt, welche Verhaltensweisen in dem jeweiligen Forum unzulässig sind.

Schreiber $=$ Derjenige, der einen Beitrag verfasst; entspricht in schriftlich realisierten Gesprächen dem Sprecher in mündlichen Gesprächen.

Thread $=$ Eine Abfolge von Beiträgen $\mathrm{zu}$ einem bestimmten Thema. In der vorliegenden Dissertation werden die beiden Threads „obo vsem i ni o čem“ (Forum 1) bzw. „flud vetka“ (Forum 2) behandelt, die kein von vornherein festgelegtes Thema aufweisen.

Zitat $=$ Mit Hilfe der Zitierfunktion bezieht sich der aktuelle Schreiber explizit auf mindestens eine Äußerung eines vorherigen Schreibers. Dies ist vor allem dann hilfreich, wenn die Äußerung(en), auf die sich der aktuelle Schreiber bezieht, länger zurückliegen. 


\section{Notationskonventionen}

Da die Transkriptionskonventionen, die für die Transkription mündlicher Konversationen gebraucht werden, vor allem lautliche Aspekte notieren (vgl. Levinson (2000, 403)), können sie für schriftlich realisierte Konversationen nicht verwendet werden. Daher wurden für die vorliegende Dissertation eigene Notationskonventionen angelegt, die alle hier wesentlichen Erscheinungen umfassen sollen:

$\mathbf{B}=$ Beitrag

$\mathbf{T}=$ Thema

non-T = explizit non-thematische Mitteilung, die keinen einschlägigen lexikalischen Inhalt hat, aber dennoch eine bestimmte Funktion, z.B. Trolling, erfüllt

$\mathbf{M}=$ Mitteilung

$\mathbf{R}=$ Reaktion (wird nur angegeben, wenn es sich nicht auf die Mitteilung davor bzw. nur auf Teile von ihr bezieht)

$\mathbf{R}_{\mathbf{v}}=$ Reaktion auf vorangehende Mitteilung/Äußerung

SK $=$ Selbstkohärenz

$\boldsymbol{\alpha}=$ Grußformel $^{1}$ (der Referenzindex i gibt dabei an, dass ein Gegengruß erfolgt)

$\boldsymbol{\omega}=$ Abschiedsformel (der Referenzindex i gibt dabei an, dass ein Gegenabschied erfolgt)

$\mathbf{a}=$ direkt adressiert (nur bei Bezeichnungen, die nicht eindeutig als Kosenamen oder Nicknames erkennbar sind)

Import $=$ importiertes Element

/ / = Kontextualisierungshinweise ${ }^{2}$

Die Themen, Mitteilungen und Äußerungen sind dabei zu Referenzzwecken durchgezählt.

\footnotetext{
${ }^{1}$ Gruß- und Abschiedsformel entsprechen den gesprächseröffnenden und gesprächsabschließenden Elementen bei Levinson (2000, 336-345).

${ }^{2}$ Hiermit werden alle potenziellen Kontextualisierungshinweise markiert, die in der vorliegenden Dissertation näher betrachtet werden.
} 


\section{Einleitung}

Die vorliegende empirisch angelegte Dissertation beschäftigt sich mit der Kommunikation in russischsprachigen Threads - einer natürlichen Kommunikation, die, wie andere Arten der Internetkommunikation auch, ein Hybridwesen zwischen geschriebener und gesprochener Sprache darstellt ${ }^{3}$. Gerade auf Grund der Beliebtheit der Internetkommunikation, die ihren Platz neben mündlicher und „klassischer“ schriftlicher Kommunikation, z.B. in Form von Briefen, einnimmt, lohnt sich eine genaue Untersuchung. Im Gegensatz $\mathrm{zu}$ anderen wissenschaftlichen Arbeiten, die sich überwiegend mit Chatraum-Kommunikation beschäftigen, wird in der vorliegenden Dissertation ausschließlich die Kommunikation in Foren analysiert. Dabei werden die Threads Obo vsem i ni o čem (in dem Subsubsubforum Boltalka) und Flud vetka (im Subsubforum Kurilka) - für russischsprachige Foren typische Plauderthreads innerhalb des off-topic-Bereichs - untersucht. Solche Threads stellen eine Besonderheit dar, da sie keiner von vornherein festgelegten Thematik oder Fragestellung folgen wie dies ansonsten in Foren üblich ist. Stattdessen können User über alles schreiben, was sie im Augenblick des Verfassens ihres Beitrags bewegt. Im Rahmen der vorliegenden Dissertation wird diese informelle Threadkommunikation mit der Kommunikation in informellen mündlichen Gesprächen verglichen.

Um an sprachliche Daten zu kommen, wurden zur Soziolinguistik ${ }^{4}$ gehörende Methoden angewandt, zu denen im Allgemeinen Interviews, Umfragen ${ }^{5}$, Gesprächsaufnahmen in Geschäften, Institutionen und die Durchführung von Tests gehören ${ }^{6}{ }^{7}$ Das Hauptproblem bei der Anwendung dieser Methoden ist das sog. Beobachterparadoxon - ein Begriff, der von William Labov, einem der Begründer der modernen Soziolinguistik, eingeführt wurde. „Этот парадокс сводиться к следующему: целью исследования является выяснение того, как

\footnotetext{
${ }^{3}$ Vgl. Baron $(2010,5)$.

4 „Социальная лингвистика (социолингвистика) - направление языкознания, изучающее общественную обусловленность возникновения, развития и функционирования языка, воздействие общества на язык и языка на общество.“ (Bondaletov $(1987,10)$ ). Allgemein beschäftigt sich die Soziolinguistik mit der Sprache in Abhängigkeit von sozialen Faktoren, wie z.B. Geschlecht, Alter, Herkunft, ethnische Zugehörigkeit, Beruf oder Bildungshintergrund. Im russischsprachigen Raum lag und liegt das Interesse der Soziolinguistik vor allem in der Arbeit mit Varietäten, zu denen die literaturnaja reč, die razgovornaja reč, das Prostorečie und die Dialekte zählen. Für genaue Informationen über die Varietäten und Dialekte siehe Gutschmidt (2006, 1854-1857). Zur Geschichte der sowjetischen Soziolinguistik siehe Gutschmidt (2006, 1852f.).

${ }_{6}^{5}$ Ein Beispiel für ein russischsprachiges Umfrageformular findet sich bei Krysin \& Belikov (2001, 289f.).

6 „Это могут быть списки слов, которые надо прочитать перед микрофоном, или связный текст, также читаемый информантом вслух; письменные задания на восстановление пропущенных фрагментов текста; ответы на устные вопросы исследователя, задаваемые в определенном временном режиме, и т.п.“ (Krysin \& Belikov (2001, 303)).

${ }^{7}$ Weiterhin können sprachliche Daten aus schriftlichen Quellen wie Tagebüchern, Briefwechsel usw. gewonnen werden (vgl. Krysin \& Belikov (2001, 312-317)).
} 
говорят люди, когда они не являются объектом систематического наблюдения; однако чтобы получить эти данные, необходимо систематическое наблюдение. Дело в том, что систематическое наблюдение само по себе создает речевую ситуацию, влияющую на стилевой регистр, побуждающую информанта стремиться к „корректности“ речи и стимулирующую значительно большую ориентацию на литературный стандарт, чем ту, которая характерна для повседневно-бытового общения.“ (Švejcer $(1977,158)$ ). Dieses sowie eine Vielzahl anderer Problem treten bei einigen Methoden deutlicher zum Vorschein als bei anderen. So wie bei der Methode der Umfrage, die trotz ihres Vorteils, von möglichst vielen Probanden die Daten zu sammeln und damit repräsentativ zu sein, zum Einen eine ,,[...] опасность приспособления опрашиваемого к нормам и ожиданиям опрашиваюшего.“ (Švejcer $(1977,158)$ ) beinhaltet und zum Anderen eine reale Gesprächssituation nicht ersetzen kann. ${ }^{8}$ Das Problem des Beobachterparadoxons besteht auch bei Interviews und Gesprächsaufnahmen. In der Soziolinguistik versucht man das Problem bei den Gesprächsaufnahmen auf mehrere Weisen zu umgehen: entweder die aufgezeichneten Personen sind sich der Aufnahme nicht bewusst ${ }^{9}$, sind an die Aufnahmen gewöhnt, weil sie lange genug durchgeführt werden ${ }^{10}$, oder der jeweilige wissenschaftliche Beobachter nimmt an dem Gespräch mit den aufgezeichneten Personen teil ${ }^{11}$. Die aus den Threads heraus

\footnotetext{
${ }^{8}$ Es gab jedoch Versuche über andere Arten der Umfragen das Problem zu umgehen. So benutzte z.B. eine Forschergruppe in der Sowjetunion zwei getrennte Fragebögen: ein Fragebogen fragte nach bestimmten sozialen Faktoren (Alter, Herkunft, Bildung, Wohnsitz, sozialer Status usw.) und ein anderer beinhaltete Fragen über sprachliche Varianten, z.B. in welchem Wort man einen Konsonanten weicher ausspricht (vgl. Švejcer (1977, 159f.)).

${ }^{9}$ Vgl. die Studien von W. Labov, die seit den 1960ern in nordamerikanischen Großstädten, bevorzugt New York und Philadelphia, durchgeführt wurden. Labov ging es vor allem darum, soziale Gruppen und ihre sprachlichen Variablen aufzudecken. So untersuchte er in einer seiner frühesten Studien die Benutzung des /r/: „The hypothesis that postvocalic /r/ usage would be correlated with social class was tested in an experiment rather more amusing than many linguistic investigations - by examinig the speech of shop assistants in three different department stores, of high, medium and low status respectively. The procedure was to find out which departments were on the fourth floor and then to ask as many assistants as possible in the rest of the shop a question like, 'Excuse me, where are the women's shoes?' The answer to this would be fourth floor - with two possible occurrences of postvocalic /r/. In this way information on /r/-usage was obtained from 264 informants (who did not know, of course, that they were being interviewed by a linguist). The results were: 38 per cent of the high-ranking store assistants used no /r/, 49 per cent in the middle store and 83 per cent in the low-ranking store." (Trudgill $(1974,48)$ ). Labovs Studien waren so erfolgreich, dass die Methoden seitdem auch auf andere Sprachen angewandt werden. Ein Beispiel aus dem russischsprachigen Raum ist die Untersuchung von Glovinskaja (1976, 100-122) zur „Variativnost“ proiznošenija zaimstvovannych slov v sovremennom russkom jazyke.", in der die Aussprache harter Konsonanten vor э, langer Konsonanten sowie die Aussprache vom unbetonten $o$ in Fremdwörtern bei Probanden unterschiedlicher Herkunft, Bildung und unterschiedlichen sozialen Status sowie Alters untersucht wurde.

${ }^{10}$ Vgl. hierzu eine Studie von Labov, Cohen, Robins und Lewis aus dem Jahr 1968, bei der die Partizipanten sich nach einiger Zeit daran gewöhnt hatten, aufgezeichnet zu werden, und so sprachen, wie sie es tagtäglich taten, anstatt sich zu verstellen.

${ }^{11}$ Vgl. hierfür die Aufzeichnungen von Zemskaja und Anderen für die Russkaja razgovornaja reč ‘, in denen die Informanten u.a. Bekannte und Freunde der Wissenschaftler waren und in einer gewohnten Umgebung, z.B. zu Hause, aufgenommen wurden (vgl. Švejcer $(1977,160)$ ).
} 
kopierten und aufbereiteten Konversationsabschnitte entsprechen der soziolinguistischen Methode der Aufnahme. Bei der Sammlung der sprachlichen Daten für die vorliegende Dissertation wurde das Problem des Beobachterparadoxons umgangen, indem nur sprachliches Material von öffentlich einsehbaren Threads, die bereits vor einiger Zeit angefangen wurden und teils mehrere Jahr alt waren, verwendet wurde. Auf diese Weise konnte sichergestellt werden, dass sich die Schreiber einer Beobachtung nicht bewusst waren und ihren Kommunikationsstil in den Threads nicht änderten. Gleichzeitig wurden keine Daten verwendet, für deren Veröffentlichung man sich von jedem Schreiber eine Erlaubnis hätte einholen müssen.

In der vorliegenden Arbeit werden erstmals die Ansätze der interaktionalen Soziolinguistik auf die Kommunikation in Internetthreads angewendet. Entsprechend liegen die Schwerpunkte dieser Arbeit auf folgenden Fragen: Wie funktioniert Kommunikation im Internet? Welche Merkmale sind charakterisch für sie? Wie laufen Konversationen ab? Wie kommen Interpretationen von schriftlich realisierten Äußerungen zu Stande, wenn lautliche und visuelle Aspekte einer Interaktion fehlen? Erfolgt immer eine korrekte Interpretation von Äußerungen oder kommt es zu Missverständnissen? Wenn es zu Missverständnissen kommt, welche Ursachen liegen ihnen zu Grunde? Dabei geht es bei der Frage wie es zur korrekten Interpretation kommt, nicht um kognitionslinguistische Prozesse, sondern darum, welcher Mittel sich der Leser bedient, um die Äußerungen des Schreibers in einer rein schriftlich realisierten Kommunikation zu verstehen.

Auf Grundlage dieser Fragen sind die zu überprüfenden Thesen:

1. schriftlich realisierte Kommunikation in Forenthreads besitzt eigene Merkmale, die sie von mündlicher Kommunikation unterscheidet;

2. da lautliche Informationen aus dem Sprachsignal nicht zur Verfügung stehen und der Leser den Schreiber nicht sehen kann, müssen sich beide anderer Kontextualisierungshinweise als in mündlicher Kommuniaktion bedienen;

3. für eine korrekte Interpretation spielen lexikalische Hinweise und solche Kontextualisierungshinweise eine Rolle, die direkt an der Wortform wahrnehmbar sind;

4. trotz des Fehlens lautlicher Aspekte (Prosodie, paralinguistische Hinweise) sowie der Mimik und Gestik können Äußerungen korrekt interpretiert werden.

Entsprechend der oben genannten Fragen und der zu überprüfenden Thesen gliedert sich die 
vorliegende Dissertation in folgende Abschnitte:

Kapitel 2 behandelt im ersten Unterkapitel theoretische Ansätze der Interaktionalen Soziolinguistik zur Frage der Interpretation von Äußerungen in mündlichen Gesprächen. Auf ihrer Grundlage werden in einem weiteren Unterkapitel eigene theoretische Überlegungen bezüglich der Interpretation schriftlich realisierter Äußerungen vorgestellt. Abgeschlossen wird es mit einer vorläufigen graphischen Darstellung des Interpretationsverlaufs.

Kapitel 3 widmet sich den Kommunikationsmerkmalen und Verläufen in mündlichen Konversationen und Konversationen in den im Rahmen der Dissertation untersuchten Forenthreads im Internet. Im ersten Unterkapitel werden die Merkmale der mündlichen Kommunikation vorgestellt und anhand mehrerer Beispiele Merkmale der Internetkommunikation in Threads herausgearbeitet. Die beiden weiteren Unterkapitel behandeln den Konversationsverlauf und den Partizipantenwechsel in den untersuchten Threads im Vergleich zu mündlichen Konversationen.

Kapitel 4 untersucht die Rolle des Hintergrundwissens und der Kontextualisierungshinweise bei der Interpretation schriftlich realisierter Äußerungen. Nachdem im ersten Unterkapitel die Bedeutung von Hintergrundwissen anhand mehrerer Beispiele untersucht wird, beschäftigen sich die weiteren Unterkapitel mit der Frage, welche potentiellen Kontextualisierungshinweise in der Threadkommunikation vorkommen können und ob sie tatsächlich als Kontextualisierungshinweise fungieren. Dies wird mit Hilfe mehrerer Versuche untersucht. Das Kapitel schließt mit der Überarbeitung der eigenen theoretischen Ansätze sowie einer graphischen Darstellung des Interpretationsverlaufs ab.

In Kapitel 5 wird anhand eines längeren Beispiels der vorgestellte Interpretationsverlauf untersucht. Dabei wird jede Äußerung einzeln analysiert.

Das letzte Kapitel 6 fasst die Dissertation sowie die Ergebnisse zusammen und bietet einen Ausblick auf weitere mögliche Forschungsfelder und -fragen.

Im Anschluss folgen die Bibliographie sowie der Anhang, der alle im Rahmen dieser Dissertation diskutierten Beispiele beinhaltet. Die Beispiele folgen der Reihenfolge ihres Auftauchens in den jeweiligen Kapiteln: (7) - (25) aus Kapitel 3, (26) - (47) aus Kapitel 4 und (48) aus Kapitel 5. 


\section{Theoretische Ansätze}

Im folgenden Kapitel werden zunächst die theoretischen Grundlagen vorgestellt. In 2.1 wird auf die interaktionale Soziolinguistik eingegangen, bevor in 2.2 ein eigener theoretischer Ansatz präsentiert wird.

\section{$\underline{\text { 2.1 Ansatz der interaktionalen Soziolinguistik }}$}

Die interaktionale Soziolinguistik ${ }^{12}$, die u.a. von John Gumperz begründet wurde ${ }^{13}$, kombiniert den Ansatz und Methoden der Soziolinguistik mit denen der Diskursanalyse und Konversations- bzw. Gesprächsanalyse. ${ }^{14}$ Der Ansatz bedient sich dabei ethnographischer Methoden $^{15}$, um die sprachlichen Daten $\mathrm{zu}$ untersuchen. Entsprechend einer der

${ }^{12}$ Die Interaktionale Soziolinguistik wird auch mit dem Begriff Interpretative Soziolinguistik bezeichnet (vgl. Glück (1993, 278)). Im weiteren Verlauf der Dissertation wird der Begriff „Interaktionale Soziolinguistik“ jedoch beibehalten.

${ }^{13}$ Eine weitere, für die Entwicklung der Theorie sehr wichtige Person ist der Soziologe Erving Goffman, der sich aus soziologischer Sicht mit dem Thema der zwischenmenschlichen Interaktion beschäftigte.

${ }^{14}$ Häufig werden die Begriffe „Diskursanalyse“ und „Konversationsanalyse“ synonym verwendet. Dass sie jedoch nicht 1:1 synonym sind, zeigen ihre Definitionen. Diskursanalyse wird bei Bußmann folgendermaßen definiert: „Aus der angloamerikan. Forschung übernommener Oberbegriff für die Analyse von Diskurs [...], der weitgehend synonym verwendet wird für «Textanalyse». Innerhalb der D. sind zwei Richtungen zu unterscheiden: (1) Angeregt durch eher formale linguistische Theorie und Verfahren (Formale Logik, Sprechakttheorie, Strukturalismus, Transformationsgrammatik) ist die Richtung primär an Wohlgeformtheit (Kohärenz, Kohäsion) und an deduktiven Regeln z.B. für Sprechakte interessiert; ihre Vertreter betrachten den Text weitgehend als statisches Produkt. (2) Beeinflusst insbesondere durch funktionale Sprachmodelle wie Funktionale Grammatik, durch Psycholinguistik, Soziolinguistik - insbes. durch Ethnographie des Sprechens und Konversationsanalyse - sowie durch Verfahren der Kognitionswissenschaft betont die andere Richtung den Charakter von Diskursen als Konstruktions- und Interpretationsprozesse durch Sprecher und Hörer, durch Schreiber und Leser. [...].“ (Bußmann $(2008,142))$. Bei Bußmann wird Konversationsanalyse folgendermaßen definiert: „Aus der Ethnomethodologie entwickelte empirische Forschungsrichtung, die sich mit der Analyse von Gesprächen beschäftigt. [...] Die Diskursanalyse stärker beeinflusst haben spätere Arbeiten zur Geordnetheit sozialer Ereignisse, die sich mit wiederkehrenden Mustern und ihren strukturellen Eigenschaften in der Organisation von Gesprächen beschäftigen; dabei gilt als dominantes und effektivstes Mittel die Organisation von »Turn« zu »Turn«, an der alle Parteien beteiligt sind. In der Weise, wie die Beteiligten die »Turns« handhaben, zeigen sie einander auf, wie sie die sie entwickelnden Aktivitäten verstehen, wie sie den vorangegangenen »Turn« und die entsprechenden Erwartungen der Partner interpretieren und welche Erwartungen sie selbst in Bezug auf den Nächsten [sic!] »Turn« haben [...]. Besonderes Interesse der K. gilt Phänomenen aus Syntax und Prosodie [...].“ (Bußmann $(2008,373))$.

${ }^{15}$ Bei ethnographischen Methoden handelt es sich um Verfahren der Ethnographie des Sprechens. „Von DELL HYMES und JOHN GUMPERZ seit Anfang der 60er Jahre eingeführte Verfahren zur Analyse des Spachgebrauchs in soziokulturellen Zusammenhängen. Im Unterschied zu linguistischen Theorie aus dem Umkreis der generativen Transformationsgrammatik geht die E. davon aus, dass die Bedeutung von Äußerungen nur in Beziehung zum jeweiligen Sprechereignis oder kommunikativen Ereignis (engl. speech event) erfasst werden kann, in das sie eingebettet sind. Solche Sprecherereignisse (z.B. >Predigt<, >Gerichtsverhandlung $>$ Telefongespräch $\measuredangle$ ) sind kulturspezifisch ausgeprägt. - Regeln des Sprachgebrauchs werden durch teilnehmende Beobachtung, durch Analyse von Spontandaten und Interviews von Muttersprachlern erfasst, vgl. Feldforschung. - Aus der E. hat sich die ethnographische Diskursanalyse entwickelt, in der konversationelle Schlussfolgerungen (engl. conversational inference) eine zentrale Rolle spielen. Diese Schlußfolgerungen sind kontextgebundene Interpretationsprozesse, in denen die Teilnehmer den Inhalt einer Äusserung und andere verbale, vokale sowie 
soziolinguistischen Methoden wird eine reale Gesprächssituation aufgezeichnet und im Ganzen, d.h. Äußerungen samt Kontext und nicht einzelne Sätze, analysiert. ${ }^{16}$ Die von Gumperz angenommene Erweiterung der soziolinguistischen Theorie und Methoden gründet darauf, dass Soziolinguistik alleine nicht ausreicht, um die Sprache in einer Sprechergruppe $\mathrm{zu}$ beschreiben, da auf Grund immer weniger deutlicher sozialer Grenzen, die ,[...] assumption that speech communities, defined as functionally integrated social systems with shared norms of evaluation, can actually be isolated thus becomes subject to serious question.“ (Gumperz (1982, 26)). Vielmehr sei ein neuer, erweiterter Ansatz nötig, der losgelöst von sozialen Faktoren und Generalisierungen agiert und dabei dennoch alle sprachlichen Varietäten (linguistic diversity) miteinschließt. „Linguistic diversity serves as a communicative resource in everyday life in that conversationalists rely on their knowledge and their stereotypes about variant ways of speaking to categorize events, infer intent and derive expectations about what is likely to ensue. All this information is crucial to the maintenance of conversational involvement and to the success of persuasive strategies. By posing the issue in this way, one can avoid the dilemma inherent in traditional approaches to sociolinguistics, where social phenomena are seen as generalizations about groups previously isolated by nonlinguistic criteria such as residence, class, occupation, ethnicity and the like, and are then used to explain individual behavior. We hope to be able to find a way of dealing with what are ordinarily called sociolinguistic phenomena which builds on empirical evidence of conversational cooperation and does not rely on a priori identification of social categories by extending the traditional linguistic methods of in-depth and recursive hypothesis testing with key informants to the analysis of the interactive processes by which participants negotiate interpretations.“ (Gumperz $(1982,130)$ ). In den Mittelpunkt dieses Ansatzes rückt

nonvokale Hinweise mit Hintergrundwissen verbinden (Kontextualisierung), um den spezifischen Austausch zu verstehen. [...].“ (Bußmann $(2008,176))$.

${ }^{16}$ Die bevorzugte Analyse ist die von Sequenzen und ihrer sequentiellen Organisation. Dabei bezeichnet die sequentielle Organisation „In der Konversationsanalyse [die] Strukturierung von Gesprächen durch Typen von Handlungsfolgen, die von verschiedenen Sprechern produziert werden. Es wird davon ausgegangen, dass sich das, was mit einer Äußerung getan wird, aus ihrer Platzierung in einer Sequenz ergibt, d.h. innerhalb einer Sequenz realisiert eine Äußerung eine der möglichen Folgehandlungen, die in einem vorangegangenen Turn aufgerufen wurden, und lässt selbst wiederum im nächsten Turn eine Handlung aus einer eingegrenzten Menge von möglichen Folgehandlungen erwarten. Auf diese Weise zeigen die Gesprächsteilnehmer sich wechselseitig auf, wie sie den jeweils vorangegangenen Turn verstanden haben. Zu solchen Sequenztypen zählen Paarsequenzen, in denen die Wahl einer ersten Handlung eine bestimmte zweite im folgenden Turn implikatiert (z.B. wird auf eine Frage eine Antwort erwartet [...]), oder Sequenzen mit präferierten Optionen (etwa Einladungs-Zusage statt -Absage [...]). Weitere Evidenz für die S.O. bieten Ausdrücke, die Abweichungen von erwartbaren Folgehandlungen in einer Sequenz markieren (z.B. die Verwendung von Diskursmarkern wie übrigens, die dem Hörer ein vom gegenwärtigen Gesprächsthema abweichendes Thema anzeigen). Gestützt wird die S.O. durch die »lokale« Handhabung des Sprecherwechsels [...]. Analysiert werden daher nicht isolierte Äußerungen, sondern Äußerungen in Sequenzen. Dieses Vorgehen unterscheidet den Ansatz der Konversationsanalyse von dem der Sprechakttheorie. [...]“. (Bußmann $(2008,622))$. 
somit nicht mehr die Sprache und Kommunikation einer Gruppe mit gleichen Normen und Werten, sondern die des Individuums, das vorher nicht nach bestimmten Kriterien ausgesucht wurde, während einer Interaktion. ${ }^{17}$ „Интеракционистский подход предполагает переход с макроуровня на микроуровень социолингвистического анализа: внимание сосредоточивается на межличностном общении, на анализе непосредственно самого процесса разговора (диалога).“ (Vahtin \& Golovko (2004, 226)). Dabei geht es allgemein um die Frage, wie mündliche Kommunikation zwischen Gesprächspartnern abläuft und was die Gründe dafür sind, dass sie gelingt bzw. misslingt. Es geht um Strategien der Gesprächspartner, um Konversationsverläufe ${ }^{18}$ und um die Frage, wie der Hörer zur korrekten Interpretation des Gesagten kommt.

Gumperz' Theorie fußt auf mehreren Grundideen. Eine seiner Grundannahmen ist, dass jeglicher Sprechvorgang als eine Interaktion ${ }^{19}$ aufgefasst wird, die aus einem gegenseitigen Aufeinandereinwirken und Beeinflussen zweier Akteure besteht. Eine weitere Grundannahme bildet die Vorstellung von der conversational inference: „Conversational inference [...] is the situated or contextbound process of interpretation, by means of which participants in an exchange assess others' intentions, and on which they base their responses. [...]

\footnotetext{
${ }^{17}$ Laut Gumperz ermöglicht dieser Ansatz, verschiedene Kommunikationsprozesse zu verstehen, die man mit den soziolinguistischen Theorie und Methoden alleine nicht verstehen bzw. untersuchen könnte. „For example, an ethnographic study of language behavior in what on the surface seemed like a relatively homogenous, isolated and therefore presumably stable Norwegian community revealed fundamental differences in social values among individual residents, all of whom were born und bred in the locality. It was this difference in values which might not have been discovered if sharing of norms had been taken for granted, and which was revealed only through in depth ethnographic work, that served to explain the basic facts of language usage in the community. All residents spoke both the local dialect and a regional variant of Bokmål, one of the two accepted forms of standard Norwegian. However, all speakers differed with respect to where and for what communicative goals they chose among the two codes. What was normal usage for some in some situations counted as marked for others. Marked forms, moreover, tended to be used to convey indirect inferences which could only be understood by someone who knew both the speaker's family background and his or her position within the local spectrum of value orientations. Language usage in situations such as these is thus not simply a matter of conforming to norms of appropriateness, but is a way of conveying information about values, beliefs and attitudes that must first be discovered through ethnographic investigation, and that in everyday situations define the underlying assumptions with respect to which participants infer what is intended (Blom \& Gumperz 1972).“ (Gumperz (1982, 26f.)).

${ }^{18}$ Wie Konversationsverläufe von ihrer Struktur her aussehen, wurde und wird weiterhin mit Hilfe der Konversationsanalyse aufgezeigt. Dabei hat die Konversationsanalyse als Methode nach Meinung von Gumperz (1982, 159) viel zeigen können: „Conversational analysis over the last few years has demonstrated beyond question that not only formally distinct speech events but all kinds of casual talk are rule governed. It is through talking that one establishes the conditions that make an intended interpretation possible. Thus to end a conversation, one must prepare the ground for an ending; otherwise, the ending is likely to be misunderstood. Or to interpret an answer, one must be able to identify the question to which that answer is related. To understand a pun, one must be able to retrieve, re-examine and reinterpret sequences that occured earlier in an interaction. Sequentiality, i.e. the order in which information is introduced and the positioning or locating of a message in the stream of talk, is clearly of great importance in interpreting daily conversation. The mechanisms which underlie speaker-listener coordination can be studied empirically by examining recurrent strategies, the responses they elicit, and the ways in which they are modified as a result of those responses."

${ }^{19}$, ,[...] speaking is interacting [...]“" (Gumperz $\left.(1982,29)\right)$.
} 
Conversational inference is part of the very act of conversing. One indirectly or implicitly indicates how an utterance is to be interpreted and illustrates how one has interpreted another's utterance through verbal and nonverbal responses, and it is the nature of these responses rather than the independently determined meaning or truth value of individual utterances alone that governs evaluation of intent.“ (Gumperz (1982, 153f.)). Das Bestehen einer Konversation aus Äußerungen und Reaktionen zeugt von dem grundsätzlichen Wunsch nach einer konversationellen Kooperation ${ }^{20}$. „One common way in which conversational cooperation is communicated and monitored by participants is through what Yngve (1970) calls "back channel signals": interjections such as, "O.K.," "right," "aha," "21 or nods or other body movements. Other signs of cooperation are implied indirectly in the way speakers formulate responses, i.e. in whether they follow shifts in style, agree in distinguishing new from old or primary from secondary information, or in judging the quality of interpersonal relationships implied in a message, and know how to fill in what is implied but left unsaid or what to emphasize or de-emphazise.“ (Gumperz (1982, 163f.)). Die Reaktion als Bestandteil der Kooperation bezieht sich darauf, wie der Hörer die Nachricht versteht (vgl. Gumperz (1982, 1)), vgl. dazu folgendes Beispiel (Gumperz (1982, 1)):

(1) Person A: Are you gonna be here for ten minutes?

Person B: Go ahead and take your break. Take longer if you want to.

Person A: I'll just be outside on the porch. Call me if you need me.

Person B: O.K., don’t worry.

Das Gespräch findet in einem Büro statt. Die beiden Personen kennen sich, sind höchstwahrscheinlich Kollegen. Person B interpretiert die Frage von Person A so, dass sie indirekt fragt, ob sie eine Pause machen kann. Person B greift dabei auf Hintergrundwissen über die Umgebung (Büro), die Umstände des Gesprächs, die Person A sowie Konventionen in einer bestimmten Gesprächssituation zurück. Einen Bestandteil dieses Hintergrundwissens

\footnotetext{
20 „Conversational cooperation [...] is always cooperation for some purpose, which means that participants must have at least some idea of the likely outcomes before they commit themselves to an interaction. Where potential outcomes are not agreed upon in advance they must be negotiated through talk. Information about interactive goals, therefore, has to be conveyed before enough has transpired to make a sequential description possible." (Gumperz $(1982,165))$.

21 Diese werden auch als Diskursmarker bezeichnet (vgl. Schiffrin (1987)). Diskursmarker (u.a. auch Diskurspartikeln genannt) sind „,...] sequentially dependent elements, which bracket units of talk.“ (Schiffrin $(1987,31) . \mathrm{Ihr},[. .$.$] Vorkommen [dient] der Organisation, Gliederung, Strukturierung vor allem gesprochener$ Sprache [...]“ (Glück $(1993,146))$, , [...] z.B. zeigen sie den Beginn einer neuen Diskurseinheit an (etwa mit übrigens in Übrigens, Lisa kommt zum Essen.) oder sie schaffen Übergänge zwischen zwei Einheiten (etwa mit einer Variante von also zu Beginn einer Wegbeschreibung in Also - da gehen Sie gleich hier rechts ...).“" (Bußmann (2008, 143)).
} 
bildet das Wissen, dass Pausen während der Arbeit gängig sind. Daraus schließt Person B, dass Person A eine Pause machen möchte. Anstatt auf die Frage von Person A direkt mit ,ja“ oder „nein“ zu antworten (obwohl es sich eindeutig um eine Ja-/Nein-Frage handelt), sagt Person B, dass Person A eine Pause machen kann und zeigt Person A damit, dass sie die Frage als einen Wunsch nach einer Pause interpretiert hat. Mit der Antwort implikatiert sie gleichzeitig, dass sie noch mindestens 10 Minuten anwesend sein wird. Dass es sich offenbar um die richtige Interpretation handelt, zeigt die Äußerung von Person A im nächsten Turn, mit der sie indirekt bestätigt, jetzt tatsächlich eine Pause machen zu wollen. Obwohl beide Gesprächsteilnehmer nicht direkt auf die Äußerungen des jeweils Anderen reagieren, gibt es keine Verständnisschwierigkeiten. Das führt zu der Frage, woran das liegt. Woher weiß Person B als Hörer wie sie die Äußerung zu verstehen hat und was die korrekte Reaktion ist? Wie kommt sie zu den entsprechenden Schlussfolgerungen?

Zunächst muss noch einmal wiederholt werden, dass Gumperz die Interpretation als einen kontextgebundenen Prozess versteht: „In conversations, we must continually make judgements at simultaneous levels of meaning, through an inferential process which both interprets what has been said and generates expectations about what is to come. The process is always situated or context bound. It begins with informed guessing based on what we know about the physical setting, the participants and their backgrounds, and how we relate the situation at hand to other known activities. These initial hypotheses are subject to constant modification by our perception of information signalled in both the form and the content of speech.“ (Gumperz (1982, 101)). Dieser Prozess ermöglicht uns als Hörer, die Interpretation im Laufe des Gesprächs zu verändern; die Interpretation ist also nichts Statisches, sondern wird immer wieder mit neuen Informationen koordiniert und auf ihrer Grundlage verändert. Diese Informationen bestehen u.a. aus eng miteinander verwobenen Faktoren, die beim Zustandekommen einer korrekten Interpretation als Hilfestellungen unerlässlich sind: geteilte Konventionen, zu denen u.a. das Wissen um den activity type, $\mathrm{zu}$ denen auch die speech activity zählt, gehört, gemeinsames soziokulturelles und sprachliches Hintergrundwissen, kommunikative Erfahrungen, der Kontext sowie die contextualization cues, die ebenfalls konventionalisiert sind.

Durch gemeinsame Konventionen oder Normen wissen Sprecher und Hörer, was in den entsprechenden Gesprächssituationen z.B. als markierter Rhythmus, Lautstärke, Tonfall, Intonation oder Wortwahl und was als angemessen und „normal“ gilt (vgl. Gumperz (1982, 
132)). ${ }^{22}$ Geteilte Konventionen unterstützen den Hörer damit bei der Interpretation, indem sie die Äußerung von vornherein in Bezug auf ihre formale Gestalt und den Inhalt begrenzen. Werden Konventionen, deren Verbreitung unabhängig von Sprachfamilien durch engen, langanhaltenden Sprachkontakt zwischen Sprechern stattfindet (vgl. Gumperz $(1982,118)$ ) und die je nach Kultur und Kontext variieren und erlernt werden müssen, nicht eingehalten, kommt es zu Kommunikationsproblemen. Dazu folgendes Beispiel (Gumperz $(1982,168)$ ): „The incident took place in London, England, on a bus driven by a West Indian driver/conductor. The bus was standing at a stop, and passengers were filing in. The driver announced, "Exact change, please," as London bus drivers often do. When passengers who had been standing close by either did not have money ready or tried to give him a large bill, the driver repeated, "Exact change, please." The second time around, he said "please" with extra loudness, high pitch, and falling intonation, and he seemed to pause before "please." One passenger so adressed, as well as others following him, walked down the bus aisle exchanging angry looks and obviously annoyed, muttering, "Why do these people have to be so rude and threatening about it?"

Hier geht es vor allem um die Prosodie, die als Kontextualisierungshinweis stark sprachsystemabhängig, und damit kulturabhängig, ist. „In the bus driver case, requesting exact change is customary so that the accent on "change" would be expected. But note that the politeness tag "please" is also accented and carries a falling tone. This goes counter to English prosodic conventions which associate falling tones with definiteness and finality, while rising tones, among other things, count as tentative and therefore tend to sound more polite. [...] "Please" spoken with a falling tone by contrast implies annoyance at something the listener did or is likely to do. [...]“ (Gumperz $(1982,169)$ ). Der Fahrer macht hier genau das und sorgt somit dafür, dass die Hörer, die sich auf die Konventionen im britischen Englisch verlassen, seine Äußerung als unhöflich empfinden und darüber verärgert sind (vgl. Gumperz

\footnotetext{
${ }^{22}$ Dies wird auch als kommunikative Kompetenz bezeichnet, vgl. „In order for societies to function smoothly, their members must have not only linguistic competence (the knowledge of the grammatical rules of their mother tongue, acquired well before adulthood) but also communicatice competence - the knowledge of what is and what is not appropriate to say in any specific cultural context." (Salzmann $(1993,193)$ ). Die linguistische und kommunikative Kompetenz sind Bestandteile von Regeln, die unsere Kommunikation leiten und deren Missachtung Folgen hat: „Communicative activity is guided by rules of interaction: Under normal circumstances, members of a speech community know what is and what is not appropriate. [...] When rules of interaction are broken or completely neglected, embarrassment results, and unless an apology is offered, future contacts between the parties may be strained or even avoided.“(Salzmann $(1993,200))$.
} 
(1982, 169)). Dass es sich bei der Äußerung des Fahrers aber keineswegs um Unhöflichkeit handelt, zeigt sich, wenn man die Konventionen in Westindien zu Rate zieht. „Our conclusion is that the West Indian bus driver's "Exact change / please //“" was simply his accustomed way of emphasizing the word "please," [...]. Therefore, his intention was, if anything, to be polite.“ (Gumperz $(1982,170))$.

Einen Teil der Konventionen stellt das Wissen um den activity type ${ }^{23}$ dar. Die Hypothese dabei ist, dass , ,[...] any utterance can be understood in numerous ways, and that people make decisions about how to interpret a given utterance based on their definition of what is happening at the time of interaction. [...] I will refer to the basic socially significant unit of interaction in terms of which meaning is assessed as the activity type or activity (Levinson 1978). The term is used to emphasize that, although we are dealing with a structured ordering of message elements that represents the speakers' expectations about what will happen next, yet it is not a static structure, but rather it reflects a dynamic process which develops and changes as the participants interact. Moreover, its basis in meaning reflects something being done, some purpose or goal being pursued [...]. Thus the activity type does not determine meaning but simply constrains interpretations by channelling inferences so as to foreground or make relevant certain aspects of background knowledge and to underplay others." (Gumperz (1982, 130f.)). Ein besonderer Bestandteil der activity types ist die sog. speech activity, die Gumperz einführt und folgendermaßen definiert: „Speech activities can be characterized through descriptive phrases such as "discussing politics," "chatting about the weather," "telling a story to someone“ [...].“ (Gumperz (1982, 166)). Ihre Bedeutung zeigt sich in dem Zweck, den das Wissen um sie erfüllt: „Knowledge of the conversational activity entails expectations about possible goals or outcomes for the interaction, about what information is salient and how it is likely to be signalled, about relevant aspects of interpersonal relations, and about what will count as normal behaviour. A minimal requirement for successful communication is that participants share these expectations [...].“ (Gumperz (1982, 101)).

\footnotetext{
${ }^{23}$ Der Begriff geht auf Stephen C. Levinson zurück, der dazu Folgendes schreibt: „I want to introduce as a term of art the notion of an 'activity type'. There are various terms that are employed by sociologists and anthropologists engaged in the study of language usage which are roughly equivalent, especially 'speech event' and 'episode' (see e.g. Hymes, 1972; Gumperz, 1972). My notion is to be preferred for present purposes because it refers to any culturally recognized activity, whether or not that activity is coextensive with a period of speech or indeed whether any talk takes place in it at all [...]. In particular I take notion of an activity type to refer to a fuzzy category whose focal members are goal-defined, socially constituted, bounded, events whith constraints on participants, setting, and so on, but above all on the kinds of allowable contributions. Paradigm examples would be teaching, a job interview, a jural interrogation, a football game, a task in a workshop, a dinner party and so on.“ (Levinson $(1979,368)$ )
} 
Eine besonders große Bedeutung für die Interpretation kommt soziokulturellem und sprachlichem Hintergrundwissen zu. „It seems clear that knowledge of grammatical rules is an essential component of the interactive competence that speakers must have to interact and cooperate with others. [...] Speakers who understand each other must conform to common grammatical rules even though the surface forms they employ may differ." (Gumperz (1981, 19)). Über das sprachliche Hintergrundwissen hinaus, spielt auch eine andere Art von Hintergrundwissen eine Rolle: „Aside from physical setting, participants' personal background knowledge and their attitudes toward each other, sociocultural assumptions concerning role and status relationships as well as social values associated with various message components also play an important role.“ (Gumperz (1982, 153)). Gemeinsames Hintergrundwissen ermöglicht es Sprechern und Hörern, in einer Gesprächssituation auf vorhandenes gemeinsames Wissen zurückzugreifen und u.a. in ein Gespräch „einzusteigen“, ohne dass der Sprecher den gesamten Kontext erläutern muss, vgl. dazu folgendes Beispiel (Gumperz $(1982,164))$ :

(3) „[...] The incident was observed at a luncheon counter, where the waitress behind the counter was talking with a friend seated at the counter:

Friend: I called Joe last night.

Waitress: You did? Well what'd he say?

Friend: Well, hi!

Waitress: Oh yeah? What else did he say?

Friend: Well he asked me out of course.

Waitress: Far out!

To participate in this exchange, the waitress, apart from having to rely on socio-cultural schemata about dating situations, must recognize that the first statement, which seems complete on the surface, is actually the lead-in for a story that she is expected to help elicit. Further, she must know that "called" refers to a telephone call; she must know who Joe is; and she must realize that the call was not routine but had special meaning for her friend." Dabei kann soziokulturelles Wissen nur in den jeweiligen Gesprächsakten selbst zum Vorschein kommen: „In a brilliant series of experiments, Garfinkel $(1967,1972)$ demonstrates that social knowledge cannot be adequately characterized in the form of statistically countable, abstract categories such as scalar ratings of role, status or personality characteristics. He argues that social knowledge is revealed in the process of interaction itself and that interactants create 
their own social world by the way in which they behave." (Gumperz $(1982,158))$. Dieser Argumentation folgt auch Gumperz.

$\mathrm{Zu}$ den weiteren Faktoren, die bei der Interpretation eine Rolle spielen, gehören Erfahrungen, die wir im Laufe all unserer kommunikativer Begegnungen sammeln und auf die wir uns als Basis für Interpretationsrahmen bei der Interpretation verlassen können: „Such frames enable us to distinguish among permissible interpretive options.“ (Gumperz $(1982,21)$ ). Besonders wichtig ist der Kontext, welcher durch den Diskurs zwischen Sprecher und Hörer während des Sprechvorgangs erschaffen wird und von dem die Interpretation, die im Laufe des Gesprächs durch interaktive Prozesse immer wieder geändert und angepasst werden muss (vgl. Gumperz (1982, 160)), abhängig ist. Im Zusammenhang mit dem Kontext steht die Kontextualisierung ${ }^{24}$. Unter dieser versteht Gumperz $(1982,162)$ einen “[...] process by which we evaluate message meaning and sequencing patterns in relation to aspects of the surface structure of the message, called 'contextualization cues' “ Auer (1992) beschreibt die Kontextualisierung folgendermaßen: „In most general terms, contextualization therefore comprises all activities by participants which make relevant, maintain, revise, cancel... any aspect of context which, in turn, is responsible for the interpretation of an utterance in its particular locus of occurrence. Such an aspect of context may be the larger activity participants are engaged in (the "speech genre"), the small-scale activity (or "speech act"), the mood (or "key") in which this activity is performed, the topic, but also the participants' roles (the participant constellation, comprising "speaker", "recipient", "bystander", etc.), the social relationship between participants, the relationship between a speaker and the information he conveys via language ("modality"), even the status of "focused interaction" itself." (Auer (1992, 3f.)). Die Merkmale, die an der „Oberfläche“ der Äußerung stehen und die Interpretation leiten, sind die sog. contextualization cues: „That is, constellations of surface features of message form are the means by which speakers signal and listeners interpret what the activity is, how semantic content is to be understood and how each sentence relates to what precedes or follows. These features are referred to as contextualization cues. For the

\footnotetext{
${ }^{24}$ Definiert wird Kontextualisierung folgendermaßen: „,... ] Der Gebrauch, den Sprecher und Hörer von verbalen und nonverbalen Zeichen machen, um das, was zu einer bestimmten Zeit und an einem bestimmten Ort gesagt wird, mit Wissen, das durch vergangene Erfahrung erworben wurde, in Beziehung zu setzen“ (Gumperz 1992, S. 230). Diese Beziehung wird durch Kontextualisierungshinweise (contextualization cues) ausgedrückt, die vor allem prosod. oder paralinguist. Art sind oder sich dem „,code switch“ oder einer spezif. Auswahl phonet., phonolog. oder morphosyntakt. Formen oder auch bestimmter lexikal. Formen oder formelhafter Ausdrücke verdanken. Dieses von Gumperz (vgl. bes. Gumperz 1982) entwickelte Konzept versteht sich als integrativer Grundlagen-Beitrag jenseits der methodolog. Diskussionen zwischen unterschiedl. Analyserichtungen von Diskursen, indem er auf den On-Line-Charakter des konversationellen Schließens und den in der Konversation selbst je neu ,interaktiv, also letzlich gesellschaftlich“ (Gumperz 1992, S. 230) hergestellten Charakter von Annahmen der Interaktanten über die Konversation verweist.“(Glück (1993, 332)).
} 
most part they are habitually used and perceived but rarely consciously noted and almost never talked about directly.“ (Gumperz $(1982,131)$ ). Es handelt sich um bestimmte Signale bzw. Zeichen, die im Zusammenspiel mit Grammatik und Lexik dazu dienen, eine kontextuelle Basis für die Interpretation zu erschaffen: zu diesen Hinweisen zählen z.B. Prosodie (z.B. Akzent, Intonation), paralinguistische Hinweise (z.B. Rhythmus, Pause, Ton der Stimme), Code- und Style-Switching und Wahl bestimmter lexikalischer Formen (vgl. Tannen $(2004,77))$. Eine besonders große Rolle weist Gumperz dabei der Prosodie und den paralinguistischen Hinweisen $\mathrm{zu}$ : „We have already talked about the role that prosodic mechanisms such as tone grouping, accent placement and tune play in segmenting the stream of talk, signalling thematic connections and providing information about activities. When basic tone grouping and accentuation conventions differ, predictability in conversation suffers [...].“ (Gumperz $(1982,143))$. Ebenfalls muss beachtet werden, dass die Wahl „[...] among lexical and syntactic options, formulaic expressions, conversational openings, closings and sequencing strategies can all have similar contextualizing functions." (Gumperz $(1982,131)$ ). Anzumerken ist, dass die Kontextualisierungshinweise selbst keine explizite Bedeutung haben: „Although such cues carry information, meanings are conveyed as part of the interactive process. Unlike words that can be discussed out of context, the meanings of contextualization cues are implicit.“ (Gumperz $(1982,131)$ ). Daraus folgt, dass nicht nur eine einzige Interpretationsmöglichkeit existiert, sondern viele ${ }^{25}$ : „The linguistic character of contextualization cues is such that they are uninterpretable apart from concrete situations. [...] The same sign may indicate normal information flow under some conditions and carry contrastive or expressive meanings under others." (Gumperz (1980, 170f.)). Die korrekte, d.h. vom Sprecher intendierte, Interpretation ergibt sich aus dem Kontext sowie aus dem Hintergrundwissen. Wie wichtig das Erkennen und korrekte Deuten von kontextuellen Hinweisen aber ist, wird deutlich, wenn es zu Missverständnissen kommt: „When all participants understand and notice the relevant cues, interpretive processes are then taken for granted and tend to go unnoticed. However, when a listener does not react to a cue or is unaware of its functions, interpretations may differ and misunderstanding may occur. It is important to note that when this happens and when a difference in interpretation is brought to a participant's attention, it tends to be seen in attitudinal terms. A speaker is said to be

\footnotetext{
25 Das Vorhandensein mehrerer Interpretationsmöglichkeiten der gleichen Äußerung und Gesprächssituation zeigt Gumperz an einigen Beispielen, die er mehreren an den Beispielkonversationen unbeteiligten Hörern vorgespielt hatte (vgl. Gumperz (1982, 134-139)). Dies beweist, dass die kontextuellen Hinweise soziokulturell gebunden und je nach persönlichem Hintergrund und Hintergrundwissen des Hörers unterschiedlich interpretiert werden.
} 
unfriendly, impertinent, rude, uncooperative, or to fail to understand.“ (Gumperz (1982, 132)). Häufig bestehen solche Probleme zwischen unterschiedlichen Ethnien mit unterschiedlichen Sprachsystemen und können vor allem bei Nicht-Muttersprachlern und Muttersprachlern beobachtet werden. Sie können aber auch innerhalb einer Ethnie vorkommen, z.B. wenn bestimmten Ausdrücken, sog. formulaic expressions [formelhafte Phrasen, Übers. d. Verf.] keine Beachtung geschenkt wird. Formelhafte Phrasen „[...] occur as part of routinized interactive exchanges, such as Goffman describes as "replies and responses" (1981). Their use signals both expectations about what is to be accomplished and about the form that replies must take. They are similar in function to code switching strategies. Like the latter they are learned by interacting with others in institutionally defined networks of relationships. Where these relationships are ethnically specific they are often regarded as markers of ethnic background.“ (Gumperz (1982, 134)). Mit ihnen können Gesprächspartizipanten sich gegenseitig zeigen, dass man z.B. das gleiche Sprachsystem und die gleichen sprachlichen Gepflogenheiten beherrscht. Zu formelhaften Phrasen gehören auch unvollendete Sätze, bei denen der Sprecher erwartet, dass der Hörer sie selbst beenden kann. Dies kann nur geschehen, wenn beide Gesprächspartner über gemeinsames sprachliches und soziokulturelles Hintergrundwissen verfügen (vgl. Gumperz (1982, 146)). Ein Beispiel für die Konsequenzen einer Nicht-Beachtung solcher formelhaften Phrasen in einem Gespräch zwischen Partizipanten der gleichen Ethnie ist das Folgende (Gumperz (1982, 133)):

(4) Ein afro-amerikanischer Student (Interviewer) wird geschickt, um eine afroamerikanische Hausfrau zu interviewen, der Kontakt wurde nicht von ihm, sondern von einer anderen Person aus dem Büro, in dem der Student arbeitet, hergestellt. Die Tür wird vom Ehemann (Husband) der Hausfrau mit einem Lächeln geöffnet: Husband: So y're gonna check out ma ol lady, hah?

Interviewer: Ah, no. I only came to get some information. They called from the office.

Daraufhin hört der Ehemann auf zu lächeln, verschwindet wortlos im Haus und ruft seine Frau. Das Interview verläuft nicht besonders erfolgreich. Das Problem besteht darin, dass der Student die erste Äußerung des Ehemannes nicht als wichtig genug erachtet und die Hinweise nicht erkennt. Sie entspricht nämlich einer formelhaften Phrase im Englischen von AfroAmerikanern zu Beginn eines Gesprächs, um zu schauen, ob der Konversationspartner mit einer formelhaften Antwort aufwarten kann, was als Hinweis auf gleichen Hintergrund 
gedeutet wird. Um dem Ehemann dies zu zeigen, hätte der Student mit einer formelhaften Antwort reagieren müssen, die typisch für das afro-amerikanische Englisch ist (z.B. „Yea, I'ma git some info“). Da er jedoch eine standardenglische Antwort gibt, wird es vom Ehemann so aufgenommen, dass er nicht zur eigenen Gruppe gehöre und deswegen nicht vertrauenswürdig sei.

Gumperz (1982, 145-150) führt auch Beispiele an, wo lautliche Kontextualisierungshinweise, wie Intonation oder Sprechpausen, je nach soziokulturellem Hintergrund und Sprachsystem der Hörer unterschiedlich interpretiert wurden. So, z.B. wenn jemand eine Sprechpause macht und von jemand anderem unterbrochen wird, weil dieser meint, jener sei mit seinem Turn fertig. Ein anderes Beispiel stammt aus einer Schule in den USA (Gumperz $(1982,147))$ :

T: James, what does this word say?

J: I don't know.

T: Well, if you don't want to try someone else will. Freddy?

F: Is that a $p$ or a $b$ ?

$\mathrm{T}$ : (encouragingly) It's a $p$.

F: Pen.

James (J) hat mit einer steigenden Intonation gesprochen, was in seinem Kulturkreis und dementsprechend seinem sprachlichen System einem Wunsch nach einer Ermutigung durch den Gesprächspartner - in diesem Fall den Lehrer - gleichkommt. Der Lehrer $(\mathrm{T}=$ Teacher $)$ hingegen interpretiert dies als Unwillen bzw. fehlenden Kooperationswunsch seitens des Kindes und fragt daher ein anderes Kind namens Freddy (F). Obwohl Freddys Frage genau den gleichen Wunsch nach Ermutigung bedeutet, kann der Lehrer in diesem Fall dem Wunsch des Kindes nachkommen, da Freddy seine Unsicherheit auf die Weise kommunikativ darbringt, die der Lehrer erwartet. Hier spielt auch die Formelhaftigkeit solcher Ausdrücke eine Rolle, deren Interpretation von soziokulturellem Hintergrund und dem Sprachsystem des Hörers abhängt. Für Afro-Amerikaner gilt, dass „The formulaicness of such utterances is marked syntactically by phrases like "I don't,“" "I can't,“ followed by a predicate and prosodically by a characteristic intonation contour. This contour accents the predicate and thus serves to identify the formula." (Gumperz $(1982,150))$. Das Erkennen und Interpretieren einer solchen Äußerung ist jedoch unterschiedlich: während Afro-Amerikaner solch einen Ausdruck mit der spezifischen Intonationskurve als formelhafte Phrase betrachten, deuten Amerikaner, die andere Sprachsysteme beherrschen und mit diesen kontextuellen Hinweisen 
nicht vertraut sind, dies als Unwillen zur Kooperation bzw. als Unfähigkeit, die Aufgabe auszuführen, wie man am vorigen Beispiel gut sehen konnte. Die Benutzung formelhafter Phrasen und weiterer kontextueller Hinweise ist Bestandteil unterschiedlicher sprachlicher Strategien, die von Sprechern in der Kommunikation genutzt werden, um ihre Ziele sprachlich zu verdeutlichen und zu erreichen. Diese Konversationsstrategien sind ebenfalls kulturspezifisch, unterliegen also kulturellen Konventionen und sind nur mit der Hilfe von soziokulturellem und sprachlichem Hintergrundwissen zu erkennen und zu verstehen.

$\mathrm{Zu}$ Kontextualisierungshinweisen gehören auch Gesten und Mimiken. „He [Ray Birdwhistell (US-amerikanischer Anthropologe); Anm. d. Verf.] demonstrated that in the act of talking, eyes, face, limbs and torso all emit automatically produced signs which tend to go unnoticed yet nevertheless convey information. These nonverbal signs are language like in the sense that they are learned through interaction, culturally specific and analyzable in terms of underlying processes. They are coordinated with verbal signs both at the micro-level of syllables [...] and at the level of clauses and longer discourse segments.“ (Gumperz $(1982,141)) .{ }^{26}$ Daher ist es nicht weiter verwunderlich, dass ,,a large proportion of misunderstandings are traceable to variant perceptions and interpretations of seemingly trivial facial and gestural signs." (Gumperz (1982, 141)). Im Bereich des außersprachlichen Kommunikationsverhaltens wurde außerdem in Studien zur Sprecher-Hörer-Koordination festgestellt, dass eine Synchronisierung der Konversation stattfindet: „When the relationship of speakership moves to listeners' responses was measured, it was found that these tend to be synchronized in such a way that moves and responses follow each other at regular rhythmic intervals. The timing of responses, moreover, is much faster than one would expect if unpredictable stimuli were responded to (Kempton 1981). This suggests that conversational synchrony requires some degree of predictability and routinization, such as is most commonly acquired by shared culture and similarity of interactive experience." (Gumperz (1982, 141)). Es wurde auch herausgefunden, dass je niedriger die Asynchronität war, desto höher die Menge der erhaltenen Informationen (vgl. Gumperz $(1982,142)$ ). Ein gemeinsamer Rhythmus und damit verbunden die Synchronisation ist ein wichtiger kulturspezifischer und durch konversationelle Erfahrung zu erlernender Bestandteil der Kontextualisierungshinweise. „The ability to

\footnotetext{
${ }^{26}$ Dazu passt auch das Konzept des framing, welches von dem Anthropologen Gregory Bateson stammt. „Anthropologist Gregory Bateson [1955] 1972 introduced the notion of frame to capture a fundamental aspect of meaning in interaction: No utterance or symbolic act can be understood except by reference to a "metamessage" that identifies the character of the interaction. Bateson explains that while watching monkeys playing in their cage at the zoo, he wondered how a monkey at play was able to understand a bite - an aggressive action - as a friendly move. Bateson concluded that a biting monkey sends not only a message - the bite - but also, at the same time, a metamessage "this is play." One might say that the metamessage is like an instruction guide to how the message is to be interpreted; that is the sense in which it frames the message." (Tannen $(2004,77)$ ).
} 
establish a common rhythm is a function, among other factors, of similarity in ethnic background.“ (Gumperz $(1982,142))$.

Auf der Grundlage dieser Annahmen, stellt sich Gumperz den Verlauf einer erfolgreichen Interaktion folgendermaßen vor: „In other words, a successful interaction begins with each speaker talking in certain mode, using certain contextualization cues. Participants, then, by the verbal style in which they respond and the listenership cues they produce, implicitly signal their agreement or disagreement; thus they 'tune into' the other's way of speaking. Once this has been done, and once a conversational rhythm has been established, both participants can reasonably assume that they have successfully negotiated a frame of interpretation [...]. Speakers continue in the same mode, assigning negotiated meanings to contextualization cues, until there is a perceptible break in rhythm, a shift of content and cues, or until a mismatch between content and cues suggests that something has gone wrong." (Gumperz $(1982,167))$. Dabei gibt es mehrere Interpretationsmöglichkeiten einer Äußerung: „The point is that at the level of conversation, there are always many possible alternative interpretations, many more than exist at the level of sentence grammar. Choice among these is constrained by what the speaker intends to achieve in a particular interaction, as well as by expectations about the other's reactions and assumptions. Yet once a particular interpretation has been chosen and accepted it must be followed. That is, an interpretive strategy holds until something occurs in the conversation to make participants aware that a change in strategy is indicated. Interpretations are thus negotiated, repaired and altered through interactive processes rather than unilaterally conveyed.“ (Gumperz (1982, 159f.)). Dies schließt den Kreis zu der Annahme, Interpretation sei situationsabhängig und im Laufe eines Gesprächs veränder- und anpassbar.

Abschließend lässt sich sagen, dass Gumperz in Konversationen stets von einer konversationellen Kooperation ausgeht, bei der Sprecher und Hörer sprachlich oder außersprachlich auf einander reagieren. Dabei bezieht sich die Reaktion des Hörers nicht unbedingt auf das, was wortwörtlich gesagt wurde, sondern darauf, wie er die Äußerung des Sprechers versteht. Dabei ist die Interpretation für Gumperz ein Prozess mit der Möglichkeit, mit dem Hinzukommen neuer Informationen im Laufe des Gesprächs verändert und angepasst zu werden. Die Bedeutung wird von Gumperz somit als etwas gesehen, das von Sprecher und Hörer zusammen in einer bestimmten Gesprächssituation erschaffen wird: sie ist eine ,,[...] 
situated interpretation [...]“" (Tannen $(2004,77)$ ). Dabei gibt es aber nicht nur eine, sondern viele Interpretationsmöglichkeiten, die seitens des Sprechers durch seine Intention und die Erwartungshaltung an die Reaktion des Hörers begrenzt werden. Der Hörer verlässt sich auf mehrere Faktoren, mit deren Hilfe er sich für eine Interpretation entscheidet. Ob diese korrekt, im Sinne von vom Sprecher beabsichtigt, ist, hängt von dem (Er-) Kennen, dem Deuten und der angemessenen Reaktion auf diese Faktoren ab. Zu diesen zählen laut Gumperz: gemeinsam befolgte Konventionen, mit deren Hilfe Sprecher und Hörer u.a. den gleichen Gesprächsverlauf oder bestimmte Art der Reaktionen auf bestimmte Äußerungen erwarten können; kommunikative Erfahrungen, auf die wir uns verlassen; der Kontext, der während der Interaktion von Sprecher und Hörer gemeinsam erschaffen wird und an den die Interpretation gebunden ist; gemeinsames soziokulturelles und sprachliches Hintergrundwissen; und schließlich Kontextualisierungshinweise, die die Äußerung in einen Kontext setzen und die Interpretation leiten. All das zeigt dem Hörer, wie die Nachricht des Sprechers zu interpretieren ist, was sein Ziel ist und was den Hörer als Nächstes in der Interaktion erwartet. Werden die eben angeführten Faktoren alle erkannt und richtig gedeutet und erfolgt eine angemessene, vom Sprecher erwartete Reaktion des Hörers, so gibt es keine Probleme (vgl. das Beispiel im Büro oder das Gespräch zwischen der Bedienung in einem Lokal und ihrer Freundin am Tresen). Wird aber einer der Bestandteile, seien es Konventionen oder Kontextualisierungshinweise, missachtet oder fehlt z.B. das Hintergrundwissen, so kommt es häufig zu einem Misslingen der Interaktion, wie an dem Beispiel mit dem Busfahrer in London oder an dem mit dem Lehrer und den beiden Schülern gezeigt wurde. „What distinguishes succesful from unsuccesful interpretations are not absolute, context-free criteria of truth value or appropriateness but rather what happens in the interactive exchange itself, i.e. the extent to which proffered context bound inferences are shared, reinforced, modified or rejected in the course of an encounter." (Gumperz $(1980,171))$.

Es lässt sich feststellen, dass für die richtige Interpretation laut Gumperz mehrere Faktoren eine Rolle spielen, die nicht alle rein sprachlicher Natur sind. Die Beherrschung der Grammatik und Lexik einer Sprache ist daher kein Garant für stets gelungene Interaktionen. Vielmehr sind auch andere, meist ungenannte Faktoren entscheidend, die von allen Sprechern im Laufe kommunikativer Begegnungen mit anderen Sprechern der jeweiligen Sprache erworben werden müssen. Die Wichtigkeit dieser Faktoren lässt ihre Untersuchung daher in den Mittelpunkt der Interaktionalen Soziolinguistik rücken und unterscheidet diese somit von der traditionellen Soziolinguistik. 


\subsection{Eigener theoretischer Ansatz}

$\mathrm{Zu}$ Beginn muss geklärt werden, was die Internetkommunikation $^{27}$ mit anderen Kommunikationsarten gemein hat bzw. was sie von diesen unterscheidet. Zur Beantwortung dieser Frage benutze ich ein vereinfachtes, aber dennoch für den Zweck der vorliegenden Dissertation ausreichendes Modell: „An early (from the 1940s) but serviceable model of communication makes use of five components: the sender (or source), the message, the channel, the receiver (or destination), and the effect. [...] These components take into account the entire process of transmitting information, namely, who is transmitting what by what means to whom and with what effect.“ (Salzmann (1993, 14)). Auch die Internetkommunikation beinhaltet all dies: der Schreiber (= Sender) schickt dem Leser (= Empfänger) eine Nachricht, der Leser empfängt diese schriftliche Nachricht über den visuellen Kanal. Die Wirkung der Nachricht sieht man als Reaktion des Lesers, die wiederum als schriftliche Nachricht zurückgesendet wird. Im Gegensatz zur mündlichen Kommunikation kann der Leser den Schreiber jedoch weder sehen noch hören. ${ }^{28}$

Durch Forschung wurde herausgefunden, dass ,[...] conversation is not a relatively unstructured form of human interaction. Conversational exchanges are subject to extremely complex procedures that regulate when and how speaking is done and how particular contributions - verbal or nonverbal - will be understood (which depends on their placement with respect to earlier contributions).“ (Levinson $(1989,408)$ ). Dabei ist eine der wichtigsten Eigenschaften der Konversation, dass sie interaktional ist, d.h. ihr Verlauf wird nicht von einer Person, sondern von allen Gesprächspartnern bestimmt (vgl. Levinson $(1989,408)$ ). Als Beispiel führt Levinson (vgl. Levinson $(1989,408)$ ) hierfür Pausen an, die in einem Gespräch nur realisiert werden können, wenn alle Gesprächsteilnehmer sie beachten. Um dies zu erreichen, muss es bestimmte Mechanismen geben, die den Ablauf einer Konversation regeln. Dazu gehört z.B. der Sprecherwechsel, das sog. turn-taking (vgl. Levinson (1989, 408)), auf das später noch eingegangen wird und das (neben anderen Faktoren) für eine Sequenzierung des Gesprächs sorgt. Des Weiteren sagt Levinson (1989, 409)): „Conversations have conventional opening sequences (of the "Hello, how are you" sort) and closing sequences and, at least in the case of telephone calls, an expectation that the overt reason for engaging in talk will be produced immediately after the opening sequence in what is recognizably "first topic" position. Thus we can say that a conversation is characterized not only by employing

\footnotetext{
${ }^{27}$ Im Rahmen dieser Arbeit meine ich unter Internetkommunikation die Kommunikation in Threads.

28 Eine Ausnahme im Bereich der mündlichen Kommunikation bilden z.B. „klassische“ Telefonate (ohne Videoübertragung), da der Hörer den Sprecher zwar hören, ihn jedoch nicht sehen kann.
} 
conversational procedures (most forms of talk use at least some of those) but also by conforming to certain expectations about how the whole exchange will be structured." Analysiert man im Internet geführte Gespräche oder nimmt an solchen teil, so erkennt man, dass diese auf ihre eigene Art und Weise die meisten der oben aufgeführten Merkmale einer Kommunikation beinhalten. Auch hier werden Gespräche von Regeln und Mechanismen bestimmt; der Ablauf entsteht aus dem Zusammenspiel aller Gesprächsteilnehmer, sie entscheiden, in welche Richtung sich ein Gespräch entwickelt und wann ein Gespräch über ein bestimmtes Thema zu Ende ist; ebenfalls finden wir von Beginn des Gesprächs an eine gewisse Erwartungshaltung an die Struktur des Gesprächs, hier vor allem die Position der first topic position, die den Zweck der Konversation verdeutlichen soll. Einen wichtigen Unterschied stellen die Kanäle dar, denn während in face-to-face-Kommunikation mehrere Kanäle, u.a. der paralinguistische und kinesische, gleichzeitig angesprochen werden (vgl. Levinson (1989, 407)), ist der Kanal über den eine Mitteilung in Internetkonversationen gesendet wird, der visuelle. Insgesamt ist die Internetkommunikation somit eine natürliche Kommunikation, die ein spezielles Medium mit teils eigenen Eigenschaften benutzt.

Allgemein stellt die Internetsprache eine Art „Hybrid“ ${ }^{29}$ zwischen geschriebener $^{30}$ und gesprochener Sprache dar. „What makes Netspeak so interesting, as a form of communication, is the way it relies on characteristics belonging to both sides of the speech/writing divide. [...] the situations of e-mail, chatgroups, and virtual worlds, though expressed through the medium of writing, display several of the core properties of speech. They are time-governed, expecting or demanding an immediate response; they are transient, in the sense that messages may be immediately deleted (as in e-mails) or be lost to attention as they scroll off the screen (as in chatgroups); and their utterances display much of the urgency and energetic force which is characteristic of face-to-face conversation. [...] But there are several major differences between Netspeak and face-to-face conversation [...]“. (Crystal (2001, 28f.)). ${ }^{31}$ Diese liegen unter anderem an technisch bedingten Problemen, z.B. ,[...] lack

${ }^{29}$ „Baron [...] calls it an ,'emerging language centaur - part speech, part writing'. (Crystal $(2001,48)$ zitiert Baron $(2000,248)))$.

30 „Der Terminus Geschriebene Sprache ist mehrfach ambig, gerade auch in der Gegenüberstellung zu Gesprochener Sprache:

- beide können als besondere Arten von Sprache verstanden werden,

- die geschriebene Sprache kann als sekundäre Erscheinung („Abbildung“) der gesprochenen (,eigentliche“ Sprache) verstanden werden,

- beide können als unterschiedliche mediale Repräsentationen der Sprache verstanden werden, die (als das sprachlich so Artikulierte) darin repräsentiert wird.“(Maas (2004, 633)).

31 Dieselbe Meinung vertritt ebenfalls Maas (2004, 635): „Ähnliche Klassifikationsprobleme wirft die elektronische Kommunikation im Internet auf, die in medialer Hinsicht schriftsprachlichen Praktiken 
of simultaneous feedback [...]“ (Crystal $(2001,30)$ ) oder ,[...] time-delay [...]“ (Crystal (2001, 31)) (auch als lag bezeichnet). Ich folge dieser Auffassug: Die Internetkommunikation enthält sowohl die Merkmale der schriftlichen als auch der gesprochenen Sprache und vereinigt je nach Gesprächssituation mal mehr, mal weniger dieser Merkmale. Darüber hinaus verfügt sie über eigene Merkmale, die sowohl der schriftlichen als auch der mündlichen Kommunikation fehlen. Dazu gehören z.B. die Verwendung von einer Vielzahl animierter und nicht animierter Emoticons oder die Möglichkeit über die Zitat-Funktion direkt auf Äußerungen jederzeit und vor allem immer wieder reagieren zu können - insgesamt hat man durch Scrollen der Unterhaltung die Möglichkeit, auch auf alte Mitteilungen antworten zu können, da alles archiviert wird und jederzeit zugänglich ist - im Gegensatz zur gesprochenen Sprache, in der die Sprecher nur auf die in ihrem Gedächtnis gespeicherten und potentiell lückenhaften Inhalte von Gesprächen zurückgreifen können. Ein weiteres wichtiges Merkmal der Kommunikation im Internet ist die Anonymität der Nutzer. „Operating behind a false persona seems to make people less inhibited: they may feel emboldened to talk more and in different ways from their real-world linguistic repertoire." (Crystal $(2001,51)) .{ }^{32}$ Auch die Akkommodation der Nutzer ist ein Merkmal, das sie jedoch mit anderen Kommunikationsarten teilt: „Although they come from many different backgrounds, and write in many different styles, their contributions progressively develop a shared linguistic character - the equivalent of a local dialect or accent.“ (Crystal (2001, 146)). Meiner Ansicht nach lässt sich dies aber nicht für jedes Forum und jede Chatgruppe annehmen, denn die Mitglieder können, müssen sich aber nicht einander sprachlich anpassen, das ist vor allem dort der Fall, wo die Mitglieder ständig wechseln.

Auf Grund der schriftlichen Realisierung der Internetkommunikation ersetze ich die Bezeichnungen „Sprecher“ und „Hörer“ durch „Schreiber“ und „Leser“, wobei der Leser selbstverständlich im nächsten Konversationsschritt auch Schreiber sein kann, so wie der Hörer der Sprecher sein kann. Auch gebrauche ich für Internetkonversationen die Bezeichnung Gesprächsteilnehmer bzw. -partizipant: „The component termed participants includes not only the sender of a message (also referred to as the speaker or addresser) and the intended receiver (hearer, addressee) but anyone who may be interested in or happens to perceive (hear, see) the message - the audience.“ (Salzmann $(1993,197))$. Der Vorteil dieser

zugerechnet werden muss, strukturell aber (vor allem beim „Chat") alle Merkmale einer situativ gesteuerten Kommunikation der „Nähe“ aufweist, die sich nicht nur durch die Ausbildung konventioneller parasprachlicher Zeichensysteme auszeichnet, sondern auch in Reaktion auf die besonderen Kommunikationsbedingungen z.B. eigne [sic!] syntaktische Muster aufweist [...].“

${ }^{32} \mathrm{Ob}$ ein User tatsächlich ein anderes Repertoire benutzt, ist nur überprüfbar, wenn man ihn persönlich kennt. 
Bezeichnung ergibt sich daraus, dass Beiträge in Foren von jedem gelesen werden können, unabhängig davon, ob er selbst angesprochen wird oder sich am Gespräch überhaupt beteiligt. Der Begriff umfasst somit mehr User als mögliche Leser als nur denjenigen/diejenigen Leser, dessen/deren Reaktion(en) erfolgt. Die Kommunikation in den untersuchten Threads der entsprechenden Internetforen bezeichne ich als Äußerung-Reaktion-Kommunikation. Einen Bestandteil dieser bildet die häufig vorkommende Frage-Antwort-Kommunikation, bestehend aus einem einzelnen Thread, in dem es nur um die Beantwortung der jeweiligen Frage geht.

Zunächst soll festgehalten werden, dass ich mich in meinem eigenen Ansatz auf Gruppen in Internetforen beziehe, die sich vor allem auf Grund des gemeinsamen Interesses ihrer Mitglieder bilden, sei es das Interesse an Computerspielen, Rollenspielen, bestimmten Büchern, Technik o.a.. Da in Internetforen sowie in ihren Subforen, Subsubforen und Threads ein Teil der Mitglieder regelmäßig wechselt, schlage ich für die hier untersuchten Threads die Annahme der „offenen“ Gruppe vor, als Gegensatz zu Threads, die nur bestimmten „geschlossenen“ Gruppen vorbehalten sind. ${ }^{33}$ Für die Annahme einer „offenen“ Gruppe sprechen meiner Meinung nach mehrere Gründe: die Mitgliederzahl ist nicht begrenzt; der Thread ist frei zugänglich für alle, die sich für das Thema interessieren und sich an den Gesprächen beteiligen wollen, d.h. die Mitglieder werden nicht nach bestimmten Kriterien ausgewählt und können selbsttätig jederzeit hinzukommen und den Thread jederzeit wieder verlassen.

Bei der Anwendung des Ansatzes der interaktionalen Soziolinguistik auf die Internetkommunikation, vertrete ich die Ansicht, dass nicht all das, was Gumperz als entscheidend für eine erfolgreiche Interpretation - und damit Interaktion in mündlicher Kommunikation $^{34}$ - annimmt, von gleich großer Bedeutung ist. Dies betrifft die lautlichen und visuellen Aspekte der Sprache, da sich diese im Medium Internet nur begrenzt darstellen lassen $^{35}$ und es dennoch in den meisten Fällen zur korrekten Interpretation seitens des Lesers kommt. Auch gibt es keine messbare Synchronisierung zwischen der Äußerung des Schreibers und Leserreaktionen, da der zeitliche Abstand nicht unmittelbar sein muss.

Grundsätzlich gehe ich von einer gewollten Kooperation zwischen Schreiber und Leser aus.

\footnotetext{
33 Als Beispiel dient hier das Forum antichat.ru, in dem man zu einigen Threads nur Zugang hat, wenn man Mitglied einer bestimmten Gruppe ist oder einen bestimmten Rang inne hat und eingeladen wird.

${ }^{34}$ Unter mündlicher Kommunikation verstehe ich im Rahmen dieser Dissertation face-to-face-Kommunikation. Das Gleiche gilt für mündliche Konversationen.

${ }_{35}$ Die lautliche und visuelle Seite einer Äußerung lässt sich potentiell durch graphische Mittel, wie Emoticons, Satzzeichen oder Groß- und Kleinschreibung andeuten.
} 
Da jedoch nicht auf jede Mitteilung eine Reaktion seitens eines Lesers erfolgt, müssen Gründe für ihr Fehlen untersucht werden. Meine These ist, dass einige Mitteilungen „untergehen“, d.h. einfach übersehen werden, während andere von den Lesern einfach als nicht interessant genug empfunden werden, um darauf zu reagieren. Ein weiterer Grund liegt in dem Fehlen eines Bezugs der Mitteilung zu anderen Mitteilungen (wie das z.B. beim Trolling $^{36}$ vorliegt). Damit die eigenen Mitteilungen nicht übersehen werden, verwenden Nutzer verschiedene Strategien, um auf sie aufmerksam zu machen. Diese Strategien sind Bestandteil anderer Konversationsstrategien in Internetkonversationen, mit denen Schreiber an ihre Ziele gelangen und die eng mit der Intention des jeweiligen Schreibers verknüpft sind. Mein Ansatz geht davon aus, dass für eine erfolgreiche Interaktion, neben einer korrekten Interpretation der Äußerungen, auch die Einhaltung von Gesprächskonventionen notwendig ist. Die im Internet existierenden Konventionen ähneln den allgemeingültigen gesellschaftlichen Konventionen. Darüber hinaus besitzt jedes Forum eigene, meist ungenannte Konventionen. Im engen Zusammenhang mit den Konventionen steht der Konversationsverlauf, von dem es in Internetthreads offenbar mehrere unterschiedliche Arten gibt: es gibt Forenthreads, in denen Konversationen ähnlich verlaufen wie in mündlichen Gesprächssituationen (z.B. Begrüßung - Gespräch - Abschied), andererseits gibt es Konversationen, in denen dies nicht der Fall ist. Auch innerhalb eines Threads selbst gibt es Variationen. Insgesamt sind Konversationsverläufe stark von Konventionen geprägt. Das Gleiche gilt für den Partizipantenwechsel ${ }^{37}$. Die in den jeweiligen Threads vorliegenden und meist ungenannten Konventionen bestimmen, ob Begrüßungen benutzt werden, ob auf jede

\footnotetext{
${ }^{36}$ Trolling ist eine Bezeichnung für „1. v.,n. [From the Usenet group alt.folklore.urban] To utter a posting on Usenet designed to attract predictable responses or flames; or, the post itself. Derives from the phrase "trolling for newbies" which in turn comes from mainstream "trolling", a style of fishing in which one trails bait through a likely spot hoping for a bite. The well-constructed troll is a post that induces lots of newbies and flamers to make themselves look even more clueless than they already do, while subtly conveying to the more savvy and experienced that it is in fact a deliberate troll. If you don't fall for the joke, you get to be in on it. See also YHBT. 2. $\mathrm{n}$. An individual who chronically trolls in sense 1; regularly posts specious arguments, flames or personal attacks to a newsgroup, discussion list, or in email for no other purpose than to annoy someone or disrupt a discussion. Trolls are recognizable by the fact that they have no real interest in learning about the topic at hand - they simply want to utter flame bait. Like the ugly creatures they are named after, they exhibit no redeeming characteristics, and as such, they are recognized as a lower form of life on the net, as in, "Oh, ignore him, he's just a troll." Compare kook. 3. n. [Berkeley] Computer lab monitor. A popular campus job for CS students. Duties include helping newbies and ensuring that lab policies are followed. Probably so-called because it involves lurking in dark cavelike corners. Some people claim that the troll (sense 1) is properly a narrower category than flame bait, that a troll is categorized by containing some assertion that is wrong but not overtly controversial. See also Troll-O-Meter. The use of 'troll' in any of these senses is a live metaphor that readily produces elaborations and combining forms. For example, one not infrequently sees the warning "Do not feed the troll” as part of a followup to troll postings. “ (http://www.catb.org/jargon/html/T/troll.html). Für die vorliegende Arbeit ist die 2. Bedeutung dieses Vorgangs relevant.

${ }^{37}$ Im Rahmen dieser Dissertation beschreibe ich mit dem Begriff Partizipantenwechsel den Wechsel zwischen Gesprächsteilnehmern in einer schriftlich realisierten Threadkonversation. Dies entspricht dem Sprecherwechsel in mündlicher Kommunikation.
} 
Mitteilung reagiert werden muss und wie man mit thematisch unpassenden Mitteilungen umgeht, die inmitten einer Konversation auftauchen (also, ob man sie beantwortet oder überliest). Durch die Konventionen entsteht beim Schreiber und Leser auch eine gewisse Erwartungshaltung an den Verlauf der jeweiligen Interaktion im Thread. $\mathrm{Zu}$ Konventionen gehören auch Regeln, die je nach Forum mal strikter mal weniger strikt ausfallen und deren Einhaltung sowie mögliche Strafe bei Verletzung von Forum zu Forum abweicht. Die Regeln sind dabei von vornherein festgelegt und stehen für alle sichtbar auf einer Seite im jeweiligen Forum. Hierbei handelt es sich um Verhaltensregeln, wie z.B. der Verbot der Benutzung von Schimpfwörtern.

Für eine korrekte Interpretation ${ }^{38}$ von Äußerungen in Internetkonversationen erachte ich vor allem Hintergrundwissen und Kontextualisierungshinweise, die die Äußerung in einen Kontext setzen, für den Leser als unerlässlich. Nehmen wir folgendes Beispiel:

(6) Person A schaut sich online einen Film an, der Onlinestream stoppt plötzlich mitten im Film und der Film muss eine Weile laden. Es ist schon spät und Person A möchte diesen Film gerne zu Ende sehen, da ein anderer zu lange dauern würde, um ihn sich ganz anzusehen.

Person A: Ich hoffe, der Film lädt jetzt endlich weiter. Der mit den drei Muscheln dauert viel zu lange, um ihn jetzt noch anzufangen.

Person B: Das stimmt.

Aus dem Kontext der vorherigen Äußerung kann man schließen, dass es sich bei dem Der in Der mit den drei Muscheln auch um einen Film handeln muss. Dies verrät unser sprachliches Hintergrundwissen, denn „,der“ aus der zweiten Äußerung bezieht sich eindeutig auf „der Film“ aus der ersten Äußerung. Es bleibt jedoch das Problem mit der Aussage zu den drei Muscheln. Hier ist vor allem das geteilte Hintergrundwissen von Person A und B entscheidend, da Person A annimmt, dass Person B den Film, auf den Person A mit den drei Muscheln Bezug nimmt, kennt und somit die Äußerung richtig versteht, was auch der Fall zu sein scheint. Dabei muss das Wissen nicht unbedingt zum allgemeinen Hintergrundwissen gehören. Das Hintergrundwissen, das nur von Menschen innerhalb einer Gruppe verstanden

\footnotetext{
${ }^{38}$ Unter einer korrekten Interpretation verstehe ich im Rahmen dieser Dissertation die vom Schreiber intendierte Interpretation seiner Äußerung.
} 
wird, bezeichne ich als gruppeninternes Hintergrundwissen ${ }^{39}$ und nehme damit noch eine weitere Art des Hintergrundwissens zusätzlich zum soziokulturellen und sprachlichem Hintergrundwissen von Gumperz an. Im Bereich der kontextuellen Hinweise darf die Bedeutung der lautlichen Seite einer Äußerung natürlich nicht unterschätzt werden, jedoch glaube ich, dass diese unter bestimmten Bedingungen „mitgelesen“ bzw. „mitinterpretiert“ wird. Meiner Ansicht nach können wir uns mit Hilfe des Kontextes, des Hintergrundwissens, der bekannten Konventionen und unserer gesammelten kommunikativen Erfahrung die lautliche Seite beim Lesen vorstellen. Dies belegen die vielen korrekten Interpretationen von Äußerungen in Internetkonversationen sowie die Tatsache, dass nur wenige Nachfragen erfolgen. Als potenzielle Kontextualisierunghinweise kommen im Internet vor allem Emoticons, Großschreibung ganzer Wörter sowie Satzzeichen in Frage. Nur mit ihrer Hilfe können nonverbale Kontextualisierungshinweise schriftlich wiedergegeben werden, ohne Wortformen zu gebrauchen. Jedoch scheinen aus meiner Sicht die Emoticons zur Darstellung der lautlichen und visuellen Seite einer Äußerung in den meisten Fällen - eventuell spielen sie bei Ironie oder Sarkasmus eine Rolle - überflüssig in dem Sinne zu sein, dass sie die sprachliche Äußerung betonen, ohne eine zusätzliche Bedeutung zur Äußerung beizutragen bzw. die Interpretation der Äußerung zu verändern. Dazu passt auch, dass sie meistens am Ende einer Äußerung stehen. In diesen Fällen sind sie für die Interpretation unerheblich, da die Interpretation der Äußerung bereits abgeschlossen ist. Das Gleiche gilt möglicherweise auch für Ausrufe- und Fragezeichen. Anders sieht es meiner Meinung nach bei der Großschreibung ganzer Wörter und den mehrfach vorkommenden Punkten aus, die von den Schreibern benutzt werden, um eine andere Prosodie (Großschreibung) oder Sprechpausen zwischen zwei Wortformen bzw. Äußerungen (mehrfach vorkommende Punkte) auszudrücken. Da solche Hinweise aber nicht in allen Äußerungen vorkommen, muss sich der Leser nur auf das verlassen können, was er schriftlich vorfindet. Seine wichtigsten Hinweise sind somit aus meiner Sicht lexikalische Hinweise. Für den Schreiber im Internet ergeben sich zwei Möglichkeiten: entweder er macht sich Gedanken darüber, wie seine Äußerung aufgefasst werden kann und formuliert sie so deutlich wie möglich oder er setzt Wissen bei den anderen Gesprächspartizipanten voraus, um sich den Kontext einer Äußerung zu erschließen und die Äußerung korrekt zu interpretieren. Dies ist besonders innerhalb von Gruppen häufig der Fall.

\footnotetext{
${ }^{39}$ Mit gruppeninternem Wissen bezeichne ich jegliche Art des Wissens, das man zwar als Gruppenmitglied hat, was aber nicht unbedingt soziokulturell gebunden ist (also von allen Menschen einer bestimmten Sprachsystems/ einer bestimmten Kultur beherrscht wird).
} 
Auch in Internetkonversationen kommt es zu misslungener Konversation. Es stellt sich die Frage, warum das passiert. Gumperz geht in seiner Theorie zwar auf Missverständnisse zwischen Sprechern mit unterschiedlichen Ethnien und damit unterschiedlichen Sprachsystemen ein, beschreibt jedoch in geringerem Umfang wie und warum es $\mathrm{zu}$ Misskommunikationen zwischen Gesprächspartnern mit gleichem Sprachsystem kommt. Nach einem seiner Beispiele, das auf einem Vorfall in einem Flugzeug basiert (vgl. Gumperz (1982, 161)), schreibt er „Americans will have no difficulty identifying this interchange as a joke [...].“ Jedoch ist es meiner Ansicht nach falsch anzunehmen, dass alle Amerikaner eine Äußerung auf die gleiche Art und Weise interpretieren würden. Aus meiner Sicht ist die gleiche ethnische Angehörigkeit kein Garant für geteilte Konventionen, gemeinsames soziokulturelles und sprachliches Hintergrundwissen, gleiche kommunikative Erfahrungen und gleiche Kontextualisierungshinweise und daraus resultierend die gleiche Interpretation einer Äußerung, denn sehr viel hängt vom privaten Umfeld und den persönlichen Umständen (sozial, sprachlich, kulturell) des Hörers ab, die bestimmen, über welches Hintergrundwissen er verfügt, welche kommunikativen Erfahrungen gesammelt wurden und dementsprechend, welche Kontextualisierungshinweise er erkennt. Nehmen wir als Beispiel Deutschland: jemand, der aus dem Norden kommt, wird nicht unbedingt eine ironisch oder lustig gemeinte Äußerung eines Bayern als solche erkennen, obwohl beide Deutsche sind und Hochdeutsch, wenn auch nicht sprechen, so zumindest verstehen. Ob wir eine Äußerung korrekt interpretieren, ob wir Kontextualisierungshinweise erkennen und korrekt deuten und ob wir angemessen reagieren, uns also an die Konventionen halten, hängt demnach vor allem mit unseren gesammelten Erfahrungen zusammen und nicht unbedingt mit unserer ethnischen Zugehörigkeit (und damit dem gleichen Sprachsystem), die zwar die Wahrscheinlichkeit, die gleichen Konventionen und gleiches soziokulturelles und sprachliches Hintergrundwissen zu besitzen, wesentlich erhöht, aber eben nicht garantiert. Daher kommt es auch zu vielen Missverständnissen von Gesprächspartizipanten, die der gleichen Ethnie angehören und das gleiche Sprachsystem beherrschen. Natürlich ist dies auch im Internet der Fall. Erschwerend kommt hinzu, dass nicht jeder Russischmuttersprachler auch ethnischer Russe ist; es gibt im Internet keine Möglichkeit, die Angaben, falls überhaupt welche gemacht werden, zu überprüfen. Daher fasse ich in der vorliegenden Dissertation alle Mitglieder der untersuchten Forenthreads zu einer Sprechergemeinschaft zusammen. Als Grund für die Missverständnisse schließe ich die fehlende lautliche Seite sowie fehlende Mimik ${ }^{40}$ und Gestik ${ }^{41}$ aus, da es sonst

\footnotetext{
${ }^{40}$ Unter Mimik versteht man den ,[...] Einsatz des Gesichtsausdrucks [...] zu kommunikativen Zwecken. $\mathrm{M}$ [imik] und Gestik sind wichtiger Bestandteil der nonverbalen Kommunikation, sie bestimmen zusammen mit
} 
ständig zu Fehlinterpretationen kommen würde, was aber nicht der Fall ist. Dagegen spricht ebenfalls die Tatsache, dass auch in mündlichen Gesprächen Fehlinterpretationen von Äußerungen vorkommen. Die Ursache muss also in der Kommunikation zwischen den Gesprächspartizipanten samt dem Konversationsverlauf selbst zu finden sein. Der Sprecher kann am Missverständnis die Schuld sein, indem er sich nicht an übliche Konventionen hält oder Hintergrundwissen voraussetzt, welches der Hörer nicht besitzt. Der Hörer hingegen kann die Schuld tragen, wenn er z.B. Kontextualisierungshinweise nicht korrekt deutet oder die Konventionen nicht einhält.

Zusammenfassend lässt sich sagen, dass mein Ansatz davon ausgeht, dass diejenigen der Gumperz'schen Kontextualisierungshinweise, die nonverbaler Natur in Form von Prosodie, Mimik und Gestik sind, in Internetkonversationen zwar durch eine begrenzte Menge graphischer Mittel (Emoticons, Satzzeichen, Großschreibung usw.) potentiell angedeutet werden können, aber nicht die gleiche wichtige Rolle spielen wie in mündlichen Konversationen. Gerade die lautliche Gestalt der Äußerung wird meiner Ansicht nach mitgelesen. Umso wichtiger für die korrekte Interpretation einer Äußerung sind lexikalische Hinweise und das sprachliche, soziokulturelle und gruppeninterne Hintergrundwissen, kommunikative Erfahrungen, mit deren Hilfe z.B. Strategien erkannt werden, und Konventionen. Beim Interpretationsvorgang gehe ich von einem Prozess aus, bei dem Wortform für Wortform analysiert und interpretiert wird und aus dem sich erst durch das Hinzukommen von immer mehr Wortformen die Bedeutung der ganzen Äußerung ergibt; die Bedeutung der Äußerung ergibt sich demnach aus den Bedeutungen aller Wortformen und aus dem Kontext. Diesen Kontext müssen die Gesprächspartizipanten im Laufe des Gesprächs gemeinsam erschaffen, er kann aber auch vom Schreiber im Laufe seiner ersten Äußerung vorgegeben oder bereits als bekannt vorausgesetzt werden. Im letzten Fall muss der Leser auf Hintergrundwissen zurückgreifen. Das Gelingen oder Misslingen einer Interpretation und damit Interaktion hängt in Internetkonversationen von den oben genannten Faktoren ab. Was

der Augenkommunikation die Art der Beziehung, die Sprecher und Hörer zu Beginn und während einer Interaktion herstellen. Argyle hebt vier verschiedene Funktionen des Gesichtsausdrucks in der sozialen Interaktion hervor: (a) er zeigt den Gefühlszustand der Interagierenden; (b) er stellt eine kontinuierl. Rückkopplung dar (ob jemand das Gesagte versteht, überrascht ist, zustimmt); (c) er zeigt die Einstellung gegenüber anderen an; (d) er kann metakommunikativ wirken, indem er das, was jeweils gesagt wird, modifiziert und kommentiert. [...].“(Glück $(1993,391))$.

41 Gestik wird folgendermaßen definiert: „Repertoire und System kommunikativ verwendeter Körperbewegungen und -haltungen, v.a. Hand- und Armbewegungen. [...] Die G[estik] ist ein wichtiger Bestandteil des Multikanalsystems Kommunikation mit unterschiedl. Funktionen: als Demonstrativgesten, Betonungsgesten, Zeigegesten (auf jdn. zeigen), Berührungsgesten begleiten oder ersetzen sie das Gesprochene. Sie können außerdem der „Interpunktion“ des Gesprochenen dienen als auch der Gesprächssteuerung. Hauptfunktionen sind Illustration und Ergänzung sprachl. Äußerungen. [...].“ (Glück (1993, 223f.). 
den Konversationsverlauf und die Strategien angeht, so gibt es sowohl Gemeinsamkeiten mit der als auch Unterschiede zur gesprochenen Sprache, die durch das schriftliche Medium Internet und die in Internetgesprächen vorliegenden Konventionen bedingt sind. An dieser Stelle soll ein vereinfachtes Modell vorgestellt werden, wie meine Vorstellung von dem Interpretationsverlauf in Internetkonversationen aussieht. Dabei orientiere ich mich grob sowohl an den Ansätzen der interaktionalen Soziolinguistik als auch an Bierwisch (1983).

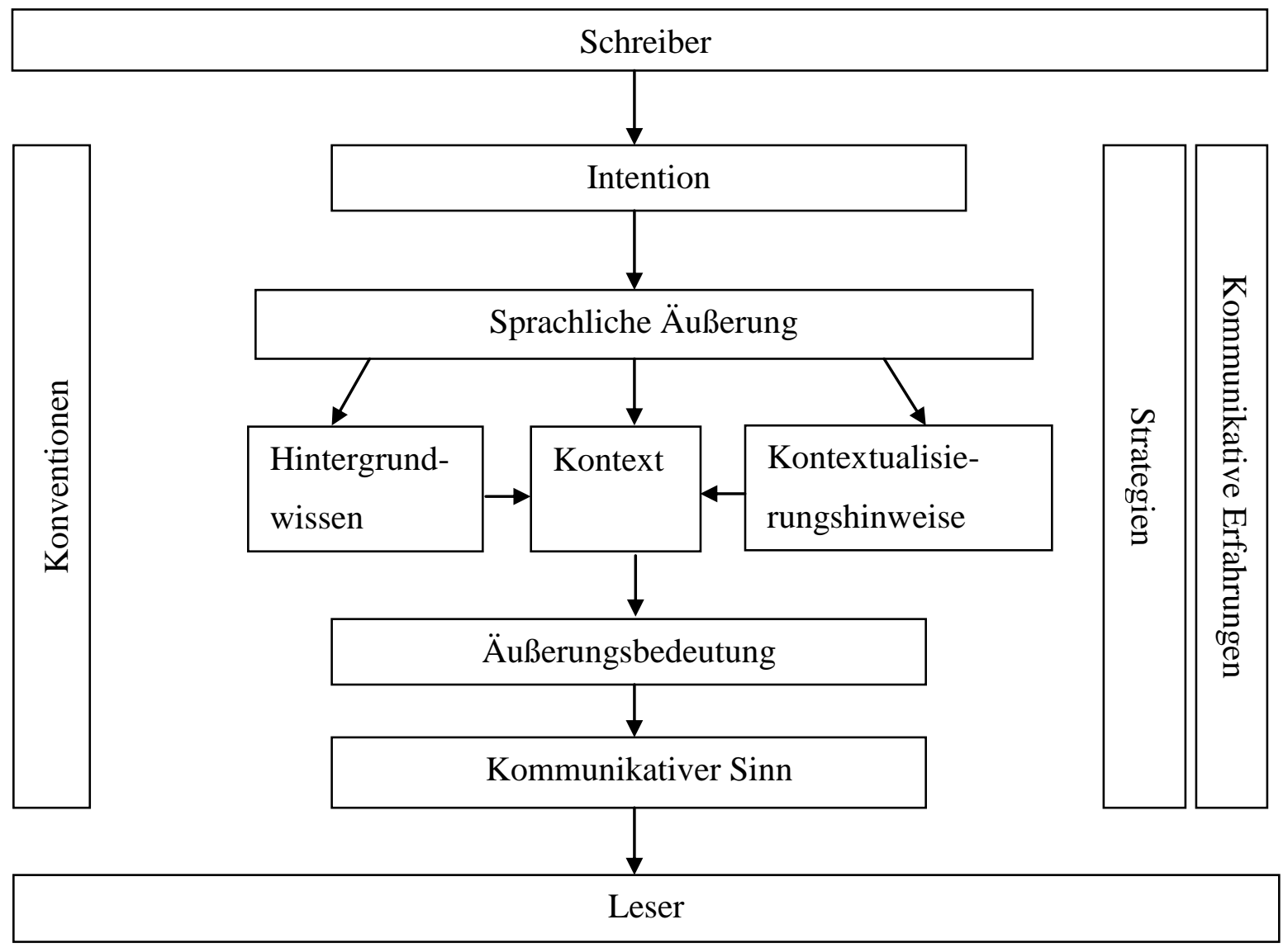

Abb.1 Grobe Vorstellung des Interpretationsverlaufs

Der Schreiber äußert sich mit einer bestimmten Intention, wobei er bestimmte Strategien benutzt und Konventionen einhält. Mit Hilfe von kommunikativen Erfahrungen kann der Leser die benutzten Strategien deuten und erkennen, welche Intention der Schreiber verfolgt. Die sprachliche Äußerung selbst besteht formal aus der sprachlichen Form und einem physikalisch messbaren Signal (vgl. Bierwisch $(1983,65)$ ). Sie muss dabei von vornherein formal und inhaltlich den gängigen Konventionen der jeweiligen Sprache entsprechen, so dass sowohl Schreiber als auch Leser die gleiche Erwartungshaltung an den Verlauf der Konversation und die Reaktionen auf bestimmte Äußerungen haben. Um die Bedeutung der Äußerung zu verstehen, wird diese in ihrem Kontext betrachtet, für den Hintergrundwissen und Kontextualisierungshinweise, die ebenfalls mit Hilfe von kommunikativen Erfahrungen 
erkannt werden, wichtig sind. Auf dieser Grundlage entscheidet sich der Leser für eine hypothetische Äußerungsbedeutung, die ,[...] kontextinterpretierte Äußerung [...] [nimmt dabei] in bezug auf einen bestimmten Interaktionsrahmen [...] einen speziellen kommunikativen Sinn [...] [an] und [wird] damit zu einem Sprechakt [...].“ (Bierwisch $(1983,65))$. In Abhängigkeit davon, ob eine weitere Äußerung erfolgt oder nicht, kann nun der Leser als neuer Schreiber reagieren.

Abschließend soll kurz die Wahl der Foren sowie die Beschaffung des sprachlichen Materials erläutert werden. Die untersuchten Foren wurden absichtlich nach mehreren Kriterien ausgewählt: 1. Das Forum muss einen frei zugänglichen „Plauder-“ bzw. „Floodthread“442 haben, der untersucht wird. Solche thematisch nicht begrenzten Threads haben den Vorteil, mit realen Gesprächssituationen, in denen Gesprächsteilnehmer sich begegnen und über alles reden können, vergleichbar zu sein; 2. Der untersuchte Thread muss über einen längeren Zeitraum laufen, um möglichst viele Beispiele für Konversationen zu enthalten; 3. Der Thread muss möglichst viele Beispiele mit gleichen Gesprächsteilnehmern enthalten; 4. Die Foren und Threads müssen thematisch unterschiedlich sein.

Ausgewählt wurden schließlich zwei Foren: ein Forum für Hacker/Programmierer („,hakerok“) und eines für Rollenspiele (,alohomora“). Ihre thematisch unterschiedliche Ausrichtung macht die Untersuchung noch interessanter im Hinblick auf Gemeinsamkeiten bzw. Unterschiede. Um an das sprachliche Material zu gelangen, bediene ich mich der Methode, die sowohl in der Soziolinguistik als auch in der interaktionalen Soziolinguistik üblich ist, nämlich der „Aufnahme“, deren sich die Gesprächsteilnehmer nicht bewusst sind. Die „Aufnahme“ bezieht sich bei mir jedoch nicht auf mündliche Kommunikation, sondern auf verschriftlichte Gesprächssituationen, die gespeichert und analysiert werden. Es muss jedoch beachtet werden, dass diese Gesprächssituationen nicht als spontane Rede gewertet werden können, da verschriftlichte Mitteilungen von den Usern beliebig oft verändert und korrigiert werden können, bevor sie abgeschickt werden; es gibt jedoch Versuche von Usern durch Diskursmarker wie hmmm... oder durch „...“ angedeutete Sprechpausen eine Art spontane Rede wiederzugeben.

\footnotetext{
${ }^{42}$ Ein solcher Plauderbereich im russischsprachigen Internet ähnelt einem Chatraum insofern, als dass kein spezifisches Thema durch bspw. eine Fragestellung, vorgegeben wird.
} 


\section{Kommunikation}

Im vorliegenden Kapitel werden anhand der Beispiele (7) - (25) aus den untersuchten Threads die Charakteristika der Internetkommunikation in Foren diskutiert. Dabei werden die Beispiele mit alltäglichen mündlichen Konversationen, wie sie z.B. zwischen Bekannten oder Freunden vorkommen, verglichen. Es geht also nicht um Konversationen zwischen Besuchern und Mitarbeitern von Institutionen oder fachliche Diskussionen in einer Gesprächsrunde o.ä.. Daher wurden aus den off-topic Plauderbereichen der Foren bestimmte Threads ausgesucht und untersucht.

\section{$\underline{\text { 3.1 Kommunikation und ihre Merkmale }}$}

Ein Grundprinzip der menschlichen Kommunikation bildet das sog. Kooperationsprinzip des Sprachphilosophen H. P. Grice: „Unsere Gesprächsbeiträge sind normalerweise keine Abfolge zusammenhangloser Bemerkungen, sonst wären sie nicht rational. Sie sind mindestens bis zu einem gewissen Grad charakteristischerweise kooperative Bemühungen, und jeder Gesprächsteilnehmer erkennt in ihnen in gewissem Maß einen gemeinsamen Zweck oder ein Bündel von Zwecken oder doch zumindest eine gemeinsam akzeptierte Richtung. Dieser Zweck oder diese Richtung können von Anfang an festgelegt sein (z. B. indem gleich zu Beginn ein Diskussionspunkt vorgeschlagen wird), sie können sich aber auch erst während des Gesprächs ergeben. Sie können ziemlich genau festgelegt sein, sie können aber auch ganz unbestimmt sein und den Gesprächsteilnehmern viel Freiheit lassen (wie in informeller Konversation). In jedem Stadium jedoch sind einige mögliche Gesprächsbeiträge ausgeschlossen, weil sie nicht in das Gespräch passen. Wir können also ein grobes Grundprinzip formulieren, dessen Befolgung man von Gesprächsteilnehmern (ceteris paribus) erwarten kann, nämlich: gestalte deinen Gesprächsbeitrag so, daß er dort, wo er im Gespräch erscheint, dem anerkannten Zweck dient, den du gerade mit deinen Kommunikationspartnern verfolgst. Man könnte dies das Kooperationsprinzip nennen.“ (Grice $(1980,113)) .{ }^{43}$

\footnotetext{
${ }^{43}$ Inwieweit das Kooperationsprinzip immer befolgt wird, ist fraglich. Eine bewusste Nichtbefolgung seitens eines der Gesprächspartizipanten kann z.B. in solchen Fällen vorliegen, in denen einer der Gesprächsteilnehmer ein bestimmtes Thema vermeiden möchte und daher plötzlich schweigt oder das Thema plötzlich wechselt, z.B.

Person A: „Und was hast du für eine Note bekommen?““

Person B: „Wir haben heute so schönes Wetter.“

In solchen Fällen erfolgt zwar eine Kooperation in dem Sinne, dass überhaupt eine Reaktion vorhanden ist,
} 
In diesem Zusammenhang geht Grice von vier Kategorien aus, die unterschiedliche Gesprächsmaximen ${ }^{44}$ nahelegen und ,[...] deren Befolgung meist zu Ergebnissen führt, die mit dem Kooperationsprinzip in Einklang stehen [...].“(Grice $(1980,113))$ :

- Kategorie der Quantität mit den beiden Maximen „Mache deinen Gesprächsbeitrag so informativ wie (für die augenblicklichen Gesprächszwecke) nötig“ und „Mache deinen Gesprächsbeitrag nicht informativer als nötig ${ }^{\star 45}$.

- Kategorie der Qualität mit der Hauptmaxime „Versuche deinen Gesprächsbeitrag so zu gestalten, daß er wahr ${ }^{46}$ ist “ $^{\text {“ }}$ und den beiden untergeordneten Maximen „Behaupte nichts, von dessen Wahrheit du nicht überzeugt bist“ und „Behaupte nichts, wofür du keine hinreichenden Beweise hast"“.

- Kategorie der Relation mit der Maxime „Sei relevant““. 47;48

- Kategorie der Modalität mit der Hauptmaxime „Sei klar“ und den weiteren Maximen „Vermeide Unklarheit im Ausdruck“, „Vermeide Mehrdeutigkeit“, „Vermeide Weitschweifigkeit“" und „Vermeide Ungeordnetheit“".

$\mathrm{Ob}$ all diese Maximen in der face-to-face-Kommunikation eingehalten werden, ist fraglich.

jedoch ist die Reaktion insofern nicht kooperativ als dass der Gesprächsbeitrag thematisch eigentlich ausgeschlossen ist.

${ }^{44}$ Eine weitere in der Wissenschaft verbreitete Bezeichnung für Gesprächsmaximen sind Konversationsmaximen (vgl. Bußmann (2008, 373f.)).

45 Über die zweite Maxime ließe sich laut Grice diskutieren, da es Argumente für und gegen sie gibt (vgl. Grice (1980, 113f.).

${ }^{46}$ Unter „wahr“ versteht Grice den Wahrheitsgehalt des Gesprächsbeitrags. Er hält die erste Maxime dieser Kategorie für die wichtigste Maxime überhaupt, der alle anderen Maximen folgen (Grice (1980, 114)).

${ }^{47}$ Grice führt an, dass es in Bezug auf diese Maxime unbeantwortete Fragen gibt: „Diese Maxime ist zwar kurz, aber ihre Formulierung birgt eine Reihe von Problemen in sich, die mir ziemlich zu schaffen machen. Es sind dies Fragen wie: Wieviel verschiedene Arten und Gesichtspunkte der Relevanz gibt es? Wie verändern sie sich im Verlauf eines Gesprächs? Wie läßt sich die Tatsache unterbringen, daß Gesprächsthemen durchaus gewechselt werden können usw." (Grice $(1980,114)$ ).

${ }^{48}$ Mit Relevanzsemantik beschäftigten sich Sperber \& Wilson (1995) und stellen fest, dass die Relevanz einer Äußerung vom Kontext, in dem diese geäußert wird, abhängt. Sie stellen folgende Definition auf: „An assumption is relevant in a context if and only if it has some contextual effect in that context. " (Sperber \& Wilson $(1995,122))$. Kontextuelle Effekte sind ,[...] a result of interaction between new and old information.“ (Sperber \& Wilson $(1995,109))$. So können durch die neue Information z.B. alte Annahmen gestärkt oder sogar bis zur Verwerfung geschwächt werden (vgl. Sperber \& Wilson $(1995,109)$ ). Ein kontextueller Effekt tritt nicht immer auf: „If all a contextualisation does is add all, some or none of the new information to the context without otherwise altering the context at all, then this contextualisation has no contextual effect. Otherwise, there is some contextual effect, in the form of an erasure of some assumptions from the context, a modification of the strength of some assumptions in the context, or the derivation of contextual implications." (Sperber \& Wilson (1995, 117)). Dabei gehen Sperber \& Wilson von mehr als einem Kontext aus: ,[...] the individual has at his disposal a particular set of accessible contexts. This set is partly ordered: each context (apart from the initial context) contains one or more smaller contexts, and each context (apart from the maximal contexts) is contained in one or more lager contexts. The set of accessible contexts is thus partly ordered by the inclusion relation. This formal relation has a psychological counterpart: order of inclusion corresponds to order of accessibility." (Sperber \& Wilson $(1995,142))$. Demnach sei eine Äußerung für ein Individuum erst dann relevant, wenn es in mindestens einem der für das Individuum zugänglichen Kontexte relevant ist (vgl. Sperber \& Wilson (1995, 144)). 
Selbst Grice (1980, 117f.) sagt, dass nicht alle Maximen, die unterschiedliches Gewicht haben (vgl. Grice (1980, 114)), stets auf eine Konversation zutreffen, denn die Befolgung dieser wird von Sprechern häufig absichtlich, z.B. zum Zwecke der Ironie oder Doppeldeutigkeit, missachtet.

Bei der Betrachtung, ob und in welchem Maße die Konversationsmaximen und das Kooperationsprinzip auch auf Threads im Internet zutreffen, erscheint es auf den ersten Blick, als würden die Konversationsmaximen, in allererster Linie die Maximen der Kategorien der Quantität und der Modalität, eingehalten werden und die Gespräche dem Kooperationsprinzip folgen. Die User bemühen sich darum, die Information in der Mitteilung möglichst kompakt, aber dennoch ausreichend, und so klar wie möglich darzustellen, damit es keine Missverständnisse gibt. Dennoch kommen unvollständige Äußerungen in einer Mitteilung vor. $^{49}$ Als unvollständige Äußerungen definiere ich solche Äußerungen, bei denen syntaktische Argumente des Verbs oder das Verb selbst fehlen. Hierbei handelt es sich teilweise um Ellipsen, teilweise aber auch um unvollständige Äußerungen, bei denen sich das fehlende Argument nicht aus dem Kontext heraus erschließen lässt. Der Leser muss somit beim Lese- und Interpretationsvorgang die fehlenden Komponenten selbst ergänzen. In der vorliegenden Dissertation habe ich dies anhand von Personalpronomen untersucht: von den 383 Äußerungen (220 aus dem ersten Thread und 163 aus dem zweiten Thread ${ }^{50}$ ), die die in diesem Kapitel vorgestellten Beispiele enthielten, findet man innerhalb des ersten Threads 45 und innerhalb des zweiten Threads 25 Äußerungen, bei denen das Personalpronomen weggelassen wurde. Das macht im ersten Thread 20,45\% und im zweiten Thread 15,38\% der Äußerungen aus. Gründe für das häufige Auslassen des Personalpronomens könnten zum Einen die Schnelligkeit und Ökonomie der Internetkommunikation sein und zum Anderen die morphologische Personenmarkierung am Verb. Häufig handelt es sich bei den ausgelassenen Personalpronomen um kontextuelle Ellipsen.

\footnotetext{
${ }^{49}$ Dabei gelten unvollendete Äußerungen als typisches Merkmal der gesprochenen Sprache, das in anderen Formen der Internetkommunikation häufig vorkommt: „Spokenness in LTC [live text commentary; Anm. d. Verf.] is further conveyed through characteristic sentence and clause structures, and numerous other phenomena more common in speech than in writing. In terms of sentence and clause structure, repetitions and incomplete sentences with ellipsis of subjects and verbs, characteristically occuring at the highpoints of the game, tend to convey a marked degree of immediacy and conversationalism in the written text. Many of the utterances manifest features that represent the constructional principles of spoken grammar (cf. Biber et al. 1999), such as prefaces and tags, non-clausal inserts, syntactic non-clausal units, and ellipsis in clausal units.

Inserts, for instance, constitute an interesting phenomenon whereby utterances can be expanded. Inserts can function as interactive devices that contribute towards increasing the dialogism of monologic texts. This concerns, above all, socalled interpolations (cf. Talbot 1995), which serve to interrupt the clause structure by means of inserting an explanatory or evaluative comment (or some other kind of a reader-oriented utterance, such as a rhetorical question)." (Chovanec $(2009,123))$.

${ }^{50}$ Die Äußerungen aus Beispiel (8) wurden dabei nicht berechnet, da sie noch einmal in (23) vorkommen.
} 
Untersucht man die Konversationsbeispiele jedoch genauer auf die Frage hin, ob alle Maximen eingehalten werden, zeigen sich gewisse Abweichungen von diesen. So wird bei der Quantität die zweite Maxime häufig nicht eingehalten, wie an Beispiel (7) zu sehen ist. In diesem Beispiel macht der User Azure seinen Gesprächsbeitrag informativer als es für die Gesprächssituation nötig ist: anstatt nur das Horoskop aufzuführen, gibt er/sie zusätzliche Informationen bezüglich seines/ihres Lieblingstages (hier: Freitag, der 13.), was für das Gespräch, in dem es um Horoskope geht, irrelevant ist.

Auch die zweite Kategorie der Qualität stellt ein gewisses Problem dar. Zwar lassen sich durch das Internet Informationen sehr schnell überprüfen, dennoch ist dies keine Garantie für den vollständigen Wahrheitsgehalt des Inhalts einer Äußerung. Dies gilt vor allem für Informationen aus dem privaten Leben, da Nutzer sich die Anonymität im Internet zu Nutze machen, um eine eigene Online-Identität zu erfinden.

Die Feststellung der thematischen Relevanz einer jeden Mitteilung und Äußerung gestaltet sich vor allem in nicht thematisch angelegten Threads als schwierig. Die Relevanz einer Mitteilung kann in solchen Threads meiner Ansicht nach nicht anhand des Themas gemessen werden, sondern daran, ob Reaktionen anderer Nutzer erfolgen: gibt es Reaktionen, ist die Mitteilung relevant. Die Missachtung dieser Kategorie hat ihren Höhepunkt bei den Vorgängen des Floodens ${ }^{51}$ und des Trollens.

Auch die Kategorie der Modalität gestaltet sich als problematisch. Zwar bemühen sich User darum, ihre Mitteilungen so klar wie möglich zu formulieren, jedoch gibt es viele Beispiele, in denen die Verfasser bewusst dagegen verstoßen. Wie in mündlicher Kommunikation kann dies dazu dienen, bewusst Ambiguität herbeizuführen: die Schreiber der Äußerungen spielen absichtlich mit der Mehrdeutigkeit der benutzten Wörter oder gebrauchen Ironie. Die Ironie stellt dabei eine andere Form der Mehrdeutigkeit dar, da ironisch gemeinte Äußerungen das Gegenteil von dem bedeuten, was gesagt wurde (vgl. Bußmann $(2008,308)$ ). (8) $\mathrm{T}_{1} \mathrm{M}_{2,2-7}$ ist ein gutes Beispiel dafür. Trotz der fehlenden lautlichen Realisierung lässt sich diese Mitteilung als Ironie erkennen. Bereits die Situation lässt auf Ironie schließen, da der User

\footnotetext{
51 To flood bedeutet im Kontext von Internetkonversation: „1. To overwhelm a network channel with mechanically-generated traffic; especially used of IP, TCP/IP, UDP, or ICMP denial-of-service attacks. 2. To dump large amounts of text onto an $\underline{I R C}$ channel. This is especially rude when the text is uninteresting and the other users are trying to carry on a serious conversation. Also used in a similar sense on Usenet.3. [Usenet] To post an unusually large number or volume of files on a related topic." (http://www.catb.org/jargon/html/F/flood.html).

Flooden lässt sich häufig dadurch erklären, dass die Nutzer auf diese Weise die Anzahl ihrer Beiträge im jeweiligen Forum steigern wollen. Denn in vielen Foren kann man bei einer bestimmten Anzahl von Beiträgen z.B. in eine hierarchisch höhere Gruppe aufsteigen.
} 
Erik seinen Vorschlag an andere User, im Falle, dass Programme etc. zum Hacken benötigt würden, die angegebene Nummer zu benutzen, in einem Forum für Hacker/Programmierer schreibt, dessen User sich in der Regel mit solchen Programmen usw. auskennen und im Falle eines Problems, sich an vertrauenswürdige Personen in anderen thematischen Threads des Forums wenden würden. Darauf spielt auch der User AgentMario mit seinen ironisch gemeinten Reaktionsäußerungen in $\mathrm{T}_{1} \mathrm{M}_{2,2-4}$ und $\mathrm{T}_{1} \mathrm{M}_{2,7}$ an, die dabei immer das Gegenteil von dem bedeuten, was geschrieben wurde: so ist z.B. das Dankeschön in $T_{1} M_{2,4}$ und $T_{1} M_{2,7}$ nicht als echtes Dankeschön für z.B. Hilfestellung gemeint. Die Prosodie wird beim Lesen mitinterpretiert. Beispiel (9) hingegen zeigt die Verwendung mehrdeutiger Wörter, die vor allem bei Anspielungen und Flirtversuchen benutzt werden. Die Mehrdeutigkeit offenbart sich hier an zwei Stellen. Die erste Stelle ist in der zweiten Äußerung der Mitteilung von Black Santa zu finden: neben der wörtlichen Bedeutung des Lexems opyжue (Waffe), kann dieses auch in einem entsprechenden Kontext eine sexuelle Konnotation bekommen (männliches Geschlechtsorgan). Die zweite Stelle eines mehrdeutig gemeinten Wortes ist $\mathrm{T}_{2} \mathrm{M}_{3,3}$ : mit игрушки (Spielzeuge) sind in diesem Kontext Waffen gemeint, was zusätzlich durch das Emoticon unterstrichen wird. Die Mehrdeutigkeit der verwendeten Lexeme ist jedoch nicht von vornherein gegeben, sondern entsteht in beiden Fällen in Beispiel (9) erst durch den entsprechenden Kontext. Neben der beabsichtigten Ambiguität, kommt es vor, dass sich die Schreiber unbewusst unklar ausdrücken, vgl. AgentMario und Klubnayk in Beispiel (10). In diesem Beispiel gibt es ein Missverständnis zwischen Klubnayk und AgentMario, weil er AgentMarios Frage $\left(\mathrm{T}_{1} \mathrm{M}_{2}\right)$ anders versteht, als sie gemeint war. Dabei scheint AgentMario als Schreiber nicht darauf zu achten, ob seine Äußerung so verstanden wird, wie er sie gemeint hat oder es ist ihm nicht bewusst, dass seine Äußerung anders verstanden werden könnte, da sie inhaltlich unvollständig formuliert ist. Außer den Gesprächsmaximen haben Gespräche für Grice folgende, seiner Meinung nach „,...] für kooperative Handlungen allgemein gültige Merkmale [...]“ (Grice $(1980,116))$ :

- „Die Teilnehmer haben ein unmittelbares, gemeinsames Ziel, z. B. die Reparatur eines Autos. Ihre Fernziele können natürlich miteinander im Konflikt stehen. Vielleicht will jeder das Auto nur deshalb wieder in Gang setzen, um damit davonzufahren und den anderen im Stich zu lassen. In Dialogen gibt es gleichfalls ein gemeinsames Ziel, selbst wenn es, wie bei Gesprächen über den Gartenzaun, nur ein zweitrangiges Ziel ist, nämlich daß sich die Gesprächspartner für den Augenblick gegenseitig mit den Gesprächsinteressen des anderen identifizieren. 
- Die Beiträge der Gesprächspartner sollten miteinander verzahnt sein, d. h. gegenseitig voneinander abhängig sein.

- Es besteht (meist stillschweigend, möglicherweise auch explizit) Einigkeit darüber, daß bei sonst gleichen Voraussetzungen die gemeinsame Aktion in angemessenem Stil weitergehen soll, solange nicht beide Teilnehmer sie beenden wollen. Man geht nicht einfach weg oder fängt mit etwas anderem an.“

Sucht man diese Merkmale in Internetkonversationen, so stellt man fest, dass sie in unterschiedlichem Maße zutreffen. Es gibt Interaktionen, die den überwiegenden Teil bzw. alle Merkmale, die Grice anführt, enthalten, vgl. Beispiel $(11)^{52}$. In dieser Interaktion sieht man die Identifikation der Gesprächsteilnehmer anhand des gemeinsamen Interesses an bestimmten Autos. Die Beiträge ${ }^{53}$ sind miteinander verzahnt und nehmen Bezug aufeinander. Auffällig ist das abrupte Ende der Interaktion. Man könnte meinen, dass damit das dritte Merkmal fehlt. Dies ist jedoch nicht der Fall, da die Übereinkunft der User über das Ende der Konversation nicht durch Worte, sondern dadurch angedeutet wird, dass sich niemand mehr zu dem entsprechenden Thema meldet. Somit ist deutlich, dass alles Relevante gesagt wurde. Gleichzeitig gibt es auch viele Situationen, auf die diese Merkmale nicht zutreffen, denn nicht aus allen Beiträgen im Internet entwickelt sich ein Dialog. Zum Einen kann es vorkommen, dass niemand auf den Beitrag reagiert, zum Anderen verfolgt nicht jeder Schreiber mit seinem Beitrag das Ziel, eine dem Kooperationsprinzip entsprechende Interaktion zu beginnen oder fortzuführen $^{54}$, d.h. nicht jeder User hat ein Gespräch zum Ziel, sondern möchte sich nur mitteilen, ohne großes Interesse an Reaktionen anderer Nutzer zu haben. In solchen Fällen haben Redebeiträge keinerlei Bezug zueinander, wie Beispiele (12) und (13) zeigen. In beiden Beispielen sind jeweils drei aufeinander folgende Beiträge zu sehen, die sich jedoch nicht aufeinander beziehen. Da die Beispiele aus beiden Threads stammen, kann man nicht sagen, es handele sich um ein Phänomen eines einzelnen Forums. Meiner Meinung nach können solche Situationen nicht als Konversationen gewertet werden, da keine Reaktion der anderen Leser wahrnehmbar ist. Ich bezeichne dies als Menge von Einzelbeiträgen. Grundsätzlich ist jeder Beitrag, der an niemanden adressiert ist, ein Einzelbeitrag. Ob sich daraus eine Konversation entwickelt, d.h. ob andere User darauf reagieren wie es in Beispiel (12) $\mathrm{T}_{4} \mathrm{M}_{2}$ $\mathrm{T}_{4} \mathrm{M}_{4}$ der Fall ist, hängt von unterschiedlichen Faktoren ab.

\footnotetext{
${ }^{52}$ Die Mitteilungen von Yodjer (Mitteilung $\mathrm{T}_{3} \mathrm{M}_{1}$ ) und Resilved (Mitteilung $\mathrm{T}_{4} \mathrm{M}_{1}$ ), die beide thematisch nicht zum Rest passen und somit nicht dem Kooperationsprinzip entsprechen, müssen außer Acht gelassen werden.

${ }^{53}$ In der vorliegenden Dissertation werden die Begriffe Redebeitrag und Beitrag synonym gebraucht.

${ }^{54}$ Vgl. z.B. Trolling, wo Äußerungen von Sprechern eingeworfen werden, um den Redefluss der anderen Forumteilnehmer absichtlich zu stören.
} 
Wir können an dieser Stelle kurz festhalten, dass die Gesprächsmaximen von Grice Idealisierungen sind, die weder in mündlichen noch in Internetkonversationen von allen Gesprächspartnern stets eingehalten und befolgt werden. Jedoch können die Merkmale, die Grice für mündliche Kommunikation annimmt, im Internet in solchen Situationen gefunden werden, in denen es eine Äußerung und eine Reaktion auf diese Äußerung gibt; diese Situationen können als Konversationen bezeichnet werden. In ihnen, wenn sie sich entwickeln, werden von den Partizipanten feste spezielle Ziele verfolgt, sei es die Beantwortung einer Frage oder die Diskussion eines Vorfalls/Problems, wenn auch nur für die kurze Zeit dieser Gespräche. Die Konversationen in den Threads besitzen also die gleichen Merkmale, die Grice für mündliche Konversationen annimmt. Jedoch muss beachtet werden, dass nicht jeder Beitrag dem Zweck einer Konversation dient. So entsteht eine Menge von Einzelbeiträgen. Hierbei handelt es sich um ein Merkmal, welches die Kommunikation in Internetthreads von anderen Kommunikationsarten unterscheidet.

Neben den oben bereits erwähnten Merkmalen, gibt es noch weitere Merkmale, die die Kommunikation in Internetthreads ausmachen und die im Folgenden anhand der beiden Threads vorgestellt werden. Auffällig ist, dass im ersten Thread ein Teil der User sich selbst und andere anders bezeichnen als mit dem entsprechenden Usernamen, so wird z.B. der User Tempest in (7) $\mathrm{T}_{3} \mathrm{M}_{2}$ mit Mendik und in (17) $\mathrm{T}_{2} \mathrm{M}_{2}$ mit Professor angesprochen. Dies deutet darauf hin, dass die User sich bereits aus einem anderen Thread kennen, aber in diesem Thread andere Identitäten annehmen und somit innerhalb eines Forums unterschiedliche Identitäten haben können. Dies lässt sich im zweiten Thread nicht finden. Auf den ersten Blick fällt auch auf, dass Grußformeln fehlen, z.B. (15): ohne zu grüßen - wie es in mündlicher Kommunikation üblich wäre - wird in der ersten Mitteilung sofort der Zweck dieses Threads erläutert, nämlich einen Floodthread zu erschaffen, in dem das Flooden ungestraft bleibt. Nach der ersten Mitteilung gibt es eine Spaltung: in einem Teil der folgenden Mitteilungen wird das Flooden $\left(\mathrm{T}_{2} \mathrm{M}_{1}, \mathrm{~T}_{2} \mathrm{M}_{2}, \mathrm{~T}_{2} \mathrm{M}_{3}\right)$ praktiziert, während in einem anderen $\left(\mathrm{T}_{1} \mathrm{M}_{2}, \mathrm{~T}_{1} \mathrm{M}_{3}\right)$ darüber diskutiert wird. Daran wird bereits deutlich, was später in dem Abschnitt zum Konversationsverlauf behandelt wird: thematisch zusammenhängende Mitteilungen müssen einander nicht direkt folgen.

Das Fehlen von Begrüßungen in diesem und in anderen Beispielen bedeutet jedoch nicht, dass sie immer fehlen, wie Beispiel (16) zeigt. Dies unterscheidet den zweiten vom ersten Thread, 
in dem insgesamt ein häufiges Fehlen jeglicher Formalia in Form von Begrüßungen oder Verabschiedungen, die typisch für eine mündliche Interaktion sind, auffällt. Von den 1081 Redebeiträgen $^{55}$ des ersten Threads, die für die vorliegende Dissertation untersucht wurden, begannen neun mit привет und zwei mit здраствуйте (oder einer davon abgeleiteten Form) und nur zwei Redebeiträge endeten mit einer Verabschiedung in Form von ,всем [...] доброго дня“ und „С уважением [...]“. Der zweite Thread enthielt hingegen im untersuchten Zeitraum 880 Beiträge, von denen 25 mit привет (oder einer orthographischen Entstellung davon wie z.B. превед), einer mit dem Verb приветствовать, fünf mit здравствуйте, einer mit доброе утро, einer mit с новым днем, einer mit хай und einer mit Всем доброго времени суток begannen und sieben Beiträge, in denen sich die User mit спокойной ночи (fünf), mit споки (einer) oder mit пока (einer) verabschiedeten. Damit enthielt der erste Thread nur 1,01\% Beiträge, die mit einer Begrüßung anfingen gegenüber 3,97\% aller Beiträge im zweiten Thread, die eine Art der Begrüßung enthielten. ${ }^{56}$ Auch was die Anzahl der Verabschiedungen, die deutlich geringer ausfällt als die Anzahl der Begrüßungen, betrifft, so zeigen sich zwischen den beiden Threads deutliche Unterschiede: $0,19 \%$ (Thread 1) gegenüber $0,79 \%$ (Thread 2). ${ }^{57}$ Das soll jedoch keinesfalls bedeuten, dass es im zweiten Thread eher als im ersten eine Konvention sei, sich zu Beginn eines Gesprächs zu grüßen und am Ende zu verabschieden; es scheint vielmehr so zu sein, dass wenn gegrüßt wird, die anderen User zurückgrüßen. Das Fehlen von Begrüßungen im ersten Thread ist jedoch kein Hinweis dafür, dass die Nutzer unhöflich oder unfreundlich seien. Vielmehr ist ein freundlicher und netter Umgang miteinander ein charakteristisches Merkmal des ersten Threads, was sich in Beispiel (17) daran zeigt, wie häufig neben Gratulationen auch liebevolle Koseformen wie солнцо (Formen davon sind in $\mathrm{T}_{2} \mathrm{M}_{2}$ und $\mathrm{T}_{2} \mathrm{M}_{4} \mathrm{zu}$ finden), конфетулечка любимая $\left(\mathrm{T}_{2} \mathrm{M}_{5}\right)$, зая моя $\left(\mathrm{T}_{2} \mathrm{M}_{8,5}\right)$ etc. vorkommen. Der Grund dafür, dass sich offensichtlich kein User an fehlenden Begrüßungen oder Verabschiedungen stört, könnte unter anderem auch an der Ökonomie liegen, die von der Kommunikation im Thread erwartet wird. Die Ökonomie gehört somit zu Konventionen in der Threadkommunikation. Das Beispiel (17) zeigt ein weiteres Merkmal: über das Kürzel „P.S.“58 bzw. „P.S.S.“ wird ein Themenwechsel markiert. Dass dieser aber nicht immer markiert wird, zeigen z.B. Beispiel (15) sowie weitere Beispiele aus dem zweiten Thread. Das Fehlen von Überleitungen von

\footnotetext{
${ }^{55}$ Für die Berechnung wurden in beiden Threads alle Beiträge im untersuchten Zeitraum miteinbezogen, da sich daran am Besten der Unterschied zwischen beiden Foren aufzeigen lässt.

56 Diese und folgende Zahlen wurde nochmal als Diagramme auf den letzten Seiten dieses Abschnitts zusammengefasst.

${ }^{57} \mathrm{Zu}$ Begrüßungen/Verabschiedungen siehe Abb.2.

${ }^{58}$ Die russische Entsprechung auf der Tastatur ist „Zy Sy“, was sich häufig statt P.S. finden lässt.
} 
einem Thema zum nächsten führt dazu, dass die Interaktionen „,bruchstückhaft“ wirken und thematisch schnell wechselnd sind. Neben P.S. als Markierung eines Themenwechsels kommen auch Einleitungen vor, vgl. (7) $\mathrm{T}_{2} \mathrm{M}_{1,4}$, wo das Lesen des Horoskops eingeleitet wird. Dabei war bei der Untersuchung der Threads festzustellen, dass die User des ersten Threads den Übergang in ein neues, inhaltlich unabhängiges Thema über „P.S.“ favorisieren. Von den in diesem Kapitel vorgestellten Beispielen stammen sieben aus dem ersten Thread und in drei davon - nämlich in den Beispielen (12), (17) und (24) - lassen sich Übergänge in ein inhaltlich neues Thema über P.S. ${ }^{59}$ finden. Das macht fast die Hälfte der sieben vorgestellten Beispiele in diesem Kapitel aus. In den in diesem Abschnitt vorgestellten Beispielen des zweiten Threads findet sich hingegen kein Beispiel für eine Überleitung in ein neues Thema über P.S. Das einzige Beispiel, in dem innerhalb eines Redebeitrags ein zweites Thema eingeführt wird, ist Beispiel (10), wo der User AgentMario in $T_{2} M_{1,3}$ von dem Thema davor zu einem anderen Thema wechselt dies durch $i$ ksati markiert. Als Alternative zum Wechsel des Themas innerhalb eines Beitrags kann entweder ein zweiter Beitrag verfasst, z.B. (7) Mitteilung $\mathrm{T}_{3} \mathrm{M}_{6}$ und $\mathrm{T}_{4} \mathrm{M}_{2}$ von Ksanka, oder im Nachhinein ein Beitrag noch durch dobavleno ergänzt werden, z.B. in (10) $\mathrm{T}_{1} \mathrm{M}_{4}$. Ein weiteres Merkmal der Kommunikation in Internetthreads ist die Möglichkeit parallel besprochener Themen wie Beispiel (18) aus dem ersten Thread zeigt. Nachdem in $\mathrm{T}_{1} \mathrm{M}_{1}-\mathrm{T}_{1} \mathrm{M}_{3}$ u.a. die Aussage des Bildes aus $\mathrm{T}_{1} \mathrm{M}_{1}$ verhandelt wird, gibt es in $T_{2} M_{1}$ und $T_{3} M_{1}$ gleich zweimal einen Wechsel des Themas: in $T_{2} M_{1}$ wechselt Black Santa das Thema zum Bereich des Essens, während in $\mathrm{T}_{3} \mathrm{M}_{1}$ Vesnuška mitteilt, dass sie bereits seit einem Monat Mitglied des Forums ist. Beide Themenwechsel sind unvorbereitet und für den Leser in dem Sinne unerwartet, als dass er statt Reaktionen auf die Mitteilungen davor neue Themen präsentiert bekommt. Erst $a b B_{6}$ bestehen die Mitteilungen aus Reaktionen auf eine bzw. auf mehrere thematisch unterschiedliche Mitteilungen davor. Hieran zeigt sich, dass es offenbar nicht ungewöhnlich für die Kommunikation in Threads ist, dass man nicht bei einem Thema bleibt, sondern sukzessive andere Themen auftauchen, die entweder bis zur nächsten Reaktion auf das erste Thema oder parallel diskutiert werden. Das liegt nicht zuletzt auch daran, dass nicht jeder Nutzer ständig online ist und somit nicht gleich reagiert. Häufig werden solche Mitteilungen oder Beiträge aber auch überlesen bzw. keiner geht auf sie ein. Erfolgt jedoch eine Reaktion, so entstehen parallele Gesprächsstränge, die wenn sie ohne Zitate oder ähnliche Verweise laufen - für ein gewisses Durcheinander im Konversationsablauf sorgen können, auch weil zwischen den Reaktionen einige Zeit vergehen

\footnotetext{
${ }^{59}$ Siehe hierzu Abb.4.
} 
kann. Die User wissen aber in der Regel, mit wem und worüber sie sich gerade unterhalten, so dass keine Probleme bestehen. Hilfreich ist dabei der dauerhafte Bestand aller Mitteilungen, die auf diese Weise jederzeit nachgelesen werden können. Darüber hinaus liegt ein schneller Wechsel der Gesprächsteilnehmer vor, die nicht von vornherein feststehen, da kein User weiß, mit welchen der anderen User genau er sich unterhalten wird. Beispiele mit auftauchenden thematisch zum ersten Thema unpassenden Mitteilungen sowie mit einem schnellen Wechsel der Gesprächsteilnehmer kommen auch häufig im anderen Thread vor (z.B. Beispiel (11)) und stellen somit ein typisches Kommunikationsmerkmal dar. Dabei steht das Auftauchen solcher thematisch unpassender Mitteilungen im Konflikt mit dem Kooperationsprinzip. Eine weitere typische Eigenschaft ist, wie oben bereits erwähnt, dass die schriftlich festgehaltenen Gespräche, technisch bedingt, länger verfügbar sind als Gespräche in der mündlichen Kommunikation, die flüchtig ist. Dies ermöglicht, immer wieder auf bestimmte Äußerungen zurückkommen zu können. ${ }^{60}$ So können Äußerungen auch nach längerer Zeit wieder aufgegriffen und von anderen Nutzern kommentiert werden, siehe den zeitlichen Abstand in Beispiel (19). Ermöglicht wird das durch die Zitatfunktion, durch die die User klar stellen, auf welche Äußerung bzw. Mitteilung (falls es die ganze Mitteilung und nicht nur eine Äußerung ist) sich ihre eigene Mitteilung bezieht. Ebenfalls durch die Technik wird es Usern ermöglicht, die geschriebenen Äußerungen zu korrigieren, so dass die anderen User vorausgesetzt man ist schnell genug mit seiner Korrektur - einen Fehler nicht bemerken. Wird der Fehler dennoch bemerkt, hängt es von den Usern ab, ob sie ihn kommentieren oder nicht. ${ }^{61}$ Während im ersten Thread sich kein Beispiel für das Kommentieren findet, gibt es einige im zweiten Thread, vgl. Beispiel (20). Hier sieht man an dem Zitat in Lelikas Mitteilung $\mathrm{T}_{1} \mathrm{M}_{3}$, dass ein Rechtschreibfehler in der Originalmitteilung vorlag und im Nachhinein durch eine Korrektur vom User geändert wurde, ohne, dass die Änderung angezeigt wird. Ebenfalls ist es in Threads technisch möglich, seine eigene Mitteilung nach einiger Zeit zu ergänzen, vgl. Beispiel (21) $\mathrm{T}_{1} \mathrm{M}_{2}$ oder non- $\mathrm{T}_{3} \mathrm{M}_{1}$. Angezeigt wird dies durch dobavleno in Kombination mit der Angabe der Zeit, nach welcher die Nachricht ergänzt wurde.Ein weiteres Merkmal ist das oben bereits erwähnte Trolling, das hier an Beispiel (21) aus dem zweiten Thread verdeutlicht werden soll. Dabei stören die beiden User Aligarh (non$\mathrm{T}_{2} \mathrm{M}_{1}$ ) und nikitaSWAT (non- $\mathrm{T}_{3} \mathrm{M}_{1}$ ) den Dialog der beiden anderen Nutzer. Auffällig ist, dass Trolling nur im zweiten Thread vorgekommen ist und somit nicht als Merkmal bezeichnet

\footnotetext{
${ }^{60}$ Dies gilt sowohl für Foren als auch für viele Chaträume, wo Gespräche in der Regel ebenfalls gespeichert werden und jederzeit abrufbar sind.

${ }^{61}$ Häufig hängt es vom Grad der Beliebtheit des jeweiligen Users ab: wird er von anderen Usern nicht gemocht, ist die Wahrscheinlichkei viel höher, dass sie einen Fehler kommentieren.
} 
werden kann, das alle Threads gleichermaßen betrifft.

Einige allgemeine Merkmale sind von vornherein durch die Zugehörigkeit zu einer bestimmten Systemart gegeben: „Another way of viewing message processing is in terms of how it relates to the technique of signal transmission. The data transmission can be "one-way" or "two-way" (Cherny, 1995; cf. Capron, 1990; Walther, 1996b). One-way transmissions are sent one message at a time. One-way transmission characterizes electronic mail messages that are created by the sender, and then sent via the network (e.g., Internet or Intranet) to the receiver who then reads the message. [...] Two-way transmissions, in contrast, are sent as a continuous stream (Capron, 1990). Two-way transmission occurs when a message created by a sender is transmitted to a receiver simultaneous with its production. (There is a delay as measured in milliseconds, due to system signal transformations of digital to analog and back to digital, but this is difficult to detect with the human senses.) The telephone is a device that sends spoken two-way transmissions.“ (Anderson et al (2010, 4)). Das System gibt aber auch weitere Merkmale von vornherein vor: „Systems can be said to predispose communication to nonsimultaneity (i.e., asynchronous, one-way systems, such as email, discussion boards, blogs), in which conversation is isolated spatially, chronologically, and contextually; near simultaneity (i.e., synchronous, one-way systems, as in instant messaging, multiparticipant chat rooms, and text chat in multiplayer games), in which users typically respond to others' comments as soon as they are received; and high simultaneity (i.e., synchronous, two-way systems, such as the VAX "phone" and the contemporaneous UNIX "talk" programs), in which not only immediate responses are possible, but also communications overlap.“ (Anderson et al $(2010,5)$ ). Nach dieser Aufteilung gehören die in der vorligenden Dissertation untersuchten Threads $\mathrm{zu}$ einem one-way transmission system, deren Konversationen nicht simultan ablaufen. ${ }^{62}$ Für solche Systeme ist ebenfalls das in den meisten Fällen fehlende sofortige Feedback charakteristisch. Das liegt daran, dass - wie oben bereits erwähnt - nicht jeder User jederzeit online ist. Als Folge von einem fehlenden sofortigen Feedback tauchen thematisch nicht dazugehörige Mitteilungen anderer User auf und sorgen auf diese Weise für ein Durcheinander bei der Reihenfolge der Schreiber und Themen. Im Gegensatz zu mündlicher Kommunikation wird das sofortige Feedback aber auch nicht erwartet.

\footnotetext{
${ }^{62}$ Es gibt jedoch auch Threads, die eine Mischung aus gar nicht simultan und simultanähnlich ablaufenden Konversationen darstellen, da es auch vorkommt, dass die User sofort auf eine Mitteilung reagieren und den Thread wie eine Art Chat mit längeren Mitteilungen verwenden, vgl. die zeitliche Abfolge der Mitteilungen auf der Seite http://www.pustoty.net/showthread.php?t=9415. Die User dieses Threads reagieren häufig sehr schnell auf Mitteilungen, so dass in solchen Fällen ein sofortiges Feedback vorliegt. Auch in den untersuchten Threads kommt so etwas gelegentlich vor, wenn auch wesentlich seltener und scheinbar eher zufällig.
} 
Zusammenfassend lässt sich feststellen, dass die Grice'schen Konversationsmaximen in einigen Fällen gut umgesetzt (vgl. kaum unvollendete, sondern möglichst klar formulierte Äußerungen) werden, während sie in anderen Fällen von vielen Usern häufig missachtet werden (vgl. z.B. die Missachtung der Maximen beim Trolling). Auch das Kooperationsprinzip wird nicht immer befolgt. Was die Kommunikationsmerkmale angeht, so gibt es sowohl Gemeinsamkeiten als auch deutliche Unterschiede zwischen der face-to-faceKommunikation und der in den untersuchten Threads. Anhand der beiden Threads lassen sich einige charakteristische Merkmale der Internetkommunikation feststellen, die für das Medium Internet spezifisch sind und auf diese Art in der „realen“ Welt nicht vorkommen könnten, ohne den oder die Gesprächspartner zu irritieren:

1) Einander folgende Redebeiträge müssen sich nicht auf einander beziehen.

2) Gespräche fangen abrupt an und hören abrupt auf. Das Gespräch ist beendet, sobald keiner mehr zu dem jeweiligen Thema schreibt. Verabschiedungen fehlen.

3) Häufig fehlende Überleitungen zwischen den Gesprächsthemen verbunden mit einem häufigen Themenwechsel.

4) Es ist für den Einzelnen nicht von vornherein ersichtlich, wer die Gesprächspartner sein werden, d.h. wer sich von den möglichen Usern, die online sind, an der Interaktion beteiligen wird.

5) Der Beitrag bzw. die Mitteilung muss an niemanden direkt gerichtet sein.

6) Möglichkeit mehrerer parallel besprochener Themen.

7) Möglichkeit auch weit zurückliegende Äußerungen neu aufzugreifen, ohne, dass diese im Zusammenhang mit dem derzeit Besprochenen stehen.

Aber auch zwischen den Threads gibt es Unterschiede. Ein einfaches Diagramm gibt einen Überblick über die Verwendung von Begrüßungen und Abschiedsformeln (die y-Achse gibt die Prozentzahl in Bezug auf alle Mitteilungen in den Threads im untersuchten Zeitraum an): 


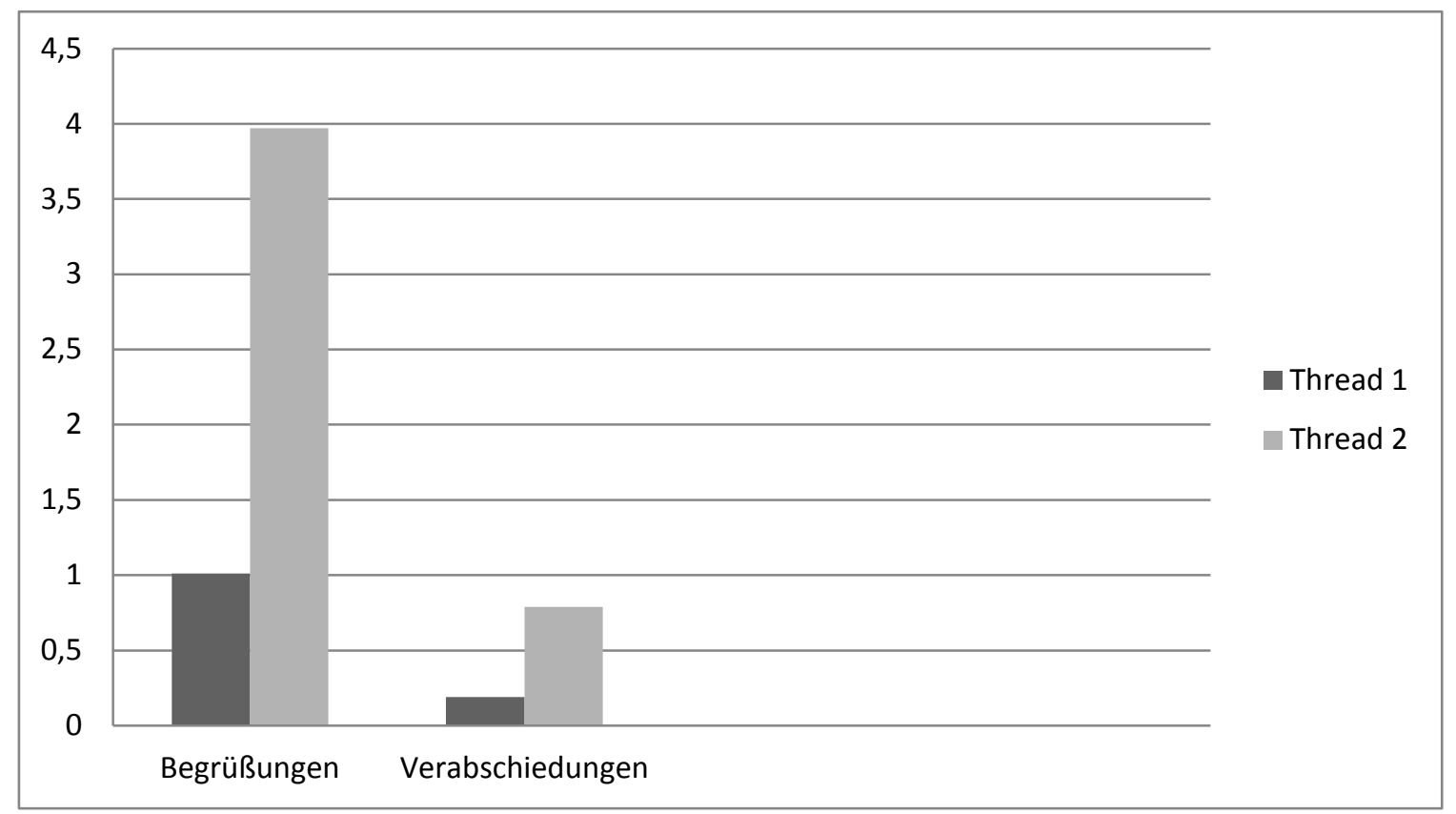

Abb. 2 Verwendung von Gruß- bzw. Abschiedsformeln in \%

Jedoch stört sich kein User an der Nichtverwendung dieser, was den Eindruck erweckt, das Fehlen von Gruß- bzw. Abschiedsformeln, das möglicherweise der Ökonomie der Kommunikation in Internetthreads geschuldet ist, sei konventionell.

Auch bezüglich anderer Merkmale gibt es Unterschiede zwischen beiden Threads, wie Abb. 3 und Abb. 4 zeigen (die y-Achse gibt die Prozentzahl in Bezug auf die im vorliegenden Kapitel untersuchten Beispiele an):

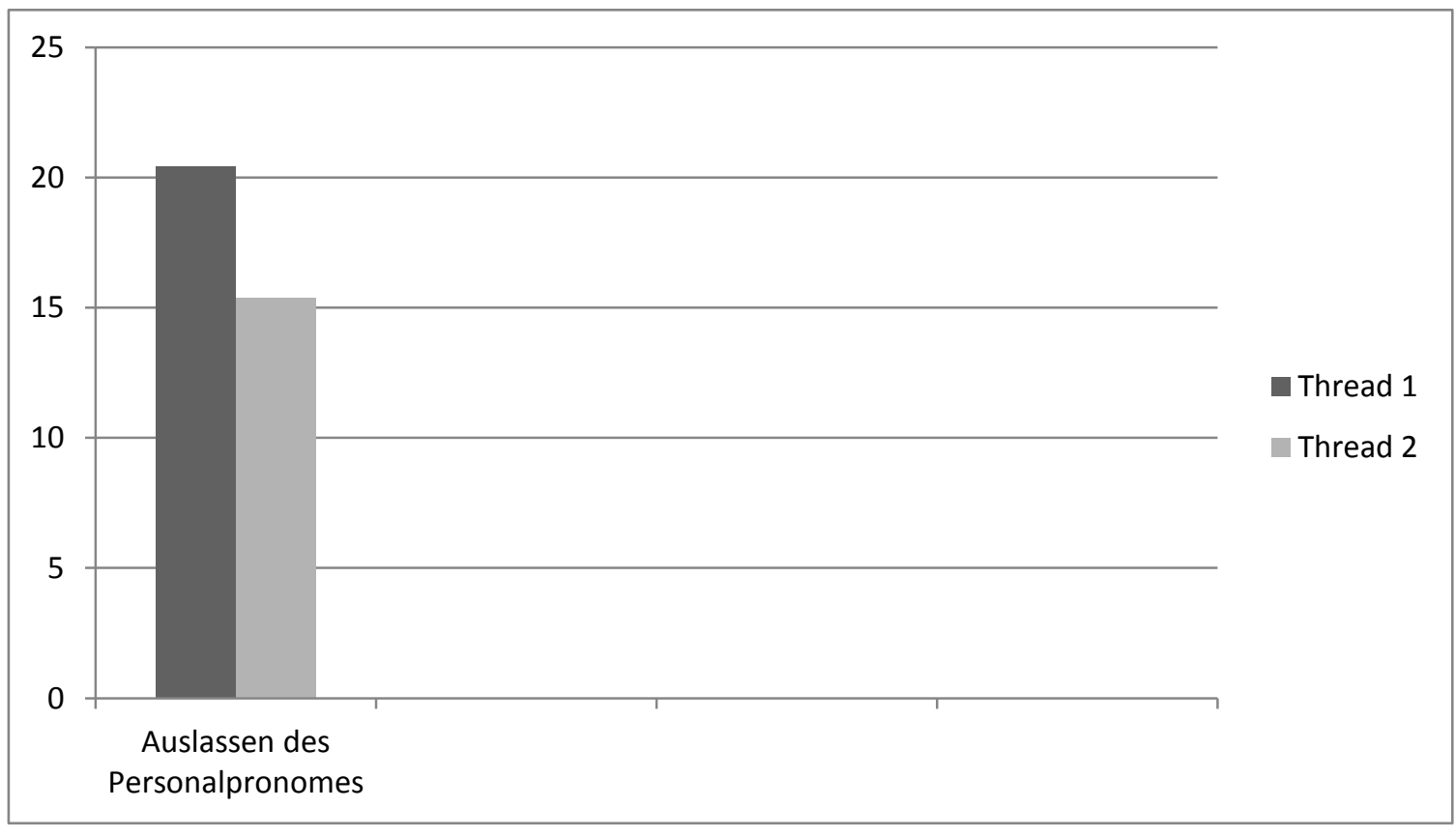

Abb. 3 Auslassen des Personalpronomens in \% 


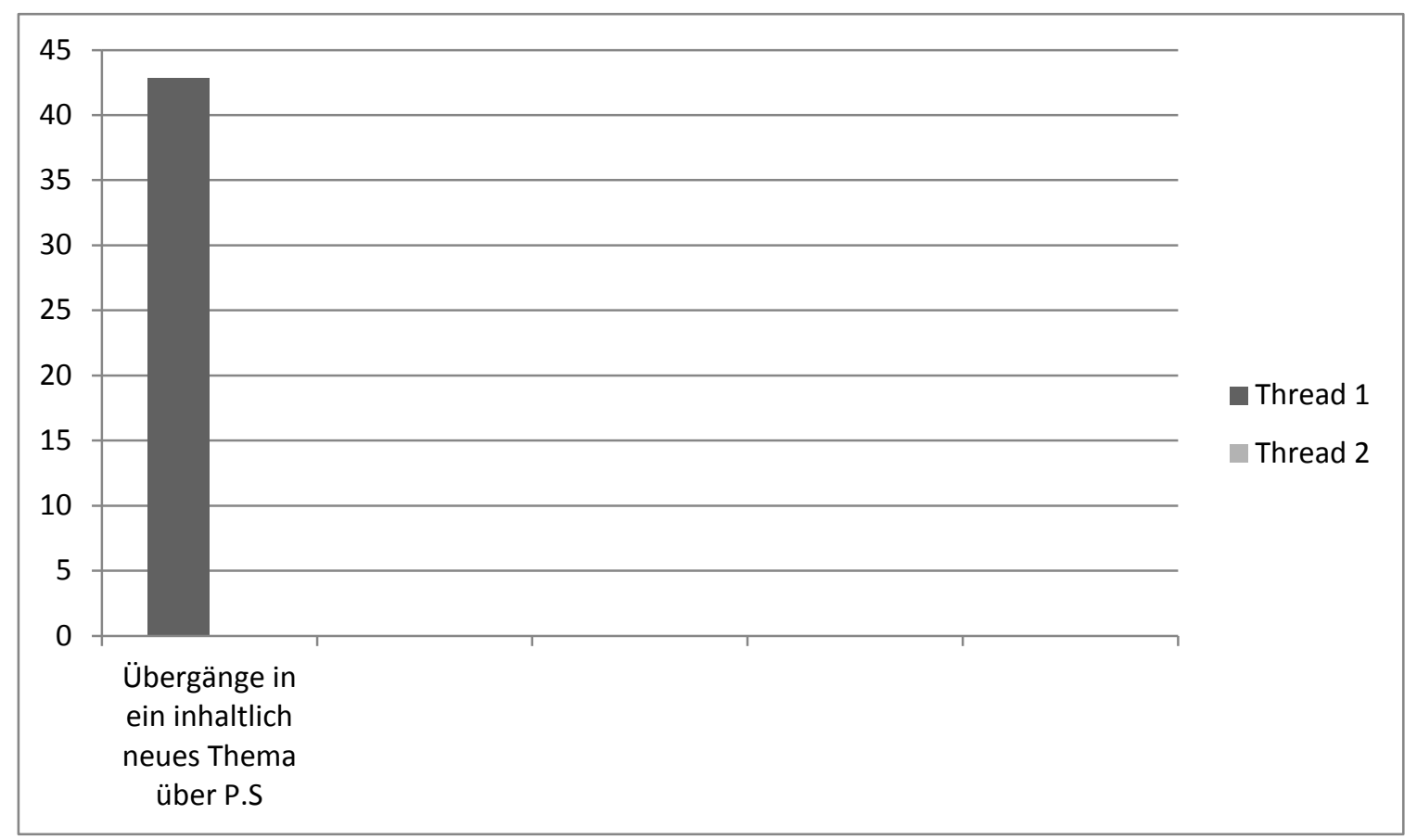

Abb. 4 Das Vorkommen von Übergängen in ein neues Thema über P.S. in \%

An den Abbildungen sieht man, dass während das Auslassen des Personalpronomens in Beispielen beiden Forenthreads vorgekommen ist, man Übergänge in ein neues Thema über P.S. nur im ersten Thread findet. Möglicherweise liegt es daran, dass die Schreiber im zweiten Forenthread befürchten, dass ein weiteres Thema überlesen wird, wenn es über P.S. eingeführt wird. Vielleicht entspricht es aber auch nicht der „Art“ der Kommunikation im zweiten Forenthread, wo die Mitteilungen von den Usern in der Regel wesentlich kürzer gehalten werden als im ersten Thread.

\section{$\underline{\text { 3.2 Konversationsverlauf }}{ }^{63}$}

Die Forschung der letzten Jahrzehnte ${ }^{64}$ hat ergeben, dass der Konversationsverlauf in der mündlichen Kommunikation bestimmten wiederkehrenden Regelmäßigkeiten unterliegt. Demnach besitzt jedes Gespräch eine Struktur, deren Grundlage Sequenzen ${ }^{65}$ sind. Die

\footnotetext{
${ }^{63}$ Da die Frage nach dem Konversationsverlauf kein zentraler Gegenstand der vorliegenden Dissertation ist, wird nicht auf alle Aspekte eingegangen.

${ }^{64}$ Vergleiche hierzu z.B. die Arbeit von H. Schegloff \& E. Sacks (1973).

65 Trotz der Uneinigkeit darüber, ab wann man von einer Sequenz sprechen kann, werden grundsätzlich zwei Arten der Sequenzen unterschieden: die sog. a-priori-Sequenzen auf der einen und kontingente Sequenzen auf der anderen Seite. „Die Struktur von a-priori-Sequenzen wird durch den logischen Zusammenhang der sie konstituierenden Sprechhandlungen gebildet und ist damit situationsunabhängig. $\mathrm{Zu}$ ihnen zählen: Nachbarschaftspaare (vgl. 3.1.), Einschub-und Expansionssequenzen (vgl. 3.4.) und Reparatursequenzen (vgl. 3.4.2.). Da diese Sequenztypen miteinander kombinierbar sind, ist es auch möglich, komplexe
} 
Sequenzen wiederum bestehen aus Gesprächsschritten, die ebenfalls als Turns ${ }^{66}$ oder Redebeiträge bezeichnet werden und durch den Sprecherwechsel begrenzt sind (vgl. Brinker \& Sager (1989, 55 und 57)). Die Gesprächsschritte lassen sich dabei grob in drei Arten gliedern: initiierend, respondierend und reaktivierend. „Mit einem initiierenden Gesprächsschritt fordert der Sprecher den Hörer zu einer bestimmten Reaktion auf. Es gehört zu den Basisregeln der Kommunikation ${ }^{67}$, daß der Angesprochene antwortet; er ist sozusagen verpflichtet, aus einer beschränkten Anzahl von Fortsetzungsmöglichkeiten ${ }^{68}$ eine bestimmte Antwort zu realisieren. Mit dem respondierenden Gesprächsschritt erfüllt er dann die mit dem initiierenden Schritt etablierten Obligationen.“ (Brinker \& Sager (1989, 69)). Der reaktivierende Gesprächsschritt ist eine Mischung aus den beiden anderen Gesprächsschritten, da er Eigenschaften beider Schritte besitzt (vgl. Brinker \& Sager (1989, 71)). Die Äußerungen der Gesprächsschritte sind dabei immer verbunden: „Äußerungen werden in der Regel spezifisch in Hinblick auf die vorangegangenen Äußerungen formuliert. Die Konversationsanalyse spricht hier von lokaler Produktion: Äußerungen sind (zumeist) keine vorgefertigten Versatzstücke, sondern werden in ihren Details zu dieser Gelegenheit für diese Gelegenheit produziert (Schegloff 1984). [...] In Gesprächen gilt das Prinzip der lokalen Kohärenz $^{69 ; 70}$ (Sacks 1987): Die unmittelbar vorangehende Äußerung bildet den Bezugsrahmen für die gegenwärtige Äußerung, sofern nicht ausdrücklich angezeigt wird, daß eine andere Äußerung den relevanten Kontext darstellt. Das Prinzip der lokalen Kohärenz ist eine unmittelbare Konsequenz des allgemeinen Sequenzialitätsprinzips. Das Prinzip der

Gesprächsstrukturen zu beschreiben. Im Gegensatz dazu sind kontingente Sequenzen durch keine internen logischen Zusammenhänge bedingt, sondern die Logik ihrer Abfolge ist durch kontextuelle Faktoren (also außersprachliche Ziele, Intentionen oder Pläne der Gesprächsbeteiligten) bedingt." (Gruber (2001, 1227)).

${ }^{66}$ Ein Turn wird folgendermaßen definiert: ,[...] Aus dem Engl. übernommener Terminus der Diskursanalyse zur Bezeichnung eines einzelnen Sprecherbeitrags. Bestimmt werden T. (a) anhand formaler Kriterien: T. eingegrenzt durch Pausen oder als syntaktische Einheit, nach der ein Sprecherwechsel möglich ist; (b) anhand funktionaler Kriterien: T. als mindestens ein «Zug» (vgl. Austausch); (c) in der Konversationsanalyse: T. als Produkt eines Prozesses, dessen Länge und Struktur interaktiv bestimmt ist (Rezipientenspezifischer Zuschnitt, Sequentielle Organisation, Sprecherwechsel); im Idealfall hat ein solcher T. eine ausgebildete triadische Struktur: mit dem ersten Teil wird eine Beziehung zum vorangegangenen T. hergestellt, mit dem dritten eine Beziehung zum nachfolgenden T. (vgl. ja und die Frageintonation im T. von B: Ja is 'das nicht zu teuer? Als Erwiderung auf A: Nimm doch 'n Taxi). "(Bußmann (2008, 756f.)).

${ }^{67} \mathrm{Zu}$ den Basisregeln zählen Brinker \& Sager das Kooperationsprinzip.

${ }^{68}$ Brinker \& Sager $(1989,80)$ benutzen dafür auch den Begriff Fortsetzungsraster.

69 „Das Prinzip der lokalen Kohärenz kann aber auch außer Kraft gesetzt werden: durch Vor- und Rückverweise (,nochmal zu ...“, „,bevor ich zu ... komme“, ,jedenfalls“; Fokuswechseloperatoren nach Kallmeyer 1978), durch Deplazierungsmarkierungen (,nebenbei gesagt“, „da fällt mir gerade ein“; ,misplacement markers“ nach Schegloff/Sacks 1973) oder durch die Initiierung von Einschubsequenzen (z.B. wenn einer Frage mit einer Gegenfrage begegnet wird, die die vorherige Abklärung einer Voraussetzung für die Antwort auf die erste Frage zum Gegenstand hat; , insertion sequence " nach Schegloff 1968). [...] Eine andere, häufig zu beobachtende Art, lokale Kohärenz aufzuheben, besteht darin, daß Sprecher nicht an ihre Vorgänger anknüpfen, sondern an ihre eigene letzte Äußerung (,Selbstkohärenz ').“(Deppermann $(2008,65))$.

${ }^{70}$ Auch Gesprächskohärenz genannt (vgl. Brinker \& Sager $(1989,72)$ ). 
lokalen Kohärenz sorgt dafür, daß zwischen direkt aufeinanderfolgenden Äußerungen auch solche Kohärenzverhältnisse hergestellt werden, die nicht explizit formuliert werden müssen. Dies gilt genauso für das Verhältnis von Äußerungen innerhalb des Beitrags eines Sprechers wie für das Verhältnis zwischen Beiträgen verschiedener Sprecher.“ (Deppermann (2008, 64)). Die Gesprächsschritte sind dabei entweder grammatisch oder thematisch verknüpft, z.B. durch die Wiederaufnahme (vgl. Brinker \& Sager (1989, 72-78)).

Als kleinste Struktureinheit, d.h. kleinste Sequenz, bilden die sogenannten Adjazenzpaare (auch Paarsequenzen genannt) die Basis: „Die Paarsequenzen sind offensichtlich eine grundlegende Komponente der Gesprächsstrukturierung - es wurde sogar behauptet, sie seien die grundlegende Komponente (siehe z.B. Goffman 1976, Coulthard 1977:70).“ (Levinson (2000, 330)). Solche Adjazenzpaare setzen sich aus einem oder auch aus zwei Äußerungspaaren zusammen. ${ }^{71}$ „Schegloff and Sacks (1973) identified the “adjacency pair” as a concept for understanding paired actions. The first action, "the first pair part" (e.g., a question) is ideally always followed by a "second pair part" (e.g., an answer). One speaker provides the first pair part, and the second pair part is typically provided by a second speaker.“ (Anderson et al $(2010,7)){ }^{72}$ Neben der Vorstellung, dass ein Adjazenzpaar aus zwei Redebeiträgen besteht, gibt es auch den Ansatz, dass solche Sequenzen aus drei Beiträgen bestehen: Person A sagt etwas (erster Beitrag), Person B reagiert entsprechend ihrer Interpretation des von Person A Gesagten (zweiter Beitrag) und Person A bestätigt diese Interpretation oder lehnt sie ab, indem sie Person B z.B. korrigiert (dritter Beitrag) (vgl. Gruber $(2001,1231)) .{ }^{73}$ Jedoch muss die Struktur nur in dem Fall aus drei Beiträgen bestehen, in dem die Interpretation des ersten Beitrags durch Person B nicht korrekt ist. „Ist das hingegen nicht der Fall, zeigt jede ,natürliche“ Weiterentwicklung des Gesprächs an der dritten Position an, daß gegenseitiges Verstehen gegeben ist. Damit wäre die Verbalisierung der dritten Position einer Sequenz ein „Notfallsystem“, das nur in bestimmten Situationen eingesetzt wird.“ (Gruber (2001, 1232)). Unabhängig davon, ob sie zwei- oder dreiteilig sind, unterliegen Adjazenzpaare dem Kriterium der bedingten Relevanz ${ }^{74}$ : „Wurde der erste Teil

\footnotetext{
${ }^{71}$ Den Zweck und Vorteil mehrerer Äußerungen behandelt der zweite Abschnitt des Artikels „Opening up closings" von Schegloff \& Sacks (1973, 289-327).

${ }^{72}$ Adjazenzpaare spielen nicht nur eine bei der Eröffnung eines Gesprächs große Rolle, sondern auch bei der Beendigung, da beide Partizipanten sich auf diese Weise vergewissern können, sich auf das gleiche Resultat geeinigt zu haben (vgl. Schegloff \& Sacks (1973, 297f.)).

${ }_{73}$ Für die Begründung einer solchen Annahme s. Gruber (2001, 1231f.). Meiner Ansicht nach, ist dieser Ansatz sehr plausibel, da er von sprachlichen Daten gestützt wird, z.B. Beispiel (10) $\mathrm{B}_{2}-\mathrm{B}_{4}$ : die Mitteilung $\mathrm{T}_{1} \mathrm{M}_{2,1}$ wird von Klubnauk in $\mathrm{T}_{1} \mathrm{M}_{3,6-7}$ offensichtlich anders interpretiert als von AgentMario beabsichtigt, woraufhin seinerseits eine Korrektur in $\mathrm{T}_{1} \mathrm{M}_{5,1}$ erfolgt.

${ }^{74}$ Eine andere Übersetzung für den in der amerikanischen Forschung etablierten Begriff conditional relevance ist bedingte Erwartbarkeit oder konditionelle Relevanz. Der Begriff conditional relevance wurde von Schegloff
} 
eines Paares realisiert, so wird die Realisierung des zweiten Teils für den nächsten Sprecher umgehend relevant und erwartbar (Schegloff 1972a: 363ff.). Taucht ein solcher zweiter Teil nicht auf, so fehlt er merklich; und erscheint an seiner Stelle irgendein anderer erster Teil, dann versteht man diesen möglichst als eine Einleitung für den zweiten Teil.“ (Levinson (2000, 333)). Die Erwartbarkeit bezieht sich dabei auf den zweiten Redebeitrag, der Konventionen entsprechen soll (vgl. Brinker \& Sager $(1989,79))$. Jedoch ist die Reaktion im zweiten Teil nicht festgelegt, viel mehr gibt es mehrere alternative Reaktionsmöglichkeiten, die scheinbar einer Präferenz unterliegen: „Der Kernpunkt dabei ist, daß die möglichen zweiten Teile zu einem ersten Teil nicht alle gleichrangig sind. Die Alternativen werden in eine bestimmte Rangfolge gebracht, so daß es zumindest eine bevorzugte und eine weniger bevorzugte Kategorie von Reaktionen gibt.“ (Levinson $(2000,334)){ }^{75}$ Dabei seien die bevorzugten Reaktion unmarkiert und die weniger bevorzugten Reaktionen markiert (vgl. Levinson (2000, 361)). Als Beispiel für eine weniger bevorzugte Reaktion lassen sich zeitliche Abstände zwischen dem ersten Redebeitrag des Adjazenzpaares und der Reaktion anführen. Ein nicht sofort erfolgtes Feedback wird vom Sprecher als Problem interpretiert, ${ }^{76}$ da eine zeitliche Pause zwischen den Bestandteilen eines Adjazenzpaares nicht erwartet wird (vgl. Levinson (2000, 347f.)). Die Pause gehört somit als Merkmal zu der Kategorie weniger bevorzugter Reaktion. ${ }^{77}$

Anzumerken ist, dass Paarsequenzen nicht immer direkt hintereinander stehen müssen: häufig werden weitere Äußerungen eingeschobenen, die z.B. für die Vorbereitung der Antwort relevant sind (vgl. Levinson (2000, 331f.)). Diese sog. Einschubsequenzen können selbst ein Adjazenzpaar bilden und können in einer größeren Anzahl zwischen den beiden Bestandteilen eines Adjazenzpaares vorkommen. Neben Paarsequenzen und Einschubsequenzen gibt es

(1968) geprägt.

75 Zur Präferenz siehe Levinson (2000, 361-375). Die folgende Tabelle (Levinson (2000, 365)) verdeutlicht dabei welche Reaktionen präferiert und welche weniger präferiert werden:

\begin{tabular}{|llllll|}
\hline ERSTE TEILE: & Bitte & Angebot/Einladung & Beurteilung & Frage & Vorwurf \\
$\begin{array}{l}\text { ZWEITE } \\
\text { TEILE: } \\
\text { Bevorzugt }\end{array}$ & Gewähren & Annahme & Zustimmung & erwartete Antwort & Abstreiten \\
$\begin{array}{l}\text { Weniger } \\
\text { bevorzugt: }\end{array}$ & Ablehnung & Ablehnung & Widerspruch & unerwartete & Zugeben \\
& & & & $\begin{array}{l}\text { Antwort oder } \\
\text { keine Antwort }\end{array}$ & \\
\hline
\end{tabular}

$A b b . i$ Präferierte und weniger präferierte zweite Teile zu ausgewählten ersten Teilen

${ }^{76}$ Für Beispiele unterschiedlicher Interpretationen von Pausen mit unterschiedlicher Dauer siehe Levinson (2000, 354-357).

${ }^{77}$ Für weitere Merkmale der weniger bevorzugten Reaktionen siehe Levinson (2000, 363). 
auch andere Sturktureinheiten (vgl. Levinson $(2000,366)$ ), z.B. Aufruf-Antwort-Sequenzen ${ }^{78}$ oder Vorsequenzen, auch Präsequenzen ${ }^{79}$ genannt. Beispiele für Vorsequenzen sind z.B. VorMitteilungen $^{80}$ oder Vor-Bitten. Vor-Mitteilungen ,[...] bestehen gewissermaßen aus zwei sich überlappenden Paarsequenzen - einem Vor-Paar (zum Beispiel A: Hast du schon das Neueste gehört?, B: Nein) und einem zweiten Paar (zum Beispiel B: Schieß los, A: Peter hat im Lotto gewonnen). Sie überlappen sich insofern, als der zweite Teil des ersten Paares und und [sic!] der erste Teil des zweiten Paares im selben Beitrag oder in derselben Position auftreten.“ (Levinson $(2000,380)) .{ }^{81}$ Vor-Mitteilungen werden z.B. dazu benutzt, um eine

78 „Aufruf-Antwort-Sequenzen unterscheiden sich von anderen Paarsequenzen (wie Gruß-Gegengruß, AngebotAnnahme/Zurückweisung) darin, daß sie stets zu etwas überleiten. Dieses Etwas ist normalerweise der Grund für den Aufruf. Demnach bestehen Aufruf-Antwort-Sequenzen aus (mindestens) drei Beiträgen [...]:

(28)

$\mathrm{T}_{1} \quad$ A: Peter?

$\mathrm{T}_{2} \quad \mathrm{~B}: \mathrm{Ja}$ ?

$\mathrm{T}_{3} \quad$ A: Gibst du mir mal das Wasser?

((Aufruf))

((Antwort))

((Grund für Aufruf))

Die dreiteilige Struktur wird auch dadurch unterstrichen, daß in $\mathrm{T}_{2}$ häufig eine Gegenfrage gestellt wird (Was?, Was ist los?, Ja?); diese ist gleichzeitig der zweite Teil zu dem Aufruf und ein erster Teil, in dem nach Gründen für den Aufruf gefragt wird, und schafft damit eine Struktur aus drei Beiträgen, die zwei Paarsequenzen ergeben.“ (Levinson $(2000,337)$ ).

${ }^{79}$ Neben Kern-bzw. Hauptsequenzen, Vorsequenzen und Einschubsequenzen gibt es ebenso Postsequenzen und Nebensequenzen. „Eine Kernaktivität (Bsp.: A lädt B zu einer Feier ein), die aus einer einfachen Paarsequenz besteht (im Bsp.: A spricht Einladung aus - B nimmt Einladung an oder schlägt sie aus), kann durch mehrere Sequenztypen erweitert werden, die jeweils unterschiedliche Funktionen haben und auf unterschiedliche Gegebenheiten antworten:

- Präsequenzen (pre-sequences, Schegloff 1980) gehen der Kernaktivität voraus; sie dienen meist dazu, Handlungsvoraussetzungen für die Kernaktivität abzuklären, Hindernisse zu sondieren etc. und somit entweder den Boden für die Kernaktivität zu bereiten oder aber dafür zu sorgen, daß sie ohne Gesichtsverlust unterlassen werden kann (im Bsp.: A fragt, was B am betreffenden Abend vorhat, bevor A die Einladung ausspricht);

- Postsequenzen folgen der Kernaktivität; sie bestehen oft aus Würdigungen und Bestätigungen („Sehr schön“, „Dann sehen wir uns also...“), in problematischen Fällen kann es zu Reparatursequenzen [...] kommen, in denen Korrekturen an der Kernsequenz vorgenommen werden (im Bsp.: schlägt B die Einladung mit der Begründung aus, er habe einen anderen Termin, korrigiert A die Einladung: „Du kannst auch später kommen“);

- Einschubsequenzen (insertion sequences, Schegloff 1972) sind in die Kernaktivität eingelagert; wenn diese aus einer Paarsequenz besteht, wird der Einschub vom Adressaten des ersten Teils der Paarsequenz initiiert (im Bsp.: A spricht Einladung aus - B fragt nach der genauen Uhrzeit); in diesem Fall schafft der Einschub selbst eine konditionelle Relevanz, die erst eingelöst werden muß (A nennt die Uhrzeit), bevor dann auch die konditionelle Relevanz der Kernaktivität erfüllt werden kann (B nimmt Einladung an oder schlägt sie aus);

- Nebensequenzen (side sequences, Jefferson 1972) sind Aktivitäten, die die Kernaktivität unterbrechen und die die Gesprächsteilnehmer so rahmen, daß sie für die Kernaktivität irrelevant seien.“ (Deppermann $(2008,76 f$.)). Nebensequenzen sind ,[...] background information, that is, information that at the current stage of the conversation is not relevant in its own right, but only as a background, or context, for some other piece of information. [...] One of the characteristic features of side sequences is that there are relevance relations between the main and the side sequences. One way we can see that the information in the side sequence is not relevant in its own right - that it is backgrounded, contextual, and dependent - is that the relevance relation between the side sequence and the main sequence is not symmetrical, so that the side sequence is relevant for understanding or contextualizing the main sequence, but not the other way around.“ (Svennevig $(1999,294)$ ).

${ }^{80}$ Für die Struktur der Vor-Mitteilungen siehe Levinson (2000, 380).

${ }^{81}$ Das angeführte Beispiel würde mit der Überlappung somit wie folgt aussehen:

A: Hast du schon das Neueste gehört?

B: Nein. Schieß los.

A: Peter hat im Lotto gewonnen. 
Geschichte oder Neuigkeit anzukündigen und abhängig von der Reaktion des Hörers (bekannte/unbekannte Neuigkeit, Geschichte usw.) die Sequenz weiterauszuführen oder nicht. Ihr Zweck liegt sowohl in der Ankündigung einen längeren Turn beim Turn-Taking beanspruchen zu wollen (z.B. bei Geschichten) als auch in dem ,,[...] Bedürfnis, den Hörern nichts zu erzählen, was sie bereits wissen.“ (Levinson (2000, 385)). Levinson (2000, 388396)) beschäftigt sich auch mit einem weiteren Beispiel für Vorsequenzen, nämlich den VorBitten. Mit Vor-Bitten wollen die Sprecher z.B. prüfen, ob ihre Bitte vom Gesprächspartner erfüllt wird. Ein weiterer Zweck ist es, mit ihnen den Gesprächspartner dazu zu bewegen, die nicht explizit genannte Bitte zu erfüllen, da ein Angebot seitens des Gesprächspartners gegenüber dem eigenen Aussprechen der Bitte präferiert wird. Bei den Vor-Bitten ergibt sich folgende Präferenzreihenfolge: an erster Stelle kommt Vor-Bitte - direkte Reaktion auf eine implikatierte Bitte, an zweiter Stelle findet sich eine dreiteilige Struktur mit Vor-Bitte Angebot - Annahme und an letzter Stelle, d.h. am wenigsten präferiert, kommt schließlich die Struktur Vor-Bitte - Bestätigung - Bitte - Reaktion (vgl. Levinson (2000, 392)).

Die Gesamtorganisation einer Konversation besteht aus mehreren Gesprächsphasen, die sich wiederum aus Sequenzen und Redebeiträgen zusammensetzen. Ein Gespräch wird durch eine Eröffnungsphase eröffnet. „Die Dauer der Eröffnungsphase kann stark variieren; sie ist von verschiedenen Faktoren abhängig: dem Bekanntheitsgrad der Teilnehmenden und deren Zeitbudget, der Öffentlichkeit oder Privatheit der Gesprächssituation, dem Zeitpunkt des letzten Kontakts etc.“ (Spiegel \& Spranz-Fogasy (2001, 1247)). Die Eröffnungsphase beinhaltet auf der verbalen Ebene in der Regel mindestens einen Austausch von Begrüßungen. Meistens besteht sie aber aus ,[...] mindestens drei Beiträgen, wobei der erste die andere Partei um ihre Aufmerksamkeit bittet, der zweite dieser anderen Partei die Gelegenheit bietet, sich auf die Interaktion einzulassen, und der dritte die Leerstelle ist, in der die erste Partei den (vorläufigen) Grund für die Interaktion nennen kann. So ergibt sich die vertraute Struktur Aufruf-Antwort-erstes Thema, die den Grundstein für eine koordinierte Zusammenarbeit bildet, den beiden Parteien für die Dauer der ersten drei Beiträge Sprecher- und Hörerrollen zuweist und so die Mindestvoraussetzungen für das Ingangsetzen der SprecherwechselMaschinerie schafft.“ (Levinson (2000, 350f.)). Die Leerstelle ist eine ,[...] sogenannte Leerstelle für das erste Thema ${ }^{82}[\ldots]^{683}$ (Levinson (2000, 339)), das in den meisten Fällen

\footnotetext{
${ }^{82}$ Als Thema definiere ich im Rahmen dieser Dissertation in Anlehnung an Brinker $(2001,169)$ das, was der „[...] Inhaltskern [...]“ z.B. eines Textes oder Gesprächs ist. Es handelt sich dabei um etwas, was unter einen grob umfassenden Begriff gefasst werden kann: „Das Textthema ist entweder in einem Textsegment realisiert, oder es
} 
noch nicht vorgegeben ist. Mit ihr wird gleichzeitig die wesentlich komplexer strukturierte Kernphase eingeleitet ${ }^{84}$, die einer Themenorganisation unterliegt, und auf diese Weise in sog. thematische Gesprächsabschnitte gegliedert wird. ${ }^{85}$ Der ersten Leerstelle folgen die anderen Themen, die nach einer bestimmten Reihenfolge eingebracht werden - sie können demnach miteinander verknüpft sein, aber auch plötzlich wechseln. Im Fall eines plötzlichen Themenwechsels, erfolgt eine Markierung ${ }^{86}$. Dabei gilt für Gespräche das Gleiche wie für Texte: „Da ein Text in der Regel mehrere Themen enthält (mit einem jeweils unterschiedlichen thematischen Stellenwert), ist es nötig, zwischen dem Textthema (als Hauptthema) und den Nebenthemen zu differenzieren, so daß sich eine Art Themenhierarchie ergibt.“ (Brinker \& Hagemann (2001, 1254)). Differenziert wird zwischen Haupt- und Nebenthemen mit Hilfe des Ableitbarkeitsprinzips ${ }^{87}$ und des Kompatibilitätsprinzips ${ }^{88}$. Jedoch gibt es auch Gespräche, in denen mehrere Hauptthemen existieren (vgl. Brinker \& Hagemann (2001, 1255), die sich an Schank $(1977,236 \mathrm{ff}$.$) orientieren). { }^{89}$ Die Konversation wird durch

muß erst aus dem Textinhalt abstrahiert werden, indem der Analysierende den Gesamtinhalt auf eine ,knappe Formel" bringt." (Brinker (2001, 169)). Dabei erweitere ich meine Definition dahingehend, dass auch ein Einzelbeitrag, der nicht in eine Interaktion eingebettet ist, da auf ihn keiner reagiert, ein Thema besitzt.

${ }^{83}$ Die Leerstelle für das erste Thema ist der sogenannte first-topic-slot (Levinson $(1983,312)$ ), der in späteren Publikationen von Levinson als first-topic-position (Levinson $(1989,409)$ ) bezeichnet wird. In der vorliegenden Arbeit wird der Begriff first-topic-position verwendet.

84 „Bei Analysen der „Gesprächsmitte“ werden zur Phasierung v. a. zwei Dimensionen bzw. Interaktionsebenen in Anschlag gebracht: die Handlungs- und die Themenebene (Henne/Rehbock 1995; Levinson 1990; Schank 1981; Techtmeier 1984). Der Aufgaben- bzw. der Themenwechsel soll danach zur Phasierung, zur Binnenstrukturierung genutzt werden, und die Analyse gesprächsorganisatorischer Eigenschaften wird als Mittel der formalen Bestimmung zur Unterstützung herangezogen. Gesprächsphasen werden aufgaben- bzw. handlungsbezogen rekonstruiert und/oder relativ zur thematischen Entwicklung als emergente Einheitenbildung erfasst.“ (Spiegel \& Spranz-Fogasy (2001, 1243)). Für die genaue Beschreibung beider Phasenmodelle siehe Spiegel \& Spranz-Fogasy (2001, 1243-1247).

${ }^{85}$ „Zur analytischen Rekonstruktion der thematischen Phasierung wurde eine Reihe von Vorschlägen entwickelt. Schank (1981) bestimmt ,thematische Abschnitte“ als das, was zwischen zwei Themenwechseln liegt, was von den GesprächsteilnehmerInnen mit Gliederungssignalen und Formulierungshandlungen markiert wird. Er knüpft dabei an den Fokusbegriff Goffmans (1971) an: „Thema ist das intentionale Objekt im Fokus einer zentrierten Interaktion, soweit dieses explizit verbalisiert wird“ (1981, 22). Brinker (1988, 27 f) kritisiert die Konzeption thematischer Abschnitte als zu unpräzise und schlägt eine semantisch-strukturelle Analyse vor, die auch implizite bzw. präsupponierte Themen systematisch erfasst: Das Thema ist der Kern des Gesprächsinhalts, der Grund- oder Leitgedanke eines Gesprächs oder einer Gesprächsphase. [...] Dittmar (1988, 71) schlägt eine Gliederung des Gesprächs in thematische Felder vor, dem je ein Schlüsselwort zugeordnet werden kann. Ein thematisches Feld wird in thematischen Etappen interaktiv bearbeitet, wobei thematische Wanderungen, die nicht als linear anzunehmen sind, auftreten.“ (Spiegel \& Spranz-Fogasy $(2001,1246))$.

${ }^{86}$ Den plötzlichen Themenwechsel markiert eine „,erhöhte Lautstärke, [ein] höherer Ton, Marker für Selbstreparatur und Zögern [...] sowie der Diskontinutätsmarker Hey.“ (Levinson $(2000,340)$ ).

87 „Als Hauptthema des Textes ist das Thema zu betrachten, aus dem sich die anderen Themen am überzeugendsten (bezogen auf das jeweilige Textverständnis) 'ableiten' lassen (nicht im logisch-deduktiven Sinn).“ (Brinker $(2001,170)$.

${ }^{88}$ „Da sich Thema und kommunikative Funktion bis zu einem gewissen Grade gegenseitig bedingen (in etwa vergleichbar mit dem Verhältnis von illokutivem und propositionalem Akt in der Sprechakttheorie), gilt als Hauptthema des Textes das Thema, das sich am besten mit der aufgrund einer pragmatischen Analyse ermittelten Textfunktion (J Art. 19, 41) verträgt.“ (Brinker $(2001,170)$ ).

${ }^{89}$ Brinker \& Hagemann erwähnen zwar in Anlehnung an Schank, dass es solche Gespräche gibt, gehen aber nicht weiter darauf ein. 
eine Beendigungsphase beendet, die ,[...] zeitlich so abgestimmt sein muß, daß keiner Partei die Gelegenheit zum Sprechen entzogen wird, obwohl sie gern noch etwas sagen wollte, und weil überhastete wie auch stark hinausgezögerte Gesprächsabschlüsse die Teilnehmer möglicherweise zu unerwünschten Schlüssen über die Art ihrer Beziehung verleiten. Die Mittel zur Strukturierung der Beendigungsphase sind auf diese Probleme fein abgestimmt.“ (Levinson (2000, 344)). Levinson (2000, 344f.) führt folgenden Verlauf einer Beendigungsphase $^{90}$ als charakteristisch $a^{91}$ :, ,(a) Abschluß eines Themas, typischerweise eines Themas, das die Beendigung nahelegt; $\mathrm{zu}$ diesen gehören das Treffen von Vereinbarungen, das erste Thema in monothematischen Anrufen, das Grüßen von anderen Familienmitgliedern usw. (b) ein oder mehrere Paare beiläufiger Beiträge mit Ausdrücken der Vor-Beendigung, wie Okay, All right, So [...] (c) gegebenfalls eine Typisierung des Anrufs als Bitte, die gewährt wurde, (Thank you) oder als Erkundigung nach dem Gesundheitszustand des Empfängers (Well I just wanted to know how you were) usw., worauf ein weiterer Austausch von Vor-Beendigungs-Ausdrücken folgt (d) ein letzter Austausch abschließender Elemente: Bye, Righteo, Cheers usw. Die wichtigsten Elemente (nach dem Erfolgen von (a)) sind (b) und (d). Mit diesen beiden Komponenten wird ein koordinierter Abschluß des Gesprächs erzielt. Mit Hilfe der themenlosen beiläufigen Beiträge in (b) wird die wechselseitige Vereinbarung getroffen, nicht weiterzureden, was zu dem Austausch der abschließenden Paarsequenz in (d) überleitet, der das Gespräch beendet. Die wechselseitige Vereinbarung erfolgt, indem eine Partei einen themenlosen beiläufigen Beitrag erzeugt, der anzeigt, daß sie nichts mehr sagen möchte, woraufhin die andere Partei - falls sie auch nichts mehr sagen möchte - ebenso einen solchen Beitrag produzieren kann. Den

\footnotetext{
${ }^{90}$ Das gilt für verbale Beendigungsphasen. Nonverbal kann ein Gespräch z.B. durch das ,,[..] Aufstehen, Zusammenpacken etc. begleitet [werden].“ (Spiegel \& Spranz-Fogasy $(2001,1248)$ ).

${ }^{91}$ Levinson $(2000,344)$ gibt dazu folgendes Beispiel:

„E: Why don't we all have lunch

A: Okay so that would be in St Jude's would it?

E: Yes

$(0.7)$

A: Okay::::

E: One o'clock in the bar

A: Okay

E: Okay?

A: Okay then thanks very much indeed George=

E: $=$ All right

A: //See you there

E: $\quad$ See you there

A: Okay

E: Okay // bye

R: Bye“
} 
technischen und sozialen Problemen von Gesprächsbeendigungen begegnet man also zunächst damit, daß der Zeitpunkt für die gesamte Beendigungsphase im Zusammenspiel der Teilnehmer festgelegt wird - das Angebot, das Gespräch zu beenden, erfolgt in der Form von Okay, Right oder ähnlichen Ausdrücken, und nur, wenn es angenommen wird, wird der Gesprächsabschluß weiter verfolgt.“ Dabei kommt es nicht selten vor, dass Sprecher in der Beendigungsphase auf Beiträge des ersten Themas oder der Eröffnungsphase referieren (vgl. Levinson (2000, 345)). Auch können Konversationen während der Beendigungsphase (und sogar direkt nach ihr) wieder eröffnet werden, z.B. ist dies möglich nach einer VorBeendigung wie okay (vgl. Schegloff \& Sacks (1973, 304 und 318f.)). Damit sieht die Gesamtorganisation eines Gesprächs vereinfacht dargestellt eine Eröffnungsphase, eine Kernphase, in dem eins oder mehrere Themen besprochen werden, und eine Beendigungsphase $^{92}$ vor. Dabei kann die Kernphase z.B. anhand ihrer Themen grundsätzlich in weitere Phasen unterteilt werden.

Im Allgemeinen verlaufen Konversationen ${ }^{93 ; 94}$ in den untersuchten Internetthreads nach bestimmten regelmäßigen Mustern. Wie in mündlicher Kommunikation basiert die Struktur der Konversationen auf Redebeiträgen bzw. Turns, die formal durch den Partizipantenwechsel begrenzt sind. Jedoch kann ein Beitrag inhaltlich noch weiter aufgesplittet sein, nämlich in thematische Mitteilungen. Auf Grundlage der regelmäßigen Muster lassen sich mehrere Verlaufsarten ableiten, so dass jede Konversation einer dieser Arten zugeordnet werden kann. Jedoch kann weder die Gesamtorganisation noch die Struktur eines mündlich ablaufenden Gesprächs eins zu eins auf die Konversation in Internetthreads übertragen werden, wie sich zeigen lässt. ${ }^{95}$ Eine grobe Aufteilung der Verlaufsarten der Konversationen in Internetthreads kann danach vorgenommen werden, ob Unterbrechungen durch thematisch unpassende Mitteilungen vorkommen. Ich bezeichne die Konversationsverläufe als kontinuierlich bzw. als diskontinuierlich, wobei kontinuierlich bedeutet, dass thematisch zusammenhängende

\footnotetext{
${ }^{92}$ Neben den oben genannten Begriffen, werden ebenfalls die Begriffe Gesprächseröffnung, Gesprächsmitte und Gesprächsbeendigung bzw. Anfangsphase, Gesprächsmitte und Beendigungsphase benutzt (vgl. Spiegel \& Spranz-Fogasy $(2001,1241))$.

${ }_{93}$ In den entsprechenden Forenthreads wurden Alltagsgespräche untersucht und mit den Erkenntnissen für mündliche Alltagsgespräche verglichen.

${ }^{94}$ Meiner Ansicht nach dauert eine Konversation so lange bis die letzte Mitteilung zu allen Themen, die sie beinhaltet, verfasst wurde, d.h. die Konversation ist nicht zu Ende sobald z.B. die letzte Mitteilung des ersten Themas verfasst wurde, sondern erst wenn alle anderen Themen, mit denen während der Verhandlung des ersten Themas angefangen wurde, abgeschlossen sind. Dadurch entsteht eine thematische „Verschachtelung“ in der Konversation.

${ }_{95}$ Mein Eindruck ist, dass dies in Chaträumen anders aussieht, da dort die Konversationsverläufe denen in mündlicher Kommunikation stark ähneln.
} 
Mitteilungen einander folgen ${ }^{96}$, während bei diskontinuierlichen Verläufen zusammenhängende Mitteilungen durch thematisch unpassende unterbrochen werden. Demnach wird deutlich, dass eine Phasierung des Gesprächs, genauer gesagt der Kernphase, anhand thematischer Abschnitte vorgenommen wird; eine Hierarchisierung wird nicht anhand von Haupt- und Nebenthema, sondern anhand eines dominanten vs. raren Themas vorgenommen. Das dominante Thema ist dabei dasjenige, zu dem mehr Mitteilungen verfasst werden.

Als ein Beispiel für Konversationen, die ohne Unterbrechungen durch thematisch fremde Mitteilungen verlaufen, soll das Beispiel (14) untersucht werden. Diese und folgende Grafiken sind folgendermaßen zu lesen: auf der y-Achse sehen wir die Anzahl der Themen, die zum Zwecke der Referenz durchnummeriert sind, während sich auf der x-Achse die Anzahl der Beiträge befinden, die nur bei Themenwechsel bzw. bei dem ersten Beitrag eines neuen Themas angegeben werden. „-“ steht für eine Mitteilung innerhalb des Beitrags. Großbuchstaben stehen für den jeweiligen Schreiber. Tiefgestellte Zahlen zeigen, auf welche Mitteilung innerhalb des Themas Bezug genommen wird. „a“ bezeichnet Grußformeln und

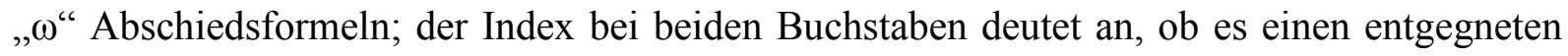
Gruß bzw. Abschied gibt, das „ $\varnothing^{“}$ hingegen gibt das Fehlen von Gruß- bzw. Abschiedsformeln an. Die Struktur der Konversation sieht dabei folgendermaßen aus:

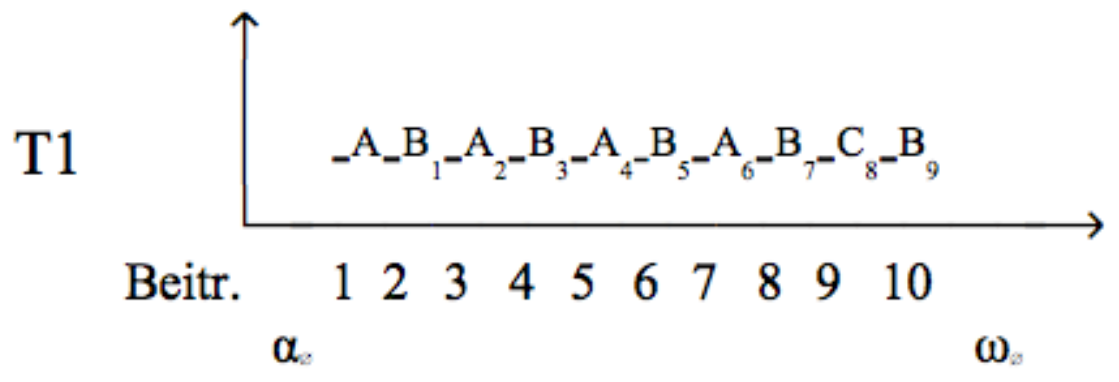

Abb. 5

Schreiber A = DragOn

$\underline{\text { Thema } 1}=\underline{\text { Funktion der Farbe im Thread }}$

$\underline{\text { Schreiber B }}=\underline{\text { bogda }}$

Schreiber C $=$ Lelika

\footnotetext{
${ }^{96}$ Das kann mit einer Perlenkette verglichen werden, bei denen die einzelnen Mitteilungen Perlen darstellen und einander wie auf einem Faden folgen.
} 
Über die Anzahl der Themen auf der y-Achse sehen wir, dass in diesem Beispiel nur ein einziges Thema $\left(\mathrm{T}_{1}\right)$ behandelt wird. Ein Themenwechsel findet somit nicht statt. Die Konversation verläuft kontinuierlich. Es beteiligen sich insgesamt drei Schreiber, wobei zwischen bogda und DragOn in den Beiträgen 1-7 ein regelmäßiger Partizipantenwechsel ${ }^{97}$ stattfindet. Dominant ist der Schreiber bogda, da er die höchste Anzahl an Mitteilungen zu dem Thema verfasst. Die fehlenden Grußformeln zeigen an, dass die Interaktion nicht mit den ersten Schritten einer Eröffnungsphase (etwa A: „Hallo.“, B: „Hallo“) beginnt, sondern mit der ersten thematischen Mitteilung, d.h. der first-topic-position - hier einer implikatierten Erinnerung des Users DragOn daran, dass der thematische Thread zu Nicknames usw. bald geschlossen wird, die damit das einzige Abschnittsthema vorgibt. Als Reaktion darauf erfolgt eine Mitteilung von bogda, mit der implikatiert wird, eine andere Farbe des Nicknames haben zu wollen. Die nächste Mitteilung bildet die Frage von DragOn in $\mathrm{T}_{1} \mathrm{M}_{3}$, die gleichzeitig als präferierte Reaktion auf $\mathrm{T}_{1} \mathrm{M}_{2}$ zu betrachten ist, da mit ihr ein Interesse an dem Farbwunsch des Gesprächspartners bezüglich des Nicknames (und der damit verbundenen Bedeutung für die Funktion im Forum) bekundet wird. Die Reaktion hierauf stellt die Antwort von bogda in $\mathrm{T}_{1} \mathrm{M}_{4}$ dar, die gleichzeitig den ersten Teil zur darauffolgenden Antwort bildet und mit der bogda DragOn indirekt auffordert bezüglich des Farbwunsches zu reagieren, was, wie erwartet, auch passiert. Die Frage in $\mathrm{T}_{1} \mathrm{M}_{5}$ bildet eine präferierte Reaktion auf $\mathrm{T}_{1} \mathrm{M}_{4}$, da DragOn, der die Antwort auf seine Frage bereits vermutet, mit ihr seine Bereitschaft signalisiert, über das Thema weiter zu sprechen. Die Antwort findet sich im nachfolgenden Redebeitrag von bogda. Untersucht man diesen Redebeitrag genauer, so stellt man fest, dass er mehrere Bestandteile unterschiedlicher Sequenzarten enthält. ${ }^{98}$ Die erste Äußerung ist Bestandteil einer Nebensequenz, die zwischen der Frage in $T_{1} M_{5}$ und der eigentlichen Antwort in $\mathrm{T}_{1} \mathrm{M}_{6,2}$, eingeschoben wird. Die Nebensequenz bleibt unvollständig, da der Schreiber bogda keine Reaktion seines Gesprächspartners abwartet, sondern seinen Redebeitrag fortsetzt. $\mathrm{T}_{1} \mathrm{M}_{6,3-4}$ stellt einen Bestandteil einer Postsequenz dar, mit der bogda versucht, sich selbst zu empfehlen und zu zeigen, dass er bereits Erfahrungen als Moderator hat. Der Grund hierfür liegt darin, dass er offenbar nicht alle Voraussetzungen erfüllt, um Moderator $\mathrm{zu}$ werden, und um eine negative Reaktion abzuwenden, benutzt er die Postsequenz, um DragOn von seiner Erfahrung und sich selbst zu überzeugen. Mit $\mathrm{T}_{1} \mathrm{M}_{6,4}$ fordert er DragOn indirekt auf zu reagieren. DragOn entspricht dieser Erwartung mit seiner

\footnotetext{
${ }^{97}$ Auf den Partizipantenwechsel komme ich im nächsten Abschnitt zu sprechen.

98 Das Vorkommen unterschiedlicher Sequenzarten innerhalb eines Redebeitrags lässt sich auch an anderen Stellen belegen (z.B. (24) $\mathrm{T}_{1} \mathrm{M}_{1}$ ), wird in der vorliegenden Dissertation aber nicht weiter untersucht.
} 
Reaktion, die sowohl eine Zusage implikatiert als auch einen Teil des ersten Schrittes der Beendigungsphase darstellt, da eine Übereinkunft erzielt wurde: bogda will Moderator werden und DragOn sagt indirekt zu. An dieser Stelle ist das Gespräch zwischen bogda und DragOn beendet, da bogda sich zwar zu diesem Thema in der nächsten Mitteilung $T_{1} M_{8}$ meldet, die Ansprechpartner jedoch andere farblich markierte User sind. Auf diese Weise wird die Konversation in der Beendigungsphase wieder aufgegriffen. Mit seiner Mitteilung $\mathrm{T}_{1} \mathrm{M}_{8}$ zeigt bogda an, dass er Moderator geworden ist, sie bildet aber auch einen Aufruf an die anderen User zu reagieren. Diesem Aufruf kommt Lelika in $\mathrm{T}_{1} \mathrm{M}_{9}$ nach, was eine präferierte Reaktion darstellt. Die letzte Mitteilung zu diesem Thema stellt $\mathrm{T}_{1} \mathrm{M}_{10}$ dar, in der bogda auf die Mitteilung von Lelika reagiert. Eine Beendigungsphase, einschließlich der Komponenten des Gesprächsabschlusses, fehlt, wie in der Grafik ebenfalls zu sehen ist. Dennoch ist das Gespräch an dieser Stelle beendet, da sich keiner der Gesprächspartner mehr zu Wort meldet. Auf dieser Grundlage lässt sich sagen, dass die User eine Konversation in Threads auch mit einer Übereinkunft beenden können, ohne Vor-Beendigungen sowie gesprächsabschließende Elemente wie Auf Wiedersehen für den Abschluß eines Gesprächs benutzen zu müssen, da diese unnötig und unökonomisch sind, wenn alles Relevante bereits gesagt wurde und sich keiner mehr zu Wort meldet. Die Unvollständigkeit der Eröffnungs- und Beendigungsphase stellt einen Unterschied zur Gesamtorganisation mündlicher Konversationen dar und zeigt, dass sich die User vor allem auf die Kernphase, also auf das Wesentliche einer Konversation, konzentrieren.

Das bisherige Beispiel war eine Konversation zwischen wenigen Usern. Häufig kommen in Threads aber Konversationen zwischen einer größeren Anzahl an Partizipanten vor, z.B. (22) mit acht Gesprächsteilnehmern. Dabei stellen sich einem mehrere Probleme: zum Einen ist nie eindeutig klar, wer auf welche Mitteilungen reagieren wird, und zum Anderen passiert es häufig, dass eine einzige Mitteilung den Bestandteil von Sequenzen verschiedener Sprecher bildet $^{99}$, z.B. kann die Mitteilung von Schreiber A sowohl den ersten Teil zu einer Paarsequenz mit der Mitteilung von Schreiber B bilden als auch gleichzeitig der erste Teil zu einer mehrgliedrigen Sequenz mit den Mitteilungen des Schreibers C sein. Ein anderes Beispiel ist, wenn die Mitteilung des Schreibers B den zweiten Teil einer Paarsequenz bildet und dann von einem Schreiber C, der an der Konversation bisher nicht beteiligt war, als erster Teil für eine neue Sequenz verwendet wird. Um vor allem dem zweiten Problem zumindest

\footnotetext{
${ }^{99}$ Das häufige Vorkommen solcher Fälle resultiert auch daraus, dass die Konversationen schriftlich realisiert sind und dauerthaft bestehen, sie können also jederzeit von jedem User gelesen und kommentiert werden. Es handelt sich hier also um ein medienspezifisches Phänomen.
} 
teilweise zu begegnen, muss die Konversation zunächst danach gegliedert werden, wer mit wem spricht. Daraus können schließlich die Sequenzen abgeleitet werden.

Die Struktur eines kontinuierlich verlaufenden Gesprächs mit mehreren Schreibern sieht dabei so aus wie in der Grafik für das Beispiel (22) dargestellt. Die Stapelung von ,-,“ zeigt an, dass der Beitrag aus mehreren Mitteilungen besteht: so sagen zwei Striche aus, dass der Beitrag zwei Mitteilungen enthält.

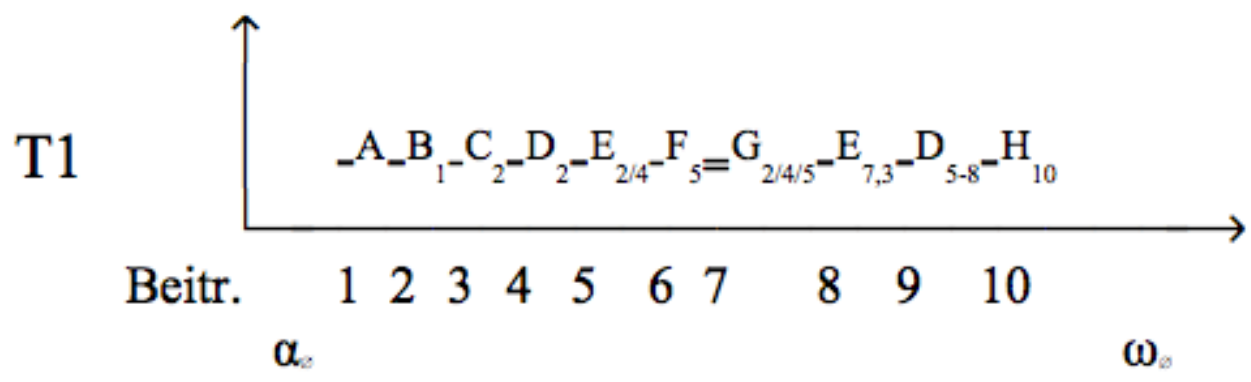

Abb. 6

Schreiber A = Agrippina

$\underline{\text { Thema } 1}=\underline{\text { Kälte (Schnee) vs. Wärme }}$

Schreiber B = Orchan

Schreiber C = June

Schreiber D = Nikitina

Schreiber E $=$ Westwood

Schreiber F = Echo

Schreiber $\mathrm{G}=$ scary boom

Schreiber H = Lenivyj

Wie in dem Beispiel davor, sehen wir an der Grafik, dass es nur ein Thema gibt, die Konversation demnach ohne Unterbrechungen durch thematisch nicht zum ersten Thema passende Mitteilungen verläuft. Es gibt einen einzigen thematischen Abschnitt. Die Konversation beginnt nicht mit einer Grußformel, die erwidert wird, sondern mit einer Mitteilung des Users Agrippina bezüglich des Zeitaums bis Silvester, auf die der User Orchan reagiert und mit seiner Reaktion eine Diskussion darüber auslöst, ob Schnee, Kälte und Winter besser sind als Sommer und Wärme. Die first-topic-position ist, wie in der Grafik zu sehen, direkt im ersten Beitrag zu finden. Dies ähnelt Beispiel (14), wo ebenfalls die ersten Schritte der Eröffnungsphase fehlen. An der Konversation beteiligen sich acht User, von 
denen zwei mehr als eine Mitteilung verfassen. Man findet sowohl zweigliedrige als auch dreigliedrige Struktureinheiten vor. Als ein Beispiel für eine Paarsequenz kann man $T_{1} M_{1}$ und $\mathrm{T}_{1} \mathrm{M}_{2}$ sehen, während $\mathrm{T}_{1} \mathrm{M}_{4}$, die Reaktionen in $\mathrm{T}_{1} \mathrm{M}_{5}-\mathrm{T}_{1} \mathrm{M}_{8}$ und $\mathrm{T}_{1} \mathrm{M}_{10}$ erweiterte Paarsequenzen ${ }^{100}$ bilden: die Mitteilung in $\mathrm{T}_{1} \mathrm{M}_{4}$ stellt dabei den ersten Teil dar und z.B. $\mathrm{T}_{1} \mathrm{M}_{7}$ den zweiten Teil zum Paar, welches durch die Mitteilung $\mathrm{T}_{1} \mathrm{M}_{10}$, in der keine neuen Informationen hinzukommen, erweitert wird. Die Erweiterung der Paarsequenz durch einen dritten Beitrag von Nikitina erfolgt hier aus dem Grund, dass die Reaktionen der anderen User nicht präferiert sind, da sie ihr nicht zustimmen, und sie ihre Ansicht nochmal betonen möchte. Wie in der Grafik zu sehen ist, finden sich mehrere Mitteilungen in einem einzigen Redebeitrag: in $\mathrm{B}_{7}$ von Sprecher 7 finden sich zwei Reaktionen auf zwei unterschiedliche Mitteilungen anderer User. Auf dieser Grundlage kann angenommen werden, dass nicht der Redebeitrag, sondern die Mitteilung die Basis jeder Sequenz ist. Die Beendigungsphase fehlt hier komplett, so dass nicht nur der Anfang der Konversation, sondern auch das Ende sehr abrupt ist. Geht man nach der Einteilung einer Konversation in einen Anfang, eine Mitte und einen Schluss, so liegt hier nur die Mitte ${ }^{101}$ der Konversation vor. Theoretisch könnte die Konversation weiter fortgesetzt werden, da nichts auf einen Abschluss hindeutet. Das wird jedoch nicht getan, weil die User offensichtlich alles Relevante zu dem Thema gesagt haben. Insofern kann hier festgehalten werden, dass eine Internetkonversation nicht durch eine Beendigungsphase abgeschlossen werden muss, um als beendet zu gelten. Stattdessen wird der Gesprächsabschluss dadurch bestimmt, dass sich niemand mehr zu dem Thema meldet, da alles Relevante gesagt wurde. Das alles unterscheidet dieses Beispiel von mündlichen Konversationsverläufen, in denen Konversationen in der Regel eingeführt und mit bestimmten

\footnotetext{
${ }^{100}$ Bei meiner Vorstellung einer erweiterten Paarsequenz greife ich zwei Annahmen auf: auf der einen Seite Grubers Vorstellung einer aus drei Beiträgen bestehenden Paarsequenz und auf der anderen Seite Postsequenzen. Im Gegensatz zu Grubers Meinung, der dritte Beitrag werde nur verbalisiert, um eine falsche Interpretation zu korrigieren, gehe ich davon aus, dass ein dritter Beitrag auch verfasst werden kann, wenn der zweite Redebeitrag sowohl eine nicht präferierte als auch eine präferierte Reaktion darstellt. Im Falle einer nicht präferierten Reaktion, kann der dritte Beitrag dazu benutzt werden, um z.B. seine Meinung noch einmal zu bekräftigen. Dabei kann der Schreiber seine Äußerung im dritten Beitrag paraphrasieren. Für Reaktionen auf präferierte bzw. nicht präferierte zweite Teile, ist sowohl charakteristisch, dass in dem dritten Beitrag keine neuen Informationen hinzukommen, die für die Weiterentwicklung des Themas relevant wären, als auch, dass die dritten Beiträge nicht nach einer weiteren Reaktion verlangen. Bei der erweiterten Paarsequenz handelt es sich also um eine dreigliedrige Struktureinheit, bei der auf den zweiten Beitrag aus der Sicht des Schreibers des ersten Beitrags eine weitere Reaktion erfolgen sollte, um z.B. seine Meinung zu bekräftigen, um zu zeigen, dass man von den Argumenten des anderen Partizipanten nicht überzeugt wurde und von seiner ursprünglichen Meinung nicht abgewichen ist oder um sich aus Höflichkeitsgründen zu bedanken. Dies findet sich auch in Postsequenzen (vgl. Fn.79). Der Grund dafür, warum ich eine erweiterte Paarsequenz statt einer Postsequenz annehme, ist der, dass es sich bei den dritten Beiträgen meiner Meinung nach um Bestandteile der Hauptsequenz handelt, da die Schreiber eine Notwendigkeit darin sehen, den zweiten Beitrag des anderen Gesprächspartizipanten noch einmal zu kommentieren.

${ }^{101}$ In der vorliegenden Dissertation werden die Begriffe Gesprächsmitte und Kernphase synonym verwendet.
} 
Phrasen beendet werden.

$\mathrm{Zu}$ linearen Konversationsverläufen gehört auch der Fall, bei dem es zwar mehrere Themen gibt, der Verlauf jedoch kontinuierlich ist: hier beginnt ein Schreiber, der bereits am ersten Thema beteiligt war, das zweite Thema erst mit dem Ende des anderen Themas. Thematisch zusammenhängende Mitteilungen stehen nacheinander. Es handelt sich jedoch nicht mehr um eine, sondern um zwei einander folgende Konversationsverläufe, da es keinen thematischen Zusammenhang zwischen den Gesprächen gibt. Der Konversationsverlauf des zweiten Themas kann dabei anders aussehen als der des ersten.

Die andere Art der Konversationsverläufe ist bei Konversationen mit Unterbrechungen durch thematisch fremde Mitteilungen zu finden, wie z.B. (21). Hier gibt es zwei Mitteilungen (non$\mathrm{T}_{2} \mathrm{M}_{1}$ und non- $\mathrm{T}_{3} \mathrm{M}_{1}$ ), die innerhalb eines Gesprächs auftauchen, aber nichts mit diesem zu tun haben.

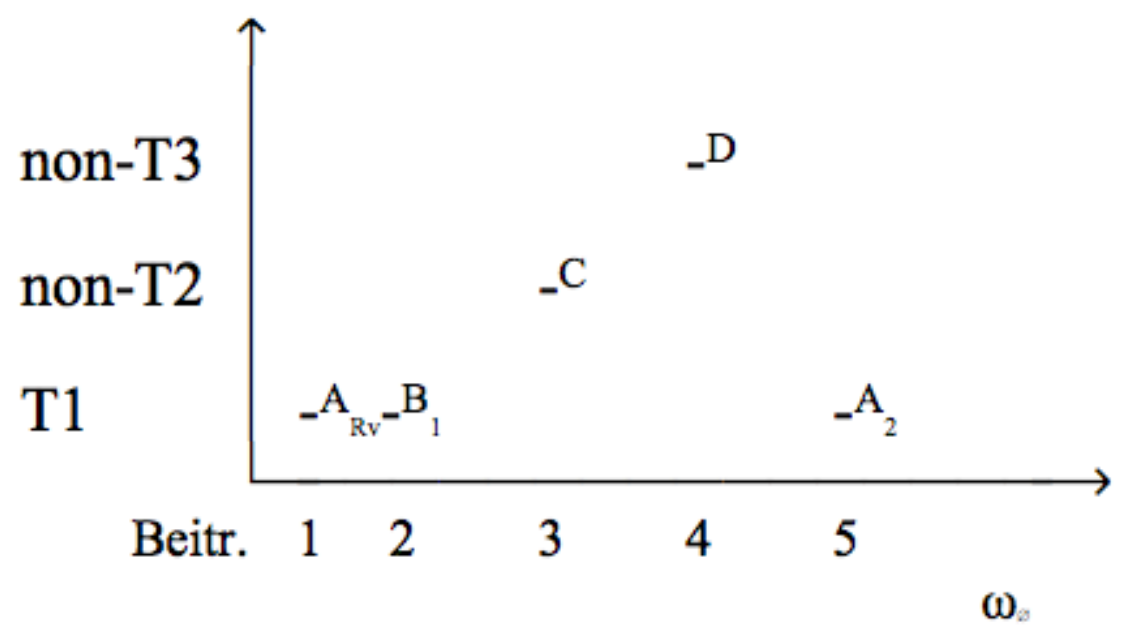

Abb. 7

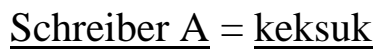

Schreiber B = Ra1n

Schreiber $\mathrm{C}=$ Aligarh

Schreiber D = nikitaSwatT
Thema $1=\underline{\text { Moderator und Umgang mit Trolls }}$

non-Thema $2=$ Trolling

non-Thema 3 = Trolling

Wie an der Grafik zu sehen ist, gibt es neben dem Thema 1 zwei non-Themen ${ }^{102}$. Es handelt

\footnotetext{
${ }^{102}$ Es handelt sich bei den beiden Mitteilungen um Troll-Mitteilungen, die den Zweck haben das Gespräch zu stören und die in diesem Fall keinen lexikalischen Inhalt haben. Dennoch vergebe ich Ihnen zwei non-Themen, da sie für ihre Schreiber einen bestimmten Zweck erfüllen und die Funktion des Störens als Metathema haben.
} 
sich bei ihnen um eingliedrige Sequenzen, da die Schreiber auf sie keinerlei Reaktion bekommen und möglicherweise auch nicht erwarten. Auf Grund des Bezugs der Mitteilungen lässt sich schließen, dass die Redebeiträge des ersten Themas zwei Paaren bilden: das Erste bilden die Mitteilungen $T_{1} M_{1}$ und $T_{1} M_{2}$, das Zweite $T_{1} M_{2}$ und $T_{1} M_{3}$. Die Frage in $T_{1} M_{2}$ ist also sowohl der zweite Teil zu $\mathrm{T}_{1} \mathrm{M}_{1}$ als auch der erste Teil zu der Antwort darauf in $\mathrm{T}_{1} \mathrm{M}_{3}$. An der Entfernung zwischen den zusammengehörenden Mitteilungen in $\mathrm{T}_{1}$ sieht man, dass die eingeworfenen Mittelungen der non-Themen die Gesprächsstruktur zwar „stören“, aber ignoriert werden; der User keksuk übergeht dabei einfach die beiden Mitteilungen der nonThemen und schreibt, ohne die Mitteilung von Ra1n, auf die er sich bezieht, zu zitieren, direkt seine Antwort. Die Antwort, die den zweiten Teil des zweiten Paares bildet, entspricht dabei einer bevorzugten Reaktion, da es sich um eine zu erwartende Antwort handelt. Anhand der Grafik sieht man, dass in diesem Beispiel ein Beitrag nicht mehr als eine Mitteilung enthält, was ihn mit einem Redebeitrag in mündlichen Konversationen vergleichbar macht. Wie in den Beispielen davor fehlen Abschiedsformeln. Das Gespräch endet somit abrupt, d.h., dass eine Beendigungsphase nicht vorhanden ist. Das Beispiel ist exemplarisch für einen Konversationsverlauf, der durch thematisch unpassende Mitteilungen unterbrochen wird, die in dieser Konversation jedoch ignoriert werden.

Die beiden Beispiele (23) und (18) beinhalten unterbrochene Verläufe, bei denen andere User auf die thematisch fremden Mitteilungen eingehen. Auf diese Weise entstehen mehrere thematische Abschnitte innerhalb einer Konversation. An Beispiel (23) ist charakteristisch, dass in das initiale Thema ein weiteres Thema eingebettet ist. Der thematische Abschnitt zum zweiten Thema endet, sobald das dominante Thema wieder aufgegriffen wird, wie an der Struktur zu erkennen ist. An $B_{17}$ in (23) sieht man, dass ein Redebeitrag mehrere thematische Abschnitte enthält. Die Grafik wird um mehrere Zeichen ergänzt: „「“ gibt an, dass der Themenwechsel innerhalb eines Redebeitrags markiert ist, während „SK“ für Selbstkohärenz steht. Bei der Selbstkohärenz handelt es sich um eine spezielle Art der Mitteilung, bei der der Schreiber an seine eigene Mitteilung nach einer bestimmten Zeit anknüpft. 


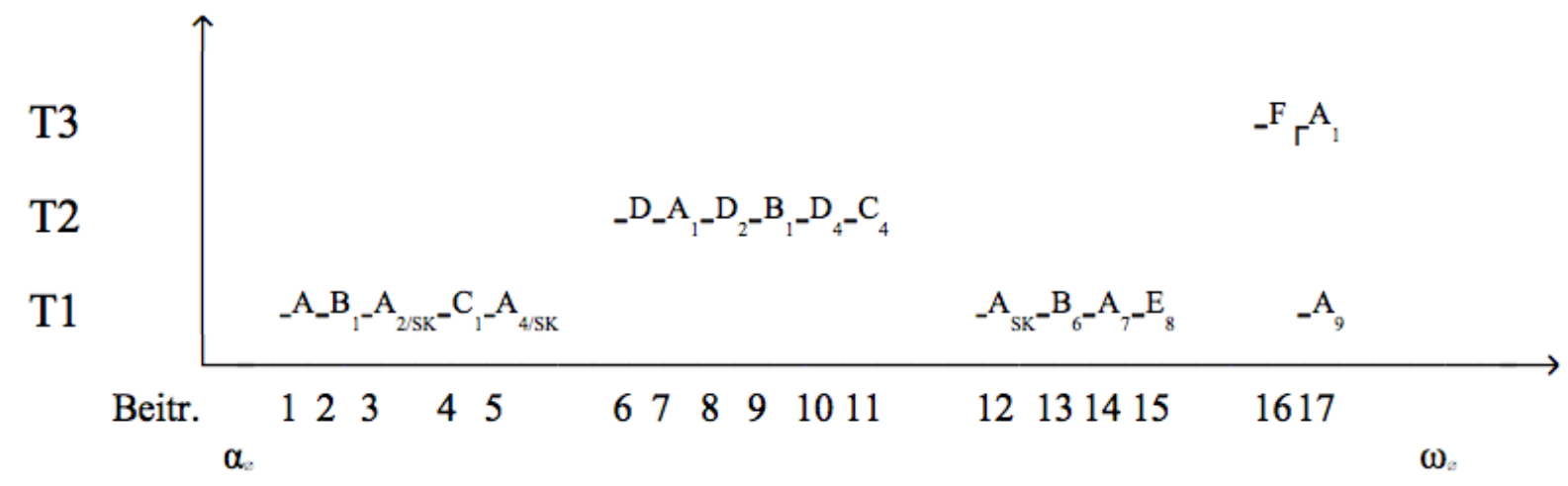

Abb. 8

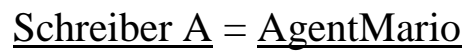

$\underline{\text { Schreiber B }}=\underline{\text { silver }}$

Schreiber $\mathrm{C}=$ Tiesto

$\underline{\text { Schreiber D }}=\underline{\text { MaxaRiaZ }}$

Schreiber $\mathrm{E}=\mathrm{Lam} *$

Schreiber F = Erik
Thema $1=\underline{\text { Experiment }}$

Thema 2 = „Rauchen“ ohne MaxaRiaZ

Thema 3 = Hilfe bei Beschaffung von

Programmen

Wie an der Grafik zu sehen ist, werden mehrere Themen verhandelt, wovon das erste Thema anhand der höheren Anzahl der Mitteilungen als dominantes Thema anzusehen ist. Als dominante Schreiber sind AgentMario, silver und MaxaRiaZ zu betrachten. Das ist nicht weiter verwunderlich, da AgentMario und MaxaRiaZ die Gesprächsinitiatoren von zwei der drei Themen sind. Der allgemeine Verlauf der Konversation ähnelt dem der anderen Beispiele. Da Grußformeln fehlen, fehlen die ersten Schritte der Eröffnungsphase. Das Gespräch fängt mit der first-topic-position in $\mathrm{T}_{1} \mathrm{M}_{1}$ an. Über den in der Grafik dargestellten Bezug der Mitteilungen, erkennt man, dass es in diesem Abschnitt des Gespräches zwei dreigliedrige Struktureinheiten gibt, die beide erweiterte Paarsequenzen darstellen: die erste bildet AgentMarios erster Redebeitrag, die Reaktion des Users silver in $T_{1} M_{2}$ sowie die nochmalige Reaktion von AgentMario auf silvers Mitteilung in $\mathrm{T}_{1} \mathrm{M}_{3}$, die zweite erweiterte Paarsequenz ergibt sich wiederum aus AgentMarios erstem Redebeitrag, der Reaktion des Users Tiesto in $T_{1} M_{4}$ sowie der nochmaligen Reaktion von AgentMario in $T_{1} M_{5}$. In beiden Fällen trägt der dritte Beitrag keine neuen Informationen bei. Die Reaktionen der User silver und Tiesto sind dabei unterschiedlich: während es sich in $T_{1} M_{2}$ um eine nicht präferierte Reaktion handelt, gehört die Reaktion in $\mathrm{T}_{1} \mathrm{M}_{4}$ zur präferierten Kategorie. Beide Reaktionen haben unterschiedliche Resultate: während die Reaktion auf $\mathrm{T}_{1} \mathrm{M}_{2}$ einer Korrektur durch den 
Gesprächsinitiator AgentMario ähnelt, folgt auf $\mathrm{T}_{1} \mathrm{M}_{4}$ eine Danksagung in $\mathrm{T}_{1} \mathrm{M}_{5}$. Dass AgentMarios Beiträge dabei immer wieder an seine initiale Mitteilung anknüpft, kann damit erklärt werden, dass er die anderen User immer wieder dazu aufruft, sich an seinem Experiment zu beteiligen. Im sechsten Redebeitrag beginnt abrupt, d.h. ohne jede Einleitung, das zweite Thema. Aufällig ist hier, dass das erste und das zweite Thema nicht parallel besprochen werden: sobald das zweite Thema einsetzt, wird das erste Thema nicht weiter verhandelt und andersherum. Das zweite Thema beinhaltet sowohl zwei- als auch dreigliedrige Struktureinheiten. Eine zweigliedrige Struktureinheit bilden die Mitteilungen $\mathrm{T}_{2} \mathrm{M}_{4}$ und $\mathrm{T}_{2} \mathrm{M}_{6}$. Die erste dreigliedrige Struktureinheit bilden $\mathrm{T}_{2} \mathrm{M}_{1}-\mathrm{T}_{2} \mathrm{M}_{3}$. Die zweite dreigliedrige Struktureinheit stellen $\mathrm{T}_{2} \mathrm{M}_{1}, \mathrm{~T}_{2} \mathrm{M}_{4}$ und $\mathrm{T}_{2} \mathrm{M}_{5}$ dar. Hier sehen wir, dass eine Mitteilung von mehreren Usern als erster Teil von unterschiedlichen Sequenzen verwendet wird. Ab Beitrag 12 gibt es wieder einen Wechsel zum ersten Thema, der ebenfalls abrupt ist. Die erste Mitteilung stammt von AgentMario und wird von ihm dazu benutzt, an seine früheren Mitteilungen anzuknüpfen und weitere User zur Reaktion im Sinne einer Beteiligung an seinem Experiment aufzufordern. In diesem Abschnitt des Themas lassen sich wieder dreigliedrige Struktureinheiten erkennen: die erste Einheit bilden die Mitteilungen von AgentMario in $\mathrm{T}_{1} \mathrm{M}_{6}$, silver in $\mathrm{T}_{1} \mathrm{M}_{7}$ und AgentMario $\mathrm{T}_{1} \mathrm{M}_{8}$ und die zweite Einheit bilden die Mitteilungen von AgentMario in $\mathrm{T}_{1} \mathrm{M}_{8}$, Lam* in $\mathrm{T}_{1} \mathrm{M}_{9}$ und wieder AgentMario in $\mathrm{T}_{1} \mathrm{M}_{10}$. Dabei wird die zweite Struktureinheit von Lam* aber forciert, da er die Mitteilung $\mathrm{T}_{1} \mathrm{M}_{8}$ von AgentMario als ersten Part für seine Mitteilung verwendet, obwohl er an der vorherigen Sequenz als Schreiber nicht beteiligt war. In Beitrag 16 fängt ohne Einleitung das dritte Thema an. Die Struktur ist zweigliedrig: AgentMarios Reaktion bildet den zweiten Teil der Paarsequenz, die jedoch von dem User Erik als solche nicht präferiert ist. Wie in der Grafik ebenfalls zu sehen ist, verfasst AgentMario zwei thematisch unterschiedliche Mitteilungen in einem einzigen Beitrag; der Themenwechsel innerhalb dieses Beitrags ist jedoch durch das Zitieren der jeweiligen Mitteilung, auf die er sich bezieht, markiert. Wie in dem Beispiel davor lässt sich auf dieser Grundlage belegen, dass ein Redebeitrag nicht als Basis einer Sequenz angesehen werden kann. Insgesamt ist die Reihenfolge der Themen beliebig in dem Sinne, dass die Themen austauschbar sind, z.B. hätte „Rauchen“ statt dem zweiten auch das dritte Thema sein können. Wie in dem Beispiel zuvor, fehlt auch hier die Beendigungsphase komplett. Die Konversation endet abrupt. Von der Gesamtorganisation her betrachtet, entspricht auch dieses Beispiel bezüglich der Gesamtorganisation eher der Kernphase einer mündlichen Konversation.

Anhand des Beispiels (18) wird eine weitere Unterart für einen Konversationsverlauf 
aufgezeigt, bei dem von den Usern auf eingeworfene, thematisch unpassende Mitteilungen eingegangen wird. Es handelt sich hier um eine längere Interaktion, in der drei Themen parallel besprochen werden: das erste Thema entwickelt sich aus der Aussage des Bildes aus der Mitteilung von Ksanka, das zweite bezieht sich auf den „Sushi“-Vorschlag von Black Santa (ab Mitteilung $\mathrm{T}_{2} \mathrm{M}_{1}$ ) und in dem dritten geht es um Vesnushka (ab Mitteilung $\mathrm{T}_{3} \mathrm{M}_{1}$ ). Die Struktur sieht folgendermaßen aus:

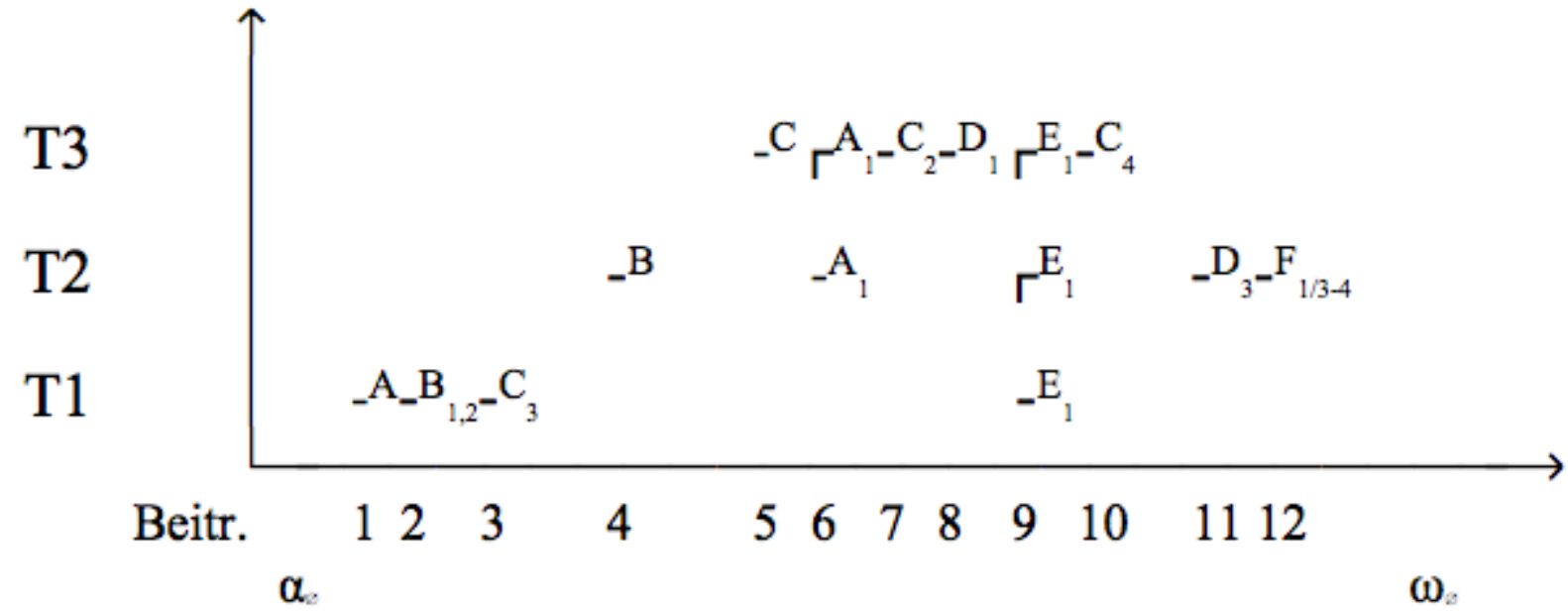

Abb. 9

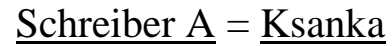

Schreiber B = Black Santa

$\underline{\text { Schreiber } \mathrm{C}}=\underline{\text { Vesnushka }}$

Schreiber D = Tempest

Schreiber E = scary boom

Schreiber F = baskervila $\underline{\text { Thema } 1}=\underline{\text { Bild }}$

$\underline{\text { Thema } 2}=\underline{\text { Essen } / \text { Sushi }}$

$\underline{\text { Thema } 3}=\underline{\text { Mitgliedsdauer von Vesnushka }}$

Wie in den Beispielen davor fehlen Grußformeln und damit Bestandteile der Eröffnungsphase. Das Gespräch setzt mit der first-topic-position im ersten Redebeitrag ein. Auf diese Weise kennen die Leser sofort den Grund für die Konversation. Auch in den beiden anderen Themen werden diese Gründe direkt in den ersten Redebeiträgen angegeben. Wie an der Grafik ebenfalls zu sehen ist, entstehen durch Reaktionen auf thematisch nicht dazugehörige Mitteilungen seitens anderer User drei parallel geführte Konversationsstränge mit drei fast gleich dominanten Themen, so dass eine Themenhierarchie nicht vorhanden ist. Eine Einbettung des zweiten und dritten Themas in das erste Thema wie in Beispiel (23) liegt 
in diesem Beispiel nicht vor. Als dominanter Schreiber (da am aktivsten) ist im ersten Thema Ksanka und im dritten Vesnushka anzusehen. Dies verwundert kaum, da beide auch die Gesprächsinitiatoren der jeweiligen Themen sind. Wie der Grafik zu entnehmen ist, bestehen einige Redebeiträge ( $\mathrm{B}_{6}$ und $\mathrm{B}_{9}$ ) aus mehreren Mitteilungen. Dies entspricht auch dem Befund aus anderen Beispielen und unterstützt die These, dass als Basis einer Sequenz in Konversationen in Internetforen nicht der Redebeitrag, sondern die Mitteilung angesehen werden muss. Über den Bezug der Mitteilungen in der Grafik lässt sich erkennen, dass das erste Thema eine zwei- und eine dreigliedrige Struktureinheit beinhaltet. Bei der zweigliedrigen handelt es sich um eine Paarsequenz aus $T_{1} M_{1}$ und $T_{1} M_{4}$, bei der dreigliedrigen um eine größere Sequenz aus $\mathrm{T}_{1} \mathrm{M}_{1,2}-\mathrm{T}_{1} \mathrm{M}_{3}{ }^{103}$. Bei der Paarsequenz sieht man, dass zusammenhängende Bestandteile eines Paares nicht nah beieinander stehen müssen. Da es sich in Forenthreads im Internet um schriftlich realisierte Konversationen handelt, die lange Bestand haben, können Mitteilungen jederzeit von anderen Usern dazu benutzt werden, um darauf zu reagieren und so eine neue bzw. weitere Sequenz anzufangen. Eine Mitteilung kann somit ein Bestandteil mehrerer unterschiedlicher Sequenzen sein. Ein Augenmerk sollte darauf gelegt werden, dass Ksanka in der ersten Mitteilung den anderen User Black Santa durch eine direkte Adressierung mit seinem Spitznamen ptičko involviert - im Gegensatz zu der selbsttätigen Involvierung, die sonst üblich ist - und auf diese Weise das Rederecht an ihn weitergibt. Das zweite Thema besteht ebenfalls aus mehreren Struktureinheiten, die zweigliedrige Paarsequenzen darstellen: 1) $T_{2} M_{1}$ und $T_{2} M_{2}$, 2) $T_{2} M_{3}$ und $T_{2} M_{4}$ und 3) $T_{2} M_{1}$ und $T_{2} M_{3}$. Sowohl $T_{2} M_{1}$ und $T_{2} M_{2}$ als auch $T_{2} M_{1}$ und $T_{2} M_{3}$ bilden Paarsequenzen, aus denen sich größere Struktureinheiten hätten entwickeln können, da $T_{2} M_{2}$ und $T_{2} M_{3}$ jeweils die zweiten Bestandteile zu $\mathrm{T}_{2} \mathrm{M}_{1}$ sind, aber auch gleichzeitig durch die Fragen, mit denen Black Santa zu einer Reaktion aufgefordert wird, die ersten Parts zu weiteren Paaren bilden, die jedoch auf Grund der fehlenden Reaktion von Black Santa unvollständig bleiben. Möglicherweise ist die fehlende Reaktion seitens Black Santa der Grund dafür, dass ein anderer User - hier Tempest - auf die Mitteilung $\mathrm{T}_{2} \mathrm{M}_{3}$ reagiert. Die letzte Mitteilung des Themas stellt $\mathrm{T}_{2} \mathrm{M}_{5}$ dar, mit der der User baskervila auf die Mitteilungen $\mathrm{T}_{2} \mathrm{M}_{1}, \mathrm{~T}_{2} \mathrm{M}_{3}-\mathrm{T}_{2} \mathrm{M}_{4}$ gleichzeitig reagiert. Beim dritten Thema liegen überwiegend dreigliedrige Struktureinheiten vor. Bei diesen handelt es sich um erweiterte Paarsequenzen, die aus der Mitteilung $T_{3} M_{1}$ von Vesnushka, einer Reaktion auf die Mitteilung (z.B. $\mathrm{T}_{3} \mathrm{M}_{2}, \mathrm{~T}_{3} \mathrm{M}_{4}$ ) und dem Dank von

\footnotetext{
${ }^{103}$ Dass eine Äußerung innerhalb einer Mitteilung den Bestandteil einer anderen Struktureinheit darstellen kann als die restliche Mitteilung, legt den Schluß nahe, dass möglicherweise eine Äußerung als kleinste Einheit angenommen werden muss.
} 
Vesnushka bestehen (z.B. $\mathrm{T}_{3} \mathrm{M}_{3}$ oder $\mathrm{T}_{3} \mathrm{M}_{6}$ ). Der Dank im dritten Beitrag ist dabei nicht erforderlich, d.h. es wird nicht vom zweiten Beitrag verlangt. Vielmehr verdeutlicht Vesnushka dadurch, dass sie weitere Reaktionen der beiden anderen User Ksanka oder Tempest $\mathrm{zu}$ dem Thema (außer vielleicht einem gern geschehen) nicht mehr erwartet. Offenbar wird dies auch so interpretiert, denn Tempest wendet sich in ihrer nächsten Mitteilung $\mathrm{T}_{2} \mathrm{M}_{4}$ einem der anderen Themen zu. Nur auf die Reaktion von scary boom in $\mathrm{T}_{3} \mathrm{M}_{5}$ erfolgt kein Dank von Vesnushka, so dass diese Sequenz nicht als erweiterte Paarsequenz zu betrachten ist. Dies ist insofern überraschend, als dass scary boom offenbar an dem importierten Chatgespräch partizipiert hat. ${ }^{104}$ Einen ersichtlichen Grund, warum Vesnushka auf $\mathrm{T}_{3} \mathrm{M}_{5}$ nicht reagiert, scheint es nicht zu geben. Möglicherweise hat sie die Mitteilung überlesen und sich deshalb nur bei Tempest und nicht bei beiden Usern bedankt.

Wie in der Grafik zu sehen ist, reagieren einige Partizipanten in einem einzigen Redebeitrag auf mehrere bzw. alle Themen, wie z.B. scary boom im Beitrag $B_{9}$. Dabei arbeiten sie mit der Zitatfunktion oder adressieren die Mitteilung direkt an die jeweiligen User, um zu zeigen, auf welche Äußerung oder Mitteilung sie sich beziehen. Auf diese Weise wird Missverständnissen von vornherein vorgebeugt, da alles übersichtlich und klar strukturiert ist. Innerhalb solcher Beiträge fungiert das Zitieren als eine Markierung des Themenwechsels. Die präferierte Reihenfolge der Reaktionen in Beiträgen, in denen mehrere thematische Abschnitte behandelt werden, entspricht der Themenorganisation, d.h. die Reihenfolge der Reaktionen in einem Beitrag erfolgt nach der Reihenfolge der Einbringung der Themen. Wie in den anderen Beispielen auch, fehlen Abschiedsformeln. Die Konversation ist hier wie in anderen Beispielen damit beendet, dass kein weiterer User mehr eine Mitteilung zu einem der Themen verfasst, weil alles Relevante gesagt worden ist. Die Gesamtorganisation des Beispiels beinhaltet auf diese Weise die Kernphase einer Konversation.

Diese Art des Konversationsverlaufs kommt vor allem im ersten Thread von allen Unterarten am häufigsten vor. Hierbei scheint es sich um den ökonomischsten Konversationsverlauf zu handeln, da mehrere Themen parallel laufen und die User auf so viele Mitteilungen wie möglich in einem einzigen Beitrag reagieren können. Dementsprechend kam in beiden untersuchten Threads kaum ein Beispiel vor, bei dem die User auf thematisch unterschiedliche Mitteilungen nicht in einem einzigen, sondern in mehreren einzelnen Redebeiträgen reagierten. Der Grund dafür liegt auf der Hand: es ist unökonomisch zwei getrennte Beiträge zu verfassen, wenn unterschiedliche Reaktionen auf unterschiedliche

\footnotetext{
${ }^{104} \mathrm{Zu}$ erkennen u.a. daran, dass scary boom direkt aus dem Gespräch heraus zitiert.
} 
Mitteilungen in einem einzigen Beitrag verfasst werden und z.B. mit Hilfe der Zitatfunktion von einander klar abgetrennt werden können.

Die beiden Beispiele (11) und (24) stellen eine Mischung aus Verläufen, in denen in das dominante Thema eingeworfene Mitteilungen anderer Themen ignoriert und nicht ignoriert werden, dar. Untersuchen wir zunächst Beispiel (11). Die Struktur des Beispiels sieht folgendermaßen aus:

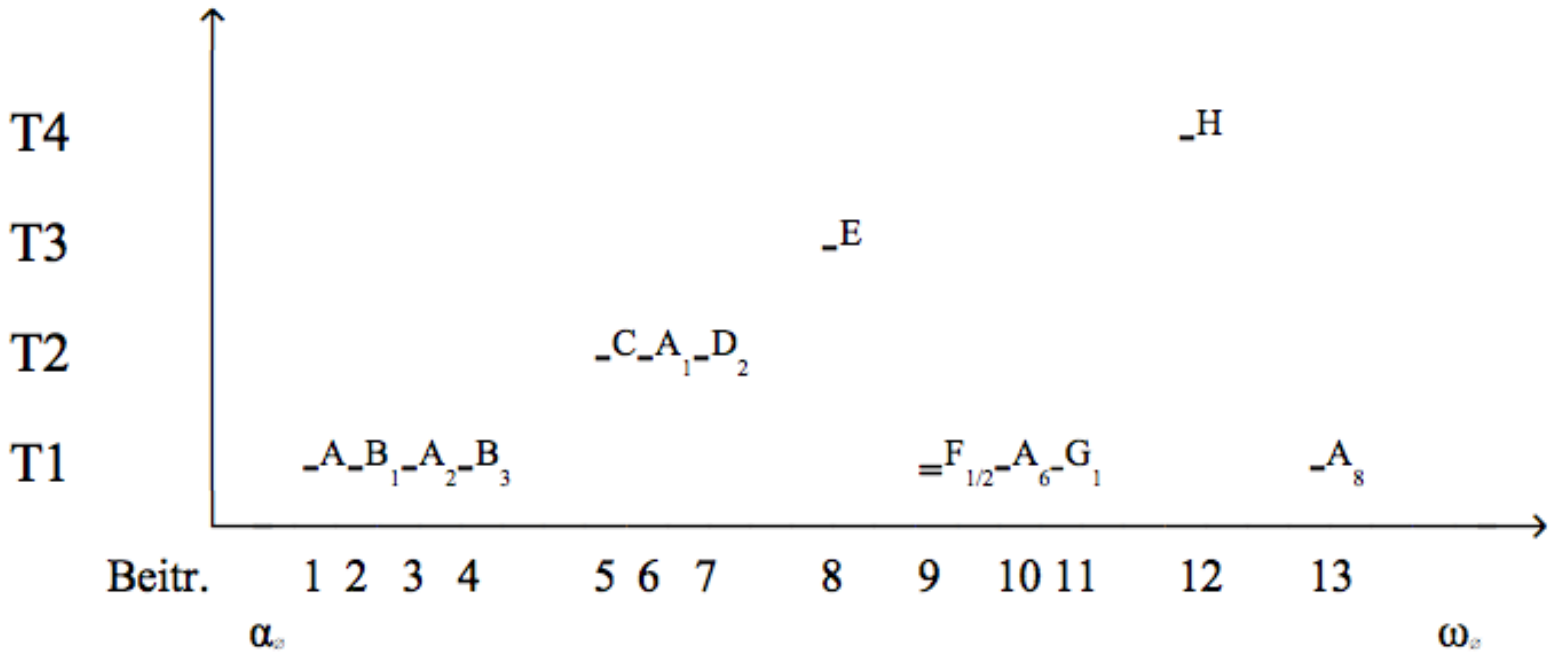

Abb. 10

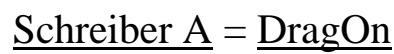

Schreiber B = Ra1n

Schreiber $\mathrm{C}=$ surpriz

Schreiber D = mironich

Schreiber E $=$ Yodjer

Schreiber $F=3 G$

Schreiber $\mathrm{G}=$ keksuk

Schreiber H = Resilved $\underline{\text { Thema } 1}=\underline{\text { Auto }}$

Thema $2=$ Geld

Thema 3 = Frage bezüglich Hackens

Thema 4 = Frage bezüglich DDOS

Wie an der Grafik zu erkennen ist, liegt hier ein Gespräch mit vier Themen vor, wovon jedoch bei zweien die Mitteilungen durch die anderen User ignoriert werden. Das dominante Thema ist das erste und der dominante Schreiber DragOn, was erwartbar ist, da er der Gesprächsinitiator ist. Die anderen Themen sind in das erste eingebettet, wodurch eine Verschachtelung, aber auch eine Aufteilung des ersten Themas in einzelne Abschnitte 
entsteht, wie z.B. auch in (23). Die einzelnen Abschnitte sind durch das gemeinsame Thema und den Gesprächsinitiator DragOn, der als einziger User in allen Gesprächsteilen vorkommt, verbunden, während die anderen Partizipanten, die sich selbst involvieren, wechseln. DragOn reagiert dabei auf jede Mitteilung, die zu seiner Frage passt. Wie in den Beispielen davor fehlen Grußformeln, so dass das Gespräch direkt mit einer Frage von DragOn in der firsttopic-position beginnt, durch die verdeutlicht wird, was der Schreiber bezwecken will. Die Konversation setzt somit mit der Kernphase ein. Über den Bezug der Mitteilungen in der Grafik sieht man, dass das erste Thema aus drei- oder mehrgliedrigen Sequenzen besteht. Die erste mehrgliedrige Sequenz bildet sich aus $\mathrm{T}_{1} \mathrm{M}_{1}-\mathrm{T}_{1} \mathrm{M}_{4}$. Es entstehen zwei Paarsequenzen: $\mathrm{T}_{1} \mathrm{M}_{1}$ ist der erste Part, $\mathrm{T}_{1} \mathrm{M}_{2}$ als Reaktion darauf der zweite, $\mathrm{T}_{1} \mathrm{M}_{3}$ ist wiederum die Reaktion auf $\mathrm{T}_{1} \mathrm{M}_{2}$ und bildet den ersten Part zum zweiten Part $\mathrm{T}_{1} \mathrm{M}_{4}$. Die zweite Sequenz stellen die Mitteilungen $T_{1} M_{1}$ und $T_{1} M_{5}-T_{1} M_{7}$ dar. Dabei greift der Schreiber $3 G$ die beiden Mitteilungen $T_{1} M_{1}$ und $T_{1} M_{2}$ auf und reagiert darauf in zwei Mitteilungen in einem einzigen Redebeitrag, obwohl er an der vorherigen Sequenz nicht beteiligt war. Den Abschluss dieser Sequenz bildet die Reaktion von DragOn in $\mathrm{T}_{1} \mathrm{M}_{7}$. Wie in den anderen Beispielen bereits zu sehen war, wird auch hier eine Mitteilung als Bestandteil mehrerer verschiedener Sequenzen von den Usern verwendet. Die letzte mehrgliedrige Struktureinheit bilden die Mitteilungen $\mathrm{T}_{1} \mathrm{M}_{1}, \mathrm{~T}_{1} \mathrm{M}_{8}$ und $\mathrm{T}_{1} \mathrm{M}_{9} . \mathrm{T}_{1} \mathrm{M}_{8}$ stellt den zweiten Part zu $\mathrm{T}_{1} \mathrm{M}_{1}$ dar. Die Sequenz wird weiter mit der Reaktion auf $\mathrm{T}_{1} \mathrm{M}_{8}$ in $\mathrm{T}_{1} \mathrm{M}_{9}$ fortgesetzt, wird jedoch nicht abgeschlossen, da das Paar auf Grund der fehlenden Reaktion von Resilved unvollendet bleibt. Das zweite Thema besteht aus zwei Sequenzen. $\mathrm{T}_{1} \mathrm{M}_{1}$ wird dabei von surpriz dazu verwendet, um ein weiteres Thema anzuknüpfen. Diese Reaktion in $\mathrm{T}_{2} \mathrm{M}_{1}$ bildet gleichzeitig den ersten Part zu $\mathrm{T}_{2} \mathrm{M}_{2}$. Die zweite Sequenz ergibt sich aus $T_{2} M_{2}$ und $T_{2} M_{3}$. Obwohl es sich bei $T_{2} M_{1}$ um eine weniger bevorzugte Reaktion handelt, da sie - indem sie vom ersten Thema zu einem anderen Thema überleitet - weder hilfreich noch erwartet ist, reagiert DragOn auf diese, während er auf die andere Mitteilung $\mathrm{T}_{2} \mathrm{M}_{3}$ nicht mehr reagiert. Möglicherweise liegt dies daran, dass die Frage in $\mathrm{T}_{2} \mathrm{M}_{1}$ als eine Aufforderung zu einer Reaktion fungiert und DragOn dieser Aufforderung nachkommt. $\mathrm{T}_{2} \mathrm{M}_{3}$ wird hingegen nicht als Aufforderung interpretiert, so dass DragOn eine Reaktion für unnötig hält. Wie oben bereits erwähnt, werden zwei thematisch fremde Mitteilungen, nämlich $\mathrm{T}_{3} \mathrm{M}_{1}$ und $\mathrm{T}_{4} \mathrm{M}_{1}$, von den anderen Schreibern ignoriert, obwohl sie als Bitten bzw. Fragen die ersten Teile von Paarsequenzen bilden. Da die Mitteilungen ignoriert werden, bleiben die Paare unvollständig. Das Fehlen der Antworten gilt dabei als weniger präferierte Reaktion und wird als Ablehnung interpretiert - zu sehen daran, dass keine weiteren Fragen dieser User kommen. Wie in den anderen Beispielen auch, fehlen 
Abschiedsformeln, und da die letzte Paarsequenz des ersten Themas unvollendet bleibt, findet das Gespräch ein abruptes Ende ohne einen einzigen Schritt der Beendigungsphase. Somit entspricht es nur der Kernphase einer mündlichen Konversation. Dies entspricht auch den vorherigen Befunden. Das Gesprächsende erfolgt auf der Grundlage, dass sich kein anderer User mehr zu dem Thema zu Wort meldet. Insgesamt ist für dieses Beispiel festzustellen, dass viel mit der Zitatfunktion gearbeitet wird. Damit zeigen die User immer an, auf welche Mitteilung sich die eigene Mitteilung bezieht. Ebenfalls finden wir hier einen Fall, in dem ein Redebeitrag aus mehreren Mitteilungen besteht.

Ein weiteres Beispiel für eine solche Unterart des Konversationsverlaufs ist (24). Die Grafik wurde um ein weiteres Zeichen ergänzt: tiefgestellte kleine Buchstaben „a,b,c...“ (B $\left.\mathrm{B}_{8}\right)$ zeigen die Reihenfolge der Mitteilungen auf, sollte ein Beitrag mehrere thematische Mitteilungen beinhalten und diese nicht der Reihenfolge der Themen entsprechen. Die Struktur sieht folgendermaßen aus:

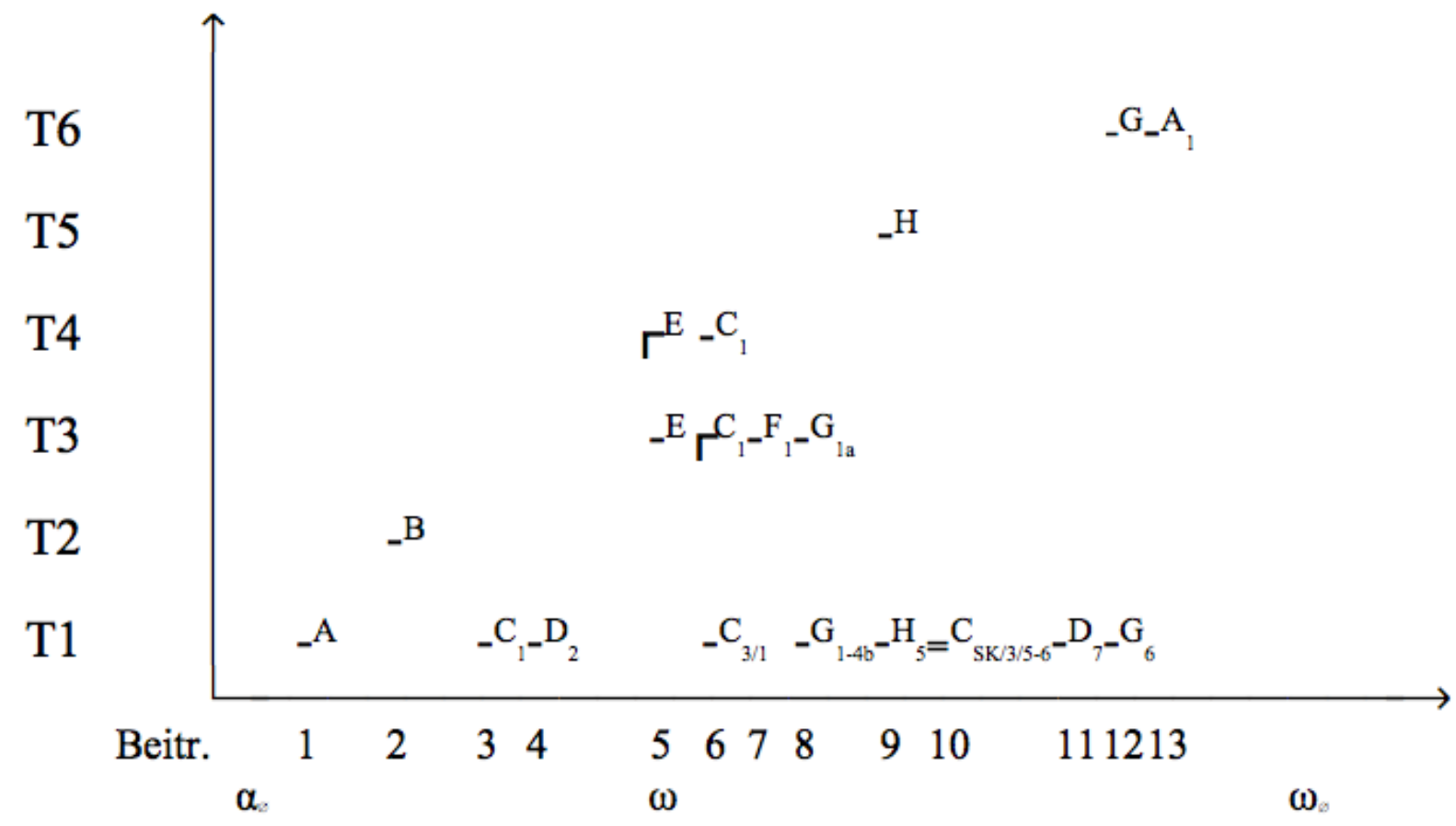

$A b b .11$

Schreiber A = Mariann Mistik

Schreiber B $=$ Jo

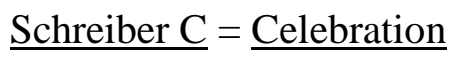

$\underline{\text { Schreiber } \mathrm{D}}=\underline{\text { Tempest }}$

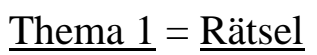

Thema $2=$ Gesprächsthemen von

Kindern

Thema 3 = „Abschied“ Heilings 
Schreiber E $=$ Heiling

Schreiber F = Imja Pol'zovatelja

$\underline{\text { Schreiber } \mathrm{G}}=\underline{\text { Stchatksi }}$

Schreiber H = Black Santa
Thema 4 = Gewicht/Figur

Thema 5 = lustiger Code

Thema $6=$ Severus Tobbiasovič

Wie an der Grafik zu erkennen ist, hat das Beispiel mehrere Themen, von denen das erste Thema als dominantes Thema anzusehen ist. Als dominante Schreiber sind in T1 Celebration, Tempest und Stchatksi anzusehen, während es in den anderen Themen keine dominanten Schreiber gibt. Das Beispiel beginnt nicht mit einer Begrüßung, sondern setzt direkt mit der first-topic-position in der ersten Mitteilung ein. Eine Eröffnungsphase ist somit wie in den anderen Beispielen auch nicht vollständig. Über den Bezug der Mitteilungen sieht man, dass das erste Thema mehrere unterschiedlich große Struktureinheiten beinhaltet. Den ersten Part bildet $\mathrm{T}_{1} \mathrm{M}_{1}$ : Mariann Mistik gibt eine Aufgabe - eine Art Rätsel - auf. Dieser Beitrag besteht aus mehreren Sequenzarten: Äußerungen 1 und 4 bilden die Hauptsequenz, in die die Äußerungen 2-3 als Nebensequenz eingeschoben werden. Die Mitteilung $\mathrm{T}_{1} \mathrm{M}_{1}$ dient dabei als erster Part für mehrere Sequenzen, z.B. stellen $\mathrm{T}_{1} \mathrm{M}_{1}$ als erster Part und $\mathrm{T}_{1} \mathrm{M}_{2}$ als zweiter Part eine Paarsequenz dar. Dabei wird $\mathrm{T}_{1} \mathrm{M}_{2}$ von dem User Tempest als ersten Part für die eigene Mitteilung $\mathrm{T}_{1} \mathrm{M}_{3}$ aufgegriffen, woraus eine mehrgliedrige Sequenz entsteht, da der User Celebration in $\mathrm{T}_{1} \mathrm{M}_{7}$ auf $\mathrm{T}_{1} \mathrm{M}_{4}$ reagiert, worauf Tempest in $\mathrm{T}_{1} \mathrm{M}_{9}$ nochmals antwortet. Zu den Besonderheiten dieser Konversation gehört die Mitteilung $\mathrm{T}_{1} \mathrm{M}_{4}$, da diese den zweiten Part zu zwei Mitteilungen gleichzeitig bildet - angedeutet auch durch die Adressierung - und zwar zu $\mathrm{T}_{1} \mathrm{M}_{1}$ und $\mathrm{T}_{1} \mathrm{M}_{3}$. Dieselbe Vorgehensweise findet man auch bei dem Schreiber Stchatksi in $\mathrm{T}_{1} \mathrm{M}_{5}$, wo er gleichzeitig auf die Mitteilungen $\mathrm{T}_{1} \mathrm{M}_{1}-\mathrm{T}_{1} \mathrm{M}_{4}$ reagiert und alle gleichzeitig mit narod adressiert. Diese Mitteilung wird aber noch für zwei andere Sequenzen benutzt: eine dreigliedrige Sequenz bilden die Mitteilungen $T_{1} M_{5}, T_{1} M_{6}$ und $T_{1} M_{10}$; eine zweiteilige Struktur entsteht aus $\mathrm{T}_{1} \mathrm{M}_{5}$ und $\mathrm{T}_{1} \mathrm{M}_{8}$, wobei unklar ist, ob die Sequenz als vollendet anzusehen ist oder der Schreiber Celebration eine Reaktion erwartet, die dann jedoch nicht erfolgt. Charakteristisch für diesen Verlauf ist das Vorkommen thematisch fremder Mitteilungen inmitten des dominanten Themas, von denen manche ignoriert werden, während auf andere reagiert wird. In diesem Beispiel wird $\mathrm{T}_{2} \mathrm{M}_{1}$ von den anderen Usern unbeachtet gelassen. Dies gilt wie in dem Beispiel (11) als nicht präferierte Reaktion, weswegen sich der User Jo nicht noch einmal meldet. Eine andere thematisch fremde Mitteilung, auf die kein Bezug genommen wird, ist $\mathrm{T}_{5} \mathrm{M}_{1}$. Auch hier gilt das als eine nicht präferierte Reaktion, so dass Black Santa sich zu diesem Thema nicht mehr meldet. Beachtet wird hingegen das dritte 
Thema, welches erstmals in $\mathrm{B}_{5}$ auftaucht. Wie in der Grafik zu sehen ist, beziehen sich die Mitteilungen innerhalb dieses Themas immer auf die erste Mitteilung, so dass wir hier mehrere Paarsequenzen vorfinden, zu denen den ersten Part immer $\mathrm{T}_{3} \mathrm{M}_{1}$ bildet: die erste Paarsequenz ist $T_{3} M_{1}$ und $T_{3} M_{2}$, die zweite $T_{3} M_{1}$ und $T_{3} M_{3}$ und die dritte $T_{3} M_{1}$ und $T_{3} M_{4}$. Dabei provoziert der User Heiling in $\mathrm{T}_{3} \mathrm{M}_{1}$ eine Reaktion der anderen User, indem er seine Mitteilung so formuliert, als würde er die Gruppe verlassen. Darüber hinaus verabschiedet er sich am Ende seiner Mitteilung. Das erklärt, warum sich alle anderen Mitteilungen dieses Themas auf die erste Mitteilung beziehen. An diesem Thema zeigt sich zum wiederholten Male, dass eine einzige Mitteilung als Bestandteil mehrerer unterschiedlicher Sequenzen fungieren kann. Die anderen beiden thematisch zum dominanten Thema nicht passenden Mitteilungen, auf die jedoch seitens anderer User reagiert wird, sind $\mathrm{T}_{4} \mathrm{M}_{1}$ und $\mathrm{T}_{6} \mathrm{M}_{1}$. Auch diese beiden Themen bestehen aus Paarsequenzen. Teils werden die unterschiedlichen Themen in dieser Konversation parallel besprochen, was dazu führt, dass mehrere thematisch unterschiedliche Mitteilungen in einem Redebeitrag vorhanden sind, z.B. B 6 . Dies unterstützt zusätzlich die These, dass ein Redebeitrag nicht mehr als die Basis einer Sequenz angesehen werden kann. Befinden sich mehrere thematisch unterschiedliche Mitteilungen in einem Redebeitrag, so folgen sie nicht immer der Reihenfolge der eingebrachten Themen in das Gespräch, wie in $\mathrm{B}_{8} \mathrm{zu}$ sehen ist. Um klar zu machen, worauf man mit der eigenen Mitteilung reagiert, zitieren die User oder adressieren ihre Mitteilungen. Im Gegensatz zu den Beispielen davor, finden wir in $\mathrm{B}_{5} \mathrm{~T}_{3} \mathrm{M}_{1}$ eine Äußerung, die als Abschiedsformel fungiert. Nichtsdestotrotz fehlen die Schritte einer Beendigungsphase, da der Schreiber Heiling sich verabschiedet, ohne die Reaktionen der anderen Schreiber abzuwarten; er eröffnet nach der Abschiedsformel sogar ein weiteres Thema im gleichen Redebeitrag. Insgesamt fehlen eine Beendigungsphase und eine vollständige Eröffnungsphase, so dass diese Konversation wie bei anderen Beispiele der Kernphase entspricht.

Die Konversationsverläufe unterscheiden sich teils von denen in mündlicher Kommunikation. Es lassen sich Regelmäßigkeiten ausmachen, auf deren Grundlage Konversationsverläufe zunächst in mehrere Typen und Unterarten nach den Merkmalen der Reaktion und der Unterbrechung des dominanten Themas durch thematisch andere Mitteilungen unterteilt werden können, vgl. dazu folgende Tabelle: 


\begin{tabular}{|c|c|c|}
\hline Reaktion & + & - \\
\hline+ & $\begin{array}{l}\text { Konversationsverlauf mit } \\
\text { Unterbrechungen des } \\
\text { dominanten Themas durch } \\
\text { Mitteilungen anderer Themen, } \\
\text { auf die reagiert wird } \\
\text { (diskontinuierlich) }\end{array}$ & $\begin{array}{l}\text { Konversationverlauf ohne } \\
\text { Unterbrechungen des } \\
\text { dominanten Themas durch } \\
\text { Mitteilungen anderer } \\
\text { Themen (kontinuierlich) }\end{array}$ \\
\hline- & $\begin{array}{l}\text { Konversationsverlauf mit } \\
\text { Unterbrechungen des } \\
\text { dominanten Themas durch } \\
\text { Mitteilungen anderer Themen, } \\
\text { auf die nicht reagiert wird } \\
\text { (diskontinuierlich) }\end{array}$ & Einzelbeitrag \\
\hline$+/-$ & $\begin{array}{l}\text { Mischung aus Reaktion und } \\
\text { Nicht-Reaktion auf Unter- } \\
\text { brechungen des dominanten } \\
\text { Themas durch Mitteilungen } \\
\text { anderer Themen }\end{array}$ & \\
\hline
\end{tabular}

$A b b .12$ Überblick über unterschiedliche Konversationsverläufe

Diese Aufteilung findet sich in der Gesamtorganisation der Beispiele wieder, denn auf diese Weise entstehen zwei Arten der Konversationsverläufe: ein kontinuierlicher Konversationsverlauf ohne Unterbrechungen [+Reaktion, -Unterbrechung] und ein diskontinuierlicher Verlauf mit Unterbrechungen, der nach dem Merkmal der Reaktion in drei Gruppen unterteilt werden kann: in die erste Gruppe fallen Konversationen, bei denen auf Unterbrechungen des dominanten Themas durch Mitteilungen anderer Themen mindestens ein weiterer User eingeht [+Reaktion, +Unterbrechung]; diese haben die weiteren Unterarten: ein Gespräch mit einem dominanten und einem raren Thema und ein Gespräch mit mehreren dominanten Themen, bei denen die User innerhalb eines Beitrags auf alle reagieren können. Dabei gilt für beide Unterarten, dass die Themen parallel verhandelt werden können. In die 
zweite Gruppe fallen Verläufe, in denen es zwar zu Unterbrechungen durch Mitteilungen anderer Themen als dem dominanten Thema kommt, aber kein User darauf reagiert [Reaktion, +Unterbrechung] und schließlich die dritte Gruppe, die eine Mischung aus Unterbrechungen, auf die keiner reagiert und Unterbrechungen, auf die jemand reagiert, darstellt [+/-Reaktion, +Unterbrechung]. Im Gegensatz zu einem kontinuierlichen Konversationsverlauf, der nur einen einzigen thematischen Abschnitt hat, haben diskontinuierliche Konversationsverläufe immer mehrere thematische Abschnitte. Der Unterschied innerhalb solcher Verläufe entsteht durch die Frage, ob auf thematisch fremde Mitteilungen, die den ersten Part einer Sequenz bilden, eine Reaktion als zweiter Part erfolgt oder nicht. Bei diskontinuierlichen Konversationsverläufen, bei denen auf thematisch fremde Mitteilungen eingegangen wird, sind diese thematischen Abschnitte entweder klar getrennt, wenn das eine Thema nur so lange behandelt wird, bis das Gespräch zum anderen Thema wieder fortgesetzt wird, oder nicht klar getrennt, wenn mehrere unterschiedliche Themen parallel besprochen werden, da die Reaktionen auf die einzelnen Abschnitte häufig in einem einzigen Beitrag verfasst werden. Solche Redebeiträge, in denen auf mehr als ein Thema eingegangen wird, stellen einen Unterschied zur mündlichen Kommunikation dar.

Anhand der hier untersuchten Beispiele lassen sich Merkmale ausmachen, die typisch für Konversationen in Internetthreads sind. Dazu gehören beispielsweise das Vorkommen thematisch fremder Mitteilungen inmitten eines Gesprächs und das parallele Abhandeln mehrerer Themen. Die Themenwechsel sind größtenteils abrupt, können aber durch P.S. oder Zitate usw. markiert sein. ${ }^{105}$ Eine präferierte Reihenfolge der weiteren Themen lässt sich nicht feststellen. Einige der Annahmen zu Merkmalen präferierter und weniger präferierter Reaktionen bei zweiten Teilen treffen auf die untersuchten Internetkonversationen nicht zu: so werden zeitliche Pausen z.B. nicht als Merkmal einer negativen Reaktion wahrgenommen, da die User keine sofortige Rückmeldung erwarten. Dagegen gilt das Ignorieren einer Mitteilung als deutlichtes Zeichen der Ablehnung. Somit gibt es Ähnlichkeiten und Unterschiede zu mündlichen Konversationen. Ein besonderes Augenmerk soll dabei auf die Gesamtorganisation und die Struktur des Gesprächs gelegt werden. Allen im Rahmen dieses Kapitels untersuchten Beispielen fehlen vollständige Eröffnungsphasen und die kompletten Beendigungsphasen ${ }^{106}$, d.h. die Konversation setzt i.d.R. mit der first-topic-position ein

\footnotetext{
105 Jedoch muss über P.S. nicht immer ein markierter Themenwechsel eingeleitet werden, in manchen Fällen handelt es sich einfach um einen Zusatz. Auch ein Zitat muss nicht unbedingt einen Themenwechsel markieren, sondern nur anzeigen, auf welche Mitteilung/Äußerung sich der Schreiber bezieht.

${ }^{106}$ Damit stimmt das vergleichsweise geringe Vorkommen von Begrüßungen und vor allem Verabschiedungen überein, die vorher behandelt wurden. Möglicherweise hängt das Fehlen der Begrüßungen und v.a. von
} 
(damit die Leser sofort wissen, worum es geht) und hört auf, wenn kein User mehr reagiert. Das trägt dazu bei, dass alles abrupt wirkt. Ein wichtiges Merkmal der Konversationsverläufe der untersuchten Beispiele, welches sie von mündlichen Konversationen unterscheidet, ist, dass sie von ihrer Gesamtorganisation her nur der Kernphase einer mündlichen Konversation entsprechen. ${ }^{107}$ Der Grund liegt in dem Wunsch die Kommunikation möglichst ökonomisch zu gestalten und da nur in der Kernphase einer Konversation das Thema abgehandelt wird, sind die restlichen Gesprächsphasen thematisch überflüssig. So wird statt einer längeren Beendigungsphase der Gesprächsabschluss dadurch angedeutet, dass kein User mehr etwas zu dem entsprechenden Thema schreibt, weil alles Wichtige bereits gesagt wurde. Parallel zur fehlenden Verbalisierung der Beendigungsphase, wird ebenfalls die Eröffnungsphase aufgelöst: so gibt es statt einer dreiteiligen Eröffnungsphase mit Aufruf-Antwort-erstes Thema nur noch die first-topic-position, die gleichzeitig der erste Bestandteil der Kernphase ist.

Die sequentiellen Strukturen, die man vorfinden kann, entsprechen auch denen in mündlicher Kommunikation. Es finden sich sowohl zweigliedrige Strukturen aus Mitteilung und Reaktion

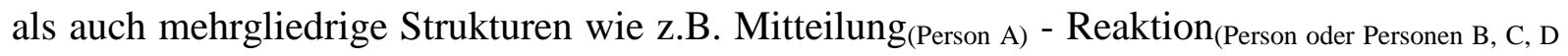
...) - Reaktion (Person A) auf Reaktion ${ }^{108}$. Auch gibt es eingliedrige Strukturen, bei denen auf den ersten Part kein zweiter seitens eines anderen Users erfolgt. ${ }^{109}$ Hierbei kann es sich sowohl um unvollständige Paarsequenzen handeln, als auch um eingliedrige Sequenzen, bei denen die Schreiber keinen zweiten Bestandteil erwarten. Im Bereich der Sequenzen gibt es auch wichtige Unterschiede zur mündlichen Kommunikation. Das Vorkommen mehrerer Reaktionen auf Mitteilungen verschiedener User und mehrerer thematisch unterschiedlicher Mitteilungen innerhalb eines Redebeitrags zeigt, dass einzelne Redebeiträge nicht mehr eins zu eins einem Sequenzbestandteil entsprechen ${ }^{110}$, dass ein Redebeitrag bzw. Turn also nicht

\footnotetext{
Abschiedsformeln mit Höflichkeitskonventionen zusammen (vgl. Schlund, K. (2009)).

${ }^{107}$ In einigen anderen Beispielen wurde zum Teil festgestellt, dass sie komplette Eröffnungsphasen enthielten (vgl. Böhnisch (in Erscheinung)). Auffällig war, dass eine komplette Beendigungsphase jedoch fast nie vorkam.

${ }^{108}$ Vgl. Beispiel (20):
}

\begin{tabular}{|c|c|c|c|}
\hline $\mathrm{B}_{1}$ & $\mathrm{~T}_{1} \mathrm{M}_{1}$ & Smoked: ${ }_{1}$ Кто пезд1т того я рот е..л & (Mitteilung) \\
\hline $\mathrm{B}_{2}$ & $\mathrm{~T}_{1} \mathrm{M}_{2}$ & MaxaRiaZ: ${ }_{2}$ кто поможет перевести вышесказанное? ) & (Reaktion1) \\
\hline $\mathrm{B}_{3}$ & $\mathrm{~T}_{1} \mathrm{M}_{3}$ & $\begin{array}{l}\text { Lelika: zЦитата: Сообщение от Smoked: Кто пезд1m } \\
\text { того я роб е..л }\end{array}$ & \\
\hline & $\mathrm{R}_{\mathrm{T} 1 \mathrm{M} 1}$ & 1интересно, где этот роб находится? & (Reaktion 2) \\
\hline $\mathrm{B}_{4}$ & $\mathrm{~T}_{1} \mathrm{M}_{4}$ & MaxaRiaZ: ${ }_{1}$ вот мне тоже интересно ) & (Reaktion 3) \\
\hline $\mathrm{B}_{5}$ & $\mathrm{~T}_{1} \mathrm{M}_{5}$ & Smoked: ${ }_{1}$ Опечаточка была=) & (Reaktion 4 auf $1-3$ ) \\
\hline
\end{tabular}


mehr als elementare Grundbasis einer Sequenz in Konversationen in Internetforen angesehen werden kann; diese Funktion wird von den Mitteilungen übernommen. Die Unterschiede in der Struktur lassen sich grob folgendermaßen darstellen:

Mündliche Konversation: $\quad[\mathbf{E}[\mathrm{s} \ldots[\mathrm{R} \ldots] \ldots]] \quad[\mathbf{k}[\mathrm{s} \ldots[\mathrm{R} \ldots] \ldots]] \quad[\mathbf{B}[\mathrm{S} \ldots[\mathrm{R} \ldots] \ldots]]$

Konversation in Internetthreads: $\quad[\mathrm{K}[\mathrm{s} \ldots[\mathrm{R} \ldots[\mathrm{M} \ldots] \ldots] \ldots]]$

$\mathrm{Zu}$ sehen ist ein Vergleich zwischen der Struktur einer mündlichen Konversation und der einer Konversation in Internetthreads. Die Abkürzungen stehen für: E = Eröffnungsphase, $\mathrm{K}=$ Kernphase, $\mathrm{B}=$ Beendigungsphase, $\mathrm{S}=$ Sequenz, $\mathrm{R}=$ Redebeitrag und $\mathrm{M}=$ Mitteilung. Während eine mündliche Konversation drei Phasen besitzt, besteht eine Konversation in Interntetthreads nur aus der Kernphase, also der Gesprächsmitte. Wie oben bereits erwähnt, wird die Basis der Sequenzen nicht mehr durch Redebeiträge, sondern durch Mitteilungen gebildet.

Ein weiteres Problem im Bereich der Sequenzen, das den Ansatz unterstützt, Mitteilungen statt Redebeiträgen als Basis einer Sequenz anzusehen, ergibt sich durch die Position der zusammengehörenden Mitteilungen, die eine Sequenz bilden. Diese stehen nämlich nicht immer eng beieinander, so dass eine klare Abgrenzung der Sequenzen - wie z.B. in mündlicher Konversation: in Redebeitrag 1 findet sich der erste Part der Paarsequenz, in Redebeitrag 2 der erste Part der Einschubsequenz, in Redebeitrag 3 der zweite Part der Einschubsequenz und in Redebeitrag 4 der zweite Part der Paarsequenz - nicht möglich ist. Dies wird vor allem durch die lange Bestandsdauer von Threadkonversationen ermöglicht, die ebenfalls dafür sorgt, dass eine einzige Mitteilung den Bestandteil mehrerer Sequenzen bilden kann. Bei all diesen Unterschieden, die häufig in der Ökonomie der Kommunikation im Internet begründet sind, handelt es sich um spezifische Eigenschaften des Mediums Internet. Auf dieser Grundlage halte ich die Annahme einer neuen Struktur, die speziell an die besondere Art von Konversationen in Internetthreads angepasst ist, für notwendig.

\subsection{Turn Taking}

Wie in Abschnitt 3.2 angemerkt, gibt es einen engen Zusammenhang zwischen der Organisation eines Gesprächs und dem System des Sprecherwechsels - auch als Turn Taking 
bezeichnet. So können sich Sprecher z.B. mit Vor-Mitteilungen eine längere Sprechdauer sichern, ohne Sorge zu haben, unterbrochen zu werden. Für die mündliche Kommunikation wird dabei folgende Funktionsweise des Sprecherwechsels angenommen: „Der Sprecherwechsel wird durch einen komplexen Regelapparat gesteuert, der auf der Grundlage zweier Komponenten funktioniert: Die Redebeiträge der Sprecher (turns) sind in Turnkonstruktionskomponenten (turn constructional units, TCUs) gegliedert, die mögliche Übergabestellen für den Sprecherwechsel (transition relevance places, TRPs) projizierbar bzw. für die Interaktionspartner erkennbar machen. ${ }^{111}$ Hierauf bauen dann die Regeln für den Sprecherwechsel als zweite Komponente auf: An einem TRP kann ein nächster Sprecher vom aktuellen Sprecher ausgewählt werden (Fremdwahl). Wird kein Folgesprecher ausgewählt, kommt es zur Selbstwahl durch einen beliebigen anderen Sprecher, der zuerst beginnt (first starter principle). Wird keine dieser Möglichkeiten realisiert, setzt der aktuelle Sprecher seinen turn fort, bis am nächsten TRP wieder die bereits genannten Optionen für den Sprecherwechsel zur Verfügung stehen.“ (Kosta \& Thielemann $(2009,1033)) .{ }^{112}$ Vor allem bei Adjazenzpaaren ist der Sprecherwechsel von vornherein geregelt: die Reihenfolge der Sprecher ist selbstdeterminiert, zu einer Selbstwahl kommt es also nicht.

In mündlichen Gesprächssituationen kann es auch zu Überlappungen der Äußerungen kommen. „Rather, turn taking seems to be organized on the following rule-governed basis: the current speaker has the right to finish a minimal linguistic unit (clause or other prosodically defined unit), at the end of which any party may choose to speak, first speaker winning rights to that next unit, which in turn is subject at its completion to competitive turns by others. This predicts, correctly, that overlap will occur typically at transition points and will be caused by competitive first starts. Or overlap can occur just before the intended transition point at which a speaker has appended a tag question, name, or other unpredictable addition. When, because of competitive simultaneous starts, two speakers find themselves speaking simultaneously, there seem to be methods for resolving who should continue to speak. These methods largely involve indicating a degree of determination to continue, signaled, for example, by amplitude

\footnotetext{
${ }^{111}$ Dies kann z.B. durch Sprechpausen angezeigt werden.

112 Diese Annahmen wurden bereits vorher vorgestellt: „Sacks, Schegloff, and Jefferson's (1974) model of turn taking focuses on two components: the "turn-constructional component" based on the speaker's choice of turn taking unit-types, and the "turn-allocational component" based on how a participant takes the next turn (p. 12). Sacks et al. note that turns in talk can be constructed out of four units of talk: (a) one word, (b) a phrase, (c) a clause, or (d) a sentence. [...] The Sacks et al. (1974) model identifies three techniques for turn allocation: (a) current speaker selects next, (b) another speaker self-selects, and (c) current speaker continues. A transitional relevance place (TRP) refers to places within talk in which participants can project where a turn might end. TRPs cue participants to a turn's end; in FTF interaction, such cues may include sentence structure, phrases, a word, silence, pitch or volume change, and body motion." (Anderson et al $(2010,7)$ ).
} 
increase and syllable lengthening. “113 (Levinson (1989, 408)). Das System des Sprecherwechsels sei laut Levinson dabei universell.

In Internetkonversationen in Threads, die ein one-way transmission system darstellen, sieht die Funktionsweise des Partizipantenwechsels teils anders aus, da es sich hier um eine rein schriftlich realisierte Kommunikation handelt, die durch unterschiedliche Aspekte begrenzt ist, vor allem durch die Technik: so gibt z.B. die Geschwindigkeit des Internetanschlusses und des jeweiligen Computers vor, wie schnell der eigene Beitrag versendet werden kann. In manchen Foren gibt es auch eine Zeichenbegrenzung, die die Länge eines Beitrags reguliert. $^{114 ; 115}$ Eine Gemeinsamkeit zwischen Internetkonversationen und mündlicher Kommunikation ist die Gliederung der Turns in TCUs. Nehmen wir hierfür das Beispiel (11) Mitteilung $\mathrm{T}_{1} \mathrm{M}_{1}$. Der Turn des Users DragOn besteht aus zwei Komponenten: die erste Frage, die nach möglichen Besitzern der entsprechenden Autos fragt, ist die erste Komponente und die zweite Frage an die Besitzer bezüglich der Autos selbst ist die zweite. Trotz der Gliederung des Turns in TCUs, gibt es keine nutzbaren TRPs innerhalb von Redebeiträgen - d.h. Stellen an denen der Turn an den nächsten Schreiber übergeben werden könnte - die mit solchen in mündlichen Gesprächssituationen vergleichbar wären. Es sind vielmehr fiktive Übergabestellen, die tatsächlich aber nicht nutzbar sind. Exemplarisch kann man hierfür Sprechpausen anführen, die - angedeutet durch die mehrfache Verwendung von Punkten - die Funktion einer möglichen Übergabestelle besitzen, jedoch in schriftlich realisierten Konversationen von den Schreibern nicht als solche realisiert werden, da der Turn des Schreibers nach der Pause weitergehen kann, vgl. (25) $\mathrm{B}_{6}, \mathrm{~B}_{7}$ oder $\mathrm{B}_{8}$. Selbst Abschiedsformeln müssen nicht immer das Ende eines Turns kennzeichnen, vgl. (24) B 5 . Erst das Ende eines Redebeitrags ist als tatsächliche Übergabestelle zu werten. Solche Annahmen können für alle Beispiele gemacht werden. Das Fehlen nutzbarer Übergabestellen stellt einen der größten Unterschiede dar. Ein anderer großer Unterschied zu mündlichen Gesprächssituationen, besteht darin, dass die Schreiber nicht unterbrochen werden können,

\footnotetext{
${ }^{113}$ In schriftlich realisierten Konversationen, wie sie u.a. in Forenthreads vorliegen, wird z.B. die Dehnung von Lauten - dargestellt durch Mehrfachvorkommen der entsprechenden graphischen Zeichen - für andere Zwecke verwendet als von Levinson für die mündliche Kommunikation genannt, da keine Überlappungen in Konversationen in Forenthreads vorkommen.

${ }^{114}$ Crystal beschreibt dies für Chatrooms: „Technologically imposed length constraints are an important factor influencing the linguistc character of chatgroup messages, therefore, over and above the pragmatic pressure on individuals to keep their contributions relatively short. Chatgroups are unlikely to be a domain where lengthy monologues or balanced dialogues - speeches, lectures, commercial presentations, formal debates, and suchlike - are found.“(Crystal $(2001,134))$.

115 Jedoch ist etwas Derartiges in den untersuchten Threads nicht der Fall gewesen, so dass die Beiträge beliebig lang sein konnten.
} 
keine hörbaren Sprechpausen bzw. „Tipppausen“ machen und es dementsprechend keine Überlappungen in Gesprächen geben kann. ${ }^{116}$ Die Dauer des Turns eines Schreibers ist somit so lang, wie der Schreiber es möchte oder die Technik es zulässt. Dabei kann der Beitrag sehr lang (vgl. (22) $\mathrm{T}_{1} \mathrm{M}_{2}$ oder $\mathrm{T}_{1} \mathrm{M}_{3}$ ) oder kurz sein (vgl. (22) $\mathrm{T}_{1} \mathrm{M}_{4}$ ). ${ }^{117 ; 118}$ Geht es um die Wahl des nächsten Sprechers, so gibt es Gemeinsamkeiten und Unterschiede zur mündlichen Kommunikation. Eine Gemeinsamkeit stellt das first starter principle, d.h. die Selbstwahl eines Gesprächsteilnehmers beim nächsten Turn, dar: wie an den vorigen Beispielen bereits erkennbar war, bringen sich die Schreiber selbsttätig in das Gespräch ein, ohne von dem vorangehenden Sprecher ausgewählt worden zu sein. ${ }^{19}$ Das kam in den untersuchten Konversationen am häufigsten vor. Ein Unterschied zum für die mündliche Kommunikation angenommenen Regelapparat ist das Fehlen einer bindenden Reihenfolge bei der Fremdwahl, also der Wahl eines nächsten Schreibers durch den jetzigen. Trotz einer direkt adressierten Mitteilung durch den jetzigen Schreiber (vgl. die direkte Adressierung von Black Santa durch Ksanka in Beispiel (18) $\mathrm{T}_{1} \mathrm{M}_{1}$ ) kommt es häufig vor, dass der Turn des Adressaten nicht der nächste ist. Denn neben dem technischen Aspekt (Geschwindigkeit des Internetanschlusses) ist zum Einen nicht jeder User rund um die Uhr online und zum Anderen reagiert häufig derjenige zuerst, der die Äußerung des Schreibers liest und darauf eingehen möchte, vgl. dazu in Beispiel (25) die Mitteilung von Lelika, die in $\mathrm{T}_{2} \mathrm{M}_{4}$ an Stelle von beebace reagiert, obwohl silvers Frage in $\mathrm{T}_{2} \mathrm{M}_{3}$ als direkte Reaktion auf die vorangehenden Mitteilungen von beepace an diesen gerichtet war.

\footnotetext{
116 Diese Annahme kann zumindest für Forenthreads gemacht werden. In Chats hingegen kann es viel wahrscheinlicher zu einer Überlappung kommen, da der zeitliche Abstand zwischen den abgeschickten Beiträgen auf Grund fast simultan ablaufender Kommunikation wesentlich kürzer ist und Chatteilnehmer ihre Beiträge nicht immer als Ganzes, sondern in mehrere Bruchstücke unterteilt, abschicken können (vgl. Fußnote 118). Welche Strategien in Chats z.B. an den Übergabestellen an den nächsten Sprecher genutzt werden, muss noch weiter untersucht werden.

${ }^{117}$ Auffällig an den untersuchten Beispielen ist, dass die Beispiele des zweiten Thread zwar mehr Redebeiträge enthalten (94 Redebeiträge in 12 Beispielen), jedoch deutlich weniger Äußerungen (163) als im zweiten Thread, wo auf 7 Beispiele 64 Redebeiträge und 220 Äußerungen kommen. Das bedeutet, dass die Redebeiträge im zweiten Thread deutlich länger sind als im ersten.

${ }^{118}$ Die Länge eines Turns in einem Forum bildet auch einen Unterschied zur Chatkommunikation, wo viele User eine Äußerung in mehrere Bruchstücke splitten, so dass sie sich über mehrere Beiträge zieht, z.B.:

„IM Transmission 1: that must be nice

IM Transmission 2: to be in love

IM Transmission 3: in the spring

IM Transmission 4: with birds chirping

IM Transmission 5: and frogs leaping“ (Baron $(2010,4)$ ).

Für weitere Informationen zu diesem Phänomen siehe Baron (2010, 1-32).

119 Auch bei einer Studie zum Turn Taking bei einem two-way-System hatten Anderson und seine Kollegen ähnliche Ergebnisse herausgefunden: „However, it appears that turn taking in the conversation was primarily accomplished through self-selection by participants. Self-selection took place when a participant took a turn without receiving any cue from another participant such as addressing by name or requesting a response." (Anderson et al $(2010,9))$.
} 
Zusammenfassend kann man sagen, dass kein Regelapparat für den Partizipantenwechsel vorliegt, der mit dem für mündliche Kommunikation vergleichbar wäre. Obwohl es TCUs gibt, sind die Übergabestellen nur fiktiv und somit nicht nutzbar, woraus fehlende Überlappungen folgen. Die Länge eines Turns eines Schreibers kann nur technisch durch eine begrenzte Zeichenanzahl reguliert werden. Ebenso ist das Fehlen einer bindenden Reihenfolge der Schreiber charakteristisch. Wenn die Mitteilung nicht direkt an einen User, sondern an alle adressiert wird, involvieren sich die anderen Gesprächspartizipanten selbsttätig. Es liegt in den meisten Fällen somit das first starter principle vor. Über die Funktionsweise des in Internetkonversationen anders verlaufenden Partizipantenwechsels gibt es offenbar eine Übereinkunft zwischen den Usern: diese wissen, was sie zu erwarten haben und passen sich entsprechend an. Der Partizipantenwechsel ist somit konventionell geregelt.

\subsection{Schlussfolgerungen}

Trotz einiger Gemeinsamkeiten unterscheiden sich Konversationen in Threads in vielen Fällen von mündlichen Konversationen hinsichtlich ihrer Merkmale. Diese decken sich nur bedingt mit denen, die Grice annimmt. Die folgende Tabelle bietet einen Überblick darüber, inwieweit die von Grice angenommenen charakteristischsten Merkmale von mündlicher Kommunikation auf die Internetkommunikation in den untersuchten Threads zutreffen $(+=$ zutreffend, - = nicht zutreffend, +/- = eher zutreffend, $-/+=$ weniger zutreffend; $\varnothing=$ Unmöglichkeit des Vorkommens):

\begin{tabular}{|l|l|l|l|}
\hline Merkmale & $\begin{array}{l}\text { Mündliche } \\
\text { Kommunikation }\end{array}$ & \multicolumn{2}{l|}{ Internetkommunikation } \\
\hline $\begin{array}{l}\text { Befolgung des } \\
\text { Kooperationsprinzips }\end{array}$ & + & Dialog & \multicolumn{2}{l|}{ Einzelbeitrag } \\
\hline $\begin{array}{l}\text { gemeinsames Ziel/Identifikation } \\
\text { mit den Interessen des Anderen }\end{array}$ & + & $+/-$ & - \\
\hline Bezug der Gesprächsbeiträge & + & + & - \\
\hline
\end{tabular}




\begin{tabular}{|l|l|l|l|}
\hline aufeinander & & & \\
\hline $\begin{array}{l}\text { Fortsetzung der Konversation, } \\
\text { bis alle Gesprächsteilnehmer sie } \\
\text { beenden wollen }\end{array}$ & + & + & $\emptyset$ \\
\hline $\begin{array}{l}\text { komplette Anonymität der } \\
\text { Partizipanten }\end{array}$ & $-{ }^{120}$ & + & + \\
\hline $\begin{array}{l}\text { Koexistierende Identitäten der } \\
\text { Sprecher/Schreiber }\end{array}$ & - & + & + \\
\hline
\end{tabular}

Abb. 13 Überblick über das Vorhandensein bzw. Fehlen von Merkmalen

Da das Koopertationsprinzip nicht immer befolgt wird, wird es mit +/- angegeben. Ebenfalls gibt es Unterschiede zwischen Dialogen und Einzelbeiträgen. Was die Anonymität und die Annahme mehrerer Identitäten der User angeht, so ist dies ein typisches Merkmal für Internetforen und -chats allgemein.

Auch die Gesamtorganisation der Konversationen unterscheidet sich in vielerlei Hinsicht von der in mündlicher Kommunikation. Da Gesprächseröffnungen fehlen, ist der Grund für das Anbahnen einer Konversation, also die first-topic-position, in Threadkonversationen meistens direkt in der ersten Mitteilung zu finden. Die Struktur der Eröffnungsphase ist daher nur auf die first-topic-position reduziert. Hinsichtlich der Themenorganisation in der Kernphase, gibt es Gespräche mit nur einem Thema und solche, in denen mehrere Themen verhandelt werden. In den Konversationen, in denen mehrere Themen verhandelt werden, lassen sich anhand der Anzahl der Mitteilungen dominante oder rare Themen ausmachen. Die Themenübergänge sind häufig abrupt. Ein gravierender Unterschied zur mündlichen Kommunikation zeigt sich darin, dass ein Redebeitrag - ermöglicht durch die Zitatfunktion - Reaktionen auf mehrere Themen sowie mehrere Reaktionen auf Mitteilungen verschiedener User enthalten kann. Beides spricht für die Annahme, die Basis einer Sequenz bilde nicht der Redebeitrag, sondern die einzelne Mitteilung. Des Weiteren fehlen in der Regel die Beendingungsphasen. Das Gespräch gilt als beendet, wenn zu dem besprochenen Thema keine Mitteilung mehr verfasst

\footnotetext{
${ }^{120}$ Eine komplette Anonymität ist nicht gegeben, da man den anderen Gesprächspartizipanten sieht oder beim Hören z.B. am Telefon anhand der Stimme, falls bekannt, erkennt; falls der Gesprächspartner unbekannt ist, stellen sich beide Gesprächspartner in der Regel vor.
} 
wird, da alles Relevante bereits gesagt wurde. Die Kommunikation scheint auf diese Weise viel ökonomischer zu sein, da sich die User nicht mit für sie sekundär gewichteten Phasen beschäftigen müssen, sondern schon mit der ersten Mitteilung jedem mitteilen, was der Zweck der Kommunikation ist, welches Ziel sie also verfolgen. Die komplette Kommunikation und ihre Organisation sind auf diesen Zweck hin ausgerichtet. Auf Grundlage von fehlenden Phasen sowie der Tatsache, dass ein Redebeitrag mehrere Mitteilungen enthalten kann, wurde weiter oben eine neue Struktur vorgeschlagen.

Der Partizipantenwechsel funktioniert ebenfalls anders als in mündlichen Gesprächssituationen, was nicht zuletzt an der Technik und der schriftlichen Realisierung liegt.

All diese Merkmale, der Verlauf und der Partizipantenwechsel sind dabei konventionalisiert, denn, wie jegliches menschliches Kommunikationsverhalten, folgen auch schriftlich realisierte Gesprächssituationen bestimmten formalen und inhaltlichen Konventionen. Diese Konventionen können trotz häufiger Gemeinsamkeiten je nach Forum unterschiedlich sein. Es zeigt sich, dass dabei viele Gesprächskonventionen aus der „realen“ Welt in die „virtuelle“ übernommen werden. So zum Beispiel, dass man sich möglichst klar ausdrücken soll, um Missverständnisse zu vermeiden - eine Konventionen, die einer der Grice'schen Konversationsmaximen entspricht. Andere Konventionen müssen erst im Laufe der Auseinandersetzung mit der Threadkommunikation und ihren Umsetzungsmöglichkeiten von den Usern gemeinsam erschaffen werden. Das betrifft z.B. den Umgang mit dem Partizipantenwechsel oder den in ein Gespräch eingeschobenen, thematisch fremden Mitteilungen. Auch, ob man sich an Formalia, die für ein höfliches, mündliches Gespräch charakteristisch sind, hält, wird über Konventionen geregelt. Erworben werden die Konventionen von den Usern über die kommunikativen Erfahrungen, die sie im Laufe ihrer Gespräche im Internet sammeln. 


\section{Der Weg zur korrekten Interpretation}

Im vorliegenden Kapitel werden die Beispiele (26) - (47) im Hinblick auf Hintergrundwissen und potenzielle Kontextualisierungshinweise analysiert. Die Untersuchung soll zeigen, welche Rolle Hintergrundwissen zukommt und welche potenziellen Kontextualisierungshinweise in Wirklichkeit als Kontextualisierungshinweise in schriftlich realisierten Konversationen fungieren können. Da es im vorliegenden Abschnitt nicht um Konversationsverläufe geht, werden nicht immer vollständige Konversationen angegeben. Ebenfallss werden keine Gruß- oder Abschiedsformeln markiert.

\section{$\underline{4.1}$ Hintergrundwissen in Internetthreads $^{121}$}

Wie oben bereits erwähnt, folge ich Gumperz in der Annahme, soziokulturelles und sprachliches Hintergrundwissen (HW) spiele eine sehr große Rolle in Interaktionen zwischen Gesprächspartnern, da diese für eine erfolgreiche Interpretation von Äußerungen des Gegenübers über das gleiche Hintergrundwissen in einem Gespräch verfügen müssen. Auf Grundlage des für diese Dissertation bearbeiteten Korpus müssen mehr Arten des Hintergrundwissens angenommen werden als in Gumperz' Theorie: universelles, soziokulturelles, sprachliches, gruppeninternes und situationsbezogenes. Universelles Hintergrundwissen beinhaltet allgemeines Weltwissen und ist kulturunabhängig. In dieses Wissen fällt beispielsweise die Kenntnis, dass die Sonne morgens auf- und abends untergeht. Im Gegensatz dazu sind soziokulturelles und sprachliches Hintergrundwissen kulturabhängig, da sie Informationen zu der jeweiligen Gesellschaft und ihrer Sprache beinhalten. Gruppeninternes Hintergrundwissen kann, muss aber nicht kulturabhängig sein. Hier geht es vielmehr darum, dass dieses nur Gruppenmitglieder besitzen, die jedoch nicht den gleichen soziokulturellen und sprachlichen Hintergrund haben müssen, sondern sich auf Grund von gleichen Interessen (z.B. Computern, Spielen, Büchern etc.) zu einer Gruppe zusammenfinden. Eine weitere Art bildet das situationsbezogene Hintergrundwissen, d.h. das Wissen über eine bestimmte Situation.

Wie wichtig das von Schreiber und Leser geteilte Hintergrundwissen für die korrekte Interpretation schriftlich realisierter Äußerungen in Internetkonversationen ist, wird erst

\footnotetext{
${ }^{121}$ Der folgende Abschnitt behandelt nicht die neurowissenschaftliche Frage, wie Hintergrundwissen in unserem Gehirn abgespeichert und wieder aufgerufen wird.
} 
deutlich, wenn es fehlt: der Leser muss in diesem Fall nachfragen, wie bspw. in den Beispielen (26) und (27). Jedoch ist der vorherige Schreiber nicht verpflichtet, die Nachfrage zu beantworten: so sehen wir, dass im Unterschied zum ersten Beispiel, wo das fehlende Wissen auf die Nachfrage hin ergänzt wird, im zweiten Beispiel keine derartige Reaktion stattfindet. Während in Beispiel (26) der Schreiber Vesnuška demnach Interesse daran hat, dass Nikitina alles in ihrer Mitteilung versteht und die Konversation fortgesetzt werden kann, ist dies bei scary boom und Ksanka in (27) nicht der Fall. Die Weigerung von scary boom das Zitat zu erklären, wird dabei vom Fragesteller Ksanka als negative Reaktion, d.h. als fehlender Wunsch seitens scary boom, sich auf eine Interaktion mit Ksanka einzulassen, aufgefasst, weswegen weiteres Nachfragen unterbleibt.

Die Bedeutung von Hintergrundwissen für die Interpretation lässt sich an den Beispielen (26) und (14) zeigen. So finden sich beispielsweise in (26) $\mathrm{B}_{2} \mathrm{~T}_{1} \mathrm{M}_{2,2}$ die Formen chto und enta, die im Standardrussischen nicht zu finden sind. Der Leser benötigt hier Hintergrundwissen, um zu wissen, dass diese Formen aus der gesprochenen Sprache stammen: chto ist eine verschriftlichte Variante der Aussprache des Pronomens kto, während enta von dem Pronomen éta stammt und neben der gesprochenen Sprache in Dialekten gebraucht wird ${ }^{122}$. In Beispiel (14) $B_{10} T_{1} M_{10}$ wird vom Schreiber ein Zitat geäußert, das nur mit Hilfe des Hintergrundwissens als ein Zitat aus dem sowjetischen Zeichentrickfilm Vinni Puch ${ }^{123}$ erkannt wird. Dieses Hintergrundwissen wird benötigt, um einen Zusammenhang zwischen der Äußerung $\mathrm{T}_{1} \mathrm{M}_{9,1}$, in der der vorherige Schreiber Lelika den aktuellen Schreiber bogda als Vinni Puch bezeichnet, herzustellen, aber auch für die Interpretation des Zitates, seine Implikatur $^{124}$ und die folgende Regieanweisung ${ }^{125}$. Um die Intention des Schreibers bogda zu erkennen, muss der Leser die entsprechende Stelle im Zeichentrickfilm kennen: Vinni Puch möchte von den Bienen Honig stehlen, damit sie ihn aber nicht bemerken, versucht er einen Trick mit zwei verschiedenfarbigen Luftballons, mit denen er zum Bienenstock hochfliegt: er benutzt zunächst einen hellblauen, damit die Bienen ihn für eine Wolke halten und als das fehlschlägt, einen grünen, damit sie denken, er sei ein Blatt. Das Zitat gibt die Stelle wieder, an der Vinni Puch sich entschließt den grünen Luftballon zu benutzen, um an den Honig zu gelangen. Dem entspricht auch die Regieanweisung. Die ganze Mitteilung von bogda lässt sich nun so interpretieren, dass er es geschafft hat die „Bienen“, d.h. die anderen User,

\footnotetext{
${ }^{122}$ Ein Wörterbucheintrag zu dieser Form lässt sich sowohl bei Dal' als auch bei Fasmer finden.

${ }^{123}$ Bei dem Zeichentrickfilm handelt es sich um die sowjetische Adaption des Kinderbuchs „Winnie-the-Pooh“ von A. A. Milne.

${ }^{124}$ Im Rahmen dieser Dissertation wird Implikatur/implikatieren in Grice'schen Sinne benutzt.

${ }^{125}$ Auf Regieanweisungen wird in Abschnitt 4.2.3.3 eingegangen.
} 
Moderatoren, Administratoren usw., zu täuschen und unbemerkt an den „Honig“ - im Sinne von einer Position als Moderator, die schwer zu bekommen ist - zu gelangen. Das Zitat passt auch insofern gut an diese Stelle, als dass sowohl der Luftballon als auch die Farbe zur Kennzeichnung von Moderatoren grün ist.

In dem vorliegenden Abschnitt sollen aber vor allem die Arten des Hintergrundwissens untersucht werden, die von Gumperz nicht erwähnt wurden: das gruppeninterne und das situationsbezogene Hintergrundwissen. Als gruppeninternes Hintergrundwissen bezeichne ich das Hintergrundwissen, über das nur eine Gruppe verfügt. ${ }^{126}$ Hierbei unterscheide ich in Abhängigkeit von der Größe der Gruppe mehrere Arten: makrogruppeninternes Hintergrundwissen und mikrogruppeninternes Hintergrundwissen. Makrogruppeninternes Hintergrundwissen lässt sich bei größeren Gruppen finden, die sich über fachliches Interesse an einem bestimmten Thema definieren. Im ersten Forum ist dies Harry Potter, im zweiten Computer, Programmieren und Hacken. Die Bezeichnung „Makro-“ bezieht sich darauf, dass die Gruppe aus wesentlich mehr Teilnehmern als nur z.B. den Mitgliedern eines bestimmten Threads innerhalb des Forums besteht. So gibt es unzählige Interessenten sowohl an Harry Potter als auch am Programmieren, die keine Mitglieder der untersuchten Forumthreads sind

\footnotetext{
${ }^{126}$ Dass es sich bei den Mitgliedern eines Forumthreads eindeutig um eine Gruppe - wobei es innerhalb einer großen Gruppe noch weitere kleinere Gruppen geben kann - handelt, zeigt die Definition des Begriffs Gruppe: „Sozialwissenschaftlich umfasst die Umschreibung von Gruppe seit Alderfer (1977) eine Anzahl von Personen, die untereinander dependente Beziehungen haben, d.h., die Mitglieder beeinflussen sich wechselseitig in ihrem Verhalten und Erleben; die Beziehungen bestehen über längere Zeit. Die Personen betrachten sich selbst als Gruppe und zu ihr zugehörig. [...] Die Mitglieder haben gemeinsame Bedürfnisse, vielleicht gemeinsame Ziele oder Aufgaben oder sind nur einfach gern beieinander. Es gibt ein Gefüge von Funktionen, differenzierten Rollen und Regeln, die das Verhalten in einer Gruppe steuern." (Fisch $(2004,423)$ ). Das Wichtigste für die Gruppen ist die Kommunikation. Sie ,[...] ist das Agens, das Gruppenprozesse initiiert, aufrecht erhält und steuert, wobei die Bedeutung und Wirkungen der nichtsprachlichen Kommunikation für die Gruppenprozesse gegenüber den sprachlich-inhaltlichen Äußerungen häufig unterschätzt werden. Gruppen, die länger bestehen, können Zeichensysteme und eine eigene Sprache mit Ausdrücken entwickeln, die nur von Mitgliedern der Gruppe richtig verstanden werden (können), als Erkennungszeichen benutzt werden und die Zugehörigkeit signalisieren, so dass Abgrenzungen möglich werden im Sinn einer „Wir"-Gruppe und einer „Die“-Gruppe.“ (Fisch $(2004,426)$ ). Im Bereich der Lexik spielt für die Gruppen sowohl die Fachsprache als auch Slang oder Jargon eine wichtige Rolle, da sie Gruppen voneinander abgrenzen, eine Gruppenidentität herstellen und nach außen und nach innen hin stärken (vgl. Hoffmann (2004, 235f.)). Die Definition von Fisch trifft so auch auf die Gruppen im Internet zu, die wegen ihres gemeinsamen Interesses zusammenkommen und sich von anderen Gruppen (z.B. durch ihre Projekte oder spezielle Interessen (vgl. Spieleforen, die sich mit unterschiedlichen Spielen/Spieleprojekten beschäftigen) abgrenzen. Die Gruppen haben gemeinsame Ziele und Aufgaben (z.B. Spieleentwicklung, Programme schreiben, Problemlösungen); dabei kann das Ziel aber auch einfach in der Kommunikation mit Menschen mit ähnlichem Interesse liegen. In jeder Gruppe im Internet gibt es Regeln und Personen, die verschiedene Funktionen und Rollen inne haben: Administratoren, Supermoderatoren, Moderatoren usw.. Auch Eigenbezeichnungen und damit eine namentlich ausgedrückte Identifikation mit der Gruppe kommen vor, z.B. war dies im ersten Forum alohomorcy (und jegliche Ableitung davon) und tuški. Die Gruppenzugehörigkeit bzw. das Fehlen hiervon wird dabei auch durch Ausdrücke wie Gast, die benutzt werden, um die eigenen Mitglieder von fremden Nicht-Mitgliedern zu unterscheiden (vgl. Crystal (2001, 60)), angezeigt. Auch aus lexikalischer Sicht bilden die Mitglieder eines Forumthreads eine Gruppe, da sie über die entsprechende „Fachsprache“ verfügen, sei es im Bereich des Programmierens oder im Bereich des Harry PotterUniverums.
} 
und dennoch auf Grund ihrer Beschäftigung mit dem entsprechenden Thema über das gleiche makrogruppeninterne Hintergrundwissen verfügen. Als exemplarisch für makrogruppeninternes Hintergrundwissen untersuche ich die Beispiele (28) aus dem ersten und (29) - (30) aus dem zweiten Thread. In (28) finden sich mehrere Fachbegriffe, deren genaue Bedeutung einem Leser, der sich nicht mit Harry Potter auskennt, fremd sein dürften. Dazu gehören gippogrifovaja ${ }^{127}$, domašnaja èl'fijka, BZDNE் und kosaja alleja. Um die Äußerung korrekt zu interpretieren, bedarf der Leser der Kenntnis dieser Begriffe. Ohne dieses Hintergrundwissen, kann die Äußerung nicht in den korrekten Kontext gesetzt werden, so dass es nur bei Vermutungen des Lesers, wie z.B., dass dem Schreiber Ksanka offenbar etwas an dem Verhalten des anderen Users missfällt, bleibt. Als Beispiele aus dem zweiten Thread dienen (29) und (30). Auch hier benötigt der Leser das entsprechende Hintergrundwissen, um die Fachlexik (z.B. in (29) bot, ban, flud, kriptor, hajd etc. in $\mathrm{T}_{1} \mathrm{M}_{1}$ ) verstehen zu können. ${ }^{128}$ Die meisten dieser Begriffe haben eine ursprüngliche Bedeutung (z.B. ,flud' - ,flood' - ,Flut', ,Überschwemmung'), bekommen aber durch den Gebrauch in einer (Fach)Gruppe eine zusätzliche Bedeutung bzw. eine Bedeutungserweiterung, z.B. brutit'/brut und vzlom in (30) $\mathrm{T}_{1} \mathrm{M}_{1,1}$ und $\mathrm{T}_{1} \mathrm{M}_{5,1}$ : bei einem brut (engl. brute) handelt es sich keineswegs um einen ,Unmenschen', ,Grobian' oder ,brutalen Menschen', sondern um einen Brute-Force-Angriff; vzlom bedeutet ,Aufbruch', ,Einbruch', im Sprachgebrauch innerhalb von Gruppen, wie die im zweiten Thread, ist damit ein Hackerangriff bzw. das Hacken gemeint.

Ein weiteres Unterscheidungsmerkmal zwischen makro- und mikrogruppeninternem Hintergrundwissen ist, dass makrogruppeninternes Hintergrundwissen bereits in den Gruppen in den untersuchten Foren von den Schreibern vorausgesetzt wird und nicht erst dort geprägt wird. Das bedeutet, dass die Leser dieses Hintergrundwissen bereits mitbringen müssen, wenn sie sich an Konversationen im Forum beteiligen möchten. Das geteilte makrogruppeninterne Hintergrundwissen konstituiert die Gruppe der Threadteilnehmer in diesen Threads mit. Im Gegensatz dazu wird mikrogruppeninternes Hintergrundwissen erst innerhalb der Gruppe der Threadteilnehmer geprägt, indem es im Laufe der Konversation entsteht. ${ }^{129}$ Hier konstituiert die Gruppe der Threadteilnehmer das Hintergrundwissen. Die Beispiele (14), (31) - (32) aus dem ersten und dem zweiten Thread werden exemplarisch für mikrogruppeninternes

\footnotetext{
${ }^{127}$ Der Gippogrif ist ein mythologisches Wesen, das nicht nur in den Romanen von Harry Potter vorkommt.

128 Des Weiteren wird Hintergrundwissen benötigt, um zu verstehen, dass in (29) $\mathrm{T}_{1} \mathrm{M}_{1}$ pocaki $<$ pacany, zaregat' < zaregistrirovat oder moder < moderator stammt.

${ }^{129}$ Eventuell ist es sogar möglich anhand des mikrogruppeninternen Hintergrundwissens Gruppenmitglieder der Kerngruppe/,engen“ Gruppe von den „entfernten“ Mitgliedern der ganzen Gruppe zu unterscheiden: wer diese Art von Hintergrundwissen besitzt, gehört dem engeren Kreis an.
} 
Hintergrundwissen untersucht.

In Beispiel (31) muss der Leser über Hintergrundwissen verfügen, um bereits die erste Mitteilung korrekt interpretieren und dem daraus resultierenden Gespräch folgen zu können: Hintergrundwissen, um zu erkennen, dass es sich bei ptičko um keine standardrussische Form handelt ${ }^{130 ; 131}$, um zu wissen, dass Kapitan Džek Vorobej (,Captain Jack Sparrow') einer der Hauptprotagonisten der Filmreihe Piraty Karibskogo morja (,Pirates of the Caribbean') und ein Pirat ist, der sich durch bestimmte Charakteristika - so ist lochmatnost' (,Zottigkeit', ,Struppigkeit', ,Ungekämmtheit') in $\mathrm{T}_{1} \mathrm{M}_{2}$ und $\mathrm{T}_{1} \mathrm{M}_{3,1-3}$ z.B. auf das Aussehen seines Haares bezogen - auszeichnet und um zu wissen, wer als ptičko bzw. pingvin bezeichnet wird. ${ }^{132} \mathrm{Um}$ $\mathrm{zu}$ wissen, auf wen ptičko und pingvin referieren, benötigt der Leser mikrogruppeninternes Hintergrundwissen. Besitzt der Leser dieses Wissen nicht, kann er erst nach mehreren Mitteilungen annehmen, dass ptičko bzw. pingvin die Ruf- bzw. Kosenamen des Users Black Santa sind, d.h. er kann erst dann die davor gemachten Äußerungen anderer Schreiber korrekt interpretieren und am Gespräch partizipieren. In diesem Beispiel jedoch besitzen die am Gespräch beteiligten Personen alle das erforderliche mikrogruppeninterne Hintergrundwissen und können an der Konversation teilnehmen, ohne den User Black Santa direkt mit seinem Usernamen anzusprechen, da jeder weiß, wer mit ptičko gemeint ist. Erworben haben sie dieses Hintergrundwissen in einer vorherigen Gesprächssituation, nach der sie diese Information gespeichert haben. Mikrogruppeninternes Hintergrundwissen benötigt der Leser in diesem Beispiel auch, um tuška zu verstehen. Ohne dieses kann der Leser mit diesem Begriff im Kontext dieser Konversation nichts anfangen, da tuška mehrere Bedeutungen hat, von denen keine in den Kontext dieser Konversation passt. Die Bedeutung des Begriffs im Rahmen des Threads wird in einer anderen Gesprächssituation erläutert (siehe (12)). Diese Information muss der Leser als mikrogruppeninternes Hintergrundwissen gespeichert haben. Auffällig ist, dass sowohl ptičko als auch tuška im Verlauf des Threads immer wieder auftauchen ${ }^{133}$. Die Leser müssen demnach immer wieder dieses Hintergrundwissen aufrufen

\footnotetext{
${ }^{130}$ Die normative Form lautet ptička und ist ein Femininum. Die -o-Endung macht daraus hingegen ein Neutrum.

${ }^{131}$ Das Gleiche gilt für die Form mnu in $\mathrm{T}_{1} \mathrm{M}_{5,1}$ und $\mathrm{T}_{1} \mathrm{M}_{6,1}$.

${ }^{132}$ Hintergrundwissen braucht der Leser z.B. auch für das Verständnis von $\mathrm{T}_{1} \mathrm{M}_{4,1}$ : hierbei handelt es sich nach der Regieanweisungen um den Liedtextausschnitt aus einem im ersten Film vorkommenden Lied. Ohne die Kenntnis des Films, d.h. ohne das entsprechende Hintergrundwissen, ist für den Leser nicht klar, wie dieser Liedtextausschnitt in den restlichen Gesprächskontext passt.

${ }^{133}$ Beispielsweise:
}

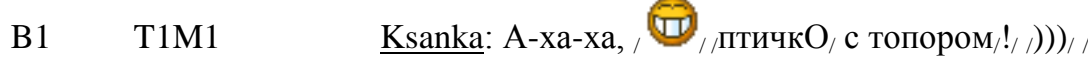


können.

In den beiden Beispielen (14) und (32) wird vom Leser mikrogruppeninternes Hintergrundwissen in Bezug auf die Farben der Nicknames im zweiten Thread vorausgesetzt. Die Farbe wird dabei vom Schreiber als Verweis auf dieses Hintergrundwissen benutzt, fungiert aber auch als Kontextualisierungshinweis ${ }^{134}$. Da die Bedeutung der Farben der Nicknames nicht aus dem aktuellen Gesprächskontext ersichtlich ist, ist eine der Voraussetzungen für eine erfolgreiche Interaktion zwischen Schreiber und Leser, dass beide das mikrogruppeninterne Hintergrundwissen bezüglich der Farben teilen. ${ }^{135}$ Die Erwartung bogdas, DragOn als Leser besäße das entsprechende mikrogruppeninterne Hintergrundwissen, dass die Nicknamefarbe Grün die Moderatoren des Threads kennzeichnet, wird durch DragOns korrekte Interpretation von $\mathrm{T}_{1} \mathrm{M}_{4}$ bestätigt. ${ }^{136}$ Die späteren Mitteilungen $\mathrm{T}_{1} \mathrm{M}_{8^{-}}$ $\mathrm{T}_{1} \mathrm{M}_{10}$ sind ohne mikrogruppeninternes Hintergrundwissen, hier bezogen auf Vinni Puch, ebenfalls nicht zu interpretieren. Um zu verstehen, auf wen die Mitteilung $\mathrm{T}_{1} \mathrm{M}_{9}$ von Lelika referiert, muss man wissen, dass bogda Vinni Puch als Avatar hat und daher von Lelika als

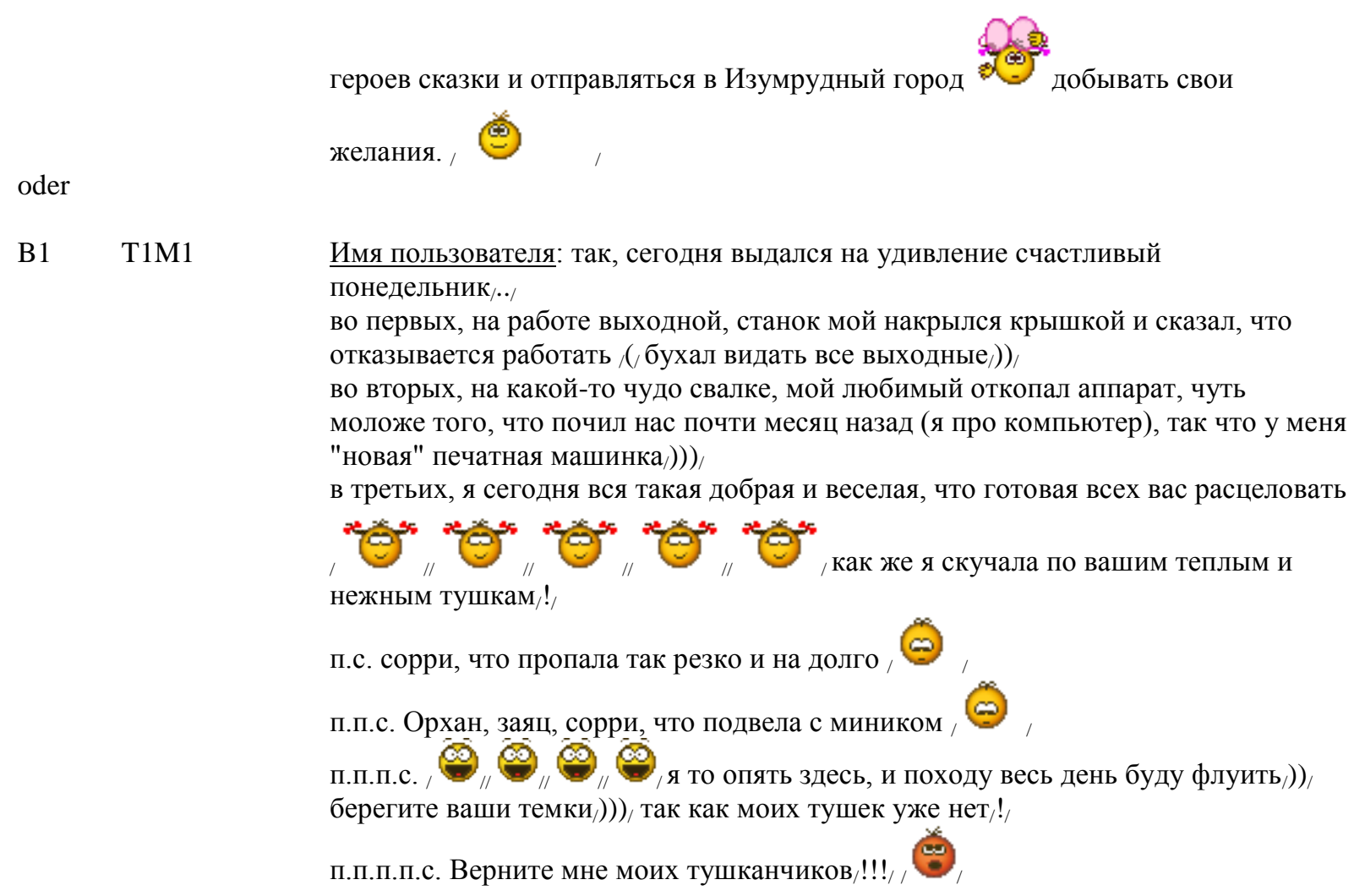

${ }^{134}$ Auf Farben als Kontextualisierungshinweise komme ich in 4.2.2 zu sprechen.

${ }^{135}$ Die Funktion im Thread wird über folgende Farben angezeigt: Administratory (rot) - Private persone (violett) - Moderatory lvl2 (blau) - Moderatory lvl1 (grün) - Pomoščniki (braun) - Obyčnye juzery (weiß) - Izgnannye (orange). Die Anmerkungen in Klammern stammen von der Verfasserin.

(vgl. http://forum.xakepok.net/showthread.php?t=5239\&highlight=Private+persone)

136 Auch ein Leser, der über dieses Hintergrundwissen nicht verfügt, kann die Äußerung verstehen, jedoch erst im Nachhinein, da die Bedeutung der Farbe durch die Nachfrage von DragOn in $\mathrm{T}_{1} \mathrm{M}_{5}$ implikatiert wird. 
Vinni Puch bezeichnet wird. Wie wichtig das mikrogruppeninterne Hintergrundwissen um die Bedeutung der Farbe in diesem Thread ist, zeigt auch das Beispiel (32). Mit der Äußerung, der User AgentMario sei grün geworden, spielt Lelika auf die Farbveränderung des Usernamens von AgentMario von blau zu grün an. Im zweiten Teil der Äußerung wird dem Leser der Grund für die Veränderung präsentiert: AgentMario hat die blaue Gruppe verlassen und ist offensichtlich zu einem Mitglied der grünen geworden. Die ganze Äußerung kann der Leser jedoch nur korrekt interpretieren, wenn er über das entsprechende mikrogruppeninterne Hintergrundwissen verfügt: der Leser muss wissen, dass die Moderatoren lvll grüne Usernamen haben, Moderatoren lvl2 hingegen blaue, Administratoren rote usw., er muss aber auch die Hierarchiestufen kennen. Auf dieser Grundlage kann er die Farbveränderung als Implikatur einer hierarchischen Abstufung interpretieren, da Moderatoren lvl1 hierarchisch im Forum unter den Moderatoren lvl2 stehen.

Bei der letzten von mir untersuchten Art des Hintergrundwissens handelt es sich um das situationsbezogene. Dieses beinhaltet die Kenntnis einer Situation (z.B. eines Vorfalls), die außerhalb der aktuellen Gesprächssituation stattgefunden hat. Fehlt den Lesern das situationsbezogene Hintergrundwissen, können sie nur Vermutungen darüber anstellen, was passiert sein könnte. Eine korrekte Interpretation der Äußerung des Gesprächspartners ist nicht möglich. So kann der Leser in (28), selbst wenn er über das entsprechende „fachliche“ Hintergrundwissen verfügt und diese Begriffe versteht, nicht wissen, ob sie positiv oder negativ gemeint sind, da ihm die Kenntnis des anderen Kontexts fehlt: für den Leser ist hier bspw. nicht ersichtlich, ob obrazina gippogrifovaja in diesem Kontext als positiv oder negativ $\mathrm{zu}$ werten ist, da obrazina eigentlich negativ konnotiert ist, während das Zauberwesen gippogrif im Harry Potter- Universum als neutral bis positiv bewertet wird. Ein Beispiel für das Fehlen von situationsbezogenem Hintergrundwissen ist das Beispiel (33). Hier fehlt dem Schreiber Lelika das Hintergrundwissen bezüglich der Situation, weswegen bogda plötzlich seine Farbe verändert hat. Auf Grund ihres mikrogruppeninternen Hintergrundwissens weiß sie, der Verlust der Farbe hängt mit dem Verlust des Moderatorpostens zusammen $\left(\mathrm{T}_{1} \mathrm{M}_{1}\right)$. Jedoch muss sie auf Grund des fehlenden situationsbezogenen Hintergrundwissens nachfragen. Als ihr AgentMario antwortet, bogda habe u.a. eine Verwarnung erhalten, teilt sie in $T_{1} M_{3,2}$ ihre Annahme bezüglich des Grundes für diese Verwarnung mit. Diese Annahme ist möglich, weil sie aus ihrem mikrogruppeninternen Hintergrundwissen heraus weiß, für welches Vergehen ein Moderator in diesem Forum eine Verwarnung erhalten könnte. So kann sie eine Erwartung an das stellen, was der Grund sein könnte. Das mikrogruppeninterne 
Hintergrundwissen reicht alleine jedoch an dieser Stelle nicht aus; da ihr situationsbezogenes Hintergrundwissen fehlt, muss sie weiter nachfragen. Zudem ist ihre Annahme in $T_{1} M_{3,2}$ und somit ihre Interpretation falsch. Dies ist an der Antwort von AgentMario in $\mathrm{T}_{1} \mathrm{M}_{4} \mathrm{zu}$ erkennen, wo er ihre Interpretation korrigiert und Beleidigung als tatsächlichen Grund für bogdas Verwarnung angibt. Beispiel (34) zeigt hingegen einen Fall, in denen die Gesprächspartizipanten über das gleiche Hintergrundwissen in Bezug auf die Situation verfügen. Dabei geht es um eine Situation in einem anderen Thread des Forums. Sowohl Lelika, die das Thema anspricht, als auch AgentMario verfügen beide über das Hintergrundwissen, dass in einem anderen Thread eine Situation vorgefallen ist, in der eine männliche Person Kleider angeboten hat. Diese Situation bildet den Auftakt zu einer Konversation und muss von Schreiber und Leser als Information gespeichert worden sein, um in dem Gespräch als situationsbezogenes Hintergrundwissen aufgerufen werden zu können. Fehlte dem Leser AgentMario dieses Hintergrundwissen, hätte er wie in dem oben vorgestellten Fall nachfragen müssen, hätte die Äußerung nicht korrekt interpretiert oder hätte sich gar nicht erst auf die Interaktion eingelassen.

Anhand der eben vorgestellten Beispiele lässt sich die Bedeutung des Hintergrundwissens für die Interpretation sehen. Meiner Ansicht nach wird es vom Leser sowohl im ersten als auch im zweiten Interpretationsschritt aufgerufen. Dabei greifen wir als Leser auf die in unserem Gedächtnis gespeicherten Informationen zurück, die unser Hintergrundwissen bilden. Wie so etwas aus meiner Sicht funktionieren könnte, lässt sich beispielsweise an gruppeninternem Hintergrundwissen verdeutlichen, auf das in den Verläufen beider Threads immer wieder zurückgegriffen wird. Nehmen wir beispielsweise (14) $T_{1} M_{4}$ und $T_{1} M_{5}$. Um den Leser DragOn darauf hinzuweisen, dass er für die korrekte Interpretation mikrogruppeninternes Hintergrundwissen benötigt, platziert der Schreiber bogda einen strategischen Marker. Dies kann, muss aber nicht gemacht werden. Der Marker ist in diesem Fall eine bestimmte Farbe, nämlich Grün. Dieser Marker zieht die Aufmerksamkeit des Lesers auf sich und gibt ihm den Hinweis, dass er in diesem Gesprächskontext eine bestimmte Bedeutung hat bzw. eine bestimmte Funktion erfüllt. Der Leser geht dann vorherige Gesprächssituationen, an die er sich erinnern kann, auf der Suche nach diesem Marker durch, d.h. der Leser prüft, ob in seinem Gedächtnis ein Gesprächskontext gespeichert ist, in dem der entsprechende Marker bereits auftauchte. Dies bildet sein Hintergrundwissen. Kann der Leser den Marker nicht identifizieren, bedeutet es, dass dem Leser das entsprechende gruppeninterne Hintergrundwissen fehlt. Die Äußerung bleibt für ihn auf diese Weise nicht korrekt 
interpretierbar. Hätte DragOn den Marker „grüne Farbe“ nicht identifizieren können, so hätte er die Äußerung von bogda so interpretiert, dass er einfach einen grün eingefärbten Usernamen haben möchte. Jedoch kann in diesem Beispiel DragOn den Marker identifizieren, da in seinem Gedächtnis die Information gespeichert ist, dass nur Moderatoren grüne Usernamen haben dürfen; diese Information stellt einen Bestandteil seines mikrogruppeninternen Hintergrundwissens dar. So kommt seine Interpretation, dass bogda Moderator werden möchte $\left(\mathrm{T}_{1} \mathrm{M}_{5}\right)$ zu Stande.

Zusammenfassend lässt sich sagen, dass anhand der Beispiele gezeigt werden konnte, dass jenseits der Arten des Hintergrundwissens, die Gumperz anführt, weitere angenommen werden müssen. Daher ergänze ich die Annahmen von Gumperz um makro- und mikrogruppeninternes und situationsbezogenes Hintergrundwissen. Beim Interpretationsvorgang spielt das Hintergrundwissen an mehreren Stellen eine wichtige Rolle: das erste Mal tritt es auf der Ebene der Interpretation der einzelnen Wortformen, das zweite Mal auf der Ebene der vollständigen Äußerung auf. Dabei trägt es zur Schaffung des Kontextes der Äußerung bei.

\subsection{Kontextualisierungshinweise in Internetthreads}

Bei den Kontextualisierungshinweisen $(\mathrm{KH})$ zeigen sich mehrere wichtige Unterschiede zwischen mündlich und schriftlich realisierter Kommunikation. Der erste Unterschied ist, dass die schriftlich realisierte Internetkommunikation nur bedingt über die gleichen Mittel verfügt wie mündliche Kommunikation und es dementsprechend keine Möglichkeit gibt, lautliche Phänomene, Gestik oder Mimik unmittelbar und uneingeschränkt wahrzunehmen. Die Schreiber sind daher auf andere Mittel angewiesen. Die Funktion solcher Mittel muss konventionalisiert sein, um vom Leser korrekt interpretiert werden zu können. Der zweite wichtige Unterschied liegt in der Wahrnehmung der Kontextualisierungshinweise: in mündlicher Kommunikation werden diese parallel zur Äußerung gehört und/oder gesehen, in schriftlich realisierten Konversationen ist dies auf Grund der Linearität einer Äußerung in den meisten Fällen nicht möglich. Auf Grund dieser Unterschiede stellen sich mehrere Fragen: 1) welche Mittel kommen in der Kommunikation in Internetthreads als Kontextualisierungshinweise in Frage und 2) erfüllen sie eine Funktion, die mit der von Kontextualisierungshinweisen in mündlicher Kommunikation vergleichbar ist? Die zu 
überprüfende These ist, dass die Internetkommunikation zwar über eine Vielzahl von Mitteln verfügt, die als potenzielle Kontextualisierungshinweise fungieren können, die meisten in schriftlich realisierten Gesprächen jedoch nicht die gleiche Funktion besitzen wie Kontextualisierungshinweise in mündlicher Kommunikation. Sie leiten die Interpretation nicht, können sie im besten Falle am Ende nur bestätigen. Diese These wurde anhand mehrerer Experimente überprüft. In den Versuchen hatten mehrere Probandinnen ${ }^{137 ; 138}$ die Aufgabe aus den Internetthreads stammende Konversationen laut vorzulesen. Dabei bekam die einzelne Probandin den jeweiligen Text zunächst z.B. im Original, in einem weiteren Experiment über ein Jahr später erhielt sie den gleichen Text, der jedoch dahingehend manipuliert wurde, dass beispielsweise Emoticons fehlten. Der vorgelesene Text wurde mit einem Diktiergerät aufgezeichnet. Für die Analyse der für die Experimente verwendeten Beispiele sowie die Erstellung der Intonationskurven ${ }^{139}$ (IK) wurde das Programm „Praat“ verwendet. Das Ziel der Experimente war es herauszufinden, ob die Mittel, die als potenzielle $\mathrm{KH}$ in Frage kommen, einen Einfluss auf die Intonation ausüben. Da die Intonation einen wichtigen Bestandteil des Interpretationsprozesses bildet, spiegelt eine IK meiner Ansicht nach die Interpretation einer Äußerung wieder: wird die Äußerung bspw. als scherzhaft verstanden und vorgelesen, ist die Prosodie anders, als wenn sie als traurig interpretiert wird. Folgt man dieser Vorstellung, so müssten feststellbare Unterschiede bei den Intonationskurven auszumachen sein, wenn die Probandinnen die gleiche Äußerung unterschiedlich interpretieren. Dabei erlaubt die Untersuchung der Intonationskurven eine aus meiner Sicht objektivere Messmethode als ein Fragebogen, da die Probanden die Konversationsbeispiele spontan vorlesen müssen und keine Zeit zum Überlegen haben, d.h. sie produzieren die Äußerungen so, wie sie ihrer Interpretation in dem Kontext entsprechen.

Die Mittel, die potenzielle KH bilden, werden in verschiedene Gruppen unterteilt. In der ersten Gruppe finden sich Hinweise auf Prosodie, in der zweiten Gruppe farbliche Markierungen und in der dritten Gruppe Hinweise auf Handlungen, Gestik und Mimik. Ein Schwerpunkt der Untersuchung liegt auf der dritten Gruppe.

\footnotetext{
137 Auf Grund der geringen Teilnehmerzahl (unter 10 Personen) sind die Experimente zwar statistisch nicht relevant, zeigen jedoch Tendenzen auf, die für die Zwecke der vorliegenden Dissertation ausreichen.

${ }^{138}$ Bei den Probanden handelte es sich um Frauen zwischen 30 und 60 Jahren. An dieser Stelle möchte ich mich nochmals bei Elena Grimmig, Marianna Leonova und Olga Liebich vom Seminar für Slavische Philologie der Universtität Göttingen sowie anderen Probandinnen für ihre Teilnahme bedanken.

${ }^{139}$ Intonationskurve wird in der vorliegenden Arbeit synonym zur Grundfrequenzkontur verwendet.
} 


\subsubsection{Hinweise auf Prosodie}

In der schriftlich realisierten Kommunikation, wie sie unter anderem in den untersuchten Internetthreads vorliegt, werden unterschiedliche graphische Mittel verwendet, um prosodische Eigenschaften ${ }^{140 ; 141}$ wiederzugeben. ${ }^{142}$ Dazu gehören neben Satzzeichen vor allem Großschreibungen sowie intendiertes Mehrfachvorkommen von graphischen Zeichen für Vokale und Konsonanten. „In the written mode, graphology takes over some of the functions which fall within the scope of phonology or prosody in the spoken mode. This concerns both the actual representation of sounds and the conventional indication of certain suprasegmental features such as intonation, stress, etc. Features evocative of the spoken mode are thus realized, among others, as the emphatic lengthening of written representations of sounds (e.g., Peep! Peep! Peeeeeep!! It's all over.; Ger-Tur [Deutschland-Türkei Europameisterschaft 2008; Anm. d. Verf.], end of game) and the excessive use of multiple punctuation marks and capital letters (cf. Jucker 2006: 125). [...] Other features include the conventional indication of emphasis by means of changing the font ( $I$ really hope he's being sarcastic [...] (Por-Gre [Portugal-Griechenland Euromeisterschaft 2004; Anm. d. Verf.], halftime)), the inclusion of various hesitation sounds and fillers (Diarra is booked for, erm, he's booked for, erm, very little in fact. (Ita-Fra [Italien-Frankreich Weltmeisterschaft 2006; Anm. d. Verf.], 76 mins)), as well as the written representation of other paralinguistic phenomena, such as pauses ([...] by winning the ball, going on a surging run down the left flank and then ... losing it again. (Gre-Cze [Griechenland-Tschechien Europameisterschaft 2004; Anm. d. Verf.], 46 mins)).“ (Chovanec $(2009,116))$. Die graphischen Mittel können dabei eine Funktion als Kontextualisierungshinweise übernehmen, müssen jedoch als solche

\footnotetext{
${ }^{140}$ Bei der Definition von Prosodie richte ich mich nach Mehlhorn, die unter Prosodie das Gleiche wie Intonation versteht: „Unter Intonation verstehe ich die prosodischen Eigenschaften von sprachlichen Äußerungen, die nicht an einen Einzellaut gebunden sind. Dieses Verständnis von Intonation ist gleichzusetzen mit dem Begriff der Prosodie.“ (Mehlhorn (2002, 139)).

${ }^{141}$ Die Intonation ist im Russischen besonders wichtig, da mit ihrer Hilfe ,,[... syntaktisch und lexikalisch gleich strukturierte Sätze disambiguiert werden [können].“ (Mehlhorn (2002, 143)). Anhand eines Experiments untersucht sie u.a. neutralen Fokus und Kontrastfokus, aber auch Verumfokus, und die akustische Realisierung in russischen Sätzen und stellt als Ergebnis fest: „Durch das Experiment konnten prosodische Korrelate für verschiedene Diskursfunktionen im russischen Satz belegt werden. Es konnte gezeigt werden, dass mit Hilfe des Kontexts und der Intonation eine Disambiguierung verschiedener Fokustypen im Satz möglich ist. Das erlaubt es, Intonationskonturen auf Grund der kommunikativen Absicht des Sprechers zu beschreiben. Informationsstrukturelle Merkmale korrespondieren tatsächlich mit einer bestimmten Position im Satz und bestimmten prosodischen Merkmalen. So konnte nachgewiesen werden, dass Informationsstrukturierung ihren Niederschlag in der Intonation findet und dass die Topikalisierung und Fokussierung syntaktischer Konstituenten mit einem bestimmten Tonhöhenverlauf einhergeht.“ (Mehlhorn (2002, 172)).

Zur Prosodie speziell von Topiks im Russischen siehe Alter \& Junghanns (2002).

${ }^{142}$ Graphische Mittel werden jedoch nicht nur zur Wiedergabe prosodischer Phänomene verwendet, sondern haben noch weitere Funktionen, z.B. die Erregung der Aufmerksamkeit des Lesers. „Distinctive graphology is also an important feature of Netspeak. [...] " (Crystal $(2001,87))$.
} 
konventionalisiert sein, um vom Leser erkannt zu werden.

Grundsätzlich dienen Ausrufe- und Fragezeichen der rudimentären Markierung von Intonation, die in schriftlich realisierten Gesprächen auf Grund des Fehlens eines Audiosignals nicht ohne Weiteres angegeben werden kann. Beide Satzzeichen können sowohl einzeln ((35) $\left.\mathrm{T}_{3} \mathrm{M}_{1,7}\right)$ als auch gehäuft ${ }^{143 ; 144}\left((35) \mathrm{T}_{2} \mathrm{M}_{1,2}\right.$ oder (36) $\mathrm{T}_{1} \mathrm{M}_{1}$ ) auftreten. Auch können beide Satzzeichen miteinander kombiniert werden: entweder folgt ein Ausrufezeichen einem Fragezeichen ((37) $\left.\mathrm{T}_{1} \mathbf{M}_{1,1}\right)$ oder andersherum ((32) $\left.\mathrm{T}_{1} \mathbf{M}_{4,1}\right)$.

Durch ihre Position am Ende der Äußerung ist es fraglich, ob ihre Funktion mit der „echter“ prosodischer Kontextualisierungshinweise, wie sie in der mündlichen Kommunikation genutzt werden, verglichen werden kann. Aus meiner Sicht spielen sie keine Rolle für die Intonation und damit Interpretation. Um diese These $\mathrm{zu}$ überprüfen, wurden mit einer Probandin zwei Experimente durchgeführt, bei dem die gleichen Konversationsbeispiele vorgelesen werden sollten. Beim ersten Experiment erhielt sie die Originalbeispiele mit den jeweiligen Satzzeichen. Beim zweiten Experiment, das über ein Jahr später durchgeführt wurde, erhielt sie die gleichen Beispiele, jedoch in manipulierter Form: die Ausrufe- bzw. Fragezeichen wurden durch Punkte und in einem Beispiel der Punkt durch ein Fragezeichen ersetzt. Dabei wurden sowohl kurze als auch längere Äußerungen, bei denen das Ausrufebzw. Fragezeichen nicht bereits von vornherein aus dem Augenwinkel heraus für den Leser zu erkennen war, untersucht. Sollten Ausrufe- und Fragezeichen als Kontextualisierungshinweise in schriftlich realisierten Äußerungen in Frage kommen, wären Unterschiede im Intonationsverlauf der Probandin $\mathrm{zu}$ erwarten gewesen, die den Unterschieden in den IK in der Akademiegrammatik von 1980 entsprechen.

Als Beispiele für kurze Äußerungen mit Ausrufezeichen wird hier die Äußerung (8) $T_{1} M_{2,4}$ präsentiert. In der ersten Abbildung finden wir die jeweilige Äußerung mit (vgl. (8)) und in der zweiten ohne das Ausrufezeichen (vgl. (8)').

\footnotetext{
${ }^{143}$ Manchmal ist die Anzahl übertrieben hoch, z.B. (36) $\mathrm{T}_{1} \mathrm{M}_{1,3}$, was unökonomisch ist und dem 'save a keystroke'-Prinzip von Crystal $(2006,90)$ auf den ersten Blick widerspricht. Geht man jedoch davon aus, dass man nur einmal die Tasten betätigen muss, unabhängig davon, ob man ein oder mehrere Satzzeichen schreiben möchte, und dass es keine technische Zeichenbegrenzung für die Mitteilungen im Forum gibt, so entspricht auch die unnötig hohe Anzahl der Satzzeichen immer noch dem 'save a keystroke'-Prinzip.

${ }_{144} \mathrm{Ob}$ das Mehrfachvorkommen der Satzzeichen für den Leser eine Rolle spielt, wurde im Rahmen der Experimente nicht abschließend geklärt und muss weiter untersucht werden.
} 


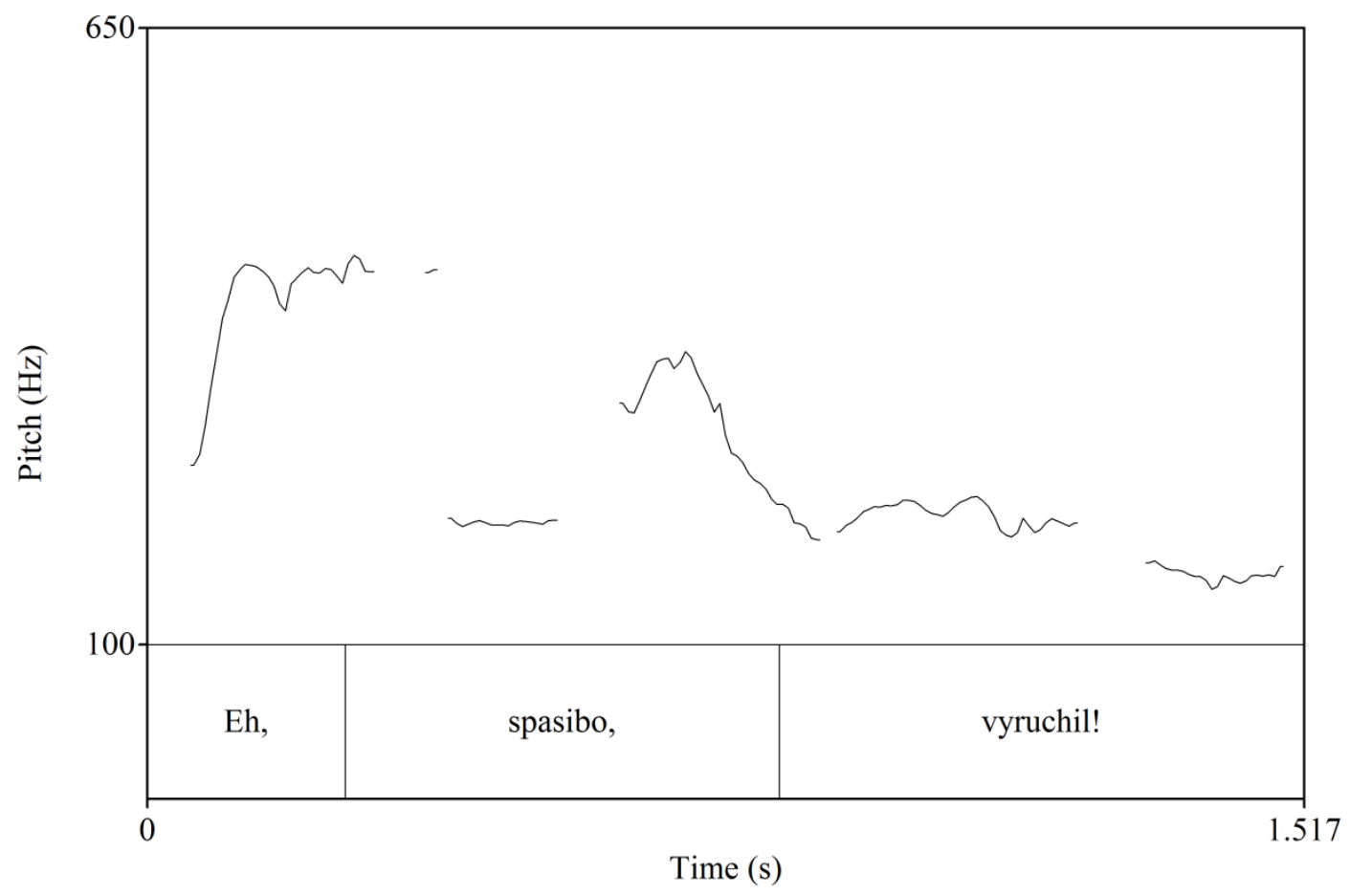

Abb. 14 Probandin 1 mit Ausrufezeichen

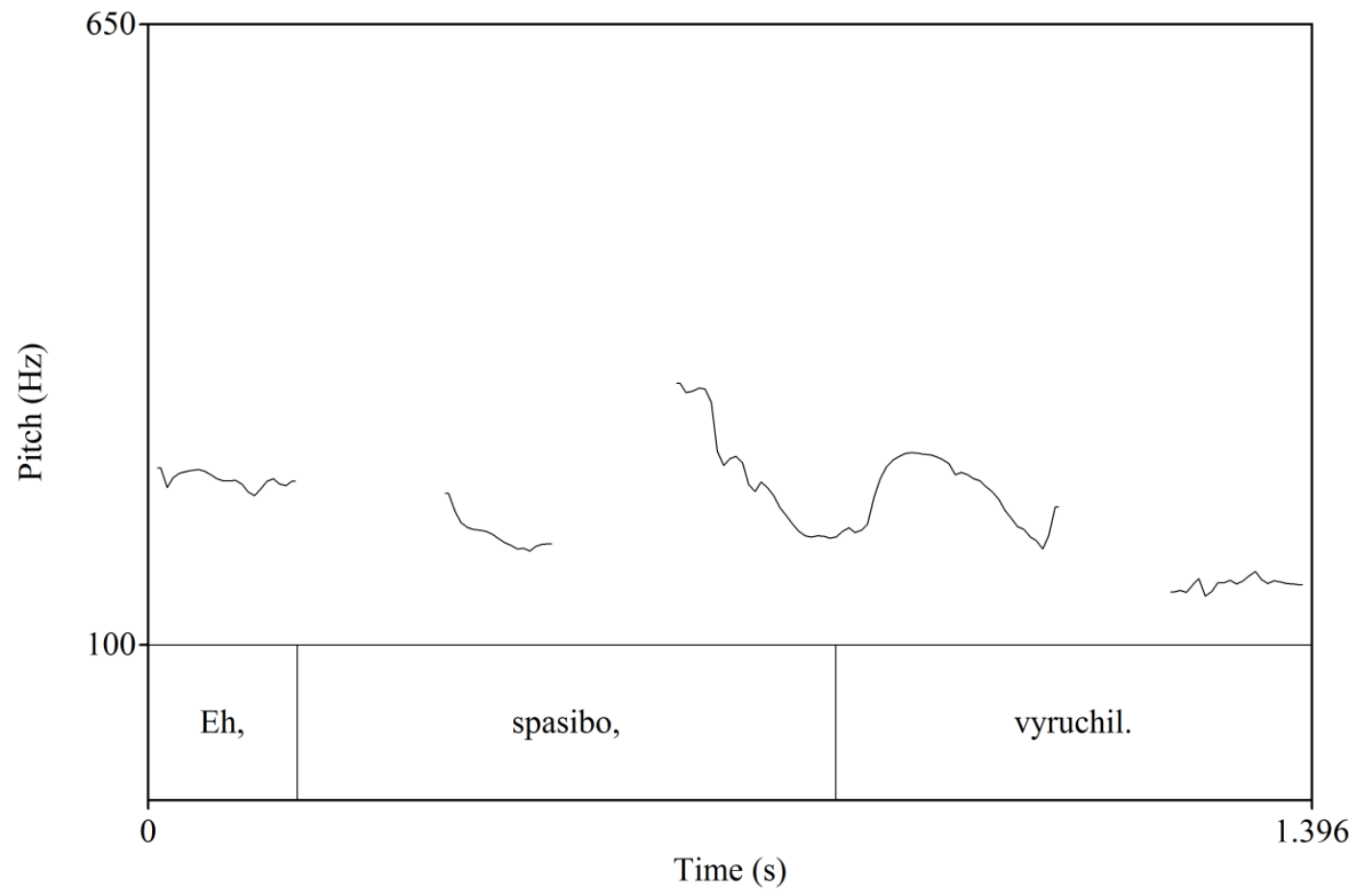

Abb. 15 Probandin 1 ohne Ausrufezeichen

In diesem Beispiel sieht man in der ersten Abbildung eine steigende Intonationskurve zu 
Beginn der Äußerung. Die Vorleserin reagiert hier auf das lexikalische Signalwort Ech. ${ }^{145}$ Einen vergleichbaren Anstieg gibt es in der zweiten Abbildung jedoch nicht. Auffällig ist der Verlauf der Intonationskurve am Ende der Äußerung: obwohl die Äußerung in der ersten Abbildung mit einem Ausrufezeichen abgeschlossen wird, gibt es keinen steilen Anstieg der Kurve - im Gegensatz zu der Intonationskurve der Äußerung ohne das Ausrufezeichen, in der ein Anstieg bei vyručil zu sehen ist. Stattdessen verläuft die Intonationskurve relativ flach wie bei einem Deklarativsatz. Obwohl es sich bei diesem Beispiel um eine kurze Äußerung handelt, gibt es seitens des Lesers keine Reaktion auf das Ausrufezeichen. Möglicherweise liegt das daran, dass diese Äußerung für den Vorleser keinen Exklamativsatz darstellt. Da das Ausrufezeichen hier keine veränderte Intonation auslöst, liegt die Vermutung nahe, dass das Ausrufezeichen von dem Leser nicht wahrgenommen wurde und auf die Interpretation keinen Einfluss ausübte. Damit kommt es als Kontextualisierungshinweis in diesem Beispiel nicht in Frage. Lexikalische Signalwörter spielen hingegen eine Rolle.

Um den Einfluss lexikalischer Signalwörter auszuschließen, wurden ebenfalls Intonationskurven von Äußerungen ohne entsprechende Signalwörter analysiert, beispielsweise (43)' und (43)' $\mathrm{T}_{2} \mathrm{M}_{2,3}$ :

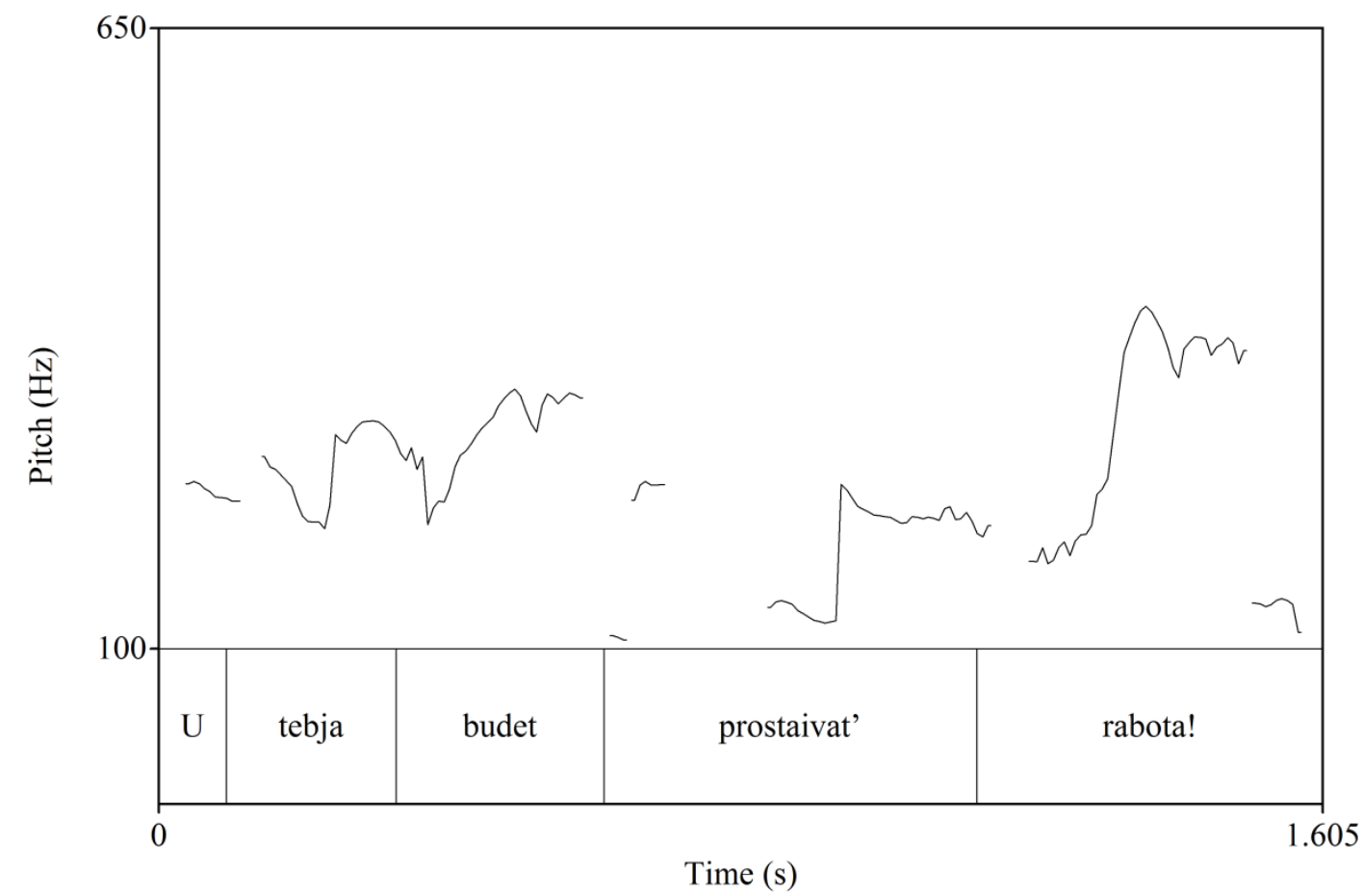

Abb. 16 Probandin 1 mit Ausrufezeichen

${ }^{145}$ Bei Signalwörtern handelt es sich um Interjektionen, z.B. Ach/Ech, und Partikel, z.B. da. 


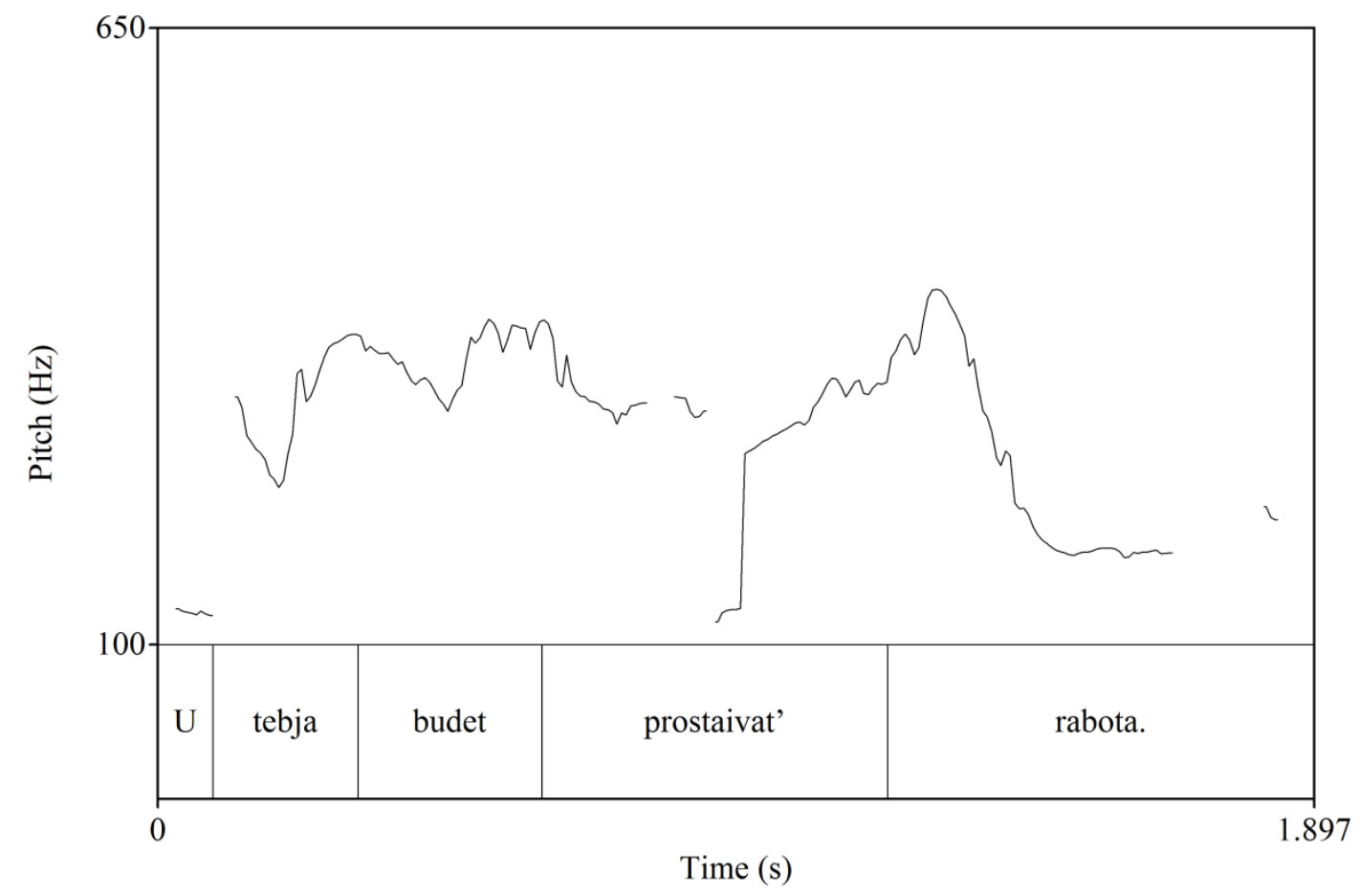

Abb. 17 Probandin 1 ohne Ausrufezeichen

In der ersten Abbildung erkennt man am Ende der Äußerung eine steil steigende Intonationskurve, die dann fällt: der Fokus liegt hier auf rabota. Als Gegensatz dazu beginnt der Anstieg in der zweiten Abbildung bereits vor rabota. Der Fokus liegt hier auf prostaivat'. Der Fokus wird in beiden Beispieläußerungen unabhängig von dem Ausrufezeichen platziert, ein Anstieg der IK findet somit unabhängig vom Vorhandensein eines Ausrufezeichens statt. Damit kann man nicht von einem Einfluss des Ausrufezeichens auf die Intonation sprechen.

Für kurze Äußerungen mit Fragezeichen wurden die Intonationskurven Äußerungen $T_{1} M_{1,3}$ oder $\mathrm{T}_{1} \mathrm{M}_{3,2}$ des Beispiels (45)' und (45)" analysiert. Zunächst wird die Äußerung $\mathrm{T}_{1} \mathrm{M}_{3,2}$ untersucht: 


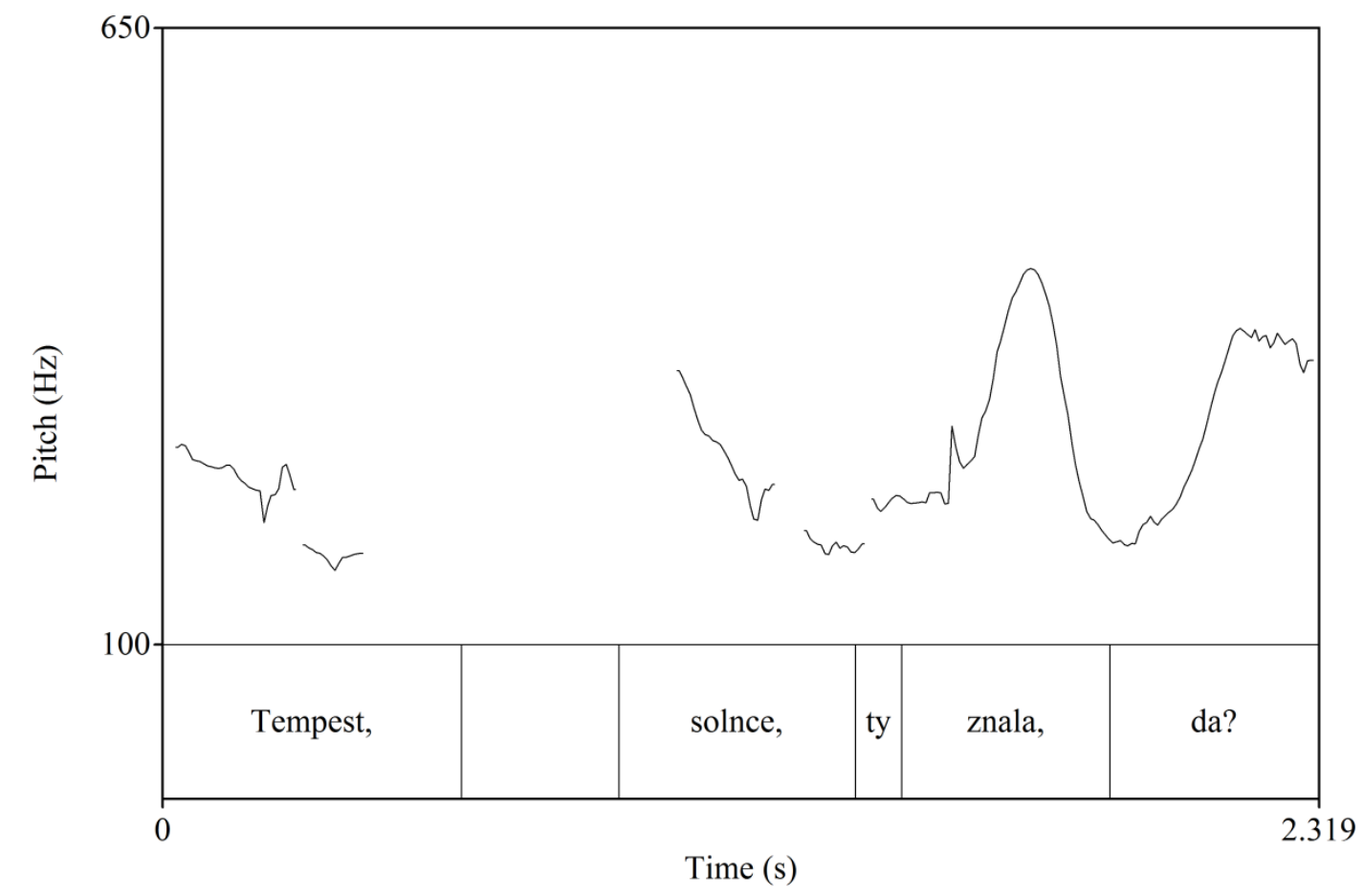

Abb. 18 Probandin 1 mit Fragezeichen

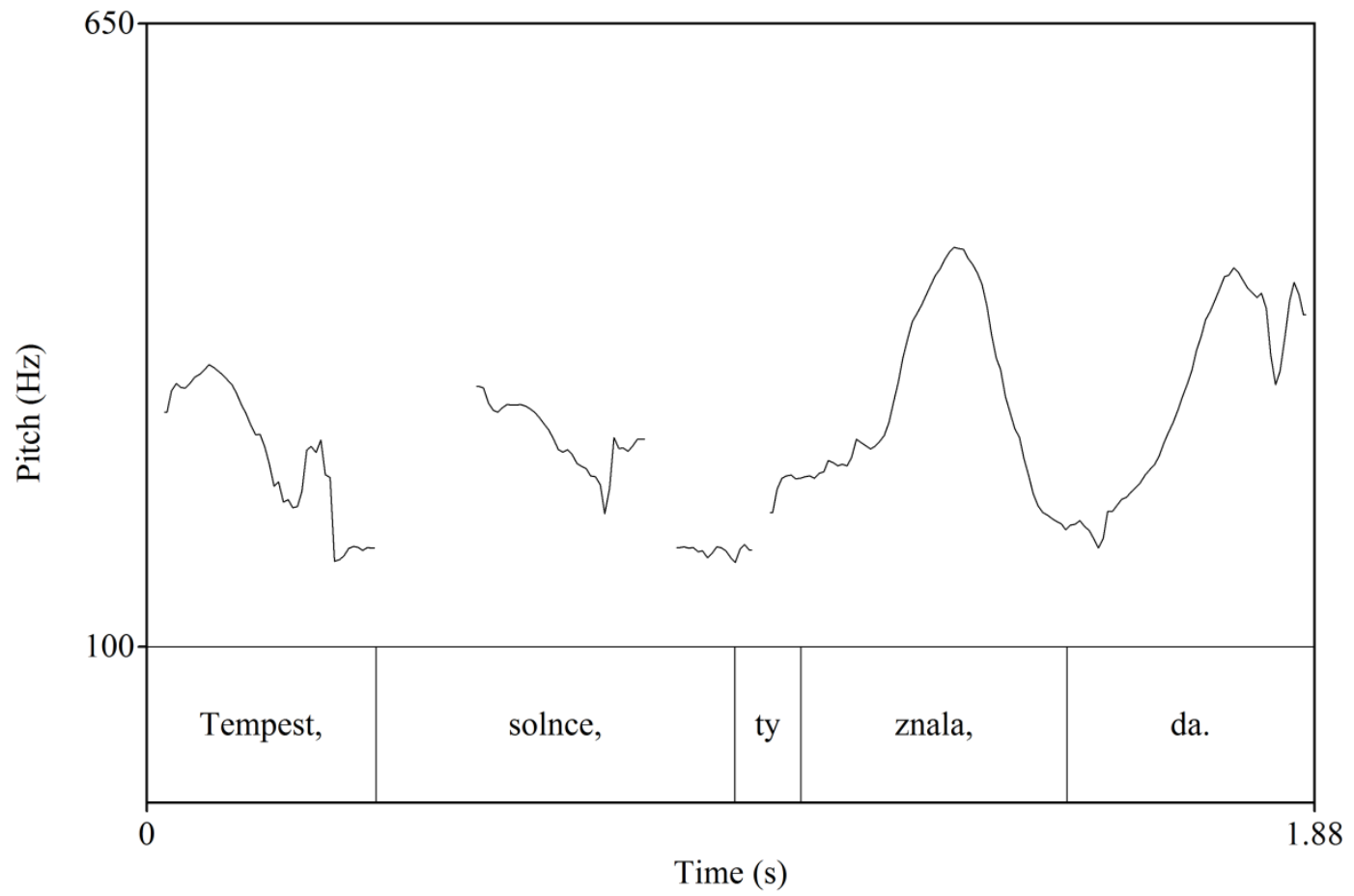

Abb. 19 Probandin 1 ohne Fragezeichen

Da diese Äußerung mehrere Intonationsbrüche enthält, wird nur das Ende der Äußerung vor 
der Partikel $d a^{146}$, d.h. ty znala, betrachtet. Aus den Abbildungen der Intonationskurven ist festzustellen, dass in beiden Fällen die Intonationskurve ansteigt. Hierbei handelt es sich um die für Frageintonation typische IK3. ${ }^{147}$ Die Äußerung wird somit unabhängig davon, ob ein Fragezeichen vorhanden ist oder nicht, auf die gleiche Art und Weise mit einer Frageintonation auf znala realisiert. Die Repräsentation eines Fragezeichens spielt für die Intonation und letztendlich auch für die Interpretation dieser Äußerung offensichtlich keine Rolle.

Abschließend lassen sich für kurze Äußerungen folgende Ergebnisse festhalten: Bezüglich der Ausrufezeichen kann man sagen, dass in keinem der Beispiele das Ausrufezeichen eine andere Intonation auslöste, als das gleiche Beispiel ohne das Ausrufezeichen. Bei den Fragezeichen zeigte sich, dass sie keinen anderen Einfluss auf die Intonation ausübten als die Äußerung ohne Fragezeichen und somit offensichtlich für die Interpretation der Äußerung keine Rolle als Kontextualisierungshinweise spielten. Aus den Experimenten kann man ebenfalls schließen, dass nicht die Satzzeichen, sondern die Signalwörter für die Interpretation wichtig sind.

Im Vergleich hierzu stehen die langen Äußerungen mit Ausrufe- und Fragezeichen, bei denen der jeweilige Vorleser das Satzzeichen nicht aus dem Augenwinkel heraus erkennen und somit eine Intonation antizipieren konnte.

Als Beispiel für eine Äußerung mit einem Ausrufezeichen habe ich die Äußerung $T_{2} M_{2,2}$ aus (43)' und (43)', genommen:

\footnotetext{
${ }^{146}$ Die Äußerung $\mathrm{T}_{1} \mathrm{M}_{3,2}$ enthält als Signalwort, das die Äußerung für den Leser als Frage kennzeichnet, die Partikel $d a$. Hierbei handelt es sich um eine typische Fragepartikel im Russischen.

${ }^{147}$ Vgl. die Tabelle der Intonationsverläufe unterschiedlicher russischer Sätze in der Akademiegrammatik Bd. II $(1980,100)$.
} 


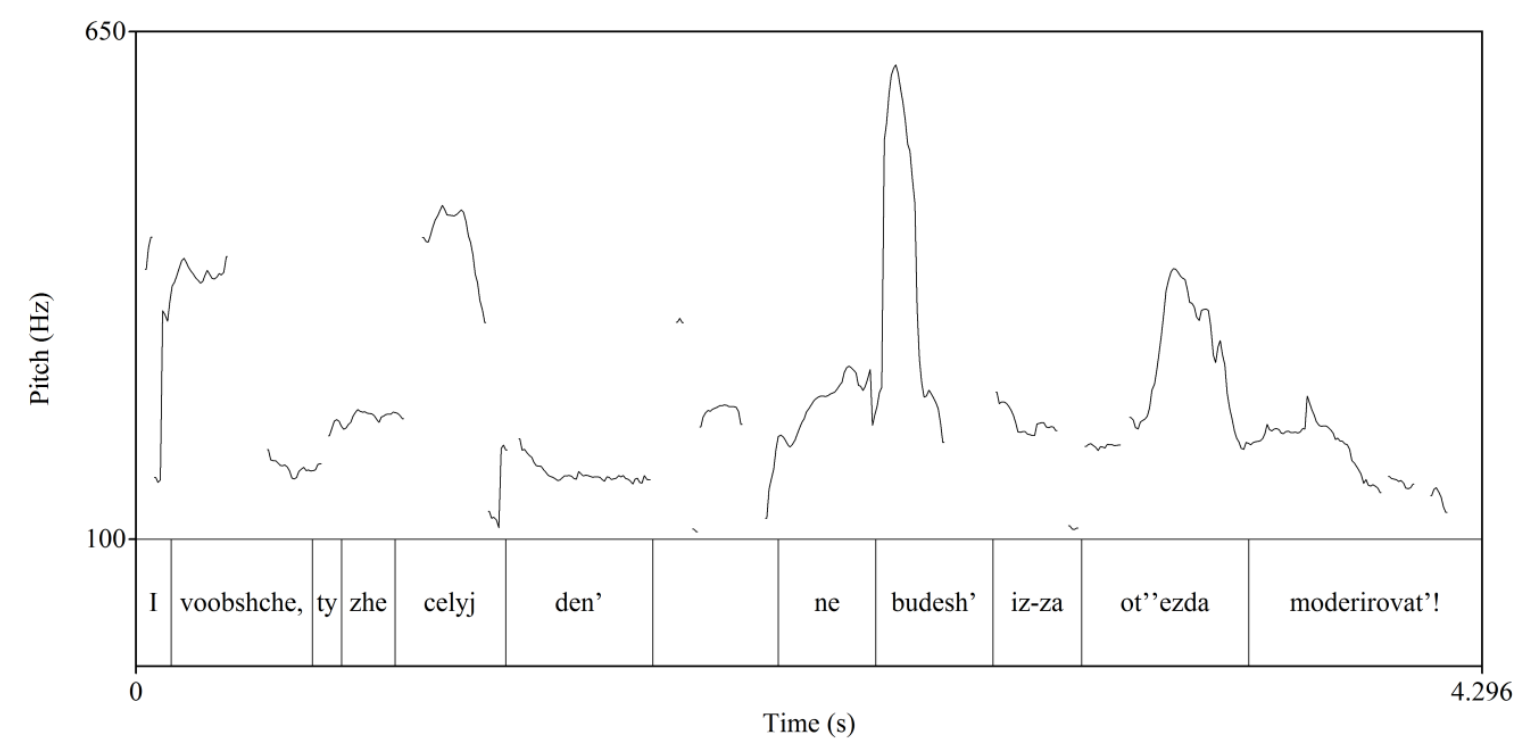

Abb. 20 Probandin 1 mit Ausrufezeichen

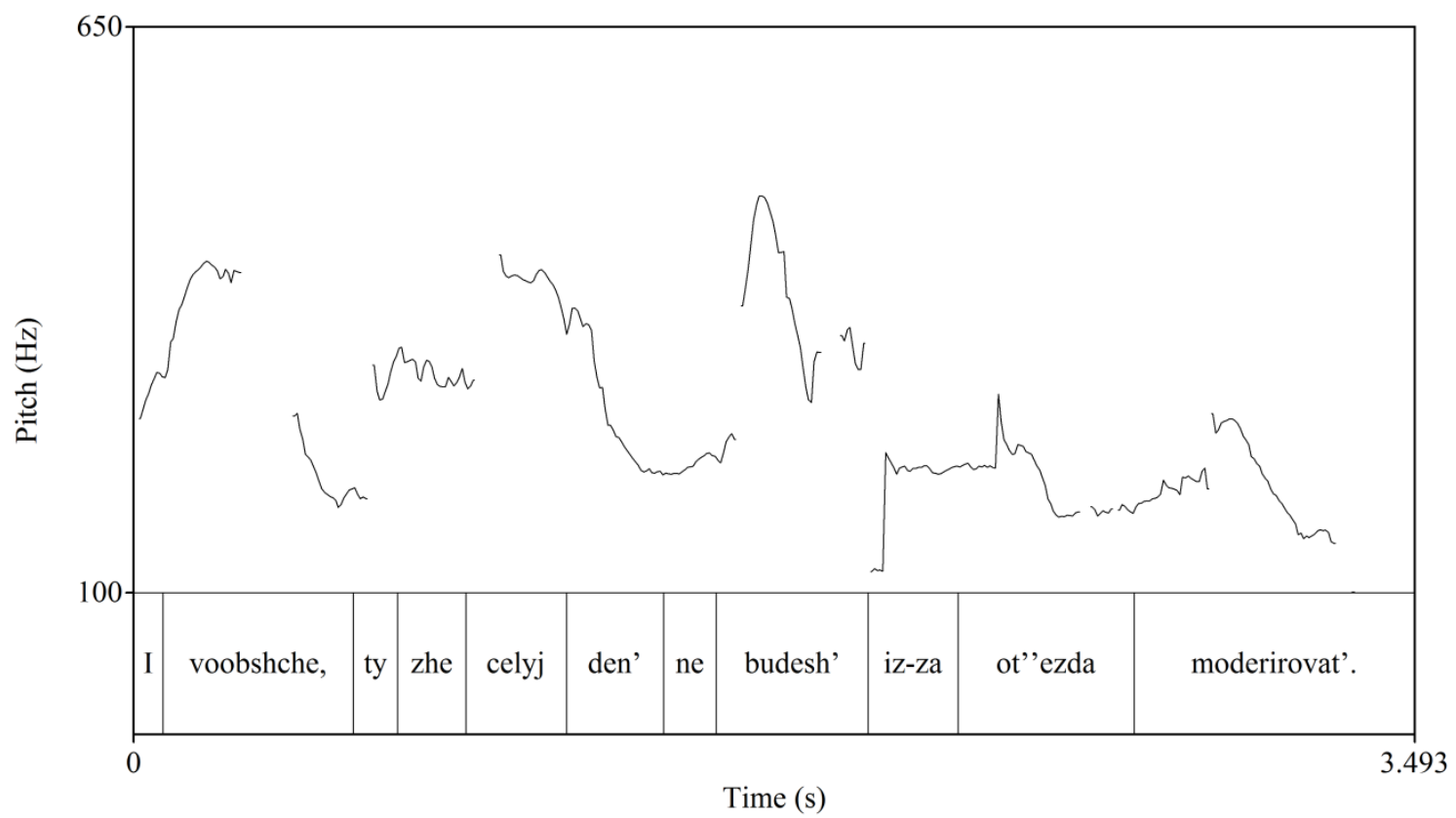

Abb. 21 Probandin 1 ohne Ausrufezeichen

Im Vergleich weisen die Intonationsverläufe große Ähnlichkeiten auf. So steigt in beiden Abbildungen die IK zu Beginn der Äußerung steil an und fällt dann steil, um wieder zu steigen und ab celyj wieder steil zu fallen. Bei budesh' gibt es erneut einen steilen Anstieg und steilen Fall. Hier liegt in beiden IK der Fokus. Den nächsten Anstieg und Fall findet man bei ot''ezda. Auch am Ende der Äußerung ähneln sich in beiden Abbildungen die Intonationskurven: ein Anstieg mit anschließendem Fall. Die Ähnlichkeiten liegen 
unabhängig vom Vorhandensein oder Fehlen des Ausrufezeichens vor. Man kann festhalten, dass es keinen Unterschied macht, ob ein Ausrufezeichen am Ende der Äußerung verwendet wird oder nicht. In beiden Fällen kann eine ähnliche Intonation realisiert werden. Das Ausrufezeichen spielt somit für die Probandin keine Rolle als KH. Dies entspricht auch dem Ergebnis der beiden kurzen Beispiele.

Als Beispiele für die Untersuchung von langen Äußerungen mit Fragezeichen dienten die Äußerung $\mathrm{T}_{1} \mathrm{M}_{2,3}$ des Beispiels (8) und (8)' und die Äußerung $\mathrm{T}_{1} \mathrm{M}_{2,6}$ des Beispiels (46)' und (46)', Bei dem ersten Experiment wurde ein Vergleich zwischen einer Äußerung mit Fragezeichen im Original und der gleichen Äußerung ohne Fragezeichen gemacht.

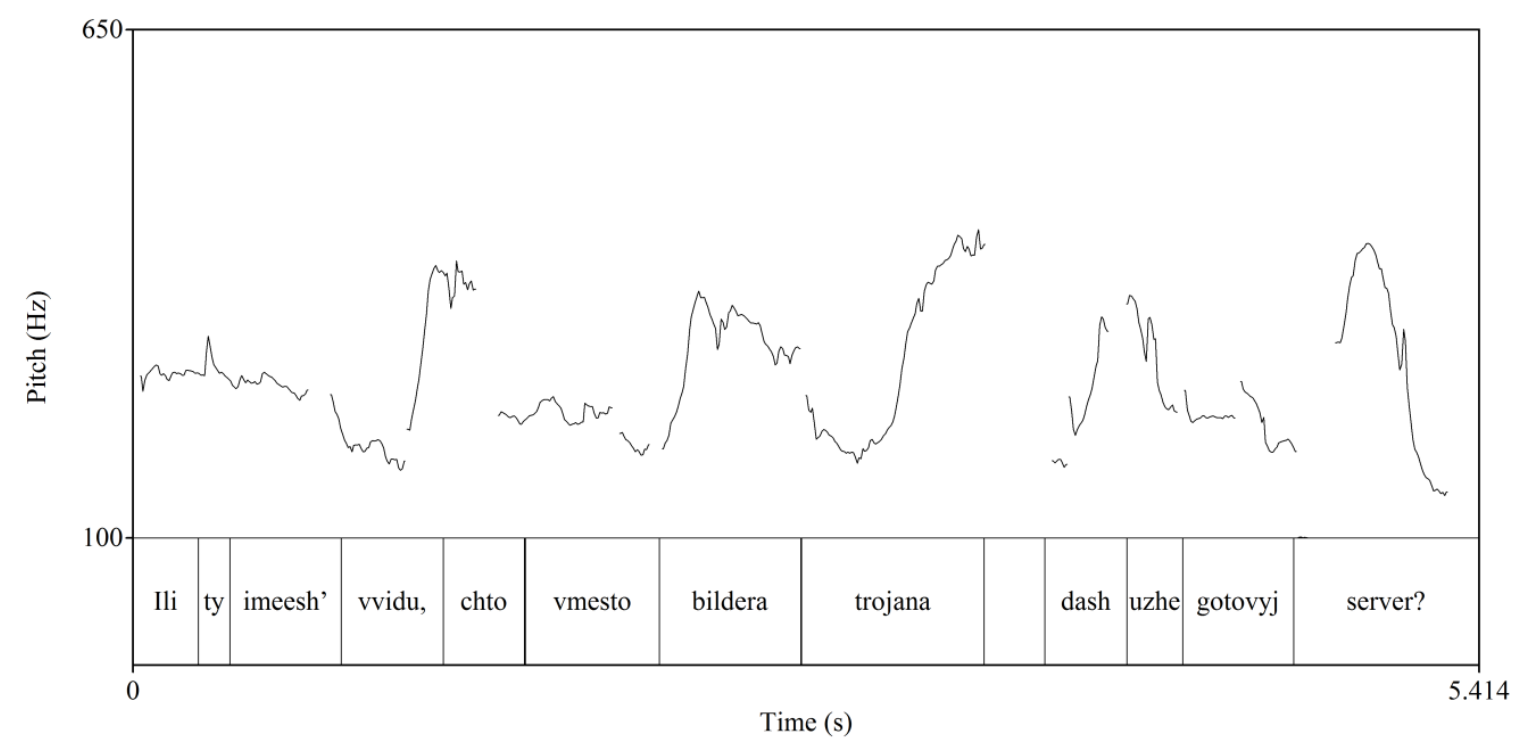

Abb. 22 Probandin 1 mit Fragezeichen

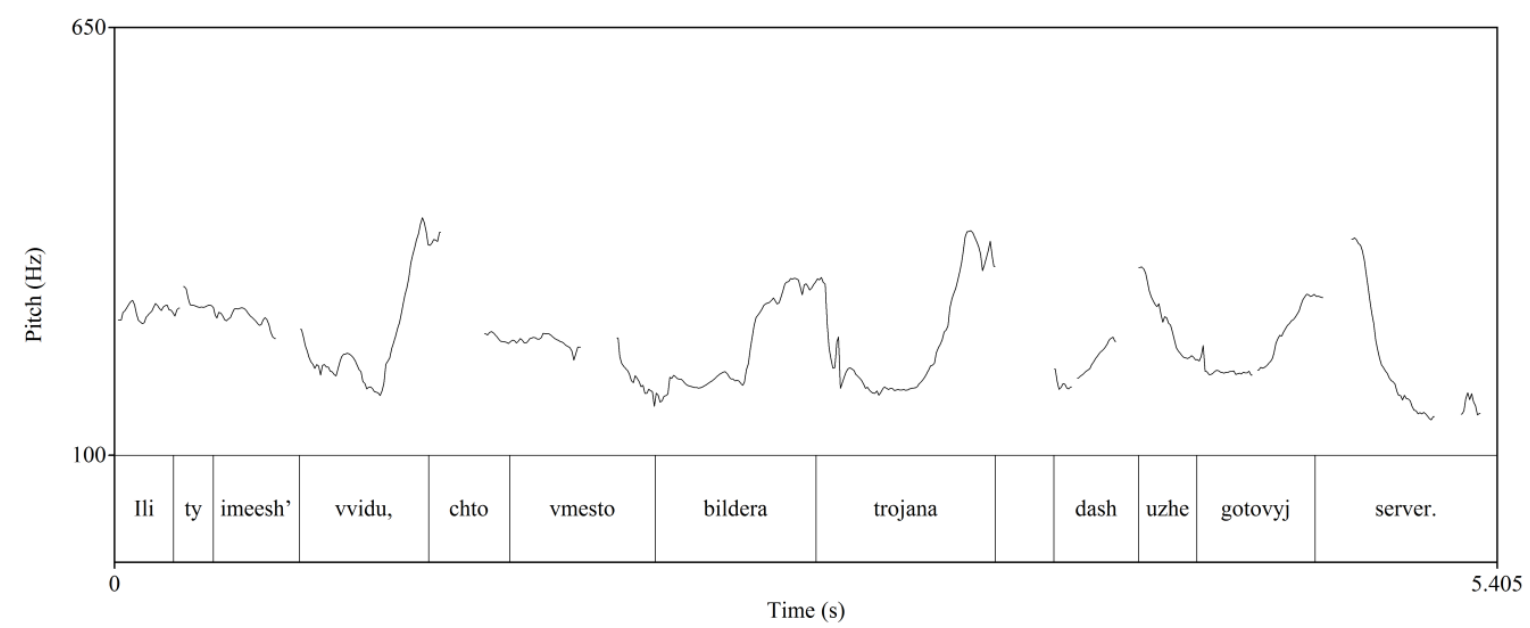

Abb. 23 Probandin 1 ohne Fragezeichen 
Diese Äußerung ist durch das initial stehende lexikalische Signalwort Ili markiert, sodass den Lesern von Anfang an klar ist, dass die Äußerung mit einer Frageintonation vorgelesen werden muss. Dies geschieht hier auch. Vergleicht man beide Abbildungen, stellt man große Ähnlichkeiten im Verlauf der Intonationskurven fest. In beiden Abbildungen steigt die IK bei vvidu steil an, um anschließend steil zu fallen. Den nächsten steilen Anstieg findet man bei bildera, mit anschließendem steilen Fall und erneutem steilen Anstieg und Fall bei trojana. Bei dash steigt die IK wieder steil an, um bei uzhe steil zu fallen. Der letzte steile Anstieg und Fall findet am Ende der Äußerung statt. Die Beibehaltung eines hohen F0 ${ }^{148}$-Niveaus über die Gipfel und vor allem der letzte steile Anstieg und Fall am Ende der Äußerung sprechen dafür, dass diese mit einer Frageintonation vorgelesen wird, unabhängig davon, ob ein Fragezeichen vorhanden ist oder nicht. Die Auswertung der Abbildungen bestätigt das vorige Ergebnis, dass das Fragezeichen keine Rolle spielt, ein lexikalisches Signalwort hingegen schon.

Zum Vergleich wurde eine Äußerung untersucht, die im Original kein Fragezeichen enthält. Diese besitzt auch keinerlei Signalwörter, die es als Fragesatz kennzeichnen würden. Beim ersten Experiment bekam die Probandin diese Äußerung ohne und beim zweiten Experiment mit einem Fragezeichen, um zu sehen, ob das Fragezeichen den Intonationsverlauf verändert. In der ersten Abbildung ist die Äußerung im Original, in der zweiten mit dem Fragezeichen zu sehen:

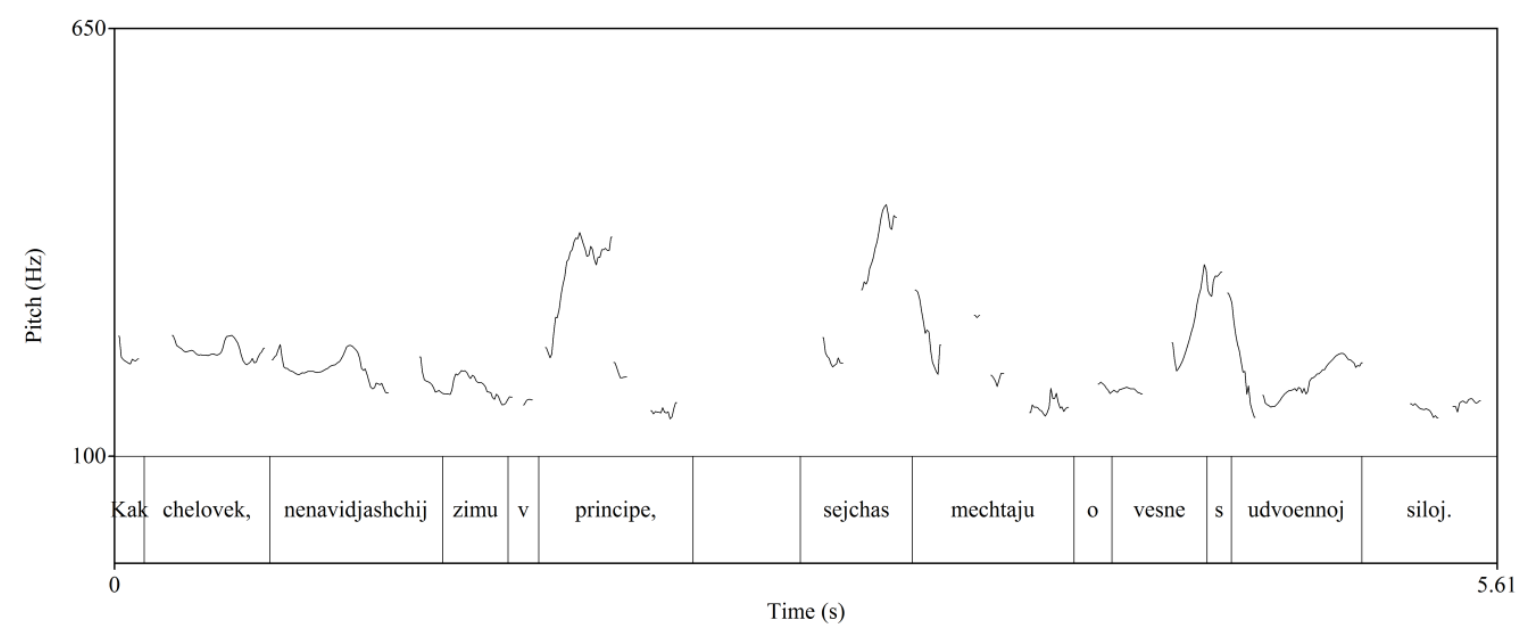

Abb. 24 Probandin 1 ohne Fragezeichen

${ }^{148}$ FO = Grundfrequenz. 


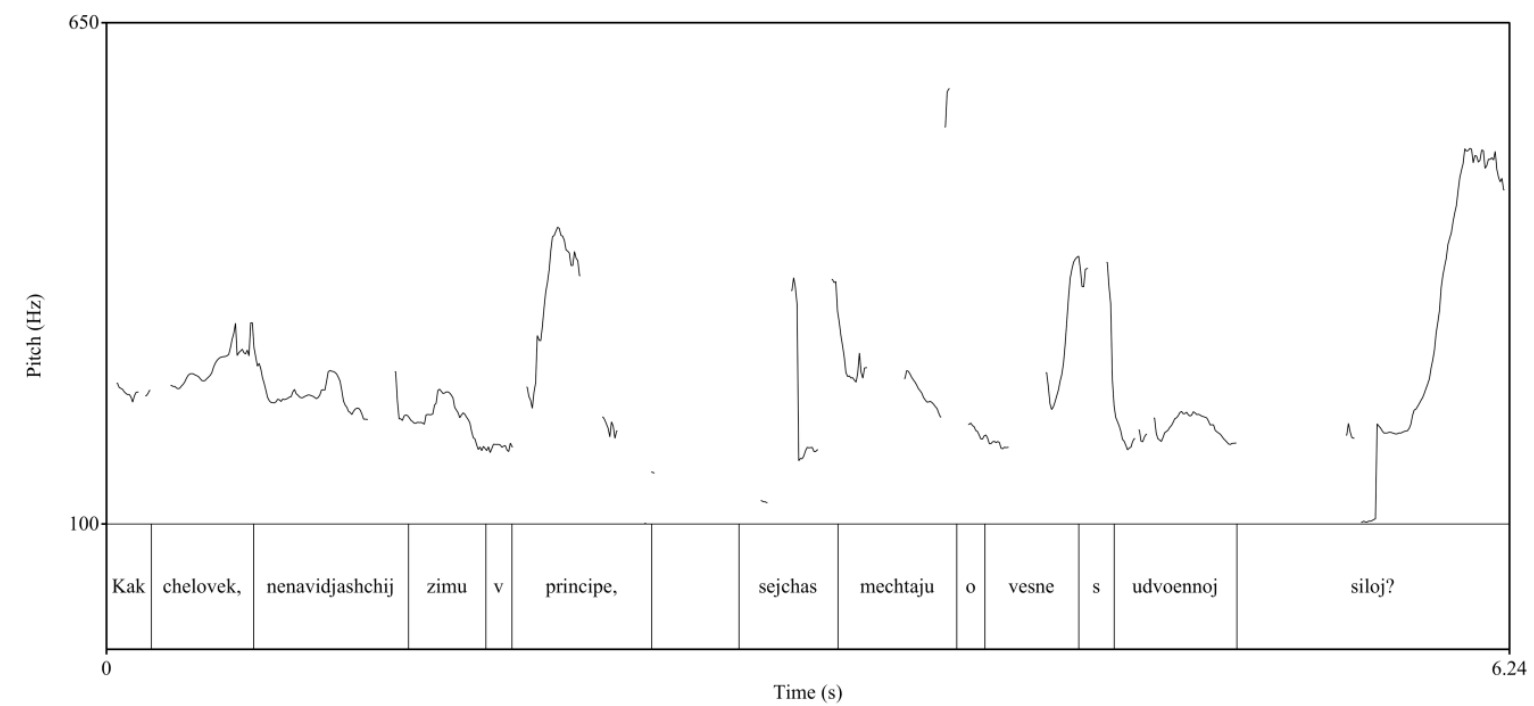

Abb. 25 Probandin 1 mit Fragezeichen

In beiden Abbildungen ist bis udvoennoj ein ähnlicher Intonationsverlauf zu erkennen. Bei principe sieht man einen steilen Anstieg und anschließenden steilen Fall der IK. Bei sejchas sieht man den nächsten steilen Anstieg und Fall, bis die Kurve bei vesne wieder steil ansteigt und danach wieder fällt. Der Fokus liegt in beiden Äußerungen auf vesne. Postfokal ist in beiden Fällen der Verlauf der IK relativ flach. Am Ende der Äußerung gibt es jedoch einen wichtigen Unterschied zwischen beiden: die IK in der manipulierten Äußerung weist einen steilen Anstieg auf. Dieser beginnt jedoch sehr spät, was sich daraus erklärt, dass sie Probandin einen Korrekturversuch unternimmt, sobald sie das Fragezeichen wahrnimmt. ${ }^{149}$ Anhand dieses Beispiels konnte gezeigt werden, dass Fragezeichen durchaus als Kontextualisierungshinweise einen Einfluss auf die Intonation schriftlich realisierter Äußerungen nehmen können. Dies passiert aber vor allem dann, wenn es keine lexikalischen Hinweise auf die Äußerung als Frage hin gibt bzw. dies aus dem Kontext nicht zu erschließen ist. Die These in Bezug auf die Fragezeichen kann weder verworfen noch bestätigt werden.

Da Ausrufe- und Fragezeichen häufig bzw. überhaupt nicht als Signal der Intonation in den Interpretationsvorgang einer Äußerung einfließen, muss der Leser bereits beim Vorgang des Lesens eine bestimmte Intonation mitinterpretieren. Die Wahl der Intonation wird durch den Kontext der Äußerung und bestimmte Signalwörter vermittelt. Dennoch können Satzzeichen in einigen Fällen eine Rolle als KH spielen, wie an dem letzten Beispiel gezeigt werden

149 Die Probandin fragte direkt nach dem Vorlesen der Äußerung, die die letzte Äußerung des Konversationsbeispiels darstellt, weswegen dort ein Fragezeichen stand, da dieses für sie völlig unerwartet war und inhaltlich nicht passte, da nichts auf die Äußerung als Frage hindeutete. 
konnte.

Einen anderen potenziellen Kontextualisierungshinweis stellen Punkte dar. Während der satzabschließende Punkt eine unmarkierte Intonation anzeigt und daher nicht immer von den Schreibern gesetzt wird, erfüllt die Verwendung von zwei, drei oder mehr Punkten eine andere Funktion. Stehen mehrere Punkte, wie z.B. in (35) $\mathrm{T}_{1} \mathrm{M}_{1,1}$ „E் ĖE் $\dot{E} \dot{E} \dot{E}$.... ${ }^{150}$, so handelt es sich um Sprechpausen. Obwohl für den Schreiber keine Notwendigkeit besteht, Sprechpausen anzugeben, da ein Turn in schriftlich realisierten Konversationen nicht unterbrochen werden kann, wird dies häufig getan. Möglicherweise soll dadurch eine intonatorische Pause in Form z.B. eines Zögerns wiedergeben werden. In den Experimenten zeigte sich, dass solche Pausen nicht von allen Probandinnen realisiert werden: während die Zeitstruktur der Abbildung 28 in diesem Abschnitt oder der Abbildungen 43-46 im Abschnitt „Emoticons“ Sprechpausen an der Stelle, an der mehrere Punkte in der Äußerung standen, erkennen lässt, trifft dies auf die Abbildungen 41, 42, 47 und 48 nicht zu. Das Ergebnis bezüglich der Punkte ist somit nicht eindeutig.

Ein weiteres Mittel zur Wiedergabe prosodischer Elemente ist die Großschreibung ${ }^{151}$ einzelner Wortformen bis hin zu ganzen Äußerungen, z.B. (36) $\mathrm{T}_{1} \mathrm{M}_{1,3}$, (38) $\mathrm{T}_{1} \mathrm{M}_{1,1}$ oder (41) $\mathrm{T}_{1} \mathrm{M}_{1}$. Der Schreiber benutzt dieses Mittel zur intonatorischen Hervorhebung einer Wortform ((38) $\left.\mathrm{T}_{2} \mathrm{M}_{1,8}\right)$ oder einer ganzen Äußerung ((38) $\left.\mathrm{T}_{1} \mathrm{M}_{1,1}\right)$. Die These ist, dass die Großschreibung vom Leser direkt an der Wortform wahrgenommen wird und einen Einfluss auf die (mitgedachte) Intonation ausübt. Damit fungiert sie als ein Kontextualisierungshinweis, der mit seinem Gegenstück in mündlicher Kommunikation nämlich der intonatorischen Hervorhebung (Prominenz) - vergleichbar ist. Um diese These zu prüfen, wurde ein Experiment durchgeführt, bei dem eine Probandin das Beispiel (44) laut vorlesen sollte, in dem im Original eine Äußerung mit einer großgeschriebenen Wortform kak vorkam $\left(\mathrm{T}_{1} \mathrm{M}_{4,1}\right)$. Die Probandin bekam zunächst die Originaläußerung mit der

\footnotetext{
${ }^{150}$ Hierbei handelt es sich um einen Diskursmarker. Für das Vorkommen von mehreren Punkten nach einem Diskursmarker gibt es noch weitere Beispiele, z.B.

$\mathrm{B}_{1} \quad \mathrm{~T}_{1} \mathrm{M}_{1} \quad$ silver: Хм...на месте богды я скорее всего поступил так же, хоть и выдал сам ему предупреждение. Не люблю когда стараются унизить...

${ }^{151}$ Im Gegensatz zu den Satzzeichen hat die Großschreibung mehrere Funktionen, von denen hier jedoch nur die Funktion als möglicher Kontextualisierungshinweis untersucht wird. Eine weitere Funktion erfüllt sie als strategisches Mittel, um die Aufmerksamkeit der Leser auf die entsprechende Äußerung zu ziehen.
} 
Großschreibung (vgl. (44)). Bei der Wiederholung des Experiments über ein Jahr später, erhielt sie den gleichen Text mit der Äußerung ohne die Großschreibung (vgl. (44)'). Die erste Abbildung zeigt die Intonationskurve der Probandin mit der Äußerung im Original und die zweite ihre Intonationskurve für die Äußerung ohne die Großschreibung.

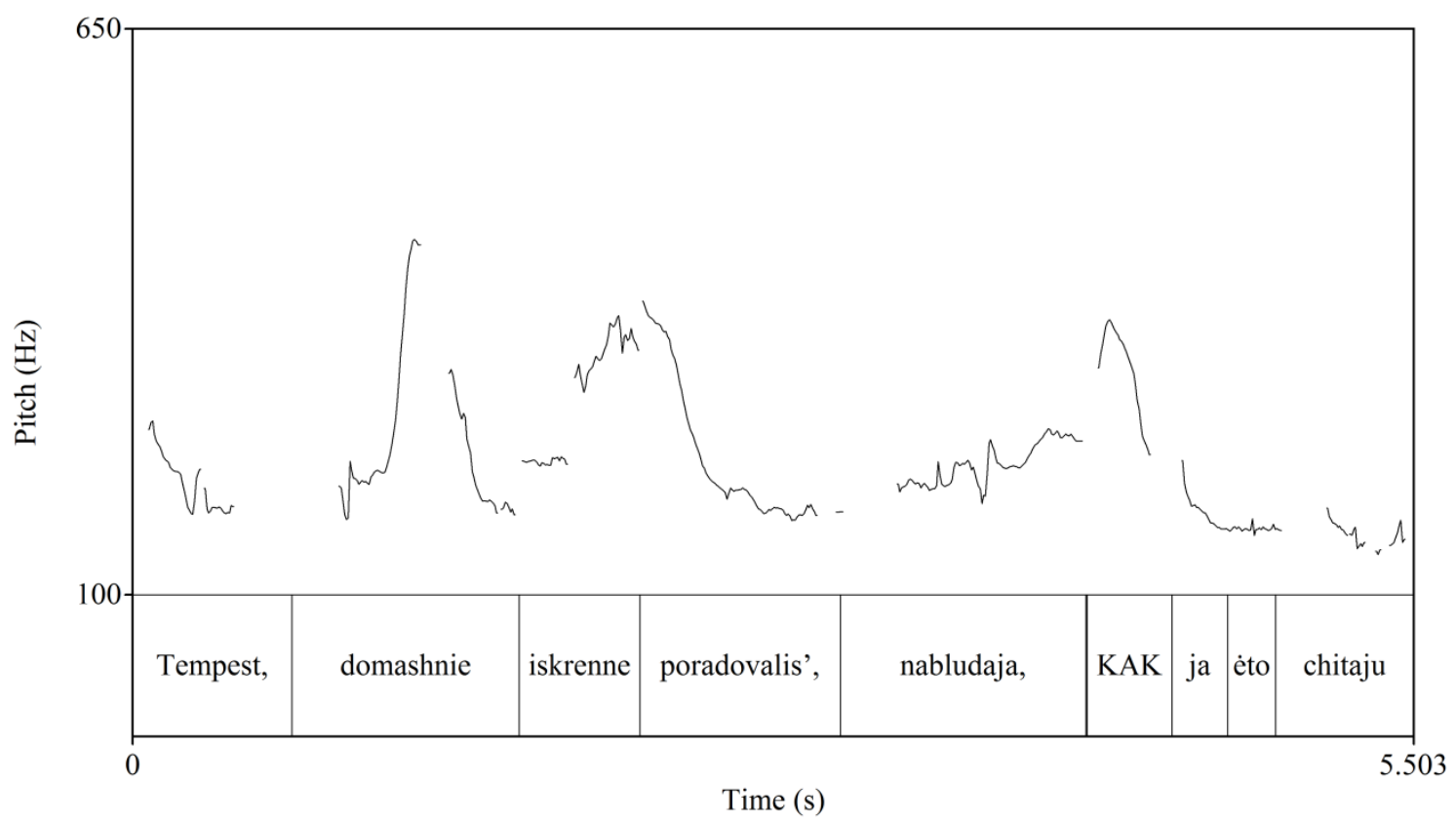

Abb. 26 Probandin 1 mit Großschreibung

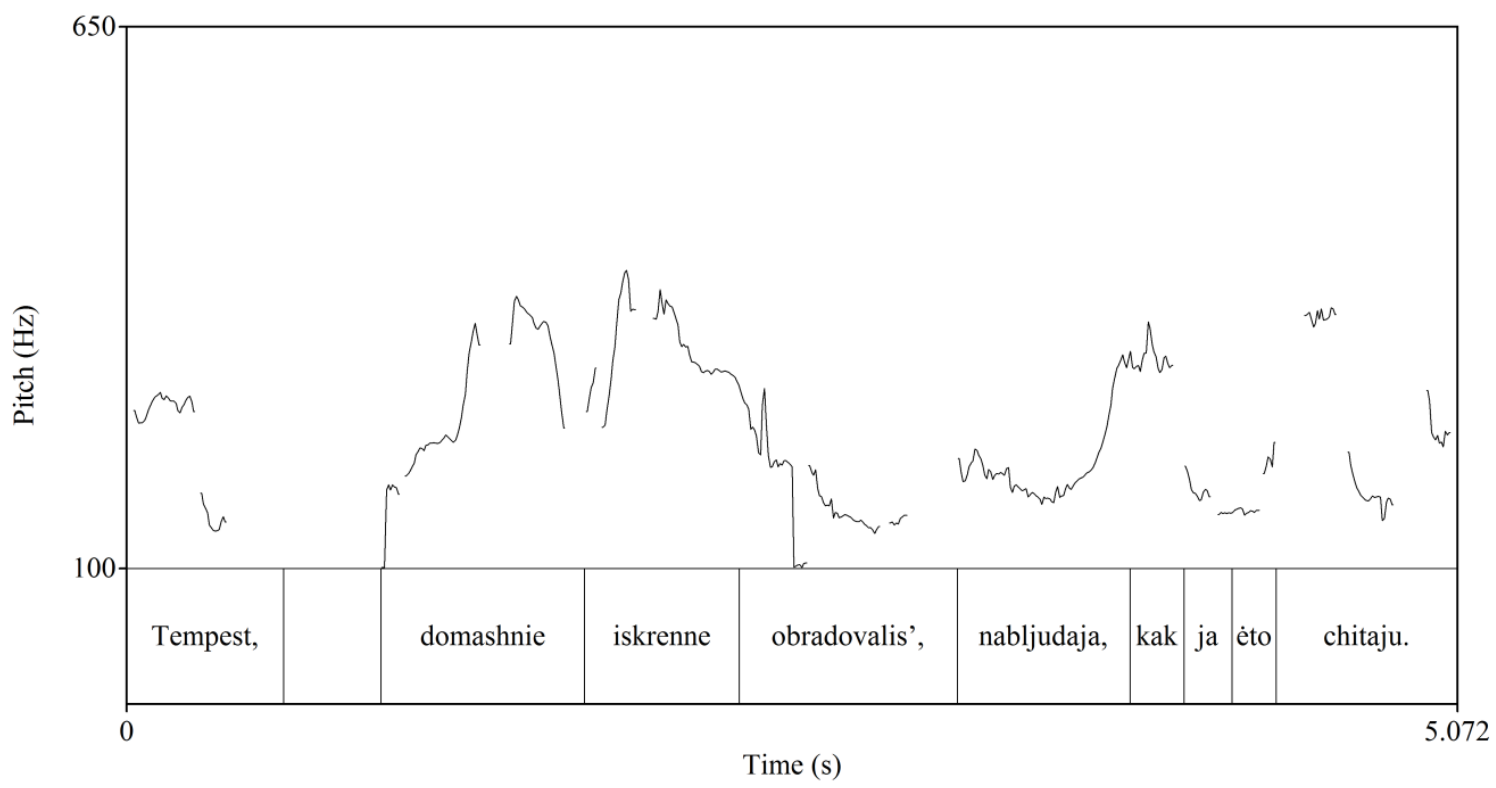

Abb. 27 Probandin 1 ohne Großschreibung

In der ersten Abbildung sieht man die deutliche Hervorhebung der Wortform kak. Die IK steigt bei kak steil an und fällt danach, ohne noch einmal zu steigen, d.h. dass die 
Intonationskurve nach der Hervorhebung von kak flach verläuft. In der zweiten Abbildung ist ein steiler Anstieg und Fall der IK unmittelbar vor kak zu erkennen, nach kak steigt und fällt die IK zum Ende der Äußerung hin erneut. Die Probandin hebt das nicht großgeschriebene kak somit nicht besonders hervor. Stattdessen liegt der Fokus der Äußerung in der Abb. 27 auf chitaju. Das Experiment zeigt, dass die Großschreibung als Hinweis von den Lesern erkannt und intonatorisch umgesetzt wird. Die Großschreibung wird dabei im Gegensatz zu Satzzeichen parallel zur Wortform wahrgenommen. Das Hervorheben durch die Großschreibung ist somit mit dem entsprechenden mündlichen Kontextualisierungshinweis der intonatorischen Prominenz vergleichbar.

Als weiteres Mittel zur Wiedergabe prosodischer Eigenschaften kommen Mehrfachvorkommen von graphischen Zeichen für Laute, vor allem Vokale, ${ }^{152}$ als potenzielle Kontextualisierungshinweise in Frage. Beispiele für solche Mehrfachvorkommen finden sich in (31) $\mathrm{T}_{1} \mathrm{M}_{6,1}$ (,aaaaa') oder (44) $\mathrm{T}_{1} \mathrm{M}_{3,1}$ (,Aaaaa'). In allen Fällen wird durch das Mehrfachvorkommen eine Dehnung der jeweiligen Vokale dargestellt. Eine besondere Funktion kommt dem Mehrfachvorkommen dabei vor allem in den Fällen zu, wo diese benutzt wird, um eine Verwechslung mit einer Konjunktion zu verhindern, vgl. (31) $\mathrm{T}_{1} \mathrm{M}_{6,1}$ und (44) $\mathrm{T}_{1} \mathrm{M}_{3,1}$. Beim Mehrfachvorkommen von graphischen Zeichen für Laute gilt die gleiche These wie bei der Großschreibung: das Mehrfachvorkommen wird von den Vorlesern an der Wortform selbst wahrgenommen und als solche lautlich realisiert, wie an der IK der folgenden Abbildung der Mitteilung $\mathrm{T}_{1} \mathrm{M}_{3}$ in (44)", ,' zu sehen ist:

\footnotetext{
${ }^{152}$ Beispiele mit Konsonantenwiederholungen kommen deutlich seltener vor, vgl. die Wiederholung des Liquids in glamurrrnaja in der folgenden Äußerung:

$\mathrm{B}_{1} \quad \mathrm{~T}_{1} \mathrm{M}_{1}$ Vesnushka: [...] ${ }_{3}$ По сему поставила форумке розовый дизайнчик и сидю тут такая гламуррнная, $\Theta_{\|} \Theta$,
} 


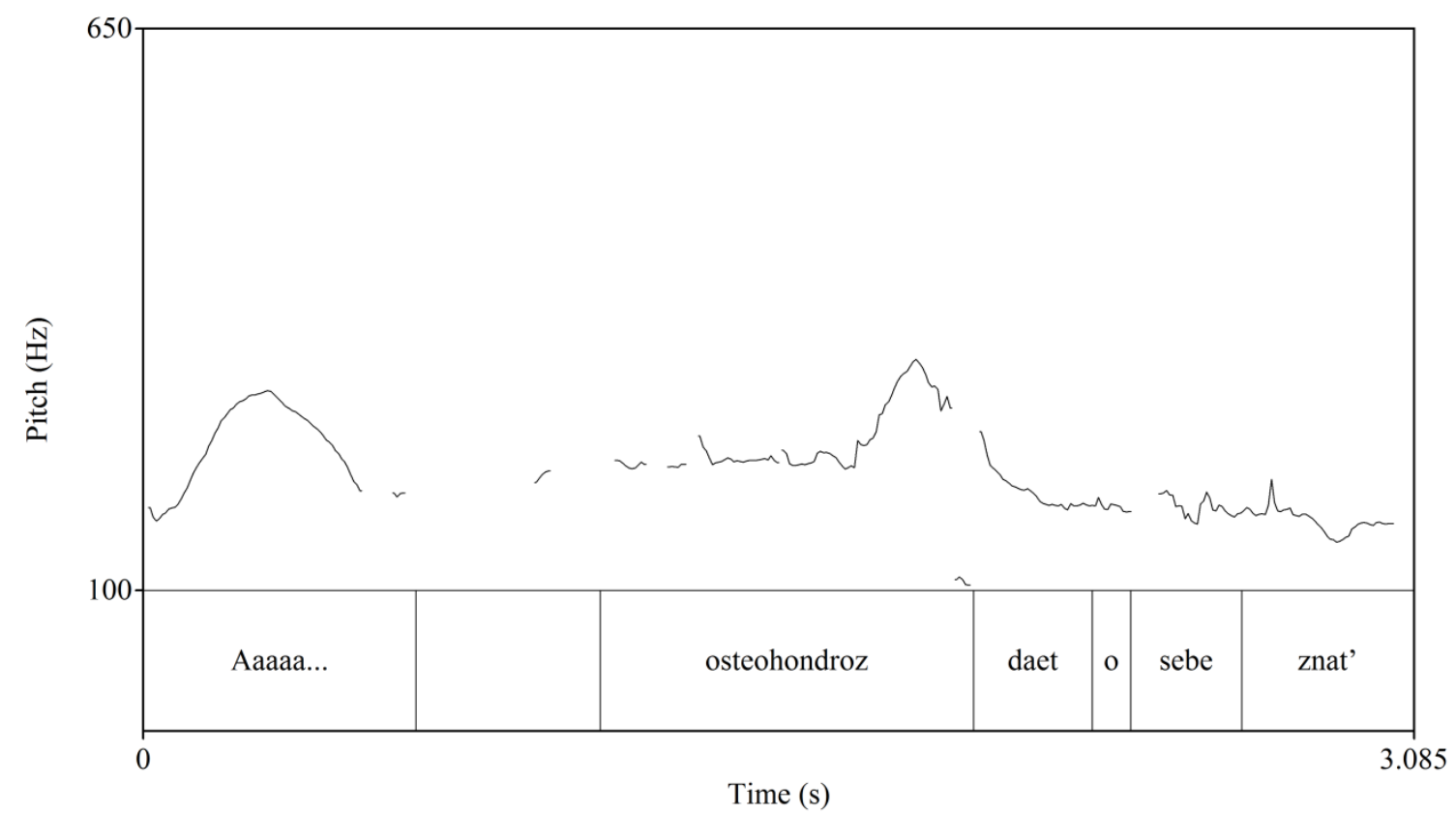

Abb. 28 Probandin 1 mit Mehrfachvorkommen von graphischen Zeichen für Laute

In der Abbildung sieht man zu Beginn der Äußerung eine steigende und fallende IK mit einer anschließenden Pause, die in der Zeitstruktur den drei Punkten der graphischen Form entspricht. Anstatt einen kurzen Laut zu realisieren, dehnt die Probandin den Vokal /a/: die zeitliche Dehnung beträgt 0,66 sek. Die Zeit, die die Probandin für die Realisierung benötigt, deutet darauf hin, dass sie das Mehrfachvorkommen von Zeichen für Laute als Kontextualisierungshinweis registriert und sofort lautlich umgesetzt hat. Wie bei der Großschreibung wird die angenommene These durch das Experiment bestätigt.

\subsubsection{Farbliche Markierung}

Farbliche Markierung bildet unter den potenziellen Kontextualisierungshinweisen eine Ausnahme, da sie keine Entsprechung in mündlicher Kommunikation besitzt. Da farbliche Markierung in schriftlich realisierten Konversationen direkt an der Wortform vom Leser wahrgenommen werden kann, wird hier die gleiche These angenommen wie für Großschreibung und das Mehrfachvorkommen graphischer Zeichen für Laute. In Internetkonversationen in den untersuchten Foren kann Farbe unterschiedliche Funktionen besitzen: einmal als potenzieller Kontextualisierungshinweis und einmal als Strategiemittel. ${ }^{153}$

${ }^{153}$ Die Farbe als Strategiemittel dient dem Zweck aufzufallen und auf diese Weise die Aufmerksamkeit des 
Als potenzieller KH liegt farbliche Markierung in (14) und (32) vor. ${ }^{154}$ Die Farbe kann aber nur dann als Kontextualisierungshinweis fungieren, wenn sie an ein bestimmtes Hintergrundwissen gekoppelt ist. Dies ist in (14) und (32) der Fall, wo ihre Bedeutung im mikrogruppeninternen Hintergrundwissen gespeichert ist. Durch die Koppelung an Hintergrundwissen wird die Farbe gleichzeitig konventionalisiert. Im Zusammenspiel mit dem entsprechenden Hintergrundwissen kann die Farbe eine Wortform oder eine ganze Äußerung in einen gewünschten Kontext setzen und so die Interpretation des Lesers in die vom Schreiber beabsichtigte Richtung leiten, wie bogda dies mit seiner Äußerung $\mathrm{T}_{1} \mathrm{M}_{4}$ in (14) tut. Hätte für DragOn als Leser die Farbe nicht als KH fungiert, wäre seine Reaktion anders ausgefallen. Dies bestätigt die angenommene These, Farbe komme als Kontextualisierungshinweis in Frage und werde vom Leser bereits an der Wortform wahrgenommen.

\subsubsection{Hinweise auf Handlungen, Gestik und Mimik}

Für die Wiedergabe von Handlungen, Gestiken und Mimiken stehen den Schreibern verschiedene Möglichkeiten zur Verfügung. Dazu gehören Akronyme, Emoticons und Regieanweisungen, die alle als potenzielle Kontextualisierungshinweise in Frage kommen. Der Schwerpunkt in diesem Abschnitt wird auf Emoticons und Regieanweisungen gelegt.

\subsubsection{Akronyme}

In Konversationen in Internetthreads lässt sich eine Vielzahl von Akronymen finden. ${ }^{155}$ Diese müssen konventionalisiert und bekannt sein, damit sie vom Leser verstanden werden. Die geläufigsten Akronyme in den untersuchten Forenthreads waren gygy, gyyy, $Y y^{156}$ oder hyyy $y^{157}$

Lesers auf sich ziehen. In solchen Fällen hat sie keine weitere Bedeutung, vgl. (24) $T_{1} M_{1}$.

${ }^{154}$ Im ersten Thread hat Farbe hingegen keine Funktion als Kontextualisierungshinweis.

155 Nach der Bedeutung von Akronymen kann man auf der folgenden Internetseite suchen: http://www.acronymfinder.com/. Eine Liste der in der Internetkommunikation gängigen Akronyme bietet auch Wikipedia: https://de.wikipedia.org/wiki/Liste_von_Abk\%C3\%BCrzungen_\%28Netzjargon\%29. Wie in der Internetkommunikation vieler anderer Länder, benutzen auch russischsprachige User vor allem englischsprachige Akronyme. So gibt es viele Seiten, die Akronyme samt Bedeutung auflisten (z.B. http://skyeng.ru/articles/sms-sokrashcheniya-4u-populyarnye-akronimy-ery-tekstovyh-soobshchenij-i-internetchatov), aber auch Diskussionsseiten über die Verwendung dieser und anderer Phänomene der Internetsprache (z.B. http://www.yaplakal.com/forum7/topic779350.html).

${ }^{156}$ Es handelt sich bei diesen Formen um die (entstellte) Wiedergabe dessen, was man sich unter der Aussprache der englischsprachigen Akronyme $g$ und $g g$, die soviel wie grin bzw. great grin bedeuten, vorstellt. 
in (7) $\mathrm{T}_{3} \mathrm{M}_{2}$, (9) $\mathrm{T}_{3} \mathrm{M}_{2}$, (18) $\mathrm{T}_{3} \mathrm{M}_{5}$, (24) $\mathrm{T}_{5} \mathrm{M}_{1}$, (33) $\mathrm{T}_{1} \mathrm{M}_{4}$ oder lol ${ }^{158}$ in (39) $\mathrm{T}_{1} \mathrm{M}_{2}$. Es gibt mehrere Sorten von Akronymen, wovon hier zwei betrachtet werden: diejenigen, die für Handlungen stehen, z.B. lol, und die, die Mimik betreffen, z.B. gg. Aus meiner Sicht kann diesen potenziell eine Rolle als Kontextualisierungshinweise zukommen. Beispielsweise sieht man das in (24) $\mathrm{T}_{5} \mathrm{M}_{1}$, wo der Schreiber Black Santa seine Äußerung mit gy einleitet: mit Hilfe das Akronyms weiß der Leser, dass der Schreiber möchte, dass seine Äußerung(en) als lustig empfunden wird (werden). $\mathrm{Zu}$ der These, Akronyme könnten als potenzielle Kontextualisierungshinweise die Interpretation beeinflussen, passt, dass viele Akronyme vor der eigentlichen Äußerung zu finden sind und sie einleiten. Diese Präferenz wurde anhand des Vorkommens des Akronyms $g g$ und davon abstammenden Formen (ohne hy) im ersten Thread untersucht: in 1081 Redebeiträgen finden sich 12 Mal gy bzw. gygy und 8 Mal yy (die Anzahl der wiederholten Vokale kann schwanken). Von den $12 \mathrm{Mal}$, in denen gy vorkam, stand es neun Mal vor der Äußerung und folgte dieser drei Mal. Das Akronym y fand sich sieben Mal vor der Äußerung und kein einziges Mal nach einer; einmal bildete es die Äußerung selbst, was zeigt, dass Akronyme als selbstständige Reaktion auftauchen können, d.h. die Mitteilung muss keinen weiteren lexikalischen Inhalt haben, wie in (39) und (40) zu sehen ist. Für die Schreiber stellt dies eine ökonomische Möglichkeit der Reaktion dar. In solchen Fällen handelt es sich bei dem Akronym aber nicht mehr um einen Kontextualisierungshinweis; stattdessen ist dies eine eigenständige non-verbale Reaktion, wie sie auch in face-to-face-Kommunikation vorkommen kann ${ }^{159}$. Im Gegensatz zu Emoticons und Großschreibung wurde der Einfluss der Akronyme auf die Intonation nicht durch Experimente untersucht. Es lässt sich jedoch vermuten, dass der Einfluss vergleichbar ist mit dem von Regieanweisungen und vor der Äußerung stehenden Emoticons.

\section{$\underline{\text { 4.2.3.2 Emoticons }}^{160}$}

Dass Emoticons eine wichtige Rolle in der Internetkommunikation zukommt, zeigt ihr häufiger Gebrauch: so enthielt in den vorgestellten Beispielen des ersten Forums

\footnotetext{
${ }^{157}$ Hier gilt das Gleiche wie für gy, gygy oder yy. Im Gegensatz zu gy usw., könnte dieses Wort jedoch von hihi stammen und würde so das Geräusch einer bestimmten Art des Lachens wiedergeben.

${ }_{158}^{158}$ Abkürzung von laughing out loud.

${ }^{159}$ Ein simples Beispiel hierfür ist, wenn Person A einen Witz erzählt und Person B lacht, ohne sich weiter zu dem Witz zu äußern.

${ }^{160}$ Es werden keine japanischen oder koreanischen Emoticons behandelt, da diese in den beiden untersuchten Threads nicht vorgekommen sind.
} 
„Alohomora“ fast jeder Beitrag mindestens ein Emoticon. Die Emoticons sind dabei ein typischer Bestandteil der Internetkommunikation, jedoch nicht früherer schriftlicher Kommunikationsarten: „What is interesting to the linguist, of course, is why these novelties [Emoticons usw.; Anm.d.Vf.] have turned up now. Written language has always been ambigious, in its omission of facial expression, and in its inability to express all the intonational and other prosodic features of speech. Why did no one ever introduce smileys there? The answer must be something to do with the immediacy of Net interaction, its closeness to speech. In traditional writing, there is time to develop phrasing which makes personal attitudes clear [...]. A rapidly constructed Net message, lacking the usual courtesies, can easily appear abrupt or rude. A smile defuses the situation." (Crystal (2001, 38f.)). Ob der gleiche Grund für die Kommunikation in Foren angenommen werden kann, ist fraglich, da die Schreiber hier im Gegensatz zu Instant-Messaging-Diensten oder Chaträumen länger über das Formulieren ihre Mitteilung nachdenken können und die Leser entsprechend mehr Zeit zum Interpretieren haben, ohne, dass eine längere Pause vom Gesprächspartner als negativ gewertet würde. Eine schnelle, zeitlich unmittelbar folgende Antwort wird in Foren grundsätzlich nicht erwartet. Es muss demnach eine andere Erklärung für die Verwendung von Emoticons in der Forumkommunikation geben. Ein möglicher Erklärungsansatz ist, dass User Emoticons aus der Chatkommunikation in die Threadkommunikation automatisch übertragen, da viele Foren auch Chaträume anbieten; User können sich so gleichzeitig sowohl in den Chaträumen als auch in den einzelnen Threads unterhalten. Ein weiterer Erklärungsansatz ist, dass die Verwendung von Emoticons eine Konvention ist und in manchen Gruppen sogar einen Bestandteil der Gruppenidentität bildet. Der wichtigste Erklärungsansatz ist aber, dass Schreiber versuchen mit Emoticons in der rein schriftlichen Interaktion bis zu einem gewissen Grad Mimik und sogar Gestik (vor allem in animierten Emoticons) wiederzugeben. Damit könnten Emoticons als potenzielle KH Hinweise auf die Realisierung einer sprachlichen Äußerung geben: bei einem lächelnden oder grinsenden Emoticon könnte sich der Leser vorstellen, wie derjenige, der sich gerade äußert, lächelt bzw. grinst, welche Intonation die Äußerung hat usw.

Mit der Rolle von Emoticons in der zwischenmenschlichen, medienbasierten Kommunikation beschäftigen sich mehrere Studien. So wurde in einer australischen neurowissenschaftlichen Studie mit 20 Probanden untersucht, wie Emoticons im Vergleich zu Gesichtern im Gehirn verarbeitet werden. „Participants were shown pictures of canonically arranged and inverted faces, emoticons in the form ":-)" and nonrepresentational character combinations (henceforth 
known as characters) in the form "*/.”. [... Sixty stimuli in each category were shown along with 30 pictures of flowers which were always presented upright. Faces were half male, half female and all showed a happy expression.“ (Churches et al. $(2014,198))$. Das Ergebnis war, dass bei kanonisch ausgerichteten Smileys im Gehirn des Lesers die gleichen Areale aktiviert werden wie bei der Verarbeitung von realen Gesichtern. Bei invertierten Smileys hingegen wurden die Verarbeitungsareale nicht so stark aktiviert. Daraus schließen die Verfasser der Studie, dass , ,...] the encoding of the smiley emoticon as a face occurs through configural processes rather than featural processes.“ (Churches et al. $(2014,197))$ „The results show that while faces are recognized as faces when canonically arranged or inverted because both configural and featural mechanisms are able to process the image, emoticons are perceived as faces only through configural processes. When the configuration is disrupted (through a process such as inversion), the emoticon no longer carries its meaning as a face." (Churches et al. (2014, 201f.)). Die Verarbeitung eines Smileys wie ein Gesicht führt auch zu dem Schluss, dass Emoticons wie Mimik eine Funktion als Kontextualisierungshinweise erfüllen könnten. Eine weitere Studie befasst sich mit Ironie in der face-to face und der computerbasierten Kommunikation. Dabei sollten 40 Partizipantenpaare zwei Konversationsaufgaben lösen, die absichtlich Ironie hervorrufen sollten. Das Ergebnis bezüglich der Emoticons war, dass sie als Hinweise auf Ironie bzw. Humor zwar benutzt wurden, jedoch viel seltener im Vergleich zu anderen Hinweisen (vgl. Hancock $(2004,460)$ ). Eine weitere Studie befasst sich speziell mit dem Thema, wie Emoticons in Onlinenachrichten interpretiert werden. Die Partizipanten 105 Schüler einer Schule - sollten E-Mail-Nachrichten lesen, die im Vorfeld manipuliert wurden: „The sender of the message was manipulated (stranger, good friend). The valence of the messages varied (positive, negative, neutral). The message was complemented with one of the three emoticons (smile, frown, wink) or left blank in the case of the control condition." (Derks et al. (2008, 383)). Anschließend sollten sie im ersten Teil der Studie einen Fragebogen zu jeder Nachricht ausfüllen, der folgende Fragen enthielt:

„How do you feel after reading this message? (1 =very negative, $7=$ very positive)

How positively do you rate the message? $(1=$ very negative, $7=v e r y$ positive $)$

How familiar was the sender of the message? ( 1 =very unfamiliar, 7 =very familiar $)$

How does the sender evaluate your performance as presenter? $(1$ =very negative, $7=$ very positive)

How ambiguous was the message? ( 1 =very unambiguous, 7 =very ambiguous $)$

How serious was the message? ( 1 =not serious at all, 7 =very serious $)$

How easy was it to understand the message? (1=very easy, 7 =very difficult $)$ 
How happy was the writer of the message? (1=very sad, 7 =very happy)

How sincere was the writer of the message? (1=very insincere, $7=v e r y$ sincere $)$

On a scale from 1 to 100 , with 1 being the least and 100 being the most, how much happiness did the writer of the message portray?

On a scale from 1 to 100, with 1 being the least and 100 being the most, how much sarcasm did the writer of the message portray?

On a scale from 1 to 100 , with 1 being the least and 100 being the most, how much humor did the writer of the message portray?" (Derks et al. $(2008,383)$ )

Im zweiten Teil der Studie wurde speziell nach dem Zweck der benutzten Emoticons gefragt: „For example, participants were asked to rate how much they agreed with the following statement: "When the writer of a message uses the emoticon 'smile', he/she wants to express his/her emotions." The motives measured for each emoticon were to express emotion, to strengthen the message, to manipulate the interaction partner, to express humor, to put a remark into perspective, to regulate the interaction, and to express irony." (Derks et al. (2008, 383)). Die Studie ergab, dass ,[...] emoticons are useful in strengthening the intensity of a message. A positive message with a smile is rated more positively than a positive pure message, and a negative message with a supporting frown is more negative than a negative pure message. The same effects are found for the amount of happiness the writer of the message portrayed. This is in line with the power of nonverbal cues to intensify a verbal message in F2F communication (Lee \& Wagner, 2002). [...] Negative verbal messages accompanied with a smile were interpreted more positively than a negative pure message but less positively than a positive pure message. Positive verbal messages accompanied with a frown were rated less positively than positive pure messages and more positively than negative pure messages. This indicates that online verbal messages have more influence than the "nonverbal" part of the message, the emoticon. Emoticons do not have the strength to turn around the valence of the verbal message. [...] A negative message accompanied by a wink emoticon indeed conveys less negativity than a negative pure message. However, the positivity of a positive verbal message with a wink was equal to a positive verbal message alone. [...] All messages accompanied with an emoticon with a different valence than the verbal message conveyed greater sarcasm than pure messages.“ (Derks et al. (2008, 385f.)). Die Verfasser der Studie kommen zu dem Schluss, dass ,[...] emoticons do have a certain impact on message interpretation and that they can serve some of the same functions as actual nonverbal behavior. [...] The overall conclusion of the studies presented in this article is that 
emoticons can serve as useful nonverbal surrogates for impact on message interpretation." (Derks et al. (2008, 386f.)). ${ }^{161}$

Trotz dieser und weiterer Studien ist der Einfluss der Emoticons auf die Interpretation einer schriftlich realisierten Äußerung nicht eindeutig geklärt. Daher sollte dieser Fragestellung in mehreren Experimenten nachgegangen werden, wobei entgegen der obigen Ergebnisse meine These lautet, dass Emoticons bezüglich ihres Einflusses auf die Interpretation nicht mit Kontextualisierungshinweisen wie Mimik oder Gestik in mündlicher Kommunikation vergleichbar sind.

Bei der Untersuchung der Emoticons in beiden Threads ließen sich drei Arten ausmachen: einfache, komplexe und animierte. Einfache Emoticons bestehen aus Klammern, Satzzeichen, teilweise Buchstaben und Ziffern - allem, was sich auf einer Tastatur wiederfindet. Beispiele hierfür sind (35) $\mathrm{T}_{2} \mathrm{M}_{1,3}$ oder (40) $\mathrm{T}_{1} \mathrm{M}_{5}$. Einfache Emoticons können aus mehreren Zeichen bestehen, z.B. :-), müssen es aber nicht, denn aus ökonomischen Gründen wird häufig nur eine Art Zeichen, z.B. eine oder mehrere Klammern, gesetzt, vgl. (40) $T_{1} M_{2}$ oder (44) $\mathrm{T}_{1} \mathrm{M}_{3} .{ }^{162}$ Je einfacher das Emoticon, desto eher muss es konventionalisiert sein, um korrekt verstanden zu werden, d.h. der Leser muss wissen, dass es sich beispielsweise bei einer nach rechts gebogenen Klammer „)“ um ein Lächeln handelt. Als komplexe Emoticons bezeichne ich die bekannten runden Gesichter, die z.B. in den Beispielen (36) $\mathrm{T}_{1} \mathrm{M}_{1}$, (37) $\mathrm{T}_{1} \mathrm{M}_{1}$, (38) $\mathrm{T}_{1} \mathrm{M}_{1}$ und $\mathrm{T}_{2} \mathrm{M}_{1}$ oder (43) $\mathrm{T}_{2} \mathrm{M}_{2}$ vorkommen. ${ }^{163}$ Komplexe Emoticons können zwar deutlich mehr darstellen als einfache, stören aber auch deutlich den Schreibfluss, weil sie nicht schnell getippt werden können. ${ }^{164}$ Da die Mimik erkennbar ist, kann die Bedeutung dieser Emoticons prinzipiell auch ohne Kenntnis der Konventionen verstanden werden. Unter animierten

\footnotetext{
${ }^{161}$ Wie die Verfasser selbst schreiben, ist die so durchgeführte Studie insofern unzureichend, als dass u.a. die Partizipanten nicht interagiert haben, sondern sich vorstellen sollten, eine E-Mail zu erhalten (vgl. Derks et al. (2008, 386f.)). Als weiteren Kritikpunkt kann man eine gewisse Vagheit von Begriffen wie „positive“ und ,negative“ anführen.

162 Wie oben bereits erwähnt, widerspricht dies jedoch nicht dem 'save a keystroke'-Prinzip, da für das mehrfache Schreiben des gleichen Zeichens der Schreiber einfach die Tatenkombination länger gedrückt halten muss, es für ihn also keinen Mehraufwand bedeutet.

${ }^{163}$ Auch mit auf der Tastatur vorhandenen Zeichen können aufwendigere Emoticons geschaffen werden, z.B.
}

$\mathrm{B}_{1} \quad \mathrm{~T}_{1} \mathrm{M}_{1} \quad$ patron: всем привет давно я тут не был
$(1 /$ l)
$(=' \cdot=)$
$\mathrm{E}[:]|||| \mid[:] 3$
(")_(")

164 Außer Acht gelassen werden diejenigen Programme und Messaging-Dienste, die bestimmte einfache Emoticons automatisch in komplexe umwandeln. 
Emoticons fasse ich solche Emoticons, die sich bewegen und mit denen vor allem Gesten und Handlungen dargestellt werden. ${ }^{165}$ Diese drei Arten werden in Abhängigkeit davon, ob sie mit einer Äußerung kombiniert werden (Gruppe 1) oder eigenständige Reaktionen ohne weiteres verbales Material in der Mitteilung sind (Gruppe 2), weiter in Gruppen unterteilt. Ein Beispiel für die zweite Gruppe ist (41) $\mathrm{T}_{1} \mathrm{M}_{2}$. Statt sprachlichem Material findet der Leser nur ein Emoticon vor. Grundsätzlich ist in solchen Fällen das Vorkommen aller drei Arten von Emoticons möglich. Eine Subgruppe innerhalb der zweiten Gruppe stellen Emoticons dar, die in Kombination mit wenig lexikalischem Material eine Geschichte erzählen, z.B. (42) $\mathrm{T}_{1} \mathrm{M}_{1}$ oder $\mathrm{T}_{1} \mathrm{M}_{3}$. An diesem Beispiel ist ein enger Zusammenhang zwischen Emoticons und Hintergrundwissen $\mathrm{zu}$ sehen. Wie wichtig das Hintergrundwissen für das korrekte Verständnis ist, zeigt sich bei dem Versuch, die Reaktion von Tempest in (42) $\mathrm{T}_{1} \mathrm{M}_{2}$ korrekt zu interpretieren. Das erste Emoticon stellt zwei Personen - eine weibliche und eine männliche - dar. Aus der Mitteilung von Black Santa in (42) $\mathrm{T}_{1} \mathrm{M}_{1}$, die den Kontext darstellt, ist ersichtlich, dass es sich um die User Lunko und Blejz handeln soll. Der Leser muss nun auf sein mikrogruppeninternes Hintergrundwissen zurückgreifen, um zu wissen, um welche User es sich handelt, da sie in diesem Thread unter anderen Namen auftauchen (Lunko ist Ksanka und Blejz ist Black Santa selbst) und in welcher Beziehung sie zueinander stehen. Weiter muss der Leser auch Hintergrundwissen bezüglich der engen Beziehung zwischen Tempest und Lunko/Ksanka besitzen. ${ }^{166}$ Das zweite Emoticon soll einen Mann, das vierte die Entführung der Ehegattin, das fünfte den verärgerten Ehegatten und die letzten vier die erfolgreiche Jagd des Ehegatten auf den Pinguin als Entführer darstellen. Der Zusammenhang zwischen Entführer und Pinguin erschließt sich dem Leser nur, wenn er weiß, dass Black Santa auf Grund seines Avatars und der Bevorzugung von Pinguin-Emoticons die Spitznamen pingvalpingvin (Pinguin) trägt. Nur mit Hilfe des Hintergrundwissens gelingt dem Leser die folgende Interpretation der Mitteilung von Tempest: die Hochzeit zwischen Ksanka und BlackSanta wird nur stattfinden, wenn er sie entführt, aber dann wird Ksankas Ehepartner Tempest Jagd auf ihn machen und ihn erschießen. An diesem Beispiel ist zu sehen, dass solche Emoticons eine andere Funktion erfüllen und keine Kontextualisierungshinweise im

\footnotetext{
${ }^{165}$ Beispiele für animierte Emoticons können in der Dissertation nicht dargestellt werden, sind jedoch in den untersuchten Foren zu finden, z.B. http://forum.xakepok.net/showthread.php?t=2532\&page=6.

166 Tempest schreibt in einem vorherigen Beitrag:

$\mathrm{B}_{1} \mathrm{~T}_{1} \mathrm{M}_{1}$ Tempest: Любит ли кто-то Лунко так, как люблю ее я,?, Сонычка моя - я тобю обожаю,!, [...]
} 
Gumperz'schen Sinne sind. ${ }^{167}$

In die erste Gruppe fallen alle Emoticons, die vor oder hinter einer Äußerung stehen und somit als potenzielle Kontextualisierungshinweise in Frage kommen. Für die Untersuchung der Bedeutung der Emoticons als Kontextualisierungshinweise wurden mehrere Experimente mit Probanden durchgeführt. Die Experimente waren so aufgebaut, dass die Probanden die Aufgabe erhielten, mehrere Konversationsbeispiele aus den untersuchten Forenthreads laut vorzulesen. Die Beispiele waren dabei in sich geschlossen, d.h. die Probanden bekamen den gesamten Kontext zur Verfügung gestellt. Auf diese Weise wurde versucht sicherzustellen, dass die Äußerung auch so verstanden wurde, wie sie vom Schreiber intendiert war. Das Vorlesen wurde mit dem Diktiergerät zum Zwecke der späteren Analyse aufgenommen. Während einige Probanden die Konversationsbeispiele im Original (d.h. so wie sie in den Foren zu finden waren) erhielten, bekamen andere Beispiele in manipulierter Form. Die Manipulation wurde erreicht durch: Weglassen des bzw. der Emoticons, Verschieben des Emoticons an die Position vor der Äußerung oder durch das „Umkehren“ des jeweiligen fröhlichen Emoticons (z.B. $)$ in ein trauriges (z.B. $)$. Die Aufnahmen wurden daraufhin mit der Software Praat analysiert und zu Äußerungen Intonationskurven erstellt. Das gleiche Experiment wurde über ein Jahr später mit den gleichen Probanden und den gleichen Konversationsbeispielen wiederholt, dieses Mal erhielten jedoch die Probanden, die vorher die Äußerungen mit Emoticons vorlesen sollten, die Beispieltexte komplett ohne Emoticons und diejenigen, die die Beispiele im ersten Experiment ohne Emoticons hatten, die gleichen Beispiele mit den ursprünglichen oder manipulierten Emoticons. Auf diese Weise konnten die Intonationskurven eines einzigen Vorlesers einmal mit und einmal ohne Emoticon miteinander verglichen werden. Auf Grund des großen zeitlichen Abstands kann davon ausgegangen werden, dass die Probanden sich an die Beispieltexte aus dem ersten Experiment nicht mehr in allen Details erinnerten.

Wie oben bereits erwähnt, liegt dem Ansatz, Intonationskurven zu vergleichen, die Idee zu Grunde, dass bei identischer Interpretation sich auch die Intonationskurven ähneln müssen. Gerade Emoticons - sollten sie als potenzielle KH bis zu einem gewissen Grad Mimik und Emotion wiedergeben und die Äußerung in einen positiv bzw. negativ empfundenen Kontext

\footnotetext{
${ }^{167}$ Aus diesem Grund werden solche Emoticons in der vorliegenden Dissertation nicht weiter untersucht.
} 
setzen können - müssten die Intonation beeinflussen können. Die Basis hierfür bildet der Zusammenhang zwischen Intonation und Emotion: „Speech not only conveys the strictly linguistic content of sentences but also the expression of attitudes and emotions of the speaker. Prosody plays a role in this, which may result in adding information to the linguistic content and/or its modification. Pitch level, pitch range, and speech rate are known to be important prosodic cues for, among others, the expression of emotions and attitudes in speech (Williams \& Stevens, 1972; Cahn, 1990; Carlson, Granström \& Nord, 1992; Kitahara \& Tohkura, 1992; Vroomen, Collier \& Mozziconacci,1993).“ (Mozziconaci \& Hermes (1997, 154)). Dies belegen verschiedene Studien: „Results show that the phonological choice of contour is of primary importance for determining the emotion perceived [...].“ (Mozziconacci 2002)). Außer der Kontur selbst beeinflussen Emotionen auch weitere Merkmale der Intonation: „A large number of studies ${ }^{168}$ have investigated vocal correlates of emotional expressions [...]. A common finding in those studies is that portrayed emotions influence global descriptors of $\mathrm{F}^{169}$, such as average F0, F0 level or F0 range. Reviews in this field show that portrayed emotions also have an effect on other broad descriptors of intonation, in particular measures derived from the acoustic intensity contour and measures related to the relative duration of various speech segments.“ (Bänziger \& Scherer $(2005,254))$. Und weiter: „[...] intonation (or more specifically F0 fluctuations) seems to be affected to some extent by the emotional state of the speakers and appear to carry information that can be used by listeners to generate inferences about the emotional state of the speakers, more or less independently of the linguistic features of the expressions.“ (Bänziger \& Scherer (2005, 256)). ${ }^{170}$ Dabei ist gerade der Faktor pitch level ${ }^{171}$ besonders wichtig: „One of the determining

${ }^{168}$ Vgl. hierzu eine Auflistung bei Bänziger \& Scherer (2005, 254f.).

${ }^{169} F 0=$ Grundfrequenz. Für die FO-Kurve wird in der vorliegenden Arbeit der Begriff Intonationskurve synonym gebraucht.

${ }^{170}$ Der Einfluss verschiedener Emotionen auf verschiedene Aspekte der Intonation wird von Cook $(2004,98)$ in einer Tabelle folgendermaßen zusammengefasst:

\begin{tabular}{lllcrcr}
\hline \multirow{2}{*}{ Emotion } & \multicolumn{4}{c}{ Pitch } & & \\
\cline { 2 - 4 } & Level & Range & Variability & & Loudness & Tempo \\
\hline Happiness/joy & High & $?$ & Large & Loud & Fast \\
Confidence & High & $?$ & $?$ & Loud & Fast \\
Anger & High & Wide & Large & Loud & Fast \\
Fear & High & Wide & Large & ? & Fast \\
Indifference & Low & Narrow & Small & & $?$ & Fast \\
Contempt & Low & Wide & $?$ & Loud & Slow \\
Boredom & Low & Narrow & $?$ & Soft & Slow \\
Grief/sadness & Low & Narrow & Small & Soft & Slow \\
Evaluation & $?$ & $?$ & $?$ & Loud & $?$ \\
Activation & High & Wide & $?$ & Loud & Fast \\
Potency & $?$ & $?$ & $?$ & Loud & $?$ \\
\hline
\end{tabular}

Abb. ii Einfluss unterschiedlicher Emotionen auf die Intonation 
factors in forming intonation is the main pitch level at which it is located, in other words, the average level of pitch extracted that forms the prosodic contour. [...] A link established by many studies on prosody attributes the high pitch levels to drawing the attention of the listener, because it establishes a contrast, and to emotions, such as joy, anxiety, or fear. Medium pitch levels account for more neutral attitudes. However, low pitch levels are related to sober emotions: sadness, calmness, or security. ${ }^{172}$ Thus, emotions carrying a high level of activity, such as joy or fear, tend to be situated in the top end of the frequency spectrum of the speaker. Conversely, sadness and desire tend overall to be formed at the lower end." (Rodero (2011, e25f.). In einer Studie wurde bezüglich der pitch range ${ }^{173}$ festgestellt, dass sie bei der Emotion „happy“ $172 \mathrm{~Hz}$ gegenüber $86 \mathrm{~Hz}$ für die Emotion „neutral“ und $60 \mathrm{~Hz}$ für die Emotion „sad“ betragen hat (vgl. Jacob \& Myhili (2012, 582)). Dies entspricht auch anderen Befunden, dass die Emotion ,sadness“ einen deutlich niedrigeren Tonhöhenumfang hat als die Emotion „Joy“ bzw. „Happiness“. ${ }^{174 ; 175}$ Auf dieser Grundlage wurde bei den Experimenten die mittlere Grundfrequenz und der Tonhöhenumfang, die Praat angibt, in Äußerungen mit und ohne Emoticon verglichen. Sollten die Emoticons eine Rolle als KH spielen, erwartet man Unterschiede zwischen zwei gleichen Äußerungen (einmal mit und einmal ohne das jeweilige Emoticon) der gleichen Probandin in der mittleren Grundfrequenz und in dem Tonhöhenumfang: die Äußerung mit einem positiven, fröhlichen Emoticon sollte eine höhere mittlere Grundfrequenz und einen größeren Tonhöhenumfang haben, als diejenige ohne das Emoticon, die Äußerung mit einem negativen, traurigen Emoticon sollte eine niedrigere mittlere Grundfrequenz bzw. einen niedrigeren Tonhöhenumfang als die Äußerung ohne Emoticon haben.

Um zu entscheiden, welche durchschnittlichen pitch level-Werte für die einzelne Probandin als hoch, mittel und niedrig einzustufen sind ${ }^{176}$, wurde ein weiteres Experiment durchgeführt,

\footnotetext{
${ }^{171}$ Der pitch level ist die average fundamental frequency (vgl. van Bezooijen $(1984,32)$ ) und entspricht in der vorliegenden Arbeit der mean pitch. Für den Begriff pitch level wird in dieser Arbeit auch der Begriff mittlere Grundrequenz verwendet.

${ }^{172}$ Kritisch anzumerken ist die Tatsache, dass die hier aufgezählten Begriffe nicht immer eindeutig sind: wie drücken sich z.B. die Emotionen calmness oder security aus? Auch bleibt unklar, was mit „more neutral attitudes" gemeint ist.

${ }^{173}$ Für den Begriff pitch range wird in der vorliegenden Arbeit auch der Begriff Tonhöhenumfang verwendet.

${ }^{174} \mathrm{Vgl}$. Abb. $i i$ in der vorliegenden Dissertation sowie Tab. 3.6 bei van Bezooijen $(1984,33)$.

${ }^{175}$ Auch in der Musikwissenschaft konnten solche Erkenntnisse gewonnen werden: so haben bspw. die Emotionen Happiness (a) und Sadness (b) in Bezug auf pitch folgende Merkmale (vgl. Juslin \& Laukka (2004, 221, Table 1)):

(a) high pitch, much pitch variability, wide pitch range, ascending pitch;

(b) low pitch, narrow pitch range, descending pitch.

176 Für Frauen werden die Werte 160/200 Hz - 340/400 Hz als Durchschnitt angenommen: „Ученые подсчитали, что мужчины говорят на частоте 85-200 гц, а женщины - 160-340 гц. Это средний тон
} 
bei dem die Probandinnen gebeten wurden, den kurzen Satz „Ach ty negodjajka“ einmal als neutral (nejtral'no), einmal als fröhlich (radostno) und einmal als traurig (grustno) vorzulesen. Auf diese Weise konnten Richtwerte für die mittlere Grundfrequenz unterschiedlicher Emotionen in Hz-Werten festgestellt werden. Gleichzeitig wurden Werte für den Tonhöhenumfang festgehalten. Die Ergebnisse sind in den folgenden Tabellen zusammengefasst:

\begin{tabular}{|l|l|l|l|l|l|l|}
\hline \multicolumn{2}{|c|}{ Probanden } & $\begin{array}{l}1 . \\
\text { Probandin } \\
\text { Emotion }\end{array}$ & $\begin{array}{l}2 . \\
\text { Probandin }\end{array}$ & $\begin{array}{l}3 . \\
\text { Probandin }\end{array}$ & $\begin{array}{l}4 . \\
\text { Probandin }\end{array}$ & $\begin{array}{l}5 . \\
\text { Probandin }\end{array}$ \\
\hline $\begin{array}{l}\text { Fröhlich } \\
\text { (hoch) }\end{array}$ & $\begin{array}{l}\text { pitch level / } \\
\text { mittlere } \\
\text { Grundfrequenz }\end{array}$ & $274,94 \mathrm{~Hz}$ & $313,04 \mathrm{~Hz}$ & $274,02 \mathrm{~Hz}$ & $212,22 \mathrm{~Hz}$ & $246,35 \mathrm{~Hz}$ \\
\hline $\begin{array}{l}\text { Neutral } \\
\text { (mittel) }\end{array}$ & $\begin{array}{l}\text { pitch level / } \\
\text { mittlere } \\
\text { Grundfrequenz }\end{array}$ & $248,38 \mathrm{~Hz}$ & $204,61 \mathrm{~Hz}$ & $189,45 \mathrm{~Hz}$ & $180,63 \mathrm{~Hz}$ & $196,91 \mathrm{~Hz}$ \\
\hline $\begin{array}{l}\text { Traurig } \\
\text { (niedrig) }\end{array}$ & $\begin{array}{l}\text { pitch level / } \\
\text { mittlere } \\
\text { Grundfrequenz }\end{array}$ & $214,29 \mathrm{~Hz}$ & $187,76 \mathrm{~Hz}$ & $181,56 \mathrm{~Hz}$ & $166,64 \mathrm{~Hz}$ & $185,75 \mathrm{~Hz}$ \\
\hline
\end{tabular}

Abb. 29 Richtwerte für die mittlere Grundfrequenz der einzelnen Probandinnen in $\mathrm{Hz}$

\begin{tabular}{|l|l|l|l|l|l|l|}
\hline \multicolumn{2}{|c|}{ Probanden } & $\begin{array}{l}1 . \\
\text { Emotion }\end{array}$ & $\begin{array}{l}2 . \\
\text { Probandin }\end{array}$ & $\begin{array}{l}3 . \\
\text { Probandin }\end{array}$ & $\begin{array}{l}4 . \\
\text { Probandin }\end{array}$ & $\begin{array}{l}5 . \\
\text { Probandin }\end{array}$ \\
\hline $\begin{array}{l}\text { Fröhlich } \\
\text { (hoch) } \\
\text { Range / } \\
\text { Tonhöhen- } \\
\text { umfang }\end{array}$ & $383,2 \mathrm{~Hz}$ & $229,8 \mathrm{~Hz}$ & $419,5 \mathrm{~Hz}$ & $234,5 \mathrm{~Hz}$ & $215 \mathrm{~Hz}$ \\
\hline Neutral & Range / & $208,3 \mathrm{~Hz}$ & $95,47 \mathrm{~Hz}$ & $127,9 \mathrm{~Hz}$ & $107,7 \mathrm{~Hz}$ & $89,68 \mathrm{~Hz}$ \\
\hline
\end{tabular}




\begin{tabular}{|l|l|l|l|l|l|l|}
\hline (mittel) & $\begin{array}{l}\text { Tonhöhen- } \\
\text { umfang }\end{array}$ & $191,4 \mathrm{~Hz}$ & $60,01 \mathrm{~Hz}$ & $102,3 \mathrm{~Hz}$ & $77 \mathrm{~Hz}$ & $80,61 \mathrm{~Hz}$ \\
\hline $\begin{array}{l}\text { Traurig } \\
\text { (niedrig) }\end{array}$ & $\begin{array}{l}\text { Range / } \\
\text { Tonhöhen- } \\
\text { umfang }\end{array}$ & & & & \\
\hline
\end{tabular}

Abb. 30 Richtwerte für den Tonhöhenumfang der einzelnen Probandinnen in $\mathrm{Hz}$

Zusammenfassend kann man formulieren, dass wenn die Emoticons als Kontextualisierungshinweise die Intonation beeinflussen sollten, die Intonationskurve, die mittlere Grundfrequenz und der Tonhöhenumfang einer Person, die eine Äußerung mit fröhlichem oder traurigem Emoticon vorliest, anders ausfallen sollten, als wenn sie die gleiche Äußerung ohne Emoticon vorliest. Anhand dieser Unterschiede kann man meiner Ansicht nach auf einen vorhandenen bzw. nicht vorhandenen Einfluss der Emoticons auf die Interpretation schließen.

Auf Grund ihrer häufigen Position am Ende von Äußerungen und somit der nicht parallelen Wahrnehmung beim Lesevorgang, war meine zu überprüfende These, dass Emoticons für die Kommunikation in den Threads nicht die gleiche Rolle spielen wie Gestik oder Mimik als Kontextualisierungshinweise in der mündlichen Kommunikation und somit auch keine Veränderung der Intonation und somit der Interpretation einer Äußerung bewirken.

Zunächst sollte überprüft werden, ob Emoticons, die hinter einer Äußerung stehen, einen Einfluss auf die Intonation haben. Exemplarisch für ein fröhliches Emoticon werden unten die Intonationskurven zu der Äußerung $\mathrm{T}_{2} \mathrm{M}_{2,4}$ in (43) und (43)' dargestellt: ${ }^{177}$

\footnotetext{
${ }^{177}$ In dieser und folgenden Abbildungen werden fröhliche Emoticons mit \# und traurige Emoticons mit \#* in der Zeile der Äußerung angegeben. Anhand der Position dieser Markierungen wird gleichzeitig angezeigt, wo sich das jeweilige Emoticon befindet.
} 


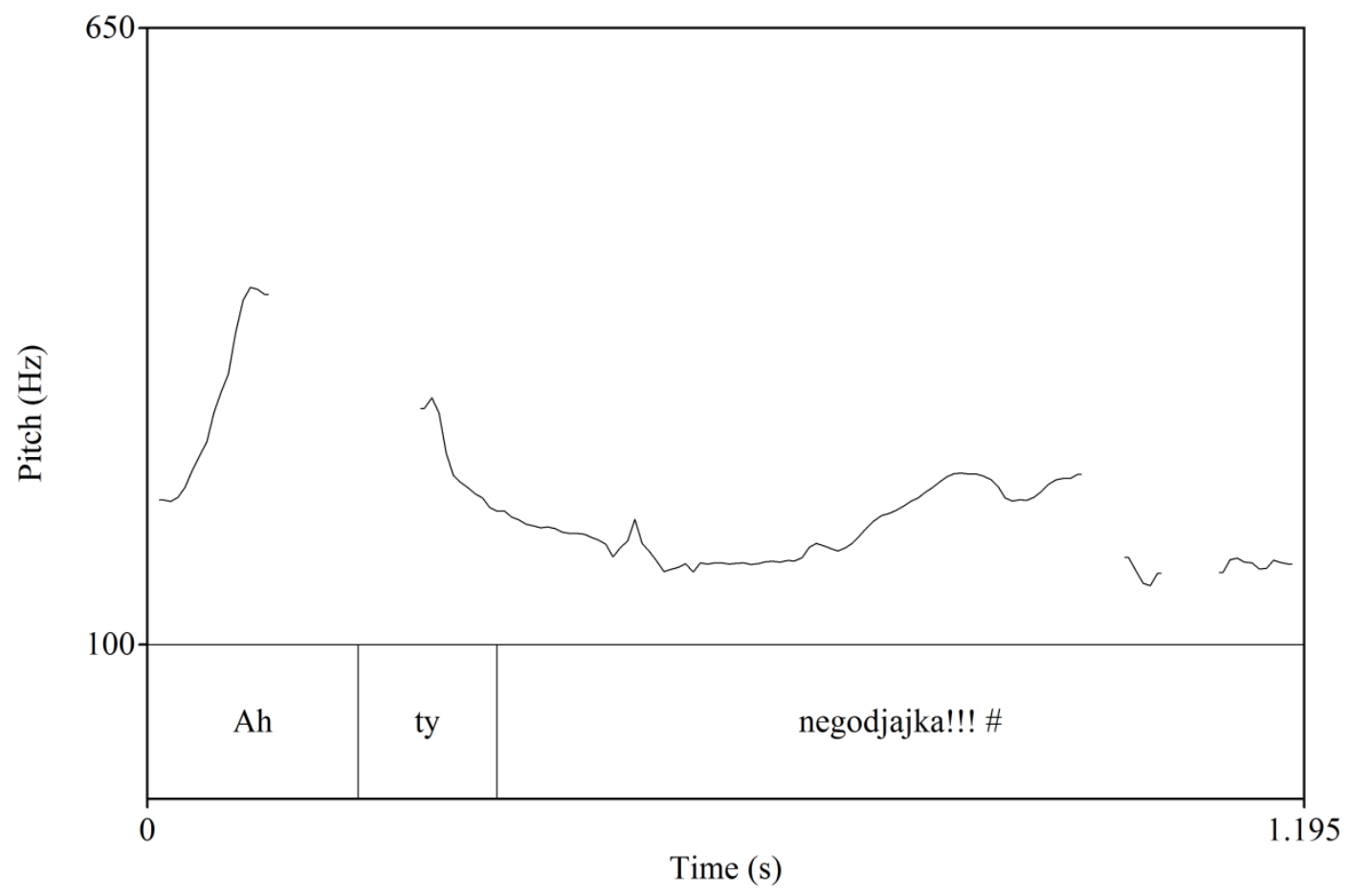

Abb. 31 Probandin 1 mit fröhlichem E hinter der Äußerung

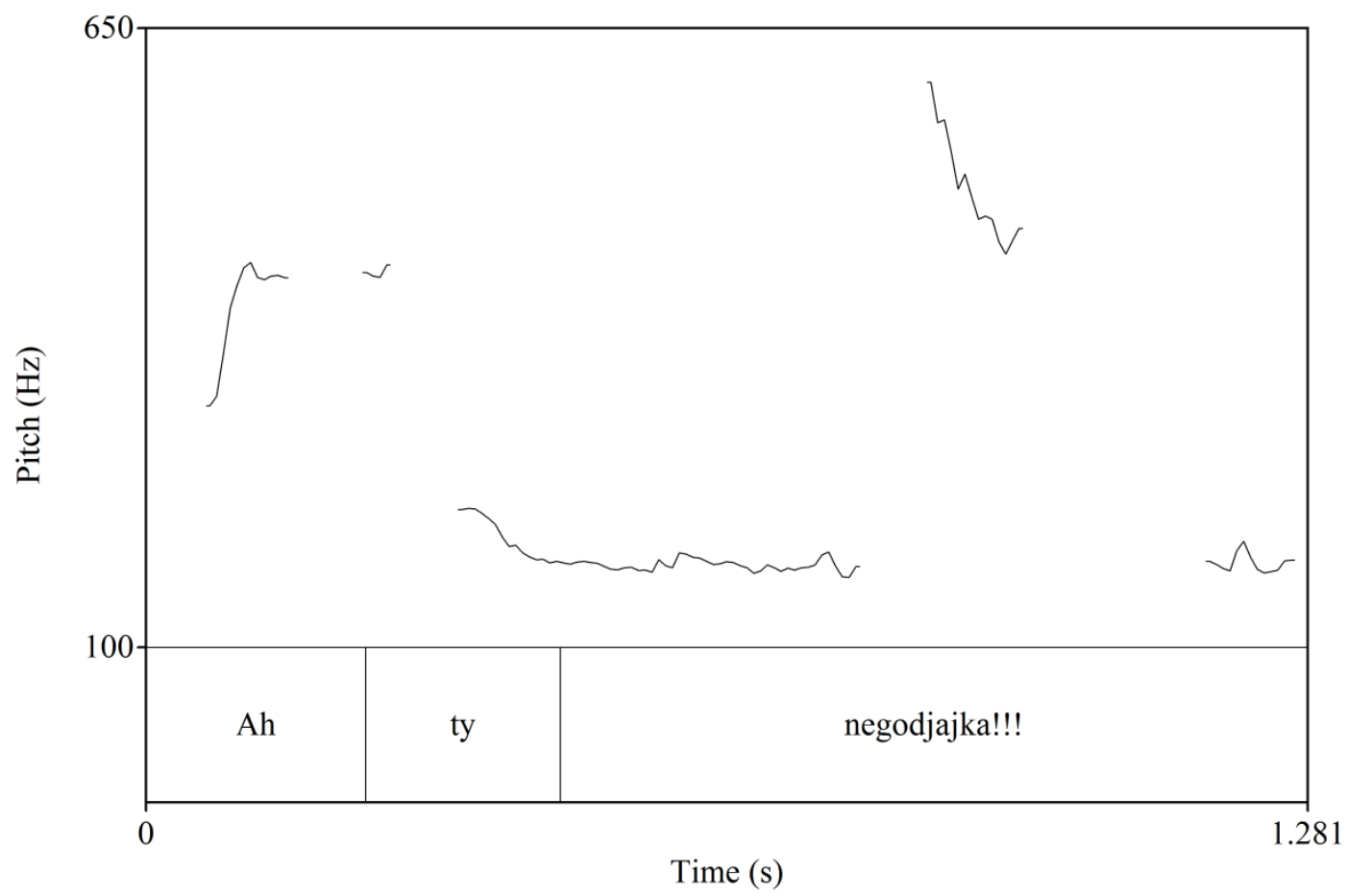

Abb. 32 Probandin 1 ohne E

Die erste Abbildung zeigt die Intonationskurve des Beispiels mit einem fröhlichen Emoticon, die zweite Abbildung die Intonationskurve des gleichen Beispiels ohne Emoticon. Ähnlich 
sind sich beide Abbildungen zu Beginn der Äußerung: in beiden Fällen steigt die IK zu Beginn der Äußerung und fällt bei ty. Einen Unterschied gibt es am Ende der Äußerung: während die IK in der ersten Abbildung zum Ende hin nur gering ansteigt und fällt, findet man in der zweiten Abbildung einen steilen Anstieg und Fall. Die Unterschiede sind meiner Ansicht nach jedoch nicht auf das Vorhandensein des Emoticons zurückzuführen. Dass das Emoticon keinen Einfluss auf die Intonation und somit Interpretation ausübt, sieht man auch, wenn man den pitch level beider IK vergleicht: in der ersten Abbildung sind es 219,41 Hz und in der zweiten 260,04 Hz. Der erste Wert liegt nah an dem Richtwert für eine niedrige mittlere Grundfrequenz der Probandin, der zweite Wer liegt zwischen dem neutralen und hohen Richtwert. Damit liegt der Wert der Äußerung ohne Emoticon höher als der Wert der Äußerung mit einem fröhlichen Emoticon. Dies spricht dafür, dass das fröhliche, lachende Emoticon nicht für die Intonation und Interpretation von Bedeutung war. Dieser Befund wird auch vom Vergleich des Tonhöhenumfangs beider Äußerungen gestützt: in der Äußerung mit Emoticon lag dieser bei $266,1 \mathrm{~Hz}$ und in der Äußerung ohne bei 439,5 Hz. Der erste Wert liegt zwischen dem mittleren und hohen Tonhöhenumfang, während sich der Wert der Äußerung ohne Emoticon über dem Richtwert für den hohen Tonhöhenumfang befindet. Das Emoticon spielt somit keine Rolle für die Interpretation der Äußerung.

Um das Ergebnis zu überprüfen, wurde das Beispiel (46) $\mathrm{T}_{1} \mathrm{M}_{1,4}$ betrachtet. Dieses Mal jedoch zum Vergleich noch die gleiche Äußerung mit einem traurigen Emoticon hinzugenommen. Die Abbildung 33 entspricht der Äußerung im Originalbeispiel mit lachendem Emoticon, die Abbildung 35 entspricht der manipulierten Äußerung in Beispiel (46)',' und die Abb. 34 und 36 der manipulierten Äußerung in $\left((46)^{\prime}\right)$. 


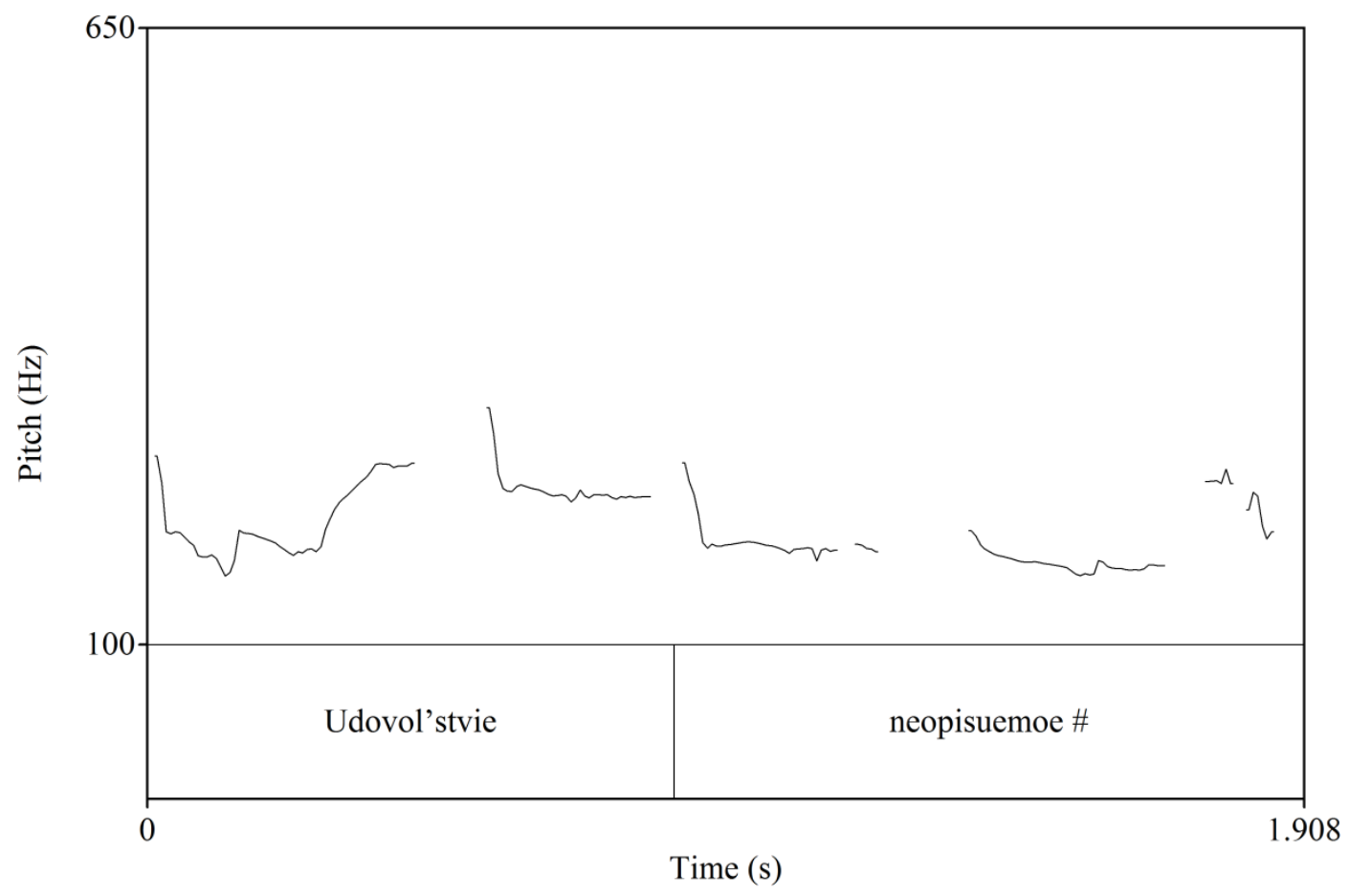

Abb. 33 Probandin 2 mit fröhlichem E hinter der Äußerung

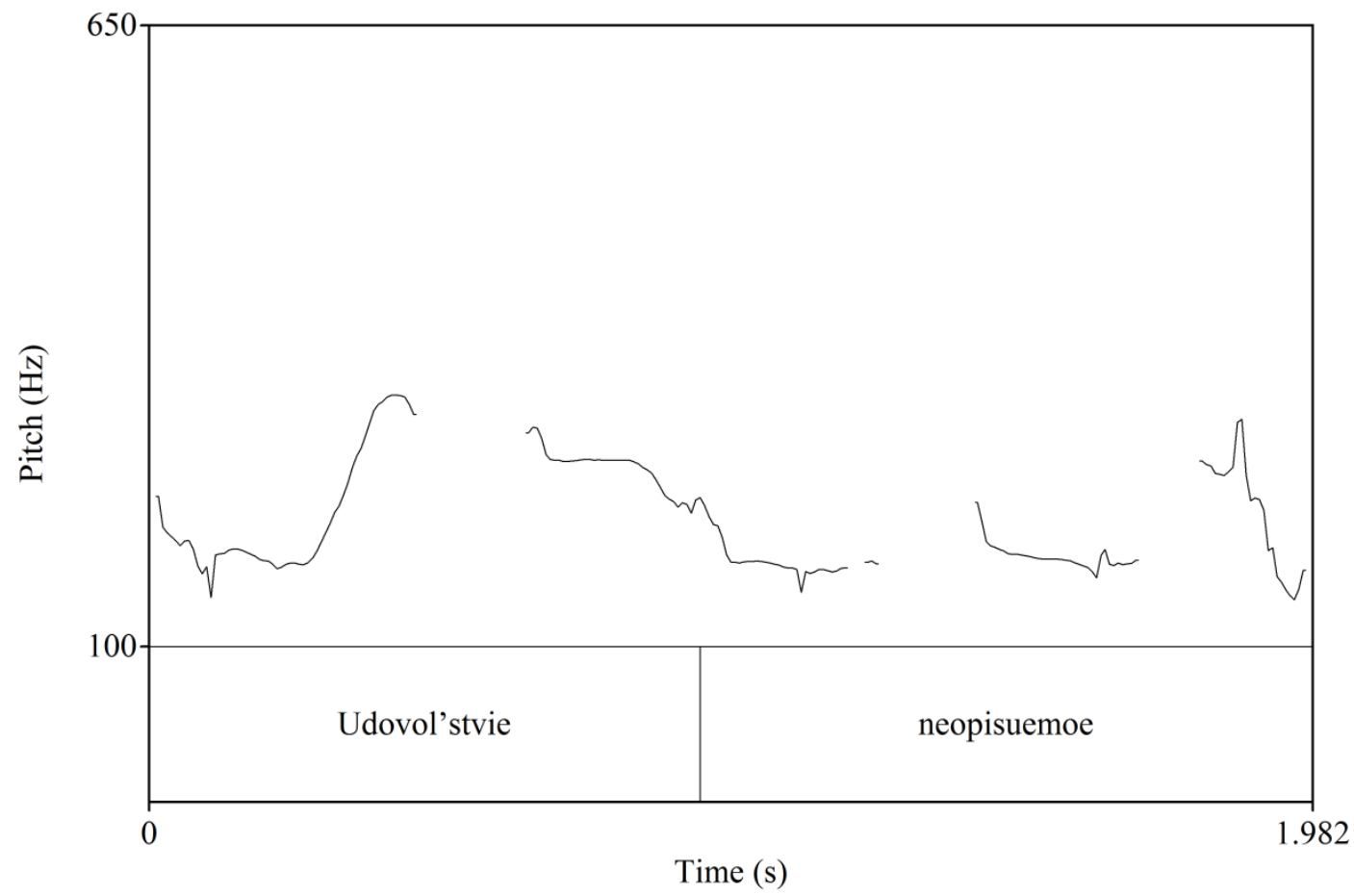

Abb. 34 Probandin 2 ohne E

Vergleicht man die beiden Abbildungen 33 und 34 stellt man Ähnlichkeiten im Verlauf der Intonationskurven fest: zu Beginn steigt die Kurve und fällt dann wieder, bevor sie wieder 
leicht steigt und fällt. Dies erfolgt in beiden Abbildungen unabhängig vom Vorhandensein eines fröhlichen Emoticons am Ende der Äußerung. Bezüglich der mittleren Grundfrequenz gibt es Unterschiede: 204,71 Hz in der Äußerung mit Emoticon gegenüber 211,28 Hz in der Äußerung ohne Emoticon. Dennoch liegen beide Werte nah an dem Richtwert für einen mittleren pitch level. Wenn das Emoticon eine Rolle spielen würde, hätte die Äußerung in der ersten Abbildung einen höheren pitch level haben müssen, da das Emoticon ein fröhliches war. Daraus kann man folgern, dass das Emoticon keinen Einfluss auf die Interpretation der Äußerung ausübt. Wie in dem vorherigen Beispiel wird dieses Ergebnis durch den Vergleich des Tonhöhenumfangs bestätigt: dieser beträgt in der Äußerung mit Emoticon 150,1 Hz und ohne Emoticon 181,2 Hz. Das Emoticon spielt somit für die Intonation keine Rolle.

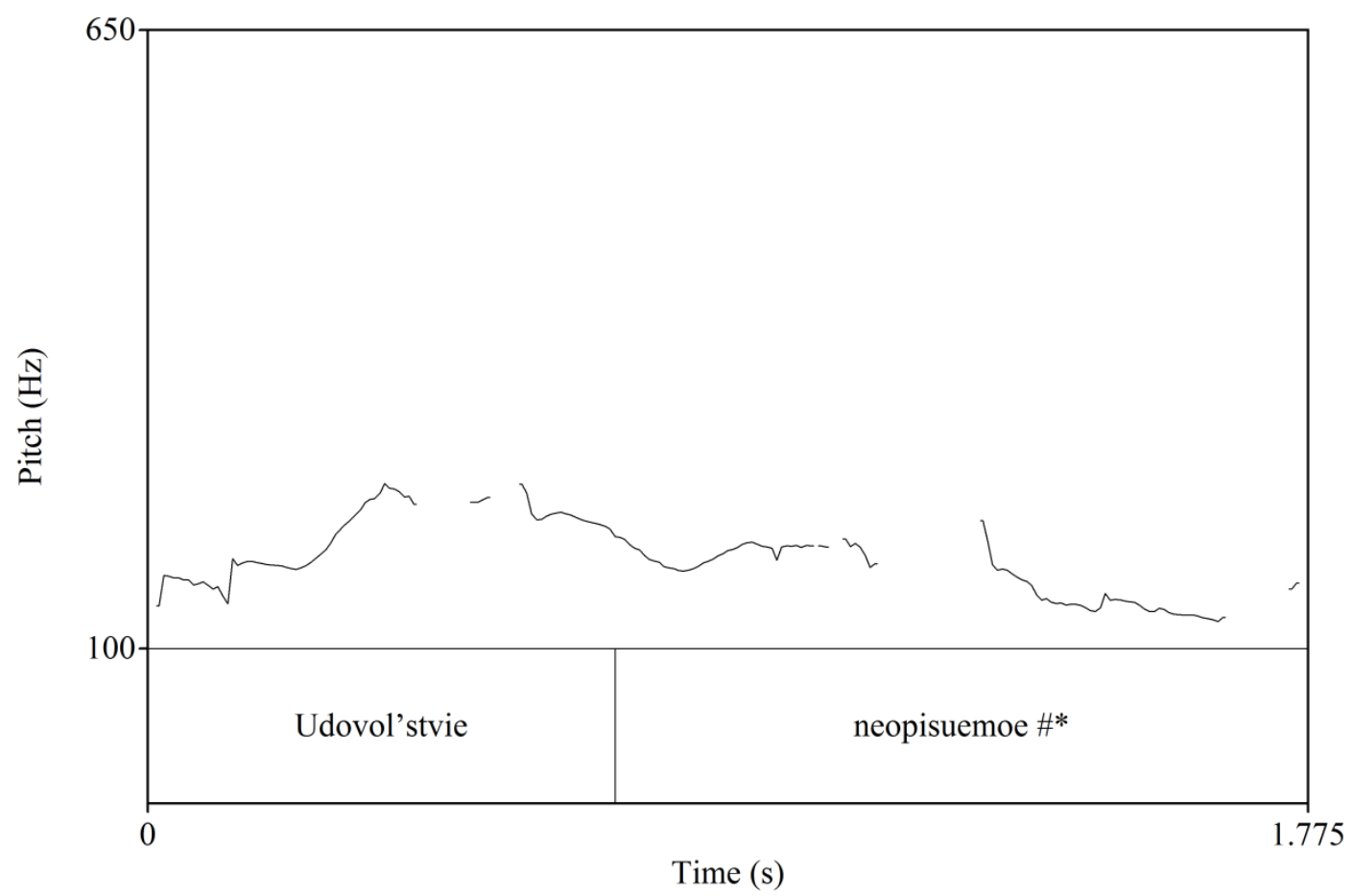

Abb. 35 Probandin 3 mit traurigem E hinter der Äußerung 


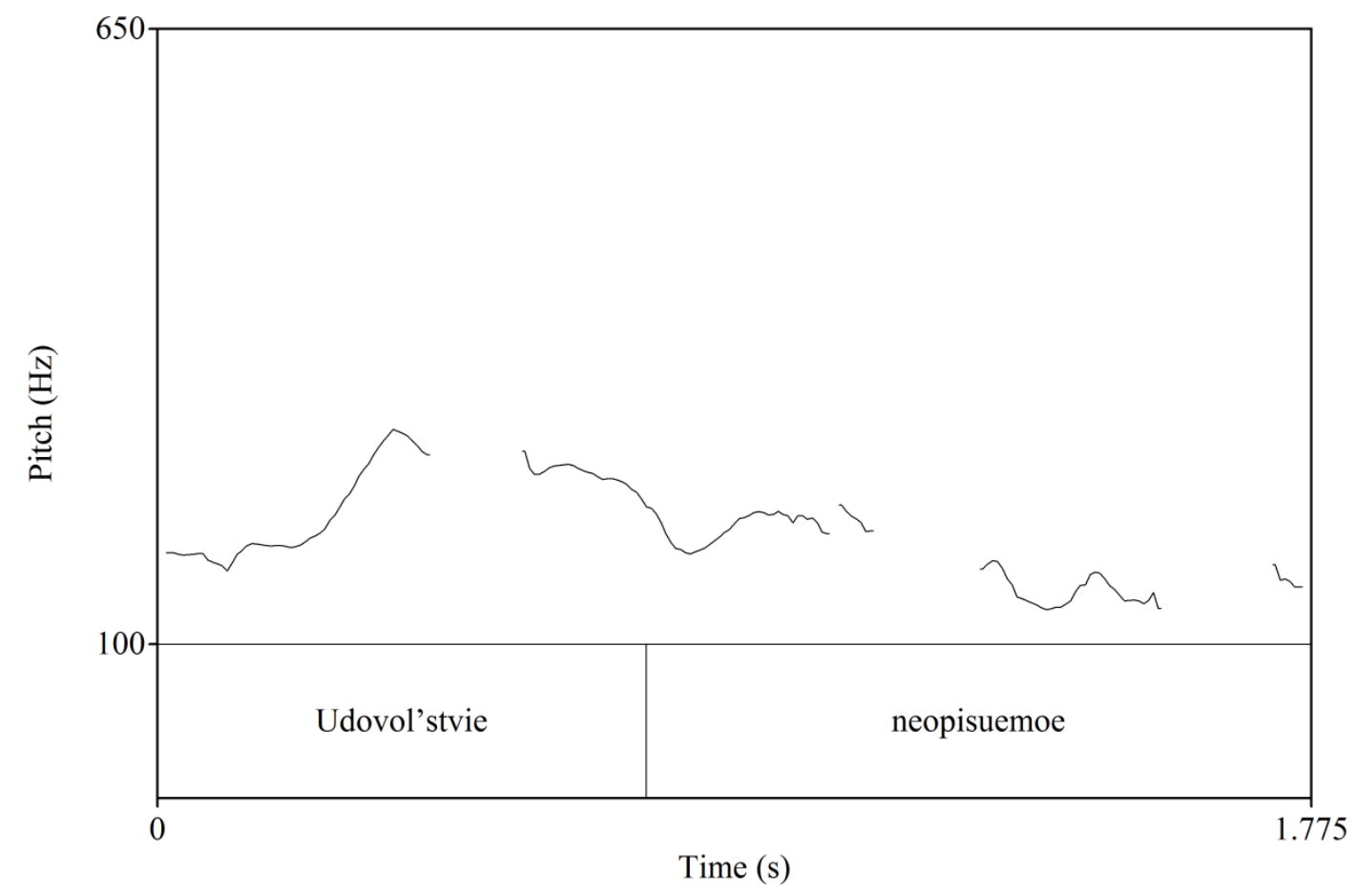

Abb. 36 Probandin 3 ohne E

Wie die bei den Abbildungen 33 und 34 liegen auch bei dieser Probandin Ähnlichkeiten im Verlauf der Intonationskurven vor: die Kurve steigt zu Beginn der Äußerung, fällt leicht, steigt wieder leicht an und fällt dann. Wie zuvor liegen die Ähnlichkeiten ungeachtet des Emoticons vor. Im Bereich des pitch levels gibt es Unterschiede: 178,78 Hz in Abb. 35 und 200,91 Hz in Abb. 36. Im ersten Fall entspricht dies dem Richtwert der Probandin für einen niedrigen pitch level, im zweiten Fall für einen mittleren. Das Emoticon könnte somit durchaus einen Einfluss auf die Intonation der Äußerung ausüben. Bezüglich des Tonhöhenumfangs liegt der Wert der Äußerung mit traurigem Emoticon bei $122,7 \mathrm{~Hz}$ und in der Äußerung ohne Emoticon bei 161,1 Hz. Der erste Wert liegt jedoch sehr nah an dem mittleren Richtwert, so dass der obige Befund weder gestützt noch widerlegt wird. Das traurige Emoticon könnte somit für die Probandin eine Rolle gespielt haben.

Durch die Auswertung der oben gezeigten Intonationskurven konnten zwei wichtige Ergebnisse herausgefunden werden:

- Auf Grund der Ähnlichkeit der Intonationsverläufe, der mittleren Grundfrequenz und des Tonhöhenumfangs in den Abbildungen 31 und 32 sowie 33 und 34 kann geschlossen werden, dass unabhängig vom Vorhandensein oder Fehlen eines fröhlichen Emoticons die Interpretation der Äußerung ähnlich ist. 
- Im Falle des traurigen Emoticons waren sich die Intonationsverläufe zwar ähnlich, jedoch gab es einen Unterschied zwischen den mittleren Grundfrequenzen, wie Abb. 35 und 36 zeigen. Somit könnte das traurige Emoticon einen Einfluss auf die Intonation ausüben.

Zusammenfassend kann man sagen, dass fröhliche Emoticons vom Leser offensichtlich nicht als Hinweis wahrgenommen werden. Da mit den Probanden im Anschluss an die Experimente weder Interviews durchgeführt noch ihnen Fragebögen vorgelegt wurden, lässt sich nicht eindeutig feststellen, ob das fröhliche Emoticon einfach nur übersehen wurde oder für die Probandinnen keine Rolle gespielt hat. Ein Argument dagegen, dass es übersehen wurde, ist die Kürze der Äußerung: der jeweilige Leser hätte das Emoticon aus dem Augenwinkel heraus sehen müssen.

In den oben vorgestellten Beispielen und Äußerungen standen die Emoticons - wie typischerweise in der Threadkommunikation, wenn man die Mehrheit die hier untersuchten Beispiele betrachtet - jedoch immer am Ende der jeweiligen Äußerung, so dass die Frage geklärt werden muss, ob Emoticons einen Einfluss auf die Intonation der Äußerung ausüben, wenn sie der Äußerung vorangehen. Dafür wurde ein weiterer Versuch durchgeführt, bei dem die Mitteilung $\mathrm{T}_{1} \mathrm{M}_{6}$ des Beispiels (44) analysiert wurde. Die jeweiligen Manipulationen an dem Beispiel sind in (44)", - (44)",", dargestellt. Die Abbildung 37 entspricht der manipulierten Mitteilung in (44)', in der das fröhliche Emoticon jeweils vor der Äußerung auftaucht. Die Abbildung 38 dem Beispiel (44)",', Die Abbildung 39 zeigt das manipulierte Beispiel (44)"', und die letzte Abbildung das Beispiel (44)",',.

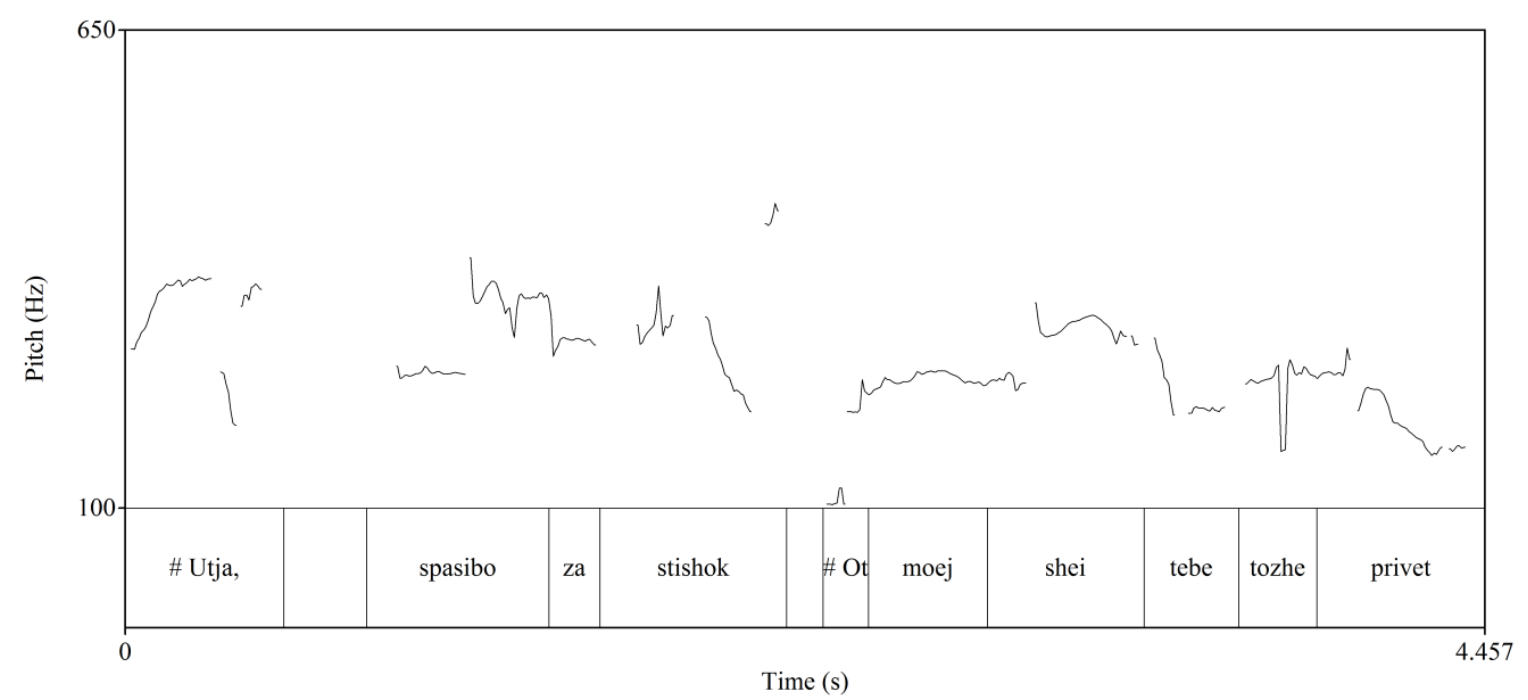

Abb. 37 Probandin 2 mit fröhlichem E vor der Äußerung 


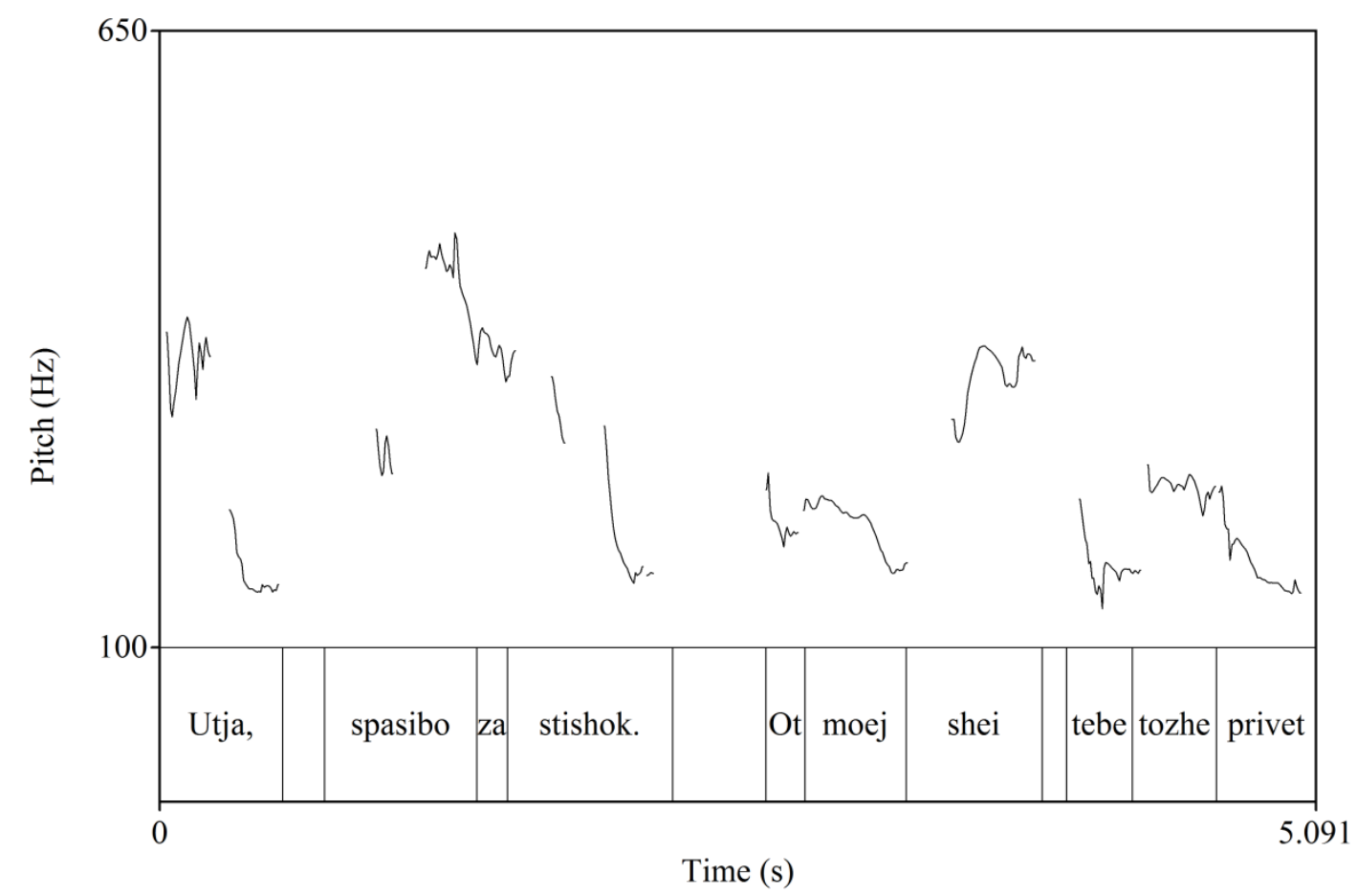

Abb. 38 Probandin 2 ohne E

$\mathrm{Zu}$ sehen ist auch hier eine Ähnlichkeit zwischen der produzierten IK mit vorangehendem fröhlichem Emoticon und der IK der Äußerungen ohne Emoticon. In beiden Fällen fällt die Äußerung zu Beginn bevor sie bei spasibo wieder ansteigt und zum Ende der Äußerung wieder fällt. Das lachende Emoticon beeinflusst dabei nicht den Verlauf der IK. In der zweiten Äußerung liegt bei shei ein Anstieg vor, wonach die IK steil fällt, dann noch einmal kurz ansteigt und am Ende der Äußerung ab privet fällt. Da die Probandin eine Sprechpause zwischen der ersten und zweiten Äußerung macht, kann man davon ausgehen, dass sie das Emoticon wahrgenommen, ihm jedoch keine Bedeutung zugemessen hat. Der pitch level liegt in beiden Beispielen nah beieinander: 269,98 Hz für die Intonationskurve der Äußerung mit dem vorangehenden fröhlichen Emoticon und $254,74 \mathrm{~Hz}$ für die Äußerung ohne das Emoticon. In beiden Fällen liegt der pitch level im mittleren Bereich. Da es auch in diesem Bereich keine großen Unterschiede gibt, kann man davon ausgehen, dass das Emoticon keine Rolle spielt. Auch beim Tonhöhenumfang liegen beide Werte nah bei einander: 346,7 Hz für die Äußerung mit und 335,6 Hz für die Äußerung ohne Emoticons. Somit wird die These unterstützt, dass Emoticons die Interpretation einer Äußerung nicht auf die gleiche Art und Weise beeinflussen wie dies z.B. Mimik in mündlicher Kommunikation tut. Um sich für eine Intonation und damit Interpretation $\mathrm{zu}$ entscheiden, ist der Kontext der Äußerung entscheidender. 


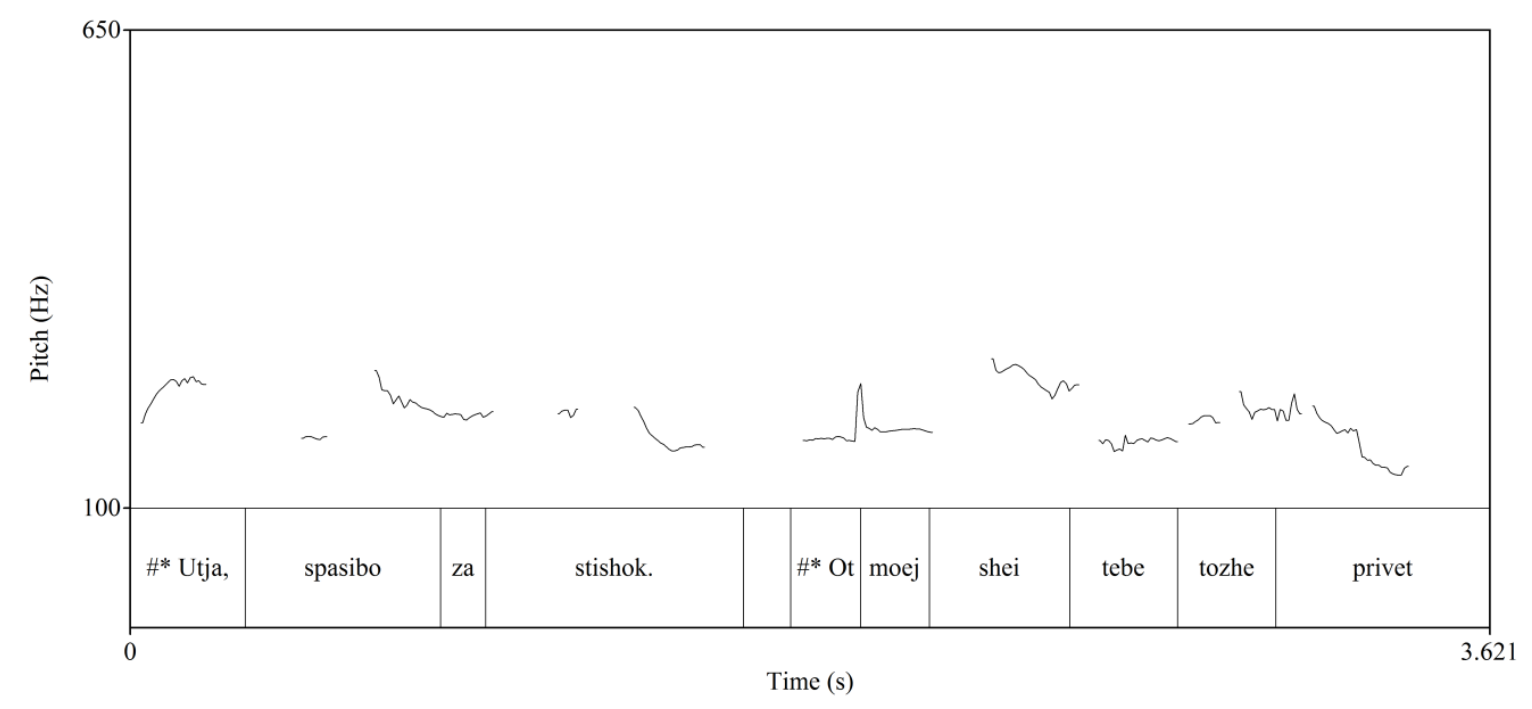

Abb. 39 Probandin 4 mit traurigem E vor der Äußerung

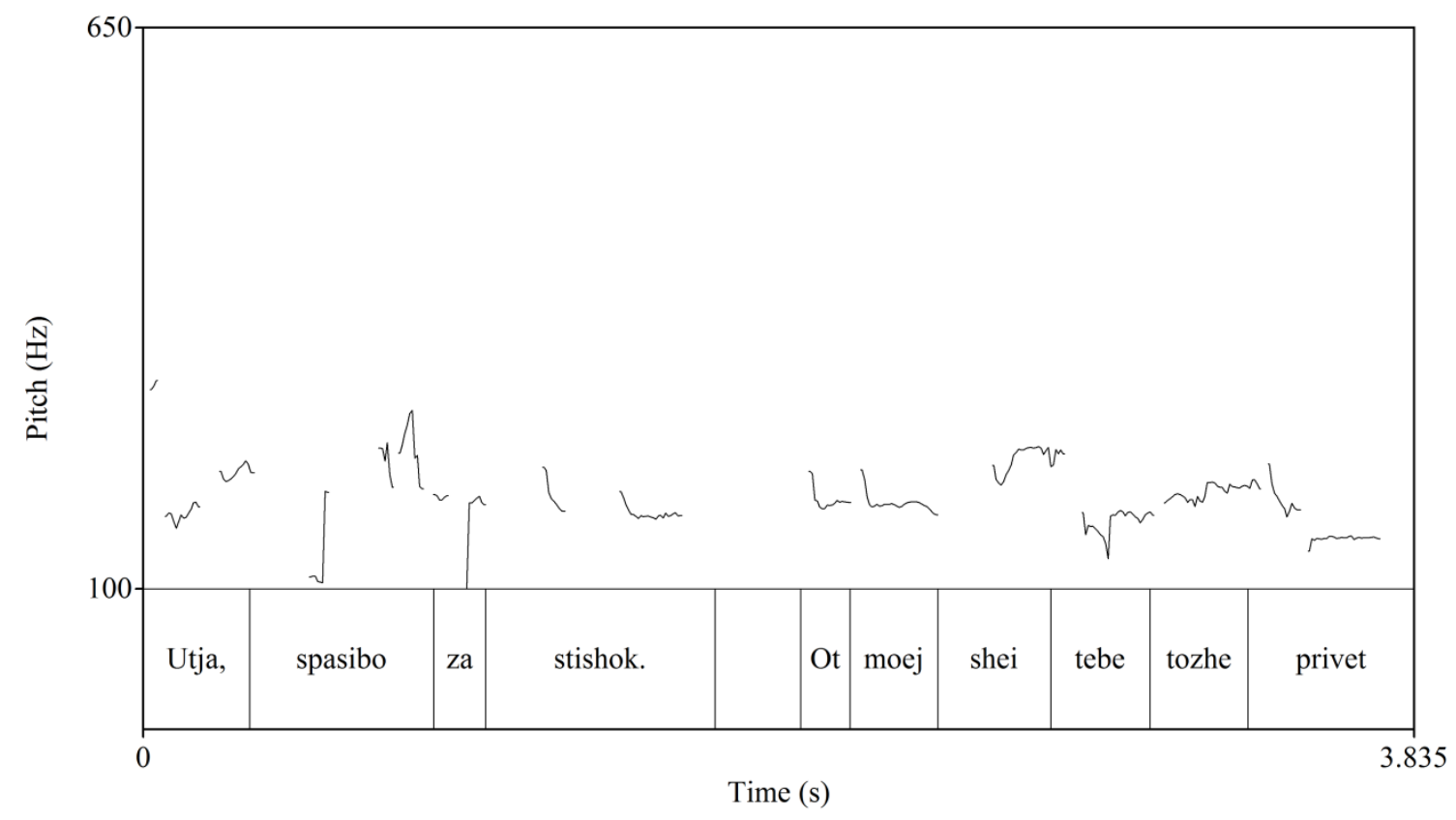

Abb. 40 Probandin 4 ohne E

Vergleicht man die beiden IK, lassen sich Ähnlichkeiten feststellen. Zu Beginn der ersten Äußerung steigt die Kurve, um nach Utja wieder zu fallen, bei spasibo wieder zu steigen und anschließend direkt wieder zu fallen. Zum Ende der ersten Äußerung fällt die IK. Auch die Intonationskurven der zweiten Äußerung sind sich ähnlich. Zu Beginn gibt es einen Anstieg, bei shei gibt es einen Anstieg und bei tebe einen Fall mit anschließendem Anstieg. Am Ende der Äußerung fällt die IK wieder. Die Ähnlichkeiten liegen unabhängig davon, ob ein trauriges Emoticon zu Beginn der beiden Äußerungen steht oder nicht, vor. Der pitch level liegt in beiden Äußerungen nah bei einander: 202,03 Hz für die Äußerung mit 
vorangehendem traurigen Emoticon und $187,81 \mathrm{~Hz}$ für die Äußerung ohne Emoticon. Da beide Werte eher im mittleren pitch level-Bereich der Probandin anzusiedeln sind, kann angenommen werden, dass die traurigen Emoticons keinen Einfluss auf die Intonation und somit Interpretation ausüben. Dies steht im Gegensatz $\mathrm{zu}$ dem vorigen Ergebnis der Probandin 3. Beim Tonhöhenumfang gibt es merkliche Unterschiede mit 133,9 Hz für die Äußerung mit vorangehenden traurigen Emoticons und 204,2 Hz für diejenige ohne. Vergleicht man diese Werte jedoch mit den Richtwerten, befinden sich beide im mittleren Bereich, so dass auch hier gesagt werden kann, dass das traurige Emoticon keine Rolle spielt.

Insgesamt kann man feststellen, dass das vorangehende Emoticon - unabhängig davon, ob es fröhlich oder traurig ist - keinen Einfluss auf die Intonation und damit Interpretation ausübt.

Zum Abschluss wird hier das Ergebnis für die Äußerung $\mathrm{T}_{1} \mathrm{M}_{3,1}$ des Beispiels (44) gezeigt: die erste Abbildung 41 zeigt die IK der Äußerung im Original an, die Abbildung 43 die IK der Äußerung mit dem vorangehenden Emoticon ((44)'), die Abbildung 45 die IK der Äußerung mit einem traurigen Emoticon ((44)',,',), das der Äußerung folgt und die Abbildung 47 die IK der Äußerung mit einem der Äußerung vorangehenden traurigen Emoticon ((44)','). Die jeweiligen Abbildungen 42, 44, 46 und 48 entsprechen der Äußerung in (44), , , .

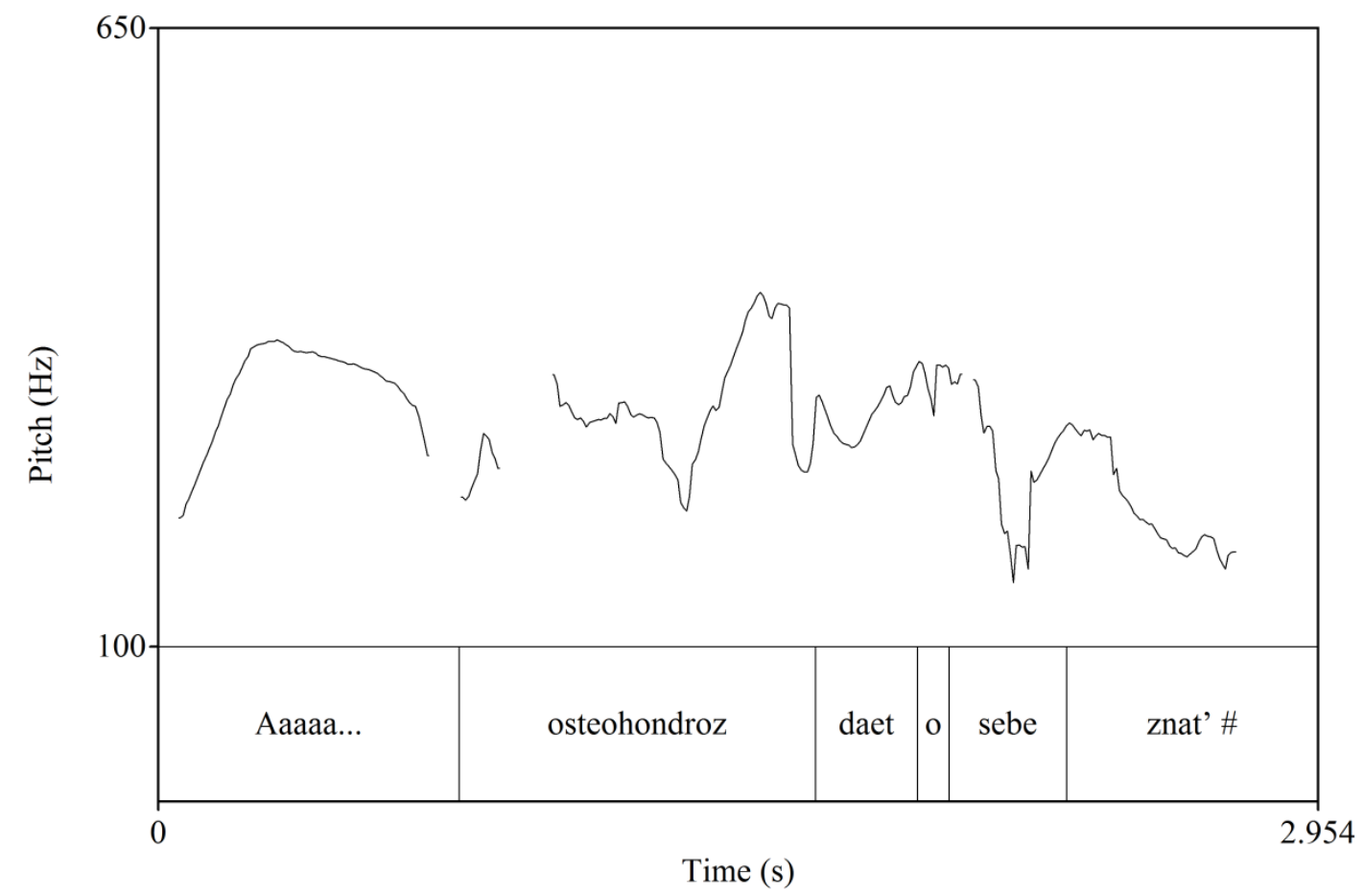

Abb. 41 Probandin 5 mit fröhlichem E hinter der Äußerung 


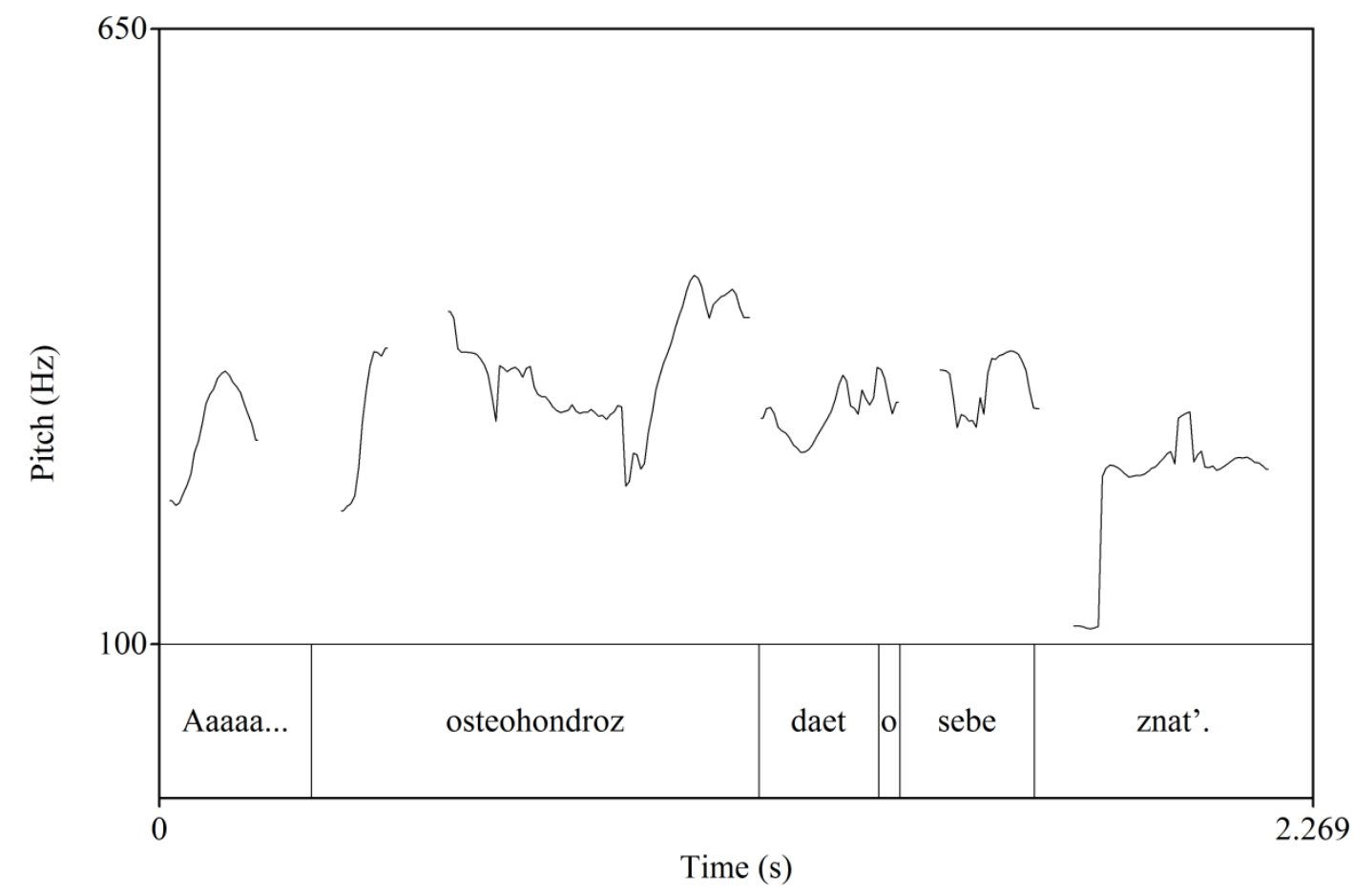

Abb. 42 Probandin 5 ohne E

Vergleicht man die IK, fallen Ähnlichkeiten und Unterschiede auf. Zu Beginn der Äußerung gibt es einen steilen Anstieg mit anschließendem Fall der IK, diesem Fall folgt ein erneuter steiler Anstieg, ein Fall und anschließender steiler Anstieg und Fall am Ende von osteohondroz, Während die IK in der ersten Abbildung bei sebe steil fällt, wieder steigt und zum Ende der Äußerung hin wieder fällt, fällt die IK in der zweiten Abbildung erst am Ende von sebe bzw. am Anfang von znat', steigt dann wieder und verläuft ohne einen Fall zum Ende der Äußerung hin. Bezüglich der pitch level gibt es Ähnlichkeiten: in der ersten Abbildung sind dies 295,71 Hz und in der zweiten 307,11 Hz. In beiden Fällen befinden sich die Werte oberhalb des Richtwertes der Probandin für einen hohen pitch level. Das Emoticon übt somit keinen Einfluss auf die Intonation aus. Dies wird durch die Werte für den Tonhöhenumfang bestätigt: 258,1 Hz für die Äußerung mit und 315,8 Hz für die Äußerung ohne Emoticon. Beide Werte befinden sich im hohen Tonhöhenumfang der Probandin. Dieses Ergebnis entspricht auch den Ergebnissen der Probandinnen 1 und 2. 


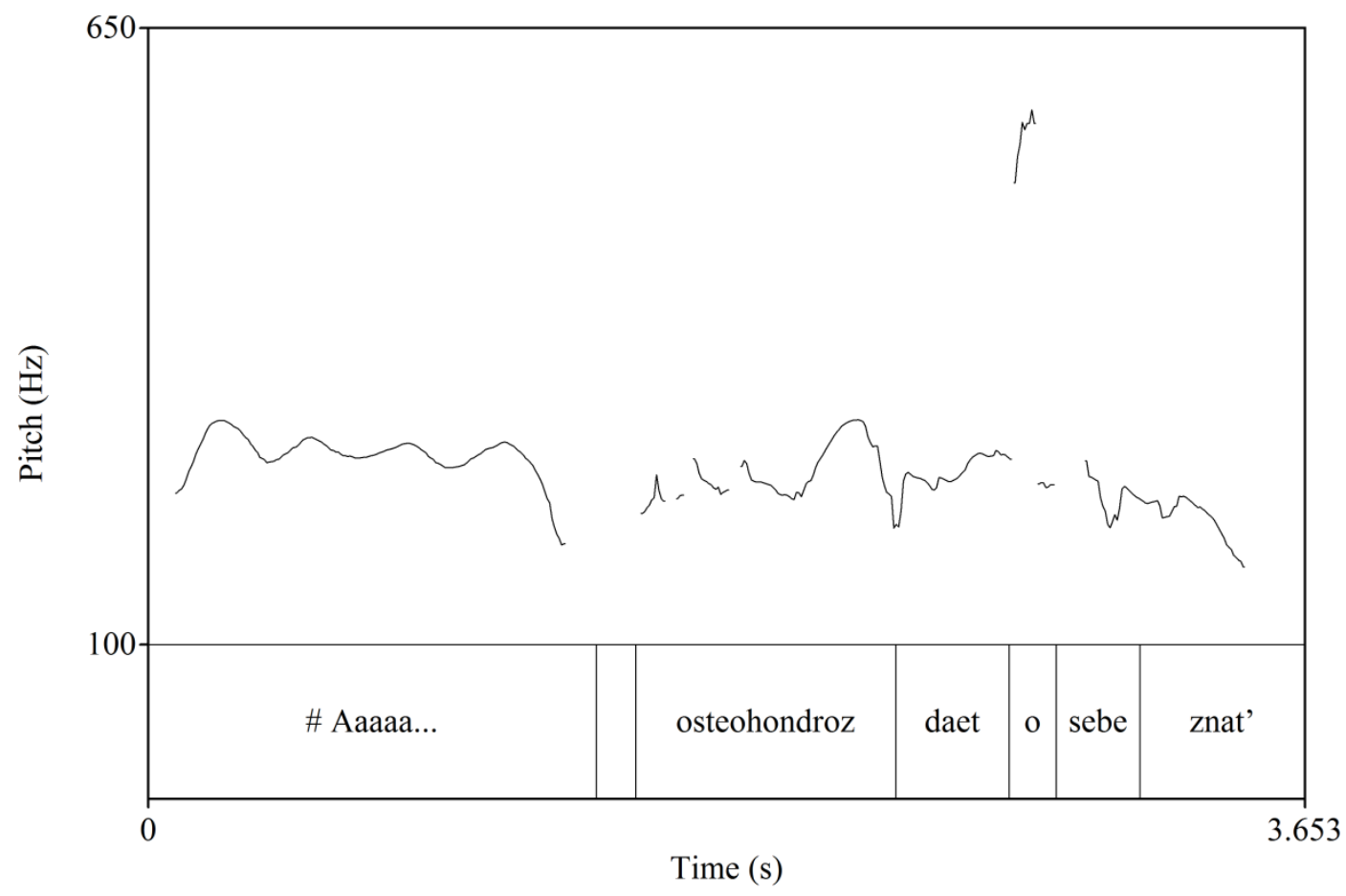

Abb. 43 Probandin 2 mit fröhlichem $E$ vor der $\ddot{A} u ß e r u n g$

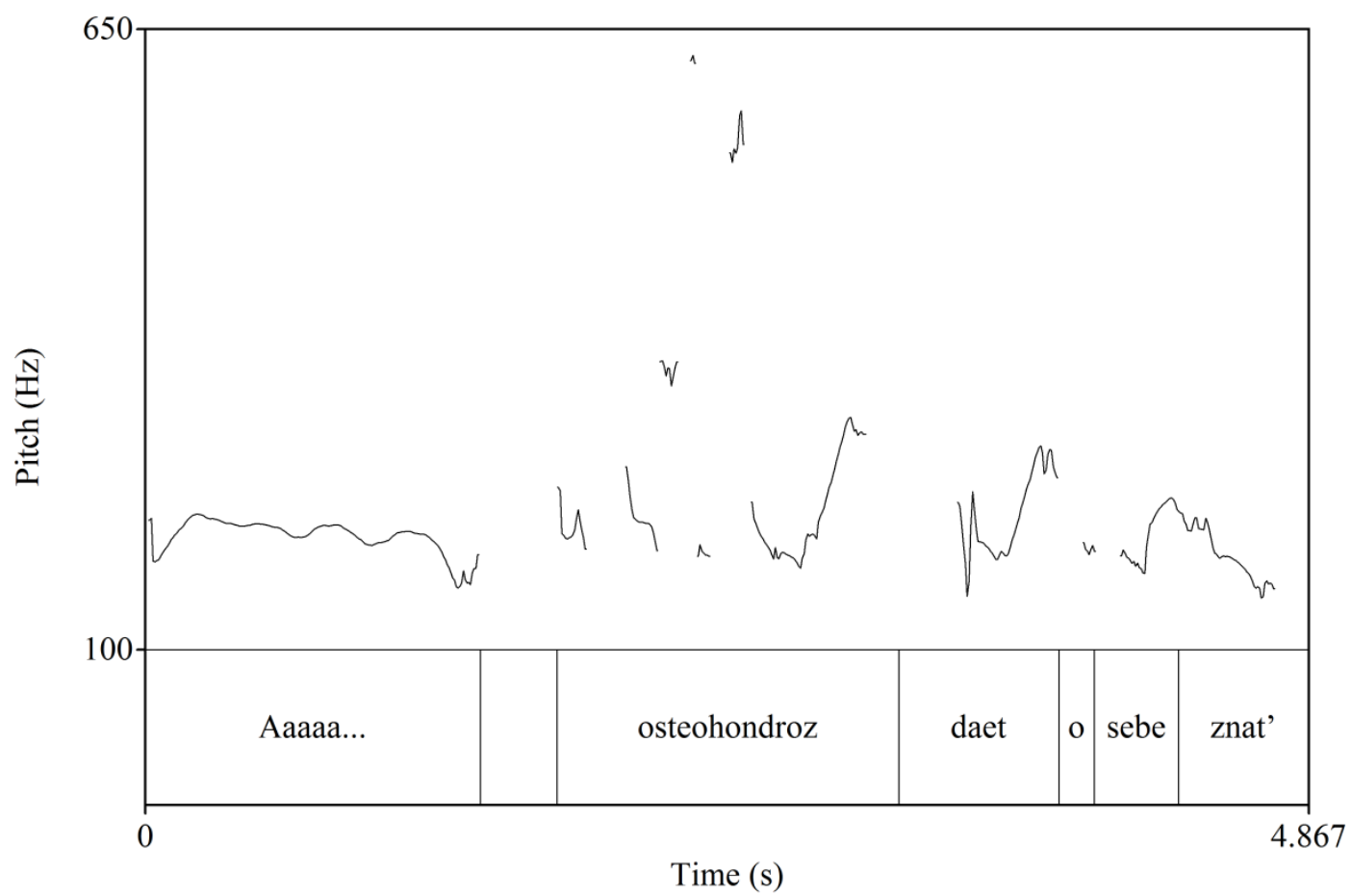

Abb. 44 Probandin 2 ohne E

Im Vergleich sieht man Gemeinsamkeiten und Unterschiede im Verlauf der IK. Zu den 
Ähnlichkeiten zählen mehrere Anstiege bei osteohondroz, wenn diese in der ersten Abbildung auch nicht so steil sind, ein Fall und erneuter Anstieg am Ende von osteohondroz bzw. bei daet und ein Fall der IK am Ende der Äußerung. Auch bei Aaaaa gibt es Ähnlichkeiten. Die pitch level beider IK unterscheiden sich: im Falle der ersten Abbildung beträgt er 260,97 Hz und im Falle der zweiten 217,29 Hz. Trotz des Unterschieds liegen beide Werte im Bereich des mittleren pitch level der Probandin. Bezüglich des Tonhöhenumfangs beträgt der Wert der Äußerung mit Emoticon 407,4 Hz und der zweite Wert 480,7 Hz. Beide Werte befinden sich über dem Richtwert für einen hohen Tonhöhenumfang. Das Emoticon übt somit keinen Einfluss auf die Intonation aus. Dies entspricht auch dem Ergebnis aus Abb. 37 und 38.

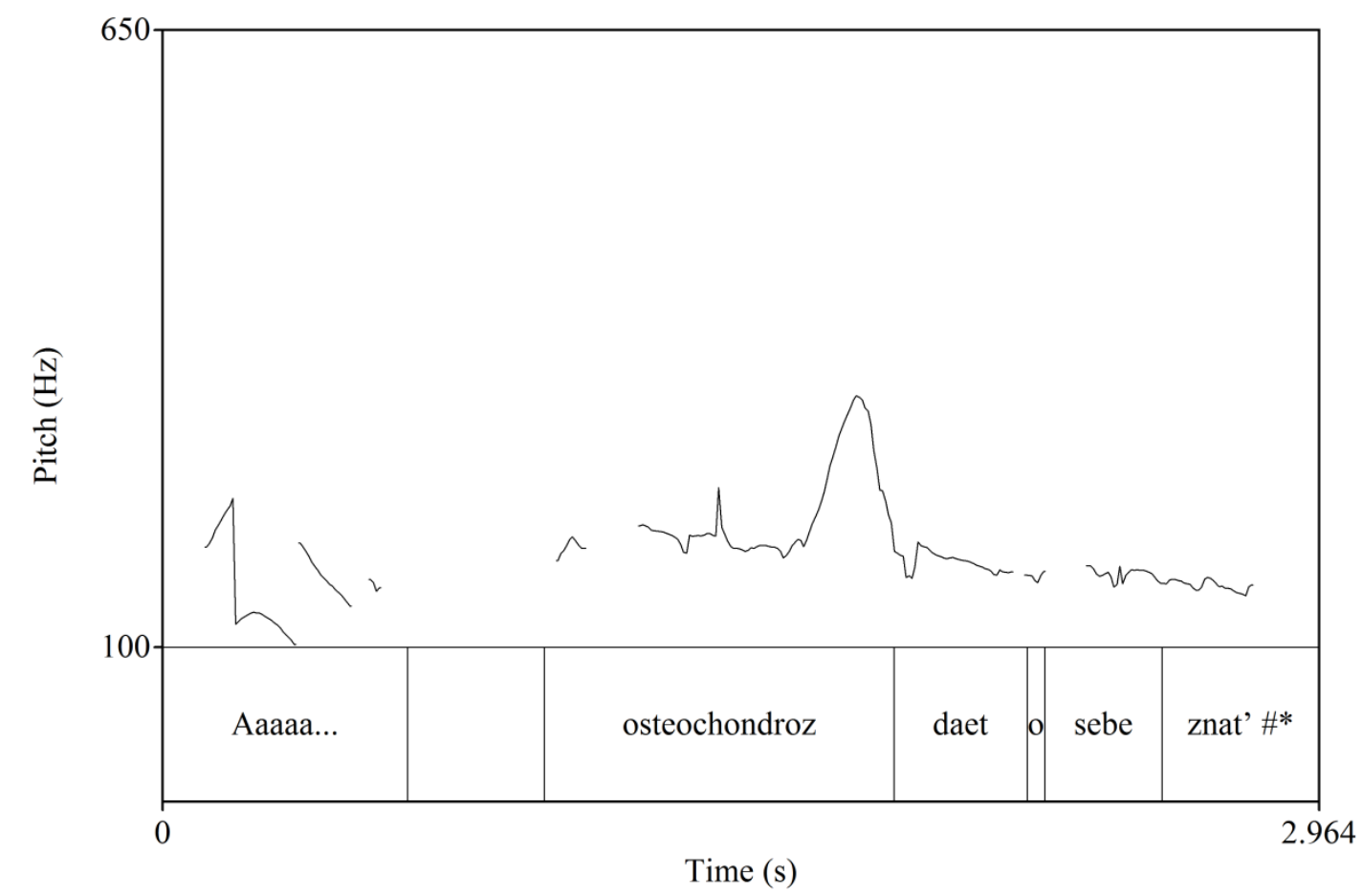

Abb. 45 Probandin 3 mit traurigem E hinter der Äußerung 


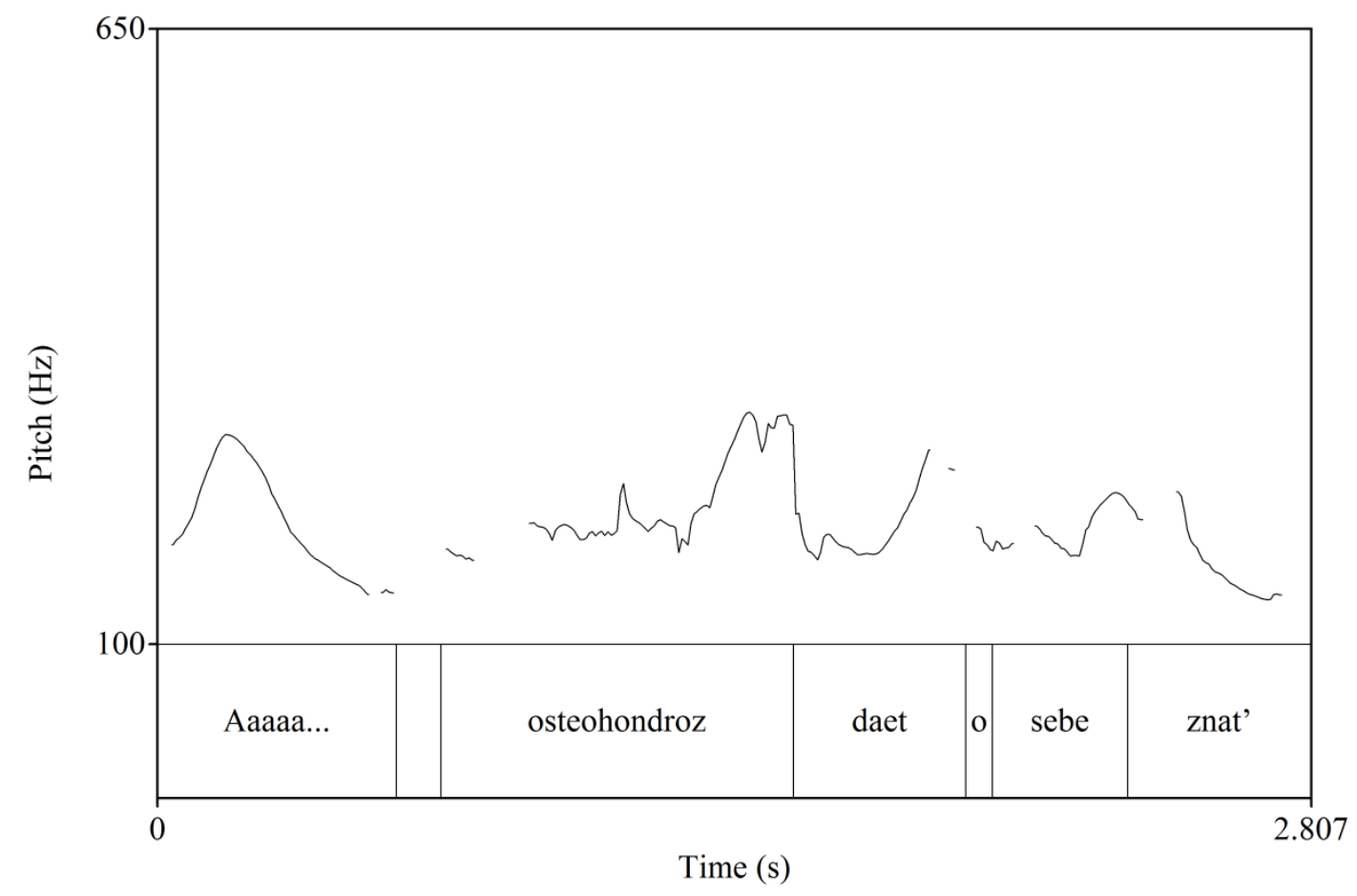

Abb. 46 Probandin 3 ohne E

Im Vergleich gibt es zwischen den IK mehr Unterschiede als Gemeinsamkeiten. Ähnlich ist in beiden Kurven der Anstieg zu Beginn der Äußerung, ein kleiner Anstieg bei osteohondroz und ein steiler Anstieg und steiler Fall am Ende von osteohondroz. Während es in der ersten Abbildung anschließend keinen großen Anstieg der Kurve mehr gibt, erkennt man in der IK der zweiten Abbildung noch mehrere Anstiege, bevor die Kurve am Ende der Äußerung fällt. In beiden Abbildungen liegt der Fokus auf osteohondroz. Zwischen den pitch level-Werten gibt es einen Abstand: 183,23 Hz in der Abbildung mit traurigem Emoticon am Ende der Äußerung und 208,13 Hz in der Abbildung ohne das Emoticon. Der erste Wert entspricht dem Richtwert der Probandin für einen niedrigen pitch level, der zweite Wert einem mittleren pitch level. Somit könnte das Emoticon in diesem Fall durchaus eine Rolle spielen. Dies stimmt auch mit dem vorigen Ergebnis der Probandin 3 überein. Beim Tonhöhenumfang gibt es Unterschiede: 221,3 Hz für die Äußerung mit traurigem Emoticon und 167,5 Hz für die Äußerung ohne. Beide Werte sind jedoch im mittleren Bereich anzusiedeln. Dies widerspricht dem Ergebnis des Vergleichs der mittleren Grundfrequenz. Es kann in diesem Fall nicht eindeutig geklärt werden, ob das traurige Emoticon einen Einfluss ausübt. 


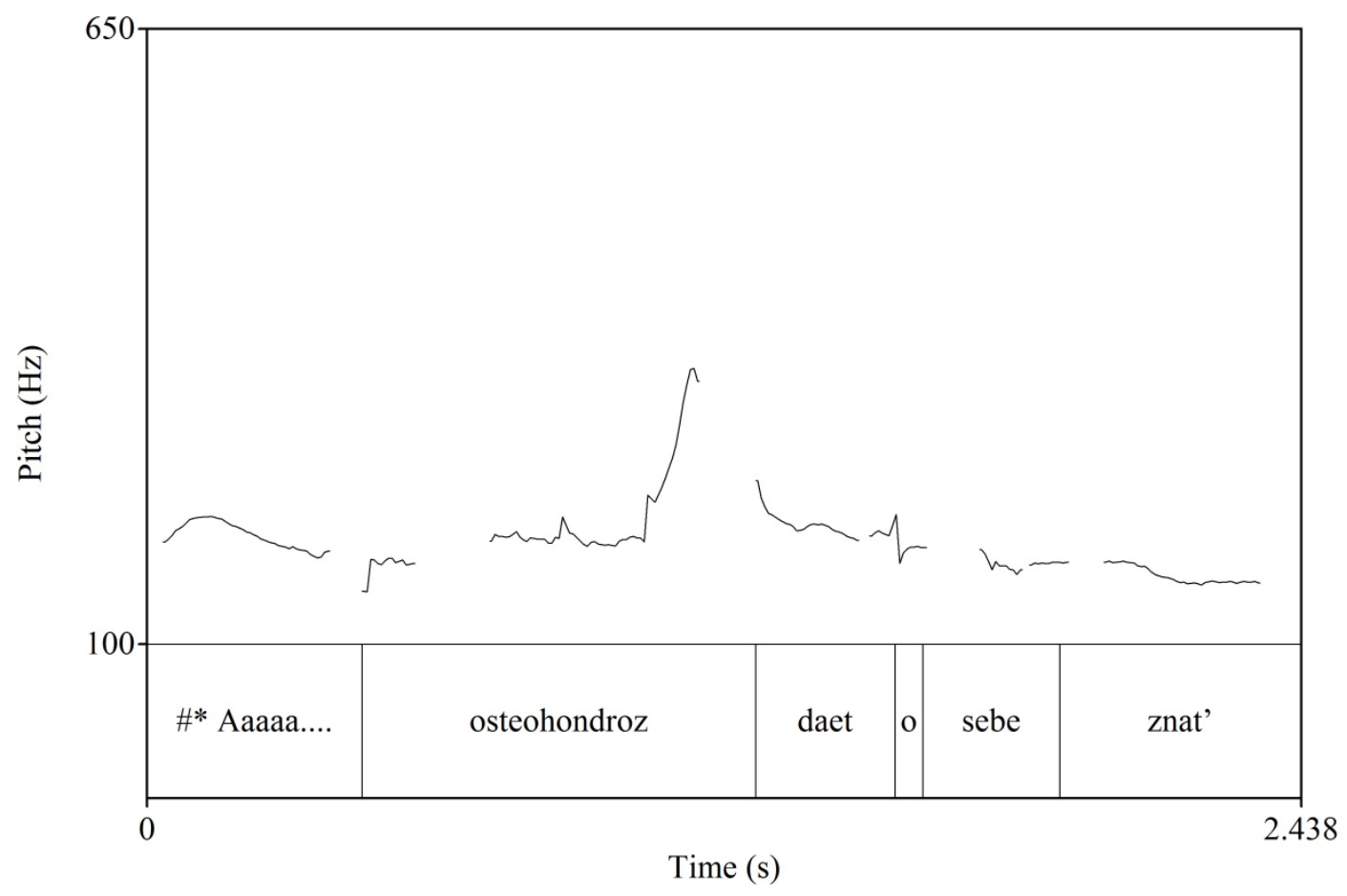

Abb. 47 Probandin 4 mit traurigem $E$ vor der Äußerung

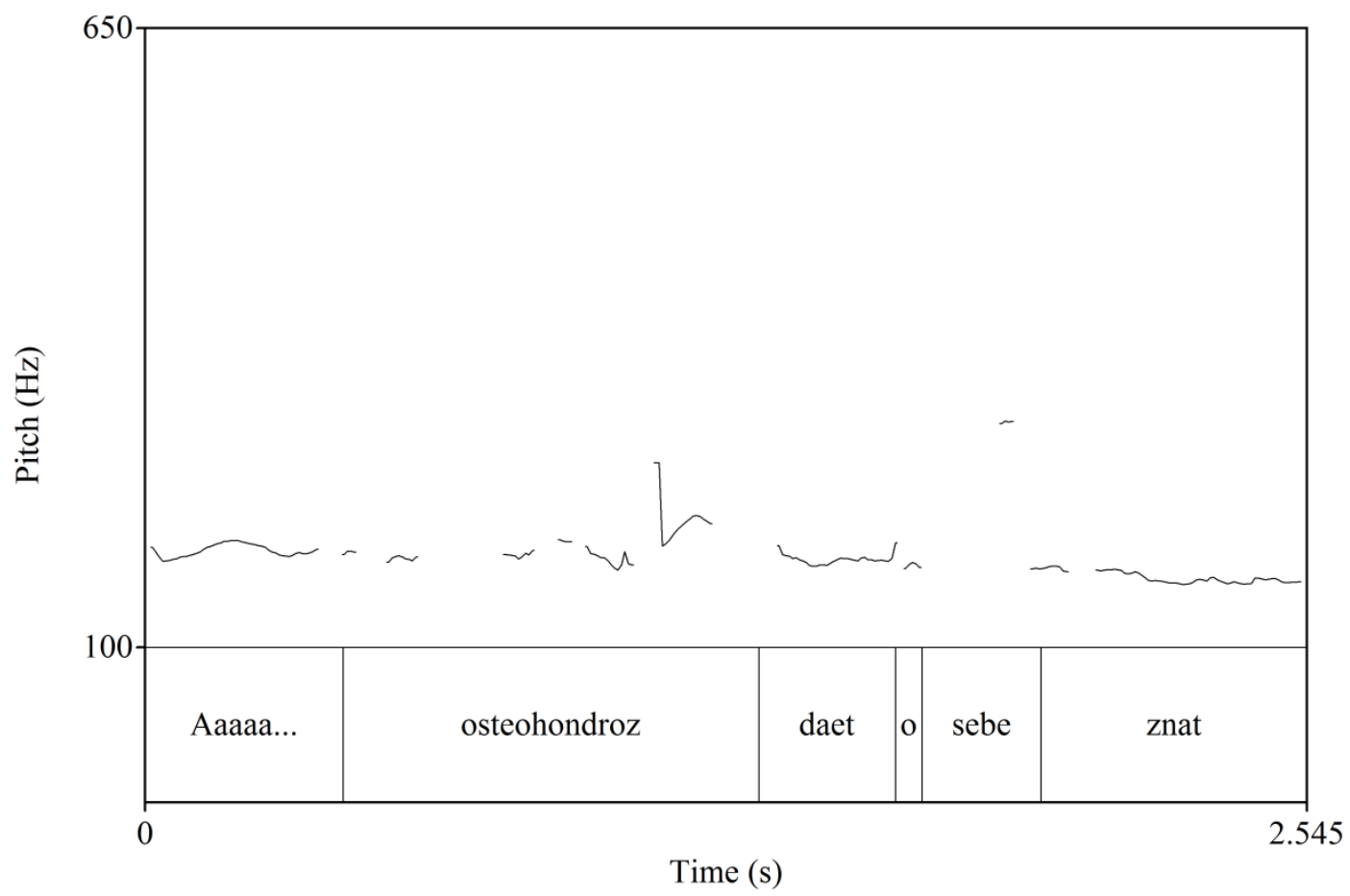

Abb. 48 Probandin 4 ohne E

Die IK beider Äußerungen ähneln sich. Einen deutlichen Anstieg der Kurve gibt es in beiden Fällen bei osteohondroz. Der pitch level liegt in der ersten Abbildung bei 192,02 Hz und in 
der zweiten bei 180, 35 Hz. Dies entspricht in beiden Fällen den Richtwerten der Probandin für den mittleren pitch level. Der Tonhöhenumfang beträgt für die Äußerung mit traurigem Emoticon 199,4 Hz und für die Äußerung ohne $145 \mathrm{~Hz}$. Beide Werte befinden sich im Bereich des mittleren Tonhöhenumfangs der Probandin. Das traurige Emoticon vor der Äußerung übt somit keinen Einfluss auf die Intonation und damit Interpretation aus. Dies entspricht auch dem vorigen Ergebnis der Probandin.

Anhand der gleichen Äußerung konnte exemplarisch gezeigt werden, dass ein fröhliches Emoticon in keinem angenommenen Fall einen Einfluss auf die Intonation und somit Interpretation ausübte. Das traurige Emoticon spielte hingegen möglicherweise für die Probandin eine Rolle, die das Beispiel mit traurigen Emoticon hinter der Äußerung vorgelesen hatte. Dafür spricht die unterschiedliche mittlere Grundfrequenz, während dies beim Vergleich des Tonhöhenumfangs nicht eindeutig ist. Die These, Emoticons spielten für die Interpretation nicht die gleiche Rolle wie Kontextualisierungshinweise in der face-to-faceKommunikation, kann zumindest für fröhliche Emoticons und traurige Emoticons, die der Äußerung vorangehen, bestätigt werden.

Zusammenfassend konnte durch die Versuche gezeigt werden, dass fröhliche Emoticons und traurige Emoticons, die der Äußerung vorangehen, keinen Einfluss auf die Intonation und damit Interpretation ausüben. Die Intonationskurven der Äußerungen wiesen unabhängig von dem Auftauchen sowie der Position eines Emoticons große Ähnlichkeiten auf. Auch die pitch level sowie der Tonhöhenumfang zwischen der jeweiligen Äußerung mit und ohne Emoticon ähnelten sich in den meisten Fällen. Es ist zu vermuten, dass Emoticons von den Lesern zwar gesehen, jedoch nicht als zusätzliche Information zur Äußerung in den Interpretationsprozess einfließen. Damit können fröhliche Emoticons und traurige Emoticons, die der Äußerung vorangehen, keine Kontextualisierungshinweise im Gumperz'schen Sinne sein. Dieses Ergebnis widerspricht den Resultaten der meisten oben erwähnten Studien. Interessant ist hingegen der Befund, dass im Falle des der Äußerung folgenden traurigen Emoticons die pitch level-Werte der Probandin unterschiedlich waren: die Äußerungen mit einem traurigen Emoticon wurden von ihr mit einem niedrigeren pitch level realisiert als die Äußerungen ohne Emoticon. Das Emoticon könnte für die Probandin somit eine Rolle gespielt hat. Dagegen sprechen jedoch der Vergleich der Werte des Tonhöhenumfangs sowie der Befund, dass das vorangehende traurige Emoticon keinen Einfluss auf die Intonation, den pitch level und den 
Tonhöhenumfang ausgeübt hat. Das Ergebnis für traurige Emoticons ist somit nicht eindeutig. Die überwiegende Mehrheit der Beispiele spricht dafür, dass die angenommene These durch die Experimente bestätigt werden kann. Bei den Emoticons handelt es sich meiner Ansicht nach um Pseudo-Kontextualisierungshinweise, die theoretisch die Intonation und damit Interpretation beeinflussen könnten, es tatsächlich aber nicht tun. Um sich für eine Intonation und damit Interpretation zu entscheiden, ist für die Probandinnen vor allem der Kontext der Äußerung entscheidend.

Warum werden Emoticons von vielen Usern dennoch so häufig verwendet? Während der Konversationsverlauf ökonomisch gestaltet ist und alles Unnötige weggelassen wird, gehen die User mancher Threads - hier vor allem im ersten Thread - bei der Verwendung der Emoticons (Anzahl und Gestalt) äußerst unökonomisch vor. Die Frage, die sich auf Grund des häufigen Vorkommens stellt, ist, welchen Zweck sie erfüllen, wenn sie keine Rolle für die Interpretation spielen. Es wäre möglich, dass der Schreiber mit ihnen Aufmerksamkeit erregt; in diesem Fall handelt es sich bei einem Emoticon um ein strategisches Mittel.

\section{$\underline{\text { 4.2.3.3 Regieanweisungen }}^{178}$}

Beim letzten potenziellen $\mathrm{KH}$ handelt es sich um ein Phänomen, welches ich als Regieanweisung bezeichne. In den untersuchten Internetthreads lässt es sich vielfach finden: z.B. (7) $\mathrm{T}_{2} \mathrm{M}_{1}$ und $\mathrm{T}_{1} \mathrm{M}_{2}$, (9) $\mathrm{T}_{2} \mathrm{M}_{1}$, (17) $\mathrm{T}_{2} \mathrm{M}_{3}$ und $\mathrm{T}_{2} \mathrm{M}_{8}$, (24) $\mathrm{T}_{3} \mathrm{M}_{4}$ und $\mathrm{T}_{1} \mathrm{M}_{10}$, (31) $\mathrm{T}_{1} \mathrm{M}_{4}$, (14) $T_{1} M_{10}$ oder (46) $T_{1} M_{1}$ und $T_{1} M_{3}$. Die Regieanweisungen werden in der Regel durch Kursivierung und die Einrahmung mit Asterisken von der restlichen Mitteilung abgesetzt. Sie werden vom Schreiber verwendet, um entweder parallel zur Äußerung ausgeführt gedachte Handlungen ( ${ }^{*}$ noemb ${ }^{*}$ in (31) $\mathrm{T}_{1} \mathrm{M}_{4}$ ) oder die Art und Weise wie eine Äußerung lautlich realisiert wird (z.B. *приторно-сладким голосом* in (31) $\mathrm{T}_{1} \mathrm{M}_{3}$ ), anzugeben. Der Schreiber versucht eine gewisse Parallelität zwischen der verbalen und non-verbalen Seite der Äußerung herzustellen, indem er solche Regieanweisungen vor der ersten Wortform der Äußerung positioniert. ${ }^{179}$ Daraus ergibt sich ein Paradoxon: die Regieanweisung wird auf Grund der Linearität vor der Äußerung interpretiert, findet aber gleichzeitig parallel zur Äußerung als Kontextualisierungshinweis statt. In den untersuchten Threads standen Regieanweisungen im

\footnotetext{
${ }^{178}$ In diesem Abschnitt werden nur Beispiele behandelt, bei denen die Regienanweisungen vor der eigentlichen Äußerung stehen. Dies ist in den meisten Fällen der Fall.

${ }^{179}$ Regieanweisungen folgen nur selten der Äußerung (z.B. (14) $T_{1} M_{10}$ ).
} 
Gegensatz zu Akronymen niemals als selbstständige Reaktion in einer Mitteilung. Meiner These nach beeinflusst ihre Position vor den Äußerungen dabei die Erwartung des Lesers an den Inhalt dieser, d.h. wenn der Schreiber wie in (31) $\mathrm{T}_{1} \mathrm{M}_{4}$,singen“ als Regieanweisung schreibt, erwartet der Leser, dass die nachfolgende Äußerung z.B. einen Ausschnitt aus einer Liedstrophe beinhaltet. Die Linearität schriftlicher Äußerungen führt dazu, dass Regieanweisungen bereits vor der Äußerung interpretiert werden und den Interpretationsprozess bereits vor der ersten Wortform in eine bestimmte Richtung leiten sowie den ganzen Interpretationsprozess über vom Leser als KH mitinterpretiert werden. Die These, Regieanweisungen würden die Interpretation von vornherein beeinflussen, wurden zwei Experimenten, bei dem mehrere Probanden die Beispiele (45) - (47) laut vorlesen sollten, unterzogen. Im ersten Experiment lasen die Probanden die Äußerungen mit Regieanweisungen vor. ${ }^{180}$ Das zweite Experiment wurde wie bei den Emoticons über ein Jahr später wiederholt: diesmal erhielten die Probanden die gleichen Beispiele ohne Regieanweisung. Es wurden absichtlich mehrere Äußerungen verschiedener Beispiele gewählt, um die Wiederholbarkeit der Ergebnisse zu überprüfen. Da es bei den Experimenten nur um Regieanweisungen ging, wurden zunächst alle Emoticons und Akronyme entfernt. Danach wurden alle Beispiele auf die ein oder andere Weise manipuliert: die Formen der 3.Ps.Sg. wurden durch die der 1.Ps.Sg. ersetzt (vgl. (45) ržet > ržu), damit für die Leser eindeutig wurde, dass die Wortform auf die schreibende Person referiert, in (46) - (47) wurden Regieanweisungen von der Vf. eingesetzt, da die Originalbeispiele keine enthielten. Daraufhin wurden wie bei den Emoticons die Intonationskurven der gleichen Äußerungen einmal mit und einmal ohne Regieanweisung miteinander verglichen. Erwartet wurden Unterschiede in den Intonationskurven, in den mittleren Grundfrequenzen und im Tonhöhenumfang zwischen den Äußerungen mit und ohne Regieanweisung. ${ }^{181}$

In den Abbildungen 49 und 50 finden wir die Äußerung $\mathrm{T}_{1} \mathrm{M}_{3,1}$ des Beispiels (45)' und (45)', in den Abbildungen 51 und 52 die Äußerung $\mathrm{T}_{1} \mathrm{M}_{1,1}$ des Beispiels (46)' und (46)' und in den letzten beiden Abbildungen die Äußerung $\mathrm{T}_{1} \mathrm{M}_{1,1}$ des Beispiels (47)' und (47)'.

\footnotetext{
${ }^{180}$ Anhand des Beispiels (45), (45)' und (45)"' werden die durchgeführten Manipulationen aufgezeigt. So ist in (45)' die Änderung des Verbs von der 3.Ps.Sg. in die 1.Ps.Sg. zu sehen, während in (45)" die jeweiligen Regieanweisungen komplett fehlen.

${ }^{181}$ Die Intonationskurve in dieser und folgenden Abbildungen dieses Abschnitts, die eine Regieanweisung haben, ist erst nach dem Ende der Regieanweisung mit der jeweiligen Intonationskurve in der Abbildung ohne die Regieanweisung zu vergleichen. Für den Vergleich der mittleren Grundfrequenz und des Tonhöhenumfangs wurden nur die Äußerungen selbst, d.h. ohne Regieanweisung, herangezogen.
} 


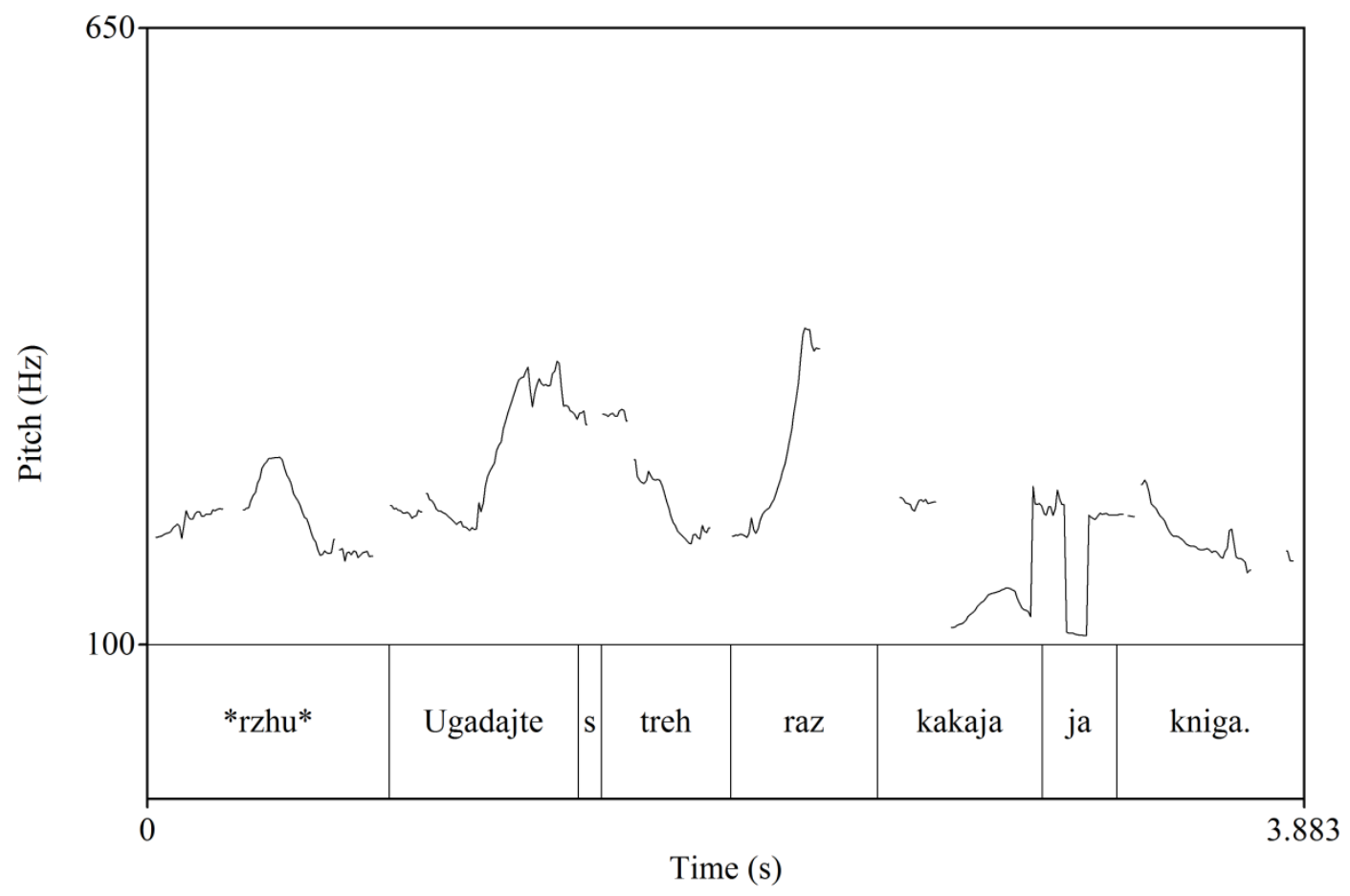

Abb. 49 Probandin 1 mit Regieanweisung

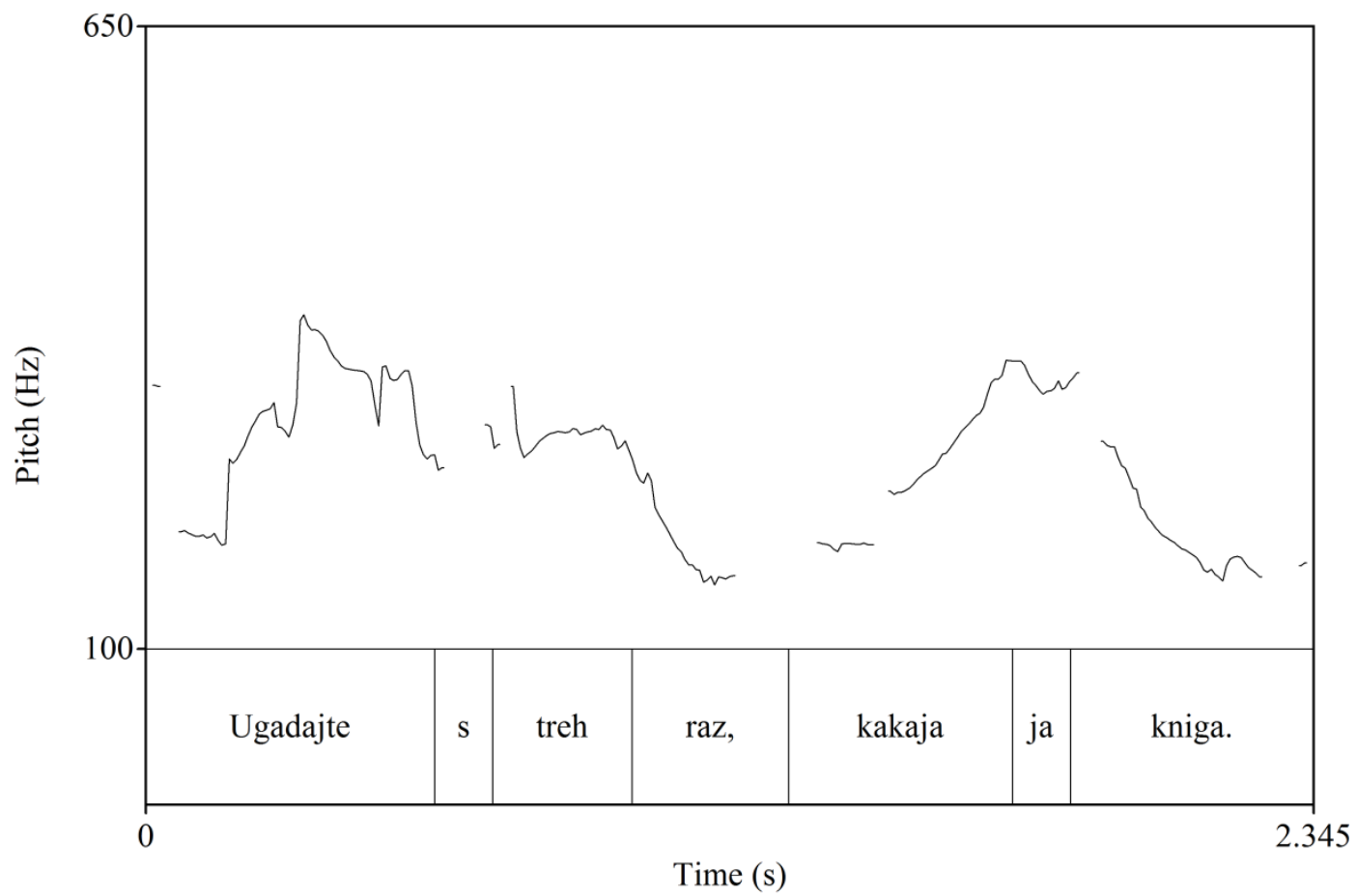

Abb. 50 Probandin 1 ohne Regieanweisung

Vergleicht man die beiden Intonationskurven, erkennt man deutliche Ähnlichkeiten zwischen 
ihnen. In beiden Fällen steigen die Kurven an und fallen bei treh bzw. bei raz, bevor sie wieder ansteigen und zum Ende der Äußerung hin wieder fallen. Dies geschieht unabhängig davon, ob eine Regieanweisung vorhanden ist oder nicht. Der Wert für die mittlere Grundfrequenz beträgt in der ersten Abbildung 224,42 Hz und in der zweiten 263,31 Hz. Der erste Wert liegt somit zwischen den Richtwerten für den niedrigen und den mittleren pitch level, der zweite zwischen den Richtwerten für den mittleren und hohen pitch level der Probandin. Die Äußerung mit der einer fröhlichen Emotion zugeordneten Regieanweisung, bei der ein höherer pitch level zu erwarten gewesen wäre, wurde mit einem niedrigeren pitch level vorgelesen als die gleiche Äußerung ohne Regieanweisung. Dies spricht dafür, dass die Regieanweisung für die Probandin keine Rolle gespielt hat. Unterstützt wird dies auch vom Ergebnis des Vergleichs des Tonhöhenumfangs: in der Äußerung mit Regieanweisung liegt dieser bei $274,1 \mathrm{~Hz}$ und in der Äußerung ohne bei 238,7 Hz. Beide Werte entsprechen dem Richtwert für einen mittleren Tonhöhenumfang. Obwohl der Wert für die Äußerung mit Regieanweisung höher ist als der andere, liegt er immer noch weit hinter dem Richtwert für einen hohen Tonhöhenumfang. Die Regieanweisung nimmt somit keinen Einfluss auf die Intonation und damit Interpretation der Äußerung.

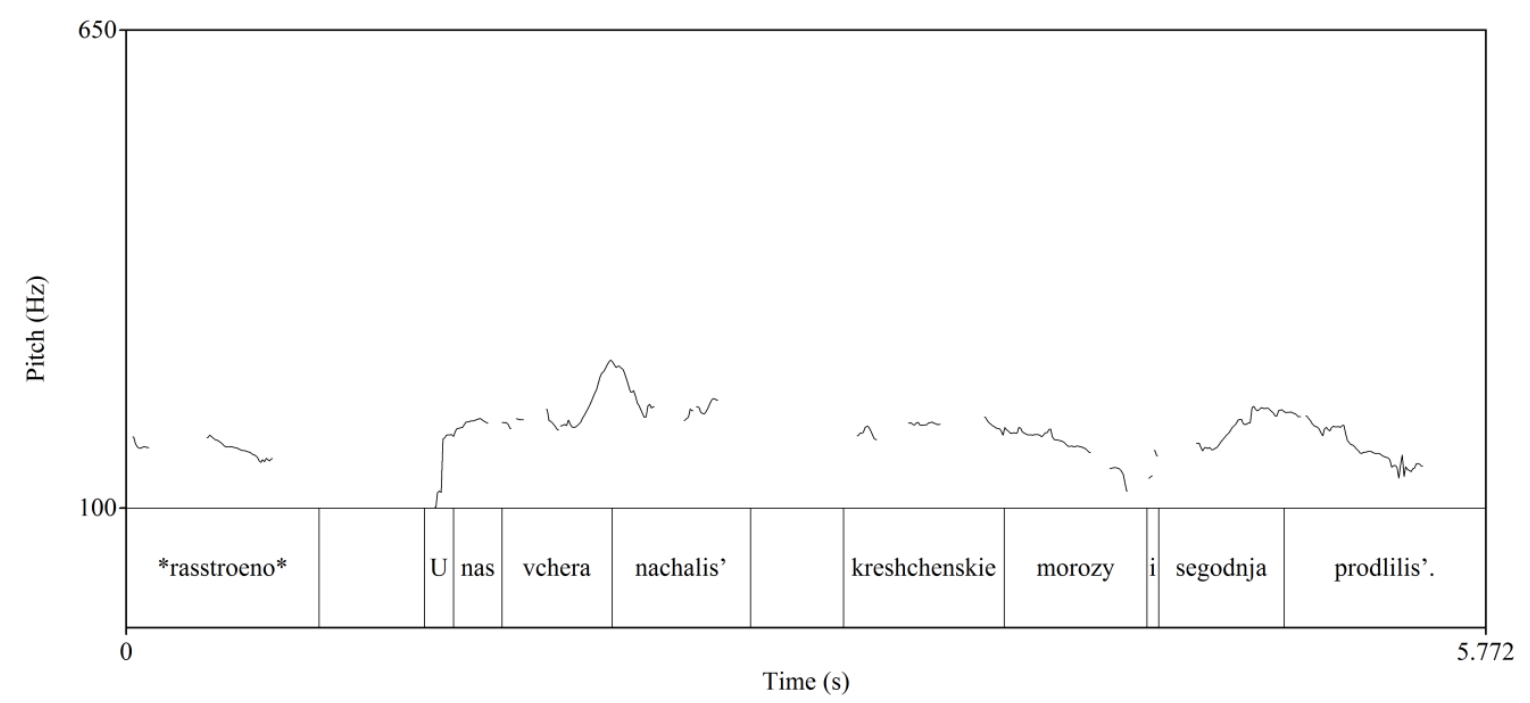

Abb. 51 Probandin 4 mit Regieanweisung 


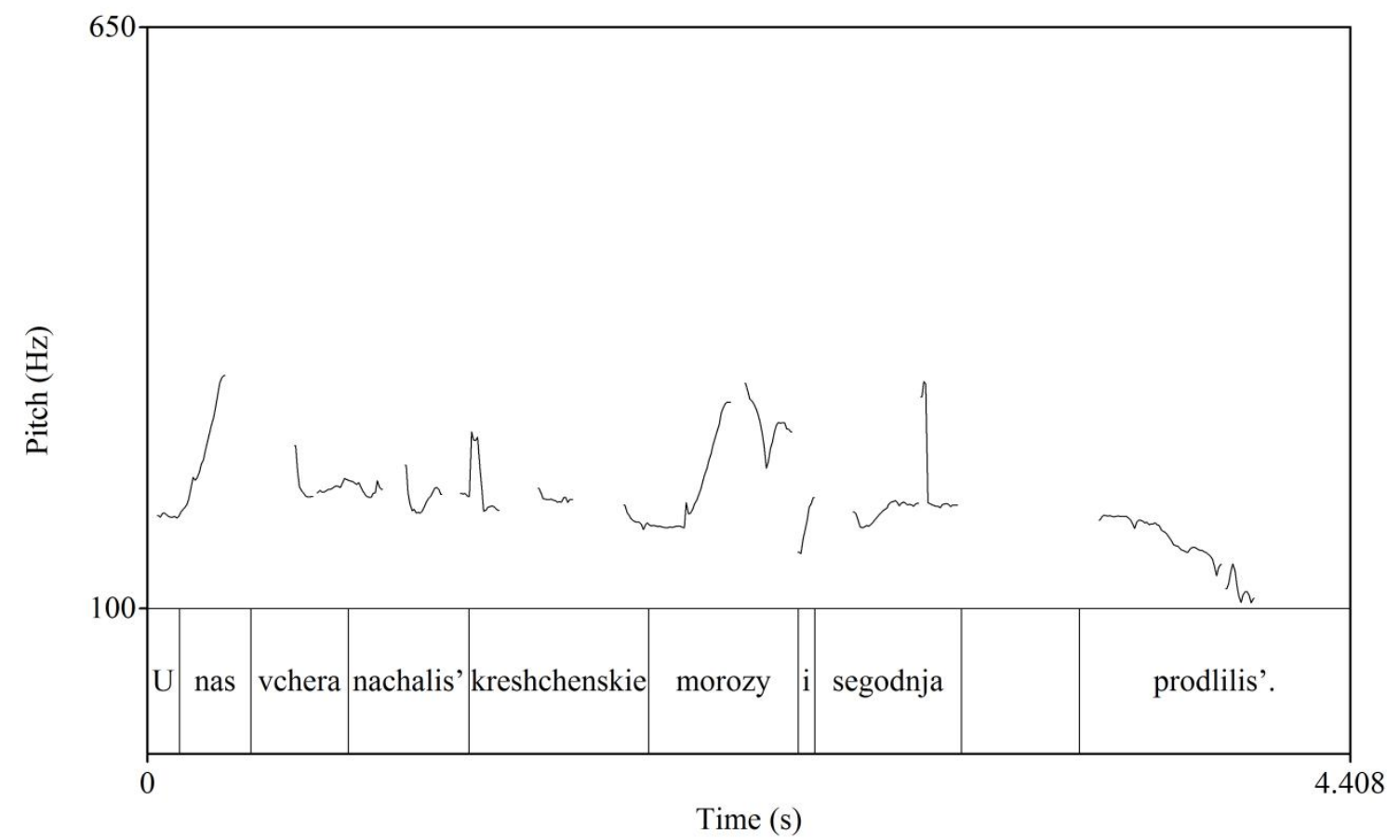

\section{Abb. 52 Probandin 4 ohne Regieanweisung}

Vergleicht man die Kurven miteinander, lassen sich mehrere Ähnlichkeiten feststellen. So steigt in beiden Abbildungen die IK zu Beginn der Äußerung an. Bei morozy fällt die Kurve und steigt dann wieder an. Am Ende der Äußerung fällt die IK in beiden Abbildungen. Die mittlere Grundfrequenz liegt für die Äußerung mit Regienanweisung bei 191,32 Hz und für die Äußerung ohne Regieanweisung bei 202,61 Hz. Trotz des Unterschieds liegen beide Werte im mittleren pitch level-Bereich. Das deutet darauf hin, dass die Regieanweisung keinen Einfluss auf die Intonation der Probandin hatte. Hätte sie einen Einfluss ausgeübt, hätte der Wert für die erste Äußerung im niedrigen pitch level- Bereich sein müssen, da die Regieanweisung auf eine negative Emotion („Enttäuschung“) hinweist. Dieser Befund wird durch den Vergleich des Tonhöhenumfangs gestützt: 169,2 Hz für die Äußerung mit und $215,1 \mathrm{~Hz}$ für die Äußerung ohne Regieanweisung. Beide Werte liegen zwischen den Richtwerten für einen mittleren und hohen Tonhöhenumfang. Obwohl der erste Wert niedriger ist, liegt er weit über dem Richtwert für einen niedrigen Tonhöhenumfang $(77 \mathrm{~Hz})$, so dass davon ausgegangen werden kann, dass die Regieanweisung nicht zu einer anderen (negativen) Interpretation der Äußerung geführt hat. 


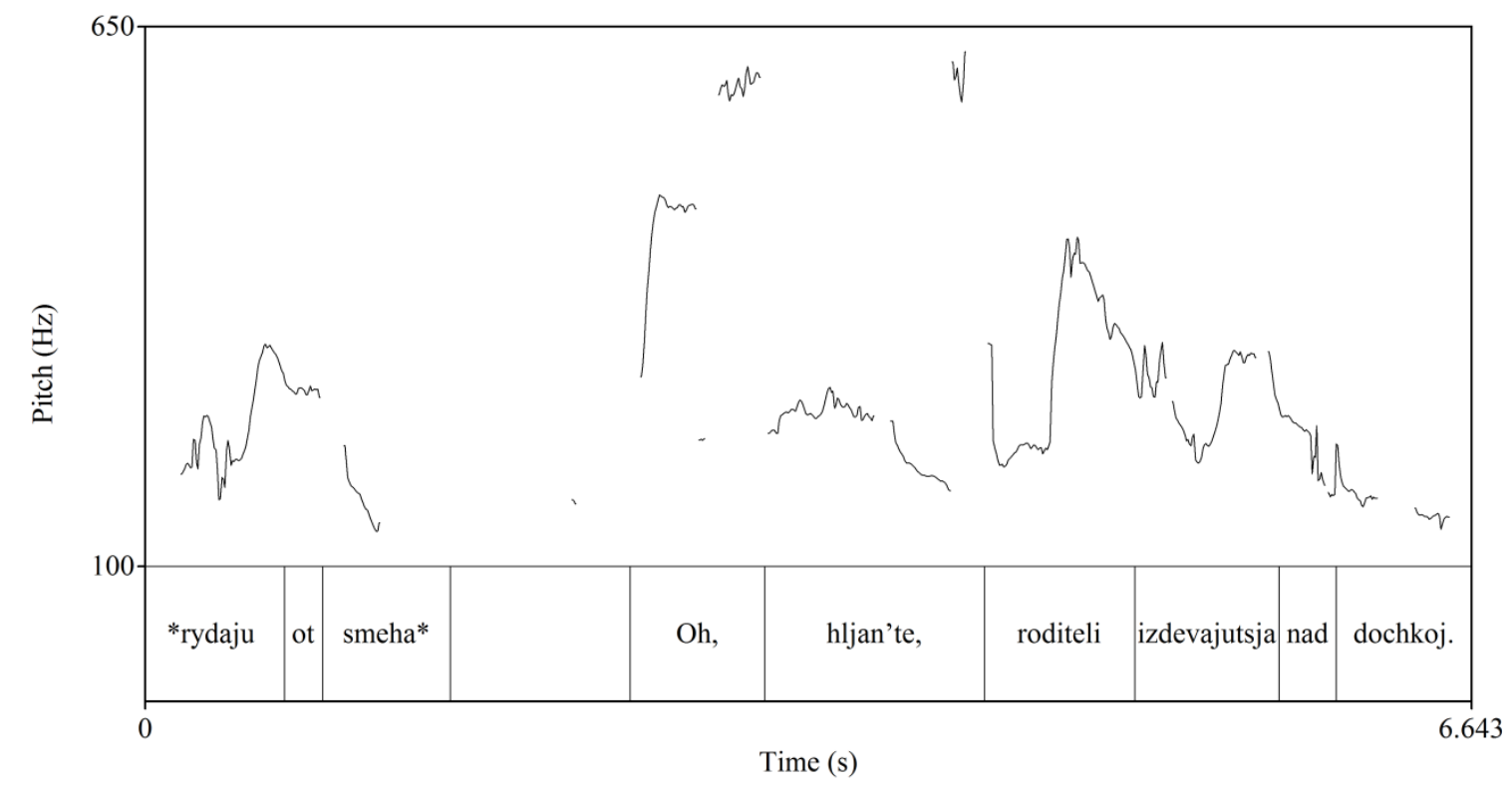

Abb. 53 Probandin 5 mit Regieanweisung

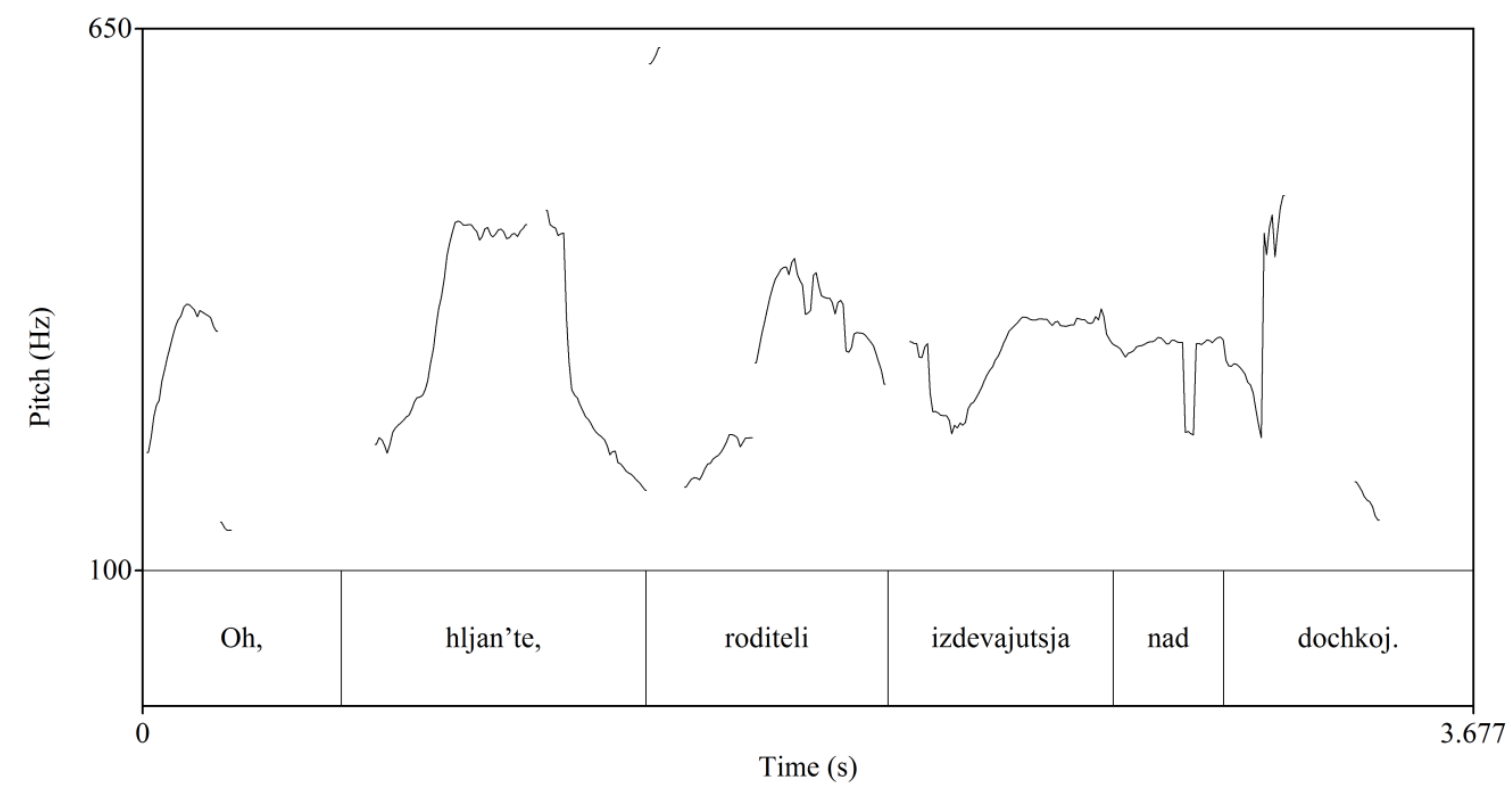

Abb. 54 Probandin 5 ohne Regieanweisung

In diesem Beispiel lassen sich mehrere Unterschiede und Gemeinsamkeiten zwischen den Intonationskurven feststellen. So steigt die Kurve zu Beginn der Äußerung steil an und fällt wieder. In der zweiten Abbildung gibt es im Gegensatz zur ersten Abbildung danach wieder einen steilen Anstieg, gefolgt von einem steilen Fall am Ende der Äußerung. In beiden Abbildungen gibt es bei roditeli einen steilen Anstieg der IK, gefolgt von einem Fall und erneutem Anstieg bei izdevajutsja. Während in der ersten Abbildung die IK danach bis zum Ende der Äußerung fällt, steigt sie in der zweiten Abbildung bei dočkoj nochmals kurz steilt 
an, bevor sie steil fällt. Die Unterschiede sind auf unterschiedliche Fokusakzente zurückzuführen: in der ersten Abbildung liegt der Fokus auf izdevajutsja und in der zweiten Abbildung auf dočkoj. Bezüglich der mittleren Grundfrequenz lässt sich feststellen, dass beide Werte über dem Richtwert für einen hohen pitch level liegen: 299,53 Hz für die Äußerung mit Regieanweisung und $321,53 \mathrm{~Hz}$ für die Äußerung ohne. Obwohl die Äußerung ohne Regieanweisung unterschiedlich interpretiert werden könnte (einmal humorvoll und einmal ernst oder traurig gemeint; zumal es sich hier um die erste Äußerung der Konversation handelt), entschied sich die Probandin für einen hohen pitch level. Dies erfolgte unabhängig von der Regieanweisung, so dass diese anscheinend keinen Einfluss auf die Interpretation nimmt. Dieses Ergebnis wird von dem Vergleich des Tonhöhenumfangs unterstützt: 492,1 Hz für die Äußerung mit Regieanweisung gegenüber 489,8 Hz für die Äußerung ohne. In beiden Fällen liegen die Werte über dem Richtwert der Probandin für einen hohen Tonhöhenumfang.

Insgesamt ist $\mathrm{zu}$ erkennen, dass sich in allen hier vorgestellten Fällen die Verläufe der Intonationskurven der Äußerungen mit und ohne Regieanweisungen im Großen und Ganzen ähneln. Das gilt auch für die Werte der mittleren Grundfrequenz sowie für die des Tonhöhenumfangs. Dies spricht dafür, dass Regieanweisungen keinen Einfluss auf die Intonation und Interpretation nehmen. Daraus lässt sich schließen, dass es für die Interpretation durch den Leser keinen Unterschied macht, ob eine Regieanweisung vor der Äußerung steht oder nicht. Die angenommene These, Regieanweisungen übten einen Einfluss auf die Intonation und somit Interpretation aus, wird durch das Experiment widerlegt. Im besten Falle können sie in einigen Fällen dem Leser dabei helfen, eine bestimmte Erwartungshaltung an den Fortgang der Äußerung stellen zu können (vgl. (31) $\mathrm{T}_{1} \mathrm{M}_{4}$ ). Hingegen wird die oben erwähnte Annahme verstärkt, dass die Intonation bereits beim Lesevorgang mitinterpretiert wird: entweder durch den vorherigen Kontext oder - falls es sich um die erste Äußerung einer Mitteilung handeln sollte - durch bestimmte Signalwörter, die einen bestimmten Intonationsverlauf markieren, vgl. Och. Insgesamt lässt sich daraus schließen, dass Regieanweisungen nicht mit „echten“ Kontextualisierungshinweisen vergleichbar sind, obwohl sie vor den Äußerungen stehen. Der Leser scheint diese nicht als KH wahrzunehmen. Da den Probanden jedoch kein Fragebogen vorgelegt wurde, lässt sich dies nicht abschließend klären. 


\subsubsection{Schlussfolgerungen}

Zusammenfassend können nun die Fragen beantwortet werden. Die Antwort auf die erste Frage ist, dass Schreibern in schriftlich realisierten Internetkonversationen eine Vielzahl an Mitteln als potenzielle Kontextualisierungshinweise zur Verfügung steht. $\mathrm{Zu}$ diesen zählen Satzzeichen, Großschreibung, Mehrfachvorkommen von graphischen Zeichen für Vokale und Konsonanten, farbliche Markierungen, Akronyme, Emoticons und Regieanweisungen. Um die zweite Frage und These zu überprüfen, wurden mehrere Experimente durchgeführt, bei denen Intonationskurven und teilweise mittlere Grundfrequenzen sowie der Tonhöhenumfang von Äußerungen mit und ohne jeweilige potenzielle KH verglichen wurden. Dabei sollte festgestellt werden, bei welchen potenziellen $\mathrm{KH}$ es sich um PseudoKontextualisierungshinweise und bei welchen um tatsächliche Kontextualisierungshinweise handelt. Die Idee, die hinter dem Vergleich dieser Faktoren steckt, ist, dass wenn die Probanden eine Äußerung gleich interpretieren, die Intonation ähnlich sein muss. Die Auswertung der Intonationskurven ergab für die Satzzeichen kein eindeutiges Ergebnis. In fast keinem Fall konnte ein Unterschied zwischen der Äußerung mit und der Äußerung ohne Ausrufezeichen oder Fragezeichen festgestellt werden. Nur in dem Beispiel, das im Original weder vom Kontext noch von einem lexikalischen Hinweis her als Frage zu erkennen war, in dem das Fragezeichen für die Probandin also unerwartet war, ließ sich ein Unterschied zwischen der Äußerung ohne und mit Fragezeichen ausmachen: die Probandin unternahm einen Korrekturversuch, sobald sie das Fragezeichen wahrgenommen hatte. Demnach könnten Satzzeichen durchaus eine Rolle als Kontextualisierungshinweise spielen. Da in der Mehrheit der Beispiele jedoch kein bedeutender Unterschied zwischen der Äußerung mit und ohne Satzzeichen feststellbar war, lässt sich abschließend sagen, dass sie meistens keinen Einfluss auf die Intonation haben und bei der Interpretation nicht beachtet werden. Im Ansatz konnte gezeigt werden, dass Signalwörtern hingegen eine Bedeutung bei der Interpretation zukommt. Bei der Großschreibung sowie bei dem Mehrfachvorkommen von Zeichen für Laute zeigte sich, dass diese Phänomene einen Einfluss auf die Intonation und somit auf die Interpretation ausübten. Das sind auch die Phänomene, die direkt an der Wortform durch den Leser wahrgenommen werden. Hierbei handelt es sich demnach um tatsächliche Kontextualisierungshinweise. Eine Besonderheit stellt farbliche Markierung dar. Sie ist das beste Beispiel dafür, dass Kontextualisierungshinweise konventionalisiert sein müssen. Die Konventionalisierung entsteht dabei durch die Koppelung an Hintergrundwissen. Der Leser muss wissen, was der entsprechende Kontextualisierungshinweis für eine Bedeutung hat, da 
der gleiche Hinweis in verschiedenen Foren auch verschiedene Bedeutung haben kann. So fungieren bestimmte Farben im zweiten Thread als Kontextualisierungshinweise, während sie im ersten Thread als Strategiemittel genutzt werden, um Aufmerksamkeit der Leser zu erregen oder sich von anderen Usern abzuheben. Handelt es sich bei ihrer Verwendung durch den User um einen Kontextualisierungshinweis, so muss eine bestimmte Farbe in dem Sinne konventionalisiert sein, dass sie mit einer bestimmten Bedeutung codiert ist, die einen Bestandteil des mikrogruppeninternen Hintergrundwissens bildet. Es lässt sich vermuten, dass farbliche Markierung die Interpretation beeinflussen kann, aber nur in dem Fall, wenn der Leser das entsprechende Hintergrundwissen, an das die Farbe geknüpft ist, besitzt. Bezüglich der Emoticons lässt sich sagen, dass sie unabhängig von ihrer Position offensichtlich keinen Einfluss auf die Intonation ausüben und somit keine Rolle für die Interpretation spielen; demnach Pseudo-Kontextualisierungshinweise sind. Dies bestätigt die angenommene These, Emoticons seien nicht mit Mimik und Gestik als Kontextualisierungshinweise vergleichbar. Entgegen der Erwartung üben Regieanweisungen keinerlei Einfluss auf die Intonation und damit die Interpretation aus, obwohl sie direkt vor der ersten Wortform der jeweiligen Äußerung stehen. Es ist $\mathrm{zu}$ vermuten, dass wenn die einleitende Position der Regieanweisungen und der Emoticons vor der Äußerung für die Interpretation keine Rolle spielt, dies auch bei Akronymen der Fall sein könnte.

Somit kann die zweite Frage folgendermaßen kurz beantwortet werden: nicht jeder in Frage kommende Kontextualisierungshinweis ist ein tatsächlicher Kontextualisierungshinweis. Zu Kontextualisierungshinweisen, deren Funktion mit denen in mündlichen Konversationen vergleichbar wäre, zählen nur die Großschreibung, das Mehrfachvorkommen graphischer Zeichen für Laute und in bestimmten Fällen farbliche Markierung. Alle anderen in Frage kommenden Phänomene sind nur Pseudo-Kontextualisierungshinweise, d.h. sie erfüllen zwar vermeintlich eine Funktion als KH, üben auf die Interpretation jedoch unabhängig von ihrer Position keine Rolle aus. Daraus ergibt sich folgende Tabelle:

\begin{tabular}{|l|l|}
\hline Tatsächliche Kontextualisierungshinweise & Pseudo-Kontextualisierungshinweise \\
\hline $\begin{array}{l}\text { Großschreibung, Mehrfachvorkommen } \\
\text { graphischer Zeichen für Laute, farbliche } \\
\text { Markierung (in bestimmten Fällen) }\end{array}$ & Emoticons, Regieanweisungen, Akronyme \\
\hline
\end{tabular}

Abb. 55 Kontextualisierungshinweise und Pseudo-Kontextualisierungshinweise 
In dieser Tabelle fehlen die Satzzeichen, da die Ergebnisse diesbezüglich nicht eindeutig waren. Die angenommene These, dass die Internetkommunikation zwar über eine Vielzahl an potenziellen Kontextualisierungshinweise verfügt, die meisten in schriftlich realisierten Gesprächen jedoch nicht die gleiche Funktion besitzen wie Kontextualisierungshinweise in mündlicher Kommunikation, kann somit bestätigt werden. Aber auch die Thesen, die für die einzeln zu prüfenden potenziellen Kontextualisierungshinweise aufgestellt wurden, können teilweise bestätigt werden.

Die Grafik ${ }^{182}$ zur eigenen Theorie muss demnach überarbeitet und ergänzt werden und sieht abschließend so aus:

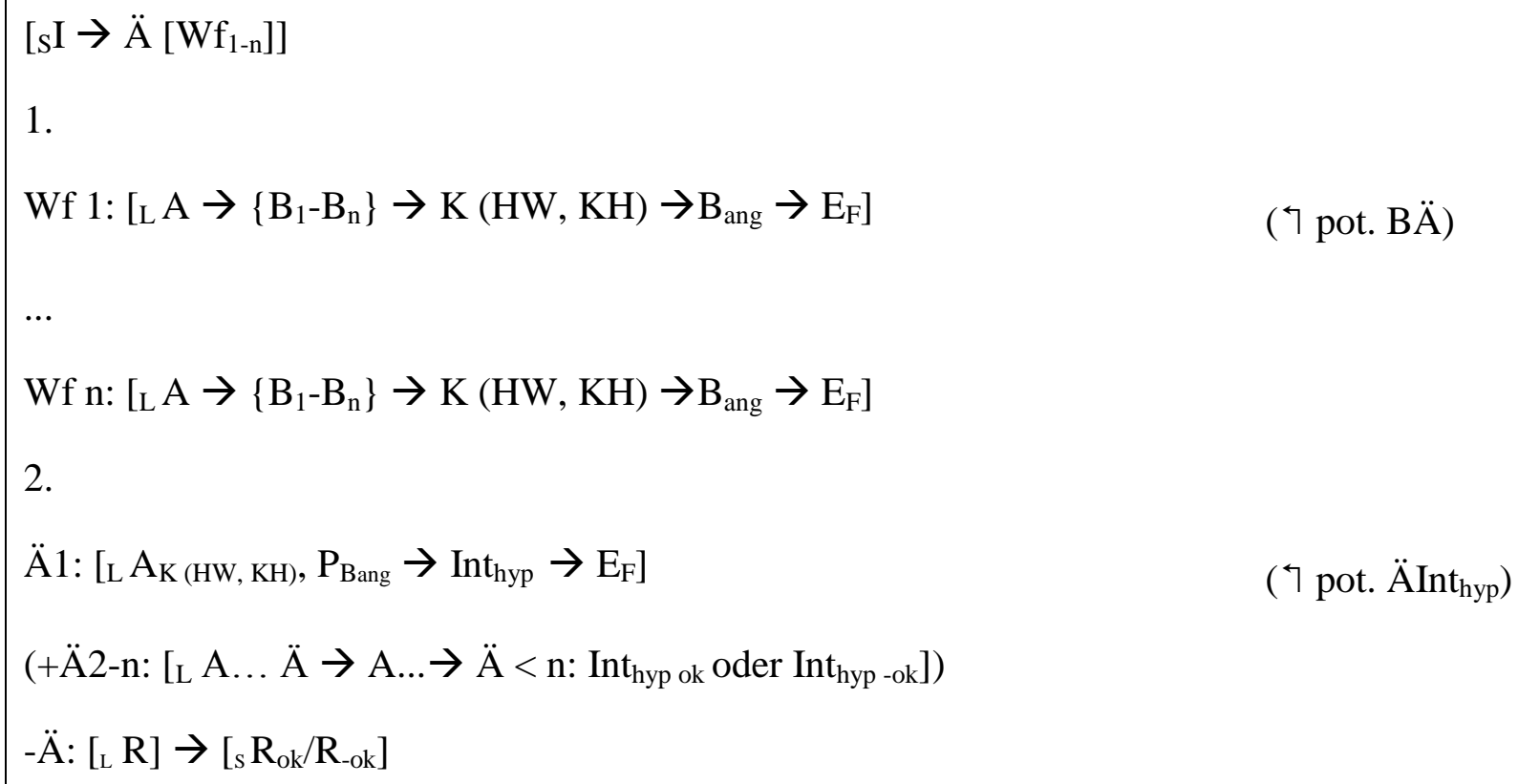

Der Schreiber (S) hat eine bestimmte Intention (I) und produziert auf ihrer Grundlage eine

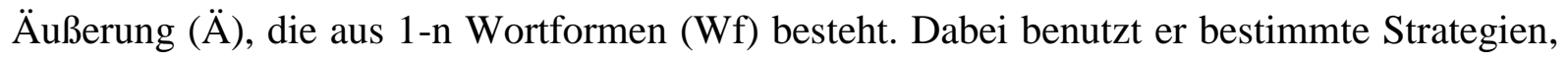
um seine Absicht zu verdeutlichen, seine Erwartungen an die Reaktion des Lesers (L) darzustellen und sein Ziel zu erreichen. Er muss jedoch Strategien verwenden, die vom Leser als solche erkannt werden. Sowohl beim Schreiber wie auch beim Leser müssen kommunikative Erfahrungen vorliegen, auf Grund derer der Leser die „Stimmung“ der Gesprächssituation (,was ist ernst gemeint und was nicht') deutet und mit derer Hilfe er aus

\footnotetext{
${ }^{182}$ Legende: $\mathrm{S}=$ Schreiber; $\ddot{\mathrm{A}}$ = Äußerung; Wf = Wortform; L = Leser; $\mathrm{A}=$ Analyse; $\mathrm{B}=$ Bedeutung; $\mathrm{K}=$ Kontext; $\mathrm{HW}=$ Hintergrundwissen; $\mathrm{KH}=$ Kontextualisierungshinweis; $\mathrm{B}_{\text {ang }}=$ angenommene Bedeutung; $\mathrm{E}_{\mathrm{F}}=$ Erwartung an Fortsetzung; pot. $\mathrm{B} \ddot{\mathrm{A}}=$ potentielle Bedeutungsänderung; $\mathrm{P}_{\mathrm{Bang}}=$ Prüfung der angenommenen Bedeutung; $\mathrm{Int}_{\text {hyp }}=$ hypothetische Interpretation; pot. ÄInt ${ }_{\text {hyp }}=$ potentielle Änderung der hypothetischen Interpretation; $\mathrm{R}=$ Reaktion.
} 
den Strategien heraus erkennt, welche Intention der Schreiber hat und welche Reaktion des Lesers gewünscht wird. Die Äußerung selbst muss dabei von vornherein formal und inhaltlich den gängigen Konventionen der jeweiligen Sprache entsprechen, damit Schreiber und Leser die gleiche Erwartungshaltung an den Verlauf der Konversation haben. Der Interpretationsvorgang der Äußerung erfordert zwei Schritte. Der erste Schritt betrifft die einzelnen Wortformen alleine und in ihrem Kontext $(\mathrm{K})$ und der zweite die vollständige Äußerung. Im ersten Schritt werden diese Wortformen nacheinander vom Leser einer phonologischen, morphologischen, syntaktischen und semantischen Analyse unterzogen. Dies ist insofern wichtig, als dass ,[d]ie semantische Repräsentation eines Wortes [...] den Beitrag dieses Wortes zur Bedeutung der Sätze (oder allgemeiner: der komplexen Ausdrücke), in denen es vorkommt [determiniert].“ (Bierwisch (1983, 61f.)). Nach der Analyse ergibt sich für den Leser mindestens eine Bedeutung (B) der Wortform. Eine Entscheidung für eine bestimmte Bedeutung der Wortform wird mit Hilfe des Kontextes, in den Hintergrundwissen (HW) und Kontextualisierungshinweise (KH) einfließen, getroffen. Dabei wird der Kontext innerhalb und außerhalb der Äußerung einbezogen, d.h. die unmittelbare und mittelbare „Umgebung“ der Wortform, da eine Wortform - genauso wie eine Äußerung - immer in Beziehung zu anderen Wortformen (und Äußerungen) steht und diese Beziehung den Kontext der Wortform ergibt. Mit Hilfe des Kontextes kann der Leser eine vorläufige Bedeutung annehmen $\left(\mathrm{B}_{\text {ang }}\right)$ und Erwartungen an den Fortgang $\left(\mathrm{E}_{\mathrm{F}}\right)$ der Äußerung stellen. Der gleiche Vorgang wird mit allen Wortformen der Äußerung wiederholt, wobei die angenommenen Bedeutungen der vorherigen Wortformen mit jeder hinzukommenden Wortform beibehalten werden können oder reanalysiert ( ७) und geändert werden müssen (pot. BÄ). Im zweiten Schritt wird schließlich die ganze Äußerung nochmals im Kontext analysiert und die angenommenen Bedeutungen geprüft. Auf dieser Grundlage entscheidet sich der Leser für eine hypothetische Interpretation der Äußerung ( $\operatorname{Int}_{h y p}$ ). Gleichzeitig bildet er Erwartungen bezüglich des weiteren Verlaufs der Konversation. Am Ende des Interpretationsvorgangs einer jeden weiteren Äußerung, wird die hypothetische Interpretation der vorherigen Äußerung entweder bestätigt oder verneint; im Fall einer Verneinung muss die vorherige Äußerung reanalysiert ( ) und die Interpretation entsprechend geändert werden. Abhängig davon, ob eine weitere Äußerung seitens des Schreibers erfolgt oder nicht, kann der Leser seine Interpretation in Form einer Reaktion äußern. Sobald der Leser sich entsprechend seiner Interpretation äußert, ist er der neue Schreiber und der Interpretationsvorgang findet auf Seite des vorherigen Schreibers und jetzigen Lesers statt. Der vorherige Schreiber kann dann in 
seinem nächsten Gesprächsschritt die Interpretation und Reaktion des vorherigen Lesers entweder bestätigen oder verneinen.

Abschließend lässt sich sagen, dass der Leser eine Äußerung bereits von vornherein mit einer bestimmten Intonation liest. Diese hängt jedoch weder von Pseudo- noch von den tatsächlichen Kontextualisierungshinweisen ab. Stattdessen wird sie offensichtlich durch Kontext und Signalwörter bestimmt.

Zusammenfassend lässt sich sagen, dass die Methode Intonationskurven zu vergleichen, eine gute Möglichkeit darstellt, den Einfluss von Satzzeichen und Großschreibung auf die Intonation und damit Interpretation messbar zu untersuchen. Die Ergebnisse im Bereich der Emoticons und gesternter Handlungen zeigen, dass sich der Vergleich der Intonationsverläufe in Kombination mit einem Vergleich von mittleren Grundfrequenzen und des Tonhöhenumfangs lohnt, um den Einfluss von Emoticons und Regieanweisungen auf die Intonation und somit Interpretation zu bestimmen. Jedoch stößt man bei dieser Methode auf Probleme: so ist bspw. nicht jeder Vorleser gleichermaßen gut geeignet, da einige sehr monoton vorlesen. Außerdem kam es gerade bei unbekannten Worten zu Leseschwierigkeiten in Form von Versprechern und Korrekturen sowie dem Abbruch des Lesevorgangs mitten im Wort und dem Ansetzen nach einer kurzen Pause. Solche Intonationskurven sind für die entsprechende Untersuchung weniger brauchbar. In den im Rahmen dieser Dissertation durchgeführten Experimenten, führte das erste Problem dazu, dass die Ergebnisse von weniger Probanden genutzt werden konnten, als Probanden involviert waren. Das zweite Problem wurde umgangen, indem eine Vielzahl unterschiedlicher Konversationsbeispiele verwendet wurde, so dass es eine große Auswahl an zu untersuchenden Äußerungen gab. Trotzdem dieser Probleme lohnt sich der Vergleich, da so festgestellt werden konnte, welche potenziellen Hinweise als ,echte“ Kontextualisierungshinweise in schriftlich realisierter Kommunikation fungieren.

In Zukunft müssen weitere Versuche mit mehr Teilnehmern durchgeführt werden, um die hier erbrachten Resultate auf den Prüfstand zu stellen. Bezüglich der Satzzeichen müssten noch weitere Experimente gemacht werden - auch mit Sprachen, in denen die Satzzeichen vor der Äußerung stehen (bspw. Spanisch). Besonders in Bezug auf die Emoticons ist die Frage zu stellen, welche Rolle das Alter und die Internetaffinität der jeweiligen Probanden spielen. Ein zukünftiger Vorlese-Test sollte zusammen mit einer Eye-Tracking-Studie durchgeführt werden, um Klarheit darüber zu erhalten, ob Emoticons beim Lesen beachtet werden, d.h. ob 
der Leser sie überfliegt oder länger bei ihnen verweilt, was auf eine Interpretation hindeuten würde, und wenn dies der Fall ist, ob ihre Position eine Rolle spielt. Weitere Versuche müssen zu der Kombinatorik von potenziellen Kontextualisierungshinweisen durchgeführt werden, die hier nicht diskutiert wurde. Auffällig ist jedoch, dass häufig z.B. mehrere unterschiedliche Emoticons oder Emoticons und Akronyme miteinander kombiniert werden, z.B. (33) $\mathrm{T}_{1} \mathrm{M}_{4}$, in dem ein einfaches Emoticon, ein Akronym und ein komplexeres Emoticon nacheinander auftauchen. Unter anderem müssten die Fragen geklärt werden, welche Funktion dies erfüllt (möglicherweise soll das Zusammenspiel mehrerer Kontextualisierungshinweise in der schriftlichen Kommunikation dem Zusammenspiel mehrerer Kontextualisierungshinweise in mündlichen Gesprächen entsprechen) und ob es einen Einfluss auf die Intonation ausübt. 


\section{Schritt-für-Schritt Diskussion eines authentischen Beispiels}

Im folgenden Kapitel werden die vorgestellten theoretischen Annahmen zum Interpretationsvorgang anhand des längeren Konversationsbeispiels (48) aus dem zweiten Forumthread überprüft. Entsprechend meines Modells erfolgt eine Schritt-für-Schritt Diskussion einer jeden Äußerung.

\section{1. $\mathrm{T}_{1} \mathrm{M}_{1,1} \quad$ bogda a ty čto polinjal?}

Intention: Die Schreiberin Lelika äußert sich mit der Intention, Information zu erhalten. Sie möchte eine Antwort auf ihre Frage bekommen und zwar von bogda, der adressiert wird. Als Strategie verwendet Lelika dabei eine Frage. Eventuell kennt Lelika, bereits die Antwort auf ihre Frage, möchte aber eine Bestätigung von bogda sowie den Grund wissen, der für sie nicht ersichtlich ist.

Wf 1: bogda: Nom. Sg.m./f.. Aus gruppenint. HW heraus ist dem Leser bekannt, dass es sich bei bogda um einen Usernamen handelt.

Wf 2: $a$ : Konj./Part. ,und', ,aber', ,sondern'; ,wie?'.

Wf 3: ty: Pron. 2.Ps.Sg. ,du’.

Wf 4: čto: Pron. Nom./Akk.Sg.n. / Konj. / Part. ,was'; ,dass'. A ty čto wird idiomatisch verwendet. Der Leser erwartet, dass es sich bei dieser Äußerung um eine Frage handelt, čto ist dabei ein lexikalischer Hinweis.

Wf 5: polinjal: 1.-3. Ps.Sg.m. Prät. pf. ,abfärben', ausbleichen', , haaren/sich mausern/sich häuten'. Auf Grund der Endung vermutet der russischsprachige Leser, dass es sich bei bogda um einen männlichen User handelt. Er weiß, dass Farbe von bspw. Kleidungsstücken durch chemische Prozesse abfärben oder ausbleichen kann und dass Tiere Fell wechseln, sich mausern (Vögel) bzw. sich häuten (Schlangen u.a). Da es keinen Bezug auf einen Menschen gibt, passt hier keine der Bedeutungen. An dieser Stelle ist gruppeninternes HW von Bedeutung: Farbe spielt in diesem Forum in Bezug auf Usernamen eine Rolle; je nach Funktion des Users (Administrator, Supermoderator, Moderator), hat sein Username eine bestimmte Farbe. Der Leser kann mit Hilfe dieses Wissens einen Bezug der Wf zur Farbe des Usernamens herstellen und vorläufig die Bedeutung ,ausbleichen“ annehmen. Gleichzeitig implikatiert ein Ausbleichen der Farbe des Usernamens, dass der User seine Funktion im 
Forum nicht mehr ausübt.

Ä 1: Den Kontext bildet ein Thread in einem Forum für Programmierer und Hacker bzw. Interessierte an diesem Thema, in dem alles besprochen werden darf. Mit Hilfe seines gruppenint. HW weiß der Leser, dass die einzelnen User, die eine Funktion ausüben, farblich gekennzeichnet sind. Čto fungiert als lexikalischer Hinweis auf eine Frage. Mit a ty čto wird gleichzeitig Erstaunen bzw. Überraschung implikatiert. Für die Schreiberin ist die Handlung, die den Adressaten betrifft, unerwartet.

Hyp. Int.: Interpretation der Äußerung als Erkundigung der Schreiberin Lelika danach, ob die Farbe des Usernamens des Adressaten bogda ,ausgeblichen“ ist, was implikatiert, dass er seine Funktion im Forumthread nicht mehr ausübt. Im weiteren Verlauf erwartet der Leser entweder eine weitere Äußerung von Lelika, die mit der ersten Frage zusammenhängt oder die Reaktion von dem Adressaten bogda, indem er die Frage beantwortet.

$+\ddot{A}:$ Der Turn des Schreibers ist noch nicht beendet, da noch eine weitere Äußerung des Schreibers folgt.

2. $\mathrm{T}_{1} \mathrm{M}_{1,2} \quad$ ili ušel s moderatorov?

Intention: Die Schreiberin Lelika präzisiert die vorherige Frage und fragt direkt danach, ob bogda kein Moderator mehr sei, wobei betont wird, dass er den Posten als Moderator selbst aufgegeben habe. Es wiederholt sich die Strategie der ersten Äußerung, da Lelika entweder bereits weiß oder zumindest vermutet, dass bogda kein Moderator mehr ist, dafür aber eine Bestätigung haben möchte und den Grund hierfür erfahren möchte, ohne direkt danach zu fragen.

Wf 1: ili: Konj. ,oder'.

Wf 2: ušel: 1-3.Ps.Sg.m.Prät.pf., weggehen'. Auf Grund der Endung vermutet der Leser, dass es sich bei bogda um einen männlichen User handelt.

Wf 3: s: Präp. ,mit', ,seit', ,ab', ,von', ,aus'. Obwohl nach der Präposition auch ein Substantiv im Instrumental stehen kann, erwartet man hier auf Grund des Kontextes ein Substativ im Genitiv. 
Wf 4: moderatorov: Gen./Akk.P1.m. ,Moderator'. Als Leser weiß man, dass es sich hierbei um eine Funktion/einen Posten in einem Forum handelt. Der Moderator wacht dabei über die Einhaltung von Verhaltensregeln bei der Kommunikation im Forum und straft Fehlverhalten $\mathrm{ab}$.

Ä 2: Den Kontext bildet Lelikas Frage danach, ob bogda ausgeblichen ist, was vom Leser vorerst so interpretiert wird, dass bogda seine Funktion im Forumthread nicht mehr ausführt. Aus seinem HW heraus, weiß der Leser, dass User von dem Posten als Moderator zurücktreten können. Da die vorherige Äußerung eine Frage war, wird durch das ili eine weitere Frage eingeleitet, das ili deutet somit als lexikalischer Hinweis auf die Äußerung als Frage hin. Da das Subjekt fehlt, ist davon auszugehen, dass es referenzidentisch mit bogda ist.

Hyp. Int.: Interpretation der Äußerung als Frage, ob bogda vom Moderatorposten zurückgetreten ist. Die angenommene hypothetische Interpretation der ersten Äußerung wird durch die zweite Äußerung bestätigt: bei der Wf polinjat' geht es tatsächlich um eine Farbänderung des Usernamens und damit verbunden um eine Funktionsänderung des Users. Der Leser erwartet eine Reaktion seitens bogda, in der er auf diese Fragen eingeht.

- ̈̈: Es folgt keine weitere Äußerung, so dass ein Partizipantenwechsel stattfindet und sich ein Leser als neuer Schreiber äußern kann.

3. $\mathrm{T}_{1} \mathrm{M}_{2,1}$ a on byl moderom? [+ Emoticon] [+ Emoticon]

Intention: Der Schreiber AgentMario äußert sich mit der Intention sein Erstauen/seine Überraschung darüber auszudrücken, dass bogda Moderator war. Anstatt eine Aussage wie „,ich wusste nicht, dass bogda Moderator war“ zu machen, stellt der Schreiber eine Frage, was sein Erstaunen/seine Überraschung deutlich zur Geltung bringt.

Wf 1: a: Konj./Partikel. ,und', ,aber', ,sondern'; ,wie?'.

Wf 2: on: Pron. 3.Ps.Sg.m. ,er'. Aus dem Kontext der vorherigen Äußerung weiß der Leser, dass on sich auf bogda bezieht.

Wf 3: byl: Verb, 1.-3.Ps.Sg.Prät. ,sein'. Formal gibt es nur die Beschränkung, dass wenn ein Substantiv oder Adjektiv folgt, es im Instrumental oder Nominativ stehen muss. 
Wf 4: moderom: Instr.Sg.m. ,Moderator'. Aus seinem Hintergrundwissen heraus weiß der Leser, dass es sich bei moder um eine gekürzte Form von moderator handelt.

Ä 1: Der Kontext der Äußerung ist, dass bogda offensichtlich kein Moderator mehr ist. Das weiß man als Leser aus den vorherigen Äußerungen von Lelika. Aus dem Kontext weiß man, dass es sich hier um eine Frage handelt, da eine Aussage mit diesem lexikalischen Gehalt nicht angemessen wäre. Es ist nicht eindeutig, ob es eine Konvention in dem Forum gibt, dass der Adressat zuerst antwortet: sollte es diese Konvention geben, hält sich AgentMario nicht an diese, sollte es sie jedoch nicht geben, verhält er sich entsprechend dem Partizipantenwechsel in der Threadkommunikation, nämlich dass sich derjenige als erster äußert, der die Mitteilung als erster liest und darauf eingehen möchte.

Hyp. Int.: Interpretation der Äußerung von AgentMario so, dass er nicht wusste, dass bogda überhaupt Moderator war. Möglicherweise war bogda nicht lang genug Moderator. Da es sich um eine Frage handelt, auf die die Antwort bereits aus der zweiten Äußerung von Lelika ersichtlich ist, erwartet der Leser auf sie keine Reaktion.

+ ̈̈: Der Turn des Schreibers ist noch nicht beendet, da noch eine weitere Äußerung des Schreibers folgt.

4. $\mathrm{T}_{1} \mathrm{M}_{2,2} \quad$ Emu kak predupreždenie vypisali, tak v zvanii i ponizili [+ Emoticon]

Intention: Der Schreiber AgentMario schreibt, dass bogda eine Verwarnung erhalten und sein Rang herabgestuft wurde. Seine Intention ist es, zu erzählen, dass es einen Vorfall gab, für den bogda bestraft wurde, woraus er folgert, dass bogda deswegen kein Moderator mehr ist.

Wf 1: Emu: Pron. 3.Ps.Sg.m. Dat. „er“满 Durch den Kontext wird vorgegeben, dass er = bogda ist.

Wf 2: kak: Konj./Adv. ,wie'.

Wf 3: predupreždenie: Nom./Akk.Sg.n. ,Verwarnung'. Aus seinem HW heraus, weiß der Leser, dass User eines Forums wegen Fehlverhaltens verwarnt werden können. Der Leser erwartet, dass ein Verb kommt, welches z.B. die Bedeutung, ausstellen' hat.

Wf 4: vypisali: 1-3.Ps.P1.Prät.pf. ,heraussschreiben', ,bestellen', , abonnieren', , aus der Liste 
streichen', ,einem Patienten den Entlassungsschein geben', ,ausschreiben', ,ausstellen'. Aus dem Kontext der vorigen Wf (und des HW) weiß der Leser, dass User auf Grund von Fehlverhalten im Forum eine Verwarnung erhalten können. Auf dieser Grundlage entscheidet sich der Leser für die Bedeutung, ausstellen’ oder , ausschreiben'.

Wf 5: tak: Konj./Adv. ,so'. Idiomatischer Gebrauch mit kak und $i$ zusammen: ,sowohl als auch'. Erwartung der Konj. $i$.

Wf 6: v: Präp. ,in’. Formale Restriktion: es folgt eine Wf im Lok.

Wf 7: zvanii: Lok.Sg.n. ,Rang', ,Dienstgrad'. Als Leser weiß man aus seinem HW heraus, dass User in Foren einen Rang besitzen. Die Rangstufe kann dabei z.B. durch die Anzahl von hilfreichen Mitteilungen (dabei bestimmen andere User, was eine hilfreiche Mitteilung ist) bestimmt werden. Unter Umständen kann es mit einem großen Aufwand verbunden sein, den nächsthöheren Rang zu erreichen. Daher ist die Herabstufung des Rangs eine Strafe für einen schwereren Regelverstoß. Erwartung des Verbs ponizit', da idiomatischer Gebrauch.

Wf 8: i: Konj. ,und', ,auch', ,sogar'. Idiomatischer Gebrauch mit kak und tak zusammen: ,sowohl als auch'.

Wf 9: ponizili: 1.-3.Ps.P1.Prät.pf. ,senken', ,herabsetzen'. In Kombination mit v zvanii trägt es die Bedeutung ,degradieren', da idiomatischer Gebrauch.

Ä 2: Den Kontext bildet Lelikas Frage danach, ob bogda kein Moderator mehr ist. Aus seinem HW heraus, weiß der Leser, dass bogda entweder selbst zurückgetreten ist oder „gefeuert“ wurde, weil er selbst gegen eine wichtige Regel/Regeln verstoßen hat. Der Regelverstoß muss dabei schwerwiegend gewesen sein. Da die Äußerung keine direkte Antwort auf Lelikas Fragen ist, handelt es sich bei ihr um eine Implikatur.

Hyp. Int.: Interpretation der Äußerung als Implikatur, dass bogda gegen mindestens eine wichtige Regel verstoßen hat, was seine Verwarnung und Degradierung zur Folge hatte. Implikatiert wird ebenfalls, dass bogda den Moderatorstatus als Folge dieser Verwarnung und Degradierung verloren hat, wobei nicht klar gestellt wird, ob ihm der Status entzogen wurde oder er selbst zurückgetreten ist. Beide Interpretationen sind möglich. Der Leser erwartet eine weitere Äußerung des Schreibers, in der der Grund für die Bestrafung erläutert wird, eine Reaktion seitens Lelika, die nachfragt, warum bogda bestraft wurde und möglicherweise woher AgentMario das weiß (= war er selbst in der Situation dabei?) oder eine Reaktion 
seitens bogda.

- ̈̈: Es folgt keine weitere Äußerung, so dass ein Partizipantenwechsel stattfindet und ein Leser sich als neuer Schreiber äußern kann.

5. $\mathrm{T}_{1} \mathrm{M}_{3,1} \quad$ za čto predupreždenie?

Intention: Intention der Schreiberin Lelika mithilfe einer Frage zu erfahren, wofür bogda verwarnt wurde.

Wf 1: za: Präp. ,hinter', ,an', während', ,bei', , außerhalb', ,für', ,wegen'. Erwartung einer Wf im Instr., Akk. oder Gen.

Wf 2: čto: Nom./Akk.Sg.n. / Konj. / Part. ,was'; ,dass'. Da čto hier ein Interrogativpronomen ist, dient es als Hinweis auf eine Frage. Vorherige Wf $z a$ hat hier die Bedeutung ,für' oder ,wegen'.

Wf 3: predupreždenie: Nom./Akk.Sg.n. ,Verwarnung'. Aus seinem HW heraus, weiß der Leser, dass User für Regelverstöße verwarnt werden können.

Ä 1: Den Kontext bildet die Äußerung von AgentMario, in der implikatiert ist, bogda sei für einen Regelverstoß verwarnt und degradiert worden. AgentMario erklärt jedoch nicht, wofür bogda verwarnt wurde. Da der Leser aus seinem HW heraus weiß, dass User für eine Vielzahl an Verstößen Verwarnungen erhalten können, kommt somit eine Vielzahl an Regelverstößen in Frage.

Hyp. Int.: Interpretation der Äußerung so, dass Lelika wissen möchte, wofür bogda eine Verwarnung ausgestellt wurde. Erwartung einer weiteren Äußerung von Lelika, in der sie vermutet, wofür bogda verwarnt wurde, einer Antwort von AgentMario oder einer Reaktion von bogda, in der er erläutert, wofür er verwarnt und degradiert.

$+\ddot{A}:$ Der Turn des Schreibers ist noch nicht beendet, da noch eine weitere Äußerung des Schreibers folgt. 
6. $\mathrm{T}_{1} \mathrm{M}_{3,2} \quad$ zaflud?

Intention: Die Schreiberin Lelika konkretisiert ihre vorherige Frage.

Wf 1: za: s.o. Präp. ,für', ,wegen'.

Wf 2: flud: Nom./Akk.Sg.m. Als Leser weiß man aus seinem HW heraus, dass flud (fludit') aus dem Englischen (flood/to flood) entlehnt wurde und dass es in Foren bestimmte Vorgänge bezeichnet: 1. das Verfassen einer thematisch unpassenden Mitteilung und 2. das Fluten eines Systems mit vielen gleichzeitigen Anfragen, was einen Zusammenbruch des Systems zur Folge hat (technischer flood $\rightarrow$ das ist die Bezeichnung, die im Englischen verwendet wird, während im Russischen flud für beides benutzt wird). Beide Vorgänge werden in Foren (unterschiedlich stark) geahndet. Gerade der erste Vorgang kommt sehr häufig vor.

Ä 2: Den Kontext bildet die letzte Äußerung von AgentMario und Lelikas Nachfrage. Aus seinem HW heraus, weiß der Leser, dass Flud in Foren immer geahndet wird. Da bogda jedoch nur eine Verwarnung erhalten haben soll, ist eher von dem Verfassen einer thematisch unpassenden Mitteilung auszugehen, da man für den anderen Vorgang aus dem Forum als Mitglied ausgeschlossen wird. Aus dem HW heraus weiß man als Leser auch, dass viele User thematisch unpassende Mitteilungen schreiben (fludit') und es sich hierbei um einen häufig vorkommenden Regelverstoß handelt.

Hyp. Int.: Interpretation der Äußerung als Mutmaßung von Lelika, dass bogda für Flud verwarnt wurde, entweder weil sie weiß, dass bogda so etwas tut oder weil das einer der häufigsten Gründe für eine Verwarnung in dem Forum ist. Erwartung einer Antwort von AgentMario oder einer Reaktion von bogda, in der einer von beiden erläutert, wofür bogda verwarnt und degradiert wurde.

- ̈̈: Es folgt keine weitere Äußerung, so dass ein Partizipantenwechsel stattfindet und ein Leser sich als neuer Schreiber äußern kann.

7. $\mathrm{T}_{1} \mathrm{M}_{4,1} \quad$ za oskoblenija.

Intention: Der Schreiber AgentMario beantwortet die Fragen, indem er klarstellt, wofür bogda verwarnt wurde. 
Wf 1: za: s.o. Präp. ,für', ,wegen’.

Wf 2: oskoblenija: Als Leser weiß man trotz der Schreibfehlers, dass es sich bei der Wf um oskorblenija Nom./Akk.Pl.n. ,Beleidigung' handelt. Er weiß, dass Beleidigungen als grober Regelverstoß in Foren gelten und geahndet werden, häufig mit einem Ausschluss vom Forum endend. Dabei wird derjenige User stärker bestraft, der als Erster eine Beleidigung äußert.

Ä 1: Den Kontext bildet die letzte Äußerung von AgentMario, einschließlich seiner Implikatur, und Lelikas Nachfrage. Aus seinem HW weiß der Leser, dass es sich bei einer Beleidigung um einen groben Verstoß gegen die Forumsregeln handelt, der wesentlich härter bestraft wird als Flud. Dabei wird vor allem derjenige härter bestraft, der angefangen hat. In den meisten Foren ist ein Ausschluss häufig die Folge.

Hyp. Int.: Die Äußerung interpretiert der Leser so, dass bogda seine Strafe, in Folge derer er laut der Implikatur von AgentMario kein Moderator mehr ist, nicht für Flud, sondern für die Beleidigung eines anderen Users oder Gastes bekommen hat. Es ist nicht klar, ob bogda als Erster angefangen hat, da seine Strafe jedoch hart war, ist dies möglich. Erwartung entweder einer Reaktion von Lelika, in der sie fragt, was vorgefallen ist, oder einer Reaktion von bogda, in der er bestätigt, kein Moderator mehr zu sein und erläutert, was passiert ist.

- ̈̈: Es folgt keine weitere Äußerung, so dass ein Partizipantenwechsel stattfindet und ein Leser sich als neuer Schreiber äußern kann.

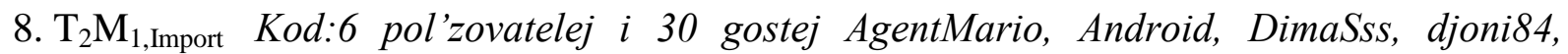
DrShaman, Lelika

Intention: Der Schreiber DimaSss intendiert anzuzeigen, wie viele User und Gäste zum Zeitpunkt des Verfassens der Mitteilung gleichzeitig online sind.

Es handelt sich hier um ein abrupt eingeschobenes, neues Thema. Ein solcher abrupter Themenwechsel ist Bestandteil der Konventionen und somit für die Gesprächspartizipanten nicht weiter ungewöhnlich.

Wf 1: Kod: Nom.Sg.m. ,Code’ Aus seinem HW heraus, weiß der Leser, dass es Foren gibt, wo nur mit Hilfe eines Codes herausgefunden werden kann, wie viele Personen und wer genau zu einem bestimmten Zeitpunkt online ist. 
Wf 2: 6: Zahl 6.

Wf 3: pol'zovatelej: Gen.Pl.m. ,Benutzer'. Als Leser weiß man, dass damit die User des Forums gemeint sind.

Wf 4: i: Konj. ,und', ,auch', ,sogar'.

Wf 5: 30: Zahl 30.

Wf 6: gostej: Gen.Pl.m. ,Gast'. Als Leser weiß man, dass es User und Gäste eines Forums gibt, wobei die User feste Mitglieder sind, die Userprofile besitzen. Gäste hingegen sind nur mit dem Profil eines Gastes registriert.

Wf 7: AgentMario: Name eines Users.

Wf 8: Android: Name eines Users.

Wf 9: DimaSss: Name des Schreibers.

Wf 10: djoni84: Name eines Users.

Wf 11: DrShaman: Name eines Users.

Wf 12: Lelika: Name eines Users.

Ä 1: Da es sich hier um ein komplett neues, abrupt eingeschobenes Thema handelt, welches mit dem davor besprochenen Thema nichts $\mathrm{zu}$ tun hat, stellen die vorherigen Mitteilungen/Äußerungen keinen Kontext dar. Den Kontext bildet ein Thread eines Forums für Programmierer, Hacker und Interessenten, in dem alles besprochen werden kann.

Hyp. Int.: Interpretation des eingeworfenen Codes so, dass der Schreiber DimaSss anzeigen möchte, wie viele User und Gäste gerade online sind. Der Leser erwartet, dass der Schreiber in einer weiteren Äußerung erklärt, warum er dies macht.

$+\ddot{A}:$ Der Turn des Schreibers ist noch nicht beendet, da noch eine weitere Äußerung des Schreibers folgt. 


\section{9. $\mathrm{T}_{2} \mathrm{M}_{1,1} \quad$ [+ Emoticon] a proekt aktivno razvivaetsja!}

Intention: Der Schreiber DimaSss intendiert mit Hilfe des zuvor zitierten Codes, der aufschlüsselt, wer zum Zeitpunkt, als er seine Mitteilung verfasst, online ist, anzuzeigen, dass aus seiner Sicht das Projekt (das Forum) stark wächst.

Alternative: Der Schreiber intendiert durch den Gebrauch von Sarkasmus anzugeben, dass sich das Projekt schlecht entwickelt.

Wf 1: a: Konj./Part. ,und', ,aber', ,sondern'; ,wie?'.

Wf 2: proekt: Nom./Akk.Sg.m.unbel. ,Projekt'. Der Leser vermutet, dass hiermit das Forum gemeint ist, was für die Gründer und Administratoren des Forums sicherlich ein Projekt darstellt, das sich erst nach und nach entwickelt. Möglicherweise ist das Forum auch als (Uni)Projekt gegründet worden.

Wf 3: aktivno: Adv. ,aktiv'.

Wf 4: razvivaetsja: 3.Ps.Sg.impf. + refl, sich entwickeln'.

Ä 2: Den Kontext dieser Äußerung bildet der zitierte Code, der angibt, wie viele User und Gäste zum Zeitpunkt des Verfassens der Mitteilung gleichzeitig online sind. Aus seinem HW heraus weiß der Leser, dass die Entwicklung eines Forums als Projekt anhand der Anzahl der User gemessen wird: je mehr User, desto positiver ist die Entwicklung eines Forums zu werten, weil eine große Zahl von der Nützlichkeit eines Forums zeugt. Das Ausrufezeichen dient als mögliches $\mathrm{KH}$.

Hyp. Int.: Die Äußerung hat zwei mögliche Interpretationen. Sollte DimaSss die Äußerung ernst meinen, ist sie so zu interpretieren, dass sich aus seiner Sicht das Forum erfolgreich entwickelt. Sollte es sich bei der Äußerung um Sarkasmus handeln, ist sie so zu interpretieren, dass sich das Forum negativ entwickelt. Der Leser erwartet eine Reaktion, in der dies bestätigt oder verneint wird. Obwohl 30 Gäste und 6 User zum Zeitpunkt des Verfassens der Mitteilung online sind, muss nicht ausschließlich eine Reaktion dieser Personen erfolgen.

- ̈̈: Es folgt keine weitere Äußerung, so dass ein Partizipantenwechsel stattfindet und ein Leser sich als neuer Schreiber äußern kann. 
10. $\mathrm{T}_{2} \mathrm{M}_{2,1} \quad$ byvalo i bol'še.

Intention: Die Schreiberin Lelika intendiert, DimaSss zu widersprechen, indem sie sagt, dass es auch Zeitpunkte gab, in denen mehr Personen gleichzeitig online waren.

Wf 1: byvalo: 3.Ps.Sg.n.Prät.impf. ,sein'.

Wf 2: i: Konj. ,und', ,auch', ,sogar'.

Wf 3: bol'še: Adv. ,mehr'.

Ä 1: Den Kontext bilden der in der vorigen Mitteilung von DimaSss angezeigte Code und seine Äußerung. Die zwei Punkte ,..“ könnten wie drei Punkte „...“ als Hinweis auf eine Sprechpause fungieren. Da die Schreiberin Lelika nur indirekt auf die eigentliche Äußerung von DimaSss reagiert, stellt ihre Äußerung eine Implikatur dar.

Hyp. Int.: Interpretation der Äußerung so, dass es schon Zeitpunkte gab, an denen mehr Personen gleichzeitig online waren als zu dem Zeitpunkt, an dem DimaSss seine Mitteilung verfasst. Lelika, die die Äußerung von DimaSss als ernst gemeint interpretiert, implikatiert, dass sie nicht der Meinung ist, dass die von DimaSss angezeigte Anzahl für eine aktive Entwicklung steht. Sollten die zwei Punkte eine Sprechpause andeuten, erwartet der Leser eine weitere Äußerung des Schreibers Lelika, ansonsten erwartet er im weiteren Verlauf eine Reaktion von DimaSss oder von einem anderen User, in der zu diesem Umstand etwas geschrieben wird.

- ̈̈: Es folgt keine weitere Äußerung, so dass ein Partizipantenwechsel stattfindet und ein Leser sich als neuer Schreiber äußern kann.

11. $\mathrm{T}_{2} \mathrm{M}_{3,1} \quad$ A DDoS emu aktivno pomogaet [+ Emoticon]

Intention: Der Schreiber AgentMario reagiert auf die Äußerung von DimaSss und intendiert durch eine nicht zutreffende Aussage (da DDoS niemals hilfreich ist) anzuzeigen, dass es Probleme gibt, die eine positive Entwicklung stören. AgentMario benutzt ebenfalls das Wort aktivno.

Wf 1: a: Konj./Part. ,und', ,aber', ,sondern'; ,wie?'. 
Wf 2: DDoS: Nom./Akk.Sg.m. ,DDoS = Distributed Denial of Service'. Als Leser weiß man, dass es sich hierbei um eine Attacke auf einen Rechner/eine Internetseite/einen Server o.ä. von vielen unterschiedlichen Rechnern aus ist. Auf diese Weise wird der Rechner/Seite/Server überlastet, was die Einstellung seiner Dienste zur Folge hat. Solche Angriffe kommen sehr häufig vor.

Wf 3: emu: Pron. 3.Ps.Dat.Sg.m. ,er'.

Wf 4: aktivno: Adv. ,aktiv'.

Wf 5: pomogaet: 3.Ps.Sg.impf. ,helfen'.

Ä 1: Den Kontext bildet DimaSss Äußerung, dass sich das Projekt (Forum) aktiv entwickelt. Da der Leser aus seinem Hintergrundwissen weiß, dass DDoS für ein Forum/eine Internetseite o.ä. niemals hilfreich ist, ist diese Äußerung als Implikatur zu verstehen.

Hyp. Int.: Interpretation der Äußerung so, dass das Forum Probleme mit DDoS-Angriffen hat, die eine stärkere Entwicklung des Forums behindern. Erwartung einer Reaktion von DimaSss oder von einem anderen Leser, der auf diesen Sachverhalt eingeht.

$+\ddot{A}:$ Der Turn des Schreibers ist noch nicht beendet, da noch eine weitere Äußerung des Schreibers folgt.

12. $\mathrm{T}_{2} \mathrm{M}_{3, \text { Zitat }}$ Zitata: Rekord odnovremennogo prebyvanija 970, èto bylo 11.09.2009 v 17:53.

Intention: Der Schreiber AgentMario intendiert, Lelika zuzustimmen, indem er durch das Zitat angibt, dass es einen Rekord bei der Anzahl der Personen, die gleichzeitig online waren, vor dem Zeitpunkt des Verfassens der Mitteilung von DimaSss gab. Gleichzeitig intendiert er, seine vorherige Äußerung zu unterstützen, dass das Forum durch Probleme an einer positiven Entwicklung gestört wird, was durch den Vergleich zwischen der Anzahl der Personen, die gleichzeitig online sind, sichtbar wird.

Wf 1: rekord: Nom./Akk.Sg.m., Rekord'.

Wf 2: odnovremennogo: Adj. Gen.Sg.m.unbel./n. ,gleichzeitig'.

Wf 3: prebyvanija: Gen.Sg.n. ,Aufenthalt', ,Verbleib'. 
Wf 4: 970: Zahl.

Wf 5: èto: Pron. Nom./Akk.Sg.n. ,das'.

Wf 6: bylo: 1.-3.Ps.Sg.n.Prät, ,sein’.

Wf 7: 11.09.2009: Datum. Das Datum liegt einige Monate vor dem Datum der Mitteilung (17.01.2010).

Wf 8: v: Präp. ,in’. Erwartung einer Wf im Akk. oder Lok.

Wf 9: 17:53: Uhrzeit.

Ä 2: Den Kontext bilden der Code von DimaSss, Lelikas Äußerung und AgentMarios eigene Äußerung. Aus seinem HW weiß der Leser, dass auf Grund von DDoS-Angriffen ein Server/eine Internetseite/ein Forum bis hin zu mehreren Wochen nicht richtig funktioniert, da es zuweilen lange dauern kann, bis alles wieder hergestellt ist. Für viele User ist das ein Grund, diese Internetseite/dieses Forum nicht mehr zu besuchen.

Hyp. Int.: Interpretation des Zitats als Angabe durch AgentMario, dass es einen Rekord bei der Anzahl der Personen, die gleichzeitig online waren, bereits vor dem Zeitpunkt des Verfassens der Mitteilung von DimaSss gab. Damit stimmt er Lelika zu und unterstreicht gleichzeitig seine vorherige Äußerung, dass das Forum Probleme hat, die dafür sorgen, dass eine stärkere Entwicklung des Forums behindert wird. Der Leser erwartet keine Reaktion darauf.

- ̈̈: Es folgt keine weitere Äußerung, so dass ein Partizipantenwechsel stattfindet und ein Leser sich als neuer Schreiber äußern kann.

13. $\mathrm{T}_{2} \mathrm{M}_{4,1} \quad$ Vo blin, a ja dumal počemu sajt tupil [+ Emoticon]

Intention: Der Schreiber DimaSss intendiert anzugeben, dass er sich bereits gefragt hat, warum die Seite Probleme hatte, weil er den Grund hierfür nicht kannte.

Wf 1: vo: stellungsbedingte Variante der Präp. $v$,in'. Als Leser weiß man aber auch, dass es eine Part. vot ,da', , eben da', ,deshalb', ,darum' , hier' gibt, die umgangssprachlich als vo realisiert werden kann. 
Wf 2: blin: Nom./Akk.Sg.m.unbel. ,Pfannkuchen'. Als Leser weiß man jedoch, dass es sich bei der Wf auch um einen Euphemismus für ein Schimpfwort (bljad') handelt und als solcher eine weitere Bedeutung (*,verdammt', ${ }^{183}$ *,Mist') hat. Auf Grund dieses Hintergrundwissens und des vorherigen Kontextes entscheidet er sich für die Bedeutung als Euphemismus. Die vorherige Wf vo hat die Bedeutung der Partikel.

Wf 3: a: Konj./Part. ,und', ,aber', ,sondern'; ,wie?'.

Wf 4: ja: Pron.1.Ps.Nom.Sg. ,ich'.

Wf 5: dumal: 1.-3.Ps.Sg.m.Prät.impf., denken'.

Wf 6: počemu: Adv. ,warum?', ,weshalb?'.

Wf 7: sajt: Nom./Akk.Sg.m.unbel. ,Site'/ ,Internetseite'.

Wf 8: tupil: 1.-3.Ps.Sg.m.Prät.impf. veralt. *,senken'; *,stumpf machen'. Vom Kontext der vorigen Wf her passt keine dieser Bedeutungen. Als Leser weiß man aber, dass das Verb im Slang noch andere Bedeutungen hat: *,langsam denken'*,nicht verstehen, wie etwas Einfaches funktioniert'; *,schlecht oder langsam funktionieren'. Auf Grundlage dieses Hintergrundwissens entscheidet er sich hier für die Bedeutung ,schlecht, langsam funktionieren'.

$\ddot{A}$ 1: Den Kontext dieser Äußerung bildet die Äußerung von AgentMario, dass die Forumsseite Probleme auf Grund von DDoS-Angriffen hat.

Hyp. Int.: Als Leser interpretiert man die Äußerung so, dass DimaSss nicht wusste (implikatiert), warum die Seite so schlecht funktionierte, es jetzt aber auf Grund der Äußerung von AgentMario versteht. Der Leser erwartet keine Reaktion darauf.

- ̈̈: Es folgt keine weitere Äußerung, so dass ein Partizipantenwechsel stattfindet und ein Leser sich als neuer Schreiber äußern kann.

${ }^{183}$ Die mit ,*“ markierten Übersetzungen stammen von der Vf. selbst. 
14. $\mathrm{T}_{2} \mathrm{M}_{5,1} \quad$ Eto že davno bylo..

Intention: Die Schreiberin Lelika intendiert, AgentMario zu widersprechen, dass die DDoSAngriffe das jetzige Problem darstellen, indem sie sagt, dass diese Probleme schon länger her sind.

Wf 1: èto: Pron. Nom./Akk.Sg.n., das'.

Wf 2: že: Part. ,aber', ,doch', ,denn', ,hingegen'.

Wf 3: davno: Adv. ,längst', ,vor langem', , seit langem'.

Wf 4: bylo: 1.-3.Ps.Sg.n.Prät. ,sein’.

Ä 1: Den Kontext bildet AgentMarios erste Äußerung. Die zwei Punkte könnten wie drei Punkte als Hinweis auf eine Sprechpause fungieren.

Hyp. Int.: Interpretation der Äußerung so, dass diese Probleme schon länger her sind. Sollten die zwei Punkte eine Sprechpause andeuten, erwartet man eine weitere Äußerung des Schreibers Lelika, ansonsten erwartet man eine Reaktion eines anderen Users, in der zu diesem Umstand geschrieben wird.

$+\ddot{A}:$ Der Turn des Schreibers ist noch nicht beendet, da noch eine weitere Äußerung des Schreibers folgt.

15. $\mathrm{T}_{2} \mathrm{M}_{5,2} \quad$ Chorošo čto sejčas netu..

Intention: Die Schreiberin Lelika intendiert anzugeben, dass es diese Probleme nicht mehr gibt, worüber der Schreiber erleichtert/erfreut ist.

Wf 1: Chorošo: Adv. ,gut'.

Wf 2: čto: Pron. Nom./Akk.Sg.n. / Konj. / Part. ,was'; , dass'.

Wf 3: sejčas: Adv. ,jetzt'.

Wf 4: netu: umgangsspr., prädikativ verwendeter Ausdruck ,nicht vorhanden', ,nicht existent'. 
Ä 2: Den Kontext bildet AgentMarios erste Äußerung und Lelikas letzte Äußerung. Chorošo fungiert als Hinweis darauf, dass der Schreiber erleichtert/erfreut ist, dass es diese Probleme jetzt nicht mehr gibt. Die zwei Punkte könnten wie drei Punkte als Hinweis auf eine Sprechpause fungieren.

Hyp. Int.: Interpretation der Äußerung so, dass der Schreiber glücklich darüber ist, dass es diese Probleme jetzt nicht mehr gibt. Sollten die zwei Punkte eine Sprechpause andeuten, erwartet man eine weitere Äußerung des Schreibers Lelika, ansonsten eine Reaktion von einem anderen User, in der zu diesem Umstand Stellung bezogen wird und sich geäußert wird, inwieweit das stimmt.

- ̈̈: Es folgt keine weitere Äußerung, so dass ein Partizipantenwechsel stattfindet und ein Leser sich als neuer Schreiber äußern kann.

16. $\mathrm{T}_{1} \mathrm{M}_{5,1} \quad$ Pomoščnik ja okazalsja nikudyšnyj, rešil ne byt' obuzoj i sam ušel v otstavku...

Intention: bogdas Intention als Schreiber ist es, auf Lelikas erste Fragen zu antworten und zu bestätigen, dass er kein Moderator mehr ist.

Es findet erneut ein abrupter Themenwechsel statt, indem das erste Thema wieder aufgegriffen wird. Ein solcher Themenwechsel ist Bestandteil der Konventionen.

Wf 1: Pomoščnik: Nom.Sg.m. ,Helfer'. Als Leser weiß man, dass damit Moderatoren gemeint sind, die eine Hilfsfunktion in Foren erfüllen.

Wf 2: ja: Pron. 1.Ps.Sg.Nom. ,ich'.

Wf 3: okazalsja: 1.-3.Ps.Sg.m. Prät. pf. ,geraten', ,gelangen', ,sich befinden', ,sich erweisen', ,sich entpuppen als', ,sich herausstellen'. In dem vorliegenden Kontext entscheidet sich der Leser für die Bedeutungen ,sich erweisen', ,sich entpuppen als' oder ,sich herausstellen'.

Wf 4: nikudyšnyj: Adj. Nom.Sg.m. ,nichtsnutzig', ,untauglich’.

Wf 5: rešil: 1.-3.Ps.Sg.m. Prät. pf. ,entscheiden'.

Wf 6: ne: Negation.

Wf 7: byt': Infintiv, sein'. Erwartung einer Wf im Instr. 
Wf 8: obuzoj: Instr. Sg. f. ,Last'. Last im Sinne einer Belastung. Aus seinem HW weiß der Leser, dass sich Last nicht nur auf das tatsächliche schwere Gewicht eines Gegenstands/einer Person bezogen sein kann, sondern auch im übertragenen Sinne (eine Bürde) gemeint sein kann.

Wf 9: i: Konj. ,und', ,auch', ,sogar'.

Wf 10: sam: Pron. Nom.Sg.m., selbst'.

Wf 11: ušel: 1.-3.Ps.Sg.m. Prät. pf. ,weggehen’.

Wf 12: v: Präp. ,in’. Erwartung einer Wf im Lok. oder Akk.

Wf 13: otstavku: Akk.Sg.f. ,Rücktritt', ,Ruhestand'. Die VP ušel v otstavku hat auf Grund idiomatischen Gebrauchs die Bedeutung, in den Ruhestand gehen' bzw. ,zurücktreten'. Durch den Kontext ist hier die Bedeutung ,zurücktreten' anzunehmen.

$\ddot{A}$ 1: Den Kontext bildet die in $\mathrm{T}_{1} \mathrm{M}_{1}$ gestellte Frage von Lelika danach, ob bogda kein Moderator mehr ist. Aus seinem HW heraus weiß der Leser, dass Moderatoren (wie alle anderen, die eine Funktion in einem Forum ausüben) sowohl entlassen werden als auch selbst zurücktreten können. Obwohl hier kein genauer Grund für den Rücktritt erwähnt wird, wird implikatiert, dass es einen Vorfall gab (sowohl auf Grund vorheriger Äußerungen von AgentMario als auch dadurch, dass bogda sich als untauglich und als Last bezeichnet), der den Grund darstellt. Die drei Punkte deuten als KH auf eine Sprechpause hin.

Hyp. Int.: Interpretation der Äußerung als bogdas Bestätigung, dass er kein Moderator mehr ist und selbst zurückgetreten ist, weil er sich als nicht tauglich für diese Funktion erwiesen hat. Bogda implikatiert, dass es für seinen Rücktritt einen Grund (Vorfall) gab. Auf Grund der drei Punkte erwartet man eine weitere Äußerung von bogda, in der er den Vorfall näher erläutert.

$+\ddot{A}:$ Der Turn des Schreibers ist noch nicht beendet, da noch eine weitere Äußerung des Schreibers folgt. 
17. $\mathrm{T}_{1} \mathrm{M}_{6,2} \quad$ Ne delaj vyvodov osnovyvajas' na svoich ubeždenijach, tot paren' pervyj načal obzyvat'sja [+ Emoticon]

Intention: Der Schreiber bogda intendiert auf AgentMarios Äußerungen zu reagieren, indem er seinen Implikaturen widerspricht, dass er in Folge eines Vorfalls und der fälligen Strafe kein Moderator mehr ist, und indem er klarstellt, dass der Andere als Erster angefangen hat.

Wf 1: Ne: Negation.

Wf 2: delaj: Imperativ 2.Ps.Sg. ,machen'. Wegen der Negation, erwartet der Leser, dass wenn eine Wf kommt, sie standardgrammatisch im Genitiv stehen wird (Genitiv der Negation).

Wf 3: vyvodov: Gen.Pl.m. ,Hinausbefördern', ,Schlußfolgerung', ,Rückschluss', ,Klemme', ,Ausgabe'. Aus dem Kontext heraus passt nur ,Schlussfolgerung' als Bedeutung, da delat' vyvody idiomatisch gebraucht wird und, Schlussfolgerung ziehen' bedeutet.

Wf 4: osnovyvajas': Adverbialpartizip Präs., refl. ,beruhen', ,entstehen', ,sich niederlassen'. Aus dem Kontext heraus passt als Bedeutung ,beruhen'. Da osnovyvajas' na idiomatisch ist, Erwartung der Präp. ,na'.

Wf 5: na: Präp. ,auf', , an', ,in', ,bei', ,mit', ,zu', ,für'; Interjektion (z.B. na tebe!). Da osnovyvajas' na idiomatisch gebraucht wird, Erwartung einer Wf im Lok.

Wf 6: svoich: Pron.Gen./Akk.bel./Lok.Pl. ,sein eigenes'. Hier ist dies auf AgentMario bezogen, der der Adressat der Mitteilung ist, daher bedeutet es ,dein eigenes'.

Wf 7: ubeždenijach: Lok.P1.n. ,Überzeugung’.

Wf 8: tot: Pron. Nom./Akk.unbel. Sg.m. ,jener'. Erwartung einer Wf im Nom.

Wf 9: paren’: Nom.Sg.m. ,Kerl', ,Bursche'.

Wf 10: pervyj: Adj. Nom.Sg.m. (prädikativ verwendet), erster'.

Wf 11: načal: 1.-3.Ps.Sg.m.Prät. pf. ,anfangen'.

Wf 12: obzyvat'sja: Infinitiv refl. ,beschimpfen'.

Ä 2: Den Kontext bildet die Implikatur von AgentMario, bogda wäre auf Grund eines Vorfalls (Beschimpfungen/Beleidigungen), für den er verwarnt und degradiert wurde, kein 
Moderator mehr. Dabei interpretiert bogda die Äußerung von AgentMario so, dass dieser implikatiert, bogda hätte als Erster mit den Beschimpfungen angefangen und hätte dafür als Folge nicht nur eine Strafe erhalten, sondern hätte auch seinen Posten als Moderator räumen müssen.

Hyp. Int.: Die Äußerung von bogda interpretiert der Leser so, dass er durch seine Äußerung, AgentMario solle keine Rückschlüsse basierend auf seinen eigenen Überzeugungen ziehen, AgentMarios Implikaturen, bogda hätte als Erster mit den Beschimpfungen angefangen und hätte dafür als Folge nicht nur eine Strafe erhalten, sondern hätte auch seinen Posten als Moderator räumen müssen (bogdas Interpretation), widerspricht. Offensichtlich weiß AgentMario nicht genau, was vorgefallen ist, da er weder in der Situation dabei war noch ihm bogda von dem Vorfall erzählt hat. Im weiteren Verlauf Erwartung einer Reaktion von AgentMario.

- ̈̈: Es folgt keine weitere Äußerung, so dass ein Partizipantenwechsel stattfindet und ein Leser sich als neuer Schreiber äußern kann.

18. $\mathrm{T}_{1} \mathrm{M}_{7,1} \quad$ Ja ne govoril, čto ty pervyj načal.

Intention: Der Schreiber AgentMario möchte bogdas Interpretation seiner Äußerungen widersprechen.

Wf 1: ja: Pron. 1.Ps.Sg. ,ich’.

Wf 2: ne: Negation.

Wf 3: govoril: 1.-3.Ps.Sg.m. Prät. impf. ,sprechen'.

Wf 4: čto: Nom./Akk.Sg.n. / Konj. / Part. ,was'; ,dass'.

Wf 5: ty: Pron.Nom.2.Ps.Sg. ,du'.

Wf 6: pervyj: Adj. Nom.Sg.m. (prädikativ verwendet) ,erster'.

Wf 7: načal: 1.-3.Ps.Sg.m.Prät. pf. ,anfangen'.

Ä 1: Den Kontext bildet bogdas Interpretation der Äußerung von AgentMario. 
Hyp. Int.: Als Leser interpretiert man die Äußerung so, dass AgentMario klarstellt, er hätte nicht behauptet, bogda habe als Erster angefangen. Erwartung einer weiteren Äußerung von AgentMario, in der er rechtfertigt, wie er zu seinem Schluss gekommen ist.

$+\ddot{A}:$ Der Turn des Schreibers ist noch nicht beendet, da noch eine weitere Äußerung des Schreibers folgt.

19. $\mathrm{T}_{1} \mathrm{M}_{7,2} \quad$ On voobšče ban polučil

Intention: Der Schreiber AgentMario möchte seine Äußerungen rechtfertigen. Er bestätigt die Äußerung von bogda, der andere User hätte ihn zuerst beschimpft.

Wf 1: On: Pron. 3.Ps.Sg.m. ,er'.

Wf 2: voobšče: Adv. ,überhaupt'.

Wf 3: ban: Nom./Akk.Sg.m. Aus seinem HW heraus weiß der Leser, dass ban aus dem Englischen (to ban s.o.) entlehnt wurde und den Vorgang bezeichnet, wenn ein User z.B. von einem Forum ausgeschlossen wird. In der Regel ist dies die höchste Strafe für einen Regelverstoß. Je nachdem, wie schwerwiegend der Regelverstoß ist, kann jemand für eine bestimmte Zeit oder auch dauerhaft des Forums verwiesen werden.

Wf 4: polučil: 1.-3.Ps.Sg.m.Prät.pf. ,bekommen'.

Ä 2: Den Kontext bildet - wie in der Äußerung davor - bogdas Interpretation der Äußerung von AgentMario. Als Leser weiß man aber, dass derjenige, der als Erster anfängt, die höhere Strafe erhält: dass der Andere des Forums verwiesen wurde, bedeutet, dass dieser als Erster angefangen haben muss.

Hyp. Int.: Interpretation der Äußerung als Bestätigung dafür, dass der Andere zuerst mit dem Beschimpfen angefangen hat und dafür von dem Forum ausgeschlossen wurde. Erwartung einer weiteren Äußerung von AgentMario, in der er rechtfertigt, wie er zu seinem Schluss gekommen ist.

$+\ddot{A}:$ Der Turn des Schreibers ist noch nicht beendet, da noch eine weitere Äußerung des Schreibers folgt. 
20. $\mathrm{T}_{1} \mathrm{M}_{7,3} \quad$ No ved' ty ušel s moderatorov $i$,,polinjal “ srazu že posle predupreždenija.

Intention: Der Schreiber AgentMario hat die Intention, seine Äußerungen zu rechtfertigen, indem er erklärt, wie er zu seinem Schluss gekommen ist.

Wf 1: No: Konj. , aber'.

Wf 2: ved': Part./Konj. ,ja', ,wirklich'; ,denn', ,doch', ,schließlich'. Aus dem Kontext heraus passt die Bedeutung, doch'.

Wf 3: ty: Pron. Nom.2.Ps.Sg. ,du'.

Wf 4: ušel: 1.-3.Ps.Sg.m. Prät. pf. ,weggehen'.

Wf 5: s: Präp. ,mit', ,seit', ,ab', ,von', ,aus'. Obwohl nach der Präposition auch ein Substantiv im Instrumental stehen kann, erwartet man hier auf Grund des Kontextes ein Substativ im Genitiv.

Wf 6: moderatorov: Gen./Akk.Pl.m., Moderator'.

Wf 7: i: Konj. ,und', ,auch', ,sogar'.

Wf 8: „polinjal“: 1.-3. Ps.Sg.m. Prät. pf. ,abfärben’ ,ausbleichen’, ,haaren/sich mausern/sich häuten'. Aus dem Kontext der vorigen Äußerungen weiß der Leser, dass sich das Verb auf das Ausbleichen der Farbe des Usernamens bezieht, was implikatiert, dass sich die Funktion des Users im Forum geändert hat. Die Anführungszeichen dienen hier als KH und deuten darauf hin, dass die Wf hier nicht auf ihre gängige Weise interpretiert werden kann.

Wf 9: srazu: Adv. ,sofort'.

Wf 10: že: Part. ,aber', ,doch', ,denn', ,hingegen'; bekräftigende Part. Da srazu že idiomatisch ist, fungiert že hier als bekräftigende Partikel.

Wf 11: posle: Adv. ,nach'. Erwartung eines Wf im Genitiv.

Wf 12: predupreždenija: Gen. Sg. n. ,Verwarnung'. Aus seinem HW heraus weiß der Leser, dass User für Regelverstöße verwarnt werden können.

Ä 3: Den Kontext bildet bogdas Interpretation der Äußerung von AgentMario $\left(\mathrm{T}_{1} \mathrm{M}_{2,2}\right)$ und seine Äußerung, AgentMario solle keine Rückschlüsse basierend auf seinen eigenen 
Überzeugungen ziehen.

Hyp. Int.: Interpretation der Äußerung so, dass AgentMario seine Rückschlüsse/Annahmen/Implikaturen darauf stützt, dass bogda direkt nach der Verwarnung seine Funktion als Moderator nicht mehr ausgeübt hat. Erwartung entweder einer weiteren Äußerung des Schreibers AgentMario oder einer Reaktion bogdas.

+ ̈̈: Der Turn des Schreibers ist noch nicht beendet, da noch eine weitere Äußerung des Schreibers folgt.

21. $\mathrm{T}_{1} \mathrm{M}_{7,4} \quad$ Kak že tut ne delat' vyvody?

Intention: Der Schreiber AgentMario hat die Intention, seine Äußerungen zu rechtfertigen, indem er rhetorisch fragt, wie man hier keine Schlüsse ziehen solle.

Wf 1: Kak: Adv./ Konj. ,wie'; ,als', ,wie’.

Wf 2: že: Part. ,aber', ,doch', ,denn', ,hingegen'; bekräftigende Part.

Wf 3: tut: Adv. ,hier'.

Wf 4: ne: Negation.

Wf 5: delat': Infinitiv impf. ,machen'. Aus dem Kontext heraus (v.a. als Reaktion auf $\mathrm{Ne}$ delaj vyvodov... in $\mathrm{T}_{1} \mathrm{M}_{6}$ ) erwartet der Leser, dass die nächste Wf vyvod ,Schlußfolgerung', ,Rückschluss' o.ä. lautet. Nach dem Verb alleine erwartet man eine Wf im Akk., durch die Negation ist standardgrammatisch jedoch eine Wf im Gen. (Genitiv der Negation) zu erwarten, wobei umgangssprachlich häufig der Akk. gebraucht wird.

Wf 6: vyvody: Nom./Akk. Pl.m. ,Schlussfolgerung', ,Rückschluss'.

Ä 4: Den Kontext bildet bogdas Äußerung, AgentMario solle keine Rückschlüsse basierend auf seinen eigenen Überzeugungen ziehen, und damit ein Zurückweisen der Implikaturen von AgentMario.

Hyp. Int.: Interpretation der Äußerung als rhetorische Frage von AgentMario, wie man mit Kenntnis des Vorfalls nicht diese oder ähnliche Schlüsse ziehen solle. Gleichzeitig bestätigt 
AgentMario indirekt bogdas Implikatur, er wäre bei dem Vorfall selbst nicht dabei gewesen. Da die Frage rhetorisch ist, erwartet man keine Reaktion bogdas oder die eines anderen Users.

- ̈̈: Es folgt keine weitere Äußerung, so dass ein Partizipantenwechsel stattfindet und ein Leser sich als neuer Schreiber äußern kann.

22. $\mathrm{T}_{1} \mathrm{M}_{8,1} \quad$ A ja gde to byla?

Intention: Die Schreiberin Lelika hat die Intention mitzuteilen, dass sie den Vorfall nicht mitbekommen hat. Das macht sie mit Hilfe einer rhetorischen oder selbstreflektierenden Frage, die ihre Überraschung vermittelt.

Wf 1: a: Konj. /Part. ,und', ,aber', ,sondern'; ,wie?'.

Wf 2: ja: Pron. Nom. 1.Ps.Sg., ich'.

Wf 3: gde: Adv. ,wo'.

Wf 4: to: Konj./ Part. / Pron. ,dann', ,denn'; Nom./Akk.Sg.n. ,jenes'.

Wf 5: byla: 1.-3.Ps.Sg.f.Prät. ,sein'.

$\ddot{A}$ 1: Den Kontext bildet der Vorfall, in den bogda verwickelt war. Durch lexikalische Hinweise ist klar, dass es sich hier um eine Frage handelt. Gleichzeitig drückt der Schreiber durch a ja gde to seine Überraschung/ sein Erstaunen aus. Da die Frage nicht direkt im Zusammenhang mit den vorherigen Äußerungen steht, jedoch einen kooperativen Beitrag darstellt, handelt es sich hier um eine Implikatur.

Hyp. Int.: Als Leser interpretiert man die Äußerung so, dass Lelika implikatiert, den Vorfall nicht mitbekommen zu haben, was sie überrascht. Da diese Frage kein anderer Gesprächspartizipant beantworten kann, erwartet man keine Reaktion seitens anderer Leser darauf. Ansonsten Erwartung einer weiteren Äußerung von Lelika, in der sie sagt, dass sie den Vorfall nicht mitbekommen hat.

+ ̈̈: Der Turn des Schreibers ist noch nicht beendet, da noch eine weitere Äußerung des Schreibers folgt. 
23. $\mathrm{T}_{1} \mathrm{M}_{8,2} \quad$ Každyj den' zachožu, a ob ètom daže ne znaju

Intention: Die Schreiberin Lelika hat die Intention mitzuteilen, dass sie den Vorfall nicht mitbekommen hat, obwohl sie das Forum jeden Tag besucht.

Wf 1: Každyj: Pron. Nom./Akk. Sg. unbel.m. ,jeder’.

Wf 2: den’: Nom./Akk.Sg.m., Tag'.

Wf 3: zachožu: 1.Ps.Sg.impf. ,hineingehen', ,vorbeikommen', ,vorbeischauen'. Aus dem Kontext heraus weiß man, dass damit das Besuchen des Forums gemeint ist.

Wf 4: a: Konj. /Part. ,und', ,aber', ,sondern'; ,wie?'.

Wf 5: ob: Präp. ,über', ,von’, ,zu’. Erwartung Wf im Lokativ.

Wf 6: ètom: Pron. Lok.Sg.m./n. ,dieser/-s'.

Wf 7: daže: Adv. ,sogar’.

Wf 8: ne: Negation.

Wf 9: znaju: 1.Ps.Sg.impf., wissen’.

Ä 2: Den Kontext bildet der Vorfall, in den bogda verwickelt war und den Lelika nicht mitbekommen hat.

Hyp. Int.: Als Leser interpretiert man die Äußerung so, dass Lelika den Vorfall nicht mitbekommen hat, obwohl sie täglich im Forum unterwegs ist. Man erwartet keine Reaktion auf diese Äußerung.

- Ӓ: Es folgt keine weitere Äußerung, so dass ein Partizipantenwechsel stattfindet und ein Leser sich als neuer Schreiber äußern kann.

24. $\mathrm{T}_{1} \mathrm{M}_{9,1} \quad$ Mog by napisat' administracii, $i$ ty by ne sletel, $i$ togo by zabanili...

Intention: Der Schreiber silver intendiert, bogda zu sagen, was er in seiner Situation (ein anderer User hat ihn beschimpft) hätte besser machen können und sollen, um den 
Moderatorposten nicht zu verlieren.

Wf 1: Mog: 1.-3.Ps.Sg.m.Prät. impf. ,können'.

Wf 2: by: Part. zur Bildung des Konjunktivs.

Wf 3: napisat': Infinitiv pf. ,schreiben'.

Wf 4: administracii: Dat.Sg.f. ,Administration'. Als Leser weiß man, dass jedes Forum über eine Administration verfügt. Die Administratoren üben in einem Forumthread die hierarchisch höchste Funktion aus: zum Einen sorgen sie für das reibungslose Funktionieren des jeweiligen Forum(-threads) und zum Anderen vergeben sie Posten (z.B. Moderator) an User, die ihnen (z.B. durch ihre Qualifikation) geeignet erscheinen. Sie sind die höchste Instanz, bei der sich User, unabhängig davon, ob sie einen Posten bekleiden, beschweren können.

Wf 5: i: Konj. ,und', ,auch', ,sogar'.

Wf 6: ty: Pron. Nom.2.Ps.Sg. ,du’.

Wf 7: by: Part. zur Bildung des Konjunktivs.

Wf 8: ne: Negation.

Wf 9: sletel: 1.-3.Ps.Sg.m. Prät. pf. ,abfallen', ,runterfallen', ,runterfliegen', ,abfliegen'. Als Leser weiß man, dass in Bezug auf einen Posten damit das Gefeuertwerden gemeint ist.

Wf 10: i : Konj. ,und', ,auch', ,sogar'.

Wf 11: togo: Pron. Gen./Akk.Sg.m.bel./Gen.Sg.n. ,jener'/,jenes'.

Wf 12: by: s.o.

Wf 13: zabanili: 1.-3.Ps.Pl. Prät.pf. ,verweisen', , ausschließen’. Als Leser weiß man, dass es aus dem Engl. ,to ban' entlehnt wurde. Man weiß, dass es in Foren (und Chaträumen) den Vorgang bezeichnet, wenn ein User für einen Regelverstoß des Forums verwiesen wird. Abhängig von der Art des Regelverstoßes kann jemand für eine bestimmte Zeit oder auch dauerhaft des Forums verwiesen werden.

Ä 1: Den Kontext bildet der Vorfall, bei dem bogda und ein anderer User sich gegenseitig beschimpft haben. Aus seinem HW heraus weiß der Leser, dass wenn bogda sich bei dem 
Administrator beschwert hätte, ohne den anderen User zu beleidigen, er nicht bestraft worden wäre. Die drei Punkte stellen als KH eine Sprechpause dar.

Hyp. Int.: Als Leser interpretiert man die Äußerung so, dass wenn bogda sich beschwert hätte, ohne auf eine Beschimpfung mit einer Beschimpfung zu reagieren, er nicht bestraft worden wäre. Für den Schreiber silver ist die Strafe dafür, dass bogda den anderen User beleidigt hat, dass bogda vom Posten des Moderators geflogen ist, d.h. silver interpretiert die vorherigen Äußerungen so, dass bogda nicht selbst zurückgetreten ist, sondern „entlassen“ wurde. Eventuell liegt dies daran, dass er überlesen hat, dass bogda selbst zurückgetreten ist $\left(\mathrm{T}_{1} \mathrm{M}_{5,1}\right)$. Da silvers Interpretation nicht korrekt ist, erwartet der Leser im weiteren Verlauf eine Reaktion bogdas, in der er diese korrigiert. Auf Grund der drei Punkte erwartet man im direkten Anschluss jedoch eine weitere Äußerung des Schreibers.

$+\ddot{A}:$ Der Turn des Schreibers ist noch nicht beendet, da noch eine weitere Äußerung des Schreibers folgt.

25. $\mathrm{T}_{1} \mathrm{M}_{9,2} \quad$ No na tupost' otvečat' tupost'ju...èto glupo.

Intention: Der Schreiber silver hat die Intention, bogdas Verhalten als dumm zu kritisieren.

Wf 1: no: Konj. ,aber'.

Wf 2: na: Präp. ,auf', ,an', ,in', ,bei', ,mit', ,zu', ,für'; Interjektion (z.B. na tebe!). Erwartung einer Wf im Akk. oder Lok.

Wf 3: tupost': Nom./Akk.Sg.f. ,Stumpfsinn'. Aus dem Kontext heraus weiß der Leser, dass sich der Stumpfsinn auf die Beschimpfung bezieht.

Wf 4: otvečat': Infinitiv impf., antworten'.

Wf 5: tupost'ju: Instr.Sg.f. ,Stumpfsinn', s.o.

Wf 6: èto: Pron. Nom./Akk. Sg. n. ,dieses’.

Wf 7: glupo: prädikativ verwendeter Ausdruck (Adv.), dumm’.

Ä 2: Den Kontext bildet der Vorfall, bei dem ein anderer User und bogda sich gegenseitig 
beschimpft haben. Die drei Punkte inmitten der Äußerung dienen als Hinweis auf eine Sprechpause. Da der Schreiber hier tupost' statt oskorblenije verwendet, handelt es sich hier um eine Implikatur.

Hyp. Int.: Die Äußerung interpretiert der Leser als Implikatur, dass es dumm ist, auf eine Beschimpfung mit einer anderen Beschimpfung zu reagieren. Der Leser erwartet entweder eine Reaktion von bogda, in der er sein Verhalten rechtfertigt, oder die Reaktion eines anderen Lesers, der silver zustimmt oder nicht.

- ̈̈: Es folgt keine weitere Äußerung, so dass ein Partizipantenwechsel stattfindet und ein Leser sich als neuer Schreiber äußern kann.

26. $\mathrm{T}_{1} \mathrm{M}_{10,1} \quad$ V smysle by ne sletel?!

Intention: bogdas Intention als Schreiber ist es, sein Unverständnis über die Äußerung von silver mitzuteilen, da er ja gesagt hat, selbst zurückgetreten zu sein.

Wf 1: v: Präp. ,in'. Erwartung einer Wf im Lok. oder Akk.

Wf 2: smysle: Lok.Sg.m. ,Sinn’.

Wf 3: by: Part. zur Bildung des Konjunktivs.

Wf 4: ne: Negation.

Wf 5: sletel: 1.-3.Ps.Sg.m. Prät. pf. ,abfallen', ,runterfallen', ,runterfliegen', ,abfliegen'. In Bezug auf einen Posten ist damit das Gefeuertwerden gemeint ist (siehe Äußerung 24).

Ä 1: Den Kontext bildet die fehlerhafte Interpretation von silver, bogda wäre vom Posten des Moderators gefeuert worden.

Hyp. Int.: Als Leser interpretiert man die Äußerung von bogda als Unverständnis angesichts von silvers Interpretation, dass bogda rausgeschmissen worden sein soll, obwohl er geschrieben hat, selbst zurückgetreten zu sein. Erwartung einer weiteren Äußerung bogdas, in der er die Interpretation silvers korrigiert.

$+\ddot{A}:$ Der Turn des Schreibers ist noch nicht beendet, da noch eine weitere Äußerung des 
Schreibers folgt.

27. $\mathrm{T}_{1} \mathrm{M}_{10,2} \quad$ ja že napisal, čto sam ušel v otstavku emae!

Intention: bogdas Intention als Schreiber ist es, silvers Interpretation, die ihn empört, da er bereits geschrieben hatte, dass er selbst zurückgetreten ist, zu korrigieren.

Wf 1: ja: Pron. Nom.1.Ps.Sg. ,ich'.

Wf 2: že: Part. ,aber’, ,doch', ,denn', ,hingegen'; bekräftigende Part.

Wf 3: napisal: 1.-3.Ps.Sg.m.Prät.pf. ,schreiben’.

Wf 4: čto: Pron. Nom./Akk.Sg.n./Konj./Part. ,was';,dass'.

Wf 5: sam: Pron. Nom.Sg.m. ,selbst'.

Wf 6: ušel: 1.-3.Ps.Sg.m. Prät. pf. ,weggehen'.

Wf 7: v: Präp. ,in’. Erwartung einer Wf im Lok. oder Akk.

Wf 8: otstavku: Akk.Sg.f. ,Rücktritt', ,Ruhestand'. Die VP ušel v otstavku hat auf Grund idiomatischen Gebrauchs hier die Bedeutung ,zurücktreten' (siehe Äußerung 16).

Wf 9: emae: Interjektion. Als Leser weiß man, dass es sich hierbei um die Interjektion $\ddot{e}$-mö̈, einen Euphemismus für ebena mat ${ }^{184}$, handelt, die allerlei Emotionen ausdrücken kann. Die Interjektion ist dabei ein lexikalischer Hinweis auf die empfundene Aufregung/Empörung/Irritation des Schreibers.

Ä 2: Den Kontext bildet die fehlerhafte Interpretation von silver. Durch die Interjektion als lexikalischen Hinweis wird für den Leser klargestellt, dass der Schreiber des Satzes sich empört.

Hyp. Int.: Interpretation der Äußerung von bogda als Wiederholung, er sei selbst vom Posten des Moderators zurückgetreten. Damit korrigiert bogda silvers Interpretation, er sei hinausgeworfen worden. Der Leser erwartet entweder eine weitere Äußerung von bogda, in

${ }^{184}$ Vgl. Kolesnikov \& Kornilov $(1996,96)$ und Elistratov $(2005,119)$. 
der er auf den Rest von silvers Mitteilung eingeht oder eine Reaktion von silver, in der er sich bspw. für seine Fehlinterpretation entschuldigt.

$+\ddot{A}:$ Der Turn des Schreibers ist noch nicht beendet, da noch eine weitere Äußerung des Schreibers folgt.

28. $\mathrm{T}_{1} \mathrm{M}_{10,3} \quad$ Esli čestno uže privyk na ponty ěšce bol'šimi pontami otvečat' [+Emoticon]

Intention: bogdas Intention als Schreiber ist es, sein Verhalten (auf eine Beschimpfung mit einer Beschimpfung zu antworten) zu rechtfertigen.

Wf 1: esli: Konj. ,wenn', ,falls'.

Wf 2: čestno: Adv: ,ehrlich'. Die Kombination esli čestno ist idiomatisch und bedeutet ,um ehrlich zu sein'.

Wf 3: uže: Adv. ,schon', , bereits'.

Wf 4: privyk: 1.-3.Ps.Sg.m.Prät.pf. ,sich gewöhnen'.

Wf 5: na: Präp. ,auf', ,an', ,in', ,bei’, ,mit', ,zu', ,für'; Interjektion (z.B. na tebe!). Erwartung einer Wf im Akk. oder Lok.

Wf 6: ponty: Akk.Pl.m. Als Leser weiß man aus seinem HW, dass das Wort ein Fremdwort aus dem Franz. ist und verschiedene Bedeutungen hat: *,Spieler, der gegen die Bank spielt'; *,Trumpfass'; *,Umstände, die von einem Diebeshelfer geschaffen werden, um einen Raub zu erleichtern'; *,Betrug'; *,Dünkel', *,Brüskierung', *,Demonstration von Überlegenheit'; *,Verstellung'; *,Vorteil'; *,Opfer eines Betrügers'; *,Erregung'. ${ }^{185}$ Da man als Leser weiß, dass es Jugendliche gibt, die durch das Benutzen möglichst vieler Schimpfwörter prahlen/angeben oder Anderen zeigen wollen, dass sie diesen überlegen sind und man sich mit ihnen nicht anlegen sollte, nimmt er als Bedeutung ,Dünkel', scheinbare/angebliche Überlegenheit', ,Brüskierung' oder ,Prahlerei', ,Angeberei' an.

Wf 7: ešče: Adv. ,noch’; ,schon; ,zusätzlich’.

\footnotetext{
${ }^{185}$ Die russischsprachigen Bedeutungen stammen aus Istoričeskij slovar' gallicizmov russkogo jazyka (http://gallicismes.academic.ru/30604/\%D0\%BF\%D0\%BE\%D0\%BD\%D1\%82; 18.01.17, 15.21 Uhr).
} 
Wf 8: bol’šimi: Adj. Instr.P1. ,groß’/ Komparativ (Adj.) Instr. Pl. ,größer'. In diesem Kontext handelt es sich um den Komparativ.

Wf 9: pontami: Instr. Pl.m. Wie oben erläutert, wird auch hier die Bedeutung ,Dünkel', scheinbare/angebliche Überlegenheit', „Brüskierung' oder ,Prahlerei', ,Angeberei’ angenommen.

Wf 10: otvečat': Infinitiv impf., antworten’.

Ä 3: Den Kontext bildet der Vorfall, bei dem bogda von einem anderen User beschimpft wurde und diesen als Reaktion darauf zurückbeschimpft hat, was silver in seiner Äußerung als stumpfsinniges Verhalten bezeichnet. Da ponty statt oskorblenija benutzt wird, handelt es sich um eine Implikatur.

Hyp. Int.: Interpretation der Äußerung als bogda Aussage, er sei es gewohnt auf Brüskierung mit einer noch größeren Brüskierung zu reagieren. Erwartung einer Reaktion von silver oder eines anderen Lesers, die sich zu diesem Verhalten äußern.

- ̈̈: Es folgt keine weitere Äußerung, so dass ein Partizipantenwechsel stattfindet und ein Leser sich als neuer Schreiber äußern kann.

29. $\mathrm{T}_{1} \mathrm{M}_{11,1} \quad$ On sam ušel, ja smotrju ne na kol-vo žalob, a umenie obščat'sja s ljudmi na forume, i normal'no bez matov vyrožat' blagodarnost' a ne "uch blja zajabis' pizdaten 'ko tak vyložili, blagodarju nach".

Intention: DragOn reagiert als Administrator des Forums auf die Äußerung von AgentMario und silver, indem er bestätigt, bogda sei selbst zurückgetreten und dass er als Administrator nur auf die Fähigkeit achtet, mit anderen Usern und Gästen des Forums umgehen zu können.

Wf 1: On: Pron. 3.Ps.Nom.Sg.m. ,er'.

Wf 2: sam: Pron. Nom.Sg.m. ,selbst'.

Wf 3: ušel: 1-3.Ps.Sg.m.Prät.pf. ,weggehen'.

Wf 4: ja: Pron. 1.Ps.Nom.Sg. ,ich'. 
Wf 5: smotrju: 1.Ps.Sg.impf. ,sehen', ,schauen’.

Wf 6: ne: Negation.

Wf 7: na: Präp. ,auf', ,an', ,in', ,bei', ,mit', ,zu', ,für'; Interjektion (z.B. na tebe!). Erwartung einer Wf im Akk. oder Lok.

Wf 8: kol-vo: Als Leser weiß man, dass es sich hier um eine abgekürzte Form von količestvo handelt. Nom./Akk.Sg.n. ,Menge'. Die vorherige Wf $n a$ hat die Bedeutung ,auf’

Wf 9: žalob: Gen.Pl.f., Beschwerde’.

Wf 10: a: Konj. /Part. ,und', , aber', ,sondern'; ,wie?'.

Wf 11: umenie: Nom./Akk.Sg.n. ,Können', ,Fertigkeit', ,Fähigkeit’.

Wf 12: obščat'sja: Infinitiv impf. + refl ,Umgang haben', ,kontaktieren', ,verkehren mit', ,kommunizieren'. Auf Grund des Kontextes passt hier ,kommunizieren’.

Wf 13: s: Präp. ,mit’. Erwartung einer Wf im Instr.

Wf 14: ljudmi: Instr. Pl. ,Menschen'.

Wf 15: na: Präp. ,auf', ,an', ,in', ,bei', ,mit', ,zu', ,für'; Interjektion (z.B. na tebe!). Erwartung einer Wf im Akk. oder Lok.

Wf 16: forume: Dat./Lok.Sg.m. ,Forum'. Hiermit ist ein Online-Forum gemeint und zwar das, in dem die Gesprächspartner verkehren.

Wf 17: i: Konj. ,und', ,auch', ,sogar'.

Wf 18: normal'no: Adv. ,normal'.

Wf 19: bez: Präp. ,ohne'. Erwartung einer Wf im Gen.

Wf 20: matov: Gen.Pl.m. ,(sport.) Matte'; ,grobe Schimpfwörter '. Aus dem vorherigen Kontext heraus weiß der Leser, dass hier die Bedeutung , grobe Schimpfwörter' angenommen werden muss.

Wf 21: vyrožat': Als Leser weiß man, dass es sich hier um einen Rechtschreibfehler handelt, 
da die Wf vyražat' lautet. Infinitiv impf., ausdrücken'.

Wf 22: blagodarnost': Nom./Akk.Sg.f. ,Dankbarkeit'.

Wf 23: a: Konj. /Part. ,und', , aber', ,sondern'; ,wie?'.

Wf 24: ne: Negation.

Wf 25: uch: Interjekt. ,Ach'.

Wf 26: blja: Interjektion, die benutzt wird, um u.a. eine Verstärkung von Emotionen anzuzeigen. Hierbei handelt es sich um die gekürzte Form von bljad'/bljat'.

Wf 27: zajabis': Als Leser weiß man, dass es sich hier um einen Rechtschreibfehler handelt, da die Wf zajebis' lautet, dennoch bleibt die Wf erkennbar. Es handelt sich bei ihr entweder um ein Imperativ 2.Ps.Sg. pf. ${ }^{186}$ *,ficken', *,sehr ermüden' oder Adv. *,sehr gut', *, hervorragend', ,qualitativ' ${ }^{187}$.

Wf 28: pizdaten'ko: Als Leser weiß man, dass diese Wf im Slang gebräuchlich und ein Adv. mit der Bedeutung *,(sehr) gut'; *, hervorragend' ist.

Wf 29: tak: Adv./Konj./Part. ,so'/,also'/,ja'.

Wf 30: vyložili: 3.Ps.Pl.pf. ,ausstellen'; , auspacken'; ,verlegen'; , ausbauen'. Als Leser weiß man, dass es in der Internetsprache die Bedeutung, auslegen' im Sinne von Fotos/Dateien hochladen und zur Verfügung stellen, hat. Aus dem Kontext heraus entscheidet man sich für diese Bedeutung.

Wf 31: blagodarju: 1.Ps.Sg.impf., danken', , sich bedanken'.

Wf 32: nach: Als Leser weiß man, dass es sich hier um die gekürzte Form des Schimpfworts nachuj handelt. Adv. *,verpiss dich' (in Kombination mit idti); *,wofür'; wird benutzt, um negative Emotionen auszudrücken. ${ }^{188}$

Ä 1: Den Kontext bildet der Vorfall, in den bogda verwickelt war und silvers

186

https://ru.wiktionary.org/wiki/\%D0\%B7\%D0\%B0\%D0\%B5\%D0\%B1\%D0\%B0\%D1\%82\%D1\%8C\%D1\%81\% D1\%8FD1\%8F; 15.01.17, 10:18 Uhr.

${ }^{187}$ https://ru.wiktionary.org/wiki/\%D0\%B7\%D0\%B0\%D0\%B5\%D0\%B1\%D0\%B8\%D1\%81\%D1\%8C; 15.01.17, 10:18 Uhr.

${ }^{188}$ Vgl. die russischsprachigen Bedeutungen: http://teenslang.su/id/10726; 15.01.17, 10:30 Uhr. 
Fehlinterpretation der Äußerung von bogda. Aus seinem HW weiß der Leser, dass DragOn als Administrator dieses Forums die Moderatoren bestimmt, d.h. er wählt aus den Bewerbern aus und kann sie auch wieder entlassen. Sollte ein Moderator zurücktreten wollen, muss er seinen Rücktrittswunsch beim Administrator einreichen. Daher weiß DragOn als Administrator am ehesten, ob bogda selbst zurückgetreten ist oder vom Posten „entlassen“ wurde. Ebenso ist ein Administrator immer im Bilde, was vorgefallen ist. Die Anführungsstriche dienen als KH, um auf das Zitat einer direkten Rede zu verweisen. Die Wf nach fungiert als lexikalischer Hinweis dafür, dass der Dank sarkastisch gemeint ist.

Hyp. Int.: Für den Leser ist der erste Teil der Äußerung so zu interpretieren, dass DragOn durch on sam ušel bestätigt, dass bogda selbst zurückgetreten ist und nicht „entlassen“ wurde. Der zweite Teil der Äußerung ist zunächst so zu interpretieren, dass eine Beschwerde (wie von silver vorgeschlagen) nichts genützt hätte, da DragOn nicht auf die Anzahl der Beschwerden, sondern nur auf den korrekten Umgang mit anderen Usern und Gästen des Forums achtet. Danach ergeben sich zwei Interpretationsmöglichkeiten, da nicht klar ist, auf welchen der beiden am Vorfall beteiligten Personen (bogda oder der andere User) DragOn sich bezieht: entweder ist bogda derjenige, der nicht mit Anderen umgehen kann und DragOn zitiert seine Äußerung oder der andere User kann es nicht, in diesem Fall wäre es die Äußerung des anderen Users. Das Zitat selbst ist so zu interpretieren, dass das Bedanken nicht erst gemeint, weil vielleicht keine Hilfe erfolgt ist. Der Leser erwartet, dass DragOn im weiteren Verlauf klarstellt, auf wen sich diese Äußerung genau bezieht und wer diese Worte geschrieben hat, oder, dass ein anderer Leser ihn danach fragt.

$+\ddot{A}:$ Der Turn des Schreibers ist noch nicht beendet, da noch eine weitere Äußerung des Schreibers folgt.

\section{0. $\mathrm{T}_{1} \mathrm{M}_{11,2} \quad$ Tak čto na nego ne naezžat'}

Intention: Der Schreiber DragOn hat die Intention bogda in Schutz zu nehmen.

Wf 1: tak: Adv./Konj./Part. ,so'/, also'/,ja'.

Wf 2: čto: Pron. Nom./Akk.Sg.n. / Konj. / Part. ,was'; ,dass'. Da tak čto hier idiomatisch gebraucht wird, hat es die Bedeutung, also'. 
Wf 3: na: Präp. ,auf', ,an', ,in', ,bei’, ,mit', ,zu', ,für'; Interjektion (z.B. na tebe!). Erwartung einer Wf im Akk. oder Lok.

Wf 4: nego: Gen./Akk.Sg.m./ Gen./Akk.Sg.n. ,er'/ ,es'. Aus dem Kontext heraus weiß der Leser, dass es sich hier um bogda handeln muss und entscheidet sich somit für die Bedeutung ,er'.

Wf 5: ne: Negation.

Wf 6: naezžat': Infinitiv impf. ,gegen etw. fahren', ,etw./jmd. anfahren'; , unerwartet ankommen'; ,aufeinander treffen'. Als Leser weiß man, dass mit ,jemanden anfahren' im übertragenen Sinne auch ein grobes Vorgehen gegen eine andere Person gemeint sein kann. Aus dem Kontext heraus passt diese Bedeutung.

Ä 2: Den Kontext bilden der Vorfall, in den bogda verwickelt war und die Äußerungen von silver.

Hyp. Int.: Als Leser interpretiert man die Äußerung so, dass DragOn den anderen User, die am Gespräch beteiligt sind, sagt, sie sollen bogda in Ruhe lassen und ihn damit in Schutz nimmt. Gleichzeitig wird die Interpretation der vorigen Äußerung in Richtung der Annahme, der andere User hätte nicht mit anderen Usern korrekt umgehen können (und hätte die zitierten Worte benutzt) gelenkt. Der Leser erwartet darauf eine Reaktion silvers, der klarstellt, dass er sich nicht mit bogda streiten wollte o.ä. oder eines anderen Lesers, der DragOns Meinung nicht teilt, dass man bogda in Ruhe lassen sollte.

- ̈̈: Es folgt keine weitere Äußerung, so dass ein Partizipantenwechsel stattfindet und ein Leser sich als neuer Schreiber äußern kann.

\section{1. $\mathrm{T}_{1} \mathrm{M}_{12,1} \quad$ Gostej nado ne pugat' (oskorbljat') nado byt' umnej i postarat'sja pomoč'}

Intention: DimaSss' Intention als Schreiber ist es, bogda zu belehren, wie man sich Gästen gegenüber zu verhalten hat. Damit intendiert er DragOns Äußerung, man solle bogda in Ruhe lassen, zu widersprechen.

Wf 1: Gostej: Gen./Akk.Pl.m. ,Gast'. 
Wf 2: nado: Präd. ,es ist nötig', ,man muss/soll'.

Wf 3: ne: Negation.

Wf 4: pugat': Infintiv impf., ,erschrecken', ,verschrecken'.

Wf: oskorbljat': Infinitiv impf. ,beleidigen'. Die Klammern geben an, dass der Schreiber mit der vorherigen Wf beleidigen implikatiert, da man Gäste auf verschiedene Art und Weise erschrecken kann und der Schreiber sichergehen wollte, dass der Leser das, was er mit erschrecken implikatiert, so versteht, wie er es intendiert hat.

Wf 5: nado: s.o.

Wf 6: byt': Infintiv, sein'.

Wf 7: umnej: Komparativ (Adj.) ,klüger'.

Wf 8: i: Konj. ,und', , auch', ,sogar'.

Wf 9: postarat'sja: Infinitiv pf. + refl. ,versuchen'.

Wf 10: pomoč': Infinitiv pf. ,helfen'.

Ä 1: Den Kontext bildet der Vorfall, bei dem bogda und ein anderer User sich gegenseitig beleidigt haben und DragOns Äußerungen, die DimaSss so interpretiert, dass bogda nicht mit anderen Usern umgehen kann. Durch die Wf in Klammern verdeutlicht der Schreiber, was er mit pugat' implikatiert: nämlich oskorbljat'.

Hyp. Int.: Als Leser interpretiert man die Äußerung so, dass DimaSss bogda belehrt, er hätte sich in der Situation klüger verhalten und helfen sollen. Es scheint keine Rolle zu spielen, dass der andere User zuerst mit den Beschimpfungen angefangen hat, sondern nur, dass bogda sich unklug verhalten hat, was von dem Schreiber DimaSss implikatiert wird. Der Leser erwartet eine Reaktion von bogda oder die eines anderen Lesers, in der dieser Kritik an bogdas Verhalten zugestimmt wird oder nicht, oder aber eine Reaktion von DragOn, in der er auf die Belehrung eingeht, obwohl er selbst gerade gesagt hat, dass man bogda in Ruhe lassen soll.

$+\ddot{\mathrm{A}}$ : Der Turn des Schreibers ist noch nicht beendet, da noch eine weitere Äußerung des Schreibers folgt. 
Intention: Der Schreiber DimaSss hat die Intention zu erklären, warum es klug ist, Usern/Gästen zu helfen.

Wf 1: Togda: Adv. ,dann'.

Wf 2: gost': Nom.Sg.m., Gast'.

Wf 3: pozovet: Als Leser weiß man, dass es sich hierbei um die Endung -ët handelt, das $\ddot{e}$ wird aus Bequemlichkeitsgründen durch $e$ ersetzt. 3.Ps.Sg. pf. ,rufen'; ,einladen'.

Wf 4: druga: Gen./Akk. Sg.m. ,Freund'.

Wf 5: i: Konj. ,und', ,auch', ,sogar'.

Wf 6: t.d.: Als Leser weiß man, dass es sich hierbei um die Abkürzung für tak dalee handelt, das in Kombination mit $i$, und so weiter' bedeutet.

Wf 7: i: s.o.

Wf 8: forum: Nom./Akk.Sg.m.unbel. ,Forum'.

Wf 9: budet: 3.Ps.Sg. Fut. ,sein'.

Wf 10: razvivat'sja: Infinitiv impf. + refl. ,sich entwickeln'.

Ä 2: Den Kontext bilden der Vorfall zwischen bogda und einem anderen User und die vorherige Äußerung des Schreibers DimaSss.

Hyp. Int.: Interpretation der Äußerung so, dass wenn man sich einem Gast/User gegenüber freundlich und hilfsbereit verhält, er seine Freunde in das Forum einlädt. So gewinnt das Forum weitere Mitglieder und kann wachsen. Erwartung entweder einer Reaktion von bogda oder der eines anderen Lesers, die dem zustimmen oder nicht.

- ̈̈: Es folgt keine weitere Äußerung, so dass ein Partizipantenwechsel stattfindet und ein Leser sich als neuer Schreiber äußern kann. 
33. $\mathrm{T}_{1} \mathrm{M}_{13,1}$ a ja čto sejčas skazal? [+ Emoticon]

Intention: Der Schreiber DragOn intendiert, durch eine Frage mitzuteilen, dass er das, was DimaSss gesagt hat, bereits gesagt hat, und DimaSss sich unnötig wiederholt.

Alternative: DragOn rüffelt DimaSss, weil DimaSss genau das macht, was DragOn gesagt hat, dass man nicht machen soll, nämlich bogda anfahren.

Wf 1: a: Konj./Part. ,und', ,aber', ,sondern'; ,wie?'.

Wf 2: ja: Pron. 1.Ps.Nom.Sg. ,ich'.

Wf 3: čto: Pron. Nom./Akk.Sg.n. / Konj. / Part. ,was'; ,dass'. Der Leser erwartet, dass es sich bei dieser Äußerung um eine Frage handeln könnte, da čto ein potenzieller lexikalischer Hinweis darauf ist.

Wf 4: sejčas: Adv. ,jetzt'.

Wf 5: skazal: 1.-3.Ps.Sg.m.Prät.pf., sagen'.

$\ddot{A}$ 1: Den Kontext bilden der Vorfall zwischen bogda und einem anderen User und die Äußerungen von DragOn sowie von DimaSss bezüglich bogda und dem richtigen Umgang mit anderen Usern und Gästen. Durch lexikalische Hinweise ist klar, dass es sich bei dieser Äußerung um eine Frage handelt.

Hyp. Int.: Interpretation der Äußerung als DragOns Implikatur, er und DimaSss hätten inhaltlich das Gleiche gesagt. Hier liegt ein Kommunikationsproblem vor, da DimaSss als Leser die vorherigen Äußerungen von DragOn offensichtlich anders interpretiert hat, als sie von DragOn intendiert waren. Als Leser erwartet man eine Reaktion von DimaSss, in der er erklärt, wie er DragOns Äußerung verstanden hat.

Alternative: Man interpretiert die Äußerung so, dass DragOn den User DimaSss rüffelt, weil DimaSss bogda nicht in Ruhe lässt, obwohl DragOn gesagt hat, dass man es soll. Erwartung einer Reaktion von DimaSss.

- ̈̈: Es folgt keine weitere Äußerung, so dass ein Partizipantenwechsel stattfindet und ein Leser sich als neuer Schreiber äußern kann. 
34. $\mathrm{T}_{1} \mathrm{M}_{14,1} \quad$ Zolotye slova DimaSssik [+ Emoticon]

Intention: Lelikas Intention als Schreiber ist es, eine Zustimmung zur Äußerung von DimaSss zu implikatieren.

Wf : Zolotye: Adj. Nom./Akk.Pl. ,Golden'. Erwartung von slova, da idiomatische Verwendung.

Wf 2: slova: Nom./Akk.Pl. ,Wort'. Idiomatische Verwendung von zolotye slova, goldene Worte'.

Wf 3: DimaSssik: Nom.Sg.m. + Diminutivsuffix. Hierbei handelt es sich um ein Hypokoristikon des Namens des Users, der sich davor bereits geäußert hat. Lelika adressiert ihn, um klar zu machen, dass sie sich auf ihn bezieht und nicht auf jemand anderen. Das Diminutivsuffix drückt eine positive Einstellung des Schreibers gegenüber dem Adressaten und Nähe zwischen beiden aus.

Ä 1: Den Kontext bilden der Vorfall zwischen bogda und einem anderen User und DimaSss' Äußerungen. Aus seinem HW weiß der Leser, dass durch den Phraseologismus Zustimmung implikatiert wird.

Hyp. Int.: Interpretation der Äußerung als implikatierte Zustimmung und Unterstützung der zuvor gemachten Äußerungen von DimaSss. Man erwartet entweder gar keine Reaktion auf diese Äußerung oder eine Reaktion, in der gefragt wird, warum Lelika diese Ansicht unterstützt (obwohl klar ist, dass der andere User zuerst bogda beschimpft hat).

$+\ddot{A}:$ Der Turn des Schreibers ist noch nicht beendet, da noch eine weitere Äußerung des Schreibers folgt.

35. $\mathrm{T}_{1} \mathrm{M}_{15,2} \quad$ Chočeš' uznat' čeloveka daj emu vlast', $i$ on pokažet sebja..

Intention: Die Schreiberin Lelika intendiert zu sagen, dass ein Mensch sein wahres Gesicht erst zeigt, wenn er bspw. durch eine Position Macht bekommen hat.

Wf 1: Chočeš’: 2.Ps.Sg. pf. ,wollen'.

Wf 2: uznat': Infinitiv ,erfahren'; , erkennen'; ,kennenlernen'. 
Wf 3: čeloveka: Gen./Akk.Sg.m. ,Mensch’. Auf Grund dieser Wf, nimmt der Leser für den Infinitiv uznat' die Bedeutung, erkennen' an.

Wf 4: daj: Imperativ 2.Ps.Sg., geben'. Erwartung einer Wf im Dat.

Wf 5: emu: Pron. 3.Ps.Dat.Sg.m. ,er'.

Wf 6: vlast': Nom./Akk.Sg.f. ,Macht'.

Wf 7: i: Konj. ,und', ,auch', ,sogar'.

Wf 8: on: Pron. 3.Ps.Nom.Sg.m., er'.

Wf 9: pokažet: 3.Ps.Sg. pf. ,zeigen'.

Wf 10: sebja: Pron. Nom./Akk.Sg. ,sich’.

Ä 2: Den Kontext bilden der Vorfall zwischen bogda und einem anderen User, das von DragOn angeführte Zitat, das Lelika als bogdas Worte und nicht die des anderen Users interpretiert und DragOns Äußerung, in der er bogda verteidigt. Die drei Punkte dienen als $\mathrm{KH}$ auf eine Sprechpause. Da es keine zu erwartende Reaktion auf die von DragOn wiedergegebene direkte Rede ist, sich aber dennoch darauf bezieht, handelt es sich hier um eine Implikatur.

Hyp. Int.: Als Leser interpretiert man die Äußerung von Lelika so, dass sich aus ihrer Sicht das wahre Gesicht eines Menschen erst zeigt, wenn er eine Machtposition erhalten hat. Damit implikatiert sie, dass sich bogdas wahres Gesicht erst zeigte, als er die Position als Moderator bekommen hatte: da er sich als Moderator, dessen Funktion es eigentlich ist, für die Einhaltung der Forumsregeln zu sorgen, erlaubt hat, einem anderen User mit Schimpfworten zu antworten, könnte sein Verhalten als Machtmissbrauch verstanden werden. Da die Schreiberin Lelika aber auch DragOns Äußerung, dass man bogda in Ruhe lassen soll, mitzitiert, kann ihre eigene Äußerung auch so interpretiert werden, dass sie auf DragOn bezogen ist, der trotz des Benutzens von Schimpfwörtern bogda nicht entlassen hat, sondern ihn in Schutz nimmt. Erwartung von Reaktionen seitens DragOns und bogdas, die auf diese Äußerung eingehen und sich bspw. verteidigen.

- ̈̈: Es folgt keine weitere Äußerung, so dass ein Partizipantenwechsel stattfindet und ein Leser sich als neuer Schreiber äußern kann. 
36. $\mathrm{T}_{1} \mathrm{M}_{16,1} \quad$ Ja takoj plochoj?

Intention: DragOns Intention als Schreiber, auf Lelikas Äußerung, die er auf sich bezieht, einzugehen und zu erfahren, ob Lelika ihn für einen so schlechten Administrator hält, weil er bogda in Schutz nimmt.

Wf 1: Ja: Pron. 1.Ps. Nom. Sg., ich'.

Wf 2: takoj: Pron. Nom.Sg.m./Akk.Sg.m.unbel. ,solch'; ,dergleichen'.

Wf 3: plochoj: Adj. Nom.Sg.m. ,schlecht'.

Ä 1: Den Kontext bilden bogdas Verteidigung durch DragOn sowie die vorige Äußerung von Lelika, dass sich das wahre Gesicht eines Menschen erst zeigt, wenn er Macht erlangt. Diese Äußerung bezieht DragOn auf sich, da er dem HW des Lesers nach als Administrator die hierarchisch höchste Machtposition in diesem Forumthread besitzt und bogda in Schutz nimmt, obwohl dieser einen anderen User beleidigt haben soll. Das Fragezeichen fungiert als KH auf eine Frage.

Hyp. Int.: Interpretation der Äußerung von DragOn, der Lelikas Äußerung auf sich selbst bezieht, als Frage danach, ob er so schlecht als Administrator ist und z.B. seine Machtposition missbraucht, weil er bogda in Schutz genommen hat. Erwartung einer Reaktion von Lelika, in der sie auf diese Frage eingeht und erklärt, wie ihre Äußerung gemeint und auf wen sie bezogen war.

- ̈̈: Es folgt keine weitere Äußerung, so dass ein Partizipantenwechsel stattfindet und ein Leser sich als neuer Schreiber äußern kann.

37. $\mathrm{T}_{1} \mathrm{M}_{17,1} \quad$ nu počemu ty vse na sebja bereš’?

Intention: Die Schreiberin Lelika intendiert durch eine rhetorische Frage zu implikatieren, dass es in ihrer vorherigen Äußerung nicht um DragOn ging.

Wf 1: nu: Interj. *, aber'/,na'; *'also', *,tja'.

Wf 2: počemu: Adv. ,warum', ,weshalb'. 
Wf 3: ty: Pron. 2.Ps.Nom.Sg. ,du’.

Wf 4: vse: Nom./Akk.Pl. ,alle'. Jedoch weiß man als Leser auch, dass häufig ein anderes Pron. auch so geschrieben wird und zwar Nom./Akk.Sg.n. ,alles'.

Wf 5: na: Präp. ,auf', ,an', ,in', ,bei', ,mit', ,zu', ,für'; Interjektion (z.B. na tebe!). Erwartung einer Wf im Akk. oder Lok.

Wf 6: sebja: Pron. Akk.Sg. ,sich’.

Wf 7: bereš’: 2.Ps.Sg. impf. ,nehmen'. Auf Grund der restlichen Wf wird für die Wf vse die Bedeutung, alles' angenommen.

Ä 1: Den Kontext bilden bogdas Verteidigung durch DragOn, Lelikas zweite Äußerung und die darauffolgende Frage von DragOn. Die Äußerung ist bereits durch lexikalische Hinweise als Frage zu erkennen. Da eine Gegenfrage keine typische Reaktion auf eine Frage darstellt und da sie auch keinerlei Antwort auf DragOns Frage liefert, handelt es sich hier um eine Implikatur.

Hyp. Int.: Interpretation der Äußerung von Lelika als rhetorische Frage, warum DragOn ihre Äußerung auf sich bezieht. Damit implikatiert Lelika, dass es in ihrer vorherigen Äußerung nicht um DragOn, sondern um jemand anderen ging. Erwartung einer weiteren Äußerung von Lelika, in der sie klarstellt, auf wen ihre Äußerung bezogen war.

$+\ddot{A}:$ Der Turn des Schreibers ist noch nicht beendet, da noch eine weitere Äußerung des Schreibers folgt.

38. $\mathrm{T}_{1} \mathrm{M}_{17,2} \quad J a$ že uže govorila, čto ty chorošen 'kij umnička, moj ljubimyj admin chakerka..

Intention: Die Schreiberin Lelika intendiert zu bekräftigen, dass es in ihrer Äußerung bezüglich des wahren Gesichts eines Menschen nicht um DragOn ging, indem sie beteuert, dass er seine Arbeit gut macht und ihr Lieblingsadministrator im Forum ist.

Wf 1: ja: Pron. 1.Ps.Nom.Sg. ,ich'.

Wf 2: že: Part. ,aber', ,doch', ,denn', ,hingegen'; bekräftigende Part. 
Wf 3: uže: Adv. ,schon', ,bereits'.

Wf 4: govorila: 1.-3.Ps.Sg.f.Prät.impf. ,sagen'.

Wf 5: čto: Pron./Konj./Part. ,was'/,dass'.

Wf 6: ty: Pron. 2.Ps.Nom.Sg. ,du'.

Wf 7: chorošen'kij: Adj. Nom.Sg.m./Akk.Sg.m.unbel. + Diminutivsuffix ,gut'.

Wf 8: umnička: Nom.Sg.f. + Diminutivsuffix , gescheiter Mensch’, ,kluger Kopf’.

Wf 9: moj: Possesivpron. Nom. Sg.m. ,mein’.

Wf 10: ljubimyj: Adj. Nom.Sg.m./Akk.Sg.m.unbel. ,Lieblings-’ .

Wf 11: admin: Nom.Sg.m. Als Leser weiß man, dass es sich hier um die Kurzform von administrator handelt, so dass man die Bedeutung ,Administrator' annimmt.

Wf 12: chakerka: Gen.Sg.m. /Nom.Sg.f./ Gen.Sg.n./Nom.Pl.m./Akk.Pl.m/ Nom. Pl.n./ Akk.Pl.n. Als Leser weiß man, dass der Name des Forums Chakerok lautet, so dass Gen.Sg.m. angenommen wird.

Ä 2: Den Kontext bilden bogdas Verteidigung durch DragOn, die Äußerung von Lelika, die Frage von DragOn und Lelikas Gegenfrage, warum er alles auf sich selbst bezieht. Die zwei Punkte könnten wie drei Punkte als Hinweis auf eine Sprechpause fungieren.

Hyp. Int.: Als Leser interpretiert man die Äußerung so, dass Lelika nochmals bekräftigt, dass ihre Äußerung nicht auf DragOn bezogen war, indem sie wiederholt, dass er seine Arbeit gut macht und ihr Lieblingsadministrator des Forums ist. Unabhängig davon, ob die zwei Punkte eine Sprechpause andeuten oder nicht, erwartet man eine weitere Äußerung des Schreibers Lelika, in der sie klarstellt, auf wen sie sich vorher bezogen hatte.

$+\ddot{\mathrm{A}}$ : Der Turn des Schreibers ist noch nicht beendet, da noch eine weitere Äußerung des Schreibers folgt. 
39. $\mathrm{T}_{1} \mathrm{M}_{17,3} \quad$ Sejčas zdes' obsuždaetsja bogda, čto on $s$ matom otvečal pol'zovateljam foruma, vot ja i napisala vyše: Citata: Chočeš’ uznat' čeloveka daj emu vlast', i on pokažet sebja..

Intention: Lelikas Intention als Schreiber ist es zu erläutern, auf wen sich ihre Äußerung bezüglich dessen, dass sich das wahre Gesicht eines Menschen erst zeigt, wenn er Macht bekommt, bezog und wie sie gemeint war. Indem sie ihre vorherige Äußerung zitiert, stellt sie einen Zusammenhang mit der jetztigen Erläuterung her.

Wf 1: sejčas: Adv. ,jetzt'.

Wf 2: zdes': Adv. ,hier'.

Wf 3: obsuždaetsja: 3.Ps.Sg. impf. + refl , besprechen', ,erörtern', , diskutieren'.

Wf 4: bogda: Nom. Sg.m. Name eines Users.

Wf 5: čto: Pron./Konj./Part. ,was'/, dass'.

Wf 6: on: Pron. 3.Ps.Nom.Sg.m. ,er'.

Wf 7: s: Präp. ,mit'. Erwartung einer Wf im Instrumental.

Wf 8: matom: Instr. Sg.m. ,grobe Schimpfwörter'.

Wf 9: otvečal: 1.-3.Ps.Sg.m.Prät.impf. ,antworten'.

Wf 10: pol'zovateljam: Dat.Pl.m. ,Benutzer'. Als Leser weiß man, dass damit die User des Forums gemeint sind.

Wf 11: foruma: Gen.Sg.m. ,Forum’.

Wf 12: vot: Part. ,eben da', ,deshalb', ,darum', ,so'.

Wf 13: ja: Pron. 1.Ps.Nom.Sg. ,ich’.

Wf 14: i: Konj. ,und', ,auch', ,sogar'.

Wf 15: napisala: 1.-3.Ps.Sg.f.Prät.pf. ,schreiben'.

Wf 16: vyše: Adv. ,höher’. Damit ist ein weiter oben stehender (und somit vorheriger) Beitrag 
gemeint.

Ä 3: Den Kontext bilden bogdas Verhalten gegenüber einem anderen User/Gast des Forums, Lelikas Äußerung, dass sich das wahre Gesicht eines Menschen erst zeigt, wenn er eine Machtposition bekommt und dass DragOn diese Äußerung auf sich selbst bezieht. Aus seinem HW weiß man als Leser, dass es sich beim Gebrauch von Schimpfwörtern um einen Regelverstoß handelt und wenn sich ein Moderator, der eigentlich für die Einhaltung der Forumsregeln zu sorgen hat, das Benutzen von Schimpfwörtern gegenüber anderen Usern erlaubt, es einem Machtmissbrauch gleichkommt. Die zwei Punkte könnten wie drei Punkte als Hinweis auf eine Sprechpause fungieren.

Hyp. Int.: Als Leser interpretiert man die Äußerung so, dass Lelika ihre Äußerung bezüglich dessen, dass sich das wahre Gesicht eines Menschen erst zeigt, wenn er Macht bekommt, auf bogda und sein Beschimpfen eines anderen Users bezogen hat. Damit korrigiert sie DragOns Interpretation, der ihre Äußerung auf sich selbst bezieht. Sollten die zwei Punkte eine Sprechpause andeuten, erwartet man als Leser eine weitere Äußerung des Schreibers Lelika, in der sie erklärt, wieso sie bogdas (Fehl-)Verhalten mit Macht in Verbindung bringt, ansonsten eine Reaktion von DragOn, in der er schreibt, dass er jetzt verstanden hat, wie sie ihre Äußerung gemeint hat.

$+\ddot{A}:$ Der Turn des Schreibers ist noch nicht beendet, da noch eine weitere Äußerung des Schreibers folgt.

40. $\mathrm{T}_{1} \mathrm{M}_{17,4}$ A èto vse $k$ tomu, čto do moderatorstva on byl chorošim forumčaninom, $a$ kogda povysilsja v zvanii počuvstvoval vlast', vot i raspušilsja..

Intention: Die Schreiberin Lelika intendiert zu erläutern, welchen Bezug ihre Äußerung zu der bisherigen Konversation zum ersten Thema hat.

Wf 1: a: Konj./Part. ,und', ,aber', ,sondern'; ,wie?'.

Wf 2: èto: Pron. Nom.Sg.n., das'.

Wf 3: vse: Nom./Akk.Pl. ,alle'; Jedoch weiß man als Leser auch, dass häufig ein anderes Pron. häufig so geschrieben wird und zwar Nom./Akk.Sg.n. ,alles'. 
Wf 4: k: Präp. ,zum’. Erwartung einer Wf im Dat.

Wf 5: tomu: Pron. Dat.Sg.m./n. ,jener/-s'.

Wf 6: čto: Pron./Konj./Part. ,was'/, dass'.

Wf 7: do: Präp. ,bis'. Erwartung einer Wf im Gen.

Wf 8: moderatorstva: Gen.Sg.m. ,Moderatorsein’. Gemeint ist die Tätigkeit als Moderator.

Wf 9: on: Pron. 3.Ps.Nom.Sg.m., ,er'.

Wf 10: byl: 1.-3.Ps.Sg.m.Prät. ,sein’.

Wf 11: chorošim: Adj. Instr. Sg. m. ,gut’.

Wf 12: forumčaninom: Instr. Sg.m. *,"Forumler". Als Leser weiß man, dass hiermit ein Forummitglied gemeint ist.

Wf 13: a: Konj./Part. ,und', ,aber', ,sondern'; ,wie?'.

Wf 14: kogda: Adv. ,wann'.

Wf 15: povysilsja: 1.-3.Ps.Sg.m.Prät.pf + refl , aufsteigen'. In diesen Kontext erwartet man $v$ zvanii, da idiomatischer Gebrauch.

Wf 16: v: Präp. ,in'. Erwartung einer Wf im Lok. oder Akk. Man erwartet zvanii ,Rang', da idiomatischer Gebrauch.

Wf 17: zvanii: Lok.Sg.n. ,Rang,, ,Dienstgrad'. In Kombination mit povysitsja $v$ bedeutet es ,im Rang aufsteigen', da idiomatischer Gebrauch. Als Leser weiß man aus seinem HW heraus, dass User in Foren einen Rang besitzen. Die Rangstufe kann dabei z.B. durch die Anzahl der Mitteilungen oder durch die Anzahl von hilfreichen Mitteilungen bestimmt werden.

Wf 18: počuvstvoval: 1.-3.Ps.Sg.m.Prät.pf ,fühlen'.

Wf 19: vlast': Nom./Akk.Sg.f. ,Macht'. Als Leser weiß man, dass Moderatoren in Foren eine gewisse Machtposition innehaben. 
Wf 20: vot: Part. ,eben da', ,deshalb', ,darum', ,so'.

Wf 21: i: Konj. ,und', ,auch', ,sogar'.

Wf 22: raspušilsja: 1.-3.Ps.Sg.m.Prät.pf *,flauschig werden'; *,sich aufplustern'; *,bedeckt werden von frischem Grün usw.'. Aus seinem HW heraus weiß man als Leser, dass auf einen Menschen nur die ersten beiden Bedeutungen bezogen werden können: die erste Bedeutung in Bezug auf seine Haare, die zweite Bedeutung hingegen nur im übertragenen Sinne, da der Mensch über kein Gefieder/ein mit anderen Säugetieren vergleichbares Fell verfügt. Damit wird ein Mensch beschrieben, der sich wichtig tut. Da es in den vorherigen Wf nicht um Haare ging, nimmt man für diese Wf die Bedeutung ,sich aufplustern' = , sich wichtigtun' an.

Ä 4: Den Kontext bilden bogdas Verhalten gegenüber einem anderen User/Gast des Forums nachdem er zum Moderator ernannt ist, Lelikas Äußerung bezüglich des Zeigens des wahren Gesichts in einer Machtposition, die DragOn auf sich selbst bezieht sowie Lelikas nachfolgende Äußerungen. Aus dem Kontext heraus schließt man darauf, dass für Lelika bogda ein guter User war, weil er andere User nicht beschimpft hat (Implikatur). Die zwei Punkte könnten wie drei Punkte als Hinweis auf eine Sprechpause fungieren.

Hyp. Int.: Als Leser interpretiert man die Äußerung so, dass bogda vor seiner Zeit als Moderator ein guter Forum-User war, der andere User nicht beschimpft hatte (implikatiert), und sich dann auf Grund seiner Machtposition aufgeplustert und angefangen hat, sich wichtig zu tun und sich bestimmtes Verhalten zu erlauben, wie z.B. andere User zu beleidigen. Wäre bogda kein Moderator gewesen, hätte er sich so nicht verhalten. Die Äußerung interpretiert man auch als Implikatur, Lelika sei von bogda enttäuscht. Sollten die zwei Punkte eine Sprechpause andeuten, erwartet man als Leser eine weitere Äußerung des Schreibers Lelika, ansonsten erwartet man eine Reaktion von bogda, in der er Stellung zu Lelikas Ansicht bezieht.

$+\ddot{A}:$ Der Turn des Schreibers ist noch nicht beendet, da noch eine weitere Äußerung des Schreibers folgt.

41. $\mathrm{T}_{1} \mathrm{M}_{17,5}$ bogda, vse čto ty zdes' čitaeš', èto vse ne $v$ obidu tebe, a urok, čtoby ponjal svoi ošibki $i$ v sledujušchij raz ne zagonjalsja 
Intention: Lelikas Intention als Schreiber ist es, bogda $\mathrm{zu}$ belehren und gleichzeitig zu besänftigen, damit er ihr (und den Anderen, die sein Verhalten kritisieren, eventuell auch) die Äußerungen nicht übel nimmt.

Wf 1: bogda: Nom. Sg.m./f.. Username.

Wf 2: vse: Nom./Akk.Pl. ,alle'; Jedoch weiß man auch, dass häufig ein anderes Pron. so geschrieben wird und zwar Nom./Akk.Sg.n. ,alles'

Wf 3: čto: Pron./Konj./Part. ,was'/, dass'.

Wf 4: ty: Pron. 2.Ps.Nom.Sg. ,du'.

Wf 5: zdes’: Adv. ,hier'.

Wf 6: čitaeš’: 2.Ps.Sg. impf. ,lesen’.

Wf 7: èto: Pron. Nom.Sg.n. ,das’.

Wf 8: vse: Pron. Nom./Akk.Sg.n. ,alles' (s.o.).

Wf 9: ne: Negation.

Wf 10: v: Präp. ,in'. Erwartung einer Wf im Lok. oder Akk.

Wf 11: obidu: Akk.Sg.f. ,Beleidigung', ,Kränkung’

Wf 12: tebe: Pron. 2.Ps.Dat.Sg. ,du'.

Wf 13: a: Konj./Part. ,und', , aber', ,sondern'; ,wie?'.

Wf 14: urok: Nom./Akk.Sg.m.unbel. ,Lehrstunde', ,Lehre'.

Wf 15: čtoby: Konj. ,um zu', ,damit'.

Wf 16: ponjal: 1.-3.Ps.Sg.m.Prät.pf. ,verstehen’.

Wf 17: svoi: Possesivpron. Nom./Akk.Pl. ,seine eigenen'.

Wf 18: ošibki: Nom./Akk.Pl. f. ,Fehler'.

Wf 19: i: Konj. ,und', , auch', ,sogar'. 
Wf 20: v: Präp. ,in’. Erwartung einer Wf im Lok. oder Akk.

Wf 21: sledujušchij: Adj. Nom./Akk.Sg.m.unbel. ,nächster’.

Wf 22: raz: Nom./Akk.Sg.m. ,Mal'.

Wf 23: ne: Negation.

Wf 24: zagonjalsja: 1.-3.Ps.Sg.m.Prät. impf. + refl. ,hineinjagen', ,hineintreiben'; ,abhetzen'; ,hetzen'. Man weiß als Leser aus seinem HW heraus, dass das Wort im Jungendslang auch noch andere Bedeutungen hat: *,etwas Unwichtiges beachten'; *, sich wegen eines anderen Menschen, der einem nicht gleichgültig ist, unwohl fühlen'; *, lügen, sich in seiner Lüge verfangen ${ }^{189}$. Aus dem Kontext heraus passt die Bedeutung, dass bogda solche Sachen wie Beleidigungen nicht beachten soll.

Ä 5: Den Kontext bilden der Vorfall zwischen bogda und einem anderen User sowie die Kritik an bogdas Verhalten bzw. die Belehrungen seitens einiger anderer User, wie er sich hätte verhalten sollen. Da Lelika in diesem Kontext ošibki verwendet, implikatiert sie ihre Einstellung zu seinem Verhalten.

Hyp. Int.: Interpretation der Äußerung so, dass Lelika möchte, dass bogda ihre Äußerungen und die der anderen Gesprächspartizipanten nicht übel nimmt, sondern daraus lernt und sich das nächste Mal anders (besser) verhält. Durch die Wortwahl (ošibki) implikatiert sie, dass sein Verhalten falsch war. Erwartung einer Reaktion von bogda, in der er auf die Belehrung eingeht.

- $\ddot{A}:$ Es folgt keine weitere Äußerung, so dass ein Partizipantenwechsel stattfindet und ein Leser sich als neuer Schreiber äußern kann.

42. $\mathrm{T}_{1} \mathrm{M}_{18,1} \quad$ Chm...na meste bogdy ja skoree vsego postupil tak že, chot' $i$ vydal sam emu predupreždenie.

Intention: Der Schreiber Silver intendiert bogdas Verhalten zu verteidigen, indem er sagt, bogdas Verhalten nachvollziehen zu können.

${ }^{189} \mathrm{Vgl}$. russischsprachige Bedeutungen im Slovar'sovremennoj leksiki, žargona i slenga (2014). 
Wf 1: Chm: Diskursmarker ,hm', mit dem eine Überlegung mit evtl. Nicht-Zustimmung signalisiert wird. Als Leser erwartet man daher, dass silver mit der Äußerung von Lelika nicht einverstanden ist.

Wf 2: na: Präp. ,auf', ,an', ,in', ,bei', ,mit', ,zu', ,für'; Interjektion (z.B. na tebe!). Erwartung einer Wf im Akk. oder Lok.

Wf 3: meste: Lok.Sg.n. ,Ort', ,Stelle'. Die vorherige Wf na hat die Bedeutung, an’.

Wf 4: bogdy: Gen.Sg.m. Username.

Wf 5: ja: Pron. 1.Ps.Nom.Sg. ,ich'.

Wf 6: skoree: Adv. ,eher'.

Wf 7: vsego: Pron. Gen.Sg.m./Akk.Sg.m.bel./Gen.Sg.n. ,alles', ,ganz'. skoree vsego wird idiomatisch gebraucht und bedeutet ,aller Wahrscheinlichkeit nach', ,höchstwahrscheinlich'.

Wf 8: postupil: 1.-3.Ps.Sg.m.Prät.pf. ,handeln’.

Wf 9: tak: Adv./Konj./Part. ,so'/, also'/,ja'.

Wf 10: že: Part. ,aber', ,doch', ,denn', ,hingegen'; bekräftigende Part. Tak že wird idiomatisch gebraucht und bedeutet, ebenso'.

Wf 11: chot': Konj. ,sogar', ,selbst'; ,nur', ,wenigstens', ,doch'; ,obwohl', ,wenn auch'.

Wf 12: i: Konj. ,und', ,auch', ,sogar'. Chot' $i$ wird idiomatisch mit der Bedeutung ,(und) wenn auch' verwendet.

Wf 13: vydal: 1.-3.Ps.Sg.m.Prät.pf. ,ausgeben', , ausstellen’.

Wf 14: sam: Pron. Nom.Sg.m., selbst'.

Wf 15: emu: Pron. 3.Ps.Dat.Sg. ,er'.

Wf 16: predupreždenie: Nom./Akk.Sg.n. ,Verwarnung'.

$\ddot{A}$ 1: Den Kontext bilden der Vorfall zwischen bogda und einem anderen User und die Belehrungen seitens Lelika und DimaSss. Aus seinem HW weiß der Leser, dass ein 
Moderator selbst einem anderen User für Fehlverhalten eine Verwarnung ausstellen kann. Die drei Punkte nach dem Diskursmarker Chm deuten als KH auf eine Sprechpause hin.

Hyp. Int.: Als Leser interpretiert man die Äußerung so, dass silver sagt, er hätte sich an bogdas Stelle höchstwahrscheinlich genauso verhalten, wenn er ihm auch selbst eine Verwarnung ausgestellt hätte. Damit implikatiert er, dass er bogdas Verhalten nachvollziehen kann. Auf Grund der drei Punkte erwartet man als Leser eine weitere Äußerung des Schreibers silver, ansonsten Erwartung einer Reaktion von Lelika oder DimaSss, die erklären, warum sie bogdas Verhalten als falsch ansehen.

$+\ddot{A}:$ Der Turn des Schreibers ist noch nicht beendet, da noch eine weitere Äußerung des Schreibers folgt.

43. $\mathrm{T}_{1} \mathrm{M}_{18,2} \quad$ Ne ljublju kogda starajutsja unizit'...

Intention: Der Schreiber Silver intendiert, zu erklären, warum er bogdas Verhalten nachvollziehen kann.

Wf 1: Ne: Negation.

Wf 2: ljublju: 1.Ps.Sg. impf. ,lieben'.

Wf 3: kogda: Adv. ,wann', ,wenn'.

Wf 4: starajutsja: 3.Ps.Pl. impf. + refl, versuchen'.

Wf 5: unizit': Infinitiv pf., erniedrigen'. Als Leser weiß man, dass eine Beschimpfung eine Form der Erniedrigung darstellt.

Ä 2: Den Kontext bilden der Vorfall zwischen bogda und einem anderen User, die Belehrungen seitens Lelika und DimaSss und silvers vorige Äußerung. Die drei Punkte weisen als KH auf eine Sprechpause hin.

Hyp. Int.: Als Leser interpretiert man die Äußerung so, dass silver höchstwahrscheinlich genauso gehandelt hätte wie bogda, weil er es nicht mag, wenn Menschen versuchen, Andere zu erniedrigen, indem sie sie beschimpfen. Auf Grund der drei Punkte erwartet man eine weitere Äußerung des Schreibers silver. 
- ̈̈: Es folgt keine weitere Äußerung, so dass ein Partizipantenwechsel stattfindet und ein Leser sich als neuer Schreiber äußern kann.

44. $\mathrm{T}_{1} \mathrm{M}_{19,1}$ esli moderator budet otvečat' matom, togda ves' forum budet "Mat na mate"

Intention: Die Schreiberin Lelika will ihre Belehrungen verteidigen, indem sie erklärt, warum bogda sich als Moderator falsch verhalten.

Wf 1: esli: Konj. ,wenn', ,falls'.

Wf 2: moderator: Nom.Sg.m. ,Moderator'.

Wf 3: budet: 3.Ps.Sg. Fut.-Auxiliar.

Wf 4: otvečat': Infinitiv impf., antworten'.

Wf 5: matom: Instr.Sg.m. ,grobe Schimpfwörter' (s.o.).

Wf 6: togda: Adv. ,dann’.

Wf 7: ves': Pron. Nom./Akk.Sg.m.unbel. ,ganz'.

Wf 8: forum: Nom./Akk.Sg.m.unbel. ,Forum'.

Wf 9: budet: 3.Ps.Sg. Fut.-Auxiliar.

Wf 10: mat: Nom./Akk.Sg.m. ,grobe Schimpfwörter' (s.o.).

Wf 11: na: Präp. ,auf', ,an', ,in', ,bei', ,mit', ,zu', ,für'; Interjektion (z.B. na tebe!). Erwartung einer Wf im Akk. oder Lok.

Wf 12: mate: Lok.Sg.m. ,grobe Schimpfwörter' (s.o.).

Ä 1: Den Kontext bilden der Vorfall zwischen bogda und einem anderen User, Lelikas (und DimaSss') Belehrungen sowie silvers Verteidigung des Verhaltens von bogda.

Hyp. Int.: Als Leser interpretiert man die Äußerung so, dass wenn ein Moderator, der eigentlich die Kommunikation „überwachen“ und Fehlverhalten bestrafen soll, auf Beschimpfungen mit Beschimpfungen reagiert, dann im Forum alle User nur noch grobe 
Schimpfwörter verwenden werden. Daher sieht Lelika bogdas Verhalten als falsch an. Erwartung einer Reaktion von silver, in der er Stellung dazu bezieht.

$+\ddot{A}:$ Der Turn des Schreibers ist noch nicht beendet, da noch eine weitere Äußerung des Schreibers folgt.

45. $\mathrm{T}_{1} \mathrm{M}_{19,2} \quad$ Inogda nužno byt' vyše.

Intention: Die Schreiberin Lelika möchte ermahnen, dass man als Moderator über Beschimpfungen stehen sollte.

Wf 1: Inogda: Adv. ,manchmal'.

Wf 2: nužno: Präd. ,es ist nötig', ,man muss/soll’.

Wf 3: byt': Infinitiv, sein'.

Wf 4: vyše: Komparativ (Adv.) ,höher'.

Ä 2: Den Kontext bilden der Vorfall zwischen bogda und einem anderen User, Lelikas (und DimaSss') Belehrungen, silvers Äußerung, er könne bogdas Verhalten nachvollziehen, sowie Lelikas vorherige Äußerung. Die drei Punkte signalisieren als KH, dass eine Sprechpause vorliegt und die Mitteilung noch nicht zu Ende ist.

Hyp. Int.: Als Leser interpretiert man die Äußerung so, dass man als Moderator über Beleidigungen stehen sollte. Auf Grund der drei Punkte erwartet man eine weitere Äußerung des Schreibers Lelika.

$+\ddot{A}:$ Der Turn des Schreibers ist noch nicht beendet, da noch eine weitere Äußerung des Schreibers folgt.

46. $\mathrm{T}_{1} \mathrm{M}_{19,3} \quad$ Chotja ne vsegda èto polučaetsja

Intention: Die Schreiberin Lelika hat die Intention, ihre Äußerungen abzumildern, indem sie eingesteht, dass es nicht immer möglich ist, über solchen Dingen zu stehen. 
Wf 1: Chotja: Konj. ,obwohl'.

Wf 2: ne: Negation.

Wf 3: vsegda: Adv. ,immer'.

Wf 4: èto: Pron. Nom.Sg.n. ,das'.

Wf 5: polučaetsja: 3.Ps.Sg. impf. + refl, gelingen’.

Ä 3: Den Kontext bilden der Vorfall zwischen bogda und einem anderen User, Lelikas (und DimaSss') Belehrungen, silvers Äußerung, er könne bogdas Verhalten nachvollziehen, sowie Lelikas vorherige Äußerungen.

Hyp. Int.: Als Leser interpretiert man die Äußerung so, dass Lelika eingesteht, dass es nicht immer möglich ist, über Beleidigungen zu stehen. Man erwartet eine Reaktion von silver oder bogda, in der auf die Äußerung(en) von Lelika eingegangen.

- Ӓ: Es folgt keine weitere Äußerung, so dass ein Partizipantenwechsel stattfindet und ein Leser sich als neuer Schreiber äußern kann.

47. $\mathrm{T}_{1} \mathrm{M}_{20,1} \quad$ Mogu pokljast'sja čem ugodno, ne bud' ja moderatorom ja by postupil $v$ točnosti tak že...

Intention: Der Schreiber bogda intendiert, Lelikas Äußerung, er hätte sich so ein Verhalten gegenüber einem anderen User nur erlaubt, weil er Moderator war, zu widersprechen.

Wf 1: mogu: 1.Ps.Sg.impf. ,können’.

Wf 2: pokljast'sja: Infinitiv, schwören'.

Wf 3: čem: Pron. Instr.Sg.n., was'.

Wf 4: ugodno: Adv./Part. ,gefällig'/ , beliebig'. Čto ugodno wird idiomatisch gebraucht und hat die Bedeutung, was auch immer'.

Wf 5: ne: Negation. 
Wf 6: bud': Imperativ 2.Ps.Sg. ,sein'. Als Leser weiß man, dass solche Formen transponiert verwendet werden (vgl. Isačenko (1968, 311ff.)).

Wf 7: ja: Pron. 1.Ps.Nom.Sg. ,ich'.

Wf 8: moderatorom: Instr.Sg.m. ,Moderator'.

Wf 9: ja: Pron. 1.Ps.Nom.Sg. ,ich'.

Wf 10: by: Part. zur Bildung des Konjunktivs.

Wf 11: postupil: 1.-3.Ps.Sg.m.Prät.pf. ,handeln’.

Wf 12: v: Präp. ,in’. Erwartung einer Wf im Lok. od. Akk.

Wf 13: točnosti: Gen./Dat./Lok.Sg.f., Genauigkeit', ,Präzision’.

Wf 14: tak: Adv./Konj./Part. ,so'/ ,also'/, ja'.

Wf 15: že: Part. ,aber', ,doch', ,denn', ,hingegen'; bekräftigende Part. Tak že wird idiomatisch gebraucht und bedeutet, ebenso'.

Ä 1: Den Kontext bilden der Vorfall zwischen bogda und einem anderen User und die Äußerung von Lelika, dass bogda sich nur erlaubt hat Schimpfwörter gegenüber einem anderen User zu benutzen, weil er ein Moderator war. Die drei Punke dienen als KH, um eine Sprechpause anzuzeigen.

Hyp. Int.: Als Leser interpretiert man die Äußerung so, dass bogda sich genauso verhalten hätte, auch wenn er kein Moderator gewesen wäre. Damit widerspricht er Lelikas Äußerung, sein Verhalten beruhe darauf, dass er als Moderator meinte, sich so verhalten zu können und dass wenn er kein Moderator gewesen wäre, er sich so nicht verhalten hätte. Auf Grund der drei Punke erwartet man eine weitere Äußerung des Schreibers bogda.

$+\ddot{A}:$ Der Turn des Schreibers ist noch nicht beendet, da noch eine weitere Äußerung des Schreibers folgt.

48. $\mathrm{T}_{1} \mathrm{M}_{20,2}$ I matom ja ego ne kryl, a skazal ja emu snačala čto to vrode: "devočka moja, rot ofni“", polučiv v otvet nagljučij post, otvetil emu... 
Intention: Der Schreiber bogda hat die Intention, sich zu verteidigen und zu erklären, dass er den anderen User nicht sofort beleidigt hatte, sondern wie es dazu kam.

Wf 1: i: Konj. ,und', ,auch', ,sogar'.

Wf 2: matom: Instr.Sg.m., grobe Schimpfwörter' (s.o.).

Wf 3: ja: Pron. 1.Ps.Nom.Sg. ,ich'.

Wf 4: ego: Pron. 3.Ps.Gen./Akk.Sg.m./Gen./Akk.Sg.n. ,er'/ ,es'. Aus dem Kontext heraus weiß man als Leser, dass das Personalpronomen auf den anderen User bezogen ist, der in den Vorfall mit bogda verwickelt war. Daher entscheidet man sich für die Bedeutung, er'.

Wf 5: ne: Negation.

Wf 6: kryl: 1.-3.Ps.Sg.m.Prät.impf., decken'; ,entgegnen'. Da idiomatischer Gebrauch hat kryt' matom die Bedeutung, jemanden beschimpfen'.

Wf 7: a: Konj./Part. ,und', ,aber', ,sondern'; ,wie?'.

Wf 8: skazal: 1.-3.Ps.Sg.m.Prät. pf. ,sagen’.

Wf 9: ja: Pron. 1.Ps.Nom.Sg. ,ich'.

Wf 10: emu: Pron. 3.Ps.Dat.Sg.m., er'.

Wf 11: snačala: Adv. ,zuerst', , anfangs'.

Wf 12: čto: Pron. Nom./Akk.Sg.n./Akk.Sg.m.unbel. /Konj./Part. ,was'/ ,dass'.

Wf 13: to: Pron. Nom./Akk.Sg.n./Konj./Part. ,jenes'/, dann'. čto-to bedeutet ,etwas'.

Wf 14: vrode: Part. ,ähnlich wie', ,in der Art von’.

Wf 15: devočka: Nom.Sg.f. ,(kleines) Mädchen’. Aus seinem HW heraus weiß der Leser, dass wenn diese Wf auf einen männlichen User bezogen wird (der andere User, der an dem Vorfall beteiligt war, war höchstwahrscheinlich männlich), dies eine deutliche Provokation darstellt. ${ }^{190}$ Mit der Wf verdeutlicht der Schreiber nicht nur seine Geringschätzung gegenüber

\footnotetext{
${ }^{190}$ Im Slang haben die Lexeme der Wortfamilie dev- (devka, devulja, devuška) häufig einen Bezug zu „passiven“ Homosexuellen (vgl. Bol’šoj slovar' russkogo žargona $(2000,150)$ ).
} 
der Referenzperson, sondern behauptet auch eine hierarchische Beziehung zwischen beiden: der Schreiber sieht sich als hierarchisch höherstehend an.

Wf 16: moja: Possesivpron. 1.Ps.Nom.Sg.f. ,meine'.

Wf 17: rot: Nom./Akk.Sg.n.unbel. ,Mund'.

Wf 18: ofni: Aus seinem HW heraus weiß man als Leser, dass diese Wf aus dem Engl. ,off abgeleitet wird und im russischsprachigen Slang/Jugendsprache als Verb mit den Bedeutungen ,schließen', ,zumachen', , ausmachen/ausschalten', , abschalten' gebraucht wird. Bei der hier vorliegenden Wf handelt es sich um Imperativ 2.Ps.Sg. mit der Bedeutung ,schließen' oder ,zumachen', auf die man auf Grund der vorherigen Wf ,Mund' kommt. Hat die gleiche Bedeutung wie zakroj rot.

Wf 19: polučiv: Adverbialpartizip ,erhalten', ,bekommen', ,beziehen', ,einnehmen'.

Wf 20: v: Präp. ,in’. Erwartung einer Wf im Lok. od. Akk.

Wf 21: otvet: Nom./Akk.Sg.m.unbel. ,Antwort'.

Wf 22: nagljučij: Adj. + Suffix ,frech'. Als Leser weiß man, dass damit in diesem Kontext ,beleidigend' gemeint ist, d.h. in der Mitteilung, die der andere User bogda geschrieben hat, hat er bogda beschimpft.

Wf 23: post: Aus seinem HW heraus weiß man als Leser, dass es sich hierbei um eine Entlehnung aus dem Engl. ,post' handelt, mit der in der Onlinekommunikation eine Mitteilung in einem Online-Forum bezeichnet wird. Die Wf hat die Form Nom./Akk.Sg.m.

Wf 24: otvetil: 1.-3.Ps.Sg.m.Prät. pf. ,antworten'.

Wf 25: emu: Pron. 3.Ps.Dat.Sg.m., ,er'.

Ä 2: Den Kontext bilden der Vorfall zwischen bogda und einem anderen User sowie die vorherigen Äußerungen, in denen es darum geht, dass bogda auf eine Beschimpfung mit einer Beschimpfung reagiert hat. Die drei Punke dienen als KH, um eine Sprechpause anzuzeigen. Da bogda nicht genau sagt, was er dem anderen geschrieben hat, sondern nur otvetil emu benutzt, handelt es sich hier um eine Implikatur.

Hyp. Int.: Als Leser interpretiert man die Äußerung so, dass bogda den anderen User zunächst 
nicht beleidigt hat, sondern ihn zurechtgewiesen hat und erst als Reaktion auf eine weitere, beleidigende Mitteilung den anderen User beschimpft hat (dass bogda ihn beschimpft hat, wird implikatiert). Auf Grund der drei Punke erwartet man eine weitere Äußerung des Schreibers.

$+\ddot{A}:$ Der Turn des Schreibers ist noch nicht beendet, da noch eine weitere Äußerung des Schreibers folgt.

49. $\mathrm{T}_{1} \mathbf{M}_{20,3} \quad$ prosto postojal za sebja, čto v etom takogo?!

Intention: Der Schreiber bogda intendiert, seine Empörung über die Kritik an seinem Verhalten kundzutun, indem er sich erkundigt, was daran falsch sei, sich selbst zu verteidigen/für sich selbst einzustehen. Gleichzeitig rechtfertigt er sein Verhalten als Verteidigung.

Wf 1: prosto: Adv. ,einfach'.

Wf 2: postojal:1.-3.Ps.Sg.m.Prät.pf. ,stehen', ,(an)halten'. Vom Kontext her erwartet man za, da idiomatischer Gebrauch.

Wf 3: za: Präp. ,hinter', , an', während', ,bei', , außerhalb', ,für', ,wegen'. Erwartung einer Wf im Instr., Akk. oder Gen.. Da idiomatischer Gebrauch von postojat' za kogo-libo, erwartet man eine Wf, die für kogo-libo eingesetzt wird; vom Kontext her erwartet man, dass dafür sebja eingesetzt wird.

Wf 4: sebja: Gen./Akk.Sg. ,sich'. Auf Grund des idiomatischen Gebrauchs bedeutet postojat' za sebja ,für sich selbst einstehen', ,sich selbst verteidigen’

Wf 5: čto: Pron. Nom./Akk.Sg.n./Akk.Sg.m.unbel. /Konj./Part. ,was'/ ,dass'.

Wf 6: v: Präp. ,in’. Erwartung einer Wf im Lok. od. Akk.

Wf 7: etom: Als Leser weiß man, dass es sich hierbei um das Pron. èto handelt. Aus Bequemlichkeitsgründen (weil die Taste für $\langle\dot{e}\rangle$ außen und weniger zugänglich beim schnellen Tippen als die Taste für $\langle e\rangle$ liegt) wird häufig e statt $\dot{e}$ geschrieben. Daher entscheidet man sich hier für die Bedeutung: Lok.Sg.n. ,das'. Da idiomatischer Gebrauch von 
čto $v$ etom takogo, Erwartung von takogo.

Wf 8: takogo: Gen.Sg.m./Akk.Sg.m.bel./Gen.Sg.n. ,solch'. Da idiomatischer Gebrauch, bedeutet čto $v$ etom takogo ,was ist dabei?'.

Ä 3: Den Kontext bilden der Vorfall zwischen bogda und einem anderen User und die Kritik an diesem Verhalten von DimaSss und Lelika. Durch lexikalische Hinweise (čto $v$ etom takogo) ist für den Leser klar, dass sich der Scheiber empört. Der zweite Teil der Äußerung ist eine Implikatur.

Hyp. Int.: Interpretation der Äußerung als Aufregung bogdas über die Kritik an seinem Verhalten, da er nur für sich selbst einstehen wollte. Durch čto v etom takogo implikatiert er, dass sein Verhalten aus seiner Sicht völlig gerechtfertigt war. Erwartung einer Reaktion von Lelika oder DimaSss, die bisher Kritik an bogdas Verhalten geübt haben, in der bogda erklärt wird, was genau an seinem Verhalten aus deren Sicht falsch gewesen sein soll.

$+\ddot{A}:$ Der Turn des Schreibers ist noch nicht beendet, da noch eine weitere Äußerung des Schreibers folgt.

50. $\mathrm{T}_{1} \mathrm{M}_{20,4}$ da nu davajte togda čmyrit' moderov a oni puskaj v trjapočku molčat, ja eto tak ponjal?

Intention: Der Schreiber bogda intendiert, seine Empörung über die Kritik an seinem Verhalten kundzutun, indem er rhetorisch fragt, ob er es richtig verstanden hat, dass andere User Moderatoren beleidigen/erniedrigen können und diese trotzdem schweigen sollen.

Wf 1: da: Part./Konj ,ja'/ ,und', ,doch', ,aber'.

Wf 2: nu: Interj. *,aber'/,na'; *'also', *,tja'.

Wf 3: davajte: Imperativ 2.Ps.Pl. ,geben'; , lasst uns'.

Wf 4: togda: Adv. ,dann'.

Wf 5: čmyrit': Infinitiv impf. *, schlagen', *,(be)schimpfen)', *, quälen', *,erniedrigen' ${ }^{191}$ In

${ }^{191}$ vgl. russischsprachige Bedeutung im Slovar ‘ russkogo argo (2002). 
diesem Kontext nimmt man als Leser die Bedeutung ,(be)schimpfen' oder ,erniedrigen' an, wobei jemanden zu beschimpfen eine Art der Erniedrigung darstellt.

Wf 6: moderov: Gen/Akk.Sg.m. ,Moderator'.

Wf 7: a: Konj./Part. ,und', ,aber', ,sondern'; ,wie?'.

Wf 8: oni: Pron. 3Ps.Nom.Pl. ,sie’.

Wf 9: puskaj: Part. ,lass', ,soll', ,möge'.

Wf 10: v: Präp. ,in'. Erwartung einer Wf im Lok. od. Akk.

Wf 11: trjapočku: Akk.Sg.f. + Diminutivsuffix ,Lappen'. Erwartung von molchat', da idiomatischer Gebrauch von molchat'v trjapochku.

Wf 12: molčat: 3.Ps.Pl.impf. ,schweigen'. Molchat'v trjapochku wird idiomatisch gebraucht und bedeutet*, seine Meinung/seine Gedanken nicht laut aussprechen ${ }^{192}$.

Wf 13: ja: Pron. 1.Ps.Nom.Sg. ,ich'.

Wf 14: eto: Als Leser weiß man, dass es sich hierbei um das Pron. èto Nom./Akk.Sg.n. ,das' handelt. Aus Bequemlichkeitsgründen wird häufig e statt $\dot{e}$ geschrieben.

Wf 15: tak: Adv. ,so'.

Wf 16: ponjal: 1.-3.Ps.Sg.m.Prät.pf., verstehen’.

Ä 4: Den Kontext bilden der Vorfall zwischen bogda und einem anderen User und die Kritik an diesem Verhalten von DimaSss und Lelika, die bogda so interpretiert, dass man Moderatoren beschimpfen kann und diese trotzdem schweigen sollen. Aus dem Kontext heraus ist klar, dass es sich bei dem zweiten Bestandteil der Äußerung um eine rhetorische Frage handelt.

Hyp. Int.: Als Leser interpretiert man die Äußerung als empörte, rhetorische Frage, ob bogda die Kritik an seinem Verhalten richtig verstanden hat, dass er als Moderator es hätte schweigend hinnehmen sollen von einem anderen User beleidigt zu werden. Erwartung einer Reaktion von DimaSss oder Lelika, womit diese auf die Kritik eingehen.

\footnotetext{
${ }^{192}$ vgl. russischsprachige Bedeutung im Frazeologičeskij slovar' russkogo literaturnogo jazyka (2008).
} 
$+\ddot{A}:$ Der Turn des Schreibers ist noch nicht beendet, da noch eine weitere Äußerung des Schreibers folgt.

51. $\mathrm{T}_{1} \mathrm{M}_{20,5} \quad$ ved juzer možet druzej pozvat'..

Intention: Der Schreiber bogda intendiert, die Kritik von DimaSss (und Lelika) zu kritisieren, die meinen, dass man als Moderator nicht trotz einer Beschimpfung schweigen soll, denn nur, wenn man einem Gast/User hilft, wirbt er für das Forum bei seinen Freunden.

Wf 1: ved': Part./Konj. ,ja', ,wirklich' / ,denn', ,doch'.

Wf 2: juzer: Nom.Sg.m. ,User'. Hiermit sind User eines Onlinedienstes wie eines Forums gemeint.

Wf 3: možet: 3.Ps.Sg.impf. ,können'.

Wf 4: druzej: Gen./Akk.P1.m. ,Freund'.

Wf 5: pozvat': Infinitiv pf., rufen'

Ä 5: Den Kontext bilden der Vorfall zwischen bogda und einem anderen User und die Kritik an diesem Verhalten von DimaSss, der meint, dass nur, wenn ein User/Gast für das Forum bei seinen Freunden wirbt, dieses sich weiterentwickelt, und deswegen User/Gäste nie beleidigt werden sollten. Die zwei Punkte könnten wie drei Punkte als Hinweis auf eine Sprechpause fungieren.

Hyp. Int.: Als Leser interpretiert man die Äußerung als implikatierte Kritik an der Äußerung von DimaSss, die bogda so interpretiert, dass ein Moderator trotz einer Beleidigung zu schweigen hat, weil der User/Gast sonst bei seinen Freunden nicht für das Forum wirbt. Sollten die zwei Punkte eine Sprechpause andeuten, erwartet man eine weitere Äußerung des Schreibers bogda, ansonsten eine Reaktion von DimaSss oder Lelika.

+ Ä: Der Turn des Schreibers ist noch nicht beendet, da noch eine weitere Äußerung des Schreibers folgt. 
52. $\mathrm{T}_{1} \mathrm{M}_{20,6} \quad$ i ksta, Dragon privel $v$ primer to ne menja, èto ne moi slova, nu eto dlja razočarovavšejsja vo mne Lelike...

Intention: Der Schreiber bogda intendiert, Lelikas Interpretation, die von DragOn zitierten Worte stamten von ihm, zu korrigieren.

Wf 1: i: Konj. ,und', ,auch', ,sogar'.

Wf 2: ksta: Aus seinem HW heraus ist dem Leser klar, dass es sich hierbei um die gekürzte Form des Adv. kstati ,übrigens' handelt. Diese gekürzte Form wird häufig von jungen Leuten verwendet.

Wf 3: DragOn: Nom.Sg.m. Username.

Wf 4: privel: 1.-3.Ps.Sg.m. Prät. pf. ,mitbringen', ,holen'; ,bewirken', ,erbringen', ,führen zu'; , angeben', ,aufführen'. Aus dem Kontext (aus dem, dass dem Leser bekannt ist, was DragOn gesagt hat $\rightarrow$ s. Zitat) heraus, entscheidet man sich als Leser zunächst für die Bedeutung, angeben'/, aufführen'. Man erwartet im weiteren Verlauf irgendetwas mit $v$ primer ,Beispiel’, da es sich bei dem Zitat von DragOn um ein Beispielzitat handelt.

Wf 5: v: Präp. ,in’. Erwartung einer Wf im Lok. od. Akk.

Wf 6: primer: Nom./Akk. Sg.m. unbel. ,Beispiel'. Da idiomatischer Gebrauch von privesti v primer ,als Beispiel anführen', korrekte Annahme der Bedeutungvon privel und Bestätigung der Ewartung.

Wf 7: to: Pron. Nom./Akk.Sg.n./Konj./Part. ,jenes'/, dann'.

Wf 8: ne: Negation.

Wf 9: menja: Pron. Gen./Akk.Sg. ,ich'.

Wf 10: èto: Pron. Nom./Akk.Sg.n. ,das'.

Wf 11: ne: Negation.

Wf 12: moi: Possesivpron. Nom./Akk.Pl.unbel. ,mein'.

Wf 13: slova: Nom./Akk.Pl.m./n., Wort'. 
Wf 14: nu: Interj. *,aber'/,na'; *'also', *,tja'.

Wf 15: eto: Aus oben bereits angeführter Gründen weiß man als Leser, dass es sich hierbei um das Pron. èto handelt. Nom./Akk.Sg.n. ,das'.

Wf 16: dlja: Präp. ,für’. Erwartung einer Wf im Gen.

Wf 17: razočarovavšejsja: Partizip Prät. Akt. ,enttäuscht sein'. Erwartung der Präp. v und einer Wf im Lok., da idiomatischer Gebrauch.

Wf 18: vo: Präp. ,in'. Als Leser weiß man, dass es sich hierbei um die korrekte stellungsbedingte Rechtschreibung der Präp. handelt. Erwartung einer Wf im Lok., da idiomatischer Gebrauch.

Wf 19: mne: Pron. Dat./Lok.Sg. ,ich'. Razocharovat'sja vo mne bedeutet, von mir enttäuscht sein'.

Wf 20: Lelike: Dat./Lok.Sg.f. Username. Hier handelt es sich jedoch um einen potenziellen Rechtschreibfehler, der als Folge eine nicht korrekte Kasusendung hat, da von der Präp. ein Genitiv verlangt wird. Auf Grund der Präposition und des Wissens, dass $e$ und $i$ häufig verwechselt werden, ist einem als Leser jedoch klar, dass es sich eigentlich um einen Gen. Sg.f. handeln sollte.

Ä 6: Den Kontext der Mitteilung bilden der Vorfall zwischen bogda und einem anderen User, das von DragOn angeführte Zitat und die Äußerungen von Lelika, vor allem die, in der sie bogdas gutes Verhalten vor der Zeit als Moderator seinem schlechten Verhalten in der Zeit als Moderator (als bogda sich erlaubt hat mit Schimpfwörtern einem anderen User zu antworten) gegenüberstellt und das darauf zurückführt, dass er seine Macht gespürt und sich daraufhin aufgeplustert hat. Bogda interpretiert diese und weitere Äußerungen von Lelika so, dass sie glaubt, er hätte die von DragOn zitierten Worte geschrieben.

Hyp. Int.: Als Leser interpretiert man die Äußerung so, dass bogda die Worte, die DragOn zitiert, nicht geschrieben hatte. Dies richtet er vor allem an Lelika, deren Äußerungen er so interpretiert, dass sie glaubt, er hätte diese Worte geschrieben und dass sie von ihm enttäuscht sei. Erwartung einer Reaktion von DragOn, in der er klarstellt, wer diese Worte geschrieben hat oder einer Reaktion von Lelika, die darauf eingeht, wie sie dieses Zitat verstanden hat und ob sie von bogda enttäuscht ist oder nicht. 
- ̈̈: Es folgt keine weitere Äußerung, so dass ein Partizipantenwechsel stattfindet und ein Leser sich als neuer Schreiber äußern kann.

53. $\mathrm{T}_{1} \mathrm{M}_{21,1} \quad$ bogda ne razočarovalas'ja v tebe

Intention: Lelika Intention als Schreiber ist es, bogda zu widersprechen, indem sie feststellt, sie sei von ihm nicht enttäuscht. Um dieser Intention Nachdruck zu verleihen, betont sie, dass das Gegenteil dessen, was bogda sagte, zutrifft. Dadurch, dass die Negation an die erste Position der Äußerung (die Adressierung besetzt als Vokativ syntaktisch eine Position außerhalb des eigentlichen Satzes) gerückt wird, betont sie, dass sie von ihm nicht enttäuscht ist.

Wf: bogda: Nom.Sg. Aus dem Kontext heraus lässt sich vermuten, dass der Name hier in vokativischer Funktion gebraucht wird und es sich hier um eine Adressierung handelt.

Wf 1: ne: Negation.

Wf 2: razočarovalas': 1.-3.Ps.Sg.f. Prät. pf. + refl ,enttäuscht sein'. Auf Grund von idiomatischem Gebrauch Erwartung der Präp. $v$ und einer Wf im Lok.

Wf 3: ja: Pron. 1.Ps.Nom.Sg. ,ich'.

Wf 4: v: Präp. ,in'.Auf Grund von idiomatischem Gebrauch Erwartung einer Wf im Lok.

Wf 5: tebe: Pron. Dat./Lok.Sg. 2.Ps. ,du'. Razocharovat'sja $v$ tebe bedeutet, von dir enttäuscht sein'.

Ä 1: Den Kontext bilden Lelikas Äußerungen bezüglich bogdas Verhaltens in dem Vorfall mit dem anderen User und bogdas Interpretation dieser so, dass sie von ihm enttäuscht ist.

Hyp. Int.: Als Leser interpretiert man die Äußerung so, dass Lelika von bogda nicht enttäuscht ist. Erwartung einer Reaktion von bogda, in der er erläutert, wie er zu seinem Schluss, sie wäre von ihm enttäuscht, kommt, und eventuell sagt, ob er ihr glaubt oder nicht.

- ̈̈: Es folgt keine weitere Äußerung, so dass ein Partizipantenwechsel stattfindet und ein Leser sich als neuer Schreiber äußern kann. 
54. $\mathrm{T}_{1} \mathrm{M}_{22,1} \quad$ Nu ja tak i ponjal, čto sovsem ne razočarovalas’..

Intention: bogdas Intention als Schreiber ist es anzugeben, dass er Lelika nicht glaubt. Dafür zitiert er sie quasi: sie habe gesagt, dass er vor dem Status als Moderator ein guter ForumUser war und sich, nachdem er Moderator geworden ist, aufgeplustert hat (d.h. sich wichtig gemacht hat, da er eine Machtposition bekommen hat) und dann kein gutes Forumsmitglied mehr war.

Wf 1: Nu: Interj. *,aber'/,na', *'also', *,tja'.

Wf 2: ja: Pron. 1.Ps.Nom.Sg. ,ich'.

Wf 3: tak: Adv./Konj./Part. ,so'/ ,also'/ ,ja'. Aus dem Kontext heraus nehme ich hier die Bedeutung , so’ an.

Wf 4: i: Konj. ,und', ,auch', ,sogar'. Tak $i$ wird idiomatisch gebraucht und bedeutet ,so auch'.

Wf 5: ponjal: 1.-3.Ps.Sg.m.Prät. pf. ,verstehen'.

Wf 6: čto: Pron./Konj./Part. ,was'/, dass'.

Wf 7: sovsem: Adv. ,ganz', ,überhaupt'.

Wf 8: ne: Negation.

Wf 9: razočarovalas': 1.-3.Ps.Sg.f. Prät. pf. + refl ,enttäuscht sein'. Erwartung der Präp. $v$ und einer Wf im Lok., da idiomatischer Gebrauch.

Ä 1: Den Kontext bilden vor allem die zitierte Äußerung von Lelika sowie ihre Behauptung, sie sei von bogda nicht enttäuscht. Die drei Punkte sind ein Hinweis auf eine Sprechpause und dadurch eine unvollständige Äußerung, da die erwartete Präp. und die Wf. im Lok. nach razočarovalas' nicht folgen, jedoch implikatiert werden. Auch die Wortwahl nu ja tak $i$ ponjal und sovsem ne fungiert als Hinweis. Da bogda nicht direkt sagt, ob er ihr glaubt oder nicht, handelt es sich hier um eine Implikatur.

Hyp. Int.: Interpretation der Äußerung von bogda als Implikatur, Lelika nicht zu glauben. Man erwartet im weiteren Verlauf eine Reaktion von Lelika, in der sie versucht bogda von der Aufrichtigkeit ihrer Äußerung zu überzeugen. 
- ̈̈: Es folgt keine weitere Äußerung, so dass ein Partizipantenwechsel stattfindet und ein Leser sich als neuer Schreiber äußern kann.

55. $\mathrm{T}_{1} \mathrm{M}_{23,1} \quad$ Vinni Puch èto bylo vyskazyvanie o tvoem moderatorstve, a ne o razočarovanii $v$ tebe, esli by razočarovalas' ja by votkrytuju prjamo ob ètom napisala

Intention: Die Schreiberin Lelika versucht, bogda zu beschwichtigen und ihn davon zu überzeugen, ihr zu glauben. Dazu benutzt sie seinen Kosenamen.

Wf 1: Vinni: Name. Da es sich hierbei nicht um den Usernamen handelt, es vom Kontext her aber eine Adressierung des Users bogda ist, muss es etwas mit ihm zu tun haben. Aus seinem Hintergrundwissen heraus weiß man als Leser, dass bogda als Avatarbild Vinni Puch hat. Auf dieser Grundlage stellt man einen Bezug zwischen den Namen und bogda her. Bei Vinni Puch handelt es sich um einen Kosenamen für bogda, der bereits an früherer Stelle in diesem Forumthread von Lelika benutzt wurde. Man erwartet entweder Puch als zweiten Bestandteil oder die Fortsetzung der Äußerung ohne Puch, da klar ist, um wen es sich handelt.

Wf 2: Puch: Bestandteil von Vinni Puch. Nom.Sg.m.

Wf 3: èto: Pron. Nom./Akk.Sg.n. ,das'.

Wf 4: bylo: 1.-3.Ps.Sg.n.Prät. ,sein’.

Wf 5: vyskazyvanie: Nom./Akk.Sg.n., Aussage'.

Wf 6: o: Präp. ,über', ,von', ,zu’. Erwartung einer Wf im Lok.

Wf 7: tvoem: Possesivpron. 2.Ps.Lok.Sg. ,dein’.

Wf 8: moderatorstve: Lok.Sg.n. *,Moderatorsein'. Damit ist die Tätigkeit als Moderator gemeint.

Wf 9: a: Konj./Part. ,und', ,aber', ,sondern'; ,wie?'.

Wf 10: ne: Negation.

Wf 11: o: Präp. ,über', ,von', ,zu’. Erwartung einer Wf im Lok. 
Wf 12: razočarovanii: Lok.Sg.n. ,Enttäuschung’. Erwartung der Präp. $v$ und einer Wf im Lok., da idiomatischer Gebrauch.

Wf 13: v: Präp. ,in’. Erwartung einer Wf im Lok., da idiomatisch.

Wf 14: tebe: Pron. 2.Ps.Dat./Lok.Sg. ,du'. Razočarovanie v tebe bedeutet ,Enttäuschung von dir'.

Wf 15: esli: Konj. ,falls', ,wenn'.

Wf 16: by: Part. zur Bildung des Konjunktivs.

Wf 17: razočarovalas': 1.-3.Ps.Sg.f.Prät.pf. + refl. ,enttäuscht sein'. Erwartung der Präp. v und einer Wf im Lok., da idiomatischer Gebrauch. Sollten sie nicht folgen, so werden sie implikatiert.

Wf 18: ja: Pron. 1.Ps.Nom.Sg. ,ich'.

Wf 19: by: Part. zur Bildung des Konjunktivs.

Wf 20: v: Präp. ,in’. Erwartung einer Wf im Lok. od. Akk.

Wf 21: otkrytuju: Adj. Akk. Sg.f. ,offen'. V otkrytuju ist idiomatisch (v otkrytuju čto-libo delat', offen handeln'). Erwartung einer Handlung, d.h. eines Verbs.

Wf 22: prjamo: Adv. ,geradeaus', ,geradewegs'.

Wf 23: ob: stellungsbedingte Variante der Präp. $o$,über', ,von', ,zu’. Erwartung einer Wf im Lok.

Wf 24: ètom: Pron.Lok.Sg.m./n. ,dieser/dieses'.

Wf 25: napisala: 1.-3.Ps.Sg.f.Prät.pf. ,schreiben’.

Ä 1: Den Kontext bilden die Äußerungen von Lelika, die bogda als Enttäuschung von ihm interpretiert, Lelikas Behauptung, dass sie von ihm nicht enttäuscht sei und die Implikatur von bogda, dass er Lelika nicht glaubt. Bei dem Namen Vinni Puch handelt es sich um einen Kosename für bogda.

Hyp. Int.: Als Leser interpretiert man die Äußerung so, dass Lelika bogdas Person und seine 
Tätigkeit als Moderator voneinander trennt und ihre Äußerungen sich auf bogdas Tätigkeit als Moderator und nicht auf seine Person bezogen. Das Benutzen des Kosenamens interpretiert man als Abmilderung, Beschwichtigung. Erwartung einer Reaktion von bogda, in der er schreibt, ob er ihr glaubt oder nicht.

- ̈̈: Es folgt keine weitere Äußerung, so dass ein Partizipantenwechsel stattfindet und ein Leser sich als neuer Schreiber äußern kann.

56. $\mathrm{T}_{1} \mathrm{M}_{24,1} \quad \mathrm{Nu}$ okej

Intention: Der Schreiber bogda intendiert einzulenken.

Wf 1: Nu: Interj. *,aber'/,na', *'also', *,tja'.

Wf 2: okejj: Interjektion: Zustimmung, ok'.

Ä 1: Den Kontext bilden die Äußerungen von Lelika, die bogda als Enttäuschung von ihm interpretiert, Lelikas Behauptung, dass sie von ihm nicht enttäuscht sei, seine Zweifel an ihrer Behauptung und ihr Versuch, bogda davon zu überzeugen, dass sie von ihm nicht enttäuscht ist. Die lexikalischen Hinweise fungieren hier als $\mathrm{KH}$, die anzeigen, dass bogda nicht komplett überzeugt ist, aber geneigt ist Lelika zu glauben.

Hyp. Int.: Als Leser interpretiert man die Äußerung so, dass bogda zwar nicht komplett überzeugt ist, aber dennoch geneigt ist, Lelika zu glauben. Man erwartet keine Reaktion auf diese Äußerung.

- ̈̈: Es folgt keine weitere Äußerung, so dass ein Partizipantenwechsel stattfindet und ein Leser sich als neuer Schreiber äußern kann.

An dieser Stelle endet die Konversation abrupt ohne Abschiedsformeln. Wie im 3. Kapitel dargelegt, handelt es sich bei einem solch abrupten Ende um ein typisches Merkmal. Auch andere Merkmale wie fehlende Eröffnungs- und Beendigungsphase, abrupte Themenwechsel und eine für das Medium Internetforum typische Art des Partizipantenwechsels lassen sich in diesem Konversationsbeispiel finden. Auf Einiges sei jedoch anhand dieses Beispiels das 
erste Mal kurz hingewiesen: Missverständnisse und die (Nicht-)Erfüllung der Erwartungshaltung des Lesers in Bezug auf die nächste Äußerung.

Wie in der mündlichen Kommunikation, kommen auch hier Missverständnisse, also Fehlinterpretationen vor. So finden wir diese z.B. zwischen den Schreibern silver und bogda oder Lelika und DragOn. Diese Missverständnisse resultieren aber nicht aus fehlendem Hintergrundwissen oder fehlenden Kontextualisierungshinweisen, wie dies Gumperz für die mündliche Kommunikation angibt, sondern daraus, dass z.B. Schreiber sich unklar ausdrücken. Entsprechende Ursachen lassen sich ebenfalls in der mündlichen Kommunikation feststellen. Bezüglich der Interpretation von Äußerungen sowie den Missverständnissen, die dabei entstehen können, ähneln sich die schriftliche und mündliche Kommunikation.

Bezüglich der Erfüllung bzw. Nichterfüllung der Erwartungshaltung des Lesers an die nächste Äußerung, kann man feststellen, dass die Erwartung des Lesers an die nächste Äußerung nicht immer erfüllt wird. Dies sieht man bspw. in Äußerungen $T_{2} M_{1 \text {,Import }}$ und $T_{1} M_{5,1}$, in denen ein plötzlicher Themenwechsel stattfindet, aber auch in Äußerung $\mathrm{T}_{1} \mathrm{M}_{9,1}$, die sich nicht auf die vorangehende, sondern auf die Äußerung $\mathrm{T}_{1} \mathrm{M}_{6,2}$ bezieht. Für die Nichterfüllung gibt es mehrere Gründe. Zum Einen ist dies einer von vornherein potenziell unbegrenzten Anzahl an Gesprächsteilnehmern geschuldet, die jederzeit einzelne Äußerungen/Mitteilungen der Konversation aufgreifen und besprechen können. Auf diese Weise entstehen „Gesprächsinseln“, die sich im Großen und Ganzen zwar um das eigentliche Thema drehen, jedoch nur einen einzelnen Aspekt des Themas zwischen wenigen Gesprächsteilnehmern behandeln, z.B. silvers und Lelikas Äußerungen $\mathrm{T}_{1} \mathrm{M}_{18,1}-\mathrm{T}_{1} \mathrm{M}_{19,3}$, in denen es darum geht, dass silver selbst höchstwahrscheinlich genauso gehandelt hätte wie bogda und Lelika ihm erklärt, warum bogdas Verhalten aus ihrer Sicht falsch war. Dass selbst innerhalb dieser „Inseln“ die Erwartungen in Bezug auf die nächste Äußerung häufig nicht erfüllt werden, liegt daran, dass ebenso wie ein Beitrag aus mehreren Mitteilungen bestehen kann (vgl. $\mathrm{B}_{10}$, der aus $\mathrm{T}_{1} \mathrm{M}_{5,1}$ und $\mathrm{T}_{1} \mathrm{M}_{6,2}$ besteht), auch eine Mitteilung aus mehreren Äußerungen bestehen kann, die die jeweiligen Äußerungen der Mitteilung davor einzeln aufgreifen: so handelt es sich bspw. bei $T_{1} M_{10,1}$ und $T_{1} M_{10,2}$ um Reaktionen auf $T_{1} M_{9,1}$ und bei $T_{1} M_{10,3}$ um eine Reaktion auf $\mathrm{T}_{1} \mathrm{M}_{9,2}$, welches auch durch die entsprechenden Zitate angezeigt wird. Dies liegt daran, dass in Threads in Internetforen in der Regel alles in einer Mitteilung verfasst wird. Ein weiterer Grund ist die Zitatfunktion. Durch diese wird ermöglicht, dass jede Äußerung zu einem beliebigen späteren Zeitpunkt aufgegriffen wird. Auf diese Weise kommt es vor, dass 
die Erwartung, die in Bezug auf die nächste Äußerung gestellt wird, später erfüllt wird, d.h. dass eine zu erwartende Reaktion in einer späteren Äußerung erfolgt. Oft liegen dazwischen nur wenige Äußerungen. Dadurch, dass aber nicht jeder Gesprächsteilnehmer stets online ist, können auch größere Abstände auftauchen, vgl. Äußerungen $T_{1} M_{17,3}-T_{1} M_{17,5}$ von Lelika, in der sie sich in Bezug auf bogda und sein Verhalten äußert, auf die die zu erwartende Reaktion von bogda, in der er zu ihren Äußerungen Stellung nimmt, erst in den Äußerungen $\mathrm{T}_{1} \mathrm{M}_{20,1^{-}}$ $\mathrm{T}_{1} \mathrm{M}_{20,6}$ erfolgt. Bei den hier aufgeführten Gründen für die Nichterfüllung der Erwartung in Bezug auf die nächste Äußerung handelt es sich um Merkmale der Onlinekommunikation in Internetforen, derer sich sowohl Schreiber als auch Leser bewusst sind, weswegen sie kein Problem damit haben.

Abschließend lässt sich sagen, dass das angenommene Modell angemessen und ausreichend ist, um den Interpretationsvorgang zu beschreiben, unabhängig davon, ob es sich bei der Äußerung um eine Implikatur handelt oder nicht. Die bisher angenommenen Thesen lassen sich alle auf das Beispiel anwenden. Die bisherigen Funde lassen sich auch hier zeigen. Wie in den vorherigen Beispielen lassen sich hier bestimmte Merkmale erkennen, z.B. der abrupte Themenwechsel, abrupter Gesprächsanfang und abruptes Gesprächsende, wenn kein User mehr etwas zu dem jeweiligen Thema beitragen möchte, so dass nur die Kernphase übrig bleibt sowie das Turn taking, bei dem der Partizipantenwechsel nicht zwingend so abläuft wie in der mündlichen Kommunikation. Darüber hinaus finden wir hier längere Abstände zwischen zusammenhängenden Äußerungen (Äußerung und Reaktion) wieder, ähnlich den Abständen zwischen zusammenhängenden thematischen Mitteilungen. Erklärbar ist dies sowohl durch die Zitatfunktion als auch dadurch, dass Schreiber alles in einer einzigen Mitteilung verfassen können. Auch wurde an diesem Beispiel wieder deutlich wie wichtig das Hintergrundwissen für die korrekte, der Intention des Schreibers entsprechende Interpretation ist und welche Kontextualisierungshinweise in der schriftlichen Kommunikation von Bedeutung sind. Als wichtigste Hinweise fungieren dabei lexikalische Hinweise bzw. Signalwörter. Obwohl einige Missverständnisse vorkommen, sind sie nicht auf ein Fehlen von lautlichen Kontextualisierungshinweisen zurückzuführen. Das deutet darauf hin, dass diese sicherlich wichtig sind, ihnen aber nicht eine so große Bedeutung beigemessen werden sollte, wie Gumperz dies für die mündliche Kommunikation tut. Möglicherweise spielen sie auch für die mündliche Kommunikation keine so große Rolle. Von viel größerer Bedeutung sind der Kontext, lexikalische Hinweise sowie das gemeinsame Hintergrundwissen von Schreiber und Leser. 


\section{Fazit}

Die vorliegende Dissertation beschäftigte sich mit der Interaktion in der Onlinekommunikation in ausgewählten russischsprachigen Foren, hier im speziellen den offtopic Threads Obo vsem i ni o čem und Flud vetka sowie der Interpretation von schriftlich realisierten Äußerungen. Die Wahl fiel auf diesen Bereich der Foren, da diese am ehesten einer Alltagskommunikation entsprechen. Auf diese Weise konnten die dort vorgefundenen Konversationen mit mündlichen Alltagsgesprächen auf unterschiedliche Annahmen, die bezüglich mündlicher Konversationen gemacht wurden, hin verglichen werden. Nachdem in der Einleitung festgestellt wurde, dass es sich bei der Internetkommunikation um ein „Hybridwesen“ - einer Mischung aus mündlicher und schriftlicher Kommunikation - handelt, bestanden die Ziele der Arbeit darin:

- die Annahmen, die für mündliche Kommunikation gemacht werden, in Bezug auf die Konversationen in Forenthreads zu überprüfen;

- die Kommunikation in mündlichen Gesprächen und in den untersuchten Forenthreads zu vergleichen und generelle Kommunikationsmerkmale in Forenthreads herauszuarbeiten;

- die Konversationsstruktur, den Konversationsverlauf und das Turn Taking in den Forenthreads zu beschreiben;

- Mechanismen und Schritte der Interpretation $\mathrm{zu}$ bestimmen und anhand von authentischem Sprachmaterial zu überprüfen.

Insgesamt ging es also darum, den besonderen Typ der Kommunikation, wie er in Forenthreads vorliegt, und seine charakteristischen Züge zu erfassen sowie Mittel und Schritte, die für eine erfolgreiche Interpretation schriftlich realisierter Äußerungen benötigt werden, herauszuarbeiten. Die allgemein zu überprüfenden Thesen waren, dass

- die schriftlich realisierte Kommunikation in Forenthreads eigene Merkmale besitzt, die sie von der mündlichen Kommunikation unterscheidet;

- sich Schreiber und Leser anderer Mittel bedienen müssen, die als Kontextualisierungshinweise dienen, da kein Sprachsignal zur Verfügung steht, dem lautliche Informationen entnommen werden können, und der Leser den Schreiber nicht sieht

- für eine korrekte Interpretation lexikalische Hinweise und solche Kontextualisierungshinweise eine Rolle spielen, die direkt an der Wortform 
wahrnehmbar sind;

- eine Äußerung trotz des Fehlens lautlicher Aspekte (Prosodie, paralinguistische Hinweise) sowie der Mimik und Gestik korrekt interpretiert werden kann.

Im zweiten Kapitel der Arbeit wurden Ansätze der interaktionalen Soziolinguistik, die die theoretische Grundlage der Dissertation bildete, vorgestellt. Als Grundlage für die interaktionale Soziolinguistik dient die Monographie von J. Gumperz „Discourse strategies“. In dieser beschreibt er u.a. wie eine korrekte Interpretation von mündlich realisierten Äußerungen zustande kommt. Laut seinen Überlegungen ist die Basis für eine erfolgreiche Interpretation der Kontext, den Sprecher und Hörer gleichermaßen kennen. Um diesen zu erzeugen, werden Hintergrundwissen, über das beide verfügen, sowie von beiden gleich verstandene Kontextualisierungshinweise benötigt. Ausgehend von diesen Ideen wurden eigene theoretische Annahmen erarbeitet. Die grundlegende Überlegung ist, dass die User sich an die Gegebenheiten in der Internetkommunikation anpassen, um sich erfolgreich zu verständigen. Da häufig nicht die gleichen Mittel wie für eine face-to-face-Kommunikation zur Verfügung stehen, werden im Zuge der Anpassung an ein begrenztes kommunikatives System andere Mittel (weiter-) entwickelt, die jene der face-to-face-Kommunikation nachahmen und sie - soweit möglich - zu ersetzen versuchen, vgl. hierzu Emoticons als Ersatz für Mimik und Gestik oder graphische Änderungen an der Wortform selbst sowie Gebrauch von Satzzeichen. So bilden Emoticons, Satzzeichen oder graphische Mittel potenzielle Kontextualisierungshinweise in der Internetkommunikation. Auch das Hintergrundwissen ist entscheidend, um eine Äußerung korrekt zu interpretieren. Am Ende des Abschnitts wurde schließlich ein erster Entwurf zum Interpretationsverlauf schriftlich realisierter Äußerungen vorgestellt, der die für die mündliche Kommunikation gemachten Annahmen aufgreift und sie auf die schriftlich realisierte Konversationen anwendet.

Das dritte Kapitel behandelte die Merkmale der Kommunikation in Threads. Diese wurden mit Merkmalen verglichen, die der Sprachphilosoph Grice für die mündliche Kommunikation anführt, wie bspw. das Kooperationsprinzip mit dazugehörigen Maximen. Das Ergebnis dieses Vergleichs war, dass in den untersuchten Onlinekonversationen einige Konversationsmaximen umgesetzt und andere nicht umgesetzt werden. Das Gleiche gilt für das Kooperationsprinzip, das nicht immer eingehalten wird. Ein weiterer Gewinn des Vergleichs war die Feststellung von Merkmalen, die Onlinekonversationen in Threads eigen sind: einander folgende Redebeiträge müssen sich nicht auf einander beziehen; Gespräche 
fangen abrupt an und hören abrupt auf; häufiger Themenwechsel; oft fehlende Überleitungen; für den Gesprächsinitiierer ist es unklar, wer von den Usern, die online sind, ein möglicher Gesprächspartner sein wird; Möglichkeit parallel geführter Gespräche zu unterschiedlichen Themen; Möglichkeit durch die Zitatfunktion auch weit zurückliegende, thematisch potentiell unpassende Äußerungen neu aufzugreifen. Es wurde jedoch festgestellt, dass die untersuchten Threads bezüglich des Ausprägungsgrades einiger Merkmale heterogen waren.

Weiter wurden Konversationsverläufe untersucht. Das Ziel war der Vergleich mit mündlichen Konversationsverläufen und die Feststellung von Gemeinsamkeiten und Unterschieden. Die theoretische Grundlage für Verläufe mündlicher Konversationen basierte hauptsächlich auf Levinson. Für die Darstellung von Konversationsverläufen in den untersuchten Forenthreads wurden eigene Notationskonventionen erarbeitet. Als Ergebnis der Untersuchung wurden mehrere Arten von Konversationsverläufen ausgemacht, die zunächst anhand der Unterbrechung des dominanten Themas durch thematisch unpassende Mitteilungen unterteilt werden konnten. So ergaben sich grob zwei Verläufe: ein kontinuierlicher Konversationsverlauf ohne Unterbrechungen und ein diskontinuierlicher Verlauf mit Unterbrechungen, der nach dem Merkmal der Reaktion weiter unterteilt werden kann. Unabhängig von der Gruppe gab es beim diskontinuierlichen Verlauf bestimmte gemeinsame Merkmale: beispielsweise das Vorkommen thematisch fremder Mitteilungen inmitten von Konversationen, das Führen paralleler Gespräche und abrupte Themenwechsel, für die es jedoch Signale (z.B. P.S. oder Zitate) geben kann. Hinsichtlich der Struktur fällt auf, dass bei allen Konversationen vollständige Eröffnungsphasen und komplette Beendigungsphasen fehlten, d.h. die Konversation setzte i.d.R. mit der first-topic-position ein und hörte auf, wenn kein User mehr reagierte. Somit unterschieden sich die Konversationsverläufe der untersuchten Beispiele aus Internetthreads von mündlichen Konversationen, dahingehend, dass sie von ihrer Gesamtorganisation her nur der Kernphase einer mündlichen Konversation entsprachen. Der Grund hierfür liegt in dem Wunsch, die Kommunikation möglichst ökonomisch zu gestalten: Da nur in der Kernphase einer Konversation das Thema abgehandelt wird, sind die restlichen Gesprächsphasen im Prinzip überflüssig. Die sequentiellen Strukturen entsprachen denen in mündlicher Kommunikation. Es ließen sich sowohl zweigliedrige Strukturen aus Mitteilung und Reaktion als auch mehrgliedrige Strukturen finden. Einen wichtigen Unterschied stellte das Vorkommen mehrerer Reaktionen auf Mitteilungen verschiedener User und mehrerer thematisch unterschiedlicher Mitteilungen innerhalb eines Redebeitrags dar, so dass einzelne Redebeiträge nicht mehr eins zu eins einem 
Sequenzbestandteil entsprechen, d.h. dass nicht ein Redebeitrag bzw. Turn, sondern eine Mitteilung als elementare Grundbasis einer Sequenz in Konversationen in Internetforen angesehen werden muss. Unterstützt wird dies durch den Befund, dass zusammengehörige Mitteilungen, die eine Sequenz bilden, auch weit voneinander entfernt stehen können, so dass eine klare Abgrenzung von Sequenzen, wie dies in mündlichen Konversationen der Fall ist, nicht möglich ist. Diese Untersuchung ergab u.a. viele Unterschiede zwischen Konversationsverläufen in der Threadskommunikation und mündlicher Kommunikation. Hieraus entsprang die Notwendigkeit, eine Darstellung der Struktur zu entwickeln, die speziell an die besondere Art von Konversationen in Internetthreads angepasst ist.

Als letzter Punkt des Kapitels wurde das Turn Taking behandelt. Das Ergebnis war, dass kein Regelapparat für den Partizipantenwechsel vorliegt, der mit dem für mündliche Kommunikation vergleichbar wäre. Charakteristisch ist, dass die Länge eines Turns nur technisch durch eine begrenzte Zeichenanzahl reguliert werden kann. Der Turn kann beliebig lang sein, ohne von anderen Gesprächspartizipanten unterbrochen werden zu können. Überlappungen kamen nicht vor. Ebenso war das Fehlen einer bindenden Reihenfolge der Schreiber charakteristisch. Als Gewinn ergibt sich die Erkenntnis, wie der Partizipantenwechsel in Forenthreads funktioniert, und die Feststellung, dass seine Funktionsweise sich von der Funktionsweise des Sprecherwechsels in mündlicher Kommunikation unterscheidet. Die Besonderheiten des Partizipantenwechsels sind somit ein weiteres spezielles Merkmal der Threadkommunikation.

Das vierte Kapitel widmete sich dem Hintergrundwissen und den Kontextualisierungshinweisen in schriftlich realisierten Konversationen. Das Ziel war die Einordnung dieser von Gumperz als wichtig eingestuften Faktoren. Die Untersuchung ergab, dass dem gemeinsamen Hintergrundwissen auch in Onlinekonversationen eine wichtige Rolle zukommt. Während Gumperz jedoch von nur sprachlichem und soziokulturellem Hintergrundwissen ausgeht, wurde anhand des in der vorliegenden Arbeit untersuchten Materials festgestellt, dass es noch weitere Sorten gibt, nämlich das situationsbezogene und gruppeninterne Hintergrundwissen. Gerade das gruppeninterne Hintergrundwissen, welches in makro- und mikrogruppeninternes Hintergrundwissen unterteilt werden kann, ist für eine erfolgreich verlaufende Kommunikation in den Threads unabdingbar. Aus soziolinguistischer Perspektive ist das gruppeninterne Hintergrundwissen auch insofern interessant, als dass bei näherer Betrachtung dieses Faktors enge und weniger enge Beziehungen zwischen Gruppenmitgliedern sowie Kernmitglieder und Nichtkernmitglieder in einer sonst völlig 
offenen Gruppe ausgemacht werden können.

Ein weiteres Augenmerk wurde auf potenzielle Kontextualisierungshinweise gelegt, da diese von Gumperz als entscheidend für die richtige Interpretation betrachtet wurden. Hier wurden die geläufigsten Mittel, die Schreibern in Internetforen zur Verfügung stehen und von ihnen genutzt werden, als potenzielle Kontextualisierungshinweise untersucht. Dazu gehören Satzzeichen, Großschreibung, Wiederholung von Zeichen für Laute, farbliche Markierungen, Akronyme, Emoticons und mit Asteriskus markierte Regieanweisungen. Ihre Bedeutung für die Interpretation schriftlich realisierter Konversationen wurde mit Hilfe von Experimenten, bei denen Intonationskurven von Äußerungen einmal mit und einmal ohne den jeweiligen potenziellen Kontextualisierungshinweis (mit Ausnahme von farblichen Markierungen und Akronymen), verglichen wurden, ermittelt. Die Befunde waren: bis auf die Großschreibung oder die Wiederholung von Lauten gab es kaum Einfluss auf die Intonationskurve. Für Satzzeichen findet sich in den meisten Fällen kein Einfluss; eine endgültige Antwort kann nicht gegeben werden. Wichtig ist die Feststellung, dass gerade Emoticons, die häufig als (wenn auch nur unvollständiger) Ersatz für Mimik oder Gestik genannt werden, keine Veränderung im Bereich der Intonationskurven, der mittleren Grundfrequenzen und der Tonhöhenumfänge im Vergleich zu den entsprechenden Intonationskurven ohne Emoticons zur Folge hatten. Dies bedeutet, dass Emoticons als Kontextualisierungshinweise für die Interpretation nicht in Frage kommen. Gleiches gilt für Regieanweisungen, die sogar mit der Äußerung mitgelesen werden. Ähnlich wie bei den Emoticons und den Regieanweisungen, ist zu vermuten, dass auch Akronyme keine Rolle bei der Interpretation spielen. Resümierend kann hier festgestellt werden, dass die Ergebnisse bezüglich der potenziellen Kontextualisierungshinweise häufig den von mir, aber nicht in allen Fällen den in der Literatur gemachten Annahmen entsprachen. Der Gewinn der Versuche war, dass auf Grund dieser Ergebnisse die potenziellen Kontextualisierungshinweise in zwei Gruppen aufgeteilt werden konnten. Die erste bildeten die echten Kontextualisierungshinweise: Großschreibung, Wiederholung von Zeichen für Laute, (unter bestimmten Umständen) farbliche Markierung. Die zweite bildeten die Pseudo-Kontextualisierungshinweise: Akronyme, Emoticons, Regieanweisungen. Die Satzzeichen wurden keiner der Gruppen zugeordnet, da die Ergebnisse nicht eindeutig waren. Insgesamt zeigte sich, dass die Probandinnen ein besonderes Augenmerk auf die lexikalischen Hinweise sowie den bereits bekannten Kontext legten, um sich für eine Intonation und somit Interpretation zu entscheiden. 
Das fünfte und letzte Kapitel hatte zum Ziel, den erarbeiteten theoretischen Rahmen und die gewonnenen Einsichten zu Eigenschaften und Struktur anhand einer authentischen Konversation zu überprüfen. Diese stammt aus dem zweiten Forum und ist ein typisches Beispiel für längere, diskontinuierliche Konversationen in den untersuchten Threads. Dies zeigt sich in den typischerweise auftretenden Merkmalen. Hauptsächlich ging es in diesem Kapitel jedoch um die Interpretation schriftlich realisierter Äußerungen. Dafür wurde jede Äußerung des Konversationsbeispiels mit Hilfe der theoretischen Annahmen bezüglich des Interpretationsablaufs, der zuvor graphisch dargestellt wurde, analysiert. Das Ergebnis war, dass die angenommenen Schritte hilfreich sind, um eine Interpretation zu gewährleisten. Darüber hinaus wurde wiederholt gezeigt, welche entscheidende Rolle vor allem das Hintergrundwissen für die Interpretation besitzt. Ebenfalls wurde deutlich, dass für eine erfolgreiche Interpretation schriftlich realisierter Äußerungen weniger Kontextualisierungshinweise in Form von Satzzeichen o.ä., sondern lexikalische Hinweise wichtig waren. Die empirische Überprüfung der im Laufe der vorherigen Kapitel gemachten theoretischen Annahmen an einem für Konversationen in Forenthreads typischen Gespräch ergab eine Bestätigung dieser Annahmen.

Die Ziele, Thesen und herausgearbeitete Ergebnisse dieser Dissertation können abschließend folgendermaßen zusammengefasst werden:

\section{Ziele:}

- Vergleich der Kommunikation in mündlichen Gesprächen mit Konversationen in Forenthreads, Prüfung der Anwendbarkeit existierender Annahmen;

- Herausarbeitung genereller Kommunikationsmerkmale in Forenthreads;

- Beschreibung der Konversationsstruktur, des Konversationsverlaufs und des Turn Takings in den Forenthreads;

- Bestimmung der Mechanismen und Schritte der Interpretation und Überprüfung an authentischem Sprachmaterial.

\section{Thesen:}

- Schriftlich realisierte Kommunikation in Forenthreads besitzt eigene Merkmale, die sie von mündlicher Kommunikation unterscheidet;

- Da lautliche Informationen aus dem Sprachsignal nicht zur Verfügung stehen und der Leser den Schreiber nicht sehen kann, müssen sich beide anderer 
Kontextualisierungshinweise als in mündlicher Kommuniaktion bedienen;

- Für eine korrekte Interpretation spielen lexikalische Hinweise und solche Kontextualisierungshinweise eine Rolle, die direkt an der Wortform wahrnehmbar sind;

- Trotz des Fehlens lautlicher Aspekte (Prosodie, paralinguistische Hinweise) sowie der Mimik und Gestik können Äußerungen korrekt interpretiert werden.

\section{Ergebnisse:}

1. Die Kommunikation in Internetthreads besitzt eigene, für sie typische Merkmale. Dazu gehören:

a. Weder alle Konversationsmaximen noch das Kooperationsprinzip werden stets eingehalten

b. Kontinuierliche Konversationsverläufe ohne Unterbrechungen vs. diskontinuierliche Verläufe mit Unterbrechungen, die nach dem Merkmal der Reaktion weiter unterteilt werden

c. Vorkommen thematisch fremder Mitteilungen inmitten von Konversationen

d. Möglichkeit parallel geführter Gespräche zu unterschiedlichen Themen

e. Häufiger Themenwechsel, oft fehlende Überleitung

f. Zitatfunktion ermöglicht es, auch weit zurückliegende, thematisch potentiell unpassende Äußerungen neu aufzugreifen

g. Einander folgende Redebeiträge müssen sich nicht auf einander beziehen

h. Für den Gesprächsinitiierer ist es unklar, wer von den Usern, die online sind, ein möglicher Gesprächspartner sein wird

i. Gespräche fangen abrupt an und hören abrupt auf: vollständige Eröffnungsphasen und komplette Beendigungsphasen fehlen, die Konversation setzt i.d.R. mit der first-topic-position ein und hört auf, wenn kein User mehr reagiert $\rightarrow$ entspricht der Kernphase einer mündlichen Konversation

j. Vorhandensein zwei- und mehrgliedriger sequentieller Strukturen. Wichtiger Unterschied zur Struktur mündlicher Konversationen: Vorkommen mehrerer Reaktionen auf Mitteilungen verschiedener User und mehrerer thematisch unterschiedlicher Mitteilungen innerhalb eines Redebeitrags + zusammengehörige Mitteilungen, die eine Sequenz bilden, können auch weit voneinander entfernt stehen, so dass eine klare Abgrenzung von Sequenzen - man vergleiche im 
Kontrast mündliche Konversationen - nicht möglich ist $\rightarrow$ einzelner Redebeitrag entspricht nicht mehr eins $\mathrm{zu}$ eins einem Sequenzbestandteil $\rightarrow$ nicht ein Redebeitrag bzw. Turn, sondern eine Mitteilung = elementare Einheit einer Sequenz in Konversationen in Internetforen $\rightarrow$ Notwendigkeit, eine neue Struktur zu entwickeln, die speziell an die besondere Art von Konversationen in Internetthreads angepasst ist

k. Kein Regelapparat für den Partizipantenwechsel, der mit dem für mündliche Kommunikation vergleichbar wäre

1. Fehlen einer bindenden Reihenfolge der Schreiber

m. Länge eines Turns nur durch Begrenzung (Festlegung) der Zeichenanzahl regulierbar $\rightarrow$ Turn könnte sonst beliebig lang werden, da nicht unterbrechbar

2. Vorschlag eigener Notationskonventionen, die die Spezifika schriftlich realisierter Konversationen berücksichtigen

3. Gemeinsames Hintergrundwissen auch in Onlinekonversationen für korrekte Interpretation unabdingbar

a. Spezifisch: situationsbezogenes und gruppeninternes Hintergrundwissen

b. Insbesondere gruppeninternes Hintergrundwissen für erfolgreich verlaufende Kommunikation in Threads essentiell

c. Unterscheidung von makro- und mikrogruppeninternem Hintergrundwissen

d. Unter dem Aspekt des gruppeninternen Hintergrundwissens lassen sich außerdem enge resp. weniger enge Beziehungen zwischen Gruppenmitgliedern sowie Kernmitglieder resp. Nichtkernmitglieder in einer sonst völlig offenen Gruppe ausmachen

4. Potentielle Kontextualisierungshinweise: Satzzeichen, Großschreibung, Wiederholung von Zeichen für Laute, farbliche Markierungen, Akronyme, Emoticons sowie mit Asteriskus markierte Regieanweisungen

a. Ermittlung deren Bedeutung für die Interpretation schriftlich realisierter Konversationen mit Hilfe von Experimenten, bei denen Intonationskurven (im Falle von Emoticons und Regieanweisungen auch mittlere Grundfrequenz und Tonhöhenumfang) von Äußerungen mit und ohne den jeweiligen potenziellen Kontextualisierungshinweis (mit Ausnahme von farblichen Markierungen und Akronymen) verglichen wurden.

b. Befunde:

i. Großschreibung und Wiederholung von Zeichen für Laute beeinflussen die 
realisierte Intonationskurve

ii. Kein eindeutiges Ergebnis bei Satzzeichen

iii. Farbliche Markierung als KH, wenn Bindung an gruppeninternes HW

iv. Kein großer Unterschied der Intonationskurven von Äußerungen mit vs. ohne Emoticons \& beim Vergleich der mittleren Grundfrequenzen und des Tonhöhenumfangs kein Hinweis (mit nur einer Ausnahme) darauf, dass Emoticons einen Einfluss auf die Intonation ausüben, obwohl diese häufig als (wenn auch nur unvollständiger) Ersatz für Mimik oder Gestik genannt werden $\rightarrow$ Emoticons kommen als Kontextualisierungshinweise für die Interpretation nicht in Frage

v. Regieanweisungen: gleiches Ergebnis wie im Falle der Emoticons

$>$ Echte Kontextualisierungshinweise: Großschreibung, Wiederholung von Zeichen für Laute, (unter bestimmten Umständen) farbliche Markierung

$>$ Pseudo-Kontextualisierungshinweise: Akronyme, Emoticons, Regieanweisungen

c. Bei der Entscheidung für eine Intonation und somit Interpretation berücksichtigen Probandinnen insbesondere lexikalische Hinweise sowie den bereits bekannten Kontext

5. Korrekte Interpretation auch ohne lautliche KH sowie Gestik und Mimik möglich: der Leser verlässt sich dabei auf andere Faktoren (lexikalische Hinweise, Kontext)

6. Entwicklung eines eigenen Schritt-für-Schritt-Modells der Interpretation von schriftlich realisierten Äußerungen und Diskussion auf der Grundlage authentischen Sprachmaterials

Der Gewinn der vorliegenden Dissertation ist nicht nur eine prägnantere, detailliertere Darstellung von Threadkommunikation, sondern auch eine Stützung der These, dass diese Kommunikationsart einen berechtigten, eigenen Platz in der modernen Welt hat. Es zeigt sich, dass die Threadkommunikation typische Merkmale von Kommunikation aufweist, bei der gleichfalls Fragen geklärt, Probleme verhandelt und diskutiert werden. Es handelt sich hier also ebenfalls um eine bewusste, gewollte Kommunikation, mit Zielen und unterschiedlichen Strategien der Gesprächspartizipanten. Die Gesprächsteilnehmer wollen kommunizieren und tun dies auch. Mehrere Faktoren begründen die Notwendigkeit, gerade die Kommunikation in Forumthreads zu untersuchen: 1. konzentriert sich die Mehrheit der bisherigen Arbeiten zur Internetkommunikation auf Chaträume, 2. funktioniert die Kommunikation in Forenthreads anders als die in Chatforen, schon alleine wegen der zeitlichen Abstände zwischen den 
Mitteilungen, 3. bieten gerade Forenthreads eine Vielfalt an Kommunikationsarten, z.B. thematisch orientierte Threads, die aus Fragen und Antworten bestehen vs. Threads, in denen jedes Thema besprochen werden kann, 4. thematisch nicht eingeschränkte Threads im offtopic-Bereich sind typisch für russischsprachige Foren. Auf der theoretischen Ebene ist ein Gewinn der Arbeit die Weiterentwicklung der interaktionalen Soziolinguistik mit entsprechenden Modifizierungen für die schriftlich realisierte Kommunikation in Forumthreads.

Diese Arbeit reißt jedoch nur einen Teilbereich an. Für zukünftige Forschung ergeben sich mögliche Felder:

- Die hier vorgestellten russischsprachigen Forenthreads können mit anderssprachigen oder themenspezifischen auf die in der vorliegenden Arbeit festgestellten Merkmale hin verglichen werden.

- Die im Rahmen dieser Dissertation herausgearbeiteten Merkmale können mit denen von Chaträumen verglichen werden.

- Man sollte sich auch mit gruppenspezifischen Fragen befassen: z.B. wie sich Gruppen zusammensetzen, ob man im Internet die gleichen Merkmale wiederfindet, die aus soziologischer Sicht für Gruppen angenommen werden oder wie es mit Hierarchie u.ä. aussieht.

- Interessant wäre auch die Untersuchung der im Rahmen dieser Arbeit nur kursorisch beschriebenen Kommunikationsstrategien, die anschließend mit denen in mündlicher Kommunikation verglichen werden können.

- Im Bereich der Kontextualisierungshinweise müssen Experimente, ähnlich denen in dieser Dissertation, mit mehr Probanden durchgeführt werden. Zusätzlich zur Untersuchung der Intonation, würden sich Eye-Tracking-Studien anbieten. In diesem Kontext stellt sich die Frage, wie Humor oder Sarkasmus in einer rein schriftlich realisierten Konversation dargestellt und verstanden wird.

Jede Untersuchung eröffnet neue Perspektiven, ergibt nächste Fragen, deren Antworten weitere nötige Details liefern sollen. 


\section{$\underline{\text { 7. Bibliographie }}$}

Akademiegrammatik Bd.II = Švedova, N. et.al. (1980): Russkaja Grammatika. Band II. Moskva: Izdat. Nauka.

Alter, K. \& U. Junghanns (2002): Topic-Related Prosodic Patterns in Russian. In: Kosta, P. \& J. Frasek (Hrsg.): Current Approaches to Formal Slavic Linguistics (= Linguistik International 9). Frankfurt am Main [u.a.]: Lang, 73-87.

Anderson et al. = Anderson, J. F., Beard, F. K., \& J.B, Walther (2010): Turn-taking and the local management of conversation in a highly simultaneous computer-mediated communication system. Language@Internet 7, article 7. (urn:nbn:de:0009-7-28048; Zugriff am 08.04.15).

Auer, P.: (1992): Introduction : John Gumperz' Approach to Contextualization. In: Auer, P. et al. (Hrsg.): The contextualization of language. Amsterdam [u.a.]: Benjamins, 1-37.

Baron, N. (2010): Discourse Structures in Instant Messaging. The Case of Utterance Breaks. Language@Internet 7, article 4. (urn:nbn:de:0009-7-26514; Zugriff am 20.04.15).

Bänziger, T. \& K. R. Scherer (2005): The role of intonation in emotional expressions. In: Speech Communication 46, 252-267.

van Bezooijen, Renée (1984): Characteristics and regognizability of vocal expressions of emotion. Foris Publications Holland: Dordrecht.

Bierwisch, M. (1983): Semantische und konzeptuelle Repräsentationen lexikalischer Einheiten. In: Růžička, R. \& W. Motsch (Hrsg.): Untersuchungen zur Semantik. Berlin: Akademie, 61-101.

Böhnisch, G. (in Erscheinung): Zur Analyse von Internetkonversationen. In: Bunčić, D. (Hrsg.): Linguistische Beiträge zur Slavistik XXIV. JungslavistInnen-Treffen in Köln, 17.19.September 2015. Frankfurt am Main [u.a.]: Lang.

Böhnisch, G. (in Vorbereitung): Der Einfluss von Emoticons auf die Interpretation schriftlicher Äußerungen. In: Böhnisch, G., Junghanns, U. \& H. Pitsch (Hrsg.): Linguistische Beiträge zur Slavistik XXV. JungslavistInnen-Treffen in Göttingen, 13.-16.September 2016. Frankfurt am Main [u.a.]: Lang.

Bondaletov, V. D. (1987): Social'naja lingvistika. Moskva: Prosveščenie.

Brinker, K. (2001): Textstrukturanalyse. In: Brinker, K. et al. (Hrsg): Text- und Gesprächslinguistik. 2. Halbband, Berlin: de Gruyter, 164-175.

Brinker, K. \& J. Hagemann (2001): Themenstruktur und Themenentfaltung in Gesprächen. In: Brinker, K. et al. (Hrsg): Text- und Gesprächslinguistik. 2. Halbband, Berlin: de Gruyter, 1252-1263.

Brinker, K. \& S. Sager (1989): Linguistische Gesprächsanalyse. Eine Einführung. Berlin: Erich Schmidt Verlag. 
Bußmann, H. (Hrsg.) ( $\left.{ }^{4} 2008\right):$ Lexikon der Sprachwissenschaft. Stuttgart: Alfred Kröner Verlag.

Churches et al. = Churches, O., Nicholls, M., Thiessen, M., Kohler, M. \& H. Keage (2014): Emoticons in mind: An event-related potential study. In: Social Neuroscience 9/2, 196-202.

Chovanec, J. (2009): Simulation of Spoken Interaction in Written Online Media Texts. In: Brno Studies in English 35/2, 109-128.

Crystal, D. (2001): Language and the internet. Cambridge: Cambridge University Press.

Crystal, D. (2006): Language and the Internet. Cambridge: Cambridge University Press.

Deppermann, A. $\left({ }^{4} 2008\right):$ Gespräche analysieren. Eine Einführung. Wiesbaden: VS Verlag für Sozialwissenschaften.

Derks et al. = Derks, D., Bos, A. \& J. van Grumbkow (2008): Emoticons and Online Message Interpretation. In: Social Science Computer Review 26/4, 379-388.

Elistratov, V.S. (2005): Tolkovyj slovar' russkogo slenga. Moskva: Ast-press kniga.

Fisch, R. (2004): Gruppe/Group. In: HSK Sociolinguistics/Soziolinguistik. Teilband I. Berlin: De Gruyter, 423-429.

Fomina, T.G. (2007): Sovremennyj ruskij jazyk. Fonetika. Učebnoe posobie. Kazan’: Kazanskij gosudarstvennyj universitet.

Glovinskaja, M. Ja. (1976): Variativnost' proiznošenija zaimstvovannych slov v sovremennom russkom jazyke. In: Krysin, L.P. \& D.N. Šmelev (Hrsg.): Social'nolingvističeskie issledovanija. Moskva: Nauka, 100-122.

Glück, H. (Hrsg.) (1993): Metzler Lexikon Sprache. Stuttgart, Weimar: Verlag J. B. Metzler.

Grice, H.P. (1980): Logik und Gesprächsanalyse. In: Kußmaul, P. (Hrsg.): Sprechakttheorie. Ein Reader. Wiesbaden: Akademische Verlagsgesellschaft Athenaion, 109-126.

Gruber, H. (2001): Die Struktur von Gesprächssequenzen. In: Brinker, K. et al. (Hrsg): Textund Gesprächslinguistik. 2. Halbband, Berlin: de Gruyter, 1226-1241.

Gumperz, J. (1982): Discourse Strategies. Cambridge: Cambridge University Press.

Gutschmidt, K. ( $\left.{ }^{2} 2006\right)$ : Die ostslawische Region. In: Ammon, U. et al. (Hrsg.): Sociolinguistics. An International Handbook of the Science of Language and Society = Soziolinguistik. Ein internationales Handbuch zur Wissenschaft von Sprache und Gesellschaft. Bd.3.3. Berlin: de Gruyter, 1851-1863.

Hancock, J. (2004): Verbal irony use in face-to-face and computer-mediated conversations. In: Journal of language and social psychology 23/4, 447-463.

Hoffmann, L. (2004): Fachsprache. In: Ammon, U. et al. (Hrsg.): Sociolinguistics. An International Handbook of the Science of Language and Society = Soziolinguistik. Ein internationales Handbuch zur Wissenschaft von Sprache und Gesellschaft. Bd.1.1. Berlin: de 
Gruyter, 232-238.

Isačenko, A. V. (1968): Die russische Sprache der Gegenwart. Teil 1: Formenlehre. München: Max Hueber Verlag.

Jacob, A. \& P. Mythili (2012): A socio friendly approach to the analysis of emotive speech. In: Procedia Engineering 30, 577 - 583.

Juslin, P. N. \& P. Laukka (2004): Expression, Perception, and Induction of Musical Emotions: A Review and a Questionnaire Study of Everyday Listening. In: Journal of New Music Research 33/3, 217-238.

Kolesnikov, N. P. \& E. A. Kornilov (1996): Pole russkoj brani. Rostov-na-Donu: Izd. Feniks.

Krysin, L. \& V. Belikov (2001): Sociolingvistika. Moskva: Rossijskij Gos. Gumanitarnyj Universitet.

Kosta P. \& N. Thielemann (2009): Gesprächsanalyse. In: Kempgen, S. et al (Hrsg.): Die slavischen Sprachen. Ein internationales Handbuch zu ihrer Struktur, ihrer Geschichte und ihrer Erforschung. = The Slavic Languages. An International Hanbook of their Structure, their History and their Investigation. Berlin: de Gruyter, 1029-1047.

Labov, W., Cohen, P., Robins, C. \& J. Lewis (1968): A study of the non-standard english of negro and puerto rican speakers in New York City. Cooperative Research Project No.3288. Volume II: The use of language in the speech community. New York City: Columbia University.

Levinson, S. (1979): Activity types and language. In: Linguistics 17, 365-399.

Levinson, S. (1983): Pragmatics. Cambridge: Cambridge University Press.

Levinson, S. (1989): Conversation. In: E. Barnouw (ed.): International encyclopedia of communications. New York: Oxford University Press, 407-410.

Levinson, S. (2000): Pragmatik. Tübingen: Max Niemeyer Verlag.

Maas, U. (2004): Geschriebene Sprache / Written Language. In: Ammon, U. et al. (Hrsg.): Sociolinguistics. An International Handbook of the Science of Language and Society = Soziolinguistik. Ein internationales Handbuch zur Wissenschaft von Sprache und Gesellschaft. Bd.1.1. Berlin: De Gruyter, 633-645.

Mehlhorn, G. (2002): Kontrastierte Konstituenten im Russischen. Experimentelle Untersuchungen zur Informationsstruktur. Frankfurt/M., Berlin, Bern, Bruxelles, New York, Oxford, Wien: Peter Lang.

Mozziconacci, S. J. L. \& D. J. Hermes (1997): A study of intonation patterns in speech expressing emotion or attitude: Production and perception. In: IPO Annual Progress Report 32, 154-160.

Mozziconacci, S. J. L. (2002): Prosody and Emotions. URL:

https://www.researchgate.net/publication/228953997_Prosody_and_emotions. Letzter Zugriff am 24.09.18. 
Rodero, E. (2011): Intonation and Emotion: Influence of Pitch Levels and Contour Type on Creating Emotions. In: Journal of Voice 25/1, 25-34.

Salzmann, Z. (1993): Language, Culture \& Society. An Introduction to Linguistic Anthropology. Boulder / Oxford: Westview Press.

Schegloff, E.A. (1968): Sequencing in Conversational Openings. In: American Anthropologist 70, 1075-1095.

Schegloff, E. \& H. Sacks (1973): Opening up closings. In: Semiotica 8/4, 289-327.

Schiffrin, D. (1987): Discourse markers. Cambridge: Cambridge University Press.

Schlund, K. (2009): Genese und Gebrauch von Höflichkeitsformeln im Serbischen und Deutschen. Ein funktionales Modell der Höflichkeit und seine Anwendung. München/Berlin: Sagner.

Searle, J. R. (1980): Indirekte Sprechakte. In: Kußmaul, P. (Hrsg.): Sprechakttheorie. Ein Reader. Wiesbaden: Akademische Verlagsgesellschaft Athenaion, 127-150.

Sperber, D. \& D. Wilson ( $\left.{ }^{2} 1995\right)$ : Relevance: communication and cognition. Oxford: Blackwell.

Spiegel, C. \& Th. Spranz-Fogasy (2001): Aufbau und Abfolge von Gesprächsphasen. In: Brinker, K. et al. (Hrsg): Text- und Gesprächslinguistik. 2. Halbband, Berlin: de Gruyter, 1241-1251.

Švejcer, A.D. (1977): Sovremennaja sociolingvistika: teorija, problemy, metody. Moskva: Nauka.

Svennevig, J. (1999): Getting acquainted in conversation. A study of initial interactions. Amsterdam/Philadelphia: John Benjamins Publishing.

Tannen, D. (2004): Interactional linguistics. In: Ammon, U. et al. (Hrsg.): Sociolinguistics. An International Handbook of the Science of Language and Society = Soziolinguistik. Ein internationales Handbuch zur Wissenschaft von Sprache und Gesellschaft. Bd.1.1. Berlin: De Gruyter, 76-88.

Trudgill, P. (1974): Sociolinguistics. An Introduction. Harmondsworth: Penguin Books

Vahtin, N. \& E. Golovko (2004): Sociolingvistika i sociologija jazyka. Sankt-Peterburg: Gumanitarnaja Akademija.

Vandergriff, I. (2010): Humor and Play in CMC. In: Handbook of Research on Discourse Behavior and Digital Communication: Language Structures and Social Interaction. Vol.1. Hershley/New York: Information Science Reference, 235-251.

Vvedenskaja, L.A. \& L.G. Pavlova (1998): Kul'tura i iskusstvo reči. Sovremennaja ritorika. Rostov-na-Donu: Izd. Feniks.

Zemskaja, E.A. (Hrsg.) (1973): Russkaja razgovornaja reč’. Moskva: Nauka. 
Quellen:

Forum 1: http://alohomora.su/topic.php?forum=59\&topic=17\&p=1; Zugriff am 28.08.2013; seit 2017: http://alohomora.forum.ru/topic.php?forum=59\&topic=17\&p=1

Forum 2: http://forum.xakepok.net/showthread.php?t=2532; Zugriff am 16.10.2014

http://www.catb.org/jargon/html/T/troll.html; Zugriff am 14.10.2014

http://www.catb.org/jargon/html/F/flood.html; Zugriff am 14.10.2014

http://forum.xakepok.net/showthread.php?t=5239\&highlight=Private+persone; Zugriff am 01.09 .2016

http://www.acronymfinder.com/; Zugriff am 28.01.2016.

https://de.wikipedia.org/wiki/Liste_von_Abk\%C3\%BCrzungen_\%28Netzjargon\%29; Zugriff am 28.01.2016.

http://skyeng.ru/articles/sms-sokrashcheniya-4u-populyarnye-akronimy-ery-tekstovyhsoobshchenij-i-internet-chatov; Zugriff am 28.01.2016.

http://www.yaplakal.com/forum7/topic779350.html; Zugriff am 28.01.2016. 


\section{Anhang}

(7) $)^{193 ; 194}$

$\mathrm{B}_{1} \quad \mathrm{~T}_{1} \mathrm{M}_{1} \quad \mathrm{R}_{\mathrm{v}}$

Tempest $^{195}:$,Гыгы - ${ }_{1}$ все ГП оказались, йа так и знала,!/,

$\mathrm{T}_{2} \mathrm{M}_{1}$

${ }_{2}$ Терь о плохом $/{ }^{*}$ с надрывом*, седня, в каких-то жалких

полчетвертого ночи подлые провайдеры , / отрубили

мну от главной артерии и системы жизнеобеспечения моего

информационного голода - инет выключили, хады, да еще и

так - без предупреждения, ( , - тыц, и все - красная

натертая ромашка и Алхи не отвечает ,

3Мну в ужасе поправила усе проводки, понажимала на все

кнопочки, вытащила у всеядного Сухаря из зубов остатки

телефонного провода, написала Сушке кучу смс-к и

расстроенная бухнулась спать , ,

$\mathrm{T}_{3} \mathrm{M}_{1} \quad{ }_{4} \mathrm{Hy}$, думаю, хоть астрологи меня порадуют - наивная /....../<smiles></smiles>

ImportВсе как всегда! (крыть)

Овнов ожидает довольно напряженный день.

Профессиональные дела не ладятся: успеть нужно многое, но возможность уделить внимание запланированным делам появляется не так часто, как хотелось бы. Возможны крупные расходы, которые нанесут серьезный удар по вашему кошельку. Однако вы не принимаете происходящее

\footnotetext{
${ }^{193}$ Alle Beispiele der vorliegenden Dissertation werden entgegen wissenschaftlichem Standard nicht kursiviert angegeben, da Kursivierung in Mitteilungen von den Usern selbst zu bestimmten Zwecken vorgenommen wird. ${ }^{194}$ Das Beispiel fängt nicht mit $\alpha$ an, da es bei $B_{1}$ nicht um den ersten Redebeitrag des Themas handelt. Das gilt für alle Beispiele, denen ein $\alpha$ fehlt.

${ }^{195}$ Die Unterstreichung der Nicknames der Nutzer wird durch die Verfasserin der Dissertation vorgenommen, um die jeweiligen Schreiber zu verdeutlichen.
} 
близко к сердцу, а потому имеете шанс изменить ситуацию к лучшему.

$\mathrm{B}_{2} \quad \mathrm{~T}_{3} \mathrm{M}_{2} \quad \mathrm{R}_{\mathrm{T} 3 \mathrm{M} 1} \quad \underline{\text { Black Santa: }}$,ГыыЫ/, ${ }_{1}$ Мэндик, я на тебя глядя тож прочел гороскоп.

Import Стрелец (крыть)

День высокого эмоционального напряжения. Все ваши неприятности этого дня будут связаны с использованием непроверенной информации. Возможен острый конфликт в семье, постарайтесь его избежать, пока гроза не переросла в бурю, которая сотрет в пыль ваши личные отношения. Сегодня лучше уделить время прогулке, съездить за город на пикник, заняться спортом в хорошей компании. Решения стоит принимать только по не терпящим отлагательства важным бытовым вопросам. Возможны неприятности с детьми, что, скорее всего, повлечет за собой непредвиденные финансовые расходы.

${ }_{2}$ И дома быть нельзя, и в школу заходить смертельно опасно ()))।

$\mathrm{B}_{3} \quad \mathrm{~T}_{3} \mathrm{M}_{3}$

Tempest: ${ }_{1}$ Black Santa - сиди дома, с половином не разговаривай, чтоб не поругаться, , чате и крути велотренажер - занимаешься спортом в хорошей компании /)))/ (9 /2B школу не ходи - ну их нафиг, всех детей в лес - на пикник /!, /

$\mathrm{B}_{4} \quad \mathrm{~T}_{3} \mathrm{M}_{4} \quad \mathrm{SK}_{\mathrm{T} 3 \mathrm{M} 2} / \mathrm{R}_{\mathrm{T} 3 \mathrm{M} 3}$ Black Santa: ${ }_{1} \mathrm{У}_{г у}$, сегодня был субботник - всем коллективом убирали территорию. / $/ 2$ Вот тебе и пикник, и спорт, и хорошая компания. / /3 Теперь разогнуться не могу. 
$\mathrm{B}_{5} \quad \mathrm{~T}_{4} \mathrm{M}_{1}$

$\mathrm{T}_{3} \mathrm{M}_{5} \quad \mathrm{R}_{\mathrm{T} 3 \mathrm{M} 1} / \mathrm{R}_{\mathrm{T} 3 \mathrm{M} 2}$

Azure: ${ }_{1}$ Сегодня мой любимый день просто /)))/2Пятница 13 е рулит/!/ , ,

${ }_{3}$ Смотрел я на вас, смотрел /))/ 4 И пошел читать свой гороскоп/.../

ImportРак (крыть)

Сегодня день благоприятен для того, чтобы заниматься домашними делами и обязанностями. Можно провести время с семьей, при этом организовав подвижные и активные игры. Благоприятно уделить время походу по магазинам, и с удовольствием потратить деньги на уютные вещи для своей квартиры. Вечер отлично подойдет для уединения, медитаций и размышления над собственной жизнью. Настроение будет прекрасным, появится много оптимистичных надежд.

${ }_{5}$ Короче, сделал вывод, что надо идти тратить то, что еще осталось от стипендии /)))/

$\mathrm{B}_{6} \mathrm{~T}_{3} \mathrm{M}_{6} \quad \mathrm{R}_{\mathrm{T} 3 \mathrm{M} 2} \quad \underline{\text { Ksanka: }}$ ЫЫ/, ${ }_{1}$ неприятности из-за использования непроверенной информации ?, ${ }_{2}$ Покажите мне этого астролога! , , *о избежание конфликтов продолжаю приворяться мебелью*, ,

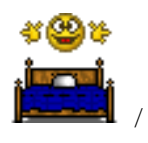

$\mathrm{B}_{7} \quad \mathrm{~T}_{4} \mathrm{M}_{2} \quad \mathrm{R}_{\mathrm{T} 4 \mathrm{M} 1,2} \quad \underline{\text { Ksanka: }}{ }_{1}$ Пятницу 13-е мы все пережили /.../ вроде.

2Тлоко после шабаша пришлось свои настоящие сущности раскрывать., ,

зТеперь вот любуюсь новым обликом /)))/,

B $\quad \mathrm{T}_{5} \mathrm{M}_{1} \quad \underline{\text { Vesnushka: }}{ }_{1}$ Я тут это/../ тоже/.../к вам $\left.\left./=\right)\right)_{/ 2}$ Возьмёте $/$ /, 
$\mathrm{T}_{1} \mathrm{M}_{2}$

$\mathrm{T}_{3} \mathrm{M}_{7} \mathrm{R}_{\mathrm{T} 3 \mathrm{M} 1-\mathrm{T} 3 \mathrm{M} 5}$ ${ }_{7}$ Начитавшись ваших гороскопов, прочитала у Санточки свой , 18 Мне тоже сбеать из дому?*

$\omega$

$(8)^{196}$

$\mathrm{B}_{1} \quad \mathrm{~T}_{1} \mathrm{M}_{1} \quad \mathrm{R}_{\mathrm{v}}$

$\mathrm{B}_{2}$

$\omega$

$\varnothing$

Erik: ${ }_{1}$ если нужны проги для взлома накрутки трояны итд пишите 444-492-434

AgentMario: Цитата: Сообщение от Lam*: AgentMario $c$ моим 16 оO

${ }_{1}$ Уже 20 , 总,

Цитата: Сообщение от Ерик: если нужны проги для взлома накрутки трояны итд пишите 444-492-434

$\mathrm{T}_{1} \mathrm{M}_{2}$ ${ }_{2} \mathrm{~A}$ я тут голову ломаю /.../ для взлома "хз чего" на хак форуме ?? 4Эх, спасибо, выручил,!/ 5Или ты имеешь ввиду, что вместо билдера трояна даш уже готовый сервер,?/ бКруто, давненько меня не ломали/../ 7 Спасибо ещё раз заранее !!,

$\varnothing$

${ }^{196}$ Das Beispiel fängt nicht mit „, $\mathrm{a}^{“}$ an, da es sich bei $\mathrm{B}_{1}$ nicht um den ersten Redebeitrag des Themas handelt (vgl. (23)). 
$(8)$

$[\ldots]$

$\mathrm{B}_{2}$

AgentMario: $[\ldots]$

$\mathrm{T}_{1} \mathrm{M}_{2}$

${ }_{2} \mathrm{~A}$ я тут голову ломаю /.../ 3 Где же мне взять троян и проги для взлома "хз чего" на хак форуме/?, чЭх, спасибо, выручил. ${ }_{5}$ Или ты имеешь ввиду, что вместо билдера трояна даш уже готовый сервер. ${ }_{6}$ Круто, давненько меня не ломали/../ ${ }_{7}$ Спасибо ещё раз заранее,!,

(9)

$\begin{array}{lll}\mathrm{B}_{1} & \mathrm{~T}_{1} \mathrm{M}_{1} \quad \mathrm{R}_{\mathrm{v}}\end{array}$

Ksanka: ${ }_{1}$ Кексик, кругом одни кролики $\left./ !_{/ /)}\right)$/

$\mathrm{T}_{2} \mathrm{M}_{1}$

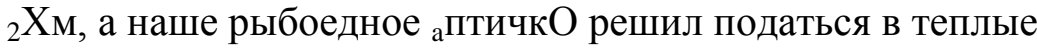

края/?,/))/, 20, /3 Уже и халатик заморский одел /))/

/*шепотом*, 4 Надеюсь, что перочинные ножички за поясом не для харакири/?, (ळ, /))/

$\mathrm{B}_{2} \quad \mathrm{~T}_{2} \mathrm{M}_{2} \quad \mathrm{R}_{\mathrm{T} 2 \mathrm{M} 1,4} \quad$ Black Santa: ${ }_{1}$ Не для харакири, а для для сеппуке ${ }^{197}$,

$\mathrm{T}_{3} \mathrm{M}_{1} \quad{ }_{2} \mathrm{~A}$ вообще буду по ночам проникать в спальни вреднючих девчонок и доставать из широких штанин длиннющее оружие и пугать их , ,

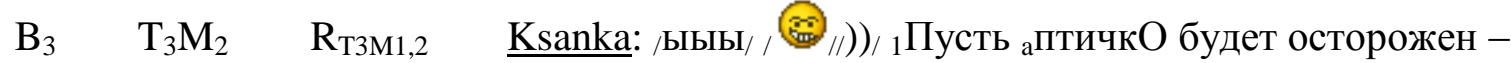
девочки не ложатся спать без своих любимых игрушек

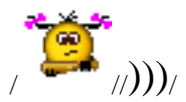

$\omega$

$\varnothing$

\footnotetext{
${ }^{197}$ Eine Form des rituellen Selbstmordes in Japan.
} 
(10)

$\mathrm{B}_{1} \quad \mathrm{~T}_{1} \mathrm{M}_{1}$

$\mathrm{B}_{2}$

$\mathrm{T}_{1} \mathrm{M}_{2}$

$\begin{array}{lll}\mathrm{B}_{3} & \mathrm{~T}_{1} \mathrm{M}_{3} & \mathrm{SK}_{\mathrm{T} 1 \mathrm{M} 1}\end{array}$

$\mathrm{B}_{4}$

$\mathrm{T}_{1} \mathrm{M}_{4}$
Klubnayk: ${ }_{1}$ А я вот флудить не буду/)/ А расскажу вам всем маленькую историю своей „ВИРТУАЛЬНОЙ/, жизни/))), ${ }_{2}$ Меня интересовало всегда 2 вапроса за последний год $\left({ }^{198}\right.$ Кстати я не нашёл ни на один из них ответа $\left({ }^{199}\right.$ 3ВОПРОС №1 Как взломать I-mail /???/ 4ВОПРОС №2 Как поиграть с читом в кс 1.6 в локальной сети Beeline C условием что: Кс пропачена и поиграть по сети можно только при включином античите sXe Injected 8.8 /?

AgentMario: Цитата: Сообщение от Klubnayk: ВОПРОС №2 Как почграть с читом в кс 1.6 в локальной сети Beeline $C$ условием что: Кс пропачена и поиграть по сети можно только при включином античите sXe Injected 8.8 ?

1Билайн UA/?,

Klubnayk: ${ }_{1}$ Блин всё зделал как написано/!/ 2 Скачял QIP 2010 зарегал себе Klubnayk@jabber.ru нашол там что то вот: xakerok@qip.ru ну добавил а он блин красный/(/ зСкажите а чё делать то а/?/ 4Как подключится/???!/ 5Пож/)/

\section{Добавлено через 5 минут 39 секунд}

Цитата: Сообщение от AgentMario: Билайн UA? 6Билайн создал свой проводной интернет,!!/ 7 Плюс в том что ещё и локальная сеть есть между пользователями/!/

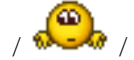
AgentMario: Цитата: Сообщение от Klubnayk: Билайн создал свой проводной интернет!! Плюс в том что ещеё и

\footnotetext{
${ }^{198}$ Es ist unklar, ob es sich hier um ein Emoticon oder eine Klammer handeln soll.

${ }^{199}$ Je nachdem, ob die vorangehende Klammer eine echte Klammer oder ein Emoticon darstellt, handelt es sich bei dieser Klammer entweder um eine verkehrt herum geschriebene Klammer, die die vorangehende Klammer schließen soll oder ein Emoticon.
} 
локальная сеть есть между пользователями!

$\mathrm{T}_{1} \mathrm{M}_{5} \quad$ Я знаю, что создал, я спрашиваю у тебя Билайн $\mathrm{UA}_{2}$ ? ${ }_{2}$ Если да, стукни в асю - могу дать пароли/...//)

$\mathrm{T}_{2} \mathrm{M}_{1} \quad$ З зарегистрированным пользователям . Зарегайся,и увидешь ссылку]!

$\mathrm{B}_{5}$

Klubnayk: Цитата: Сообщение от AgentMario: $Я$ знаю, что создал, я спрашиваю у тебя Билайн UA? Если да, стукни в асю - могу дать пароли...)

И кстати, сегодня обновился наш [Ссылки доступны только зарегистрированным пользователям . Зарегайся,u увидешь ссылку]!

$\mathrm{T}_{1} \mathrm{M}_{6} \quad{ }_{1} \mathrm{У}$ меня нету аски/!/ 2Стукни ты пож/)/ No_nekotin@ mail.ru $\varnothing$

(11) $\varnothing$

$\mathrm{B}_{1} \quad \mathrm{~T}_{1} \mathrm{M}_{1}$

$\mathrm{B}_{2}$
DragOn: ${ }_{1}$ Есть ли у нас владельцы лансеров 9-10 или маздочки 3,6,?/ 2отпишитесь как машина сама по себе, как по расходникам и пр/?,

Ra1n: Цитата: Сообщение от DragOn: Ecmь ли у нас владельиьы лансеров 9-10 или маздочки 3,6? отпишитесь как машина сама по себе, как по расходникам и пр?

[Ссылки доступны только зарегистрированным пользователям • Зарегайся,и увидешь ссылку]

$\mathrm{T}_{1} \mathrm{M}_{2}$ 
невьебаца в поворот тачила. 2 Лучшее из этих 4-ёх вариантов.

$\mathrm{B}_{3} \quad \mathrm{~T}_{1} \mathrm{M}_{3}$

$\mathrm{B}_{4}$

$\mathrm{T}_{1} \mathrm{M}_{4}$

$\mathrm{B}_{5}$

$\mathrm{T}_{2} \mathrm{M}_{1}$

$\mathrm{B}_{6} \quad \mathrm{~T}_{2} \mathrm{M}_{2}$

$\mathrm{B}_{7}$

$\mathrm{T}_{2} \mathrm{M}_{3}$

$\mathrm{B}_{8} \quad \mathrm{~T}_{3} \mathrm{M}_{1}$

$\mathrm{B}_{9}$

DragOn: ${ }_{1}$ механику смотрю только на лансере 9-ом, там нету автомата вообще в выборе. 2 не хочу сидеть мудить в пробках тех же самых.

Ra1n: Цитата: Сообщение от DragOn: механику смотрю только на лансере 9-ом, там нету автомата вообще в выборе. не хочу сидеть мудить в пробках тех же самых.

${ }_{1}$ Уверен/?/2 Могу привести пример, что это не так/)/ [Ссылки доступны только зарегистрированным пользователям • Зарегайся,и увидешь ссылку]

surpriz: Цитата: Сообщение от DragOn: Ecmь ли у нас владельцы лансеров 9-10 или маздочки 3,6? отпишитесь как машина сама по себе, как по расходникам и пр?

1деньги с сео пошли $/ / /=)_{/}$

DranOn: ${ }_{1}$ B сео денег нет

mironich: Цитата: Сообщение от DragOn: В сео денег нет

1Мне скоро это фраза снится будет

Yodjer: ${ }_{1}$ Кто нибудь залейте видео взлома компьютера через ИП

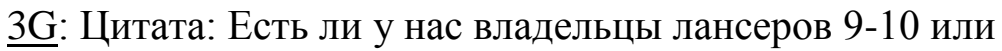
маздочки 3,6? отпишитесь как машина сама по себе, как по расходникам и пр?

$\mathrm{T}_{1} \mathrm{M}_{5}$ Митцуба однозначно дороже в обслуживании Цитата: Мазда 3 предыдущего поколения, 2.0 на механике невьебаца в поворот тачила. Лучшее из этих 4-ёх 


\section{вариантов.}

$\mathrm{T}_{1} \mathrm{M}_{6}$

${ }_{2} \mathrm{EVO} 9$ унылый сток, и нифига ни едит, њ

$\mathrm{B}_{10} \quad \mathrm{~T}_{1} \mathrm{M}_{7}$

DragOn: 1 да мне пофиг как и что едет, я для семьи беру авто, а не для гонок. 2 мне немного отжечь на дороге хватало 110 лошадок.

$\mathrm{B}_{11} \quad \mathrm{~T}_{1} \mathrm{M}_{8} \quad \mathrm{R}_{\mathrm{T} 1 \mathrm{M} 1} \quad \underline{\text { keksuk: }}{ }_{1}$ Ланцер 10 - у знакомого. $23 \mathrm{a} 4$ года обслуживания поменял только лампочки.

зМинусы - низкая подвеска и шумоизоляция стремная (уделали в ланцерах 2011 г.в.)

${ }_{4} \mathrm{~B}$ целом ничего, но советую 9 - лучше. 5 по эксплуатации хз. ${ }_{6}$ На счет мазд - не такие надежные они/../ 7хотя мне по дизайну нравятся конечно/../

$\mathrm{B}_{12} \quad \mathrm{~T}_{4} \mathrm{M}_{1} \quad \underline{\text { Resilved: }}{ }_{1}$ Возможно вопрос глупый, но все же, можно ли ддосить собственные ресурсы/?,

$\mathrm{B}_{13}$

DragOn: Цитата: Ланцер 10 - у знакомого. за 4 года обслуживания поменял только лампочки.

Минусы - низкая подвеска и шумоизоляция стремная (уделали в ланцерах 2011 г.в.)

В целом ничего, но советую 9 - лучше. по эксплуатации хз. На счет мазд - не такие надежные они.. хотя мне по дизайну нравятся конечно..

$\mathrm{T}_{1} \mathrm{M}_{9} \quad \mathrm{R}_{\mathrm{T} 1 \mathrm{M8}, 1-3} \quad{ }_{1}$ спроси про масло, жрет или нет. 2 читал в отзывах что после 100к пробега начинает кушать немного.

$\omega$ $\varnothing$ 
(12)

$\alpha$

$\mathrm{B}_{1} \quad \mathrm{~T}_{1} \mathrm{M}_{1}$

$\mathrm{T}_{2} \mathrm{M}_{1}$ $\varnothing$

guest2: ${ }_{1} У$ нас тут подарки для куратора красной группы.

${ }_{2}$ Мистер Брифор, надеемся, что вам понравятся. /

Import Спойлер (Отобразить $)^{200}$

Import Спойлер (Отобразить)

Import Спойлер (Отобразить)

Import Спойлер (Отобразить)

${ }_{2}$ Еще два подарка забыла/)/ звоть.

Import Спойлер (Отобразить)

Import Спойлер (Отобразить)

${ }_{4}$ На выходные форум вымирает, и этому есть множество причин, одна из которых - поход в кинотеатр.

5Представляем вашему вниманию несколько афиш, которые помогут определиться с выбором фильма.

6Пянтица, 13-ое уже закончилась, но кто запретит

пощекотать себе нервы в любой другой день??

Import Спойлер (Отобразить)

${ }_{7}$ Вам нужны новые аватарки/?/ 8 Хотите взрыв красок в профиле ?, 9 Обратитесь к Азуркину и Ди. 10Или просто пригласите их в кино.

\footnotetext{
${ }^{200}$ Der Inhalt wird hier nicht angezeigt, da es sich überwiegend um Bilder und Fotos handelt, auf die keiner der anderen User eingeht. Bei Bedarf sind diese unter folgender Internetadresse zu finden: http://alohomora.forum.ru/topic.php?forum=59\&topic $=17 \& \mathrm{p}=11$.
} 
Import Спойлер (Отобразить)

11 "Синяя" группа не может проиграть/!/ 2 Они захватят базу и запустят ракету, потому что так велел командир,!/

Import Спойлер (Отобразить)

${ }_{13}$ Вы проголодались или ваше имя Том/?/ 14 Тогда обязательно сходите на этот фильм.

Import Спойлер (Отобразить)

${ }_{15}$ Приятного просмотра, а в случае с последним фильмом, и аппетита, $)_{1}$

$\mathrm{B}_{2} \quad \mathrm{~T}_{3} \mathrm{M}_{1}$

Black Santa: ${ }_{1}$ Пингва пищит от радости / $/ 2$ В Липецке 17 градусов/!!!! зпосле вчерашних 32 и полутора месяцев по $35-40$

$\mathrm{B}_{3} \quad \mathrm{~T}_{4} \mathrm{M}_{1}$

Echo: ${ }_{1}$ Внимание, форумчане, Вашему вниманию предоставляется ВикиТушкоСловарь/

${ }_{2}$ Существительное ТУШКА употребляется в отношении персонажей:

31) в отношении любого персонажа любого игрока и заменяет то же самое слово «персонаж»;

42) у которого на данный момент нет хозяина;

53) у которого есть хозяин, но он временно в отъезде или проч. 6 Иными словами, на данный момент от данного персонажа нет и в ближайшее время не будет игровых постов.

7Существительное ТУШКА употребляется в отношении игроков:

81) в качестве обыкновенного обращения;

92) когда игроки тушкуют (см. значение слова тушковать в той же категории) 
10 Глагол ТУШКОВАТЬ употребляется в отношении персонажей:

111) у которых давно не было игровых постов;

122) от которых пока не стоит ждать игровых постов.

13Опять же по тем же причинам: ${ }_{14}$ Нет хозяина, хозяин занят, а может просто лениться / ${ }_{\text {/. } 15}$ А может персонаж тушкует потому что ожидает ответа перса-собеседника.

${ }_{16}$ Глагол ТУШКОВАТЬ употребляется в отношении игроков:

$\left.{ }_{17} 1\right)$ когда они «висят» в чате, то есть не проявляют активности.

${ }_{18} \mathrm{Hа} \mathrm{основании} \mathrm{вышесказанного} \mathrm{делаем} \mathrm{ВЫВОД:}$ ${ }_{19}$ ТУШКА 201 - слово универсальное.

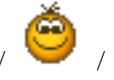

$\mathrm{B}_{4} \quad \mathrm{~T}_{4} \mathrm{M}_{2}$ Azure: 1 Эхчик, это шикарно /

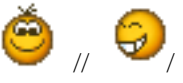

$\mathrm{B}_{5} \quad \mathrm{~T}_{4} \mathrm{M}_{3} \quad \mathrm{R}_{\mathrm{T} 3 \mathrm{M} 1} \quad \underline{\text { Tempest: }}{ }_{1}$ Викулий - это бомба, ты така умница

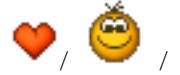

$\mathrm{B}_{6} \mathrm{~T}_{4} \mathrm{M}_{4} \quad \mathrm{R}_{\mathrm{T} 3 \mathrm{M} 1} \quad \underline{\text { Ksanka: }}{ }_{1}$ Викусик, тушечка моя умненькая, замечательные разъяснения! , (1) (I))),

$\mathrm{T}_{3} \mathrm{M}_{2}$ P.S. И о погоде , зГотовлюсь к осенним дням и наслаждаюсь остатками летних $/$ )/

$\mathrm{B}_{7} \quad \mathrm{~T}_{4} \mathrm{M}_{5} \mathrm{R}_{\mathrm{T} 3 \mathrm{M} 2, \mathrm{~T} 3 \mathrm{M} 3, \mathrm{~T} 3 \mathrm{M} 4,1}$ Echo: ${ }_{1}$ Azure, Tempest, Ksanka ну прям уж засмущали 2пасяп , (

$\omega$ $\varnothing$

\footnotetext{
${ }^{201}$ Die Wortform ist blau geschrieben.
} 
$\mathrm{B}_{1} \omega \quad \underline{\text { ProNIgZa: }}{ }_{1}$ все спокойной ночи

$\alpha$

$\varnothing$

$\mathrm{B}_{2} \quad \mathrm{~T}_{1} \mathrm{M}_{1}$

LSD66: 1 Хороша когда девушки занимаются таким

позновательным делом как хакерсто (2 Уважаю таких)/•./

$\omega$

$\varnothing$

$\mathrm{B}_{3} \quad \alpha$

ANGE1: 1 привет всем /

$\alpha$

$\varnothing$

$\mathrm{B}_{1} \mathrm{~T}_{1} \mathrm{M}_{1} \quad$ DragOn: ${ }_{1}$ короче потихоньку съезжаем сюда/.../ 2тема ники и прочее скоро закроются/)))/

$\mathrm{B}_{2} \quad \mathrm{~T}_{1} \mathrm{M}_{2} \quad$ bogda: ${ }_{1}$ Еси че, голубой ник - отсосно ${ }^{202} / \ldots /$

$\mathrm{B}_{3} \quad \mathrm{~T}_{1} \mathrm{M}_{3} \quad$ DragOn: 1 а какого цвета хочешь/?

$\mathrm{B}_{4} \quad \mathrm{~T}_{1} \mathrm{M}_{4} \quad$ bogda: ${ }_{1}$ Вот такого $\left./ . / /=\right)_{l}{ }^{203}$

$\mathrm{B}_{5} \quad \mathrm{~T}_{1} \mathrm{M}_{5} \quad$ DragOn: ${ }_{18}$ модераторы хочешь/?/)/

B $6 \quad \mathrm{~T}_{1} \mathrm{M}_{6} \quad$ bogda: ${ }_{1}$ DragOn да ты экстрасенс/..//O_O $\mathrm{O}_{2}$ Ну да, думал об этом, только вот меня остановила заморочка с помощниками/../

${ }_{3}$ Ну если что, я тут модератор [Ссылки доступны только зарегистрированным пользователям . Зарегайся,и увидешь ссылку], на подфоруме GTA. 4 Это вроде рекомендации/.../=)/

${ }^{202}$ Hier ist otsosno negativ konnotiert, im Sinne von etwas, was der Schreiber nicht haben möchte.

${ }^{203}$ Die Äußerung ist grün geschrieben. 
$\mathrm{B}_{7} \quad \mathrm{~T}_{1} \mathrm{M}_{7} \quad \mathrm{R}_{\mathrm{T1M}, 2-4} \quad \underline{\text { DragOn: }}$ 1подавай заявку оффициально и отстучи в асю

$\mathrm{B}_{8} \quad \mathrm{~T}_{1} \mathrm{M}_{8}$

$\mathrm{B}_{9} \quad \mathrm{~T}_{1} \mathrm{M}_{9}$

$\mathrm{B}_{10} \quad \mathrm{~T}_{1} \mathrm{M}_{10}$

$\omega$

(15)

$\alpha$

$\mathrm{B}_{1} \quad \mathrm{~T}_{1} \mathrm{M}_{1}$

$\mathrm{B}_{2} \quad$ (non-) $\mathrm{T}_{2} \mathrm{M}_{1}$

$\mathrm{B}_{3} \quad \mathrm{~T}_{1} \mathrm{M}_{2} \quad \mathrm{R}_{\mathrm{T} 1 \mathrm{M} 1,2} \quad \underline{\text { kotovskij: }}$ 1курилка сама по себе флуд ветка/../просто

$\mathrm{B}_{4} \quad$ non- $\mathrm{T}_{2} \mathrm{M}_{2} \mathrm{SK}_{\mathrm{T} 2 \mathrm{M} 1} \quad \underline{\text { King: }}{ }_{1}$ ффыва фвыа фыва фыва

$\mathrm{B}_{5}$ большая!

bogda: 1 Эй розовые, а зеленым круче..$/ /=\mathrm{P}_{\text {/ }}$

Lelika: 1ух ты Винни Пуха покрасили /)))/

bogda: Import"И вот, значит, если шар будет зелёный, они могут подумать, что это листик, и не заметят"

,*пошел за медом*//=),

$\varnothing$

$\varnothing$

Spam: ${ }_{1}$ Чувствовал пятой точкой что чего-то нехватает и понял! 2 Флуд ветки /!/

${ }_{3}$ Кому охота пофлудить, пишите тут $!$,

${ }_{4} \mathrm{PS}$ модераторы этого раздела просьба при переполнении темы её чистить

King: 1 фывафывафывафывафыва

2а что это такое,<smiles>[C+]1=CC=CC=C1</smiles>

Spam: Цитата: Сообщение от shnurd ${ }^{204}$ : курилка сама по себе флуд ветка...просто большая!

${ }^{204}$ An den Namensänderungen von shnurd > kotovskij und Heartbreakers > \}\{VostIk sieht man, dass die Personen im Laufe der Zeit ihre Nicknames geändert haben Dies kann entweder grundlos geschehen sein oder weil sie verbannt wurden und sich mit einem anderen Account neu eingeloggt haben, der jedoch auch auf alte Mitteilungen verweist. 


\begin{tabular}{|c|c|}
\hline $\mathrm{T}_{1} \mathrm{M}_{3}$ & ${ }_{1}$ Просто здесь безнаказанно, $(9)$ \\
\hline \multirow[t]{6}{*}{ non- $\mathrm{T}_{2} \mathrm{M}_{3}$} & Heartbreakers: ${ }_{1}$ Бла бла бла бла \\
\hline & апмикрмкармигавимгнуап745e978438ас4кмни48732и0м8гс \\
\hline & аурмиавгщимшаыовипартыаопапчрчр \\
\hline & Spam: Цитата: Сообщение от \}\{VostIk: Бла бла бла бла \\
\hline & апмикрмкармигавимгнуап745е978438ас4кмни48732u0м8гс \\
\hline & аурмиавгщимшаыовипартылаопапчрчр \\
\hline \multirow[t]{2}{*}{$\mathrm{T}_{2} \mathrm{M}_{4}$} & 1Плющит, понимаю/!!!/ 2 Сам иногда траву курю/.../ \\
\hline & зШудга ${ }^{205} / !$ \\
\hline & $\varnothing$ \\
\hline
\end{tabular}

(16)

$\mathrm{B}_{1} \alpha_{\mathrm{i}} \quad$ Lelika: ${ }_{1}$ Всем приветик,!

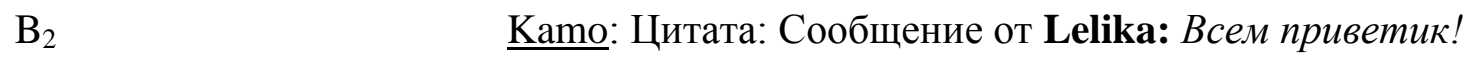

$\alpha_{\mathrm{i}}$ 13дравствуй/!/ 2наше милое создание , (9)

$\mathrm{B}_{3} \quad \alpha_{\mathrm{i}} \quad$ SenatoR: ${ }_{1}$ Всем привет $/$,

$\left.\mathrm{B}_{4} \quad \alpha_{\mathrm{i}} \quad \underline{\text { Tavares: }}{ }_{2} \mathrm{hi} /:\right) /$

(17)

$\mathrm{B}_{1} \quad \mathrm{~T}_{1} \mathrm{M}_{1} \quad \mathrm{R}_{\mathrm{v}} \quad \underline{\text { Tempest: }}{ }_{1}$ Тедька $\left.\left.\left.\left./\right)\right)\right)\right)_{/ /} \Theta_{/ 2}$ Жжоте с товарищем Горлышковым /))), (

$\mathrm{T}_{2} \mathrm{M}_{1}$ P.S. Йа сдала фсьо, что тока мона сдать, и

$205=$ šutka [Anm. der Verf.] 
защитилася - наканец-таааа,!/ /)))),

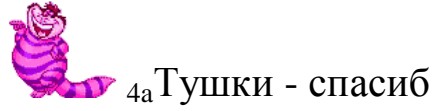

всем вам - без вас и вашей поддержки утя бы точно

крякнулО, , ${ }_{5}$ Лююю вас - от перьевого хвоста до

любопытного клюва /,

$\mathrm{T}_{3} \mathrm{M}_{1}$

${ }_{6}$ P.S.S. Товарищ Темперанс $\odot$ - кажеццо, нас заждалсО

костер на свежем воздухе/.../, (尺,

$\mathrm{B}_{2} \quad \mathrm{~T}_{2} \mathrm{M}_{2} \quad \mathrm{R}_{\mathrm{T} 2 \mathrm{M} 1,3-5}$ Nikitina: ${ }_{1}$ Профессор, сонц, мои поздравления,))/

${ }_{2}$ Отмучилась/))/

$\mathrm{B}_{3} \quad \mathrm{~T}_{2} \mathrm{M}_{3} \quad \mathrm{R}_{\mathrm{T} 2 \mathrm{M} 1,3-5} \quad \underline{\text { Ksanka: }}{ }_{1}$ Мармулядка ${ }_{\text {, }}^{206 ! !, ~} \odot \odot{ }_{2}$ Мы верили, что ты выплывешь /, /

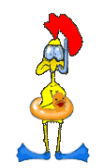

, Прячу чучелка членов комиссии, утыканные острыми иголочками, и отправляюсь мариновать тушки для барбекю*, ,

$\mathrm{B}_{4} \quad \mathrm{~T}_{2} \mathrm{M}_{4} \quad \mathrm{R}_{\mathrm{T} 2 \mathrm{M} 1,3-5} \quad$ Black Santa: $1 \mathrm{a}$ Гусена, солнцык наш носатый, заучка кривоногая, телка с монтировкой и клон кросавчега, и всевсе твои атушки, которых нам так не хватало из-за твоей хрЕновой учебы , $\Theta\left(\Theta_{12}\right.$ Теперь, я надеюся, они воскреснут,!!!, зАллилуйя,!!!!, 4Возрадуйтесь, дети мои, ибо грядет пришествие /УТИ//!!!/

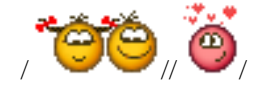

${ }_{5 \mathrm{a}}$ Мозговитая, поздравляю, я те грила, что все будет перьевым пучком/))/,

\footnotetext{
${ }^{206}$ Die Wortform ist dunkelrot geschrieben.
} 
$\mathrm{B}_{5} \quad \mathrm{~T}_{2} \mathrm{M}_{5} \quad \mathrm{R}_{\mathrm{T2M1}, 3-5} \quad \underline{\text { Rina: }}{ }_{1}$ Конфетулечка любимая/!/ 2 Со сдачей тебя/!!!!/ 3 Ты просто молодчина,!!! 4Теперь отрывайся постописанием на форуме $/)))_{\prime}$

$\mathrm{B}_{6} \quad \mathrm{~T}_{3} \mathrm{M}_{2} \quad \mathrm{R}_{\mathrm{T} 3 \mathrm{M} 1,6} \quad \underline{\text { Temperans: }}{ }_{1}$ Tempest Товарищ Tempest все готово, осталось только спичку поднести и напитки открыть.

$\mathrm{T}_{2} \mathrm{M}_{6} \quad \mathrm{R}_{\mathrm{T} 2 \mathrm{M} 1,3-5} \quad{ }_{2}$ Йулько поздравляю тебя с таким великим свершением

3$О$ Отметь за каждую сессию в отдельности основательно.

${ }_{4}$ Поздравляю тебя,

дипломированный специалист , ,

$\mathrm{B}_{7} \quad \mathrm{~T}_{2} \mathrm{M}_{7} \quad \mathrm{R}_{\mathrm{T} 2 \mathrm{M} 1,3-5} \quad \underline{\text { Scary boom: }}{ }_{1}$ Салют в честь а/ЙулькоООооОоо $/ !_{0} /{ }^{207}$

$$
\text { \%丸 }
$$

$\mathrm{B}_{8} \quad \mathrm{~T}_{2} \mathrm{M}_{8} \quad \mathrm{R}_{\mathrm{T} 2 \mathrm{M} 1,3-5} \quad \underline{\text { Azure: }}{ }_{1}$ Юлько, майне либлих конфетина,!/ 2 Мон шер, от всей души празд/../ паздр/.../ поздравляю/!!!/, *сжимает в лапке бокал с чем-то жутко алкогольным*/зВыпьем за упокой/.../ эээ/.../ здравие товарисчей членов комиссии (чтоб им там сейчас икнулось) и за нашу дорогую, абажаемую и несравненную умничку, которая (наконец-то/!!!/) все сдала, и теперь будет с нами/!/ 4Я ошшень соскучилась, просто нереально, невозможно и невыносимо/!, 5Зая моя, я тобой горжусь

$\mathrm{B}_{9} \quad \mathrm{~T}_{4} \mathrm{M}_{1} \quad \underline{\text { scary boom: }}$ Import Смешное видео. Коты.

$\mathrm{B}_{10} \mathrm{~T}_{4} \mathrm{M}_{2} \quad \underline{\text { Westwood: }}{ }_{1}$ Вволю поржал с клипа, $)_{/ 2} \mathrm{~A}$ еще рекомендую посмотреть видео про пуську по имени Мару/)))/ ХХотя думаю, этот клип видели многие /)/ Import Нажми на меня

\footnotetext{
${ }^{207}$ Die Äußerung ist rot geschrieben.
} 
$\mathrm{B}_{11} \quad \mathrm{~T}_{4} \mathrm{M}_{3} \quad \mathrm{R}_{\mathrm{T} 4 \mathrm{M} 1} \quad$ Nikitina: ${ }_{1}$ Скэри, ток засекла про грузчика, это ппц/)))//

$\mathrm{B}_{1} \quad \mathrm{~T}_{1} \mathrm{M}_{1}$

Ksanka: ${ }_{1}$ Послание всем приходящим, когда меня нет/.../
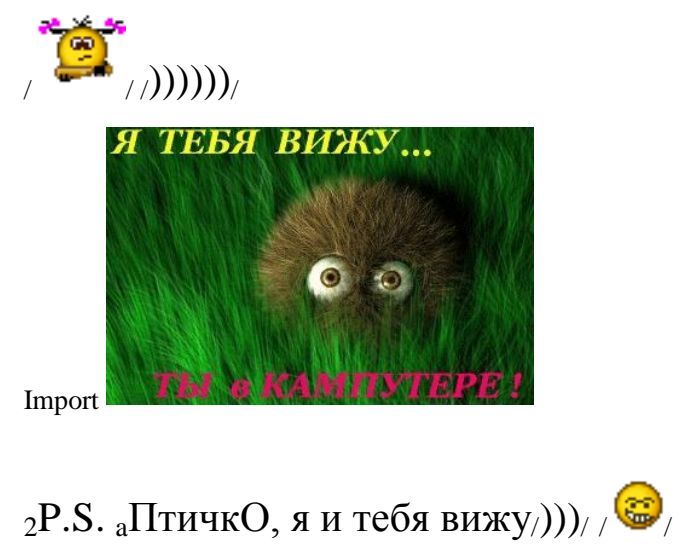

В $_{2} \mathrm{~T}_{1} \mathrm{M}_{2} \quad \mathrm{R}_{\mathrm{T} 1 \mathrm{M} 1,2}$ Black Santa: 1 А я всех вас вижу, //

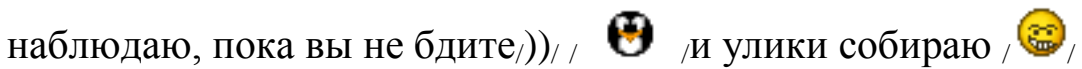

$\mathrm{B}_{3}$

Ksanka: Black Santa пишет:...и улики собираю

$\mathrm{T}_{1} \mathrm{M}_{3}$

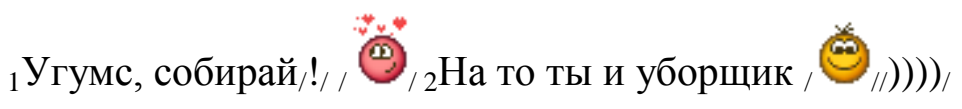

$\mathrm{B}_{4} \quad \mathrm{~T}_{2} \mathrm{M}_{1}$

Black Santa: ${ }_{1}$ КолбаСу и Сусисочки Блейзик будет нарезать, да , (잉, /, как только осилит пост 190

$\mathrm{B}_{5} \quad \mathrm{~T}_{3} \mathrm{M}_{1}$

Vesnushka: ${ }_{1}$ Я с вами уже ровно месяц, чему очень и очень рада

${ }_{2}$ Поэтому вот в самом начале. 
3Итак, где-то начало ноября. "Пьянка, дом и катлетки из

Гаррика" , , ,

4Действующие лица:

Джинни-Crazygirl

Мегги-я, Vesnushka

Teo-Scary boom

Также Дракошка и Мэндик /

Import Спойлер (Скрыть)

$[\ldots]^{208}$

$\mathrm{B}_{6}$

Ksanka: Black Santa пишет: КолбаСу и Сусисочки Блейзик будет нарезать, как только осилит пост

$\mathrm{T}_{2} \mathrm{M}_{2}$

${ }_{1}$ Нее, хочу натурального мяса/!/ / $\left.\left.\left.\otimes_{/ /}\right)\right)\right)_{/}$

2а ПтичкО-Росомаха, а на хвосте при превращении у тебя тоже стальные лезвия вместо перьев будут ?/, ( ${ }_{3}$ Если да, то можно делать шашлык из клубкопуха и еще одного «кудрявого мачО», нанизывать на хвостовые лезвия и жарить на огне до полной готовности. (

Vesnushka пишет: Итак, где-то начало ноября. "Пьянка, дом и катлетки из Гаррика"

$\mathrm{T}_{3} \mathrm{M}_{2}$

4Это дело помню очень смутно - летала/... очень рада, что Пятнышка с нами уже месяц /)))),

$\mathrm{B}_{7}$

Vesnushka: Ksanka пишет: Но я очень рада, что Пятнышка с нами уже месяц ))))

$\mathrm{T}_{3} \mathrm{M}_{3}$

${ }_{1}$ Спасибки, зай , ${ }_{2} \mathrm{~A} \mathrm{я} \mathrm{то} \mathrm{как} \mathrm{рада,} \mathrm{что} \mathrm{я} \mathrm{с} \mathrm{вами}$

${ }^{208}$ Die lange Konversation wird hier nicht weiter angezeigt, da diejenigen User, die auf einen Beitrag aus diesem Gespräch reagieren, den entsprechenden Beitrag auch zitieren. 


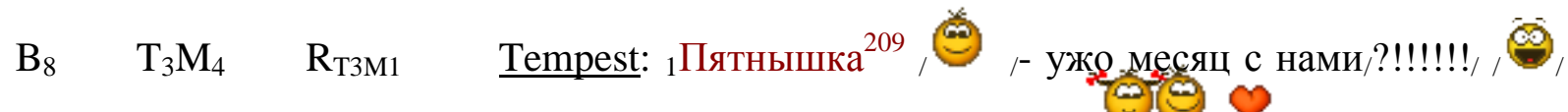
${ }_{2}$ Солнышка - мы очень рады /)))/, , /

$\mathrm{B}_{9} \quad \mathrm{~T}_{1} \mathrm{M}_{4} \quad \mathrm{R}_{\mathrm{T} 1 \mathrm{M} 1} \quad \underline{\text { scary boom: }}{ }_{1}$ Ksanka, хыыы/ потрясающая картинко. ${ }_{2}$ Напомнила рекламу про "легкий голод". , ${ }_{33}$ В нашем случае голод по алохоморе. ,

Black Santa пишет: КолбаСу и Сусисочки Блейзик будет нарезать, да 9 как только осилит пост 20

$\mathrm{T}_{2} \mathrm{M}_{3}$

Vesnushka пишет: [23:42:27] Теодор Нотт: Меган Джонс: сыми, надень, сыми, надень)))

$\mathrm{T}_{3} \mathrm{M}_{5} \quad \mathrm{R}_{\mathrm{T} 3 \mathrm{M} 1}{ }_{5}$ Ну надо же, ты это сохранила. / месяцем, солнц,! 7Желаю чтоб этих месяцев было целое множество $/)))_{/}$, (,

$\mathrm{B}_{10} \quad \mathrm{~T}_{3} \mathrm{M}_{6} \quad \mathrm{R}_{\mathrm{T} 3 \mathrm{M} 4,2} \quad \underline{\text { Vesnushka: }}{ }_{1}$ Tempest

$\mathrm{B}_{11}$

Спасибо, солнышка , (ङ) ,

Tempest: scary boom пишет: А для вегетарианцев подадут СУши?

$\mathrm{T}_{2} \mathrm{M}_{4}$ 1Для вегетарианцев - /СУшки/ и капустный /ЛИст/ /)))/,<smiles>[Te][Te]</smiles>

В ${ }_{12}$ baskervila: Black Santa пишет: КолбаСу и Сусисочки Блейзик будет нарезать

${ }^{209}$ Die Wortform ist dunkelrot geschrieben. 
scary boom пишет: А для вегетарианцев подадут СУши?

Tempest пишет: Для вегетарианцев - СУшки и капустный ЛИст )))

$\mathrm{T}_{2} \mathrm{M}_{5}$

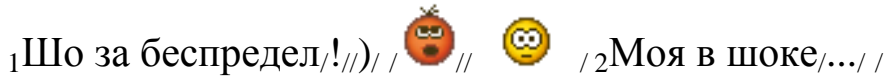

$\omega$

$\varnothing$

$(19)^{210}$

$\mathrm{B}_{1}$

Silver 18.01.2010, 10:54: Цитата: Сообщение от bogda: mom парень первый начал обзываться... $=($

$\mathrm{T}_{1} \mathrm{M}_{1}$ ㄱг бы написать администрации, и ты бы не слетел, и того бы забанили/.../

${ }_{2}$ Но на тупость отвечать тупостью/... это глупо.

$[\ldots]$

$\mathrm{B}_{2} \quad$ Logolped 04.10.2010, 17:52: Цитата: Сообщение от silver: Но на тупость отвечать тупостью...это глупо.

$\mathrm{T}_{1} \mathrm{M}_{2} \quad \mathrm{R}_{\mathrm{T} 1 \mathrm{M1}, 2} \quad{ }_{1}$ Нужно сделать тогда еще тему "слив", где и будут только такие глупости

$\omega$

$\varnothing$

(20)

$\alpha$

$\varnothing$

$\mathrm{B}_{1} \quad \mathrm{~T}_{1} \mathrm{M}_{1} \quad \underline{\text { Smoked: }}{ }_{1}$ Кто пезд1т того я рот е..л

${ }^{210}$ Das Beispiel fängt nicht mit „, $\mathrm{a}^{“}$ an, da es sich bei $\mathrm{B}_{1}$ nicht um den allerersten Redebeitrag des Themas handelt. 


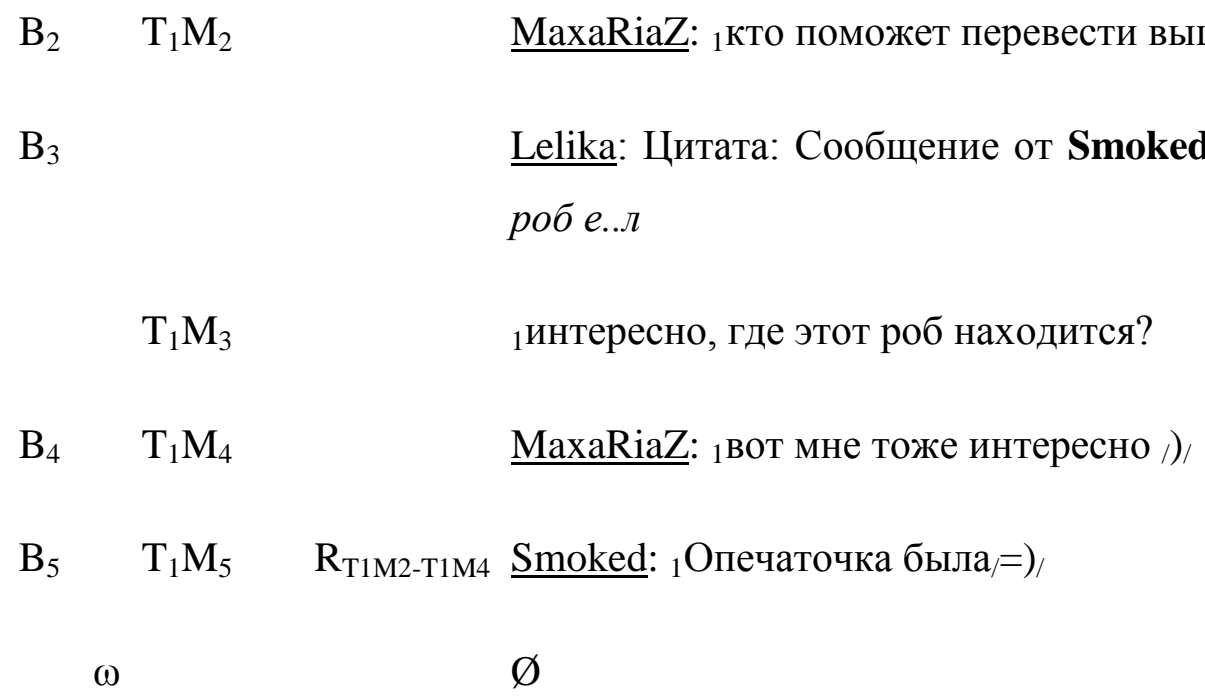

$(21)^{211 ; 212}$

$\mathrm{B}_{1} \quad \mathrm{~T}_{1} \mathrm{M}_{1} \quad \mathrm{R}_{\mathrm{v}} \quad$ keksuk: , странно мля /:D/ как меня бесят флудерасты/../

$\mathrm{B}_{2} \quad \mathrm{~T}_{1} \mathrm{M}_{2} \quad \underline{\mathrm{Ra} 1 \mathrm{n}}:{ }_{11}$ ты тоже хочешь модерку, кексик/?,

Добавлено через 1 час 47 минут 35 секунд

213

$\mathrm{B}_{3} \quad$ non- $\mathrm{T}_{2} \mathrm{M}_{1} \quad$ Aligarh: ijhfodsi

$\mathrm{B}_{4} \quad$ non- $\mathrm{T}_{3} \mathrm{M}_{1} \quad$ nikitaSWAT:1z.lskjnbjia.owjk.ban.gz.

bstoan;epanbwefdlpabndkplba

Добавлено через 1 минуту 2 секунды

${ }_{2}$ abaggsbbgsfbdsbfg

$\mathrm{B}_{5} \quad \mathrm{~T}_{1} \mathrm{M}_{3} \quad \mathrm{R}_{\mathrm{T} 1 \mathrm{M} 2,1} \quad$ keksuk: 1да я думаю мне не дадут/)/ 2 но флудеров я бы покарал)))।

\footnotetext{
${ }^{211}$ Das Beispiel fängt nicht mit „a“ an, da es sich bei $\mathrm{B}_{1}$ nicht um den ersten Redebeitrag des Themas handelt. Die Vorgeschichte sieht so aus, dass sich mehrere Teilnehmer über Flooder beschweren, darunter auch der User keksuk wie in $\mathrm{B}_{1} \mathrm{zu}$ erkennen ist.

${ }^{212}$ Bei non-Themen handelt es sich um thematische Ebenen, die jedoch explizit kein Thema haben. Ich habe mich bei diesem Beispiel für die Annahme zweier unterschiedlicher non-Themen $T_{2}$ und $T_{3}$ entschieden, da nicht mit Sicherheit geklärt werden kann, ob diese Mitteilungen thematisch zusammenhängen.

${ }^{213}$ An dieser Stelle steht ein Emoticon, das sich hier nicht darstellen lässt.
} 
$\alpha$

$\mathrm{B}_{1} \quad \mathrm{~T}_{1} \mathrm{M}_{1}$

$\mathrm{B}_{2}$

$\mathrm{T}_{1} \mathrm{M}_{2}$

$\mathrm{B}_{3} \quad \mathrm{~T}_{1} \mathrm{M}_{3}$

$\begin{array}{lll}\mathrm{B}_{4} & \mathrm{~T}_{1} \mathrm{M}_{4} & \mathrm{R}_{\mathrm{T} 1 \mathrm{M} 2}\end{array}$

$\begin{array}{lll}\mathrm{B}_{5} & \mathrm{~T}_{1} \mathrm{M}_{5} & \mathrm{R}_{\mathrm{T} 1 \mathrm{M} 2} / \mathrm{R}_{\mathrm{T} 1 \mathrm{M} 4}\end{array}$

$\mathrm{B}_{6} \quad \mathrm{~T}_{1} \mathrm{M}_{6}$$$
\text { бортом солнще жарит на } 20 \text { градусов }
$$$$
\text { дует на }-5
$$

${ }_{3} \mathrm{~A} \mathrm{если} \mathrm{и} \mathrm{снега} \mathrm{нет} \mathrm{(а} \mathrm{чувствую} \mathrm{я,} \mathrm{не} \mathrm{будет} \mathrm{нам} \mathrm{такой}$ радости зимой на Кавказе, прошлой зимой снег выпал аж в конце января, если мне не изменяет память), и радость от приближающегося Нового года уже не такая, какой ей следовало бы быть/.../

June: ${ }_{1} \mathrm{~A}$ без внешних условий взрастить радость ожидания

НГ в душе слабо,?, / , 2 Хотя тоже очень люблю снега (правда, уже в этом месяце на меня покушались падающие с крыш, непонятно, как накопившиеся там уже залежи мокрого снега) /))

Nikitina: ${ }_{1}$ И я снежок обожаю, а его все /нееет/ / ((// $)$,

Westwood: ${ }_{1}$ Какой снежок, глобальное потепление, скоро вместо елки будем пальму наряжать/)))),

Echo: ${ }_{1}$ Подписываюсь под словами Вествуда / $\Theta_{-\infty}$ / 23a

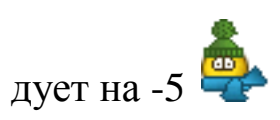

$\mathrm{B}_{7} \quad \mathrm{~T}_{1} \mathrm{M}_{7} \quad \mathrm{R}_{\mathrm{T} 1 \mathrm{M} 2} / \mathrm{R}_{\mathrm{T} 1 \mathrm{M} 4} \quad$ scary boom: ${ }_{1}$ Нафиг вам снег/?/ 2 Холод, мороз и тому подобное. зЗабыли как мерзли прошлой зимой/?//)))/ / 


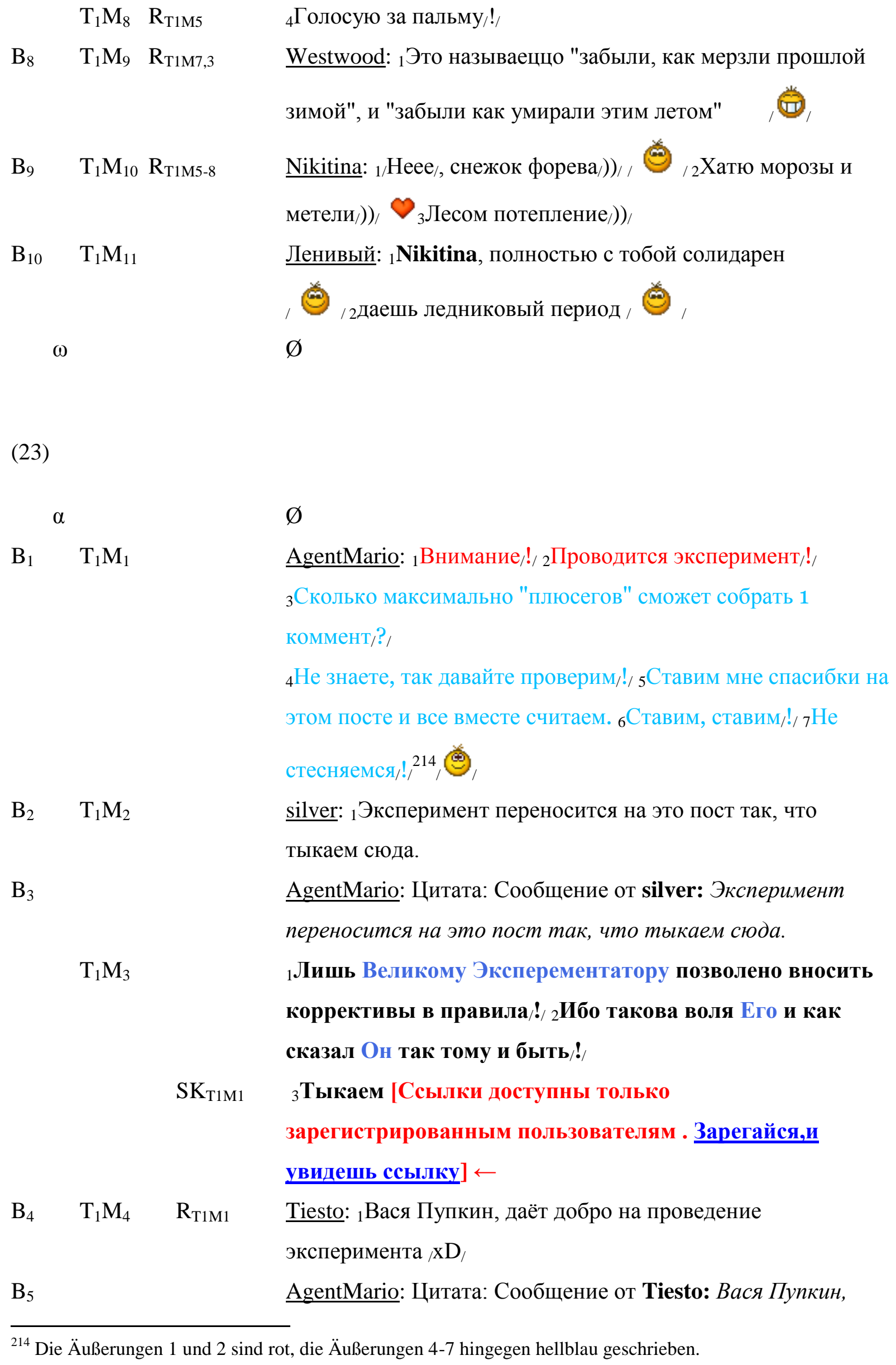

${ }^{214}$ Die Äußerungen 1 und 2 sind rot, die Äußerungen 4-7 hingegen hellblau geschrieben. 


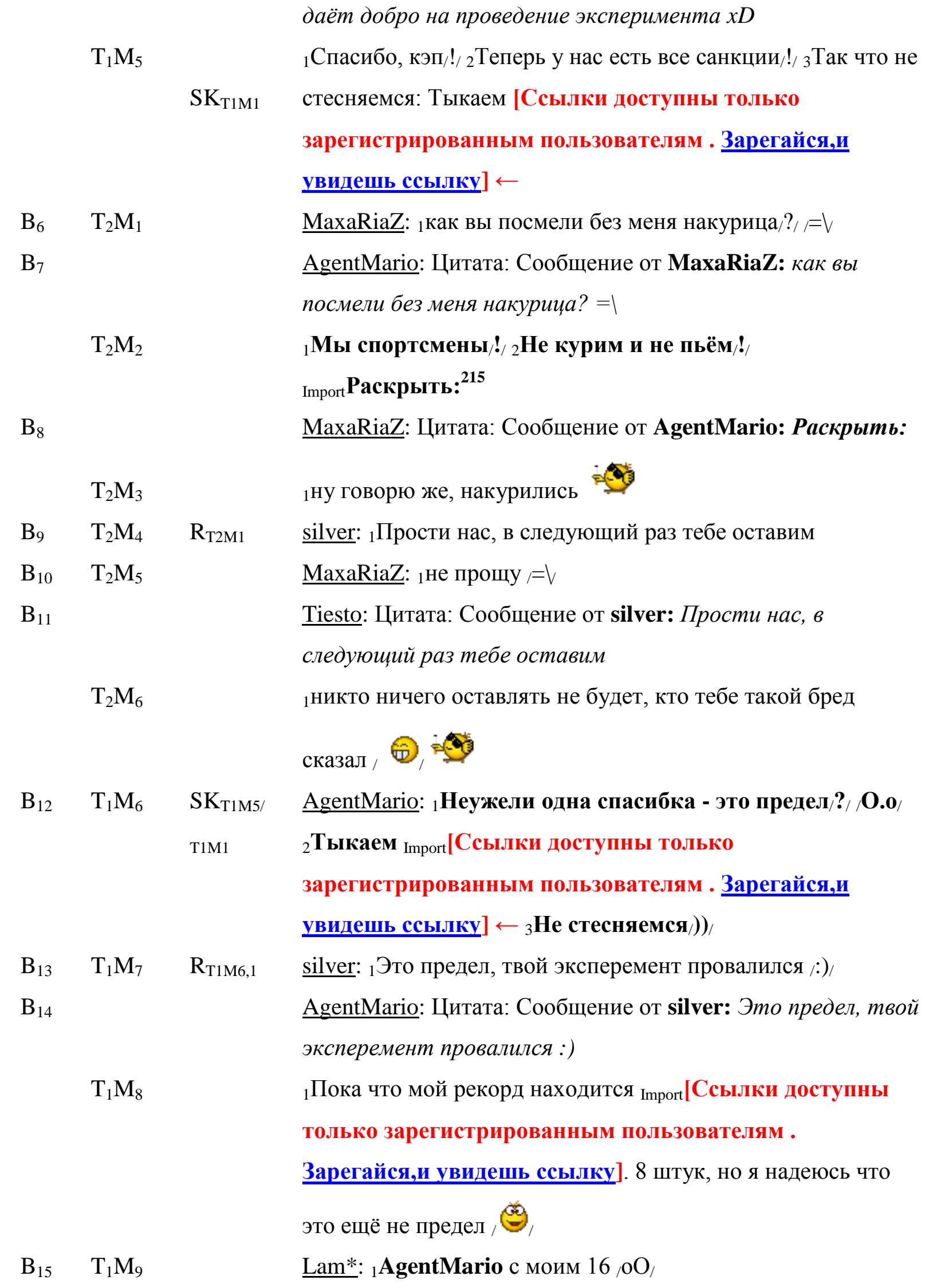


$\mathrm{B}_{16} \quad \mathrm{~T}_{3} \mathrm{M}_{1}$

$\mathrm{B}_{17}$

$\mathrm{T}_{1} \mathrm{M}_{10}$

$\mathrm{T}_{3} \mathrm{M}_{2}$

$\omega$

(24)

a

$\mathrm{B}_{1} \quad \mathrm{~T}_{1} \mathrm{M}_{1}$

$\mathrm{B}_{2} \quad \mathrm{~T}_{2} \mathrm{M}_{1}$

Erik: ${ }_{1}$ если нужны проги для взлома накрутки трояны итд пишите 444-492-434

AgentMario: Цитата: Сообщение от Lam*: AgentMario $c$ моим $16 \circ O$

${ }_{1}$ Уже $20, \Theta$

Цитата: Сообщение от Ерик: если нужны проги для взлома накрутки трояны итд пишите 444-492-434

${ }_{2} \mathrm{~A}$ я тут голову ломаю/... 3 Где же мне взять троян и проги для взлома "хз чего" на хак форуме,?/ 4Эх, спасибо, выручил/!/ 5Или ты имеешь ввиду, что вместо билдера трояна даш уже готовый сервер/? бККруто, давненько меня не ломали/../ 7 Спасибо ещё раз заранее,!/

$\varnothing$

$\varnothing$

Mariann Mistik: Задачка. 2 Не спешите гуглить ответ, попробуйте подумать.зПервоклассник с ней справится легко, старшеклассник будет долго думать, а профессор наверняка не сможет решить.

${ }_{4}$ Итак, что означает:

\section{одТЧІШСВДД}

??

Jo: ${ }_{1}$ Сидим с однокурсником в кафешке, обсуждаем всевозможные исходы встречи Волдеморта и Доктора/.../ 2 А за соседним столиком ребятишки, лет по 8, не больше, поминают Джобса, строят планы как выкупить его компанию/.../ 
${ }_{3}$ Но апофеозом всего были рассуждения о том, куда катится наша медицина и какая она за границей. 4Таким идиотом я себя ни разу в жизни не ощущала /(/.

$\mathrm{B}_{3} \quad \mathrm{~T}_{1} \mathrm{M}_{2}$

$\mathrm{B}_{4} \quad \mathrm{~T}_{1} \mathrm{M}_{3} \quad \mathrm{R}_{\mathrm{T} 1 \mathrm{M} 2}$

$\mathrm{B}_{5} \quad \mathrm{~T}_{3} \mathrm{M}_{1}$

$\omega$

$\mathrm{T}_{4} \mathrm{M}_{1}$

$\mathrm{B}_{6} \quad \mathrm{~T}_{1} \mathrm{M}_{4} \quad \mathrm{R}_{\mathrm{T} 1 \mathrm{M} 3 / \mathrm{T} 1 \mathrm{M} 1}$ Celebration: ${ }_{1}$ Tempest, Mariann Mistik чувствую себя ну ооочень умным студентом или даже профессором 2мозги окостенели напрочь. ${ }_{3} К y \partial а$, куда вы удалились, весны моей златые дни? , ,

$\mathrm{T}_{3} \mathrm{M}_{2}$

Celebration: ${ }_{1}$ Mariann Mistik, одтчпшсвдд - это значит набор букофф,

Tempest: , прочим и легко /))),

23.Ы. сказал уть, который бессовестна погуглил ,

Heiling: ${ }_{1}$ Кхм, дорогие мои форумчане/.../ мне очень приятно было играть с вами здесь, на этом чудесном форуме Алохомора. ${ }_{2}$ Я никогда не забуду те весёлые деньки и ситуации, что были между нами и нашими персонажами/.../ зтак что я хочу продолжения,!/ 43наю, немного эгоистично, но кажись сам я ничего не напишу сейчас, пока меня никто не пнёт , /. 5 Заведите со мной сценку. бЛюбую. 7 Где угодно.

${ }_{8} \mathrm{C}$ уважением, Heiling

9Зы.Сы. кста, вам не кажется, что при росте в 190 иметь вес 100 немного многовато? ${ }_{10} Э$ то прям какой-то совсем бодибилдер накаченный/.../, ,

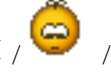

4Heiling, блин, убить тебя за такое вступление , / 5я уж думала лыжи наточил куда. 6 Чуть со стула не звезданулась. 
7Заведу с тобой сценку, готовься

$\mathrm{T}_{4} \mathrm{M}_{2}$

${ }_{8}$ Не знаю как насчет 190/100 а вот человек с параметрами 184/95 на бодибилдера ни разу не похож, просто крупный, склонный к полноте товарищ , $\odot$, 9 Х3 короче

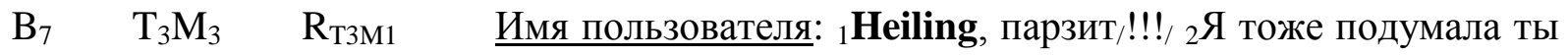
уже свалить собрался,!!!/ 3 Не шу ти так, а то моё нервное сердечко не выдержит/))/

$\mathrm{B}_{8} \quad \mathrm{~T}_{3} \mathrm{M}_{4} \quad \mathrm{R}_{\mathrm{T} 3 \mathrm{M} 1} \quad \underline{\text { Стхаткси: }}{ }_{1}$ ВоУка,!/ , ${ }^{*}$ от обилия чувствов потрясла кулаком в воздухе*, ${ }_{2} Я$ ж - девушка нервная, психованная, кардиологически не вполне здоровая. зТак что с такими заявками ты рискуешь убить Хатика,!!!,

$\mathrm{T}_{1} \mathrm{M}_{5} \quad \mathrm{R}_{\mathrm{T1M1-T1M4}}, \Theta$, ${ }_{4}$ Народ, а я совсем ненормальная, если задачку с буковками сходу решила/?, 5 В детский сад пора сдавать, да ??

$\mathrm{B}_{9} \quad \mathrm{~T}_{1} \mathrm{M}_{6}$

Black Santa: ${ }_{1}$ Стхаткси, йа тоже решила, как завсегдатай разных олимпиад в детстве, все что более-менее легкое сразу обнаруживаю, даже не интересно /((/ 2 Пошли в детский сад вместе /))),

$\mathrm{T}_{5} \mathrm{M}_{1} \quad$ Гыыы/, 3 сейчас нашла в 1С вот такой кусок кода: Import CODE:

Функция глВзятьКонтекст(КонтекстЧегоНибудь) Экспорт // чтобы вернуть что-нибудь - надо сначала вязть чтонибудь

Возврат КонтекстЧегоНибудь;

КонецФункции

${ }_{4}$ Надеюсь, никто не зайдет в офис и не увидит, как я ржу, 
$\mathrm{B}_{10} \quad \mathrm{~T}_{1} \mathrm{M}_{7} \quad \mathrm{SK}_{\mathrm{T} 1 \mathrm{M} 4} / \quad$ Celebration: ${ }_{1}$ Сил моих держаться больше не было, я тоже

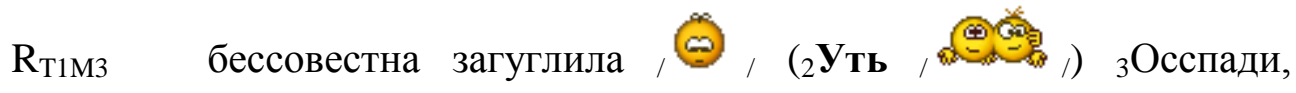
таким дауном я себя еще не чувствовала. ${ }_{4} \mathrm{У}$ нас в школе физичка так над отличниками любила глумиться, давать самую простую задачку и смотреть, как они над ней пыхтят, ища подвох /))))/

$\mathrm{T}_{1} \mathrm{M}_{8} \quad \mathrm{R}_{\mathrm{T1M5}}$-T1м6 5 Хатик, это говорит лишь о том, что у вас с Сантьяго соображалка как надо работает /)))/ бИзвестно же, что дети самый креативный народ /)/

$\mathrm{B}_{11} \quad \mathrm{~T}_{1} \mathrm{M}_{9} \quad \mathrm{R}_{\mathrm{T} 1 \mathrm{M7}, 1-3} \quad \underline{\text { Tempest: }}{ }_{1}$ Celebration, зато мы профессора /))))/(

$\mathrm{B}_{12} \mathrm{~T}_{1} \mathrm{M}_{10} \quad \mathrm{R}_{\mathrm{T} 1 \mathrm{M6}, 2} \quad$ Стхаткси: Black Santa пишет: Пошли в детский сад вместе 1/НИ, ЗА, ,ЧТО/І!!!!/ 2 Ни за какие коврижки мира,!/ З Я лучше музыкалку переживу еще раз, но в этот кошмар не вернусь/!, , , *гордая мордочка комсомолки на допросе*,

$\mathrm{T}_{6} \mathrm{M}_{1}$ Товарищи !/ ${ }_{5}$ Северус Тоббиасович был замечен $\mathrm{c}$ женщинами $!$ !

Importhttp://www.youtube.com/watch?v=8...;feature=related

\section{, (крыть $)$}

${ }_{6} \mathrm{O}$, Мерлин/!/ 7Разве танго с Рикманом - это так много/?/ 8/НЕ/ всемирного господства прошу, Господи. Вещей элементарных/.../

$\mathrm{B}_{13} \quad \mathrm{~T}_{6} \mathrm{M}_{2}$

Mariann Mistik: ${ }_{1}$ Стхаткси, вот для таких обделенных как мы / $\Theta$, это видео (+ губозакатывательная машинка в комплекте , $\Theta$,) Importhttp://www.youtube.com/watch?v=bnorSlIx 8cU 
$\mathrm{B}_{1} \quad \mathrm{~T}_{1} \mathrm{M}_{1}$

$\mathrm{B}_{2} \quad \mathrm{~T}_{1} \mathrm{M}_{2}$

$\mathrm{B}_{3} \quad \mathrm{~T}_{1} \mathrm{M}_{3}$

$\mathrm{B}_{4} \quad \mathrm{~T}_{1} \mathrm{M}_{4}$

$\mathrm{B}_{5} \quad \mathrm{~T}_{1} \mathrm{M}_{5}$

$\mathrm{B}_{6} \quad \mathrm{~T}_{1} \mathrm{M}_{6}$

$\begin{array}{lll}\mathrm{B}_{7} & \mathrm{~T}_{1} \mathrm{M}_{7} & \mathrm{R}_{\mathrm{T} 1 \mathrm{M} 5} / \mathrm{R}_{\mathrm{T} 1 \mathrm{M} 6}\end{array}$

bogda: ${ }_{1} У \mathrm{p} я / ! !$

${ }_{2}$ Вот и пришел новый, 2010 год,!!/ зВсех поздравляю/!!/

GANSTER777: ${ }_{1}$ И ТЯ ТОЖ С НОВЫМ ГОДОМ/!!!!!!!!!!

DragOn: ${ }_{1}$ ага когда сидишь пьяным за компом как то даже не тянет форум чистить/...../

silver: ${ }_{1}$ С наступившем всех $/ \mu^{216}$

bogda: ${ }_{1} \mathrm{~A}$ я еще спать не ложился/.../ нэ-нэ-нэ/..//=P/

Re\$et: ${ }_{1} \mathrm{~A}$ я наоборот лег спать в прошлом году/.../ /:)/ ${ }_{2}$ Проснулся бодрый и веселый без всякого похмелья/..../;;)/

3DragOn тебя снегом не замело /?

$\mathrm{B}_{8} \quad \mathrm{~T}_{1} \mathrm{M}_{8}$

DragOn: 1 чуть чуть/.../2я в сугробе валялся

$\mathrm{B}_{9} \quad \mathrm{~T}_{1} \mathrm{M}_{9}$

King: ${ }_{1}$ а я пьяный на кровати

$\mathrm{B}_{10} \quad \mathrm{~T}_{1} \mathrm{M}_{10}$

DragOn: ${ }_{1}$ кто не пил поднимите руку/)/

$\mathrm{B}_{11}$

Lelika: Цитата: Сообщение от DragOn: кто не пил поднимите руку)

$\mathrm{T}_{1} \mathrm{M}_{11}$

1а кто пил ,что поднять /?

$\mathrm{B}_{12} \quad \mathrm{~T}_{1} \mathrm{M}_{12}$

silver: ${ }_{1}$ ногу

$\begin{array}{lll}\mathrm{B}_{13} & \mathrm{~T}_{1} \mathrm{M}_{13} & \mathrm{R}_{\mathrm{T} 1 \mathrm{M} 11} \quad \underline{\text { DragOn: }} \text { 1лицо }\end{array}$

\footnotetext{
${ }^{216}$ Die Äußerung ist hellgrün geschrieben.
} 


$\begin{array}{lll}\mathrm{B}_{14} & \mathrm{~T}_{1} \mathrm{M}_{14} & \text { beebace: 1а каким образом поднять лицо то/?, } \\ \mathrm{B}_{15} & \mathrm{~T}_{2} \mathrm{M}_{1} & \text { beebace: 1/00о0ооооооооой/ } \\ \mathrm{B}_{16} & \mathrm{~T}_{2} \mathrm{M}_{2} & \text { beebace: 1/Поооооооооооофлудим/ } \\ \mathrm{B}_{17} & \mathrm{~T}_{2} \mathrm{M}_{3} & \text { silver: 1у тебя с головой совсем проблемы/? } \\ \mathrm{B}_{18} & \mathrm{~T}_{2} \mathrm{M}_{4} & \text { Lelika: / } \\ & \varnothing\end{array}$

$\mathrm{B}_{1} \quad \mathrm{~T}_{1} \mathrm{M}_{1} \quad$ Vesnuška: ${ }_{1 /} \mathrm{BO} /$ какие у людей бывают странности<smiles>[C-]=[Co]</smiles>

Importтыкаем беленькой штучкой, которая ползает по экрану прям сюда! ,

Селин Дион. Певица потребовала, чтобы температура воздуха в ее гримерке была ровно 23 градуса по Цельсию, иначе выступать она не будет.

2. Эми Вайнхаус. Она сама по себе ходячая нелепость, но однажды певица заявила, что в условия концерта входит трезвая команда. Организаторы должны были проследить за всеми людьми Вайнхаус, которые могли напиться.

3. Бритни Спирс как то потребовала провести ей в гримерку отдельную телефонную линию, с которой она будет осуществлять только исходящие звонки, а за каждый входящий, который ей не нужен-штраф с организатора в 3000 долларов.

4. Принц. Он требует в свою гримерку витамин В12. Непременно, только его. А также вся еда должны быть покрыта пластмассовыми крышками, которые нельзя 
открывать пока он сам это не сделает.

5. Дженнифер Лопес. O ее причудах знают, пожалуй, все. Обязательным условием для Дженнифер является наличие только белого цвета в интерьере. Все, вплоть до свечей, должно быть белоснежным.

6. Группа Van Halen. Их требование довольно милое, но действительно очень глупое. Они требовали конфеты $\mathrm{M} \& \mathrm{Ms}$, а из пачки должны быть убраны все коричневого цвета драже.

7. Моби. Никто не знает зачем, но Моби требует, чтобы у него всегда было много-много запасного нижнего белья.

8. Мадонна. O ее капризном характере слышал каждый. Звезда требует, чтобы ей ставили новые туалетные сиденья на каждом концерте.

9. Игги Попу зачем-то нужны семь карликов за сценой. Может он любит играть в "Белоснежку"??

10. Мэрайя Кэри. Ее гипертрофированная любовь ко всему пушистому и блестящему не позволяет ей проводить свои концерты без огромного количества мягких игрушек, конфет и шампанского "Кристал".

11. Пол МакКарти не зайдет в номер отеля, если там будет хоть что-то из шкуры животного, а в холодильнике окажется еда не для вегетарианца.

12. Мэрилин Мэнсон жить не может без жевательных мишек Haribo, попкорна и чипсов. Вот уж воистину, король тьмы.

13.Mötley Crüe всегда требуют в гримерку майонез, масло и горчицу.

14. Дэвид Хессельхофф переплюнул по странности многих. Он требует, чтобы в гримерках всегда стояла его фигура из картона в полный рост.

15. Барбра Стрейзанд - женщина до самых кончиков пальцев. В ее список входят лепестки роз, которые обязательно должны лежать в туалете. 
$\mathrm{B}_{2} \quad \mathrm{~T}_{1} \mathrm{M}_{2}$

$\mathrm{B}_{3} \quad \mathrm{~T}_{1} \mathrm{M}_{3}$

$\mathrm{B}_{4} \quad \mathrm{~T}_{1} \mathrm{M}_{4}$

$\mathrm{B}_{5} \quad \mathrm{~T}_{1} \mathrm{M}_{5}$

$\mathrm{B}_{1} \quad \mathrm{~T}_{1} \mathrm{M}_{1}$

$\mathrm{B}_{2} \quad \mathrm{~T}_{1} \mathrm{M}_{2}$

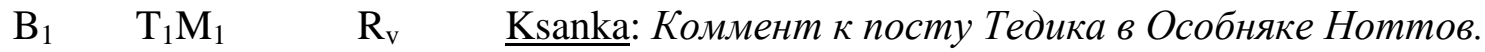

1Образина ты гиппогрифовая, , (仓), что ж ты домашнюю эльфийку своим купанием в ванной смущаешь/, , ${ }_{2}$ БЗДНЭ на тебя нет,!, , ,

33.Ы. Давай скорее в Косую аллею, мы ж скучаем /))) 
$\mathrm{B}_{1} \quad \mathrm{~T}_{1} \mathrm{M}_{1}$

$\mathrm{B}_{2} \quad \mathrm{~T}_{1} \mathrm{M}_{2}$

$\mathrm{B}_{3} \quad \mathrm{~T}_{1} \mathrm{M}_{3}$

$\mathrm{B}_{4} \quad \mathrm{~T}_{1} \mathrm{M}_{4}$

$\mathrm{B}_{5} \quad \mathrm{~T}_{1} \mathrm{M}_{5}$

notes: ${ }_{1}$ Поцаки специально зарегал бота, чтоб сказать или выразить благодарность за Код:

Вы были заблокированы по следующей причине:

Подумай прежде чем флудить!

Дата снятия блокировки: 09.05.2010, 22:00

${ }_{2}$ Смотрим внимательно 09.05.

${ }_{3} \mathrm{~B}$ общем хочу сказать спасибо тому модеру который влепил мне бан.4Спасибо за то что не пермомент. 5 Ведь я реально тогда флуд устроил чтоб криптор взять. ${ }_{6}$ Кстати за криптор отдельное спасибо, не помню уже кто выложил но он оказался рабочим из десятков мной перепробованх. 7Закругляюсь /:)/ 8Бота можете забанить я через пару дней из бана уже выйду/....../

${ }_{9} \mathrm{C}$ уважением сrys01 ${ }_{10}$ Ps.Администрации предлагаю пересмотреть свое отношение к тегу [hide]. 110 он реально только к флуду приводит і:(/ ${ }_{12}$ Pss.Специально сейчас глянул кто меня забанил и кто выложил криптор, в общем DimaSss спс кстати подумай на счет хайда/.../

GANSTER777: ${ }_{1}$ если хайда не будет- люди будут заходить и скачивать то что им надо,а регистрироваться не будут/!

MAxaRiaZ: ${ }_{1}$ в тег url суй, гостям будет вылетать сообщение типа "хочеш качать - регайся"

GANSTER777: 1лучше под хайд,т.к. если зарегается каконить новичёк и скачает,потом на вирустал зальёт $/$,

MAxaRiaZ: 1 а так новичок зарегаец, нафлудит и зальет на вирустотал /), 
$\mathrm{B}_{1} \mathrm{~T}_{1} \mathrm{M}_{1} \quad \underline{\text { GANSTER777: }}{ }_{1}$ мне нужно акки игры побрутить,кто может помочь,подсказать брут какой либо,напишите в ЛС/../20т меня несколько спасибок/)))/

$\mathrm{B}_{2} \quad \mathrm{~T}_{1} \mathrm{M}_{2} \quad$ silver: ${ }_{1}$ Какой игры ?,

$\mathrm{B}_{3}$

GANSTER777: Цитата: Сообщение от silver: Какой игры??

$\mathrm{T}_{1} \mathrm{M}_{3} \quad$ 1игра называется фабрика футбола,вот сайт этой игры[Ссылки доступны только зарегистрированным пользователям • Зарегайся,и увидешь ссылку]

$\mathrm{B}_{4} \quad \mathrm{~T}_{1} \mathrm{M}_{4} \quad \mathrm{SK}_{\mathrm{T} 1 \mathrm{M} 3}$ GANSTER777: ${ }_{1 \text { некто не знает } ? \text {, }}$

$\mathrm{B}_{5} \quad \mathrm{~T}_{1} \mathrm{M}_{5} \quad$ DimaSss: ${ }_{1}$ Попробуй самые обычные для взлома веб форм.

$\mathrm{B}_{1} \mathrm{~T}_{1} \mathrm{M}_{1} \quad$ Black Santa: ${ }_{1} \mathrm{~A}$ птичко теперь не только пингвин, но еще и

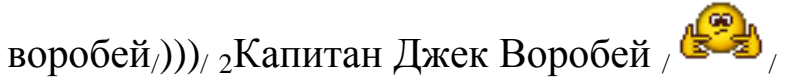

$\mathrm{B}_{2} \quad \mathrm{~T}_{1} \mathrm{M}_{2} \quad \mathrm{R}_{\mathrm{T} 1 \mathrm{M} 1} \quad$ Ksanka: „Ыы/, / 1 воробей хотя и стреляный, но все равно птичкО, только лохматость повысилась/))), (

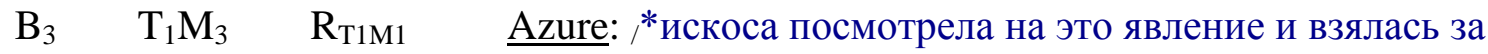
ножницы*, 1 ПтичкО, иди сюда/!, / приторно-сладким голосом*/2Я тебе модную стрижку сделаю/))/ 3 А то пингвоворобей с повышенной лохматостью - этого моя нежная впечатлительная тушка не переживет/)))/ ${ }^{217}$

$\mathrm{B}_{4} \quad \mathrm{~T}_{1} \mathrm{M}_{4} \quad \mathrm{R}_{\mathrm{T} 1 \mathrm{M} 1} \quad \underline{\text { Tempest: }}{ }_{1}$ Black Santa , 2 ,

${ }^{217}$ Der Beitrag ist dunkelblau geschrieben. 
*поеть*, Пятнадцать человек на сундук мертвеца, йо-хо-хо и бутылка рома/..../ ,

${ }_{2} \mathrm{~B}$ который раз убеждаюсь - подлецу все к лицу /)))/,

/3А такому, как Джек, вообще можно простить все/..../ /

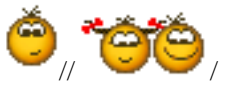

$\mathrm{B}_{5} \quad \mathrm{~T}_{1} \mathrm{M}_{5} \quad \mathrm{R}_{\mathrm{T} 1 \mathrm{M} 1} \quad \underline{\text { Ksanka: }}{ }_{1} \mathrm{~A}$ у мну уже собственная птичка есть/)))/, ${ }_{2}$ Принесли сегодня в клеточке раненную крошку костогрыза (дубонос обыкновенный). зТак что не надо нам всяких там заморских гостей в черно-белой раскраске, то ли пингвинов, то ли пиратов/))/

$\mathrm{B}_{6} \quad \mathrm{~T}_{1} \mathrm{M}_{6}$

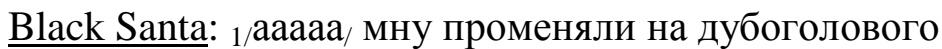
спиногрыза,!!!!!, /

$\mathrm{B}_{7} \mathrm{~T}_{1} \mathrm{M}_{7} \quad \underline{\text { Ksanka: }}$ 1аптичкО, а ну вернись в гнездо на льдину,!

, (9) 12 Джека Воробья все равно в птичий монастырь не возьмут/)))/,

$\mathrm{B}_{8} \quad \mathrm{~T}_{1} \mathrm{M}_{8} \quad \mathrm{R}_{\mathrm{T1M}} \quad \underline{\text { Azure: }}{ }_{1} \mathrm{~B}$ какой монастырь $?$ ! $_{2} \mathrm{~B}$ женский/?/ ${ }_{3}$ Не возьмут, адназначна,))/ 4Да и мы не отдадим,

$\mathrm{B}_{1} \quad \mathrm{~T}_{1} \mathrm{M}_{1} \quad$ Lelika: :shok: ${ }_{1}$ AgentMario ты $/$ позеленел/ ${ }^{219} / . . /$ ушел из нашей

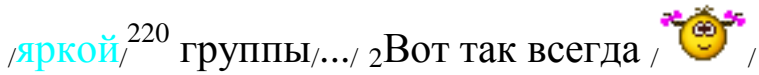

$\mathrm{B}_{2} \quad \mathrm{~T}_{1} \mathrm{M}_{2} \quad$ AgentMario: ${ }_{1}$ Я уже скучаю/!//../2 Как вы там/?/ 3 Никто не обижает $/ /)$ )

\footnotetext{
${ }^{218}$ Der Beitrag ist dunkelblau geschrieben.

${ }^{219}$ Die Wortform ist grün geschrieben.

${ }^{220}$ Die Wortform ist blau geschrieben.
} 
$\mathrm{B}_{3} \quad \mathrm{~T}_{1} \mathrm{M}_{3}$

$[\ldots]$

$\begin{array}{lll}\mathrm{B}_{4} & \mathrm{~T}_{1} \mathrm{M}_{4} & \mathrm{R}_{\mathrm{V}}\end{array}$

$\mathrm{B}_{1} \quad \mathrm{~T}_{1} \mathrm{M}_{1}$

$\mathrm{B}_{2}$

$\mathrm{T}_{1} \mathrm{M}_{2}$

$\mathrm{B}_{3} \quad \mathrm{~T}_{1} \mathrm{M}_{3}$

$\mathrm{B}_{4} \quad \mathrm{~T}_{1} \mathrm{M}_{4}$

$[\ldots]$

$\mathrm{B}_{1} \quad \mathrm{~T}_{1} \mathrm{M}_{1}$

$\mathrm{B}_{2} \quad \mathrm{~T}_{1} \mathrm{M}_{2}$

$\mathrm{B}_{3} \quad \mathrm{~T}_{1} \mathrm{M}_{3}$
Lelika: ${ }_{1}$ мы тоже скучаем с Kingoм , возвращайся/../ мы тебя ждем

Lelika: ${ }_{1}$ как это Шаг назад - расстрел!! ? Там же мы , твои родные голубые береты /

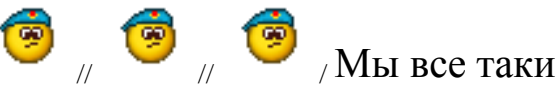
ждем твоего возвращения / ,

Lelika: ${ }_{1}$ bogda а ты что полинял /?/ 2или ушел с модераторов/?,

AgentMario: Цитата: Сообщение от Lelika: bogda a mbl что полинял ? или ушел с модераторов?

1А он был модером/?/), (O_о,

${ }_{2}$ Ему как предупреждение выписали, так в звании и понизили,

Lelika: 1за что предупреждение /?/ 23а флуд /?/

AgentMario: 1 1за оскобления.

Lelika: ${ }_{1}$ на форуме большинство мужчин, а вы в группе платья предлагаете

AgentMario: ${ }_{1} \mathrm{OH}$ думал, что это шотландский форум. ${ }_{2} \mathrm{Hy}$ ошибся парень чуток/.../

Lelika: ${ }_{1}$ нет уж Дорогие мои/!/ 2вступайте в группу, покупайте платье и за комп,$! ! ! /$ 
$\mathrm{B}_{4} \quad \mathrm{~T}_{1} \mathrm{M}_{4}$

$\mathrm{B}_{5} \quad \mathrm{~T}_{1} \mathrm{M}_{5}$

$\mathrm{B}_{6} \quad \mathrm{~T}_{1} \mathrm{M}_{6}$

$\mathrm{B}_{7} \quad \mathrm{~T}_{1} \mathrm{M}_{7}$

$\mathrm{B}_{1} \quad \mathrm{~T}_{1} \mathrm{M}_{1} \quad \mathrm{R}_{\mathrm{v}} \quad \underline{\text { Alex}}$ : 1/ЭЭЭЭЭЭЭЭ просто уржался/.../

$\mathrm{T}_{2} \mathrm{M}_{1} \quad \mathrm{R}_{\mathrm{v}} \quad{ }_{2}$ Бельченыш, а где продолжение истории по лошадку,????, 3Мне очень понравилось начало/)))/

$\mathrm{T}_{3} \mathrm{M}_{1}$ А я вот вчера был в караоке-баре с партнерами и один из моих недомерков решил исполнить Nikita Элтона Джона. ${ }_{5}$ Клип вы все помните, я надеюсь/... 6 С красивой девушкой в военной форме/... 7 И тут вдруг до меня медленно доходит, что ни фига !! в Песня-то изначально слэшная. Элтон ее писал, думал он о реальном парне, которого видел на границе. ${ }_{10} \mathrm{~A}$ потом уже песенку довели до ума и восприятия рядового меломана. 11Да так, что я, например, ни разу не усомнился даже, что Никита - девушка. ${ }_{12}$ Опять же, Бессон нимало способствовал.

${ }_{13}$ Смотрю я на своего голосистого приятеля и меня такой ржач разбирает. ${ }_{14}$ Еле высидел с каменной рожей, слушая как он спьяну распинается в любви к Никите/.../ 15У них там в Китае, кажется, до сих пор вышка за гомосячество/.../ 
(36)

$\mathrm{B}_{1} \quad \mathrm{~T}_{1} \mathrm{M}_{1}$

Mariann Mistik: 1 Девочки, вы такие умнички/!!!/ /

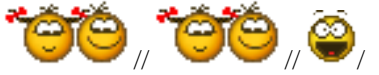

${ }_{2}$ Очень-очень-очень рада возвращению газеты/!!!/ з/ДАЕШЬ/ ППОРОК $/$ !!!!!!!!!!!!

$\mathrm{B}_{1} \quad \mathrm{~T}_{1} \mathrm{M}_{1} \quad \underline{\text { Echo: }}{ }_{1}$ Ну и чё, как всегда, ни одного шкафа, кроме „миняяяя//???!!!, , 光,

$\mathrm{B}_{1}$

Стхаткси: Black Santa пишет: Пошли в детский сад вместе

$\mathrm{T}_{1} \mathrm{M}_{1}$

1/НИ/, ЗА, ЧТО//!!!!/ 2Ни за какие коврижки мира/!/ зЯ лучше музыкалку переживу еще раз, но в этот кошмар не вернусь/!,

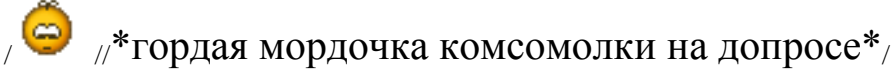

$\mathrm{T}_{2} \mathrm{M}_{1} \quad{ }_{4}$ Товарищи $!$, 5 Северус Тоббиасович был замечен с женщинами !!

http://www.youtube.com/watch?v=8...;feature=related

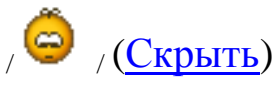

${ }_{6} \mathrm{O}$, Мерлин/!/ 7 Разве танго с Рикманом - это так много/?/ ${ }_{8} / \mathrm{HE} /$ всемирного господства прошу, Господи. 9 Вещей элементарных/.../ 
$\mathrm{B}_{1} \mathrm{~T}_{1} \mathrm{M}_{1} \quad \underline{\text { mironich: }}{ }_{1}$ Увидел комент в блоге у вазонеза я плакал/...../

$[\ldots]$

$\mathrm{B}_{2}$

crow_by: Цитата: Сообщение от mironich

Увидел комент в блоге у вазонеза я плакал.....

$\mathrm{T}_{1} \mathrm{M}_{2}$

(40)

B $\mathrm{T}_{1} \mathrm{M}_{1} \quad \underline{\text { Klubnayk: }}{ }_{1}$ Ой кстати мая ася 20318004/))/

$[\ldots]$

$\mathrm{B}_{2}$

DragOn: ЦЦитата: Ой кстати мая ася 20318004))

$\mathrm{T}_{1} \mathrm{M}_{2}$

1У кого там аськи не было,?//)/

$\mathrm{B}_{3} \quad \mathrm{~T}_{1} \mathrm{M}_{3}$

Klubnayk: ${ }_{1}$ Не было а теперь есть/))/ 2 У меня,!/

$\mathrm{B}_{4} \quad \mathrm{~T}_{1} \mathrm{M}_{4}$

AgentMario: Цитата: Сообщение от Klubnayk Не было а теперь есть)) У меня!

${ }_{1}$ Расскажи сколько ты её настраивал/.../

$\mathrm{B}_{5} \quad \mathrm{~T}_{1} \mathrm{M}_{5} \quad \mathrm{R}_{\text {T1м3 }}$ ? $\quad$ zwerweb:

Добавлено через 36 секунд

Цитата: Сообщение от AgentMario

Расскажи сколько ты её настраивал...

$\mathrm{T}_{1} \mathrm{M}_{6} 3$ дня и 3 ночи

(41)

$\mathrm{B}_{1} \quad \mathrm{~T}_{1} \mathrm{M}_{1} \quad$ Lelika: ${ }_{1} \mathrm{~B}$ нашем полку флудеров /ПОПОЛНЕНИЕ//)))/

$\mathrm{B}_{2} \quad \mathrm{~T}_{1} \mathrm{M}_{2} \quad \underline{\text { Day }}$ (Gast):, 
$\mathrm{B}_{1} \quad \mathrm{~T}_{1} \mathrm{M}_{1}$

Black Santa: ${ }_{1}$ Небольшая подборка личных смайлов для игроков и персов:

Темперанс

Tempest

Nikitina

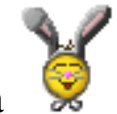

Профессор Биннс

Профессор Синистра ๑1

Профессор Спраут 요룰

Профессор МакГонагалл

Профессор Снейп

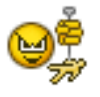

Снова Снейп

Профессор Трелони

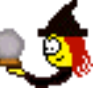

39

Гриффиндорское трио

(9)

Голдштейн и Эва

Лаванда и Гермиона

Луна

Парвати

Будущее Лунко и Блейза

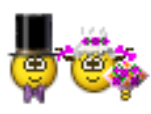

Рон и Лаванда 
Мэнди $\square$

Драко

Теодор

Ирма Пинс

Миссис Норрис

Дамблдор

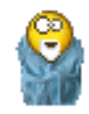

Мариса

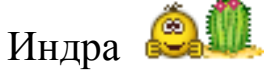

Мариса и Марсель

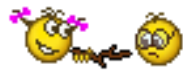

Ючи

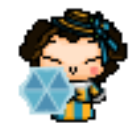

Байрас

(ca)

Хавьер ras

Аснейт

Марсель

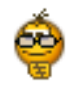

HILFE

Кристиан

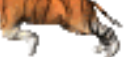

Гургени

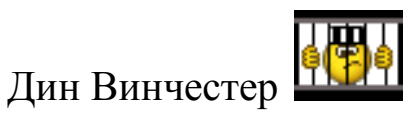

Эш

Сэм и Дин 野

Наши администраторы

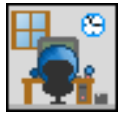


Марселю, Роберте, Айзеку, Пейдж и мадам Помфри

посвящается

\section{$[\ldots]$}

$\mathrm{B}_{2} \quad \mathrm{~T}_{1} \mathrm{M}_{2}$

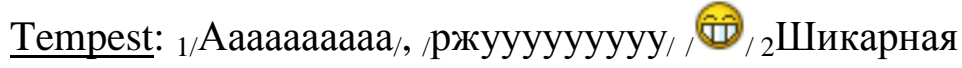
подборка /))), $@$,/ ,

зТолько, Сантик, вон чего тут выходит /

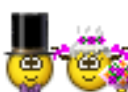

буит, только если этот тип мою Лунку

украдет , но тада, разгневанный муж

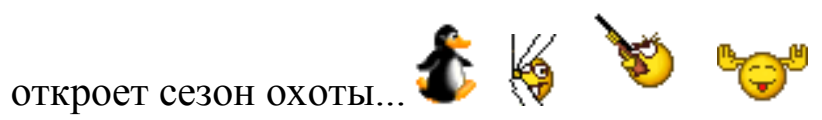

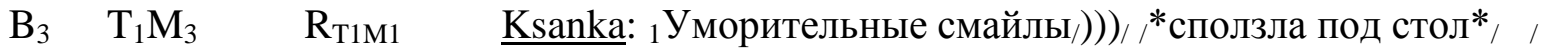
Q)

$\mathrm{T}_{2} \mathrm{M}_{1}$

23.Ы. вот как выглядит начало дня у мну

$(43)^{221}$

$\mathrm{B}_{1} \mathrm{~T}_{1} \mathrm{M}_{1} \quad \mathrm{R}_{\mathrm{v}} \quad$ Темперанс: ${ }_{1}$ Tempest Спасибо. ${ }_{2}$ Смеялась долго и громко, коллеги косятся, но в целях самосохранения молчат, дабы фыркая и хихикая я не начала им все зачитывать.

\footnotetext{
${ }^{221}$ Das Originalbeispiel ist (XX) und das manipulierte (XX)'- (XX)',"’.
} 
$\mathrm{T}_{2} \mathrm{M}_{1}$

3Постараюсь на один день стать толковым Сусаниным и провести всех нужным маршрутом. ${ }_{4}$ Ваша поддержка мне очень нужна, так что скрестите пальчики, сожмите кулачки и ругайте меня. 5(для этого могу не написать во время пост и все из-за меня (нужное вставить) застрянут на платформе)

$\mathrm{B}_{2} \quad \mathrm{~T}_{2} \mathrm{M}_{2}$ Black Santa: ${ }_{1}$ Темперанс, ты знаешь, я всегда найду за что поругать, даже если ты напишешь все посты вовремя. ${ }_{2}$ И вообще, ты же целый день не будешь из-за отъезда модерировать/!/ $У$ тебя будет простаивать работа/! 4Ах ты негодяйка,!!!, , „(

$(43)^{, 222}$

$[\ldots]^{223}$

$\mathrm{B}_{2} \quad \mathrm{~T}_{2} \mathrm{M}_{2}$

Black Santa: ${ }_{1}$ Темперанс, ты знаешь, я всегда найду за что поругать, даже если ты напишешь все посты вовремя. ${ }_{2}$ И вообще, ты же целый день не будешь из-за отъезда модерировать! ${ }_{3} У$ тебя будет простаивать работа! 4 Ах ты негодяйка!!!

$(43)^{\prime}$,

$[\ldots]$

$\mathrm{B}_{2} \quad \mathrm{~T}_{2} \mathrm{M}_{2}$

Black Santa: ${ }_{1}$ Темперанс, ты знаешь, я всегда найду за что поругать, даже если ты напишешь все посты вовремя.

${ }_{2}$ И вообще, ты же целый день не будешь из-за отъезда

\footnotetext{
${ }^{222}$ Da die (XX)'- (XX)"','-Beispiele denjenigen entsprechen, die die Probanden beim Experiment erhalten haben, werden keine Kontextualisierungshinweise durch Schrägstriche markiert.

${ }^{223}$ Aus Platzgründen werden hier und in folgenden Beispielen Beiträge ausgelassen, die Probanden haben das jeweilige Beispiel jedoch vollständig erhalten.
} 
модерировать. ${ }_{3}$ У тебя будет простаивать работа. $4 \mathrm{Ax}$

ты негодяйка.

(44)

$\mathrm{B}_{1} \quad \mathrm{~T}_{1} \mathrm{M}_{1} \quad \underline{\text { Tempest: }}$
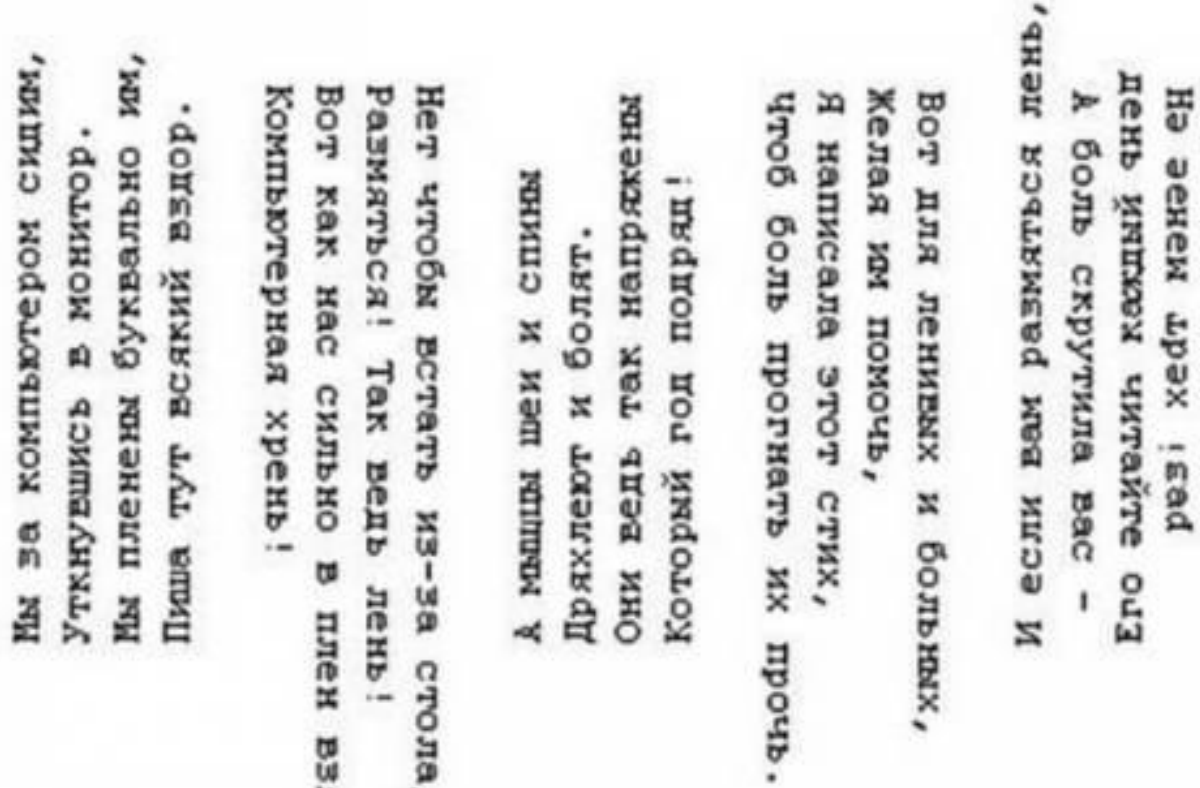

Import

$\mathrm{B}_{2} \quad \mathrm{~T}_{1} \mathrm{M}_{2}$

Echo: ${ }_{1}$ Tempest, браво, я се чуть шею не свернула, истерично посмеиваясь,,

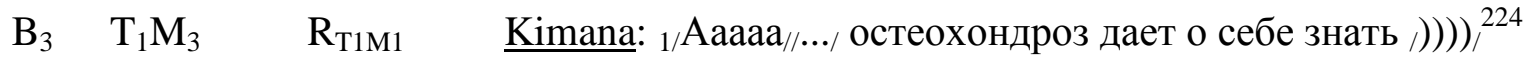

${ }_{2}$ Темпик, это что-то /))))/

$\mathrm{B}_{4} \quad \mathrm{~T}_{1} \mathrm{M}_{4} \quad \mathrm{R}_{\mathrm{T} 1 \mathrm{M} 1} \quad \underline{\text { So-Le: }}{ }_{1}$ Tempest, , $($ / домашние искренне

порадовались, наблюдая, „КАК, я это читаю ,

\footnotetext{
${ }^{224}$ Um sicherzustellen, dass die Leser die Emoticons als solche erkennen, wurden in allen für die Experimente verwendeten Beispielen die jeweiligen einfachen Emoticons durch komplexe ersetzt. Auf diese Weise sind die Emoticons auch für Leser erkenntlich, die die Konventionen in russischen Foren nicht kennen, weil sie nur selten im Runet, wie das russischsprachige Internet genannt wird, unterwegs sind.
} 
$\mathrm{B}_{5} \quad \mathrm{~T}_{1} \mathrm{M}_{5} \quad \mathrm{R}_{\mathrm{T} 1 \mathrm{M} 1} \quad$ Heiling: ${ }_{1}$ Tempest, я повеселился , $($ // ${ }_{2}$ И отправил картинку всем друзьям, чтобы поразмялись ,

$\mathrm{B}_{6} \quad \mathrm{~T}_{1} \mathrm{M}_{6} \quad \mathrm{R}_{\mathrm{T} 1 \mathrm{M} 1} \quad \underline{\text { June: }} 1$ УТя, спасибо за стишок, 2 , 2 От моей шеи тебе тоже привет , ,

(44)

$[\ldots]$

$\mathrm{B}_{4} \quad \mathrm{~T}_{1} \mathrm{M}_{4} \quad \mathrm{R}_{\mathrm{T} 1 \mathrm{M} 1} \quad \underline{\text { So-Le: }}{ }_{1}$ Tempest, $\odot$ домашние искренне порадовались, наблюдая, как я это читаю

$[\ldots]$

(44)"

$[\ldots]$

$\mathrm{B}_{2} \quad \mathrm{~T}_{1} \mathrm{M}_{2}$

Echo: $12{ }_{1}$ Tempest, браво, я се чуть шею не свернула, истерично посмеиваясь

$\mathrm{B}_{3} \quad \mathrm{~T}_{1} \mathrm{M}_{3} \quad \mathrm{R}_{\mathrm{T} 1 \mathrm{M} 1} \quad \underline{\text { Kimana: }}$ (А) ${ }_{1}$ Аааа... остеохондроз дает о себе знать ${ }_{2}$ Темпик, это что-то

$\mathrm{B}_{4} \quad \mathrm{~T}_{1} \mathrm{M}_{4} \quad \mathrm{R}_{\mathrm{T} 1 \mathrm{M} 1} \quad$ So-Le: $\odot$ (2) порадовались, наблюдая, КАК я это читаю

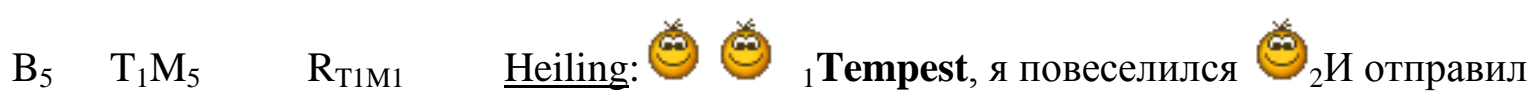
картинку всем друзьям, чтобы поразмялись 
$\mathrm{B}_{6} \mathrm{~T}_{1} \mathrm{M}_{6} \quad \mathrm{R}_{\mathrm{T} 1 \mathrm{M} 1} \quad \underline{\text { June: }} \bigodot_{1} \mathrm{У}_{\mathrm{T}}$, спасибо за стишок ${ }_{2}$ От моей шеи тебе тоже привет

$(44),$,

$\mathrm{B}_{3} \mathrm{~T}_{1} \mathrm{M}_{3} \quad \mathrm{R}_{\mathrm{T} 1 \mathrm{M} 1} \quad \underline{\text { Kimana: }}{ }_{1}$ Ааaаa... остеохондроз дает о себе знать

$[\ldots]$

$\mathrm{B}_{6} \mathrm{~T}_{1} \mathrm{M}_{6} \quad \mathrm{R}_{\mathrm{T} 1 \mathrm{M} 1} \quad \underline{\text { June: }}{ }_{1} \mathrm{У}_{\mathrm{T}}$, спасибо за стишок ${ }_{2}$ От моей шеи тебе тоже привет

$(44),,$,

$[\ldots]$

В $\mathrm{T}_{1} \mathrm{M}_{3} \quad \mathrm{R}_{\mathrm{T} 1 \mathrm{M} 1} \quad \underline{\text { Kimana: }}{ }_{1}$ Ааaаa... остеохондроз дает о себе знать

[...]

$\mathrm{B}_{6} \mathrm{~T}_{1} \mathrm{M}_{6} \quad \mathrm{R}_{\mathrm{T} 1 \mathrm{M} 1} \quad \underline{\text { June: }}{ }_{1} \mathrm{У}_{\mathrm{T}}$, спасибо за стишок. 2 От моей шеи тебе тоже привет

$(44),,,$,

$[\ldots]$

$\mathrm{B}_{3} \mathrm{~T}_{1} \mathrm{M}_{3} \quad \mathrm{R}_{\mathrm{T} 1 \mathrm{M} 1} \quad \underline{\text { Kimana: }}{ }_{1}$ Ааaаa... остеохондроз дает о себе знать

$[\ldots]$

$\mathrm{B}_{1}$

Tempest: 1 scary boom, Nikitina, добро пожаловать/!, / , , 

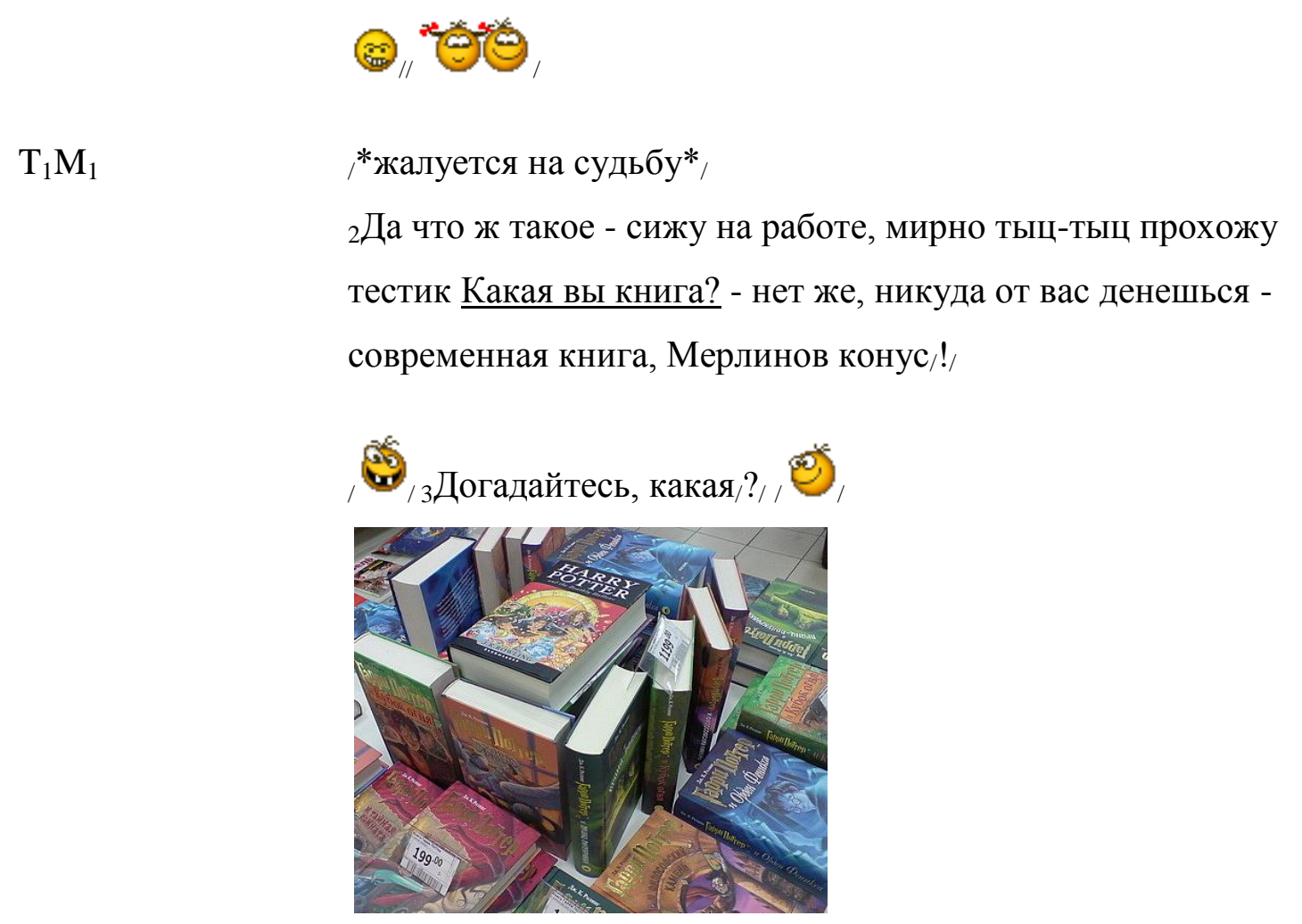

$[\ldots]$

$\mathrm{B}_{2} \quad \mathrm{~T}_{1} \mathrm{M}_{2}$

Nikitina: ${ }_{1}$ Мэндик ${ }^{225}$, мну прошел тест, и я точно такая же современная книжка)) $/ /$ Гы/ /

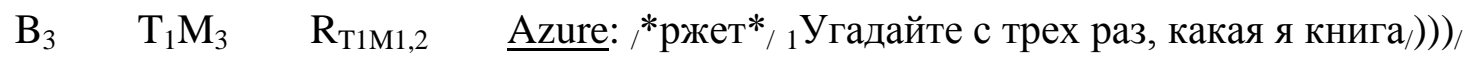
${ }_{2}$ Мэндик, солнце, ты знала, да/?/ 3 Когда подсовывала этот тест/)))/

(45)'

$\mathrm{B}_{1} \quad \mathrm{~T}_{1} \mathrm{M}_{1}$

Tempest: /*алуюсь на судьбу*,

1Да что ж такое - сижу на работе, мирно тыц-тыц прохожу тестик Какая вы книга? - нет же, никуда от вас денешься современная книга, Мерлинов конус!

2Догадайтесь,какая

\footnotetext{
${ }^{225}$ Bei Mèndik handelt es sich um einen anderen Usernamen von Tempest. Möglicherweise stammt dieser Name aus einem anderen Thread des Forums.
} 
$\begin{array}{ll}\mathrm{B}_{2} & \mathrm{~T}_{1} \mathrm{M}_{2}\end{array}$

$\mathrm{B}_{3} \quad \mathrm{~T}_{1} \mathrm{M}_{3} \quad \mathrm{R}_{\mathrm{T} 1 \mathrm{M} 1,2}$

$(45)^{\prime}$,

$\mathrm{B}_{1} \quad \mathrm{~T}_{1} \mathrm{M}_{1}$

$\mathrm{B}_{2} \quad \mathrm{~T}_{1} \mathrm{M}_{2}$

$\begin{array}{lll}\mathrm{B}_{3} & \mathrm{~T}_{1} \mathrm{M}_{3} & \mathrm{R}_{\mathrm{T} 1 \mathrm{M} 1,2}\end{array}$ (46)
Nikitina: ${ }_{1}$ Tempest $^{226}$, я $^{227}$ прошел тест, и я точно такая же современная книжка

Azure: /*ржу* / 1 Угадайте с трех раз, какая я книга ${ }_{2}$ Tempest $^{228}$, солнце, ты знала, да. ${ }_{3}$ Когда подсовывала этот тест

Tempest: 1 Да что ж такое - сижу на работе, мирно тыц-тыц прохожу тестик Какая вы книга? - нет же, никуда от вас денешься - современная книга, Мерлинов конус!

2Догадайтесь,какая?

$[\ldots]$

Nikitina: ${ }_{1}$ Tempest, я прошел тест, и я точно такая же современная книжка

Azure: ${ }_{1} У$ гадайте с трех раз, какая я книга 2 Tempest, солнце, ты знала, да? ${ }_{3}$ Когда подсовывала этот тест

Black Santa: ${ }_{1}$ У нас вчера начались крещенские морозы и сегодня продлились. 2 От -5 в воскресенье до -20 сегодня. 3 Я отморозила все что можно, /4 Удвольствие неописуемое

\footnotetext{
${ }^{226}$ Um die Probanden nicht zu verwirren, wurde in den Experimenten der Name Mèndik von vornherein zu Tempest geändert.

${ }^{227}$ Die Wf mnu wurde in (45)' und (45)', von der Vf. geändert, da es sich dabei um eine Form handelt, die im russischen Internet zwar häufig Gebrauch findet, aber nicht-internetaffinen Probanden fremd ist.

${ }^{228}$ Der Name wurde in (45)' und (45)'" von der Vf. geändert, damit die Probandinnen nicht nachfragen mussten, welcher User Mèndik ist.
} 
$\mathrm{B}_{2} \quad \mathrm{~T}_{1} \mathrm{M}_{2}$

$(46)^{\prime}$

$\mathrm{B}_{1} \quad \mathrm{~T}_{1} \mathrm{M}_{1}$

$\mathrm{B}_{2} \quad \mathrm{~T}_{1} \mathrm{M}_{2}$

$(46)^{\prime \prime}$

$\mathrm{B}_{1} \quad \mathrm{~T}_{1} \mathrm{M}_{1}$

$\mathrm{B}_{2} \quad \mathrm{~T}_{1} \mathrm{M}_{2}$

Горбатый: ${ }_{1}$ В условиях того, что я официально подмосквич, живущий в частном доме с отдельным отоплением, с ужасом взираю на столбик термометра, ибо понижение температуры чревато увеличением расхода газа и моих денег на него. 2 Это во-первых. ${ }_{3}$ А во-вторых, я не успела среагировать на понижение температуры своевременно, в результате чего дом успел промерзнуть ине хочет прогреваться толком уже второй день. / , ${ }_{4} \mathrm{~B}$ электричках холодно, по пробкам долго, от станции пешком по сугробам и гололеду - двадцать минут. 5 Мерзну. 11 ${ }_{6}$ Как человек, ненавидящий зиму в принципе, сейчас мечтаю о весне с удвоенной силой.,

Black Santa: 1 У нас вчера начались крещенские морозы и сегодня продлились. 2 От -5 в воскресенье до -20 сегодня. ${ }_{3}$ Я отморозила все что можно. ${ }_{4}$ Удовольствие неописуемое Горбатый: [...] ${ }_{6}$ Как человек, ненавидящий зиму в принципе, сейчас мечтаю о весне с удвоенной силой.

Black Santa: *расстроено* ${ }_{1} У$ нас вчера начались крещенские морозы и сегодня продлились. 2 От -5 в воскресенье до -20 сегодня. зЯ отморозила все что можно. *саркастично* ${ }_{4}$ Удовольствие неописуемое Горбатый: *недовольно ворчу* ${ }_{1}$ В условиях того, что я официально подмосквич, живущий в частном доме с 
отдельным отоплением, с ужасом взираю на столбик термометра, ибо понижение температуры чревато увеличением расхода газа и моих денег на него. 2 Это вопервых. зА во-вторых, я не успела среагировать на понижение температуры своевременно, в результате чего дом успел промерзнуть и не хочет прогреваться толком уже второй день.

*хмурюсь* ${ }_{4} \mathrm{~B}$ электричках холодно, по пробкам долго, от станции пешком по сугробам и гололеду - двадцать минут. ${ }_{5}$ Мерзну. ${ }_{6}$ Как человек, ненавидящий зиму в принципе, сейчас мечтаю о весне с удвоенной силой?

$(46)$,

$\mathrm{B}_{1} \mathrm{~T}_{1} \mathrm{M}_{1} \quad \underline{\text { Black Santa: }}{ }_{1} \mathrm{~V}$ нас вчера начались крещенские морозы и сегодня продлились. 2 От -5 в воскресенье до -20 сегодня. ${ }_{3}$ Я отморозила все что можно ${ }_{4}$ Удовольствие неописуемое Vesnushka: ${ }_{1} \mathrm{Ox}$, хляньте, родители издеваются над дочкой / Q 2Доча попросила папу сделать ей костюм конарейки: «25-го утром, примерив папин наряд у зеркала, я поняла, что, во-первых, орнитолог из родителя так себе, а во вторых, жизнь закончена.

В школу мы ехали молча, и возвращались тоже в тишине.» 
Import Птичко конарейко ,

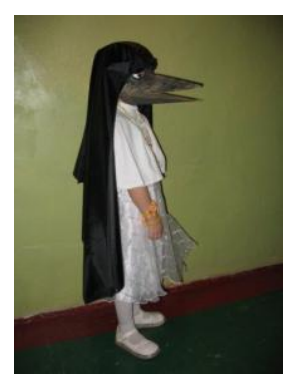

(47)'

$\mathrm{B}_{1} \quad \mathrm{~T}_{1} \mathrm{M}_{1} \quad$ Vesnushka: ${ }_{1} \mathrm{Ox}$, хляньте, родители издеваются над дочкой $[\ldots]$

(47)'

$\mathrm{B}_{1} \mathrm{~T}_{1} \mathrm{M}_{1} \quad \underline{\text { Vesnushka: }}$ * рыдаю от смеха* ${ }_{1} \mathrm{Ox}$, хляньте, родители издеваются над дочкой $[\ldots]$

(48)

$\alpha$

$\varnothing$

$\mathrm{B}_{1} \mathrm{~T}_{1} \mathrm{M}_{1} \quad \underline{\text { Lelika: }}{ }_{1}$ bogda a ты что полинял /?, 2 или ушел с модераторов/?/

$\mathrm{B}_{2}$

AgentMario: Цитата: Сообщение от Lelika: bogda a mbl что полинял? или ушел с модераторов?

$\mathrm{T}_{1} \mathrm{M}_{2}$
${ }_{1} \mathrm{~A}$
$\mathrm{OH}$
был
модером/?/)/
/O_o/
${ }_{2}$ Ему как предупреждение выписали, так в звании и понизили , 


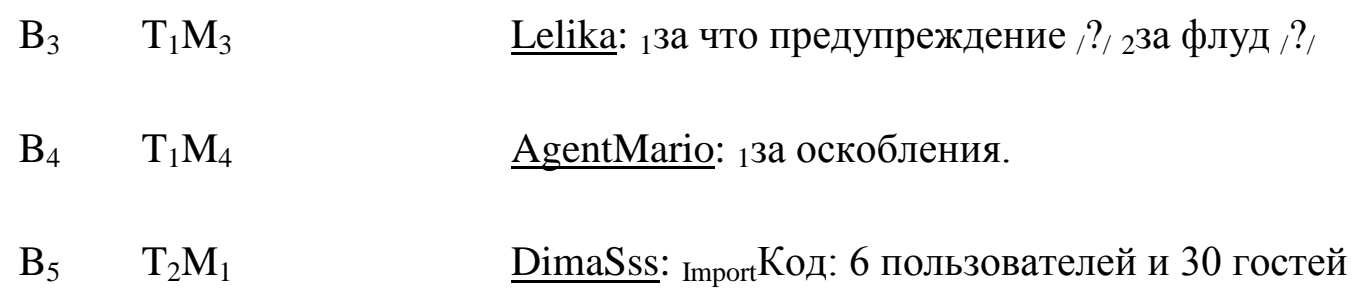

AgentMario, Android, DimaSss, djoni84, DrShaman, Lelika

/O_o/ 1а проект активно развивается/!/

B $\mathrm{T}_{2} \mathrm{M}_{2} \quad \underline{\text { Lelika: }}{ }_{1}$ бывало и больше/../

$\mathrm{B}_{7}$

$\mathrm{T}_{2} \mathrm{M}_{3}$

$\mathrm{B}_{8} \quad \mathrm{~T}_{2} \mathrm{M}_{4}$

$\mathrm{B}_{9}$

$\mathrm{T}_{2} \mathrm{M}_{5}$

$\mathrm{B}_{10}$

$\mathrm{T}_{1} \mathrm{M}_{5}$

$\mathrm{T}_{1} \mathrm{M}_{6}$

AgentMario: Цитата: Сообщение от DimaSss: проект активно развивается!

${ }_{1} \mathrm{~A} \mathrm{DDoS}$ ему активно помогает, 田,

Цитата: Рекорд одновременного пребывания 970, это было 11.09.2009 в 17:53.

DimaSss: 1 Во блин,а я думал почему сайт тупил //

Lelika: Цитата: Сообщение от AgentMario: $A$ DDoS eмy активно помогает

1Это же давно было/../ 2Хорошо что сейчас нету/../

bogda: Цитата: Сообщение от Lelika: bogda a mbl что полинял? или ушел с модераторов?

1Помощник я оказался никудышный, решил не быть обузой и сам ушел в отставку/.../

Цитата: Сообщение от AgentMario: А он был модером?)O_o Ему как предупреждение выписали, так в звании и понизили $\theta$

${ }_{2}$ Не делай выводов основываясь на своих убеждениях, тот парень первый начал обзываться /...//=(/ 
$\mathrm{B}_{11}$

$\mathrm{T}_{1} \mathrm{M}_{7}$

$\mathrm{B}_{12} \quad \mathrm{~T}_{1} \mathrm{M}_{8}$

$\mathrm{B}_{13}$

$\mathrm{T}_{1} \mathrm{M}_{9}$

$\mathrm{B}_{14}$

$\mathrm{T}_{1} \mathrm{M}_{10}$

$\mathrm{B}_{15} \quad \mathrm{~T}_{1} \mathrm{M}_{11} \quad \mathrm{R}_{\mathrm{T} 1 \mathrm{M} 9}$

$\mathrm{B}_{16} \quad \mathrm{~T}_{1} \mathrm{M}_{12}$
AgentMario: Цитата: Сообщение от bogda: Не делай выводов основываясь на своих убеждениях, тот парень первый начал обзываться... $=($

${ }_{1}$ Я не говорил, что ты первый начал. 2 Он вообще бан получил. ${ }_{3}$ Но ведь ты ушёл с модераторов и "полинял" сразу же после предупреждения. 4Как же тут не делать выводы/?/ (9)

Lelika: 1 а где я то была /?/ 2каждый день захожу , а об этом даже не знаю

silver: Цитата: Сообщение от bogda: тот парень первый начал обзываться... $=($

1Мог бы написать администрации, и ты бы не слетел, и того бы забанили/.../ 2 Но на тупость отвечать тупостью/...это глупо.

bogda: Цитата: Сообщение от silver: Мог бы написать администрации, и ты бы не слетел, и того бы забанили..

${ }_{1}$ В смысле бы не слетел/?!/ 2я же написал, что сам ушел в отставку емае,$!$

Цитата: Сообщение от silver: Но на mупость отвечать тупостью...это глупо.

${ }_{3}$ Если честно уже привык на понты еще большими понтами отвечать $/=)$,

DragOn: ${ }_{1}$ он сам ушел,я смотрю не на кол-во жалоб,а умение общатся с людьми на форуме,и нормально без матов вырожать благодарность а не "ух бля заябись пиздатенько так выложили,благодарю нах".2Так что на него не наезжать

DimaSss: ${ }_{1}$ Гостей надо не пугать (оскорблять) надо быть 
умней

2Тогда гость позовет друга и т.д и форум будет развиваться. ${ }^{229}$

$\mathrm{B}_{17} \quad \mathrm{~T}_{1} \mathrm{M}_{13}$

$\mathrm{B}_{18}$

$\mathrm{T}_{1} \mathrm{M}_{14}$

$\mathrm{T}_{1} \mathrm{M}_{15}$

$\mathrm{B}_{19}$

$\mathrm{T}_{1} \mathrm{M}_{16}$

$\mathrm{B}_{20} \quad \mathrm{~T}_{1} \mathrm{M}_{17}$
DragOn: 1 а я что сейчас сказал/?/)/

Lelika: Цитата: Сообщение от DimaSss: Гостей надо не

пугать (оскорблять) надо быть умней и постараться помочь

Тогда гость позовет друга и т.д и форум будет развиваться.

13олотые слова DimaSssik , (9),

Цитата: Сообщение от DragOn:"ух бля заябись пиздатенько так выложили,благодарю нах".Так что на него не наезжать

${ }_{2}$ Хочешь узнать человека дай ему власть, и он покажет себя/../

DragOn: Цитата: Хочешь узнать человека дай ему власть , и он покажет себя..

1я такой плохой/?,

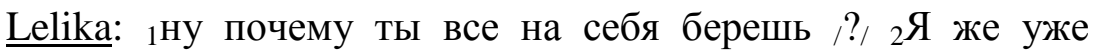
говорила, что ты хорошенький умничка, мой любимый админ хакерка/../ 3 Сейчас здесь обсуждается bogda, что он с матом отвечал пользователям форума, вот я и написала выше :

Цитата: Хочешь узнать человека дай ему власть, и он покажет себя..

${ }_{4} \mathrm{~A}$ это все к тому ,что до модераторства он был хорошим

\footnotetext{
${ }^{229}$ Der Beitrag ist orange geschrieben
} 
форумчанином , а когда повысился в звании почувствовал власть, вот и распушился/../

5bogda, все что ты здесь читаешь, это все не в обиду тебе , а урок, чтобы понял свои ошибки и в следующий раз не загонялся

$\mathrm{B}_{21} \quad \mathrm{~T}_{1} \mathrm{M}_{18}$

silver: ${ }_{1}$ Хм/...на месте богды я скорее всего поступил так же, хоть и выдал сам ему предупреждение. ${ }_{2}$ Не люблю когда стараются унизить/../

$\mathrm{B}_{22} \quad \mathrm{~T}_{1} \mathrm{M}_{19}$

Lelika: ${ }_{1}$ если модератор будет отвечать матом , тогда весь форум будет " Мат на мате"

${ }_{2}$ Иногда нужно быть выше/../ Хотя не всегда это получается $\mathrm{B}_{23}$ bogda: Цитата: Сообщение от Lelika: Хочешь узнать человека дай ему власть , и он покажет себя..

$\mathrm{T}_{1} \mathrm{M}_{20}$ 1Могу поклясться чем угодно, не будь я модератором я бы поступил в точности так же/... ${ }_{2}$ И матом я его не крыл, а сказал я ему сначала что то вроде:"девочка моя, рот офни", получив в ответ наглючий пост, ответил ему/.../ зпросто постоял за себя, что в етом такого/?! 4да ну давайте тогда чмырить модеров а они пускай в тряпочку молчат, я ето так понял/?/ 5вед юзер может друзей позвать/./

Цитата: "yx бля заябись пиздатенько так выложили,благодарю нах"

${ }_{6}$ и кста, Драгон привел в пример то не меня, это не мои слова, ну ето для разочаровавшейся во мне Лелике/.../

$\mathrm{B}_{24} \quad \mathrm{~T}_{1} \mathrm{M}_{21} \quad$ Lelika: ${ }_{1}$ bogda не разочаровалась я в тебе 
$\mathrm{B}_{25}$

$\mathrm{T}_{1} \mathrm{M}_{22}$

$\mathrm{B}_{26} \quad \mathrm{~T}_{1} \mathrm{M}_{23}$

$\mathrm{B}_{27} \quad \mathrm{~T}_{1} \mathrm{M}_{24}$

$\omega$ bogda: Цитата: Сообщение от Lelika: $A$ это все к тому, что до модераторства он был хорошим форумчанином , а когда повысился в звании почувствовал власть, вот $u$ распушился.

${ }_{1}$ Ну я так и понял, что совсем не разочаровалась/../

Lelika: ${ }_{1}$ Винни Пух это было высказывание о твоем модераторстве, а не о разочаровании в тебе, если бы разочаровалась я бы в открытую прямо об этом написала

bogda: ${ }_{1}$ Ну окэй

$\varnothing$ 


\section{Versicherung}

Hiermit versichere ich an Eides statt, dass ich die eingereichte Dissertation („Interaktion und Interpretation in russischsprachigen Threads") selbständig und ohne unerlaubte Hilfe verfasst habe. Anderer als der von mir angegebenen Hilfsmittel und Schriften habe ich mich nicht bedient. Alle wörtlich oder sinngemäß den Schriften anderer Autorinnen oder Autoren entnommenen Stellen habe ich kenntlich gemacht. Die Abhandlung ist noch nicht veröffentlicht worden und noch nicht Gegenstand eines Promotionsverfahrens gewesen.

Genia Böhnisch, M.A. 